\title{
Coordinated Detection of Micro- and Nanoparticles using Tuneable Resistive Pulse Sensing and Optical Spectroscopy
}

\author{
by \\ Peter Hauer

\begin{abstract}
A thesis
submitted to the Victoria University of Wellington in fulfilment of the requirements for the degree of

Doctor of Philosophy in Physics.
\end{abstract}

Victoria University of Wellington 2016 



\section{Abstract}

The detection and characterisation of micro- and nanoscale particles has become increasingly important in many scientific fields, spanning from colloidal science to biomedical applications. Resistive Pulse Sensing (RPS) and its derivative Tuneable Resistive Pulse Sensing (TRPS), which both use the Coulter principle, have proven to be useful tools to detect and analyse particles in solution over a wide range of sizes. While RPS uses a fixed size pore, TRPS uses a dynamically stretchable pore in a polyurethane membrane, which has the advantages that the pore geometry can be tuned to increase the device's sensitivity and range of detection. The technique has been used to accurately determine the size, concentration and charge of many different analytes.

However, the information obtained using TRPS does not give any insight into the particle's composition. In an attempt to overcome this, an experimental technique was developed in order to obtain simultaneous, time-resolved, high-resolution optical spectra of particles passing through the pore. Due to the ordered and controllable fashion in which the particles are guided through the sensing region, this approach has an advantage over diffusion based optical techniques. The experimental setup for the coordinated electrical and optical measurements involves many underlying physical phenomena, e.g. microfluidics, electrokinetic effects, and Gaussian beam optics. A significant proportion of this work was therefore devoted to the development and the optimisation of the experimental setup by adapting a commercial TRPS device and a spectrometer with an attached microscope. Methods to engineer the spot size of a Gaussian beam to account for the different pore diameters, and the development of algorithms to filter, analyse and coordinate the recorded data are essential to the technique.

The results using fluorescently labelled polystyrene particle sets with diameters from $190 \mathrm{~nm}$ to $2 \mu \mathrm{m}$ show that matching rates between the electrical and optical measurements of over $90 \%$ can repeatedly be achieved. Mixtures of particle species with similar diameters but with different fluorescent labels were used to demonstrate the technique's capability to characterise the analyte on a particle-by-particle basis and extend the information that can be obtained by TRPS alone. It was also shown that the data acquired with the electrical and optical measurements comple- 
ment each other and can be used to better understand the TRPS technique itself. The influence of experimental parameters, such as the particle velocity, the beam size and the optical detection volume, on the intensity of the optical signals and the matching rates was studied intensively. These studies showed that the technique requires a careful experimental design to achieve the best results. Overall, the developed technique enhances the particle-by-particle specificity of conventional RPS measurements, and could be useful for a range of particle characterization and bio-analysis applications.

Alongside the experiments, semi-analytic modelling and simulations using the Finite Element Method (FEM) were used to understand the particle motion through the pores, to interpret the experimental data, and predict the optical signals. The models were also used to assist the design and the optimization of the experiments. The FEM models were implemented with increasing physical detail and show superior understanding of the TRPS signals compared to the semi-analytic model, which is conventionally used in the TRPS field. The physical phenomena considered included off-axis trajectories, particle-field interactions for both fluid and electric fields, and the non-homogeneous distribution of ions close to the charged membrane and particle interfaces. Several effects which have been observed experimentally could be explained, including the intrinsic pulse height distribution, the current rectification, and the occurrence of bi-phasic pulses, demonstrating the benefits of FEM methods for RPS. 
La lutte elle-mâme vers les sommets suffit à remplir un cœur d'homme; il faut imaginer Sisyphe heureux.

The struggle itself toward the heights is enough to fill a man's heart. One must imagine Sisyphus happy.

Albert Camus, The Myths of Sisyphus 



\section{Acknowledgements}

The last years here in Wellington, New Zealand, were a very enjoyable time. Apart from working on my $\mathrm{PhD}$, I got to enjoy this beautiful country together with many great people from all around the world. My thanks goes to all of them.

I was lucky to have Geoff, Pablo and Eric, as supervisors. They were always very helpful when I had a question or problem and interested to discuss my research at any time. I want to thank them for that. Thanks to Geoff this script in now in a readable form. Unfortunately, Pablo passed away, which was one of the saddest moments in the last years. I am very grateful to have met such an inspirational man, may he rest in peace.

I also want to thank all the members of the Raman group at Victoria University, who contributed to this thesis in one way or another, and also made the work pleasurable and fun. Thank you Brendan, Matthias, Baptiste, Chris, Stefan, Camille, Walter, Johan and all the others who stayed here for a visit! Special thanks to Alan, Nick and Manu from the workshop.

As there is also a life apart from aligning lasers and fighting air bubbles in nanopores, I enjoyed many good moments with friends, who I want to thank for their company. Thanks to Hani, James, Eva, Harry, Stefanie, Felicia, Christin, Lina, Jonnel, Hayden, Balam and the many more who have come and gone again over the last years. Thanks also go to Sarah, Dorothee and Carys who each accompanied me for a part of the $\mathrm{PhD}$ and had to put up with me spending long nights in the lab.

And last but not least I want to thank my family, my sister Heidi and mother Ulrike, and friends, Lukas, Georg, Denis, Christoph and Paula, from back home in Austria. The video calls with them were always a highlight for me. I also want to thank my father who sparked the interest in science in me when I was young. Sadly he passed away much too early. I think he would enjoy reading this script. 



\section{Contents}

1 Introduction $\quad 1$

1.1 Structure of this Thesis . . . . . . . . . . . . . . . . . . . 2

2 Background and State of the Art 5

2.1 Resistive Pulse Sensing . . . . . . . . . . . . . . . . 5

2.2 Tuneable Resistive Pulse Sensing . . . . . . . . . . . . . . . 6

2.3 Synchronous Electrical and Optical Detection . . . . . . . . . . . 7

2.4 Competing Technologies . . . . . . . . . . . . . . . . 8

2.5 Relevance of This Work . . . . . . . . . . . . . . . . . . . . 8

3 Theory and Modelling $\quad 11$

3.1 Introduction . . . . . . . . . . . . . . . . . . . . . . . 11

3.2 A Semi-Analytic Model . . . . . . . . . . . . . . . . . . 12

3.2.1 The Electrical Field in a Conical Pore . . . . . . . . . . . . . . 12

3.2.2 The Fluid Velocity in a Conical Pore . . . . . . . . . . . . . . 17

3.3 Transport Mechanisms of Particles in Solution . . . . . . . . . . . . . 21

3.3.1 Pressure-Driven Transport . . . . . . . . . . . . . 23

3.3.2 Electrokinetic Transport . . . . . . . . . . . . . . . 23

3.3.3 Other Transport Mechanisms . . . . . . . . . . . . . . . 30

3.4 FEM Modelling . . . . . . . . . . . . . . . . . . 36

3.4 .1 Introduction to FEM . . . . . . . . . . . . . . 37

3.4.2 FEM Simulation of the Electric Field . . . . . . . . . . . . . 38

3.4.3 The Resistive Pulse in the FEM Model . . . . . . . . . . . . . 45

3.4.4 FEM Simulation of the Fluid Flow . . . . . . . . . . . . . 52

3.4.5 Particle Transport and Resistive Pulse in the FEM Method . . 58

3.4.6 Modelling the Ion Distribution in Charged Conical Pores . . . 72

3.4.7 The Mechanical Eigenfrequencies of a Pre-stretched Membrane 82

3.5 Conclusion . . . . . . . . . . . . . . . . . . . . . . 84

4 Materials and Methods $\quad 87$

4.1 Izon's qNano . . . . . . . . . . . . . . . . . . . 87 
4.1 .1 The Adapted Setups . . . . . . . . . . . . . . . . . 89

4.1 .2 The Electronic Noise . . . . . . . . . . . . . . . . . . . . . 94

4.2 Horiba's LabRAM . . . . . . . . . . . . . . . . . . . . . 105

4.2 .1 Lasers, Objectives and Lenses . . . . . . . . . . . . . . 107

4.2 .2 Aligning the System . . . . . . . . . . . . . . . . 108

4.2.3 The Detection Efficiency Profile of the Setup . . . . . . . . . . 113

4.2.4 The Total Detection Efficiency of the Spectrometer . . . . . . 117

4.2.5 The Time-Step Calibration in the LabRAM . . . . . . . . . . 118

4.2 .6 Noise of the CCD . . . . . . . . . . . . . . . . . 122

4.3 Pores, Solutions and Particles . . . . . . . . . . . . . . 123

4.3 .1 Pores . . . . . . . . . . . . . . . . . . . . 123

4.3 .2 Solutions . . . . . . . . . . . . . . . . . . . . . . 128

4.3 .3 Particles . . . . . . . . . . . . . . . . . . . 129

4.4 Modelling of the Optical Signals . . . . . . . . . . . . . . . . . 140

4.5 Methods and Algorithms for the Data Analysis . . . . . . . . . . . . 145

4.5.1 Removing the Laser-induced Noise . . . . . . . . . . . . . 146

4.5.2 The Event Finding Algorithms . . . . . . . . . . . . . . 146

4.5.3 Aligning the Measurements . . . . . . . . . . . . . . . . 148

4.5.4 Spectral Analysis of the Detected Events . . . . . . . . . . . . 150

4.6 Conclusion . . . . . . . . . . . . . . . . . . . . . . . . . . 151

5 Engineering the Size of a Gaussian Beam 153

5.1 Introduction . . . . . . . . . . . . . . . . . . . . . . 153

5.2 The Properties of a Gaussian Beam . . . . . . . . . . . . . . . . 154

5.3 Focussing of Gaussian Beams . . . . . . . . . . . . . . . . . . 157

5.3.1 Gaussian Beam Optics vs. Geometric Optics . . . . . . . . . . 157

5.3.2 Self's Formalism for Focussing a Gaussian Beam . . . . . . . . 157

5.3.3 A Fast Method to Calculate the Spot Size . . . . . . . . . . . 160

5.3.4 Real Gaussian Beams . . . . . . . . . . . . . . . . . 161

5.3.5 The Beam Width at the Focal Plane of the Objective . . . . . 162

5.3.6 Modifications to Self's Formalism for Immersion Objectives . . 163

5.3.7 Spot Sizes and Gaussian Shifts in a Laboratory Situation . . . 164

5.4 Engineering the Spot Size . . . . . . . . . . . . . . . . 167

5.4.1 Back Focal Plane Lens . . . . . . . . . . . . . . . . . . . . . . 167

5.4.2 Beam Expander as Back Focal Plane Lens . . . . . . . . . . . 171

5.4.3 Beam Size Engineering for the Coordination Technique . . . . 173

5.5 Experimental Verification . . . . . . . . . . . . . . . 176

5.6 Conclusion . . . . . . . . . . . . . . . . . . . . . . . . 178 
6 Detection of Microparticles - First Setup $\quad 179$

6.1 Experimental Details . . . . . . . . . . . . . . . . . . . . . 179

6.1.1 qNano and Pore Specimen . . . . . . . . . . . . . . 179

6.1.2 Optical Setup . . . . . . . . . . . . . . . . . . . 180

6.1.3 Sample. . . . . . . . . . . . . . . . . . . . . 181

6.1.4 Control of the Particle Transport . . . . . . . . . . . . . . 182

6.2 Results and Discussion . . . . . . . . . . . . . . . . . . . . . 182

6.3 Further Discussion and Possible Improvements of the System . . . . . 192

6.4 Conclusion . . . . . . . . . . . . . . . . . . . . . . 195

7 Coordinated Detection using the Second Setup 197

7.1 Introduction . . . . . . . . . . . . . . . . . . . . . 197

7.2 Coordinated Detection of $2 \mu \mathrm{m}$ Particles . . . . . . . . . . . 198

7.2.1 Experimental Details . . . . . . . . . . . . . . . 198

7.2.2 Results using a Low Magnifying Objective . . . . . . . . . . . 201

7.2.3 Using a Higher Magnifying Objective . . . . . . . . . . . . . . 206

7.2.4 Particle Sizing and Concentration Measurement . . . . . . . . 217

7.3 Co-ordinated Detection of a $1 \mu \mathrm{m}$ Particle Mixture . . . . . . . . . . . 218

7.3.1 Spectral Identification of the Species . . . . . . . . . . . . . . 218

7.3 .2 Results . . . . . . . . . . . . . . . . . 220

7.3.3 Repetition at a Lower Molarity . . . . . . . . . . . . . . 224

7.4 Coordinated Detection of $500 \mathrm{~nm}$ Particles . . . . . . . . . . . . . . 232

7.4.1 Expected Problems for Sub-um Particles . . . . . . . . . . . . 232

7.4 .2 Experimental Details . . . . . . . . . . . . . . 233

7.4 .3 Results . . . . . . . . . . . . . . . . . 233

7.5 Co-ordinated Detection of $200 \mathrm{~nm}$ Particles . . . . . . . . . . . . . 239

7.5.1 Results for One Particle Set . . . . . . . . . . . . . . 241

7.5.2 Results for Mixed Particle Sets . . . . . . . . . . . . . 247

7.6 Conclusion . . . . . . . . . . . . . . . . . . . . 250

8 Conclusion and Outlook 255

8.1 Future Work . . . . . . . . . . . . . . . . . . . 256

$\begin{array}{ll}\text { A Publications and Presentations } & 259\end{array}$ 



\section{Chapter 1}

\section{Introduction}

The recent boom and increasing use of micro- and nanoparticles in various fields in science and their more and more frequent implementation in our day to day lives demands the development of new sensing platforms that can detect and characterize such particles. Established techniques like electron microscopy or dynamic light scattering often require intensive sample preparation, are costly, have a low throughput and/or provide only information about a population of particles. Inspired by nature's solution for regulating the ion and molecular transport into and out of our cells via pores in the membrane, the resistive pulse sensing technique (RPS) has become a promising candidate to fight these shortcomings.

The invention of RPS dates back to the 1950s when, during the cold war, the demand for blood tests spiked out of fear of nuclear radiation. Wallace H. Coulter observed nurses counting blood cells under the microscope by hand and thought that there must be a better and faster way to do so. The result of his research was a device containing a membrane with a small pore that separates two chambers which are filled with the analyte solution [1]. The particles are pushed through the pore together with the fluid and are detected by a brief change in the electrical resistance across the membrane. The apparatus quickly became popular and its principle is nowadays still applied in one of the most used standard medical tests, the complete blood count.

Due to the advances in micro- and nanofabrication and measurement techniques over the last decades, the detection of single molecules with the RPS principle became possible recently, opening up a wide range of possible applications $[2,3]$. One of those is DNA sequencing, which has become the primary goal of the field $[4,5]$. Promising results using RPS suggest that the technique could reach the often cited goal of a $\$ 1000$ genome.

Optical spectroscopy is another powerful and broadly used analytical tool. Due to the molecular origin of the emitted radiation it provides information about the composition of a sample. Many biosensing applications make use of the intrinsic 
fluorescence of molecules as well as introduced fluorescent labels to identify the analyte. Raman spectroscopy has gained increasing popularity as, although it is intrinsically much weaker than fluorescence, it is a label-free method which can reveal the distinctive fingerprint of a molecule.

The aim of this thesis was to combine a derivative of RPS, namely Tuneable Resistive Pulse Sensing (TRPS), with spectroscopic measurements and use the information gained by the coordinated measurement for very specific characterization of the analyte particles. The possible applications of the coordinated sensing methodology are broad, especially in the life sciences, since detection in such a RPS device can be made in physiological conditions.

\subsection{Structure of this Thesis}

This thesis consists of eight chapters, starting with this introduction. In the next chapter, a review of relevant literature is given to establish the novelty and strengths of this work in the context of previous studies. Chapter 2 is kept relatively brief because most of the literature used in the thesis is introduced where it is specifically relevant, in later chapters. The third chapter introduces theoretical descriptions of TRPS, as well as models of the sensing process. These models use either a semi-analytic approach or the numerical Finite Element Method (FEM). They are developed in order to understand the particle motion through a pore due to different transport mechanisms relevant in TRPS. Several models were developed, with an increasing accuracy of the physical reality, including the consideration of particlefield interactions and the Electric Double Layer (EDL).

The fourth chapter describes the experimental materials and methods used and discusses two developed techniques in detail. The aim is to point out all the physical effects involved and technical issues encountered, and to give the reader all the necessary information to conduct such experiments her/himself. A model to predict the optical signals and estimate the detection limit of the technique is presented at the end of the chapter. Originating from the need to adjust the size of the laser beam for the different pore sizes used in the experiments, the fifth chapter first describes the peculiarities of the transformation of a Gaussian beam and then proposes several methods to engineer the spot size in a typical laboratory situation.

In the sixth chapter the results using the initially developed setup and fluorescently labelled polystyrene beads are presented. With this setup, the detection is limited to $\mu \mathrm{m}$-sized particles. A second, improved, setup which is capable of detecting nm-sized particles, was therefore designed. The results using various particle sets, including mixtures with different fluorescent labels, are discussed in the seventh chapter. A summary of the most important findings of the thesis, a critical 
reflection on the work, and possible improvements and further work are the subjects of the final chapter. 


\section{Chapter 2}

\section{Background and State of the Art}

\subsection{Resistive Pulse Sensing}

The idea behind resistive pulse sensing (RPS) is as simple as it is powerful. A membrane containing a micro- or nanoscale pore is placed in an aqueous electrolyte and a constant voltage is applied across the membrane. Particles traversing the pore alter the resistance and are detected as short-lived pulses in the ionic current. The technique is capable of measuring the concentration, size, and charge of the particles, as these properties are linked to the frequency, magnitude and duration of the current pulses. In comparison to competing techniques like dynamic light scattering [6], which measures the average properties of particles and is biased towards larger particle sizes, RPS probes each single particle.

RPS was first applied in the Coulter counter [1] for sizing and counting cells in blood. The underlying principle behind resistive-pulse generation is that the change of the current is related to the physical volumetric blocking of the electrolyte solution [7]. Deblois and co-workers refined the technique and were able to measure particles with diameters of $\sim 100 \mathrm{~nm}$ with pores made in polycarbonate membranes $[8,9]$. Since then RPS has developed to be capable of detecting single molecules $[2,10,11]$. It has been used to investigate many particle types, e.g. synthetic nanoparticles, proteins [12], viruses [13], and liposomes [14], over a wide range of length scales $[11,15]$. Methods such as track etching [16], electron beam lithography [17], and ion beam sculpting [18] have been used to make pores in materials like silicon, silicon nitride, glass, and polycarbonate [19] down to an opening of a few nanometers.

Inspired by the high selectivity of biological nanopores, more specific detection of target molecules can be achieved with chemically functionalised pores [20, 21]. Kasianowicz et al. [22] were the first to use an $\alpha$-haemolysin pore to detect single DNA strands, with the ultimate aim to sequence DNA, which remains the most active field in RPS science $[23,24]$. It has recently been claimed that this goal has 
been achieved with a commercial device (MinION, Oxford Nanopores). The first results obtained with the device have been reported [25-28]. RPS is an active field and as there are many physical phenomena involved, it is still of interest both for basic research [29] and constant technological improvements [29].

\subsection{Tuneable Resistive Pulse Sensing}

Sowerby et al. [30] were the first to use pores made in a stretchable material in order to reversibly tune the pore size [31, 32]. The technique was consequently commercialised by the New Zealand based manufacturer Izon Science Ltd. Tuneable pores are fabricated by puncturing an elastomeric polyurethane "cruciform" membrane with a chemically etched tungsten needle [30], leading to a typical conical pore shape (Fig. 2.1) and a resulting asymmetric shape of the current pulse caused by a traversing particle.

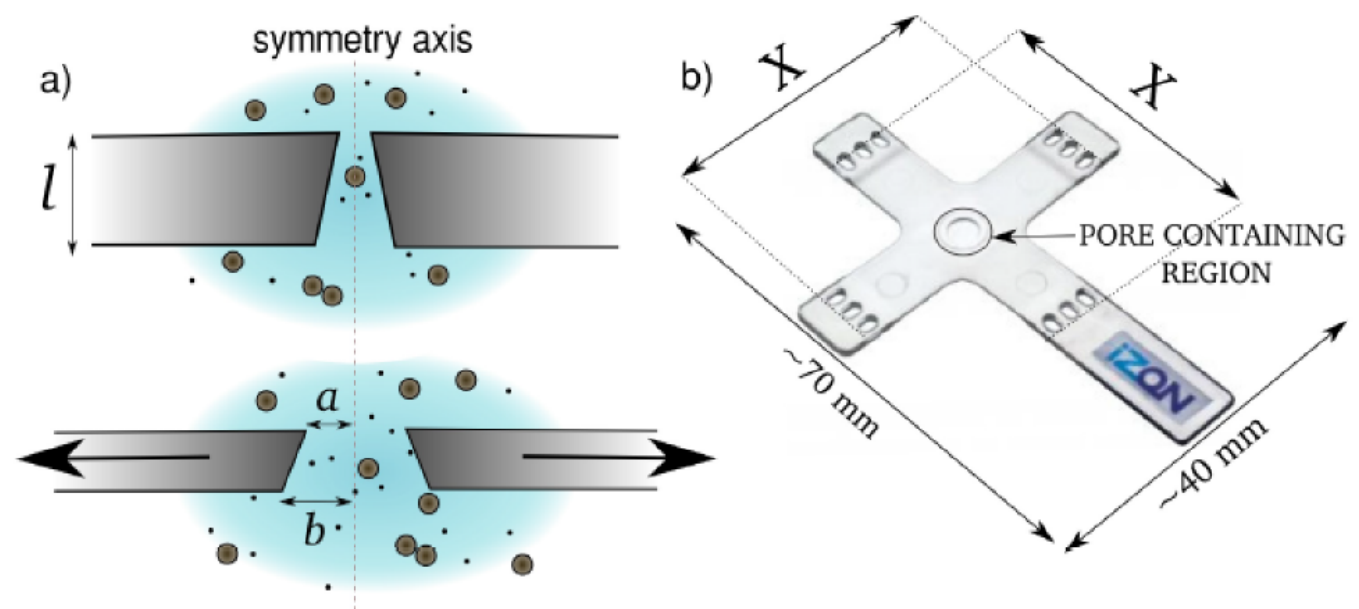

Figure 2.1: (a) A schematic illustration of the pore geometry in TRPS and its change due to the application of a radial mechanical stress. (b) The cruciform with the pore specimen in the centre. The holes in the arms are used to hold the specimen in place and apply the stretch. Image from [33].

TRPS is able to measure the concentration [34,35] as well as the size [36] and the $\zeta$-potential [37-39] of a wide range of biological and synthetic particle types down to a size of $50 \mathrm{~nm}$ in diameter. As the pore geometry is not exactly known the measurements are conventionally done using calibration particles. The technique has recently been used to study microbeads in a magnetic field [40] and to monitor aptamer-protein interactions [41, 42] among other applications. TRPS has the advantage over pores of fixed size that the pore to particle size ratio can be optimized, which allows for highly accurate measurements even for polydisperse particle mixtures [43]. 
Although TRPS has been successfully used to detect DNA molecules [30], the technique is not generally used for molecular detection but aims at the size range from $50 \mathrm{~nm}$ to several $\mu \mathrm{m}$, with numerous potential applications, especially in the life sciences [44]. It has been used to detect bacteria [35], viruses [36], liposomes [45], exosomes [46] and extracellular vesicles [47, 48]. It was used in binding assays and has proven to have a sufficient resolution to differentiate between bare particles and particles that are functionalized with streptavidin or $\lambda$-DNA [43]. For further information the reader is directed to two recent reviews $[49,50]$, a book chapter [51] and a study comparing the technique to competing technologies [52].

\subsection{Synchronous Electrical and Optical Detection}

Fluorescence is a broadly used tool in biosensing [53] and makes use of the intrinsic fluorescence of biomolecules as well as introduced fluorescent labels to identify the analyte, e.g. to distinguish between cancerous and normal cells [54]. Recently several studies that combine resistive pulse sensing with optical detection have emerged in an attempt to acquire complementary information about the analyte. Attempts in which fluorescence intensities were measured, but without detailed spectral information, have included the detection of single molecules [55-60].

Heron et al. [57] achieved synchronised detection using $\alpha$-haemolysin pores, while Soni et al. [58] obtained good signal agreement using fluorescently labelled DNA traversing a solid-state nanopore. Song et al. [59] showed that simultaneous detection is possible using carbon nanotubes and charged dye molecules. Kurz et al. [60] could visualize the translocation of DNA molecules through a silicon-nitride pore using scanning confocal microscopy, and correlated the intensity with the current signal. Another group [61] made the observation that a laser can be utilized to modulate the surface charge and therefore the translocation dynamics in solid-state nanopores in order to significantly slow down the translocation speed of DNA. Apart from the usual top down approach in which the optical technique is implemented by looking down the pore axis, several studies have been conducted where the signal is collected along the side of a microfluidic channel with a small constriction [62-64]. The most recent work [65-68] has involved the detection of DNA and biomolecules using solid state pores.

Raman [69] spectroscopy and its derivative Surface Enhanced Raman Spectroscopy (SERS) [70] are also candidates for the coordinated detection. Chen et al. [71] built a $5 \mathrm{~nm}$ wide nanoslit equipped with Bragg mirror gratings resulting in a high enough enhancement to detect adenine molecules, although the integration time was about a minute long. Theoretical predictions using a plasmonic nanopore have been made by [72], concluding that DNA could be moved in discrete steps 
through the pore by switching the laser and therefore the plasmonic field on and off, leaving sufficient time to potentially measure the Raman signal of individual nucleotides.

In all the above studies (apart from [71]) the overall intensity of the optical signal without spectral information was measured. Particles with similar fluorescence intensities but different emission profiles could therefore not be distinguished. The technique developed in this work increases the specificity of co-ordinated resistive pulse and optical data by acquiring high resolution spectra instead of an overall fluorescence intensity.

\subsection{Competing Technologies}

Fluorescence Correlation Spectroscopy (FCS) is able to acquire information about individual particle size and the optical properties of analytes down to molecular size $[73,74]$. However as with all other diffusion-based techniques it does not allow for a defined pathway of the analyte and therefore controllable throughput through the detection volume. Spectral flow cytometry addresses this issue and is now an established tool in the biosciences [75] and is often included as part of cell sorting systems. However it is limited in size to particles above $\sim 0.5 \mu \mathrm{m}$, while TRPS covers length scales from tens of micrometers down to less than $100 \mathrm{~nm}$. It also does not give information about the particle charge, a information which is accessible with the TRPS technology. Flow cytometry devices are also usually large and expensive, while the developed device is portable and can temporarily be attached to any spectrometer.

\subsection{Relevance of This Work}

A schematic illustration of the coordination technique is shown in Fig. 2.2. The addition of an optical measurement to the TRPS technique allows for similar sized particles, which are not distinguishable with RPS only, to be further characterised optically. In this thesis the technique is applicable to particles ranging from hundred $\mathrm{nm}$ to several $\mu \mathrm{m}$ in size. Since low Reynolds number flow through a pore is laminar and can be precisely controlled, RPS provides a useful means to guide particles through the optical detection region with a defined pathway and speed, which is an improvement over the stochastic particle motion prevalent in conventional solutionbased optical detection methods. Apart from practical aspects, the two techniques can complement each other in understanding fundamental aspects of both methods. The use of tunable pores also provides some particular advantages, such as the ability to measure poly disperse particle mixtures and the experimental flexibility provided 


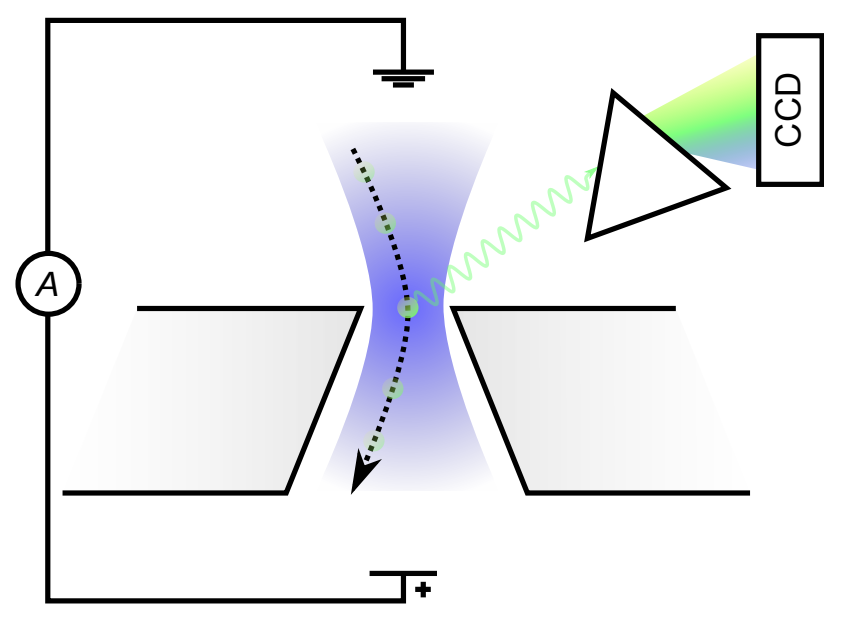

Figure 2.2: Schematic illustration of the proposed experiment in cross-section. The trajectory of a particle through the pore is indicated by the dotted line. The laser beam used for excitation has a Gaussian profile with a width comparable to the pore diameter and is represented by the blue shading. Emitted light is dispersed into spectral components with a diffraction grating and detected with a liquid nitrogencooled CCD chip.

by tuning the pore, which could lead to a new range of experimental possibilities. The coordinated detection could be useful for the characterisation of drug delivery vehicles, extracellular vesicles viruses, emulsions, and synthetic particles used in binding assays. In many of these cases, measurement and analysis of the physical and chemical properties present central research questions.

Aspects of this thesis have been published or were presented as listed in the appendix. 


\section{Chapter 3}

\section{Theory and Modelling}

\subsection{Introduction}

The understanding of the movement of particles through a micro- or nanopore and the resulting transient current pulse is of basic interest for the RPS technique. For a quantitative interpretation of resistive pulse data, the origin of the size and shape of a transient pulse has to be understood on a fundamental level. This involves several disciplines including fluid mechanics, electrostatics, electrokinetics, particle-field and electrolyte-surface interactions. In fact many of the problems involved have been of research interest on their own for many years already. One of the first attempts to calculate the effective resistivity of an electrolyte containing a dilute suspension of insulating spheres dates back to Maxwell and was published in his famous treatise on electricity and magnetism [76]. The fluid dynamic problem of a flow through a conical pore has been investigated as far back as 1850 when Stokes published first results about this particular problem. However, an exact simulation of all the physical mechanisms involved in a resistive pulse experiment is very complicated and often simplifications have to be made.

At the start of this chapter, a semi-analytic model for the electric field and the flow field in conical pores, which has been extensively used in the TRPS literature $[36,40,77]$, is introduced. The model makes many simplifications of the physical reality but has been proven useful to predict the current, the fluid flow and the resistive pulses for particles moving through conical pores. In the next section, the significance of pressure-driven, electrokinetic and other transport mechanisms of spherical particles through a conical pore with dimensions relevant to the experimental work of this thesis are discussed. The Finite Element Method (FEM) is then introduced to numerically solve the electrostatic and hydrodynamic problem in conical pores typical for TRPS. Phenomena which are beyond the capability of the semi-analytic model, including particle-field interactions, off-axis particle trajectories and charge 
polarisation in the pore can be accounted for with the FEM method. Several effects, which have been observed experimentally in TRPS, can be understood with the help of the developed models.

The models presented in this chapter are applied and tested with mainly two pore geometries, which reflect the lower and upper pore size and consequently particle size used in the experiments. The pores, a "NP200" and a "NP2000"-rated pore (compare Sec. 4.3.1) are suited to detect particles in the range from $100 \mathrm{~nm}-$ $400 \mathrm{~nm}$ and $1000 \mathrm{~nm}-4000 \mathrm{~nm}$ respectively. Both have the TRPS typical conical shape (Fig. 3.1), and while the large pore opening $b$ and the pore length $l$ are similar for all tuneable pores and are left constant at $25 \mu \mathrm{m}$ and $150 \mu \mathrm{m}$, the small pore opening $a$ is $250 \mathrm{~nm}$ for the NP200 and $2.5 \mu \mathrm{m}$ for the NP2000 pore. In reality the values for $a, b$ and $l$ may differ from the numbers used in this chapter. However, the conclusions drawn from the simulations are still valid. In the following the pore names instead of their dimensions are used.

\subsection{A Semi-Analytic Model}

\subsubsection{The Electrical Field in a Conical Pore}

The knowledge of the electric field in and around a pore is of basic importance for understanding RPS. The electric field determines the current through the pore and is also linked to the strength of the electrokinetic forces on the particles via electroosmosis and electrophoresis. For a given pore geometry, an assumed uniform electrolyte conductivity $\sigma$ and negligible surface charge, the electric field $\boldsymbol{E}=-\nabla \psi$ can be calculated from the electric potential $\psi$, which is determined by the Laplace equation $\nabla^{2} \psi=0$. For some specific geometries an analytic solution can be found, e.g. Kowalczyk [78] did so for a double-hyperboloid shaped pore. However, for most pore geometries including conical shaped pores, which are used as a model geometry in this work, an exact analytical solution is not available. Moreover, the addition of an insulating particle close to or inside the pore complicates the calculation of an exact solution even further.

For this reason a semi-analytical model to determine the electric field, which has been used in recent RPS and TRPS literature [40, 79], is introduced here. The method is based on a resistance approach, where the pore is modelled as a homogeneous, isotropic conductor with variable cross section $A(z)$, with $z$ the coordinate axis parallel to the normal of the membrane surfaces. The total resistance of the pore with length $l$ is then given as the summation over slices with infinitesimal thickness $d z$ 


$$
R_{\text {pore }}=\int_{0}^{l} \frac{\rho d z}{A(z)}
$$

This method has the advantage that there is no inherent geometrical limitation to the pore shape. However, it is implicitly assumed that $A(z)$ varies slowly with $z$, so that the radial component of the electric field is negligible compared to the axial component. Another assumption is that the resistivity of the ionic solution is homogeneous over the entire pore. This seems reasonable as the pore dimensions are several orders of magnitude larger than the double layer thickness that forms close to the pore walls and the particle surface. A FEM model that takes the ionic distribution into account will be introduced and discussed in Sec. 3.4.6 of this chapter.

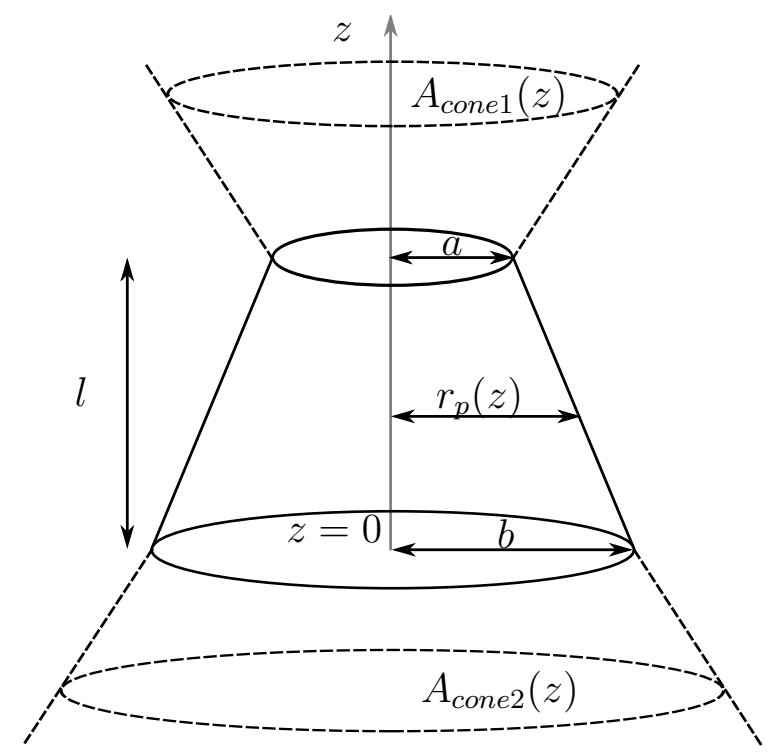

Figure 3.1: The conical pore geometry (-) used for the semi-analytic model, with parameters as defined in the text. The cones above and below the pore indicated by broken lines were introduced artificially and used to model the field in these regions.

The shape of the pores used in the experiments and modelled here is assumed to be conical (Fig. 3.1), which is a simplification as it has been shown that the cross sections of the pores fabricated by Izon are not perfectly round in shape [34, 80]. The radius $r_{p}(z)$ of the pore (Fig. 3.1) with a small pore opening with radius $a$, a large pore opening $b$ and a pore length of $l$ is given by $r_{p}(z)=b-(b-a) z / l$, with $z=l$ at the small and $z=0$ at the large pore opening. Inserting the pore's cross section $A(z)=r_{p}^{2}(z) \pi$ into Eq. 3.1 and evaluating the integral yields the expression 


$$
R_{\text {pore }}=\rho \frac{l}{\pi a b}
$$

for the resistance of the pore. The electrodes in a RPS experiment are in general not directly attached to the pore openings. Therefore an additional resistance between the electrodes and the pore openings occurs. Hall [81] found an analytic expression for the potential drop between a circular disc opening of radius $a$ and the semi-infinite half space, which is given by $\rho /(4 a)$. As in a TRPS setup the dimensions of the pore are several orders of magnitude smaller than the electrodes and are located more than $1 \mathrm{~mm}$ away from the pore, this expression can be used to account for the access resistance $R_{a c c}$ between each pore opening and the corresponding electrode [82]. Polarization potentials at the electrodes are assumed negligible, which is justified for $\mathrm{Ag} / \mathrm{AgCl}$ electrodes [83]. Therefore the total resistance between the electrodes is given by

$$
R_{0}=R_{a c c 1}+R_{\text {pore }}+R_{a c c 2}=\frac{\rho}{4 a}+\frac{\rho l}{\pi a b}+\frac{\rho}{4 b}
$$

The current $I_{0}$ through the open pore, also know as the baseline current, can then simply be calculated using Ohm's law $I_{0}=V_{0} / R_{0}$. It has to be mentioned that this procedure implicitly assumes that the pore orifices are equipotential surfaces. When the equipotential surface is a hemisphere at the pore entrance, Hille [84] derived a value of $\rho /(2 \pi a)$ for the access resistance. This result and Hall's expression for a disc $(\rho /(4 a))$ were used by Kowalczyk [78] to obtain an upper and lower limit for the total resistance of a hyperboloid pore geometry.

Accounting for the access resistance is important for the pores used in the experiments. The ratio $R_{\text {acc } 1} / R_{\text {pore }}=\pi b / 4 l$, which for typical values of $l$ and $b(l \sim$ $150 \mu \mathrm{m}$ and $b \sim 25 \mu \mathrm{m})$ gives $\sim 1 / 8$, cannot be neglected in the calculation of the baseline current and consequently the size of the resistive peaks. As the values for $b$ do not change significantly throughout the range of tuneable pores due to their production process, the relative contribution of $R_{a c c 1}$ is expected to be constant for all pore sizes. On the contrary the ratio $R_{\text {acc } 2} / R_{\text {pore }}=\pi a / 4 l$ is strongly dependent on the pore size and can be estimated to be about $\sim 1 / 100$ for a NP2000 and $\sim 1 / 1000$ for a NP200 pore. It shall be noted in this context that in Eq. 3.3 the approximation $\pi / 4 \sim 0.8$ can be made so that the total resistance becomes $R_{0} \approx \frac{\rho(l+0.8(a+b))}{\pi a b}$, the expression usually used in the TRPS literature [34].

With the knowledge of $I_{0}$, the electric field in the pore can then be calculated using the expression 


$$
E(z)=-\nabla \psi=-I_{0} \frac{d R}{d z}=-I_{0} \frac{\rho}{\pi r_{p}(z)^{2}}
$$

As each pore cross section is assumed to be an equipotential surface the electric field only depends on $z$ in this model and is uniform in the $z$-direction across the radius. This is a good approximation inside the pore, but is not valid any more at the edges of the pore openings, as it will be shown in Sec. 3.4.2. The electric field outside, i.e. above and below the pore membrane, can be calculated in the semi-analytic model by introducing an artificial cone (Fig. 3.1) whose cross sectional area follows the general expression $A_{\text {cone } 1}(z)=\pi(a+G z)^{2}$. The parameter $G$ can be obtained using the condition that the access resistance must match Hall's expression so that

$$
\int_{l}^{\infty} \frac{\rho}{\pi(a+G(z-l))^{2}} d z=\frac{\rho}{4 a} .
$$

Evaluating the integral determines $G=4 / \pi \approx 1.25$. An analogous cone is constructed below the pore and the expressions for the electric field $E(z)$ in the three cone regions are then given by a geometric term multiplied with the voltage $V_{0}$

$$
E(z)=\frac{V_{0}}{\frac{1}{4 a}+\frac{l}{\pi a b}+\frac{1}{4 b}} \times \begin{cases}\frac{1}{\pi\left(a+\frac{4}{\pi}(z-l)\right)^{2}} & \text { if } z \geq l, \\ \frac{1}{\pi\left(b-\frac{b-a}{l} z\right)^{2}} & \text { if } l \leq z \geq 0 \\ \frac{1}{\pi\left(b-\frac{4}{\pi} z\right)^{2}} & \text { if } z \leq 0\end{cases}
$$

\subsubsection{Addition of a Particle}

When an insulating particle is added in the pore, conductive solution gets displaced and the resistivity is expected to rise. To determine the resistance change and the resulting resistive pulse the integral method, based on Eq. 3.1, can still be used. To obtain the total resistance and thus the current through the pore an integral of the form Eq. 3.1 is calculated for each of the regions indicated by Roman numbers in Fig. 3.2. Here $r_{s}^{\prime}(z)$ is the radius of the particle cross section at position $z$ and $z_{s}$ is the position of the centre of the particle. For region III the area $\pi r_{s}^{\prime 2}(z)$ obstructed by a spherical particle with radius $r_{s}$ has to be excluded. The resistance of the other regions is assumed to be unaffected by the addition of the particle. Special care has to be taken when the particle overlaps with the pore openings so that the integration is split up correctly between the artificial cone and the pore. The integrals can either be evaluated with standard numerical methods or analytically. 
For a spherical particle positioned at $z_{s}$ within a conical pore $r_{s}^{\prime 2}(z)=r_{s}^{2}-\left(z-z_{s}\right)^{2}$ the expression for the corresponding integral is given by

$$
\frac{\rho}{\pi} \int_{z_{s}-r_{s}}^{z_{s}+r_{s}} \frac{1}{\left(r_{p}^{2}(z)-r_{s}^{\prime 2}(z)\right)} d z=\frac{\rho l}{\pi C}\left(\operatorname{arctanh} \frac{A}{C l}-\operatorname{arctanh} \frac{B}{C l}\right)
$$

with

$$
\begin{gathered}
A=b^{2}\left(l-z_{s}-r_{s}\right)-a b\left(l-2 z_{s}-2 r_{s}\right)-l^{2} r_{s}-a^{2}\left(z_{s}+r_{s}\right), \\
B=b^{2}\left(l-z_{s}+r_{s}\right)-a b\left(l-2 z_{s}+2 r_{s}\right)+l^{2} r_{s}-a^{2}\left(z_{s}-r_{s}\right), \\
C=\sqrt{l^{2} r_{s}^{2}-b^{2}\left(l^{2}-2 l z_{s}+z_{s}^{2}-r_{s}^{2}\right)+a^{2}\left(r_{s}^{2}-z_{s}^{2}\right)-2 a b\left(l z_{s}-z_{s}^{2}+r_{s}^{2}\right)}
\end{gathered}
$$

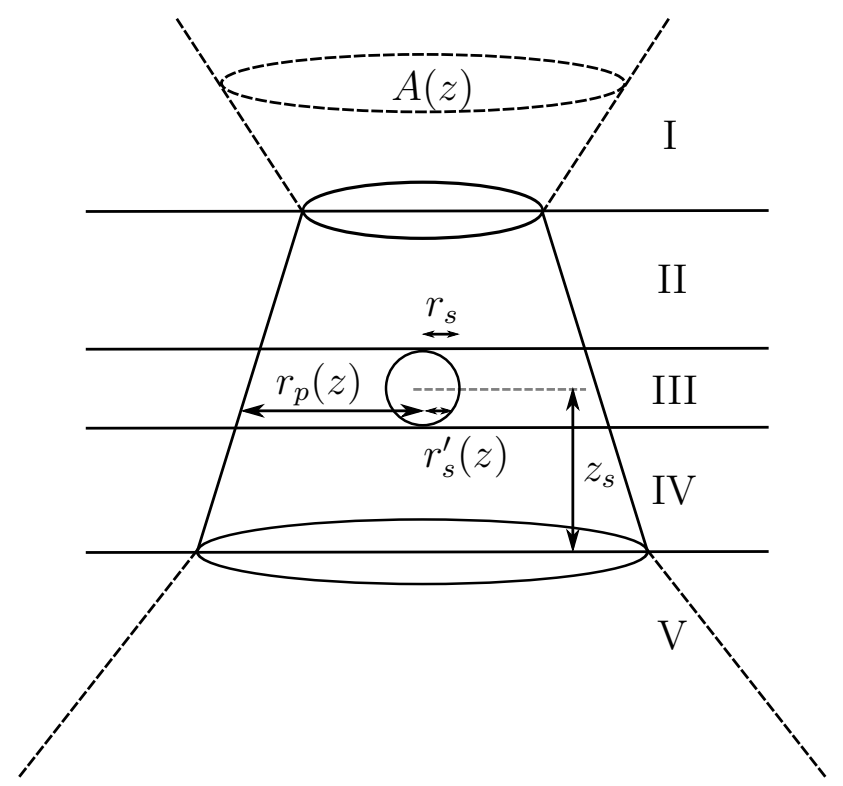

Figure 3.2: The total resistance of the pore with the addition of a spherical particle with radius $r_{s}$ positioned at $z_{s}$ can be calculated by dividing the geometry into separate regions (Roman numerals). For each region an integration over the cross section $A(z)$ is carried out, either numerically or analytically.

Analogous expressions, which are not explicitly listed here, can be obtained for each of the other regions. Heins et al. [85] found similar expressions for the same pore geometry, however, with a different arrangement of the terms. The advantage of an 
analytic evaluation of the integrals over numerical methods is a faster computation, which becomes relevant when a resistive pulse signal is calculated at a high time resolution. For the prediction of the resistive pulses with the semi-analytic model the analytic evaluation of the integrals was chosen throughout this thesis.

One clear advantage of the resistance method is its simplicity and it has been used in RPS and TRPS to predict the resistive pulse [86, 87]. It is also not restricted to any certain pore or particle shape, which has been exploited to model various hypothetical geometries [88]. With the addition of an insulating particle within the pore, the time-dependency of the resistive pulse can be calculated when combined with the model for the transport mechanisms, which will be explained in Sec. 3.3. However, the radial component of the electric fields is neglected, which is clearly not valid at the pore entrance. As a result only particles which move along the pore axis should be simulated with this method.

\subsubsection{The Fluid Velocity in a Conical Pore}

The fluid flow through micro- and nanopores of various shapes is of basic interest for the RPS technique. Whether continuum mechanics or statistical mechanics in form of molecular dynamics simulations have to be used is determined by the Knudsen number [89]. Travis et al. [90] found that continuum mechanics can still be applied for channels with a diameter of only 10 times the size of a water molecule. This is much smaller than the pore dimensions in this work and the application of continuum mechanics is therefore justified. The Navier-Stokes equations are the governing formalism for the hydrodynamic problem and, assuming an incompressible fluid, have the form [91]

$$
\rho\left(\frac{\partial \boldsymbol{u}}{\partial t}+\boldsymbol{u} \cdot \nabla \boldsymbol{u}\right)=-\nabla P+\mu \nabla^{2} \boldsymbol{u}+\boldsymbol{F},
$$

with $\boldsymbol{u}$ the fluid velocity, $P$ the pressure, $\rho$ the fluid density and $\mu$ the dynamic viscosity of the fluid $\left(1.002 \times 10^{-3} \mathrm{Pas}\right.$ for water at $293 \mathrm{~K}$ [92]). The terms from left to right correspond to the inertial, pressure, viscous and external body forces $\boldsymbol{F}$ applied to the fluid. Equation 3.11 represents the conservation of momentum and is solved together with the continuity equation, which for incompressible fluids is given by

$$
\nabla \cdot \boldsymbol{u}=0
$$

and represents the conservation of mass. Equation 3.11 is a non-linear partial dif- 
ferential equation, which makes most problems impossible to solve analytically. The non-linearity is also the reason for the turbulence of a flow. However, when the inertial forces are small compared to the viscous forces, Eq. 3.11 can be linearised by neglecting the inertial term on the left side, resulting in the Stokes equation

$$
0=-\nabla P+\mu \nabla^{2} \boldsymbol{u}+\boldsymbol{F}
$$

for the steady-state. It describes the so called Stokes or creeping flow, which is purely laminar and time-reversible. The regime where Eq. 3.13 can be used instead of Eq. 3.11 is determined by the Reynolds number Re. It represents the ratio between the inertial and viscous forces and, for a flow in a cylindrical pipe, is given by

$$
R e=\frac{\rho \bar{v} D_{H}}{\mu}
$$

with $\bar{v}$ the mean velocity. $D_{H}$ is the hydraulic diameter, which is commonly used for non-circular channels and is defined as four times the cross-sectional area divided by the perimeter of the fluid conduit. For low Reynolds numbers $(R e<0.1)$, a flow is mostly laminar and the Stokes equations can be used, which is often fulfilled in micro-and nanofluidics. For the experiments conducted in this thesis an upper limit of $\sim 0.5$ is estimated for the Reynolds number and is reached at the pore constriction for the largest pores (NP2000) and the highest hydrostatic pressure $\left(100 \mathrm{~mm} \mathrm{H}_{2} \mathrm{O}\right)$. For most experiments the Reynolds number is much smaller and a highly laminar flow can be expected. Note that in practice the pressure was measured relative to the point where no fluid flow through the pore could be observed (see Sec. 4.1.1). Therefore gravitation, which is usually implemented as a body force in Eq. 3.11, is included in the value for the pressure and does not have to be considered separately.

The Stokes equations are linearisable and can be solved analytically for certain geometries and boundary conditions. For an incompressible Newtonian fluid flowing through a long cylindrical pipe with a constant cross section, no radial flow component and zero velocity at the walls, an exact solution in form of the Hagen-Poiseuille equation exists. It predicts a parabolic, axial symmetric flow profile with the maximal velocity in the centre of the pipe. The pressure drop between the ends of a cylindrical pipe is given by

$$
P=\frac{8 \mu L Q}{\pi r_{c y l}^{4}}
$$

with $L$ the length of the pipe, $Q$ the volumetric flow rate and $r_{c y l}$ the radius of 
the pipe. It has to be noted that in reality the parabolic flow profile does not develop instantaneously at the pipe entrance but needs a certain entrance length $L_{e}$ to build up. Durst [93] found an expression for the ratio $L_{e} /(2 r)=\left(0.619^{1.6}+\right.$ $\left.\left.(0.0567 R e)^{1.6}\right)\right)^{1 / 1.6}$, which only depends on the Reynolds number. The maximal value for the entrance length in our experiments is $\sim 3 \mu \mathrm{m}$. Because the pore radius is changing slowly and the typical pore length in TRPS is usually $150 \mu \mathrm{m}$, it is therefore justified to assume a parabolic flow profile in the pore. This is also supported by Dagan et al. [94], who came to the conclusion that entrance effects are only significant near the pore opening and decay rapidly within the pore.

Assuming a slowly varying pore radius, Eq. 3.15 can be applied across infinitesimal elements. The total pressure drop between the openings of a conical pore can then be evaluated by integrating Eq. 3.15 with the expression for the pore radius $r_{p}(z)=b-\frac{(b-a) z}{l}$, which yields the expression

$$
P_{\text {pore }}=\int_{0}^{l} \frac{8 \mu Q}{\pi\left(b-z \frac{b-a}{l}\right)^{4}} d z=\frac{8 \mu l Q}{3 \pi} \frac{b^{3}-a^{3}}{(b-a) a^{3} b^{3}} .
$$

In a TRPS experiment the hydrostatic pressure is exerted between the two reservoirs attached to the pore opening. In analogy to the method applied to the electric field calculation entrance effects have to be taken into account. Sampson [95] found the analytic expression for the pressure difference between the semi-infinite half spaces with a thin circular orifice (radius $a$ ) in the middle to be $3 \mu Q / a^{3}$. Similar results were obtained by Dagan [94] and Wang [96] for the pressure drop between the half space and the entrance of a cylindrical tube of finite and infinite length. The pressure drop from an infinite half-space to the pore entry is half the value given by Sampson so that the relationship between volumetric flow rate and total pressure becomes

$$
P_{\text {total }}=P_{a c c 1}+P_{\text {pore }}+P_{a c c 2}=\frac{3 \mu Q}{2 a^{3}}+\frac{8 \mu l Q}{3 \pi} \frac{b^{3}-a^{3}}{(b-a) a^{3} b^{3}}+\frac{3 \mu Q}{2 b^{3}} .
$$

For the pores used in the experiments $b>>a$ so that the ratio $P_{a c c 1} / P_{\text {pore }}$ becomes $\pi / 32 \approx 0.1$, which indicates that it is important to account for the pressure drop from infinity to the small pore opening. The pressure drop to the large pore opening is several orders of magnitude smaller and could be neglected. Rearranging Eq. 3.17 for the total volumetric flow yields 


$$
Q=\frac{P}{\mu}\left(\frac{a^{3} b^{3}}{\frac{3}{2}\left(a^{3}+b^{3}\right)+\frac{8 l}{3 \pi}\left(a^{2}+a b+b^{2}\right)}\right) .
$$

The average fluid velocity in the pore can then simply be calculated by dividing $Q$ by the pore's cross section $\pi r_{p}^{2}$. Under the assumption $b>>a$, it can be shown the velocity at the small pore opening is proportional to $a$. This differs from the flow in cylindrical pores where the velocity is proportional to the square of the pore radius, which is a result of the conservation of mass in a fluid system.

With a similar approach as for the electric field calculation the flow field outside the pore is determined by constructing an artificial cone (Fig. 3.1) with a parameter $H$ that fulfils the condition

$$
\frac{8 \mu Q}{\pi} \int_{l}^{\infty} \frac{d z}{(a+H(z-l))^{4}} d z=P_{a c c 1}=\frac{3 \mu Q}{2 a^{3}} .
$$

Evaluating the integral yields $H=16 /(9 \pi)$, which sets the pitch of the cone above and below the pore. Unlike the case for the electric field, where the field was uniform across the pore or cone diameter, the flow velocity has a parabolic profile. The maximal velocity $v_{\max }$ is at the pore axis and is two times the average velocity $\bar{v}$, which is given by

$$
\bar{v}=\frac{1}{r^{2} \pi} \int_{0}^{r} \int_{0}^{2 \pi} v_{\max }\left(1-\left(\frac{\rho}{r}\right)^{2}\right) \rho d \rho d \phi=\frac{1}{2} v_{\max }
$$

The centre velocity in the three cone regions is then

$$
v_{\max }(z)=2 Q \times \begin{cases}\frac{1}{\pi\left(a+\frac{16(z-l)}{9 \pi}\right)^{2}} & \text { if } z>l, \\ \frac{1}{\pi\left(b-\frac{b-a}{l} z\right)^{2}} & \text { if } l \leq z \geq 0, \\ \frac{1}{\pi\left(b-\frac{16 z}{9 \pi}\right)^{2}} & \text { if } z<0 .\end{cases}
$$

The major advantage of this flow model is its simplicity and that it is easily adoptable to other pore shapes. As will be shown in Sec. 3.4, the semi-analytic models for both the electric and the flow field are able to predict the total current and flow rate through a conical pore in good agreement with computationally expensive FEM simulations. However the exact field distribution, especially the non negligible 
radial component at the pore constriction, cannot be studied with these simple models so that the particle trajectories and resulting resistive pulses are restricted to on-axis particles only.

\subsection{Transport Mechanisms of Particles in Solu- tion}

The RPS technique measures the current over time and translocating particles are identified by their current pulse. To obtain the full current-time relationship with the semi-analytic model the resistance calculation has to be combined with a model for the movement of the particle. Using the electric and the fluid fields calculated above, a model for the main transport mechanisms in TRPS, namely pressuredriven, electroosmotic, and electrophoretic transport has been developed. It has proven to be successful in the prediction of the time-dependence of the resistive pulses in TRPS [77]. In this section, first the main transport mechanisms relevant to the TRPS technique are discussed from a simplified point of view using the semianalytic approach. Then additional mechanisms, including the force caused by a focussed laser beam on a particle, are discussed. Additional physical detail to the transport model is then added in Sec. 3.4.5 using the FEM method.

There are numerous different mechanisms which affect the transport of particles through micro- and nanofluidic channels [83, 97]. The general description of the movement of a particle (hydrodynamic radius $r$ ) in a solution with viscosity $\mu$, under laminar conditions subjected to a force $F$ is the equation of motion

$$
m \frac{d v}{d t}=-\alpha v+F
$$

For low Reynolds numbers, Stokes' law is valid and the drag coefficient becomes $\alpha=6 \pi \mu r$. The equation has the solution

$$
v(t)=v_{\max }\left(1-\exp \left(\frac{-t}{\tau}\right)\right)
$$

and the particle will reach the final velocity

$$
v_{\max }=F / \alpha,
$$

with the time constant $\tau=m / \alpha$. $\tau$ determines how fast a particle gets to $v_{\max }$ and 
therefore how quickly it reacts to changes of the force $F$. For particles used in the experiments of this work the upper limit of $\tau$ is $\sim 1 \mu \mathrm{s}$, which is about three orders of magnitude faster than the time of a typical current pulse. The particle movement can therefore be modelled under quasi-steady state conditions, an approach often used in microfluidics [97, 98].

$\tau$ is related to the Stokes number

$$
S=\frac{\tau v_{\text {fluid }}}{L_{0}}
$$

where $v_{\text {fluid }}$ is the fluid velocity and $L_{0}$ the characteristic obstacle length, i.e. the diameter of the particle. For the experiments relevant to this work the maximal Stokes number will be reached for the largest particles $\left(r_{s}=1 \mu \mathrm{m}\right)$ and the fastest fluid velocities $\left(\sim 220 \mathrm{~mm} \mathrm{~s}^{-1}\right)$, yielding an upper limit for the Stokes number of $\sim 0.1$. As $S<<1$, particle inertia can be neglected and the particle trajectories closely follow the streamlines of the fluid [99]. However, if the force $F$ is exerted on the particle rather than the fluid, e.g. electrophoresis, streamline crossing can occur and particles may be transported against the direction of the fluid flow.

If the steady-state condition is valid, the Nernst-Planck equation is a more convenient way to calculate the particle transport rather than solving the equation of motion directly. In the form used in Eq. 3.26 it describes the total particle flux $\boldsymbol{J}$ of a charged species in a fluid moving at velocity $\boldsymbol{u}$ under the influence of a concentration gradient $\nabla c$ and an electric field $\boldsymbol{E}$ and is given by the expression

$$
\boldsymbol{J}=\boldsymbol{J}_{d i f f}+\boldsymbol{J}_{e p h}+\boldsymbol{J}_{c o n v}=-D \nabla c-\frac{z e}{k_{B} T} D c \boldsymbol{E}+c \boldsymbol{u} .
$$

The terms $\boldsymbol{J}_{\text {diff }}, \boldsymbol{J}_{\text {eph }}$ and $\boldsymbol{J}_{\text {conv }}$ represent the particle flux due to diffusive, electrophoretic and convective transport. $D$ is the diffusion constant, $z$ the particle valence, $e$ the electron charge and $c$ the particle concentration. With the Einstein relation $D=k_{B} T \nu$ ( $\nu$ is the general mobility, which is defined as the ratio between the drift velocity $u_{D}$ and the applied force on the particle), the particle flux due to the electrophoretic transport can also be written in the form $u_{D} c$ (compare also Eq. 3.38). Equation 3.26 covers a wide range of particles and is applied to ions as well as to microscopic particles. At the different length scales the relative magnitude of the terms vary strongly, e.g. convective transport is often dominant over diffusion and electrophoretic transport at the $\mu \mathrm{m}$-scale. The formalism is flexible so that additional transport mechanisms, e.g. dielectrotphoretic and magnetic transport can be incorporated in Eq. 3.26. An elaborate study of the relative strength of various 
transport mechanisms relevant to TRPS has been conducted by Willmott et al. [40].

\subsubsection{Pressure-Driven Transport}

With the assumption that the particle centre moves with the same velocity as the fluid and that its presence does not disturb the flow field significantly, the pressuredriven particle transport can be deduced with the semi-analytical model described above (Eq. 3.21). As the radial component of the fluid field is neglected, the model is limited to on-axis trajectories. For an NP2000 pore and an applied pressure of $100 \mathrm{~mm} \mathrm{H}_{2} \mathrm{O}(981 \mathrm{~Pa})$ a velocity of $\sim 220 \mathrm{~mm} \mathrm{~s}^{-1}$ is reached at the pore orifice. The velocity is directly proportional to the pressure and approximately inversely proportional to the size of the small pore opening. While pressure-driven transport is dominant at the upper range of the pore size used in the experiments, it competes with electrokinetic transport at the lower size range when small pressures $(<10 \mathrm{~mm}$ $\left.\mathrm{H}_{2} \mathrm{O}\right)$ are applied. In practice, the pressure is the main parameter in the coordination experiments that determines the particle velocity and therefore the throughput. Both proved to be crucial to the quality of the optical measurement (see Chaps. 6 and 7$)$.

\subsubsection{Electrokinetic Transport}

Electrokinetic transport covers a range of effects which are connected to a force acting on the electrical double layer that forms at a fluid-solid interface. In this work the main electrokinetic mechanisms are electroosmosis, the motion of a liquid in a channel induced by an electric field, and electrophoresis, the transport of charged species under the influence of an electric field. Dielectrophoresis, the particle transport due to a non-uniform electric field, is intrinsically weaker but can play a role in certain situations as it will be shown further below. In order to understand electrokinetic effects, the concepts of the double-layer and the $\zeta$-potential of a charged surface in an ionic solution are essential.

\subsubsection{The Potential of a Charged Surface in an Ionic Solution}

When a charged solid surface comes into contact with an ionic solution, counterions are attracted while co-ions are repelled, leading to a high and low concentration close to the surface respectively. The resulting structure is usually referred to as the Electrical Double Layer (EDL) [100] and is connected to an electrostatic potential. Far from the surface the ion concentration will reach its bulk value and the potential will decay to zero. A qualitative sketch of the ion distribution and the resulting potential close to a negatively charged surface is shown in Fig. 3.3. 


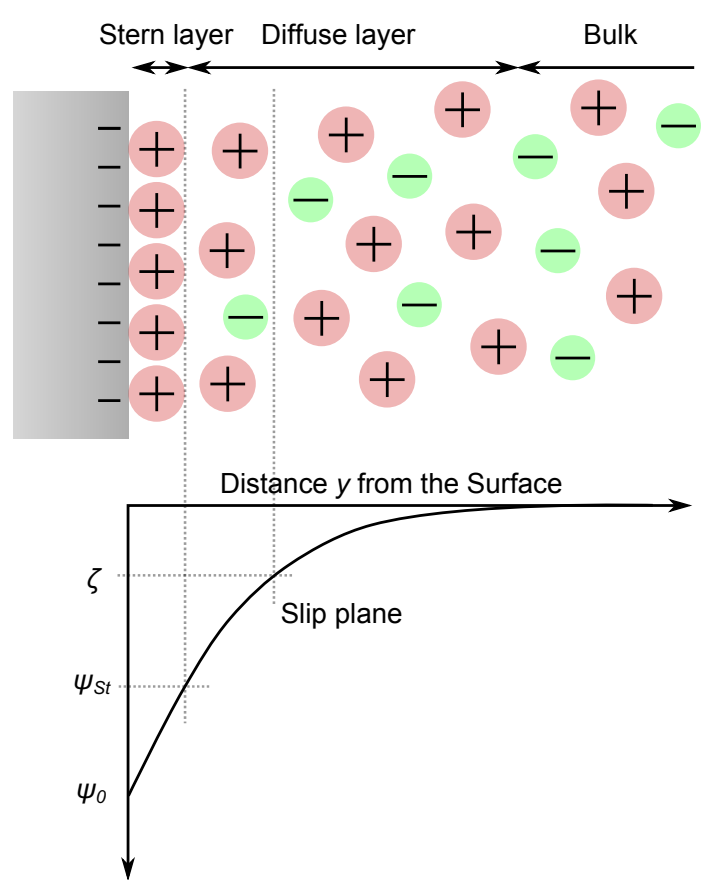

Figure 3.3: Schematic illustration of the EDL and the resulting electric potential close to a negatively charged surface (adapted from [83]). The Stern layer consists of immobile counter-ions closely attached to the surface, the diffuse layer of loosely attached co- and counter-ions with a surplus of counter-ions. The ion concentrations reaches its bulk value with equal amounts of co- and counter-ions (for a 1:1 electrolyte) at some distance away from the surface. The potential at the surface is $\psi_{0}$, at the Stern layer $\psi_{S t}$ and at the slip plane, which is located within the diffuse layer, is defined as the $\zeta$-potential.

A widely-used method to describe the EDL close to a charged surface in an ionic solution is the Poisson-Boltzmann equation. It was first used by Gouy and Chapman $[101,102]$ to determine the electrostatic potential and the ion concentration as a function of the distance from a charged surface. The equation is derived by relating the volumetric charge density given by

$$
\rho_{c}=e \sum_{i} z_{i} n_{i}
$$

with the electrostatic potential $\psi z_{i}$ is the valence and $n_{i}$ the concentration of each ion species. $\psi$ is determined by the Poisson equation

$$
\nabla^{2} \psi(y)=-\frac{\rho_{c}}{\epsilon_{0} \epsilon_{f l}}
$$

with $y$ the distance from the charged surface and $\epsilon_{f l}$ the relative permittivity of the surrounding media. For water at room temperature the value for the permit- 
tivity is 80.1 [103]. Equation 3.28 cannot be solved directly as the ion distribution is dependent on the potential itself. Assuming thermodynamic equilibrium, the concentration of each species follows a Boltzmann distribution given by

$$
n_{i}(y)=n_{i}^{0} \exp \left(-\frac{e z_{i} \psi(y)}{k_{B} T}\right)
$$

Combining Eqs. 3.27, 3.28 and 3.29 then leads to the Poisson-Boltzmann equation

$$
\nabla^{2} \psi(y)=-\frac{e}{\epsilon_{f l} \epsilon_{0}} \sum_{i} n_{i}^{0} z_{i} \exp \left(-\frac{e z_{i} \psi(y)}{k_{B} T}\right)
$$

The model is intrinsically a mean field approximation, in which ions interact with the average electrostatic field of all the neighbouring ions instead of with each ion individually. The method also neglects non-Coulombic interactions and does not take the finite ion size and molecular nature of water into account. The permittivity of the solvent is assumed to be constant, which is debatable close to a charged surface as polar molecules are prevented from freely moving around in a strong electric field. Therefore the topic is still the subject of recent research with frequent additions and improvements to the model $[104,105]$. However, the model works well for most situations relevant in colloidal science $[83,106]$. Note the close connection with the DLVO theory for colloid stability discussed in Sec. 4.3.3.

Equation 3.30 has, in its most general case, no analytic solution. For small potentials (below $25 \mathrm{mV}$ ), it can be linearised and solved analytically, which is known as the Debye-Hückel approximation [107]. The expression for the potential then simply becomes

$$
\psi=\psi_{0} \exp (-\kappa y)
$$

with the surface potential $\psi_{0}$ and the decay rate $\kappa .1 / \kappa=\lambda$ is called the screening or Debye length and is given by

$$
\lambda=\frac{1}{e N_{A}} \sqrt{\frac{\epsilon_{0} \epsilon_{f l} R T}{\sum_{i} z_{i}^{2} c_{m_{i}}}} .
$$

For practical reasons the molar concentration $c_{m_{i}}$ of each ion species, Avogadro's number $N_{A}$ and the gas constant $R$ are used in Eq. 3.32. The ion concentrations are within $0.5 \%$ of their bulk value for distances larger than $5 \lambda$. The upper bound for 
the Debye length relevant to the experimental section is $\sim 3 \mathrm{~nm}$, which is reached for an ion concentration of $c_{K^{+}}=c_{C l^{-}}=0.01 \mathrm{M}$. A uniform ion distribution in most parts of the pore is therefore expected.

The surface potential is linked to the surface charge density $\sigma$ in the linear approximation via the expression

$$
\sigma=\frac{\psi_{0} \epsilon_{f l} \epsilon_{0}}{\lambda}
$$

An analogous equation can be derived for the non-linear, one dimensional PoissonBoltzmann approach, called the Grahame-Equation [108, 109]

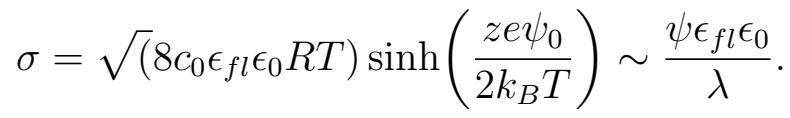

Its first order approximation is equivalent to Eq. 3.33.

The $\zeta$-Potential The ion distribution close to a charged surface is usually described with Stern's addition to the Gouy-Chapman Model [83, 110]. Stern introduced a layer of immobile, not hydrated counter-ions whose distance to the surface is limited only by their finite size. The tightly bound ions in the Stern layer are followed by more loosely attached ions in the diffusive layer (Fig. 3.3). Both contribute to the shielding of the surface charge. At some point in the diffusive layer the electrostatic force and the shear force, exerted from a tangential fluid flow, will be in balance so that the fluid velocity is zero. The electric potential at this "slip" plane is defined as the $\zeta$-potential $[100,111]$. Ions beyond the plane can move freely relative to the surface. The $\zeta$-potential is arguably more significant than the surface potential which, due to the shielding mechanisms, is beyond an experimental determination. It is a relevant parameter in colloid science and determines electrokinetic effects, colloidal stability and interactions. $\zeta$ depends on the surface charge density, the fluid viscosity and the ion molarity and $\mathrm{pH}$ of the solvent. Although under certain assumptions it can be calculated, a fully theoretical approach is not feasible in many situations, as the exact location of the slip plane remains unknown. In practice the $\zeta$-potential is measured indirectly by quantifying the electrophoretic mobility, which can be achieved using various techniques [111]. It has been shown that TRPS can be used to measure the $\zeta$-potential and the size of individual particles at the same time $[37,39]$. 


\subsubsection{Electroosmotic Flow}

The motion of liquid induced by an electric field across a fluid conduit is called electroosmotic flow. It originates from the Coulomb force exerted on the mobile ions beyond the slip plane, which consequently start moving along the field lines and drag the surrounding water along due to its viscosity. The resulting electroosmotic velocity can be obtained from the Navier-Stokes equations with the electric field times the charge distribution acting as an external body force. For the one dimensional case and a charge distribution defined by the Gouy-Chapman model the problem can be solved analytically. Rice and Whitehead [112] obtained the solution for the electroosmotic fluid velocity $v_{e o s}$ tangential to a charged surface with potential $\psi_{0}$ as a function of the distance $y$. It is given by the expression

$$
v_{\text {eos }}(y)=-\frac{\epsilon_{0} \epsilon_{f l} \psi_{0} E_{t}}{\mu}(1-\exp (-\kappa y))
$$

with $E_{t}$ the electric field component tangential to the surface. For $y>>1 / \kappa$ the exponential term can be neglected and the velocity becomes independent of $y$. This approximation is valid in the regions of a micro- or nanofluidic channel where the distance to the wall is much larger than the Debye length. When $\psi_{0}$ can be identified with $\zeta_{\text {wall }}$ of the surface, Eq. 3.35 becomes equivalent to the Smoluchowski formulation $[100,113]$ for the electroosmotic velocity

$$
v_{e o s}=-\frac{\epsilon_{0} \epsilon_{f l} \zeta_{w a l l} E_{t}}{\mu}
$$

In a cylindrical channel with a uniform electric field parallel to the walls this results in the typical "plug" flow profile. The electroosmotic fluid velocity adds to the convective term $J_{\text {conv }}$ in Eq. 3.26.

Streaming Potential and Current When a charged surface is subjected to a pressure-driven flow a streaming potential $U_{\text {str }}$ will build up between the ends of a channel [107] resulting in a corresponding streaming current. The origin of this effect is the electrical double layer at the channel walls, which leads to a net charge transport in a pressure-driven fluid field. The expression for the streaming potential for a cylindrical channel is given by

$$
U_{s t r}=-\frac{\epsilon_{0} \epsilon_{f l} \zeta_{w a l l} \Delta P \rho}{\mu}
$$


with $\Delta P$ the pressure difference and $\rho$ the electrical resistivity of the fluid. A similar expression could be obtained for a conical pore geometry, where a smaller value compared to Eq. 3.37 is expected due to a slower fluid flow at the same applied pressure. However, to estimate if the effect is relevant for the TRPS technique the calculation of an upper limit is sufficient. For the highest applied pressure of $100 \mathrm{~mm}$ $\mathrm{H}_{2} \mathrm{O}$ and a typical resistivity of $0.86 \Omega \mathrm{m}$ ([92]) for a solution of $100 \mathrm{mM} \mathrm{KCl}$, the streaming potential using Eq. 3.37 is $\sim 10 \mu \mathrm{V}$. This is negligible compared to the trans-membrane voltage used in TRPS, which is at least $20 \mathrm{mV}$.

\subsubsection{Electrophoretic Flow}

The migration of charged particles relative to a fluid under the influence of an electric field is called electrophoresis. It is again due to the formation of a double layer close to the particle's surface. The Coulomb force acts on the mobile ions beyond the slip plane, causing them to migrate and drag water molecules along. The surplus of counter-ions in the diffusive layer results in a net force on the particle in the opposite direction of the counter-ion movement. Electroosmosis and electrophoresis are therefore equivalent phenomena. In the thin layer or Smoluchowski approximation, which assumes that the radius $r_{s}$ of the particle is much larger than the Debye length the particle surface can be treated as a flat surface. The electrophoretic velocity of a particle with $\zeta_{\text {part }}$ is then given by

$$
\boldsymbol{v}_{e p h}=\frac{\epsilon_{0} \epsilon_{f l} \zeta_{\text {part }} \boldsymbol{E}}{\mu}
$$

The Smoluchowski approximation can be applied in TRPS where the smallest particle radius is $\sim 50 \mathrm{~nm}$ and the Debye length is on the order of $\sim \mathrm{nm}$. If the thin layer limit is not valid, a pre-factor which depends on the exact value of $r_{s}$ and $\lambda$ has to be added to Eq. 3.38 [83]. In the thick layer limit or Onsager approximation, where $r_{s}<<\lambda$, the hydrodynamic drag is significantly increased due to the higher apparent radius of the particle, and the pre-factor has a value of $2 / 3$.

$\zeta_{\text {part }}$ can be connected to an effective charge $Q_{\text {eff }}$ [114]. Although the total charge of the entire electrical double layer is neutral, the particle appears to carry a net charge because the surface charge is not fully compensated within the slip plane. Under the influence of an electric field the particle reaches its steady state velocity when the drag force $\boldsymbol{F}_{\text {drag }}=6 \pi \mu r_{s} \boldsymbol{v}_{e p h}$ is equal to the accelerating force $Q_{\text {eff }} \boldsymbol{E}$. Therefore the effective charge is connected to $\zeta_{\text {part }}$ through the expression

$$
Q_{e f f}=6 \pi r_{s} \epsilon_{0} \epsilon_{f l} \zeta_{p a r t}
$$


The effective surface charge density $\sigma_{\text {eff }}$ then becomes

$$
\sigma_{e f f}=\frac{3 \epsilon_{0} \epsilon_{f l} \zeta_{\text {part }}}{2 r_{s}}
$$

and is inversely proportional to the particle radius. The effective surface charge should not be confused with the surface group density of the bare particle, which is several orders of magnitude larger and can experimentally be determined by titration experiments [104]. With Eq. 3.39, ze $=Q_{\text {eff }}$ and the expression for the diffusion constant $D=k_{B} T /\left(6 \pi \mu r_{s}\right)$, the term $\boldsymbol{J}_{\text {eph }}$ in Eq. 3.26 becomes $\epsilon_{0} \epsilon_{f l} \zeta_{s} \boldsymbol{E} / \mu$, in agreement with Eq. 3.38.

Electrosmotic vs. Electrophoretic Transport Due to the similarity of the two transport mechanisms and the governing equations, the electrophoretic and electrosmotic velocity can be combined into one expression, which for a cylindrical channel with the electrodes attached to both ends $\left(|\boldsymbol{E}|=E_{t}\right)$ becomes

$$
v_{e k i n}=-\frac{\epsilon_{0} \epsilon_{f l}\left(\zeta_{\text {wall }}-\zeta_{\text {part }}\right) E_{t}}{\mu}
$$

Therefore, in the thin layer limit, a particle's net electrokinetic velocity will vanish if the $\zeta$-potentials of the channel walls and the particle are equal.

\subsubsection{Relevance for the Coordination Experiments}

The typical value for the $\zeta$-potential of the polystyrene beads used in the experiments is $-30 \mathrm{mV}$ [39]. The electrophoretic velocity can be obtained with Eq. 3.38 and the electric field calculated with the semi-analytic model (Eq. 3.6). For example, at an applied voltage of $1 \mathrm{~V}$ a value of $\sim 12 \mathrm{~mm} \mathrm{~s}^{-1}$ for $v_{e p h}$ is obtained at the orifice of a NP200 pore. For larger pores the absolute electrophoretic velocity is smaller because the electric field (Eq. 3.6) is inversely proportional to the pore size and also the applied voltage is lower for larger pores.

The $\zeta$-potential of a polyurethane surface for the molarity and $\mathrm{pH}$ typically used in TRPS is $\sim-20 \mathrm{mV}$ [115]. The electroosmotic velocity is therefore $\sim 2 / 3$ of the magnitude of the electrophoretic velocity and directed in the opposite direction. In the standard experimental configuration where a positive bias is applied to the lower electrode, the electrophoretic velocity for a negatively charged particles is directed downwards, while the electroosmotic velocity is directed upwards.

Together with the pressure-driven transport, electroosmosis and electrophoresis are the main transport mechanisms in TRPS. A comparison of their relative 


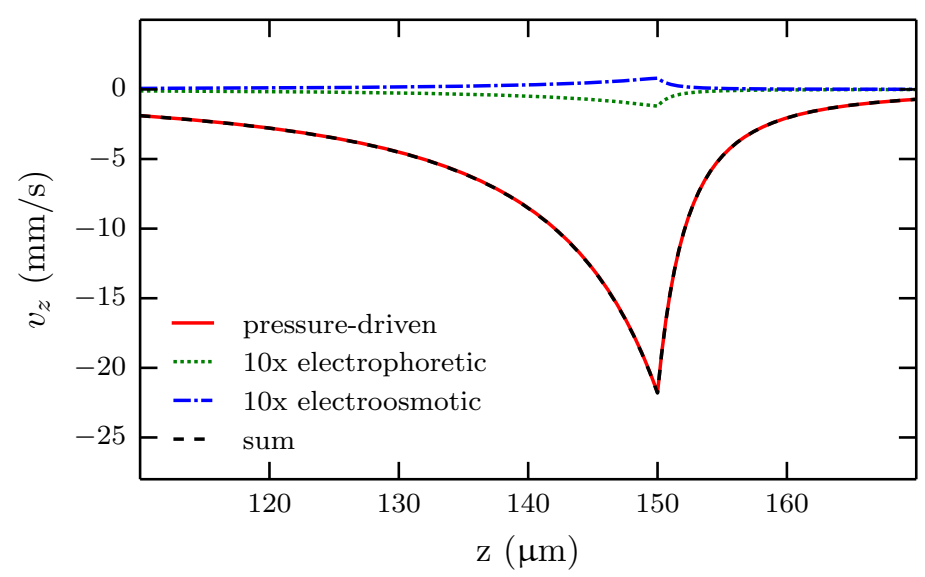

Figure 3.4: The pressure-driven, electroosmotic and -phoretic velocities of a negatively charged particle $\left(\zeta_{\text {part }}=-30 \mathrm{mV}\right)$ travelling through a NP2000 pore $\left(\zeta_{\text {wall }}=\right.$ $-20 \mathrm{mV}$ ), calculated with the semi-analytical model. A potential of $0.1 \mathrm{~V}$ and a pressure of $10 \mathrm{~mm} \mathrm{H}_{2} \mathrm{O}$ were applied. The electrokinetic velocities are multiplied by a factor of 10 to make them visible in comparison to the dominating pressure-driven velocity.

magnitudes is shown in Figs. 3.4 and 3.5 for NP2000 and NP200 pores. Pressuredriven transport dominates at the $\mu \mathrm{m}$-scale and electrokinetic effects can usually be neglected. For example, in a NP2000 pore the pressure-driven velocity at the centre of the orifice is $22 \mathrm{~mm} \mathrm{~s}^{-1}$ at an applied pressure of $10 \mathrm{~mm} \mathrm{H}_{2} \mathrm{O}$, which is two orders of magnitude higher than the electrophoretic velocity $\left(0.12 \mathrm{~mm} \mathrm{~s}^{-1}\right.$ at $0.1 \mathrm{~V})$. This value corresponds to a hydrodynamic velocity for an applied pressure of $\sim 0.05 \mathrm{~mm} \mathrm{H}_{2} \mathrm{O}$, a value far below the resolution of the pressure measurement. However, electrokinetic transport increases in relative strength with decreasing pore size so that for a NP200 pore the pressure can be used to balance electroosmosis and electrophoresis in order achieve a slow particle velocity (Fig. 3.5).

To obtain the time-current relationship with the semi-analytical model, the pressure-driven (Eq. 3.21), electrophoretic (Eq. 3.38) and electroosmotic (Eq. 3.36) velocities are calculated first for a starting particle position $z_{0}$ outside the pore. The particle position is then iteratively updated using a 4th order Runge-Kutta algorithm, so that the particle trajectory $z(t)$ is obtained for discrete time steps. The time-current relationship $I(t)$ can then be derived from $z(t)$ using the resistive pore model described in Sec. 3.2.1.

\subsubsection{Other Transport Mechanisms}

There are many other transport mechanisms in micro- and nanofluidics, e.g. transport based on magnetic, acousto- and thermophoretic forces. Here, only effects which are relevant to TRPS and the coordination technique, namely diffusion, grav- 


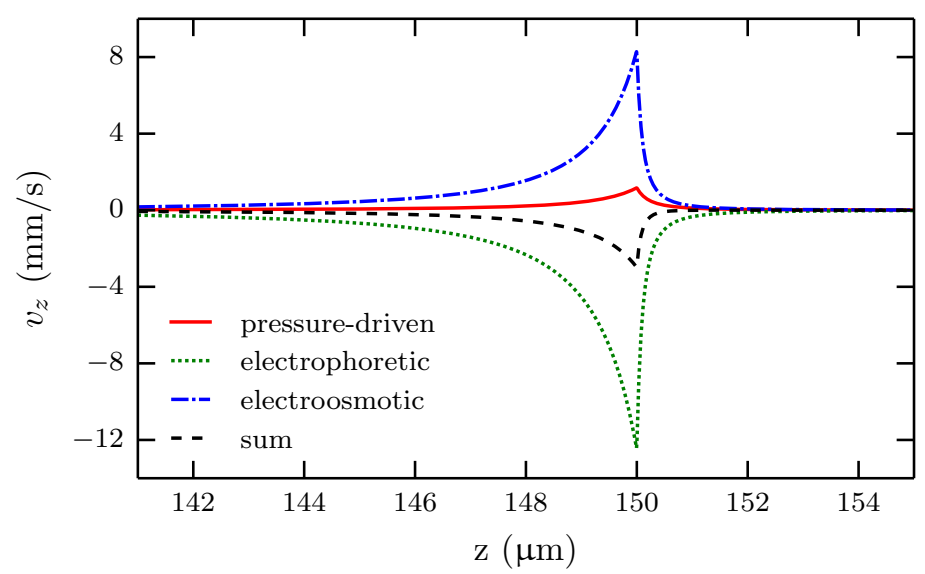

Figure 3.5: The pressure-driven, electroosmotic and -phoretic velocities of a negatively charged particle $\left(\zeta_{\text {part }}=-30 \mathrm{mV}\right)$ travelling through a NP200 pore $\left(\zeta_{\text {wall }}=\right.$ $-20 \mathrm{mV}$ ), calculated with the semi-analytical model. A potential of $1 \mathrm{~V}$ and a pressure of $-5 \mathrm{~mm} \mathrm{H}_{2} \mathrm{O}$ were applied.

ity, dielectrophoresis and the velocity due to the radiation pressure are discussed and an estimation of their strength is given.

Diffusion Particles undergo a constant stochastic Brownian motion due to their intrinsic thermal energy. Diffusion is a directed process if a concentration gradient exists in a solution and is described by Fick's first law of diffusion. The relevant parameter is the diffusion constant, which for a freely moving sphere in solution is given by

$$
D=\frac{k_{B} T}{6 \pi \mu r_{s}}
$$

It is indirectly proportional to the particle diameter so diffusion can play an important role for the particle transport in nm-sized pores [116, 117].

For the particles used in this work the diffusion constant is in the range from $0.2 \mu \mathrm{m}^{2} \mathrm{~s}^{-1}$ (1 $\mu \mathrm{m}$ particle radius) to $2 \mu \mathrm{m}^{2} \mathrm{~s}^{-1}$ (100 $\mathrm{nm}$ radius). The relationship between the observed time length $t$ and the one dimensional diffusion length is given by $L=\sqrt{2 D t}$. The time a particle with $r_{s}=100 \mathrm{~nm}$ takes to cross a characteristic length in TRPS pore diameter (500 nm for NP200) is $6 \mathrm{~ms}$. This translates into a "diffusion velocity" of $0.1 \mathrm{~mm} \mathrm{~s}^{-1}$, which is at least two orders of magnitude smaller than the typical velocity reached through electrokinetic transport at this length scale so that diffusion can be neglected in the transport model. The diffusion is accounted for in the Nernst-Planck equation by the term $J_{\text {diff }}$ (Eq. 3.26). Usually the particle concentration is equally distributed on both sides of the pore and diffusion does not contribute to a net transport in TRPS. However, the stochastic movement will 
contribute to the noise seen in the resistive pulse signals, especially at small length scales.

Gravity A spherical particle in a fluid under the influence of gravity reaches the settling velocity given by

$$
v_{\text {sett }}=\frac{2}{9} \frac{\left(\rho_{\text {part }}-\rho_{f l}\right) g r_{s}^{2}}{\mu}
$$

$g$ is the acceleration due to gravity, $\rho_{\text {part }}$ and $\rho_{f l}$ the densities of the particle and the surrounding fluid. For a polystyrene particle $\left(\rho_{\text {part }}=1.05 \mathrm{~kg} \mathrm{~m}^{-3}\right)$ with a radius of $1 \mu \mathrm{m}$ the settling velocity is $0.11 \mu \mathrm{m} \mathrm{s}^{-1}$, indicating that gravity is not a relevant transport mechanism in TRPS. However, the velocity is large enough that a $\mu \mathrm{m}$-sized particle close to a surface settles within several min and becomes immobile. This was used to investigate the photo-bleaching dynamics of single fluorescent labelled particles (Sec. 4.3.3).

Dielectrophoresis Dielectrophoresis is caused by the force on a dielectric particle in an electric field gradient and has been used to move and focus beads in microfluidic channels $[118,119]$. The highest field gradients in the axial and radial directions in a conical pore are expected to occur at the pore orifice and its edge respectively. For a homogeneous sphere in a medium the analytic expression for the dielectrophoretic force is

$$
\boldsymbol{F}_{\text {dep }}=2 \pi r_{s}^{3} \epsilon_{0} \epsilon_{f l} f_{C M} \nabla|\boldsymbol{E}|^{2}
$$

$f_{C M}=R e\left(\frac{\epsilon_{\text {part }} *-\epsilon_{f l} *}{\epsilon_{\text {part }}^{*}+2 \epsilon_{f l} l^{*}}\right)$ is the Clausius-Mossotti factor with the complex permittivity of the particle $\epsilon_{\text {part }} *$ and the fluid $\epsilon_{f l} *$. Depending on the relative permittivities of the particle and the solution $f_{C M}$ can be positive or negative and determines in which direction the force is directed. For a perfectly insulating particle it becomes $-1 / 2[120]$ and the resulting motion is directed away from regions of high field density. In the case of a conical pore the dielectrophoretic force will push the particle out of the orifice and into the middle of the pore. With the identity $\nabla|\boldsymbol{E}|^{2}=2 \boldsymbol{E} \cdot \nabla \boldsymbol{E}$ the force can be written in the form

$$
\boldsymbol{F}_{d e p}=-2 \pi r_{s}^{3} \epsilon_{0} \epsilon_{f l} \boldsymbol{E} \cdot \nabla \boldsymbol{E}
$$

In order to estimate the magnitude of the dielectrophoretic force in TRPS, data 
from FEM calculations for the field were used as the semi-analytic model does not allow for a calculation of the field gradient due to the discontinuity at the orifice (see Fig. 3.9 (a)). The semi-analytic model also neglects the radial components of the electric field, which is necessary to determine the radial force. The evaluation of the electric field and the gradients for a NP2000 pore with $V_{0}=0.1 \mathrm{~V}$ at the closest point to the pore edge that a particle can approach yields a force of $3.4 \times 10^{-14} \mathrm{~N}$ in the axial and $0.55 \times 10^{-14} \mathrm{~N}$ in the radial direction. The $z$ and $r$-components of the resulting velocity calculated with Eq. 3.24 are $v_{d e p_{z}}=2 \mu \mathrm{ms}^{-1}$ and $v_{d e p_{r}}=$ $0.32 \mu \mathrm{m} \mathrm{s}^{-1}$. These values are several orders of magnitude below the velocities resulting from the pressure-driven transport (compare Fig. 3.4) and dielectrophoretic transport can therefore be neglected at these length scales and range of voltages.

For a NP200 pore and $V_{0}=0.1 \mathrm{~V}$ the field in Eq. 3.45 is 10 times and the gradient 100 times larger. However, as the particle is also 10 times smaller, the factor $r_{s}^{3}$ in Eq. 3.45 cancels out this gain and the forces have the same value as above. The resulting velocity increases by a factor of 10 (see Eq. 3.24), which is still negligible compared to the other transport mechanisms (Fig. 3.5). If a voltage of $1 \mathrm{~V}$ is applied both the field and the gradient will gain by a factor of 10 and $v_{d e p_{z}}=2 \mathrm{~mm} \mathrm{~s}^{-1}, v_{d e p_{r}}=0.32 \mathrm{~mm} \mathrm{~s}^{-1}$. The $z$-velocity is then comparable to the other transport mechanisms and will reduce the translocation speed through the pore as it is directed towards the pore constriction. The $r$-component of the force pushes the particle closer to the middle of the pore. Assuming the particle is in the constriction region for about $0.1 \mathrm{~ms}$ it will move $\sim 30 \mathrm{~nm}$ towards the pore centre. Although this is only a rough estimate it shows that dielectrophoresis can lead to a focussing effect in pores used in TRPS, and the effect is proportional to the applied voltage in a quadratic fashion.

The estimate above uses the electric field $\boldsymbol{E}$ without the disturbance of a particle. It has been shown that this approach only delivers correct results if the particle size is much smaller than the characteristic length scale of the electric field [121]. This is not the case in the situation discussed above and a more realistic approach to derive the involved forces incorporates the integral over the Maxwell stress of the particle surface. A FEM simulation that takes that into account is presented in Sec. 3.4.5.

The Radiation Pressure on a Spherical Particle When an electromagnetic wave hits an object, part of the energy will be absorbed by the matter while the other part will be scattered. Apart from energy, light also carries momentum in the direction of propagation with a magnitude of $E_{f} / c$, with $E_{f}$ the energy flux and $c$ the speed of light. Both absorption and scattering transfer momentum from the electromagnetic field to the object. The resulting force is called radiation pressure and is usually negligible in everyday circumstances. However, it is well established 
to manipulate particles with tightly focussed laser beams [122]. The effect may be of relevance for the coordination technique. Experimentally, a pushing force can be observed via the microscope when the laser beam at maximal power hits a $\mu \mathrm{m}-$ scale particle freely diffusing in solution. As an estimation of the particle velocity is difficult to obtain from this observation, a calculation of the force is presented in the following in order to clarify if the effect is comparable to the other transport mechanisms in TRPS.

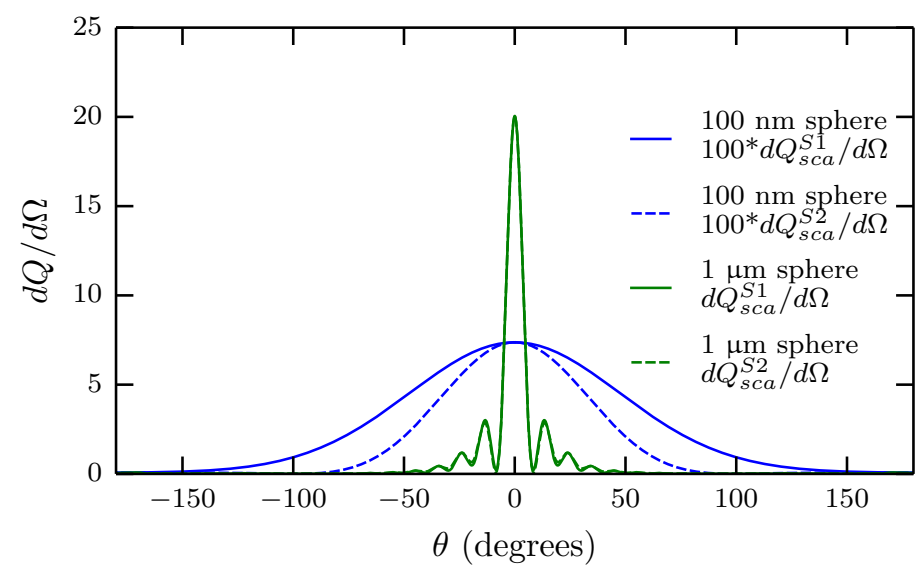

Figure 3.6: The normalized differential scattering cross section as a function of the scattering angle $\theta$ for a $100 \mathrm{~nm}$ (blue) and a $1 \mu \mathrm{m}$ (green) spherical polystyrene particle, with polarization parallel (-) and perpendicular (- -) to the scattering plane.

Under the assumption that the amplitudes of the incident and scattered fields are constant in time, the force $\boldsymbol{F}$ on an object with surface $S$, averaged over the period $2 \pi / \omega$, is given by

$$
\boldsymbol{F}=\int_{S} d S<\stackrel{\leftrightarrow}{T}(\boldsymbol{r})>\hat{\boldsymbol{n}}
$$

where $\stackrel{\leftrightarrow}{T}$ is the Maxwell stress tensor and $\hat{\boldsymbol{n}}$ is the unit vector pointed in the outward direction normal to $S$. For spherical particles with sizes comparable to the wavelength of the light, Mie-theory can be applied to this problem. An introduction to the topic can be found in [123], and here only the relevant expressions are given. The force $\boldsymbol{F}$ in the direction of the propagation $\hat{\boldsymbol{n}}^{\boldsymbol{i n c}}$ of the incoming plane wave with intensity $I^{i n c}$ can be expressed in terms of the extinction cross section $C_{e x t}=C_{a b s}+C_{s c a}\left(C_{a b s}\right.$ is the absorption cross section, $C_{s c a}$ is the scattering cross section) and the asymmetry parameter $<\cos \theta>$ 


$$
\begin{aligned}
\boldsymbol{F} \cdot \hat{\boldsymbol{n}}^{i n \boldsymbol{c}} & =\frac{1}{c} I^{i n c} C_{e x t}-\frac{1}{c} I^{i n c} \int_{4 \pi} d \hat{\boldsymbol{r}}\left(\hat{\boldsymbol{r}} \cdot \hat{\boldsymbol{n}}^{i n c}\right) \frac{d C_{s c a}}{d \Omega} \\
& =\frac{1}{c} I^{i n c}\left(C_{e x t}-C_{s c a}<\cos \theta>\right) .
\end{aligned}
$$

Photons which get absorbed transfer a momentum per unit time of $\frac{I^{i n c}}{c} C_{a b s}$ to the particle. For the particles used in the experimental sections this term can be assumed to be zero as polystyrene has no absorption bands in the visible region. Photons which are elastically scattered at the object transfer part of their momentum, and the net rate of momentum exchange is $\frac{I^{i n c}}{c} C_{\text {sca }}(1-<\cos \theta>)$. Here $<\cos \theta>$ is the average cosine of the scattering angle $\theta$ and is called the asymmetry factor. It is positive for a particle scattering more in the forward than in the backward direction and vanishes for isotropic scattering. The radiation profiles of two different sized polystyrene particles in an aqueous medium show that forward scattering increases with particle size (Fig. 3.6). The expressions for $C_{\text {ext }}$ and $C_{s c a}<\cos \theta>$ for a spherical particle are given by a sum over scattering coefficients, which consist of Bessel functions and can be found in [123]. The calculations were conducted using a freely available Matlab code developed by Le Ru and Etchegoin [124]. Usually the cross sections are normalized to the geometric cross section $G=\pi r_{s}^{2}$ of the particle and the radiation-pressure efficiency factor is then defined as

$$
Q_{p r}=\frac{C_{p r}}{G}=\frac{C_{e x t}-C_{s c a}<\cos \theta>}{G} .
$$

The radiation force $F_{\text {rad }}$ of a laser beam on a particle that is located at the waist position of the beam can then calculated with the equation

$$
F_{r a d}=\pi r_{s}^{2} \frac{Q_{p r}}{c} \frac{2 P}{\pi w_{0}^{2}}
$$

with $P$ the total power of the laser and $w_{0}$ the size of the waist. For Eq. 3.49 to be valid it is assumed that the power density is approximately uniform over the particle's cross section. Apart from scattering also the absorption by the fluorescent dyes embedded in the particle matrix will contribute to the radiation pressure. However, as the absorption cross section of a $100 \mathrm{~nm}$ sphere labelled with "Surf-Green" dye molecules is $2.1 \times 10^{-12} \mathrm{~cm}^{2}$ (Sec. 4.3.3) and about a magnitude smaller than $\pi r_{s}^{2} Q_{p r}$ $\left(2.51 \times 10^{-11} \mathrm{~cm}^{2}\right)$, it will only contribute marginally to the force. Re-emission will have no net effect to the particle momentum as the fluorescence is isotropic over all angles. 
In the experiments a maximal laser power of $P \sim 10 \mathrm{~mW}$ is available. For a $100 \mathrm{~nm}$ polystyrene particle (refractive index $n=1.6$ ) in aqueous medium the value of $Q_{p r}$ was calculated to be 0.08 . With a laser beam waist of $0.4 \mu \mathrm{m}$ the force on the particle is $F_{p r}=3.35 \times 10^{-13} \mathrm{~N}$ and yields a particle velocity of $0.18 \mathrm{~mm} \mathrm{~s}^{-1}$. As usually laser powers of at least one order of magnitude lower are used, the pushing effect can be neglected in the experiments. For $1 \mu \mathrm{m}$ particles $\left(Q_{p r}=0.32\right)$ and a beam waist of $2 \mu \mathrm{m}$ the maximal velocity becomes $0.31 \mathrm{~mm} \mathrm{~s}^{-1}$. This value agrees qualitatively with the visual observation using the microscope camera that the beads can be pushed when hit by the laser beam at full power, but is too small to have a strong influence on the particle dynamics in the coordination experiments.

Although the calculation above is physically accurate, only an approximate estimate is often sufficient to decide if the effect is relevant for a specific experiment. This can be done by calculating the upper limit for the radiation force assuming maximal momentum transfer, which, for a fully reflected beam, is two times the total momentum carried by the light. The resulting velocity is then simply given by

$$
v_{\max _{\text {rad }}}=\frac{2 P}{6 \pi \mu r_{s} c}
$$

With a maximal laser power of $10 \mathrm{~mW}$ and a particle radius of $100 \mathrm{~nm}$ this results in a velocity of $17.7 \mathrm{~mm} \mathrm{~s}^{-1}$, which is comparable to the particle velocity in TRPS. However as the used laser power in this work does not exceed 1/50 of the maximal power, an influence of the radiation pressure on the particle transport can be excluded.

\subsection{FEM Modelling}

The FEM method has become an important tool for modelling the electric field, the fluid flow, the ion distribution and the resistive pulse in RPS devices [125-132]. This is because it is very difficult or even impossible to find analytic solutions of the underlying equations for arbitrary shaped pores, especially with the addition of a particle. With the recent increase in computational power even complex coupled FEM models can be solved on a desktop PC in a reasonable amount of time. A detailed discussion of the FEM method can be found in [133], and here only a brief introduction will be given. The method is then applied to model the electric and fluid velocity fields in and around conical micro- and nanopores similar to the ones used in the experimental section. The results are compared to the semi-analytic models described above.

In the next step, a spherical insulating particle is introduced into the pore and 
the resistive pulse is calculated as a function of its position. The results are compared to the semi-analytic model and various expressions used in the RPS literature. Then, the implementation of pressure-driven and electrokinetic transport in the FEM model is discussed. The use of the Arbitrary Lagrangian Euler (ALE) method makes it possible to account for the non-zero particle size and influences of the particle on the field, which can result in significant difference to a model that treats the particle as a point. The effect of the particle trajectory on the resistive pulse and the strength of flow focussing and dielectrophoretic focussing are discussed. In a next step the ion distribution is calculated by a model that solves the Poisson-Boltzmann, Nernst-Planck and the Navier-Stokes equation simultaneously. It successfully predicts current rectification and bi-phasic pulses, both of which have been observed experientially in TRPS [134]. At the end of this section, the method was used to find the mechanical Eigenfrequencies of a pre-stretched thermoplastic polyurethane membrane as these were suspected to play a role in the origin of the laser induced current noise observed in the experiments.

\subsubsection{Introduction to FEM}

Many problems in science and nature can be described in terms of Partial Differential Equations (PDE). These systems can often not be solved analytically over arbitrary shaped domains and numerical methods to find approximate solutions have to be used instead. One of these is the Finite Element Method (FEM), which was first proposed by Courant in 1943 when he introduced special linear functions defined over small triangular regions to solve mechanical torsion problems [135]. With the possibility to use computers the method became widespread in 1960 and the expression "finite element" was first used [136]. Over the years many important contributions by several authors [137-139] made the method more popular, so that FEM is nowadays not only applied in structural mechanics but in a wide variety of physical disciplines, including heat conduction, fluid dynamics, electromagnetism and many others.

A typical work flow for the method involves drawing the geometry of the problem, assigning the material properties and defining the boundary conditions. Then, the computational domain is divided up into small elements of usually triangular, but also square or polygon shape. This step is called meshing and is crucial for the stability and convergence of the solver algorithm. According to the underlying PDEs each of these mesh elements is assigned respective equations, either of linear or polynomial form. These element equations are then recombined into a global system of equations, which can be solved numerically with common algorithms by minimizing an associated error function. 
For this work the popular FEM software COMSOL (version 4.4) was used, which has predefined modules for electrical, mechanical, fluid flow, chemical applications, etc. and is capable of solving coupled problems. It has a graphical user interface for convenient handling and provides tools for post-processing steps like visualization of the results, plotting and data manipulation. Data can also be exported for further processing.

\subsubsection{FEM Simulation of the Electric Field}

The governing equation for the calculation of the electric field in any geometric domain with conductivity $\sigma$ can be deduced as follows. With Ohm's law the current density $\boldsymbol{j}$ is given by $\boldsymbol{j}=\stackrel{\leftrightarrow}{\sigma}(\boldsymbol{r}) \boldsymbol{E}$. Using the continuity equation $\frac{\partial \rho}{\partial t}+\nabla \cdot \boldsymbol{j}=0$ and the field-potential relation $\boldsymbol{E}=-\nabla \psi$, under the assumption that there are no current sources or sinks in the domains the governing equation for the problem is $\nabla \cdot(\stackrel{\leftrightarrow}{\sigma}(\boldsymbol{r}) \nabla \psi)=0$. For an isotropic conductivity this becomes the Laplace equation

$$
\nabla^{2} \psi=0
$$

which has to be solved within the specified geometry. To ensure that there is a unique solution to the problem boundary conditions have to be imposed on the interfaces.

\subsubsection{Model Parameters and Boundary Conditions}

Due to the cylindrical symmetry of the pore the initial simulations are done using an axial symmetric geometry, which mimics the actual 3D shape, but reduces the computational time significantly. The simulated geometry consists of a conical shaped pore and two cylindrical reservoirs attached to its openings as shown in Fig. 3.7. The origin of the axial coordinate is at the large pore opening. A value of $z$ between $0 \mu \mathrm{m}$ and $150 \mu \mathrm{m}$ is therefore within, values lower than $0 \mu \mathrm{m}$ or higher than $150 \mu \mathrm{m}$ outside the pore.

The pore membrane is made of thermoplastic polyurethane and is assumed to be an ideal insulator in the simulation. As no current can flow into or out of an insulator, the associated boundary condition is $\boldsymbol{n} \cdot \nabla \psi=0$, meaning that the component of the electric field normal to the boundary has to vanish. Apart from the pore and membrane walls, the vertical walls of the reservoirs, which are made out of Polyether Ether Ketone (PEEK), are also assigned this condition. COMSOL's "Electric Currents" module is used to define the boundary conditions and compute the solutions in this section. Analogous to the experimental setup, the top and 


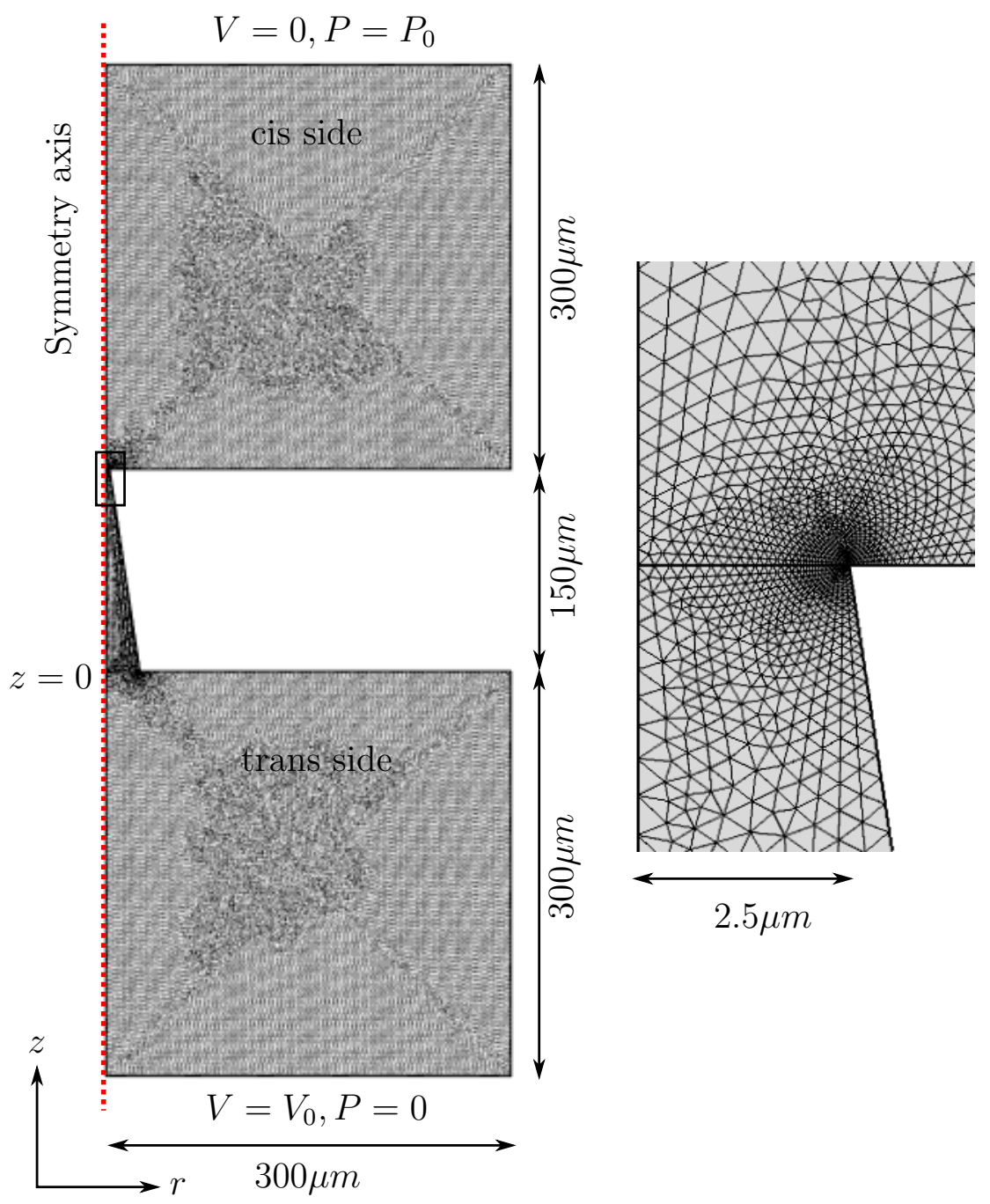

Figure 3.7: The 2D axial symmetrical geometry of a conical pore (NP2000) with the boundary settings and a typical mesh used for the FEM simulations. On the right side a magnification of the pore orifice shows that the mesh density increases towards the pore edge.

bottom boundaries of the model are the electrodes and their potentials are set to 0 and $V_{0}$ respectively. The pore and the reservoirs are filled with an electrolyte solution, which for most experiments contains $100 \mathrm{mM} \mathrm{KCl}$ and has a resistivity $\rho=$ $1 / \sigma$ of $0.86 \Omega \mathrm{m}[92]$. As the pore dimensions are much larger than the thickness of the EDL the electrolyte can be seen as a conductor with homogeneous conductivity. However, the exact ion distribution has to be taken into account under certain circumstances, which will be further discussed in Sec. 3.4.6.

In all simulations it was tested that a further refinement of the mesh resolution has no significant (less than 0.1\%) influence on the results. COMSOL's inbuilt adaptive mesh refinement algorithm can be used to locate areas where a better mesh resolution is required. Typically the predefined mesh size "fine" is sufficient for most parts of the simulated geometry. However, a finer mesh is needed at the 
corners of the pore orifice. Therefore the total number of mesh elements along the pore openings is set to 25 and distributed in a geometric sequence (element ratio of $5)$ so that the most dense mesh is at the pore edge. In simulations where a particle is involved the same can be achieved by setting the corner refinement factor to 0.01. A detailed image of the generated mesh close to the small pore opening of a NP2000 is shown in Fig. 3.7.

In a real TRPS experiment the reservoirs above and below the pore are several $\mathrm{mm}$ large. An exact representation of the real geometry is not feasible in the simulations due to the large number of mesh elements and therefore long computational time. However it can be expected that the influence of the reservoir sizes on the results can be neglected if they are large enough. It was found that reservoirs with a side length of $300 \mu \mathrm{m}$ are sufficient. A larger reservoir size has no significant (less than $0.1 \%$ ) influence on the results. Similar proportions for pore and reservoir size have been successfully used in the literature [128, 140, 141] before. The simulations have $\sim 125000$ degrees of freedom and the calculation takes $\sim 3 \mathrm{~s}$ on a PC with a $2.7 \mathrm{GHz}$ "Intel i5"-processor and 8 GB RAM.

\subsubsection{Results}

To test the validity of the model setup the electric field in a cylindrical channel (no attached reservoirs) with a radius $r_{c y l}=2.5 \mu \mathrm{m}$ and a length $l=150 \mu \mathrm{m}$ was simulated. Between top and bottom boundaries a potential difference of $V_{0}=0.1 \mathrm{~V}$ was applied. It was found, as expected, that the potential between the electrodes decreases in a linear fashion and the electric field lines are normal to the cross section of the cylinder. The current $I_{0}$, obtained by integrating the current density over the lower boundary, has a value of $15.2210 \mathrm{nA}$. This value agrees very well with the theoretical prediction $\left(I_{0}=V_{0} \pi r_{c y}^{2} /(\rho l)\right)$ of $15.22099 \mathrm{nA}$.

The results for a NP2000 pore and an applied voltage of $V_{0}=100 \mathrm{mV}$ are shown in Figs. 3.8, 3.9, 3.10 and 3.11. The electric potential (Fig. 3.8) along the pore's symmetry axis shows good agreement with the semi-analytic results. Most of the potential drop occurs close to the small pore orifice, but the access resistance, especially from the upper boundary to the small pore orifice, is not negligible. The values for the potential at the small/large pore orifices are $12.63 \mathrm{mV} / 98.68 \mathrm{mV}$ for the FEM and $12.04 \mathrm{mV} / 98.88 \mathrm{mV}$ for the semi-analytic model. The current $I_{0}$ through the pore is extracted from the simulation by integrating the normal current density $\boldsymbol{j}=\sigma \boldsymbol{E}$ over the lower electrode in the axial symmetric geometry. The current is slightly lower in the FEM model (131.252 nA) compared to the semi-analytical model (133.051 nA). This can be understood as the semi-analytic model assumes an infinite size of both electrodes. The different curvature of the potentials, especially close to the small pore orifice (inset in Fig. 3.8), leads to a discrepancy of the electric 


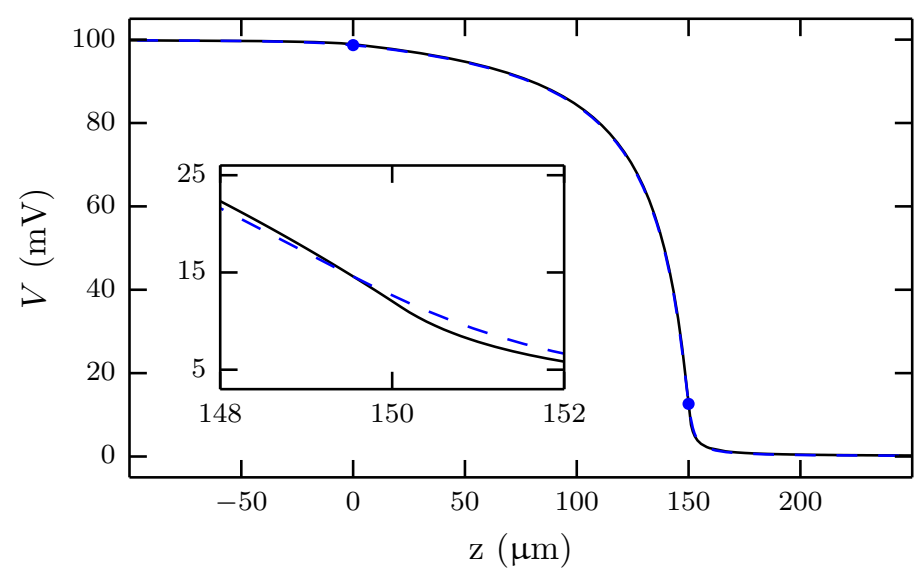

Figure 3.8: The electric potential along the $z$-axis of a NP2000 pore $\left(V_{0}=0.1 \mathrm{~V}\right)$ calculated with the semi-analytic model $(-)$ and the FEM simulation (- -). The values at the pore openings are indicated by the blue dots. The region around the small pore opening is magnified (inset).

field between the models.

Figure 3.9 (a) shows the axial component of the electric field along the $z$-axis. The semi-analytic model, which assumes a uniform electric field across the pore, varies from the FEM model both in absolute value and curve shape. Note that the discontinuity of the field-gradient at the pore orifice for the field that appears in the semi-analytic model does not appear in the FEM results, making the calculation of dielectrophoretic transport possible. The FEM simulations show that the field increases steadily towards the pore edge, reaching more than twice its on-axis value. The radial component (Fig. 3.9 (b)), which vanishes along the pore axis, has its highest value, about half of the maximal axial component, at the pore edge. A change in the direction of the radial component is observed when moving out of the pore. Both the axial and the radial components are also presented in a $2 \mathrm{D}$ intensity plot in Fig. 3.10, showing a high variability across the pore orifice and a rapid decrease outside the pore. To additionally support the simulation outcomes and prove that these are not due to an artefact created by the sharp pore edge, which can lead to numerical instabilities in FEM simulations, the same geometry with a rounded pore edge $(0.125 \mu \mathrm{m}$ radius $)$ was simulated. The results, shown in Fig. 3.10 (b), do not differ significantly, with only the position of the highest field moving slightly inside the pore.

The equipotential lines depicted in Fig. 3.11 (a) show a non-trivial shape. The pore cross sections do not, especially close to the pore orifice, reflect equipotential surfaces as assumed in the semi-analytical model. The electric field lines (Fig. 3.11 (b)) have a radial component, which even exceeds the axial component close to the pore edge. 


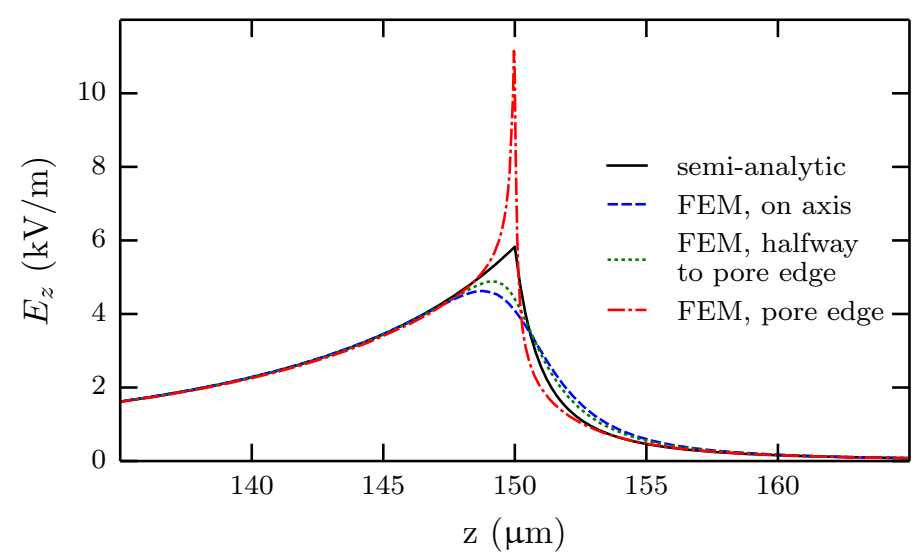

(a)

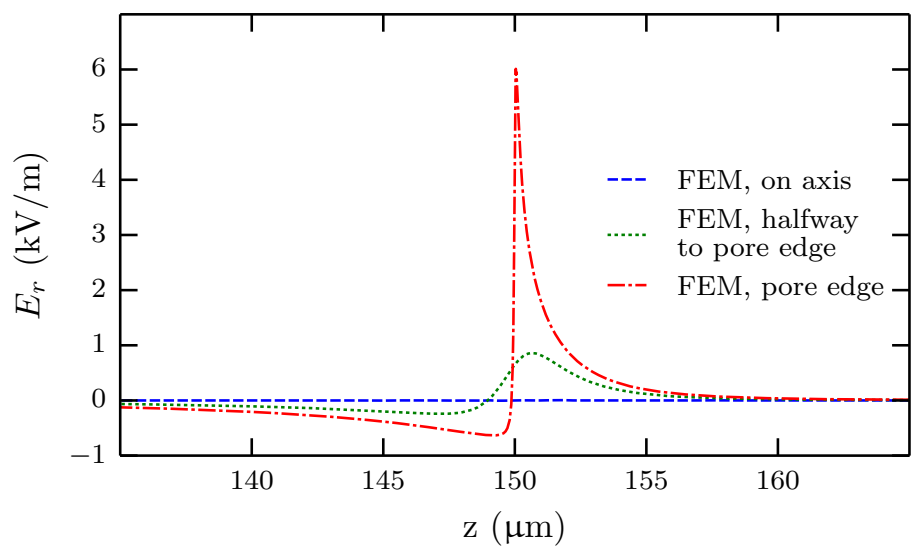

(b)

Figure 3.9: (a) The axial component of the electric field along the $z$-axis of a NP2000 pore $\left(V_{0}=0.1 \mathrm{~V}\right)$ obtained with the semi-analytic and the FEM model for three radial positions. (b) The radial component of the electric field obtained with the FEM model.

Influence of the Electrode Shape The size and shape of the electrodes used in the experiments are not exactly specified and can also vary for different setups. While the original qNano setup has a toroid with $2 \mathrm{~mm}$ inner and $3 \mathrm{~mm}$ outer radius as the upper electrode, the adapted setup for the coordination experiment uses a toroid with $6 \mathrm{~mm}$ inner and $7 \mathrm{~mm}$ outer radius. A structure made of silver paste resembling a cone with a hole in the middle is used as lower electrode. In all cases, the electrode dimensions are in the order of $\mathrm{mm}$ and are placed several $\mathrm{mm}$ away from the pore.

In order to estimate the influence of the electrode size and shape on the results, four different electrode geometries were simulated. Apart from the circular shaped electrodes used above, a circular ring geometry (inner radius $200 \mu \mathrm{m}$, outer radius $300 \mu \mathrm{m}$ ), a "point" (actual circular geometry with $1 \mu \mathrm{m}$ radius centred around the symmetry axis) and a hemispherical geometry with $500 \mu \mathrm{m}$ radius were used as electrodes. Apart from the "point" electrode, which shows a significant potential 


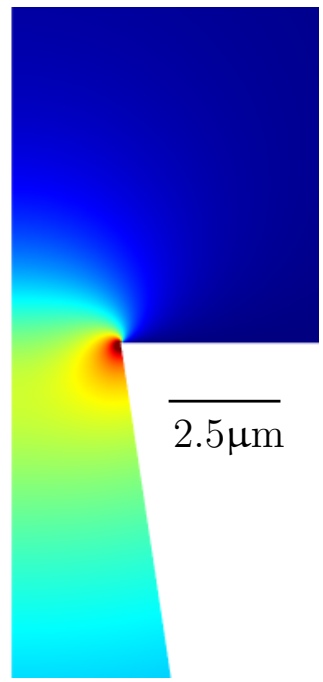

(a)

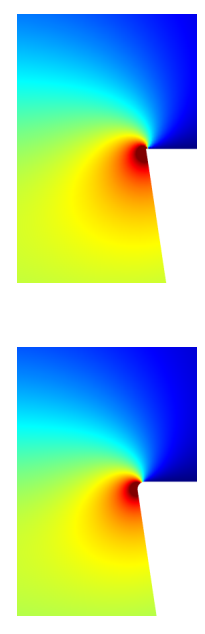

(b)

)

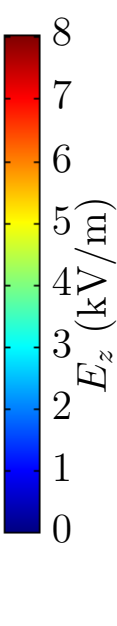

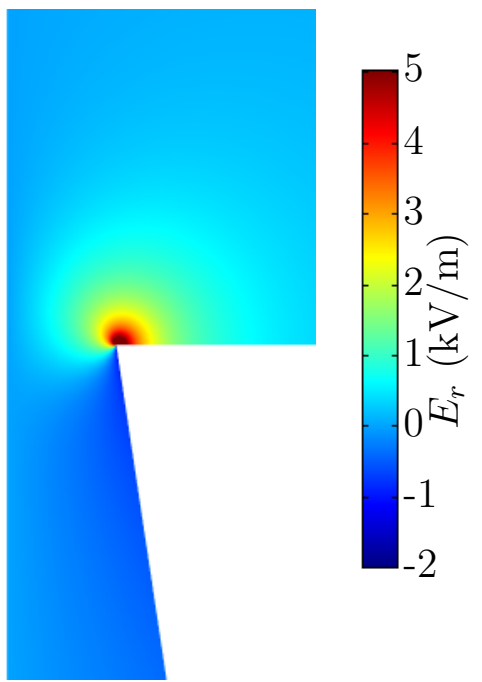

(c)

Figure 3.10: 2D intensity plots of the (a), (b) axial and (c) radial components of the electric field in an NP2000 pore $\left(V_{0}=0.1 \mathrm{~V}\right)$. The results for the axial component between a sharp (top) and a rounded (bottom) pore edge are compared in (b).

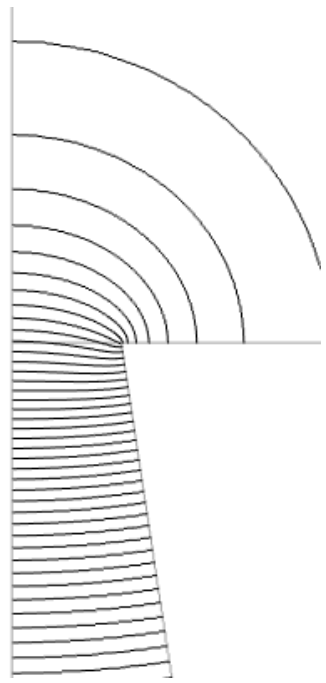

(a)

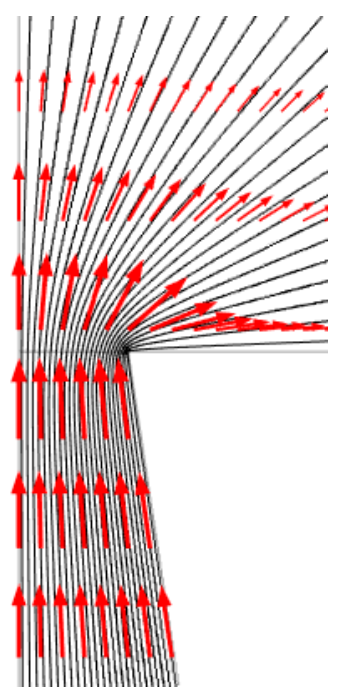

(b)

Figure 3.11: (a) The equipotential and (b) electric field lines close to the small opening of a NP2000 pore $\left(V_{0}=0.1 \mathrm{~V}\right)$. The red arrows in (b) illustrate the direction and the magnitude of the electric field with a logarithmic scale.

drop outside the pore and therefore a smaller current $(95.638 \mathrm{nA})$, all the other geometries result in almost identical values (within 1\%) for the electrical field and the current. Therefore a significant influence of the electrode shape and size on the electric field in and around the pore can be excluded as long as the electrode surfaces are much larger than both of the pore openings and are placed sufficiently far away from them. For all the following simulations in this chapter the upper and lower boundary of the geometry depicted in Fig. 3.7 are used as electrodes. 
Influence of the Size of the Small Pore Opening The simulations above were conducted with a small pore radius of $2.5 \mu \mathrm{m}$. To quantify the influence of the pore size the simulation was also conducted with a radius of $1 \mu \mathrm{m}$ and $250 \mathrm{~nm}$. The membrane thickness and the large pore opening were left constant in the simulation. The resulting potentials in Fig. 3.12 (a) show that most of the potential drop is localised closer to the orifice for smaller pores. As discussed in Sec. 3.2.1 the relative contribution of the access resistance from infinity to the small pore opening is roughly independent of $a$, which is confirmed in the simulation and indicated in Fig. 3.12 (a) by the green dot positioned where the curves overlap at $z=150 \mu \mathrm{m}$.

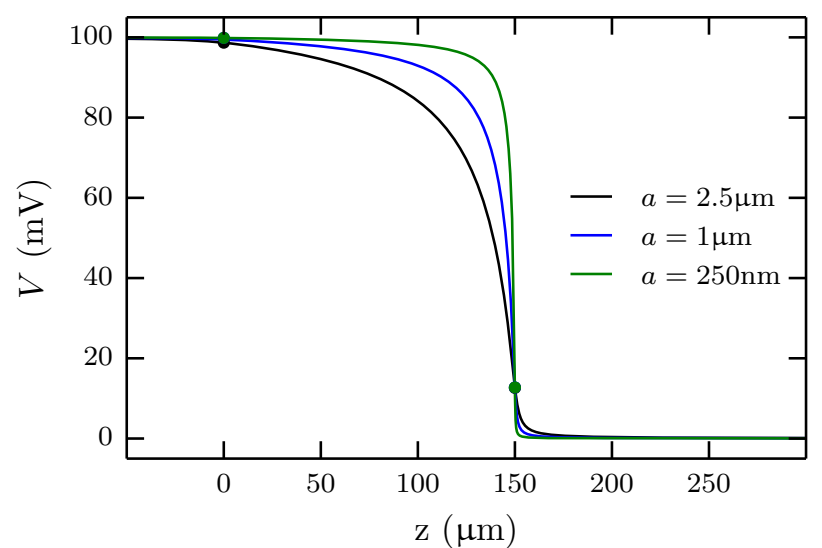

(a)

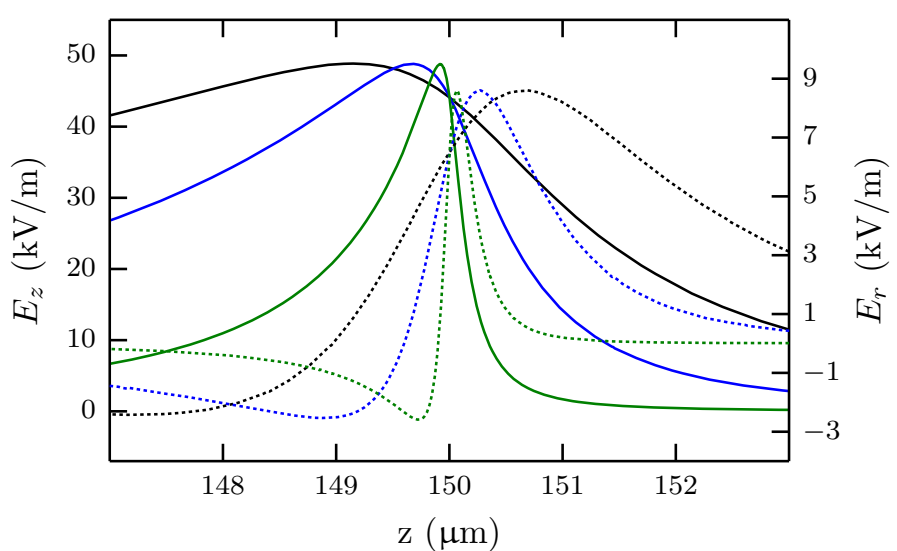

(b)

Figure 3.12: (a) The electric potential along the $z$-axis for three conical pores with different small pore openings but otherwise identical parameters $(b=2.5 \mu \mathrm{m}, l=$ $\left.150 \mathrm{~nm}, V_{0}=0.1 \mathrm{~V}\right)$. The potentials at the pore openings are indicated by green dots. (b) The axial (-) and radial $(\cdots)$ components of the corresponding electric fields for the different pore sizes (same color scheme as in (a)). The results for $a=$ $1 \mu \mathrm{m}$ and $2.5 \mu \mathrm{m}$ are multiplied by 4 and 10 respectively to obtain similar values as for the $250 \mathrm{~nm}$ pore (green).

The maximal electric field in both the axial and radial components increases with smaller pore size. The resulting fields for a $1 \mu \mathrm{m}$ and $2.5 \mu \mathrm{m}$ pore are multiplied by 4 and 10 respectively in Fig. 3.12 (b) and show a linear trend between field strength 
and the radius of the small pore opening. Interestingly, the maximal field strength is not located at the pore orifice but slightly inside the pore for the axial and outside the pore for the radial component. This displacement becomes more pronounced with larger pore size. As $b>>a$ the current $(13.241 \mathrm{nA}, 52.808 \mathrm{nA}, 131.252 \mathrm{nA})$ scales approximately linearly with $1 / a$ in agreement with the semi-analytic model (13.444 nA, 53.588 nA, $133.051 \mathrm{nA})$. As the TRPS device used in the experiments has an optimized signal to noise ratio at approximately $120 \mathrm{nA}$, the voltage has to be adjusted to reach this value. Typically a voltage of $V_{0}=1 \mathrm{~V}$ is applied for a NP200 pore, so the electric field is $\sim 100$ times higher compared to a NP2000 with $V_{0}=0.1 \mathrm{~V}$. This, and the slower fluid velocity for smaller pores are the reasons why electrokinetic transport mechanisms become more important for smaller pores.

In summary, although the semi-analytical model neglects the radial components of the electric field, it is capable of predicting the correct current, the approximate potential shape and the on-axis electric field in a conical pore. However, the FEM simulations clearly show that the electric field is not constant over the pore cross section and has a large radial component at the pore edges. This is firstly of interest for modelling off-axis particle trajectories and suggests that these will exhibit larger resistive peaks and different pulse shapes. For these reasons the implementation of a FEM model is important for the understanding of a RPS device and further results will be discussed in the following.

\subsubsection{The Resistive Pulse in the FEM Model}

When a particle is introduced into the domain, the condition for the current continuity $\sigma_{\text {fluid }}(\boldsymbol{n} \cdot \nabla \psi)_{\text {fluid }}=\sigma_{\text {particle }}(\boldsymbol{n} \cdot \nabla \psi)_{\text {particle }}$ has to be fulfilled at the fluid particle interface. Here $\sigma_{\text {fluid }}$ and $\sigma_{\text {particle }}$ are the conductivity of the fluid and the particle respectively. If the particle is insulating the condition becomes $\boldsymbol{n} \cdot \nabla \psi=0$. The other boundary conditions and parameters are left unchanged from the ones above. A refined mesh at the pore entrance is again critical for accurate results. The simulation is repeated for a sufficient number of particle positions close to the pore orifice to obtain the resistive pulse, with the minimal value identified as the pulse height.

\subsubsection{Comparison with Literature and the Semi-Analytic Model}

In his famous treatise on electricity and magnetism [76], Maxwell calculated the effective resistivity $\rho_{\text {eff }}$ of an electrolyte (resistivity $\rho$ ) containing a dilute suspension of insulating spheres and obtained the result 


$$
\rho_{\text {eff }}=\frac{2+f}{2(1-f)}=\rho\left(1+\frac{3 f}{2}+\frac{3 f^{2}}{2}+\ldots\right)
$$

in terms of the volume fraction $f$ of the spheres. This result can be used to calculate the resistance change when a single sphere with radius $r_{s}$ is introduced into a long cylindrical channel with radius $r_{c y l}$. For $r_{s}<<r_{c y l}$ the expression becomes simply [8]

$$
\Delta R_{\text {Maxwell }}=\frac{2 \rho r_{s}^{3}}{\pi r_{c y l}^{4}}
$$

The above equation, which will be referred to as the Maxwell approach in the following, is not valid for particles which are comparable to the pore size. To account for this issue DeBlois et al. [142] used the solution of the Laplace equation for a sphere in a uniform field to derive a correction factor and obtained the modified equation

$$
\Delta R_{\text {DeBlois }}=\Delta R_{\text {Maxwell }}\left(1-0.8\left(\frac{r_{s}}{r_{c y l}}\right)^{3}\right)^{-1}
$$

Gregg and Steidley [7] used the resistance model and the integral approach analogous to the semi-analytic model presented in Sec. 3.2.1. For the resistance change caused by a insulating spherical particle in a long cylinder they obtained the analytic expression

$$
\Delta R_{\text {Gregg-Steidley }}=\frac{2 \rho}{\pi r_{c y l}}\left(\frac{\sin ^{-1}\left(r_{s} / r_{c y l}\right)}{\sqrt{1-\left(r_{s} / r_{c y l}\right)^{2}}}-\frac{r_{s}}{r_{c y l}}\right)
$$

As Maxwell had already noted [76], this approach gives a lower bound for the resistance change as the current distribution is assumed uniform in the annular region around the particle and neglects the deviation of the electric field by the particle. Any non-uniformity will lead to a higher overall resistance. Note that all of the above equations are independent of the channel length and are only valid if it is long enough that entrance effects do not play a role.

Results for a Cylindrical Channel The relative resistance change for a cylindrical channel of length $l_{c y l}=150 \mu \mathrm{m}$ and radius $r_{c y l}=2.5 \mu \mathrm{m}$ in the presence of a spherical particle (particle radius $r_{s}$ from $0.25 \mu \mathrm{m}$ to $2 \mu \mathrm{m}$ ) positioned at the centre of the channel was simulated with the FEM method and compared to the analytic 


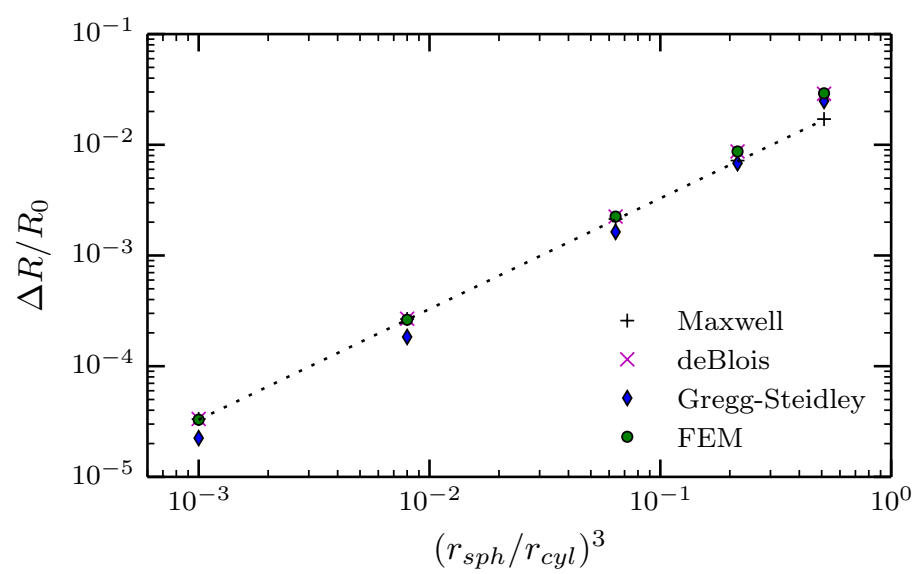

Figure 3.13: The relative resistive change in a cylindrical channel (radius $r_{c y l}=$ $2.5 \mu \mathrm{m}$, length $l_{c y l}=150 \mu \mathrm{m}$ ) as a function of the particle to channel volume. The FEM results are compared to various analytic expressions described in the text. The ... line indicates a cubic relationship between resistance change and particle radius.

expressions above. Due to the large ratio $l_{c y l} / r_{c y l}$ the length of the channel has no significant influence on the simulation. The resulting relative resistive change for five different particle to channel ratios is shown in Fig. 3.13. For relatively small particles $\left(10 r_{s}<r_{c y l}\right)$ the FEM results agree very well with the values obtained with Maxwell's and DeBlois et al.'s expressions. Gregg and Steidley's approach underestimates the resistance change by a factor of 1.5 for small particles [7]. For larger particles, Maxwell's results significantly deviate from the FEM model, while DeBlois et al.'s expression still agrees with the FEM result to within 1\%. For particles with similar size to the channel diameter the conducting part of the cross section changes slowly so that the values obtained with Gregg-Steidley's expression approach the FEM and DeBlois et al.'s results, as expected [8]. The calculations and simulations have been repeated with a channel size ten times larger and smaller than the one above and corresponding particle sizes. The results show no difference to the conclusions above, confirming that the ratio of the particle to the channel size is relevant, rather than the actual dimensions.

These observations, using the FEM method, confirm that while a cubic relationship between particle volume and pulse height in a cylindrical channel is valid for small particles, it can lead to a significant overestimation of the particle radius when the particle is comparable to the pore size. For example, for $r_{s} / r_{c y l}=0.8$ DeBlois et al.'s correction factor is 1.71, meaning that Maxwell's expression overestimates the particle size by $\sim 20 \%$. The correction factor introduced by DeBlois et al. is suitable to account for that issue and the results agree with the FEM model over the entire particle range within less than $1 \%$. 
Results for a Conical Pore While several models for the resistance change due to a spherical particle in a cylindrical channel have been developed, the case of a truncated conical pore has not been studied so intensively. Using the integral method Heins et al. [85] obtained an analytic expression for the resistance change in a conical pore similar to the one presented in Sec. 3.2.1, without considering end effects. FEM was used to investigate the dependence of the current on the particle position in conical pores [128]. In TRPS literature [36], Maxwell's or DeBlois et al.'s expressions are commonly used to deduce the particle size from the current pulse height. This is problematic as the assumption that end effects are negligible, which was used to derive the above expressions, is expected not to be valid for the resistive pulse caused by a particle in a conical pore. In this section, the resistive pulse caused by a spherical particle in conical pores with dimensions relevant to this work are obtained with the FEM method and compared to the semi-analytic approach and Maxwell's and DeBlois et al.'s expressions, which were developed for a cylindrical channel.

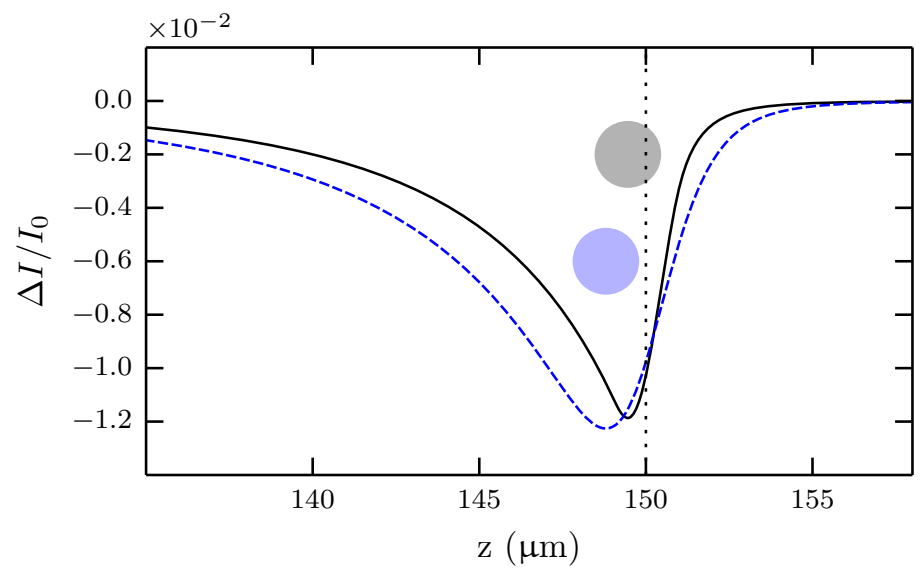

Figure 3.14: The relative current change in a conical pore (NP2000) as a function of the particle (radius $r_{s}=1 \mu \mathrm{m}$ ) position obtained with the semi-analytic model $(-)$ and the FEM simulation (- -). The particle position at the highest resistive pulse is illustrated by the black (semi-analytic) and the blue (FEM) sphere. The $\cdots$ line represents the location of the pore orifice.

The $z$-dependence of the current signal is shown in Fig. 3.14 for the FEM and the semi-analytic model. While the maximal current change is similar for both approaches, the shape and the peak location of the pulse is different. The pulse maximum for both models is expected to occur near the pore orifice where the pore cross section is minimal. However, as entrance effects are also expected near the orifice an exact prediction is difficult. For the semi-analytic model the pulse maximum occurs when the sphere is still about one quarter of its diameter outside the pore. This position stays approximately constant for smaller and larger particles 


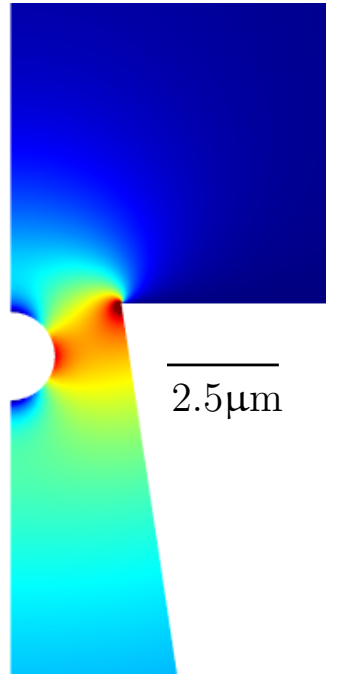

(a)

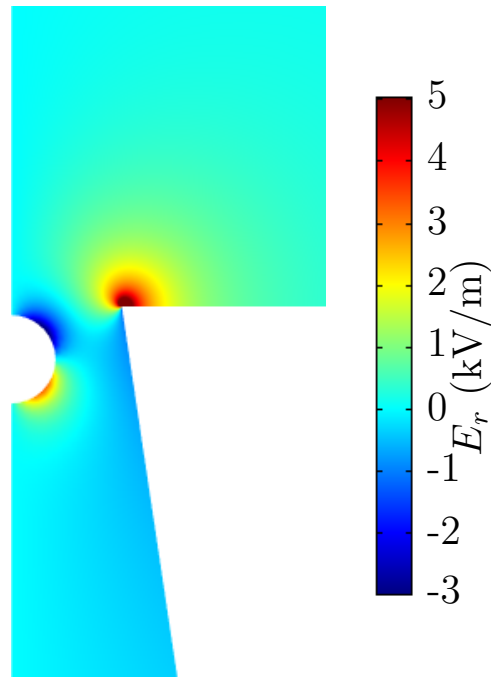

(b)

Figure 3.15: 2D intensity plots of the (a) axial and (b) radial electric field in a conical pore (NP2000, $V_{0}=0.1 \mathrm{~V}$ ) with a insulating particle positioned where the highest resistive pulse is observed in the FEM simulation.

and also for pores with different values of $a$. In the FEM model the maximal current pulse occurs when the particle is further inside the pore. This can be explained with the different distribution for the electric field obtained by both models, shown in Figs. 3.9 and 3.12. While for the semi-analytic model the highest field is always at the pore orifice, the FEM simulation reveals that it is slightly inside the pore, moving closer to the orifice with decreasing $a$. In the FEM simulation $\Delta I$ returns to half its maximal value when the particle is just twice the small pore radius $a$ away from the orifice. As the semi-analytic approach results in a higher field gradient (Fig. 3.9) at the orifice, the pulse becomes narrower. The electric field with a particle present (Fig. 3.15) changes largely compared to the "empty" pore (Fig. 3.10). The effect on the particle transport model will be discussed in Sec. 3.4.5.

Figure 3.16 shows the relative current change determined with the FEM model for various sized particles (from $0.25 \mu \mathrm{m}$ to $2 \mu \mathrm{m}$ ) as a function of the particle position $z$ in a conical pore (NP2000). The signals are multiplied by the particle's volume ratio to allow for comparison. If the relationship between particle size and signal would be cubic the curves should have the same minima. In the simulations a slight deviation from the cubic relation is observed. The particle position where the pulse maximum occurs moves inside the pore with decreasing $r_{s}$. For a particle with $0.25 \mu \mathrm{m}$ radius this position is $\sim 5$ radii inside the pore. This is in a way counterintuitive as the pore has already widened by $8 \%$ and a smaller ratio of the pore's cross section is blocked when the particle moves further into the pore.

A comparison of the peak height for different ratios between particle radius $r_{s}$ and small pore opening $a$ obtained by the various methods is shown in Fig. 3.17. The 


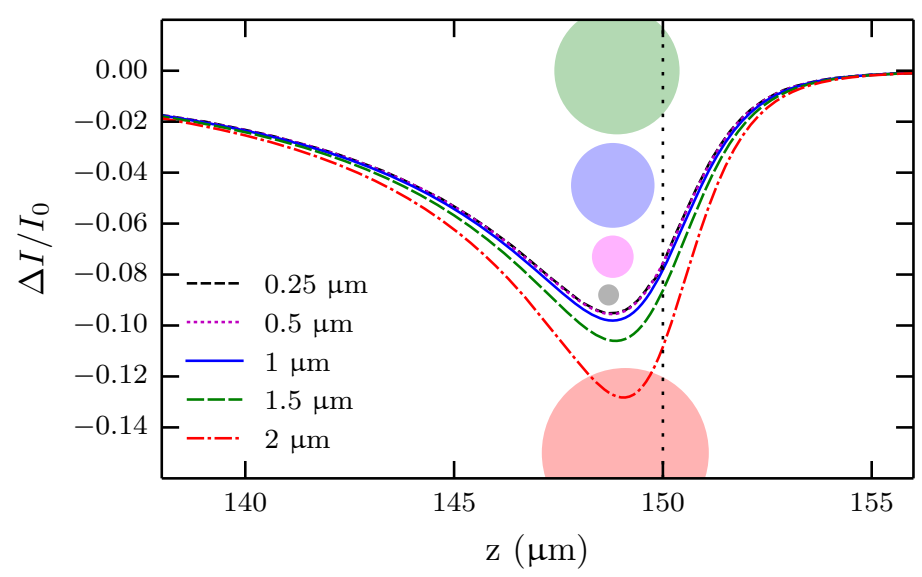

Figure 3.16: The relative current change as a function of the position of various sized particles in a conical pore (NP2000, $V_{0}=0.1 \mathrm{~V}$ ) obtained with the FEM model. To allow for comparison with the signal caused by the largest particle (red, - - - ) the values for the other particles are multiplied by 512, 64, 8 and 2.37 (in increasing size order), as expected when a cubic relationship between resistive pulse and particle size is assumed.

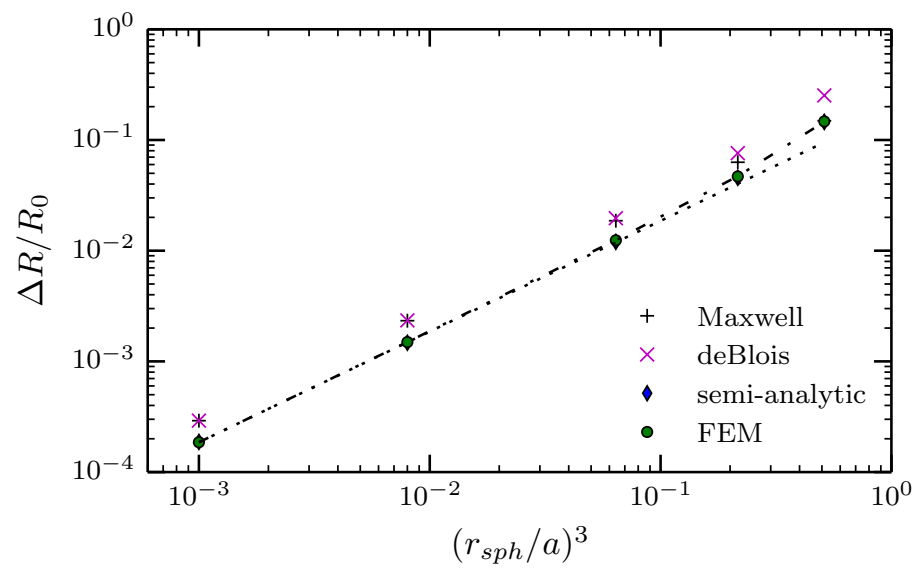

Figure 3.17: The relative resistive change in a conical pore due to the presence of a spherical particle in a NP2000 pore as a function of $r_{s} / a$ obtained with the various methods described in the text. The $\cdots$ line indicates a cubic relationship between peak and particle size. The $-\cdot-$ line incorporates an empirical correction factor based on DeBlois et al.'s correction term.

results obtained from Maxwell's and DeBlois et al.'s expressions deviate significantly from the FEM model. This is expected as both models were derived for a spherical particle within a long thin cylinder and do not account for end effects, which are relevant at the pore orifice. While DeBlois et al.'s expression results in a overestimation of the resistance change for all sizes, Maxwell's expression approaches the FEM model at large particle to pore ratios. Surprisingly, the integral or semi-analytic approach, which was underestimating the resistive change in the case of a cylinder, agrees very well with the FEM model over the whole particle range. This seems to be 
more of a coincidence connected to the divergence of the pore than actual physical agreement of the models, as the models show a large difference for a cylindrical pore (Fig. 3.13). The relationship between particle size and resistance change shows an increasing discrepancy from the cubic assumption for large particles $\left(r_{s}>1 / 10 a\right)$. With a correction term analogous to Deblois et al.'s but with a factor of 0.68 instead of 0.8 an accurate fit can be obtained for the FEM results. The calculations were repeated with a smaller pore $(a=0.25 \mu \mathrm{m})$ and correspondingly smaller particle sizes. The resulting graph shows no difference to Fig. 3.17, and confirms once more that the ratio of the particle to the channel size and not the absolute dimension is relevant for the results.

In this context it shall be noted that the ratio $\Delta I / I_{0}$, which is usually measured in TRPS experiments, is not equal to $\Delta R / R_{0}$. With $V_{0}$ the applied voltage and assuming an Ohmic conductor, this becomes clear with $V_{0}=I_{0} R_{0}=\left(I_{0}+\Delta I\right)\left(R_{0}+\right.$ $\Delta R)$ and it follows

$$
\frac{\Delta I}{I_{0}}=-\frac{\Delta R}{R_{0}}-\frac{\Delta I \Delta R}{I_{0} R_{0}} \neq-\frac{\Delta R}{R_{0}}
$$

However, the values for $\Delta R$ and $\Delta I$ in a usual TRPS experiment are $\sim 1 \%$ of the baseline resistance and current and in this case the two ratios can be assumed equal.

Table 3.1 uses the results from the FEM simulations to compare the methods (Maxwell's, DeBlois et al.'s and a calibration method) for calculating the particle size from the resistive pulse. To be able to calculate the particle size from Eqs. 3.53 and 3.54, the size of the small pore opening is assumed to be known exactly. The calculations show that both methods lead to an underestimation of the particle size, and while Maxwell's equation approaches the correct value for large particles, the correction term in De Blois et al.'s expression increases the discrepancy. This, however, is only true for a conical pore, as the method has proven to be very accurate for cylindrical channels. As the pore geometry is usually not exactly known in TRPS a method using calibration particles is commonly employed [36]. Hereby the signals of a known calibration particle set are compared to the pulse heights of the unknown particles, assuming a volumetric dependency of the resistive pulses. The data show that the method works well for smaller particles, but larger particles are overestimated in size, even more so when the resistive change instead of the current change is used. This is relevant for the TRPS sizing technique and shows that it increases the measurement accuracy when calibration particles close to the measured particles are chosen. 
Table 3.1: The particle radii in $\mu \mathrm{m}$ derived from the FEM pulse heights (NP2000, $0.1 \mathrm{~V}$ ) using Maxwell's (Eq. 3.53), DeBlois et al.'s (Eq. 3.54) and the calibration methods using $1 \mu \mathrm{m}$ calibration particles and an assumed volumetric dependency for both $\Delta R$ and $\Delta I$.

\begin{tabular}{ccccc}
\hline $\begin{array}{c}\text { Particle } \\
\text { Radius } \\
(\mu \mathrm{m})\end{array}$ & $\begin{array}{c}\text { Size from } \\
\text { Maxwell }\end{array}$ & $\begin{array}{c}\text { Size from } \\
\text { DeBlois }\end{array}$ & $\begin{array}{c}\text { Calib. } \\
\text { Method } \\
\text { using } \Delta R\end{array}$ & $\begin{array}{c}\text { Calib. } \\
\text { Method } \\
\text { using } \Delta I\end{array}$ \\
\hline 0.25 & 0.216 & 0.215 & 0.247 & 0.248 \\
0.5 & 0.433 & 0.433 & 0.494 & 0.496 \\
1 & 0.877 & 0.867 & - & - \\
1.5 & 1.365 & 1.311 & 1.557 & 1.540 \\
2 & 1.999 & 1.784 & 2.280 & 2.188 \\
\hline
\end{tabular}

\subsubsection{FEM Simulation of the Fluid Flow}

The governing equations for the hydrodynamic problem are the Navier-Stokes equations (Eqs. 3.11 and 3.12). Both pressure-driven and electroosmotic transport can be modelled with COMSOL's "Laminar Flow" module, which is used to define the boundary conditions and set up the simulations. To account for the abrupt velocity change close to solid surfaces, a fine mesh of several boundary layers around any interface is required to ensure numerical stability. Otherwise the mesh is constructed similarly to the simulation of the electric field described above. A typical simulation has $\sim 300000$ degrees of freedom and takes $\sim 30 \mathrm{~s}$ to solve. This time is reduced by $\sim 2 \mathrm{~s}$ when the option "neglect inertial term" is chosen, which represents the case of a creeping flow (Eq. 3.13). The difference in the results is $\sim 1 \%$ for a NP2000 pore and a pressure of $100 \mathrm{~mm} \mathrm{H}_{2} \mathrm{O}$. As the gain in computational speed is relatively small and the Reynolds numbers for the largest pores and highest pressures used $(R e=0.5)$ are slightly above the value for the Reynolds number that justifies Stokes flow $(R e<0.1)$, the option is not enabled in general.

\subsubsection{The Pressure-Driven Fluid Flow through a Conical Pore}

The pressure-driven flow is modelled analogous to the electric field simulation in a 2D axial symmetric geometry depicted in Fig. 3.7. The top and bottom boundaries are assigned pressures $P_{0}$ and 0 respectively. The "no-slip" boundary condition $(\boldsymbol{u}=0)$ is assigned to the remaining boundaries. To test the validity of the FEM model the fluid flow through a cylindrical pore (no additional reservoirs) with radius $r_{c y l}=$ $2.5 \mu \mathrm{m}$ and a length $l_{c y l}=150 \mu \mathrm{m}$ was simulated and compared to the analytic result described by Hagen-Poiseuille. The pressure between upper and lower boundary was set to $P_{0}=10 \mathrm{~mm} \mathrm{H}_{2} \mathrm{O}$. An entrance length of $100 l_{c y l}$ was chosen in the simulation 
in order to achieve a fully developed flow at the inlet and avoid end effects. It was found that the flow profile is parabolic, has a central velocity of $1.01985 \mathrm{~mm} \mathrm{~s}^{-1}$ and a total flow rate through the channel of $1.00123 \times 10^{-14} \mathrm{~m}^{3} \mathrm{~s}^{-1}$. The values agree very closely with the predicted ones, which are $1.01984 \mathrm{~mm} \mathrm{~s}^{-1}$ for the velocity and $1.00122 \times 10^{-14} \mathrm{~m}^{3} \mathrm{~s}^{-1}$ for the flow rate.

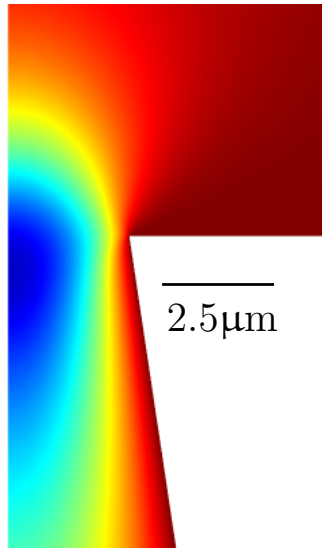

(a)

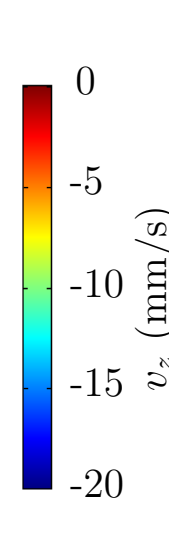

$-20$

\section{(1)}

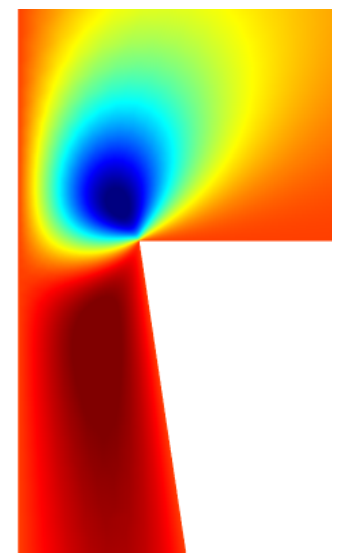

(b)

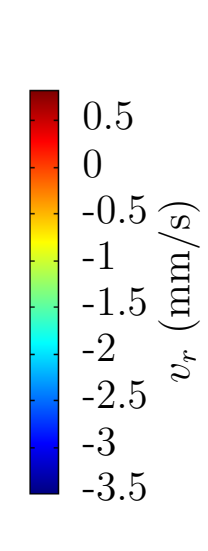

(c)

Figure 3.18: 2D plot of the (a) axial and (b) radial components of the fluid velocity in a NP2000 pore $\left(P_{0}=10 \mathrm{~mm} \mathrm{H}_{2} \mathrm{O}\right)$. (c) Visualization of the streamlines with the arrows representing the velocity direction and magnitude in a logarithmic scale.

The results for a NP2000 pore with an applied pressure of $10 \mathrm{~mm}_{2} \mathrm{O}$ are shown in Figs. 3.18, 3.19 and 3.20. The axial component of the velocity has a maximum of $\sim 20 \mathrm{~mm} \mathrm{~s}^{-1}$ close to the orifice and is rapidly decreasing outside the pore. The radial component has its highest value at the pore edge. It is directed inwards to the pore centre outside and at the entrance of the pore and changes direction inside the pore. The streamlines (Fig. 3.18 (c)) have a high density at the edge of the pore. The hydrodynamic and elecrostatic field (Fig. 3.11) lines have a very similar appearance.

The semi-analytic model, which neglects the radial component, agrees well with the FEM results inside the pore (Fig. 3.19). However, while the semi-analytic model has its maximal flow velocity at the pore orifice, the FEM model reveals that the actual position is slightly inside the pore and has a smaller value. Additionally, a detailed comparison shows that the artificial cone method used in the semi-analytic model is not capable of predicting the velocity outside the pore accurately. While the flow profiles just a few $\mu \mathrm{m}$ inside the pore show good agreement between the two models, they differ in absolute value and shape outside the pore orifice. This is illustrated in Fig. 3.20, also showing that the profile obtained with FEM is not parabolic at the orifice. As mentioned above, a parabolic flow needs a certain entrance length to develop and the estimation made in Sec. 3.2.2 agrees well with the results from the FEM simulation, suggesting an entrance length of less than $2.5 \mu \mathrm{m}$. 


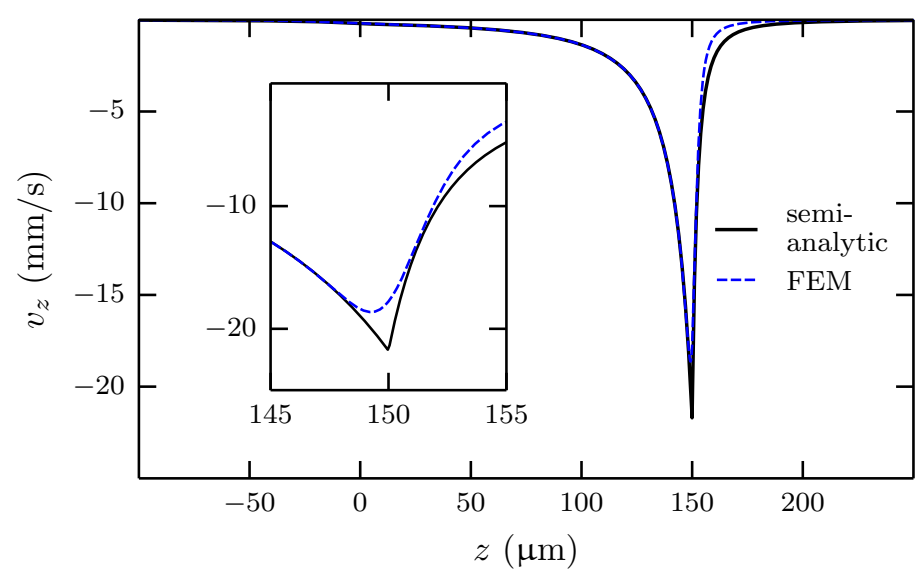

Figure 3.19: The axial velocity along the symmetry axis through a NP2000 pore $\left(P_{0}=10 \mathrm{~mm} \mathrm{H}_{2} \mathrm{O}\right)$. The results obtained with the semi-analytic and the FEM model show significant differences in the region close to the small orifice of the pore, which is shown in the inset.

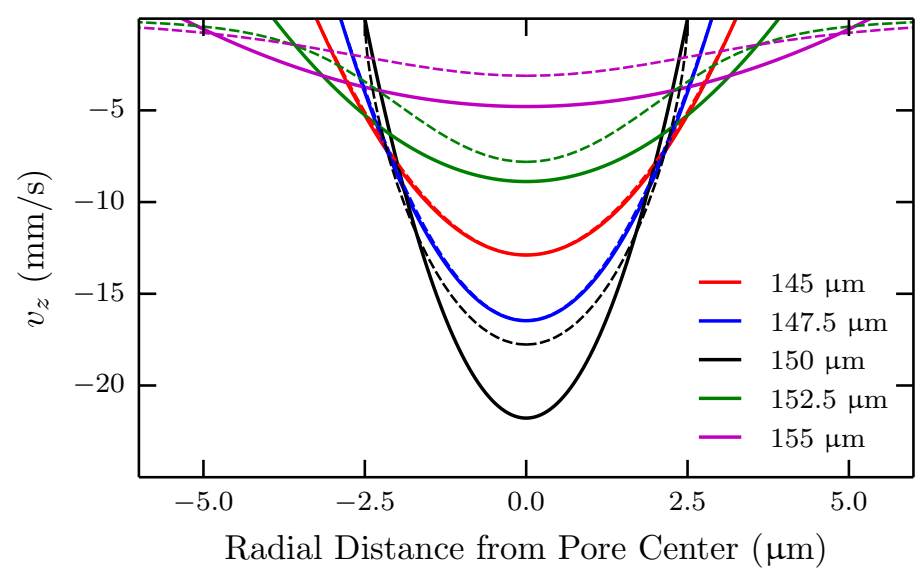

Figure 3.20: The axial velocity as a function of the radial distance at various $z$ planes of a NP2000 pore $\left(P_{0}=10 \mathrm{~mm} \mathrm{H}_{2} \mathrm{O}\right)$. While the semi-analytic $(-)$ and the FEM model (- -) show almost identical results a few $\mu \mathrm{m}$ inside the pore, they differ significantly at the pore orifice and outside the pore.

The flow rate through the pore agrees to within $2 \%$ difference between the two models (see Tab. 3.2). Although the FEM model shows that the pore orifices are not isobaric surfaces as assumed in the semi-analytic model, the differences in the absolute pressures at their centres of the pore orifices are small. The values are $7.980 \mathrm{~mm} \mathrm{H}_{2} \mathrm{O}$ at $z=d$ and $0.0021 \mathrm{~mm} \mathrm{H}_{2} \mathrm{O}$ at $z=0$ for the FEM and $7.906 \mathrm{~mm}$ $\mathrm{H}_{2} \mathrm{O}$ and $0.0021 \mathrm{~mm} \mathrm{H}_{2} \mathrm{O}$ for the semi-analytic model. This also shows that the pressure drop between "infinity" and the small pore opening is significant and cannot be neglected in the calculations. Simulations with various pressures confirm the expected linear relationship of both the flow rate and the velocity on $P_{0}$. 
Influence of the Size of the Small Pore Opening The flow rate and the velocity in a cylindrical pipe are proportional to the fourth and the second power of the pipe radius respectively. For a conical pore with constant large pore radius $b$ and length $l$, an approximately cubic and linear dependency of $Q$ and $v$ on the small pore radius $a$ can be derived from the semi-analytic Eq. 3.18 under the assumption that $b>>a$. To prove this, the same geometry as above with different small pore radii but otherwise constant parameters is modelled for both the FEM and semi-analytic models. The results are summarized in Tab. 3.2 and verify the expected dependencies. A slight deviation from the cubic and linear trend becomes pronounced with increasing $a$. The FEM results also show that the entrance length decreases with smaller $a$ so that a parabolic flow profile is developed closer to the entrance of the pore. The point of maximal axial velocity also moves closer to the pore orifice. The general appearances of the flow fields (Fig. 3.18), the streamline patterns and the relative ratios of radial and axial velocity components are very similar among all the simulations.

Table 3.2: The flow rate $Q$ and the maximal on-axis velocity $v_{\max }$ for different pore radii $a$, both for the FEM and the semi-analytic model. The number in parenthesis is the ratio compared to the smallest pore size.

\begin{tabular}{ccccc}
\hline & \multicolumn{2}{c}{ FEM } & \multicolumn{2}{c}{ Semi-analytic } \\
$\mathrm{a}(\mu \mathrm{m})$ & $Q$ & $v_{\max }$ & $Q$ & $v_{\text {max }}$ \\
& $\left(10^{-13} \mathrm{~m}^{3} \mathrm{~s}^{-1}\right)$ & $\left(\mathrm{mm} \mathrm{s}^{-1}\right)$ & $\left(10^{-13} \mathrm{~m}^{3} \mathrm{~s}^{-1}\right)$ & $\left(\mathrm{mm} \mathrm{s}^{-1}\right)$ \\
\hline 2.5 & $2.098(932)$ & $18.64(9.4)$ & $2.138(929)$ & $21.78(9.3)$ \\
1 & $0.1408(63)$ & $7.78(3.9)$ & $0.1439(63)$ & $9.16(3.9)$ \\
0.25 & 0.00225 & 1.98 & 0.00230 & 2.35 \\
\hline
\end{tabular}

In summary, the semi-analytic model, which is very easy to implement and does not need any specialised software, is able to predict the total flow through and the flow profile a few $\mu \mathrm{m}$ in a conical pore with good accuracy despite its simplification of the physical reality. The FEM model shows that the radial component close to the pore orifice is not negligible. While this may not be of great importance for on-axis particles, the semi-analytic model is not able to predict the trajectories for off-axis particles, for which the FEM model has to be used instead.

\subsubsection{Electroosmotic Flow}

The electroosmotic effect gives rise to a convective flow and can be implemented in the FEM flow model by assigning the boundary condition $\boldsymbol{u}_{\boldsymbol{w a l l}}=\boldsymbol{v}_{\boldsymbol{e o}}=\left(\epsilon_{0} \epsilon_{f l} \zeta_{\text {wall }} / \mu\right) \boldsymbol{E}_{\boldsymbol{t}}$ to the membrane walls. $\boldsymbol{E}_{\boldsymbol{t}}$ is the electric field tangential to the wall surface. In 


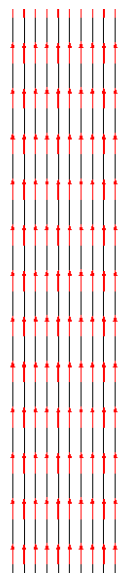

(a)

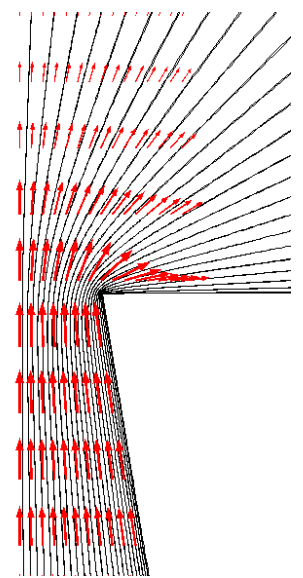

(b)

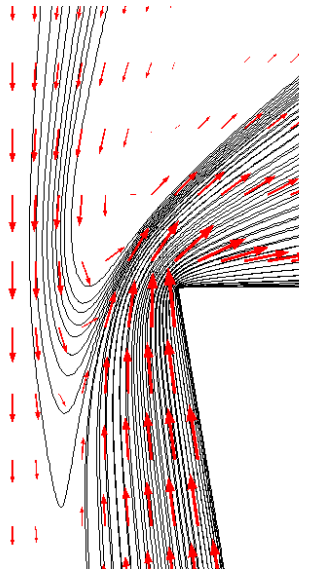

(c)

Figure 3.21: The streamlines of the electroosmotic flow in (a) a cylindrical channel $\left(r_{c y l}=250 \mathrm{~nm}, V_{0}=1 \mathrm{~V}, \zeta_{\text {wall }}=-20 \mathrm{mV}\right)$ and $(\mathrm{b})$ a conical pore (NP200, $V_{0}=$ $1 \mathrm{~V}, \zeta_{\text {wall }}=-20 \mathrm{mV}$ ) with arrows indicating the flow direction and strength in a logarithmic scale. (c) The flow in a NP200 pore resulting from a combination of pressure-driven and electroosmotic flow, which are opposite in their directions $\left(P_{0}=\right.$ $\left.38 \mathrm{~mm} \mathrm{H}_{2} \mathrm{O}, V_{0}=1 \mathrm{~V}\right)$.

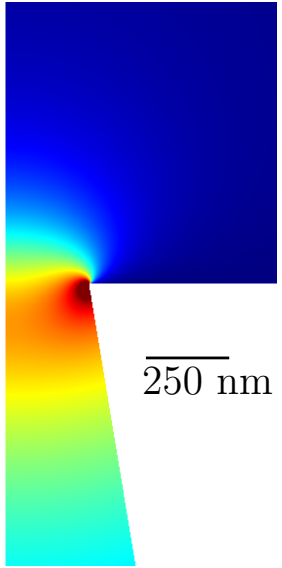

(a)

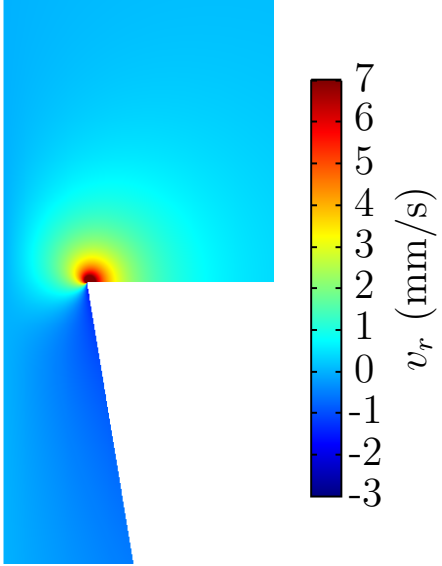

(b)

Figure 3.22: 2D plot of the (a) axial and (b) radial components of the electroosmotic velocity through a NP200 pore $\left(V_{0}=1 \mathrm{~V}, \zeta_{\text {wall }}=V_{0}=-20 \mathrm{mV}\right)$.

the case of a cylinder the simulation yields the expected plug flow with a constant velocity across the channel as illustrated in Fig. 3.21 (a). For a conical pore the flow velocity deviates from a plug flow in Fig. 3.21 (b), especially close to the pore orifices. Figures 3.22 (a) and (b) show that both the axial and radial components of the flow velocity are maximal at the pore edges, which is a result of the electric field distribution (Fig. 3.10). The components reach similar absolute values and the streamlines pattern (Fig. 3.21 (b)) shows denser flow lines close to the horizontal membrane walls compared to a pressure-driven flow profile (Fig. 3.18 (c)). This leads to greater relative outflux/influx in the radial direction which is expected to 


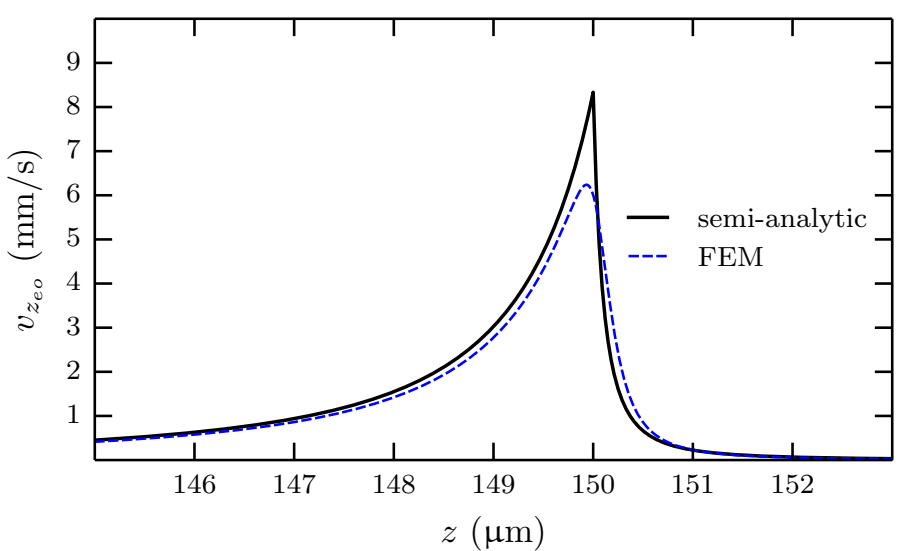

(b)

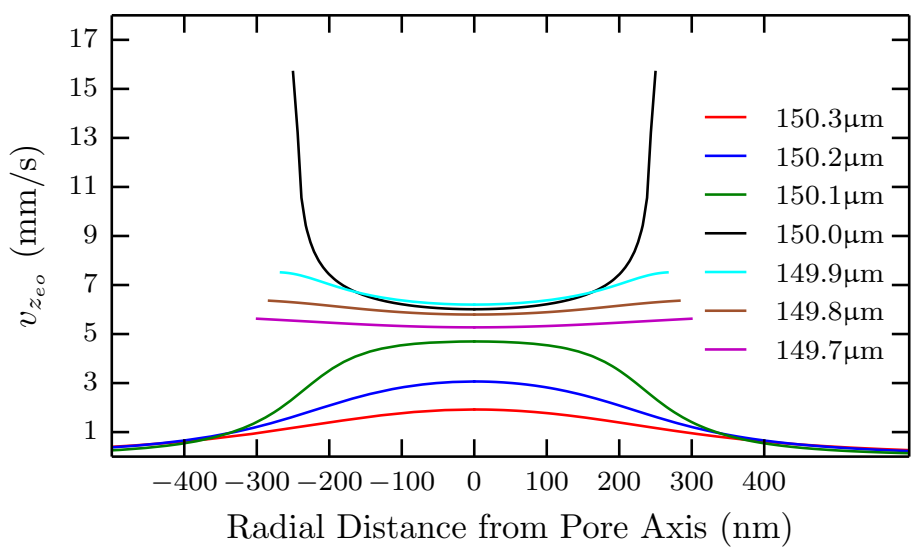

(b)

Figure 3.23: (a) The $z$-component of the electroosmotic velocity along the symmetry axis of a conical pore (NP200, $\left.V_{0}=1 \mathrm{~V}, \zeta_{\text {wall }}=-20 \mathrm{mV}\right)$ obtained with the semianalytic and the FEM model. (b) The $z$-component of the flow as a function of the radial distance to the axis at different $z$-values.

have an impact on the particle trajectories.

The semi-analytic model shows reasonable agreement with the FEM results and only slightly overestimates the on-axis velocity (Fig. 3.23 (a)) at the pore orifice. The FEM simulation also reveals that the flow close to the pore orifice deviates significantly from a plug flow and that the $z$-component of the velocity at the pore edge is about twice as high as its value at the centre (Fig. 3.23 (b)). Inside the pore, an almost perfect plug flow develops within just $300 \mathrm{~nm}$, while the flow more resembles a Gaussian profile outside the pore.

Depending on the direction of the applied voltage the electroosmotic flow can be directed parallel or antiparallel to an additional pressure driven flow. If none of the flows are clearly dominating and they are directed against each other a resulting flow that changes direction within the pore can be achieved. The streamline pattern in Fig. 3.21 (c) shows such a situation and is obtained with a hydrostatic pressure of $38 \mathrm{~mm} \mathrm{H}_{2} \mathrm{O}$ and $1 \mathrm{~V}$ applied to the lower electrode. 


\subsubsection{Particle Transport and Resistive Pulse in the FEM Method}

With the knowledge of the electric and the fluid fields, the trajectory of a particle can be derived assuming that the particle moves with the velocity of the fluid at the particle centre. The electrophoretic velocity is calculated with Eq. 3.38, the dielectrophoretic velocity by combining Eqs. 3.45 and 3.24. The particle trajectory can then be obtained from the total velocity using a numerical algorithm like the Runge-Kutta method.

Alternatively COMSOL's "Particle Tracing Module" can be used, which derives the trajectory of a particle under the influence of the corresponding forces, i.e. hydrodynamic drag, electrophoretic and dielectrophoretic forces, by solving the equation of motion. The simulated electric and fluid fields serve alongside the particle properties $\left(r_{s}, m_{\text {part }}, Q_{e f f}, \epsilon_{\text {part }} *\right)$ as input parameters. Note that for the electrophoretic force, $Q_{\text {eff }}$ is calculated from $\zeta_{\text {part }}$ with Eq. 3.39. The trajectory is derived for a particle, with defined initial position and velocity (usually 0), for a selected time range and step size. As discussed in Sec. 3.3, particle inertia does not play a role at this length scale and both methods obtain the same results. For convenience COMSOL's particle tracing is used instead of post-processing the data. As the particle transport is derived using the electric and fluid field at the particle centre and the influence of the particle on the fields is not accounted for, the approach is called the FEM-point particle method in the following. After the particle trajectory has been determined, the electrostatic problem is solved for a selection of particle positions to obtain the current-time relationship of the resistive pulse. In this step the particle dimension is accounted for so that the calculation of the resistive pulse in the FEM-point particle method is actually a "hybrid" approach.

The validity of the point particle approach is debatable for the following reasons. Firstly, as the particle is of comparable size to the small pore radius an influence of its presence on the fields seems likely. While the influence on the flow field in purely convective transport is expected to be small due to the low Stokes number of the system, the electric field in the pore is expected to be more affected. Secondly, the FEM simulations showed that both the electric and the flow fields at the pore orifice are non-uniform and the particle is therefore exposed to different fields strengths over its entire surface. For these reasons a method that accounts for the particlefluid interaction and determines the velocity via the calculation of the surface forces acting on the particle was developed and is presented in the following. 


\subsubsection{Non-Zero Particle Size - FEM-ALE Method for Hydrodynamic Transport}

In continuum mechanics usually either the Lagrangian or the Eulerian description of motion is used. While in Lagrangian algorithms each individual node of the mesh follows the associated material particle and is mainly used in structural mechanics, the Eulerian description works with a fixed mesh and the continuum moves relative to the grid, making it useful for fluid mechanics. The Arbitrary Lagrangian Euler (ALE) method combines these two techniques and allows fixed and movable mesh nodes, making it a widely used method to describe solid-fluid interactions [143]. With increases in computational power the application of the method in microand nanofluidics has become increasingly popular. D'Avino et al. [144] have used the method to predict particle trajectories in a shear flow of a cylindrical channel, while Ai et al. [131] applied it to pressure driven transport in a converging-diverging channel.

Governing Equations A particle with the mass $m_{\text {part }}$ moves according to the equation of motion

$$
m_{\text {part }} \frac{d \boldsymbol{u}_{\text {part }}}{d t}=\boldsymbol{F}
$$

with $\boldsymbol{F}$ the net force acting on the particle and $\boldsymbol{u}_{\text {part }}$ the resulting particle velocity. In a fluid field the force $\boldsymbol{F}_{\boldsymbol{h} \boldsymbol{y d}}$ originates from the variation of the pressure and viscous forces on the particle surface and is determined by the surface integration over the hydrodynamic stress tensor $\stackrel{\leftrightarrow}{\sigma}[131]$

$$
\boldsymbol{F}_{\boldsymbol{h y d}}=\int_{S} d S \stackrel{\leftrightarrow}{\sigma} \cdot \hat{\boldsymbol{n}}
$$

$\hat{\boldsymbol{n}}$ is the unit vector normal to the particle surface. $\stackrel{\leftrightarrow}{\sigma}$ is given by the expression [91]

$$
\stackrel{\leftrightarrow}{\sigma}=P \stackrel{\leftrightarrow}{I}+\mu\left(\nabla \boldsymbol{u}+\nabla \boldsymbol{u}^{T}\right)
$$

where $\stackrel{\leftrightarrow}{I}$ is the identity tensor, $P$ and $\boldsymbol{u}$ are the position-dependent pressure and velocity of the fluid field. In the present case the particle is assumed to be rigid and cannot be deformed by the fluid pressure. However, the method could also be implemented for deformable particles. 
Simulation Setup The geometry, mesh parameters and boundary conditions for the hydrodynamic and electrostatic problem are the same as those described in Secs. 3.4.2 and 3.4.4. A "moving wall" boundary condition with the velocity $\boldsymbol{u}_{\text {part }}$, determined by Eq. 3.57, is assigned to the particle boundary. For the electrostatic problem, which is calculated simultaneously, the insulation boundary condition $\boldsymbol{n}$. $\nabla \psi=0$ is assigned to the particle boundary. COMSOL's "Moving Mesh" module is used to implement the ALE method. The condition "free mesh deformation" is assigned to the whole domain. A zero displacement constraint is set to the walls of the geometry. The mesh displacement for the particle surface corresponds to the particle movement $d \boldsymbol{x}_{\text {part }}$.

The particle is initially positioned a distance away from the pore orifice where the fluid velocity is less than $1 \%$ of the maximal velocity in the pore, so that the influence of the initial velocity can be neglected. For a NP2000 pore a position $15 \mu \mathrm{m}$ from the pore orifice is sufficient. The flow field and the resulting force on the particle are calculated for each time step and the particle position is then updated by integrating the kinematic equation $d \boldsymbol{x}_{\text {part }} / d t=\boldsymbol{u}_{\text {part }}$. The time steps are chosen automatically by the solver algorithm so that the solution converges within a predetermined relative error (usually $10^{-4}$ ).

After the particle is moved to its new position, the mesh is adapted with one of the mesh smoothing algorithms described in detail in [145]. The distortion of the mesh is then determined using a COMSOL internal function and in case its value exceeds a value of 2 the geometry is re-meshed (Fig. 3.24 (b)). For a 2D axial symmetric geometry the mesh smoothing type "Yeoh" with a stiffening factor of 100 is used, which has the advantage that the mesh deformation is spread over a larger area and the re-meshing step has to be done less frequently. However, as this smoothing type caused convergence problems for the 2D models the "Laplace" smoothing type is used instead, which is numerically more stable for transient solvers [145].

On-axis Trajectory For an on-axis particle the 2D axial symmetric geometry can be used. The resulting flow field for a NP2000 pore geometry is shown in Fig. 3.18 (c). No significant distortion of the fluid field due to the presence of the particle is observed. The $z$-component of the fluid/particle velocity obtained by the semianalytic, the FEM-point particle and the FEM-ALE methods for the same pore geometry and initial particle position are shown in Fig. 3.25. With the presence of the particle the velocity at the pore orifice is $\sim 10 \%$ smaller compared to the undisturbed flow. 


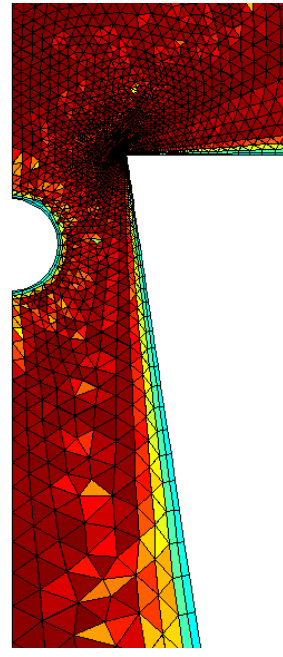

(a)

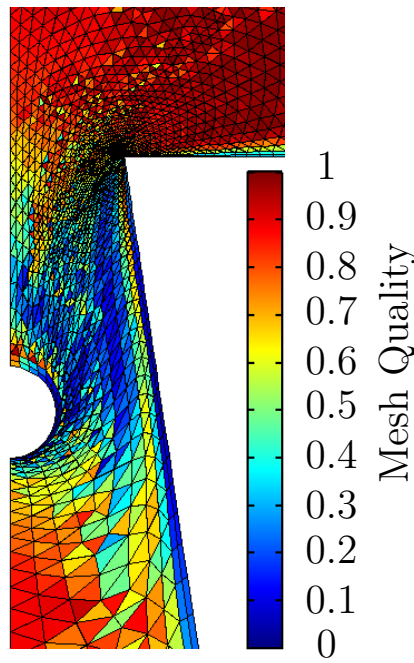

(b)

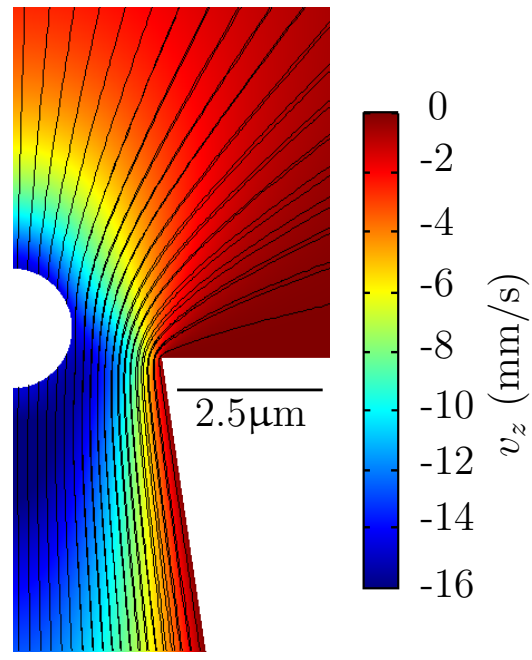

(c)

Figure 3.24: (a) Original and (b) distorted mesh caused by the particle movement using the FEM-ALE method (NP2000, $r_{s}=1 \mu \mathrm{m}, P_{0}=10 \mathrm{~mm} \mathrm{H}_{2} \mathrm{O}$ ). The mesh in (b) is highly distorted and no longer fulfils the pre-set mesh quality condition and the geometry will be re-meshed before the next time step. (c) 2D intensity plot of the $z$-component of the fluid velocity.

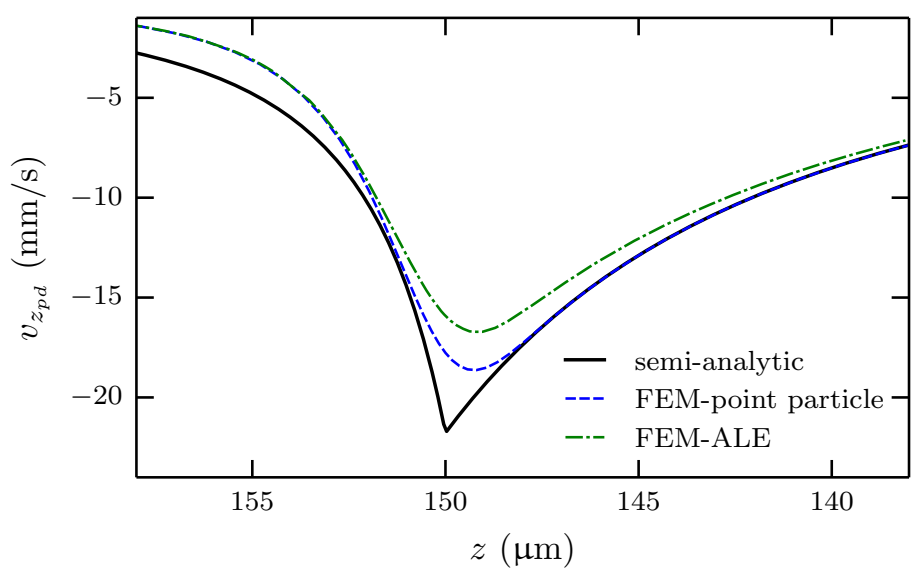

Figure 3.25: The on-axis velocity of the fluid/particle in a NP2000 pore $(10 \mathrm{~mm}$ $\left.\mathrm{H}_{2} \mathrm{O}, r_{s}=1 \mu \mathrm{m}\right)$ as predicted from the semi-analytic model, the FEM-point particle model and the FEM-ALE method in a 2D axial symmetric model.

Off-axis Trajectories - Hydrodynamic Focussing Segre and Silverberg [146, 147] were the first to experimentally observe the radial displacement of particles in a Poiseuille flow and found that mm-sized spheres accumulate in a narrow annular ring about 0.6 times the radius of the channel. The effect was consequently investigated further [148-150] with the conclusion that it is inertial in nature and scales with the Reynolds number. Since then, the orthogonal force in non-uniform flow fields has been studied intensively both experimentally [151], theoretically [152] and numerically $[144,153]$ as it opens the possibility of hydrodynamic focusing and 
particle sorting in microfluidic devices $[154,155]$.

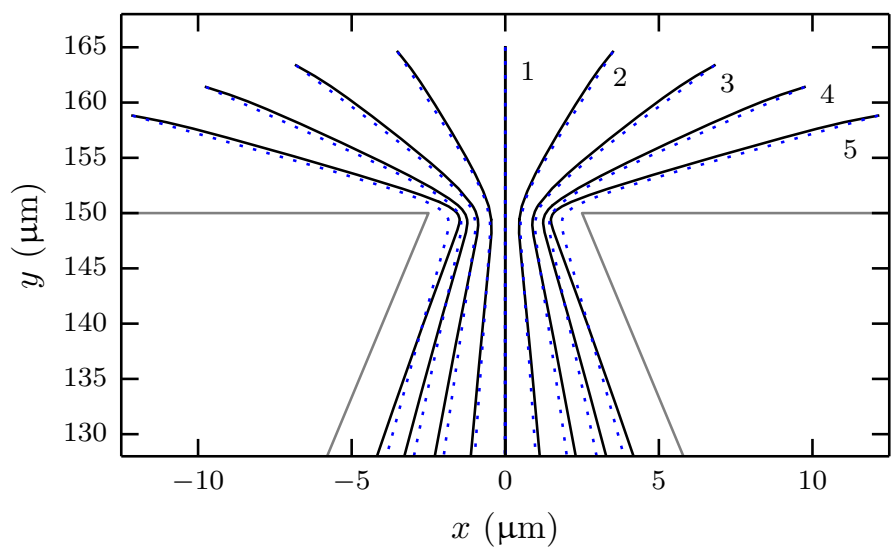

(a)

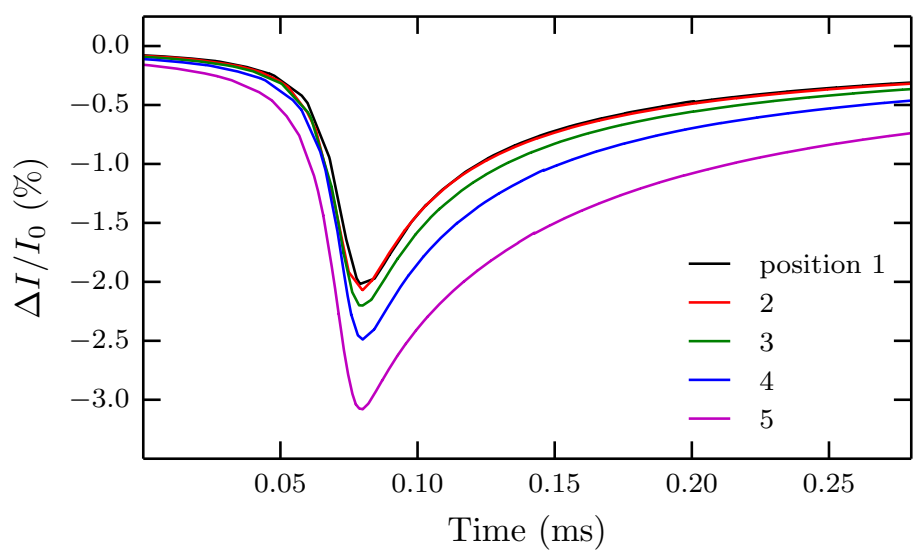

(b)

Figure 3.26: (a) The particle trajectories calculated with the ALE (-) and the point particle model $(\cdots)$ for five different start positions in the range from $0^{\circ}$ to $54^{\circ}$ from the pore axis in steps of $13.5^{\circ}$. A NP2000 and $r_{s}=0.5 \mu \mathrm{m}, P_{0}=100 \mathrm{~mm}$ $\mathrm{H}_{2} \mathrm{O}, V_{0}=0.1 \mathrm{~V}$ were used. (b) The resulting resistive pulses predicted with the ALE model.

With the addition of an off-axis particle the symmetry of the geometry is broken. Due to computational limitations, the ALE method could not be implemented in 3D and was simulated as a 2D model (Cartesian coordinates) instead. The results therefore do not provide the physically accurate values for the flow and electric field but are still useful to determine whether hydrodynamic focussing is significant under the conditions used for TRPS. As the orthogonal force in non-uniform flow field increases with the Reynolds number a geometry and pressure at the upper limit was chosen. The trajectories are calculated for five different starting positions using the ALE algorithm and compared to the ones obtained with COMSOL's "Particle Tracing Module".

A maximal radial displacement of $0.4 \mu \mathrm{m}$ towards the pore centre is observed for particles that travel close to the pore edge (Fig. 3.26 (a)). Particles that travel closer 
to the pore centre experience a smaller displacement as the difference in the fluid velocity between opposing sides is less pronounced. The effect is relatively small and is expected to be negligible for smaller pores and lower fluid velocities. Both the only slightly disturbed field in the presence of a particle and the small focussing effect imply that the assumptions underlying the semi-analytic and FEM-point particle model are acceptable. The corresponding current pulses for each trajectory obtained with the ALE method in Fig. 3.26 (b) show that the resistive pulses increase in height and duration for off-axis particles. This can be understood because the electric field increases while the fluid velocity decreases towards the pore edge, and is discussed in the following section.

Off-Axis Trajectories - Pulse Height Distribution In Coulter counters with cylindrical cross sections the influence of the particle trajectories on the resistive pulses has been studied and quantified [132, 156-158], showing that off-axis particles result in a larger resistive pulse. This implies that a certain distribution of pulse heights is intrinsic to the method. To obtain a theoretical distribution, the trajectories of a sufficiently large number of initial particle positions has to be simulated. Due to computational limitations this was executed with the FEM-point particle model, which is justified as the radial movement of the particle is not large, especially for a lower fluid velocity $\left(P_{0}=10 \mathrm{~mm} \mathrm{H}_{2} \mathrm{O}\right)$.

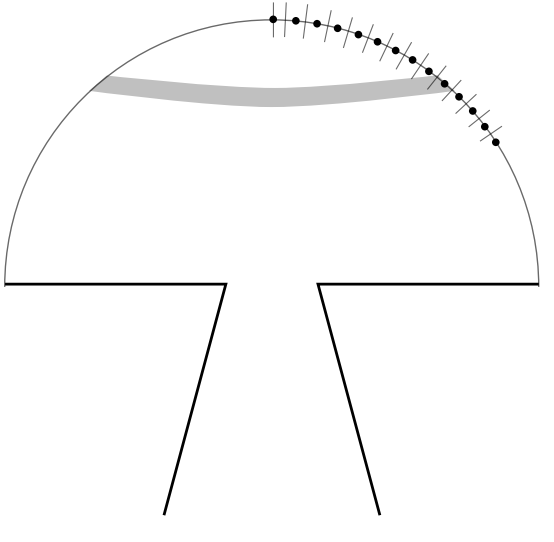

(a)

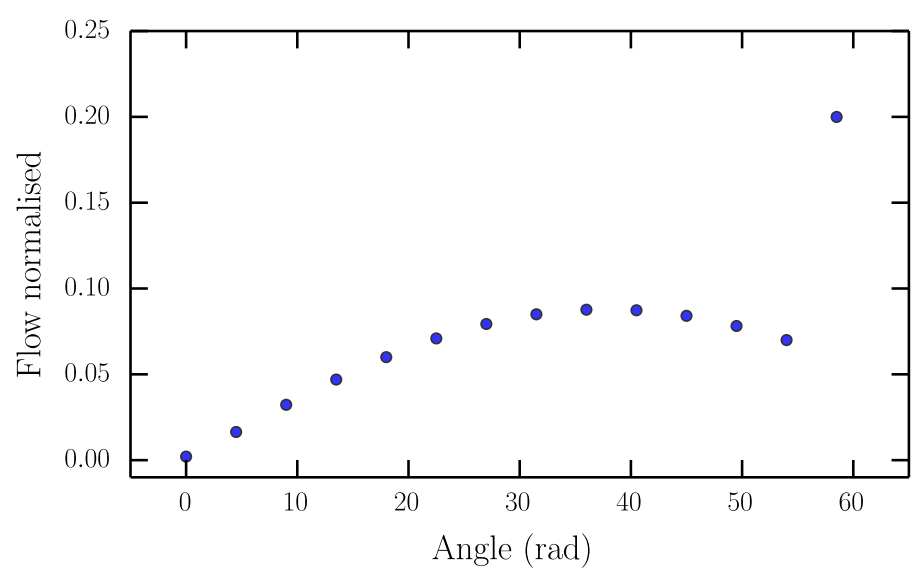

(b)

Figure 3.27: (a) The initial positions for 13 particles (marked with $\bullet$ ) used to derive the pulse distribution evenly space at $4.5^{\circ}$ intervals. The angle to the pore axis for the furthest initial position was $58.5^{\circ}$ from the pore axis. A NP2000 pore, $r_{s}=$ $500 \mathrm{~nm}$ and $P_{0}=10 \mathrm{~mm} \mathrm{H}_{2} \mathrm{O}$ were used for the simulation. (b) The normalized, relative fluid flux through each of the segments depicted in (a).

Figure 3.27 (a) shows the initial positions of 13 simulated particle trajectories. Assuming a uniform bulk concentration of the particles, the relative fraction of particles (Fig. 3.27 (b)) that move on very similar trajectories is calculated by the 
flow through the corresponding segments depicted in Fig. 3.27 (a). The particle with an initial position of $58.5^{\circ}$ from the pore axis travels through the pore at the physically closest possible distance to the edge. Particles at higher angles are expected to result in similar resistive pulses as they will "slide" into the pore.

The resulting histogram in Fig. 3.28 (a) shows that, apart from the dominating peak, a second, smaller peak at a $\Delta I$ value about $1.5 \times$ higher is predicted. The shape of the distribution can be understood as the electric field of the "empty pore" first increases slowly towards the pore edge, before it rapidly rises to its maximum at the pore edge (Figs. 3.9 and 3.10). Therefore particles which travel off-axis but do not block the region of the highest current density close to the edges contribute to the first peak and the region between the peaks. The second peak then originates from particles travelling through the pore at a very close distance to the pore edge. Experimentally this second peak is usually observed for $\mu \mathrm{m}$ sized pores at relatively low pressure, which indicates that at higher pressures particles are pushed towards the pore centre due to flow focussing so that the second peak disappears in the distribution. Figure 3.28 (b) shows the resistive pulse distribution for an experiment that uses similar parameters to the simulation. Although the exact distribution is slightly different, a second peak at approximately the predicted value can be observed. The results of this section were part of a recent publication [159].

\subsubsection{Non-Zero Particle Size - FEM-ALE Method for Electrophoretic Transport}

While at the $\mu \mathrm{m}$-scale the pressure-driven flow is dominant, the relative strength of electrokinetic effects increases with decreasing pore size. The simulations in Sec. 3.4.2 showed that the electric field is not uniform across the pore orifice and a net force towards the pore centre for negatively charged particles is expected. To study the potential effect the FEM-ALE method is used for purely electrophoretic $\left(P_{0}=0 \mathrm{~mm} \mathrm{H}_{2} \mathrm{O}\right)$ transport through a NP200 pore.

The domain, mesh and boundary conditions are constructed analogous to the section above. The electrophoretic force $\boldsymbol{F}_{\boldsymbol{e p h}}$ is calculated as the product of the electric field multiplied by the surface charge density $\sigma_{\text {eff }}$ (Eq. 3.40) integrated over the particle surface $S$

$$
\boldsymbol{F}_{\text {eph }}=\sigma_{e f f} \int_{S} d S \boldsymbol{E} .
$$

The hydrodynamic drag, which is directed in the opposite direction to the par- 


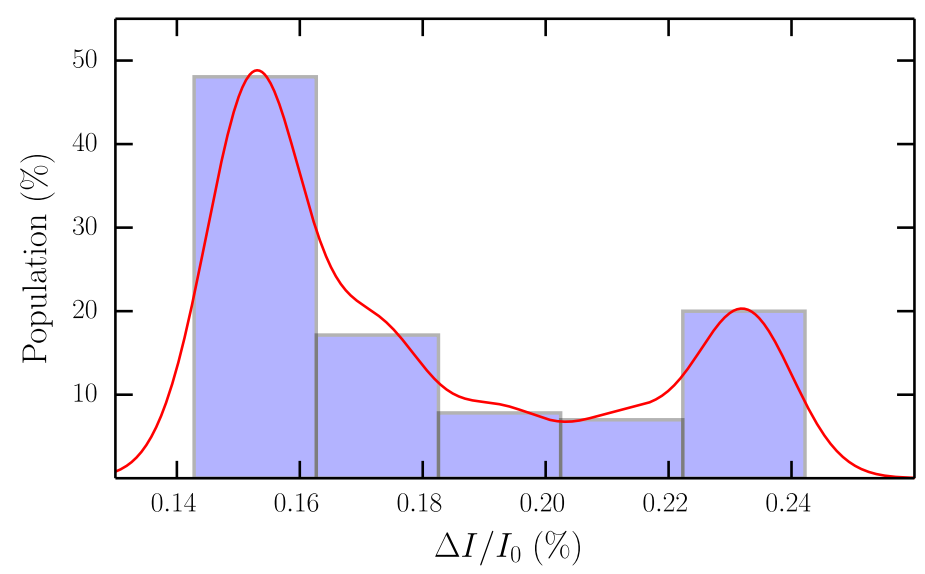

(a)

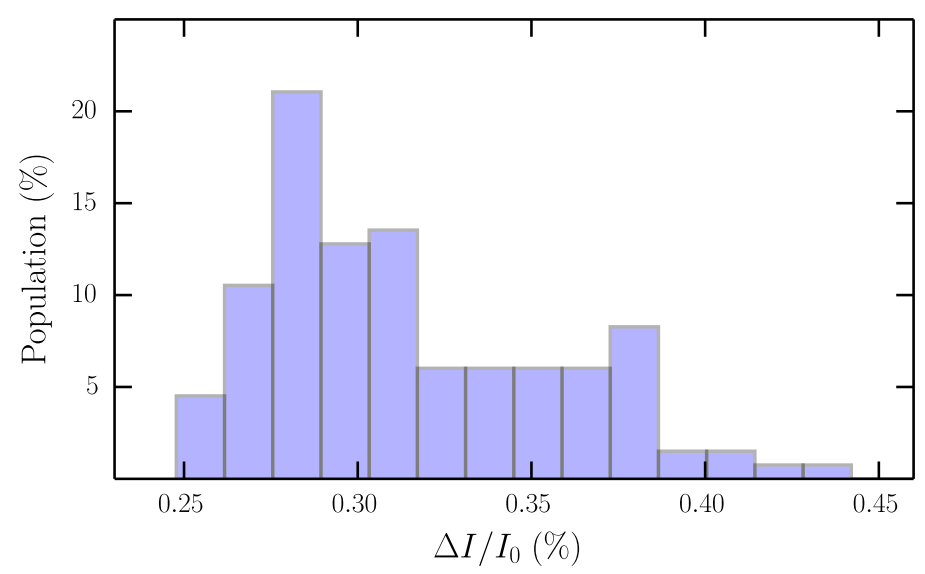

(b)

Figure 3.28: (a) The resistive pulse distribution as derived from the FEM model with a discrete number of particle start positions (NP2000 pore, $r_{s}=500 \mathrm{~nm}, P_{0}=$ $10 \mathrm{~mm} \mathrm{H}_{2} \mathrm{O}$ ). The size of the bins is 0.02 and the red line represents a continuous distribution and was obtained by the summation of Gaussian functions fitted to each bin. (b) The resistive pulse distribution originating from 133 events in an experiment using a NP1000 $\left(r_{s}=485 \mathrm{~nm}, V_{0}=0.16 \mathrm{~V}, 45 \mathrm{~mm}\right.$ stretch, $P_{0}=10 \mathrm{~mm}$ $\left.\mathrm{H}_{2} \mathrm{O}\right)$.

ticle velocity, is calculated with Eq. 3.58. Figure 3.29 shows the on-axis particle electrophoretic velocities calculated with the semi-analytic model, the FEM-point particle model and the FEM-ALE method. The first two models overestimate the electrophoretic velocity significantly, proving that the presence of an insulating particle in the pore has a non-negligible effect on the electric field. The velocity in the FEM-ALE method is approximately half of what is predicted from the semi-analytic and the FEM-point particle models. This suggests that in the pore constriction electrophoresis cannot accurately be predicted without accounting for the particle-field interactions. 


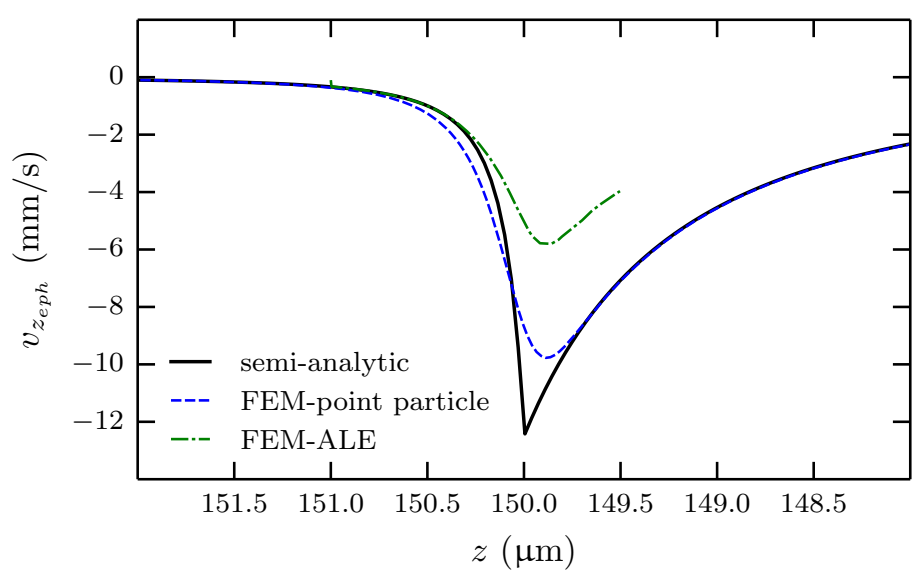

Figure 3.29: The on-axis electrophoretic velocity of a particle $\left(r_{s}=100 \mathrm{~nm}, \zeta_{\text {part }}=\right.$ $-30 \mathrm{mV})$ in a NP200 pore $\left(V_{0}=1 \mathrm{~V}\right)$ as predicted from the semi-analytic, the FEM-point particle and the FEM-ALE methods. The FEM-ALE simulation was terminated soon after the pulse maximum for computational reasons.

Electrophoretic Focussing Analogous to the study of the off-axis trajectories for hydrodynamic transport a 2D simulation of several trajectories was conducted and compared to the result obtained with the FEM-point particle method.

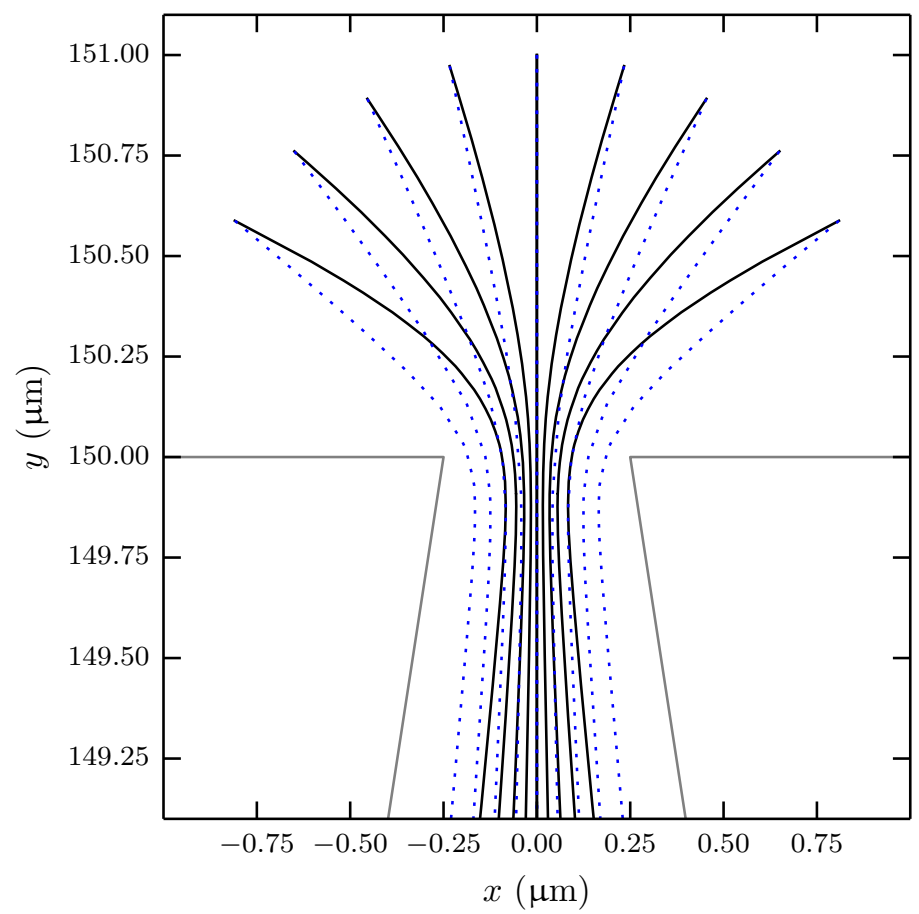

Figure 3.30: The trajectories for five different start positions of purely electrophoretically driven particles through a NP200 pore obtained with the ALE (-, black) and the point particle $(\cdots$, blue $)$ methods $\left(V_{0}=1 \mathrm{~V} r_{s}=0.1 \mathrm{~nm}\right)$. The starting positions were in a range from $0^{\circ}$ to $54^{\circ}$ from the pore axis in steps of $13.5^{\circ}$.

The particle trajectories for nine starting positions are shown in Fig. 3.30. It shows 
that the electrophoretic focussing is strong with the outermost particle moving almost one particle radius ( $95 \mathrm{~nm}$ ) towards the pore centre. The high radial component of the electric field between the negatively charged surface of the particle and the pore wall results in a net force directed to the pore centre, so that the particles do not follow the electric field lines. The difference of the results obtained with the ALE and the point particle methods is significant and it can be concluded that the latter method is not suitable to accurately predict the off-axis particle trajectories in electrophoretic driven flow through conical pores. It has to be mentioned that the focussing effect is not to be mistaken with the dielectrophoretic force, for which no net particle charge is required. The application of the FEM-ALE method to dielectrophoresis is discussed in the next section.

\subsubsection{Non-Zero Particle Size - FEM-ALE Method for DC Dielec- trophoresis}

In a non-uniform electric field an additional force on a particle arises due to the interaction of the local field with induced or permanent dipole moments in the dielectric medium. The electrical forces on these dipoles generate a stress on the particle surface which gives rise to a net force. The effect is expected to occur in conical pores as the field is non-uniform both in the axial and the radial directions. For insulating particles the force is pointed in the direction of the decreasing electric field so that a particle at the edge of the pore orifice will be pushed out and towards the centre of the pore. The dielectric force on a sphere in a non-uniform field is often used in the form of Eq. 3.44. However, it had been shown that this expression should not be used if the particle is of comparable size to the characteristic length of the electric field and can lead to an inaccurate prediction of the particle trajectories [121]. In this case the dielectrophoretic force $\boldsymbol{F}_{\boldsymbol{d e p}}$ on a particle should be calculated with the surface integral over the Maxwell stress tensor $T_{e l}[160]$

$$
\boldsymbol{F}_{\boldsymbol{d e p}}=\int_{S} d S \stackrel{\leftrightarrow}{T_{e l}} \cdot \hat{\boldsymbol{n}}
$$

with $\hat{\boldsymbol{n}}$ the unit vector normal and outward to the particle surface. $\stackrel{\leftrightarrow}{T}_{e l}$ is given by the expression

$$
\stackrel{\leftrightarrow}{T}_{e l}=\epsilon_{0} \epsilon_{f l}\left(-\frac{1}{2}|\boldsymbol{E}|^{2} \stackrel{\leftrightarrow}{I}+\boldsymbol{E} \boldsymbol{E}\right)
$$

with $\boldsymbol{E}$ the electric field at the particle surface and $\stackrel{\leftrightarrow}{I}$ the identity tensor. For an 
insulating particle with the boundary condition $\boldsymbol{n} \cdot \boldsymbol{E}=0$ at the surface the second term in the integral vanishes and the force becomes

$$
\boldsymbol{F}_{\text {dep }}=-\frac{1}{2} \epsilon_{0} \epsilon_{f l} \int_{S} d S|\boldsymbol{E}|^{2} \hat{\boldsymbol{n}}
$$

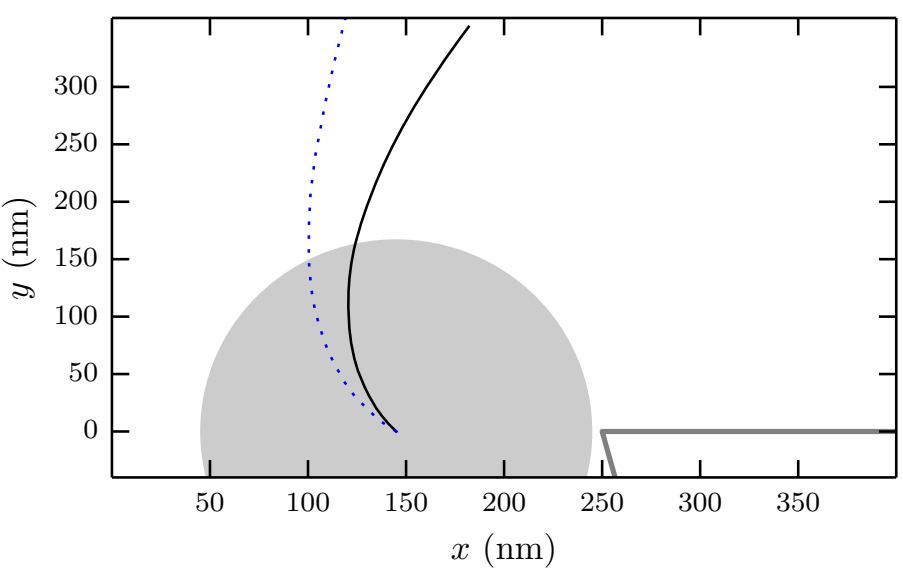

Figure 3.31: The trajectories of an initially resting, uncharged particle due to dielectrophoresis in a NP200 pore $\left(r_{s}=100 \mathrm{~nm}, V_{0}=1 \mathrm{~V}\right)$ obtained with the FEM-ALE $(-)$ and the FEM-point particle $(\cdots)$ models.

First, the trajectory of a particle initially resting at the pore edge and moved by a dielectrophoretic force is calculated. Figure 3.32 (a) shows that the particle is initially pushed to the pore centre and out of the orifice before it moves away from the symmetry axis towards a low axial and radial electric field. A simulation using the FEM-point particle model shows a very different trajectory, proving that this model should not be used when the particle size is comparable to the characteristic length of the field.

To determine whether dielectrophoresis will alter the particle trajectories for parameters typical for a TRPS experiments the simulation discussed in the previous section is repeated with the addition of the dielectrophoretic force at three different voltages. The resulting trajectories are plotted in Fig. 3.32. The first finding is that the trajectories without DEP are independent of the applied voltage. This can be understood as the ratio between the axial and radial components is only dependent on the pore geometry and not on the absolute voltage. The particle will move faster with increasing voltage but still takes the same trajectory as long as particle inertia does not play a role. The second finding is that the dielectrphoretic force does push the particle further to the pore centre compared to electrophoretic focussing alone. Since the force increases quadratically with the field strength the effect becomes more pronounced at higher voltages. This implies that particles in 


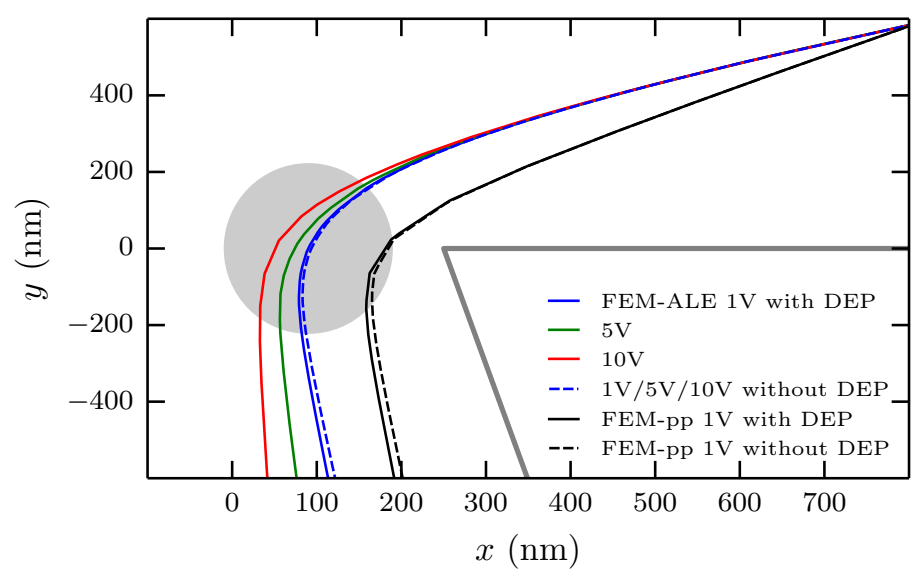

Figure 3.32: The trajectories of electrophoretically driven particles through a NP200 pore $\left(r_{s}=100 \mathrm{~nm}\right)$ obtained with the FEM-ALE method and the FEM-point particle model with $(-)$ and without $(\cdots)$ dielectrophoresis for various voltages $V_{0}$.

conical pores could be focussed with sufficiently high voltages, which would lead to a more uniform trajectory and size distribution. However, the upper limit of the voltage for TRPS and RPS in general is usually $\sim 1 \mathrm{~V}$ to avoid undesired effects like hydrolysis. For this value the particle is pushed $\sim 5 \mathrm{~nm}$ further to the pore centre, so that the dielectrophoretic transport is not expected to contribute noticeably to the particle transport in TRPS.

\subsubsection{Combination of the Transport Mechanisms}

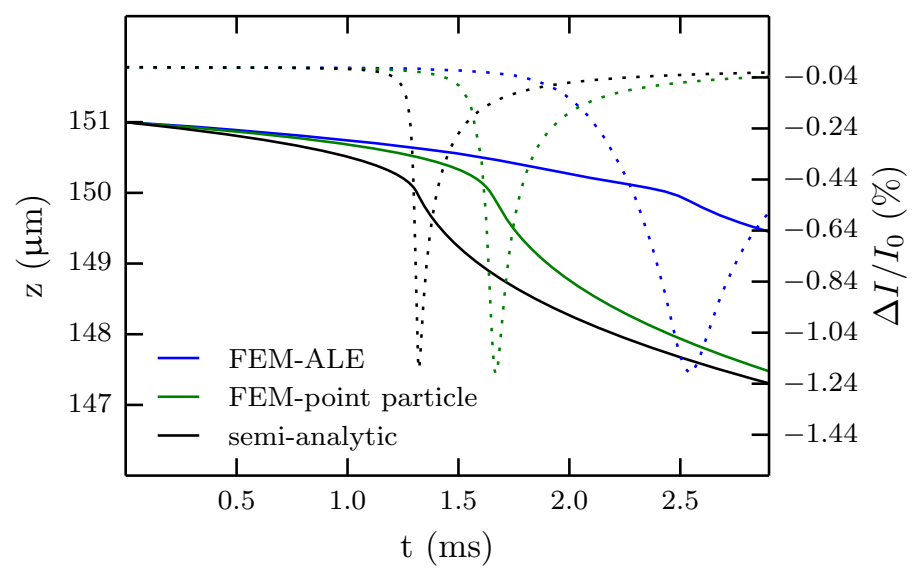

Figure 3.33: The time-position relationship $(-)$ and resistive pulse $(\cdots)$ for an onaxis particle $\left(r_{s}=100 \mathrm{~nm}\right)$ moving through a NP200 pore due to a combination of pressure-driven $\left(10 \mathrm{~mm} \mathrm{H}_{2} \mathrm{O}\right)$, electroosmotic $\left(\zeta_{\text {wall }}=-20 \mathrm{mV}\right)$, and electrophoretic $\left(\zeta_{\text {part }}=-30 \mathrm{mV}\right)$ transport, calculated with the different models.

The transport of particles through a TRPS pore is usually due to a combination of the transport mechanisms discussed above. To illustrate the difference between 
the developed models, the time-dependent position and the resulting current pulse for a particle travelling through a NP200 pore is shown in Fig. 3.33. While the current height is in good agreement for all three models, the pulse shape in the semianalytic and FEM-point particle model is significantly narrower than predicted with the FEM-ALE model. This is mainly due to the overestimation of the electrophoretic velocity in the semi-analytic and FEM-point particle model. The pulse in the semianalytic model is shorter compared to the FEM-point particle method, as already observed in Sec. 3.4.3 (Fig. 3.14), and this can be attributed to the different shape of the electric field between the models (Fig. 3.9) and a slower fluid velocity in the FEM model (Fig. 3.19). As a conclusion it shall be noted that the FEM-ALE method should be used for detailed studies of the particle trajectories.

Relevance for the Coordination Experiments The particle transport is critical for the quality and throughput of the coordination technique. In practice, as usually the voltage is kept at a value where the signal to noise ratio is maximal, this is achieved by regulating the hydrostatic pressure. Figure 3.34 shows the influence of the pressure on the on-axis particle velocities close to the orifice of NP200 and NP2000 pores using the FEM-point particle method.

For an NP200 pore and at a typical applied voltage of $1 \mathrm{~V}$, the strength of the electrokinetic transport corresponds to a pressure of several $10 \mathrm{~mm} \mathrm{H}_{2} \mathrm{O}$ and the pressure can be used to balance electroosmosis and -phoresis in order to achieve the desired particle velocity and frequency. Interesting effects can be observed for certain combinations of parameters. For example, at an applied pressure of $-15 \mathrm{~mm} \mathrm{H}_{2} \mathrm{O}$, the net velocity is positive outside and negative inside the pore, so that no particle flux through the pore will be observed (Fig. 3.34 (a)). At the $\mu \mathrm{m}$-scale, the total velocity shows a symmetric behaviour in respect to the pressure as electrokinetic transport does not play a major role. It has to be kept in mind that the transport mechanisms have a radial dependency. While the pressure-driven velocity decreases to zero at the pore walls, electroosmosis and -phoresis increase towards the pore edge due to the higher electric field.

A certain distribution of resistive pulses due to off-axis trajectories is intrinsic to the TRPS technique. Particles that travel closer to the pore edge cause, due to a higher electric field and a slower velocity, pulses with a higher peak height and duration. This correlation could also be observed in the experiment presented in Chaps. 6 and 7. Electrical and optical signals showed a negative correlation as particles close to the pore edge are subjected to a smaller laser intensity.

Simulations from a Practical Point of View While a physically accurate representation of the model is necessary to study the fundamentals of the RPS 


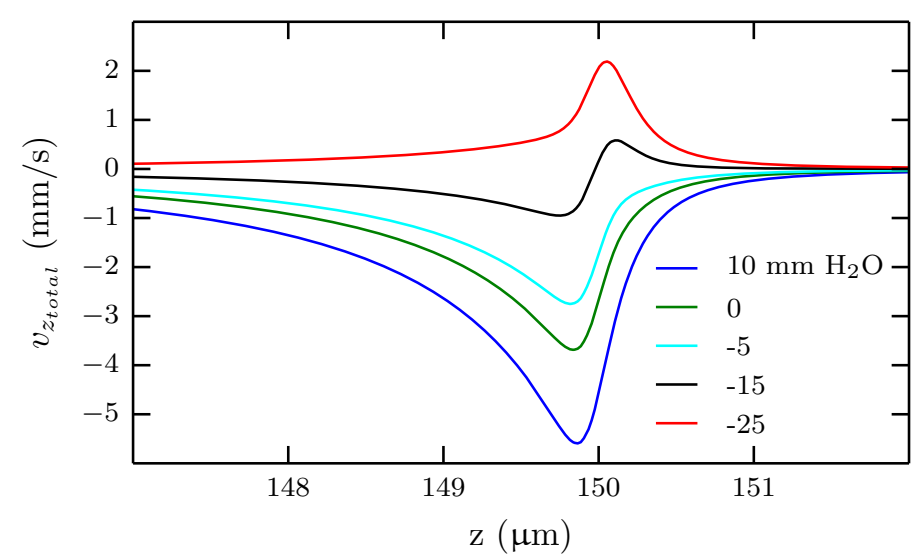

(a)

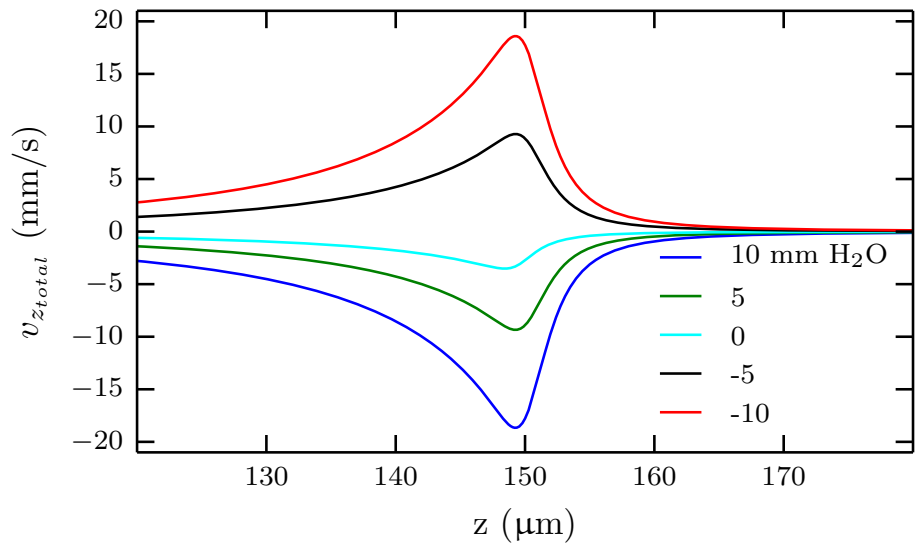

(b)

Figure 3.34: The total particle velocity at various hydrostatic pressures for a (a) NP200 pore $\left(V_{0}=1 \mathrm{~V}\right)$ and a $(\mathrm{b}) \mathrm{NP} 2000$ pore $\left(V_{0}=1 \mathrm{~V}\right)$. The velocity for $P_{0}=$ $0 \mathrm{~mm}$ in (b) is multiplied by 100 to allow for comparison. The $\zeta$-potential in both simulations is $\zeta_{\text {wall }}=-0.20 \mathrm{~V}$ and $\zeta_{\text {part }}=-0.30 \mathrm{~V}$ for the pore wall and particle respectively.

technique, in practice, a fast and simple method to calculate approximate results is often more important. Additionally, as the geometry for the pores used in TRPS is not exactly conical and also slightly varies for each pore even if they have the same classified size, the results from a simulation will not exactly agree with the measurement. The settings for the voltage and pressure are usually adjusted during an experiment to achieve the desired particle direction and frequency. In practice the time advantage that the semi-analytic and the FEM-point particle model offer therefore outweighs their intrinsic inaccuracy.

Figure 3.35 shows the contributions of the pressure-driven, electroosmotic and -phoretic transport to the total particle velocity in a NP200 pore obtained with the semi-analytic and FEM-point particle model. In the FEM simulation the velocities are slightly smaller and also show a smoother, more realistic transition between the pore in- and outside. However, overall the models show good agreement, implying 


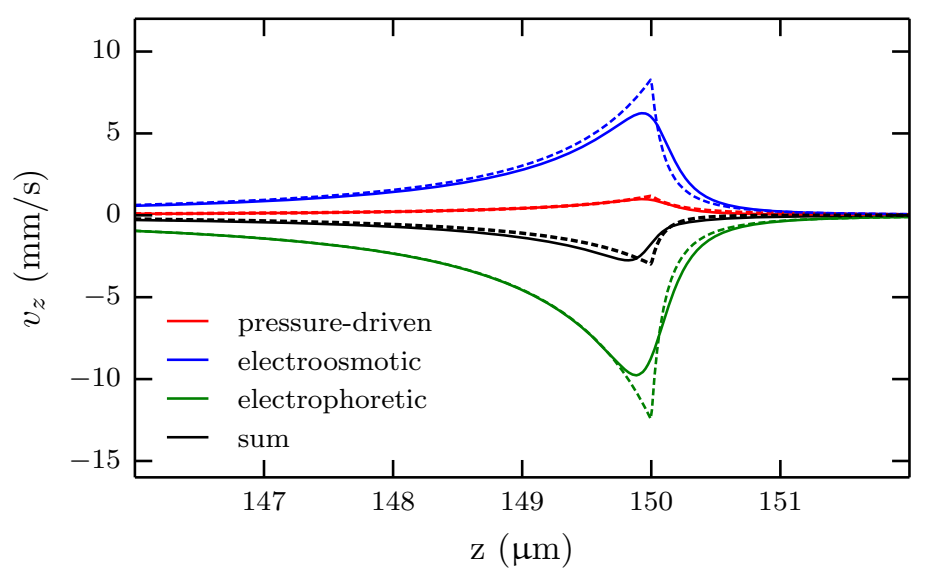

Figure 3.35: The total particle velocity in a conical pore (NP200, $P_{0}=-5 \mathrm{~mm}$ $\left.\mathrm{H}_{2} \mathrm{O}, V_{0}=1 \mathrm{~V}\right)$ and the contributions due to pressure-driven, electroosmotic and electrophoretic transport calculated with the FEM point particle model $(-)$ and the semi-analytic model (- -). The zeta potential of the particle and pore wall are $-30 \mathrm{mV}$ and $-20 \mathrm{mV}$ respectively.

that the simple semi-analytic model can be used to estimate the required parameters needed for a specific experiment. It has the advantage that it is easy to implement, does not require any specialised software and is computationally very fast.

While the simulation of a resistive pulse, which involves calculating the fields, determining the trajectory and deriving the time-current relationship, takes $\sim 10 \mathrm{~s}$ in the semi-analytic model, this value is much higher for both FEM models. The calculation of the fields in the FEM-point particle model takes $\sim 30 \mathrm{~s}$ for a $2 \mathrm{D}$ and $\sim 5$ min for a 3D geometry on an average desktop PC. The consequent calculation of the resistive pulse then takes $\sim 5 \mathrm{~min}$ and $\sim 50 \mathrm{~min}$. The time to simulate one trajectory with the FEM-ALE method can be several hours. The method is also only feasible for 2D, including the axial symmetric, geometries as for a 3D model the memory of a desktop PC is not sufficient.

\subsubsection{Modelling the Ion Distribution in Charged Conical Pores}

The influence of the EDL on the particle transport for nm-scale pores is well established [161] and opens the possibility to use nanopores to probe surface charge fluctuations [162] or use the pore as a diode [163]. In all the preceding simulations the EDL was completely neglected and isotropic conductivity and therefore an Ohmic response of the pore was assumed. The Debye length for typical ion concentrations used in TRPS is at least two orders of magnitude smaller than the smallest pore diameter, so this assumption seems at first reasonable. However, recent publications show that the ion distribution also plays a role in similar sized 
pores where effects like current rectification [140] and bi-phasic pulses [141, 164] have been observed. Lan et al. [140] has shown that the current rectification in glass pores is a direct result of a non-uniform ion distribution. Bi-phasic pulses have been observed and described in NP200 pores by Weatherall and Willmott [134], and the conductive part of the peaks was attributed to a locally enhanced conductivity due to an accumulation of ions.

FEM is the method of choice to account for the ion distribution in charged pores under the influence of an electric potential and a fluid flow as it is capable of coupling the governing equations $[141,165]$. As the particle dynamics are much slower than the dynamics of the ions due to the size difference, the ion distribution can be assumed to be at equilibrium at all times in such a simulation [98].

\subsubsection{Governing Equations}

The underling equations have been described above but are repeated here to illustrate the coupling between them. The electric potential is determined by the ion distribution through the Poisson equation

$$
\Delta \psi=-\frac{e N_{A}}{\epsilon_{f l} \epsilon_{0}} \sum_{i} z_{i} c_{i} .
$$

The Navier-Stokes equations

$$
\rho(\boldsymbol{u} \cdot \nabla) \boldsymbol{u}=-\nabla P+\mu \nabla^{2} \boldsymbol{u}+\boldsymbol{F}
$$

and

$$
\nabla \cdot \boldsymbol{u}=0
$$

couple directly to the Poisson-equation via the volume force term $\boldsymbol{F}=e N_{A} \sum_{i} z_{i} c_{i} \boldsymbol{E}$, which is used to implement electroosmotic flow in the model. The Nernst-Planck equation

$$
\boldsymbol{J}_{\boldsymbol{i}}=-D_{i} \nabla c_{i}-\frac{z_{i} e}{k_{B} T} D_{i} c_{i} \boldsymbol{E}+c_{i} \boldsymbol{u},
$$

calculates the steady-state flow of ions due to diffusion, migration in an electric field and convection and is coupled to both Eqs. 3.64 and 3.65. An analytic solution for 
this complex system of equations in an arbitrary domain is far from being feasible, but they can be solved numerically with the FEM method.

\subsubsection{Model Setup and Boundary Conditions}

The COMSOL modules "Electrostatics", "Laminar Flow" and "Transport of Diluted Species" were used to implement the corresponding equations and appropriate boundary conditions. Additionally to $P_{0}$ and $V_{0}$, the ion concentration $c_{0_{i}}$ was assigned to the top and bottom boundary of the domain (Fig. 3.7). The pore walls and the particle surface are assigned the boundary conditions $\boldsymbol{n} \cdot\left(\boldsymbol{E}_{\boldsymbol{f l}}-\boldsymbol{E}_{\boldsymbol{s}}\right)=\sigma_{\text {wall }}$ and $\sigma_{\text {part }}$. Here $\boldsymbol{E}_{\boldsymbol{f} \boldsymbol{l}}$ is the electric field in the fluid and $\boldsymbol{E}_{\boldsymbol{s}}$ at the solid surface.

To avoid convergence issues, the corners of the pore and the reservoirs were rounded with a radius $a / 100$. Another crucial point for the simulation is that the mesh size close to the charged surfaces is sufficiency small. This can be achieved using a sequence of at least 8 boundary layers, with the thickness of the first layer $~$ $1 / 3$ of the Debye length and a boundary layer stretching factor of 1.2. As usually $\mathrm{KCl}$ is used as electrolyte the diffusion constants $D_{i}$ in Eq. 3.67 are given by $1.957 \mathrm{~m}^{2} \mathrm{~s}^{-1}$ for the $\mathrm{K}^{+}$and $2.032 \mathrm{~m}^{2} \mathrm{~s}^{-1}$ for the $\mathrm{Cl}^{-}$ions [166] at $293 \mathrm{~K}$. The ions have a valence $z_{i}$ of +1 and -1 .

\subsubsection{The Ion Distribution close to a Charged Surface}

Figure 3.36 shows the mesh (a) and the 2D intensity plots of the ion concentrations for both positive (b) and negative (c) ion species close to a negatively charged surface. The Debye length is $0.975 \mathrm{~nm}$ so that the concentrations reach the bulk value within $\sim 5 \mathrm{~nm}$ distance from the surface. The resulting electrostatic potentials for different surface charge densities are plotted in Fig. 3.37 and show the expected exponential decay.

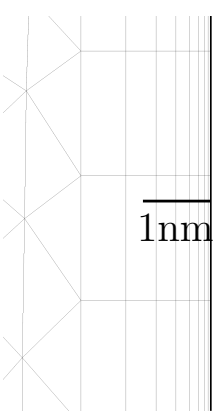

(a)

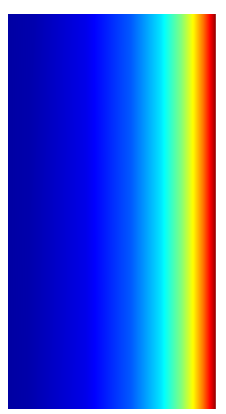

(b)

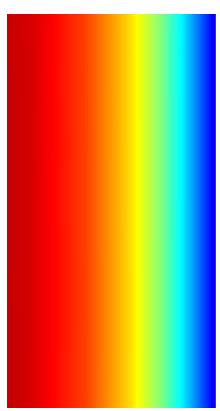

(c)

Figure 3.36: (a) The mesh boundary layer used in the simulation. The ion concentrations for the (b) counter-ion and (c) co-ion close to a charged surface in a $2 \mathrm{D}$ intensity plot $\left(\sigma_{\text {wall }}=-0.0149 \mathrm{C} \mathrm{m}^{-2}, c_{0 i}=\right.$ is $\left.100 \mathrm{mM}\right)$. 
For model verification the surface potential of a charged wall without fluid flow or external electric field is used. The results from the simulations agree very well with the Grahame equation (Eq. 3.34) and are listed in Tab. 3.3. The linear approximation of the equation (Eq. 3.33) is only accurate within an error of $5 \%$ for potentials below $-30 \mathrm{mV}$.

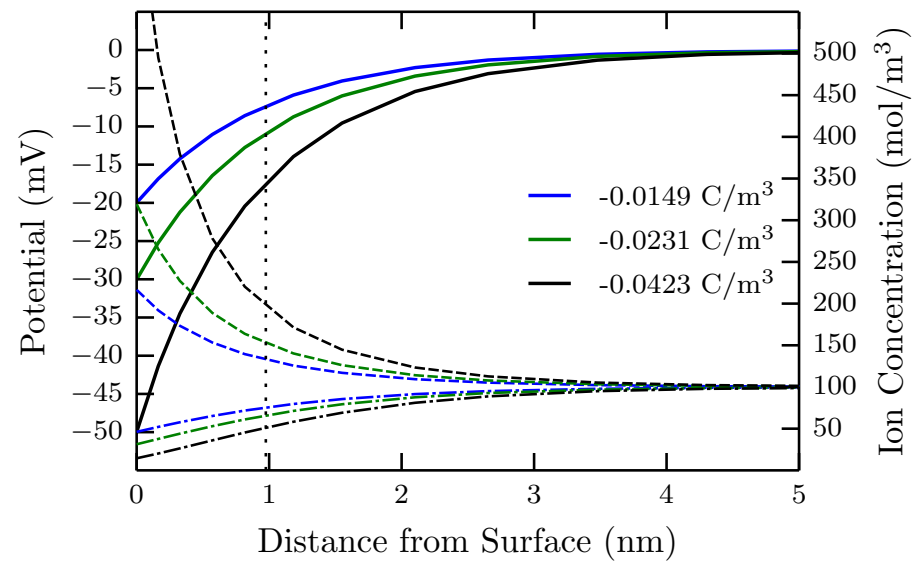

Figure 3.37: The ion concentrations (- - for positive, - - - for negative species) and electric potential $(-)$ close to a surface with the labelled charge densities $\sigma_{\text {wall }}$ for $c_{0_{i}}=100 \mathrm{mM}$. The Debye length is indicated by the $\cdots$ vertical line.

For the range of surface charge densities and molarities typical for TPRS the $\zeta$ potential can be assumed to be approximately equal to the surface potential, which will be verified further below using the electroosmotic flow in a cylindrical channel. This also explains the choice of the $\sigma_{\text {wall }}$ used in the simulations above as the surface charge densities of $-0.0149 \mathrm{C} \mathrm{m}^{-2},-0.0231 \mathrm{C} \mathrm{m}^{-2}$ and $-0.0423 \mathrm{C} \mathrm{m}^{-2}$ result in a $\zeta$ potential of $-20 \mathrm{mV}$ (pore wall), $-30 \mathrm{mV}$ (polystyrene beads) and $-50 \mathrm{mV}$ for $c_{0_{i}}=$ $100 \mathrm{mM}$.

\subsubsection{The Ion Distribution in a Conical Pore}

The Debye lengths in ionic solutions with $100 \mathrm{mM}, 50 \mathrm{mM}$ and $10 \mathrm{mM}$ concentrations are $0.975 \mathrm{~nm}, 1.379 \mathrm{~nm}$ and $3.082 \mathrm{~nm}$ respectively. All these values are almost two orders of magnitude below the smallest pore cross section used in TRPS and the ion concentrations will therefore be equal to the bulk value for most of the pore cross section. For this reason the assumption of a homogeneous ion distribution and therefore conductivity seems to be applicable. This is true for a pore without an applied voltage (Fig. 3.38 (a)). However, when an external voltage is applied the ions redistribute due to the electric field gradient inherent to the pore geometry and the ion distribution becomes non-uniform far from the charged surface. Depending on the direction of the electric field regions of ion depletion or accumulation form close to the pore orifice (Figs. 3.38 (b) and (c)). 
Table 3.3: The potential of a surface with various $\sigma_{\text {wall }}$ in a solution with ion bulk concentration $c_{0_{i}}$ and an ionic valence of $+1 /-1$. The FEM results are compared to the analytic values derived with the Grahame equation (Eq. 3.34) and its linear approximation (Eq. 3.33).

\begin{tabular}{cccc}
\hline $\begin{array}{c}\sigma_{\text {wall }} \\
\left(\mathrm{mC} \mathrm{m}^{-2}\right) \rightarrow\end{array}$ & -14.9 & -23.1 & -42.3 \\
$c_{0_{i}}(\mathrm{mM}) \downarrow$ & \multicolumn{3}{c}{$\begin{array}{c}\text { FEM/Grahame/Approx. } \\
(\mathrm{mV})\end{array}$} \\
\hline & & & \\
\hline 10 & $-54.2 /-54.2 /-64.8$ & $-73.2 /-73.3 /-100.4$ & $-102.3 /-102.4 /-$ \\
50 & $-27.6 /-27.6 /-29.0$ & $-40.6 /-40.6 /-44.9$ & $-64.3 /-64.3 / 82.2$ \\
100 & $-20.0 /-20.0 /-20.5$ & $-30.0 /-30.0 /-31.7$ & $-50.0 /-50.0 /-58.1$ \\
\hline
\end{tabular}

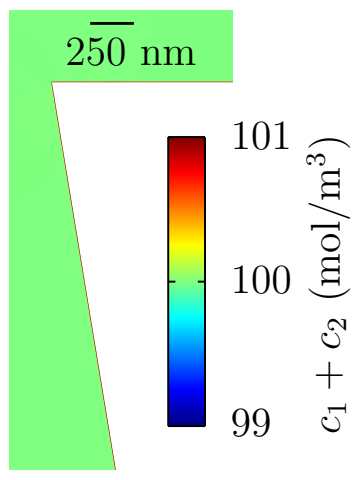

(a)

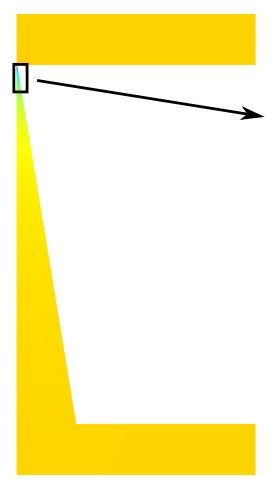

(b)

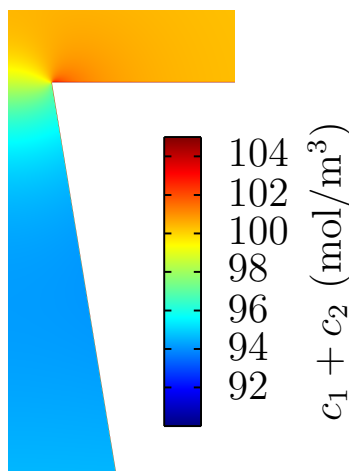

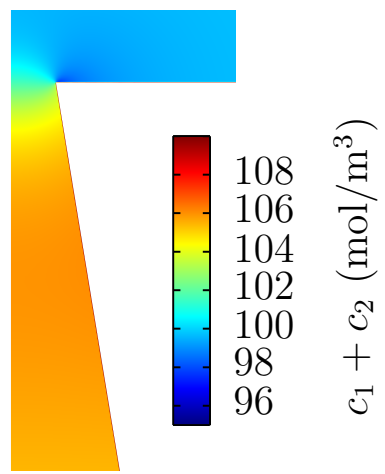

(c)

Figure 3.38: The total ion concentration in a NP200 pore at an applied voltage of (a) $0 \mathrm{~V}$, (b) $1 \mathrm{~V}$ and (c) $-1 \mathrm{~V}$ for $c_{0_{i}}=50 \mathrm{mM}$.

As the conductivity is directly related to the ion concentration, this will result in a different total resistance and current through the pore than the values expected with homogeneous conductivity models. The concentrations of the ion species are different from each other only within a few Debye lengths from the surface (Fig. 3.37). $c_{K}$ and $c_{C l}$ outside this region are exactly half of the value shown in Fig. 3.38.

The ion distribution in a NP200 pore is calculated for various parameters and plotted in Fig. 3.39 relative to the bulk concentration. The height of the accumulation/depletion region within the pore depends on the applied voltage in a linear fashion (Fig. 3.39 (a)). In case of ion depletion inside the pore, an ion concentration slightly higher than the bulk value can be found outside the pore and vice versa. The ion depletion/accumulation is more pronounced for low bulk ion concentrations and high surface charge densities (Figs. 3.39 (b) and (d)). A fluid flow through the pore, which constantly brings bulk solution into the orifice region, changes the 


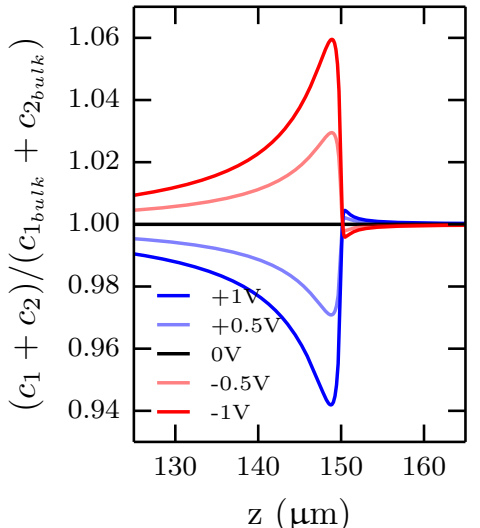

(a)

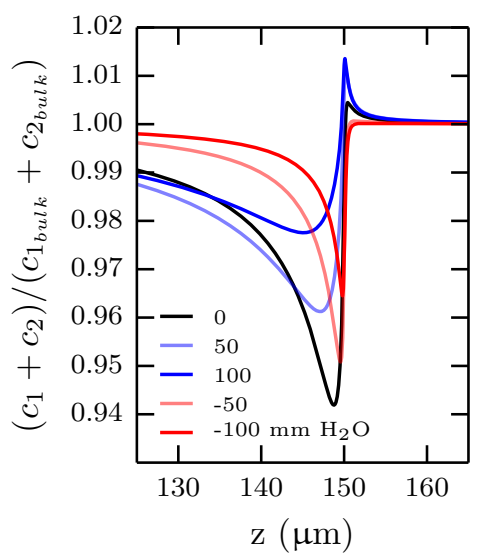

(c)

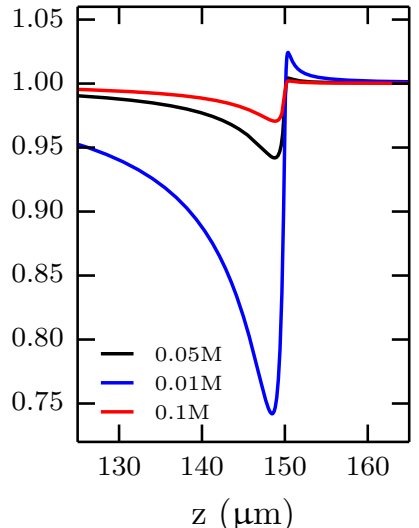

(b)

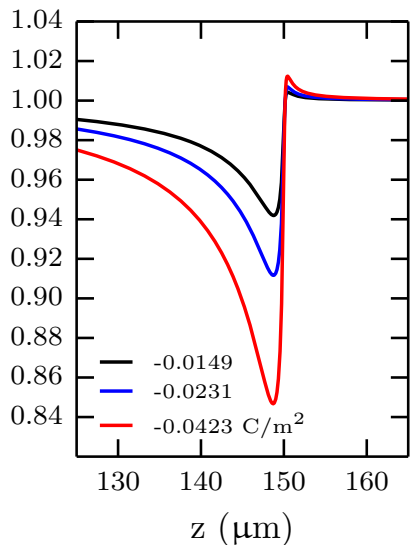

(d)

Figure 3.39: The relative ion depletion/accumulation along the symmetry axis of a NP200 pore for a variation of (a) voltages (b) ion molarities (c) pressure and (d) surface charge. The default parameters are $\sigma_{\text {wall }}=-0.0149 \mathrm{C} \mathrm{m}^{-2}, c_{0_{i}}=50 \mathrm{mM}$, $V_{0}=1 \mathrm{~V}$ and $P_{0}=0 \mathrm{~mm} \mathrm{H}_{2} \mathrm{O}$.

steady-state ion distribution (Fig. 3.39 (c)), which has been reported for similar pores [128]. The flow reduces the depletion or accumulation of ions in the pore but the effect largely depends on the flow direction. Electroosmotic flow, which is not taken into account here, might further change the distribution.

For a NP2000 pore the depletion/accumulation is much less pronounced than for the smaller pore (Fig. 3.40 (a)) and the ion distribution is almost homogeneous for typical parameters used in TRPS. A moderate pressure-driven flow reduces the ion concentration gradient further (Fig. 3.40 (b)), so that charge effects only play a small role, especially at high molarities. In summary, the build-up of an ion concentration gradient in charged conical pores is favoured by a small pore diameter, a high pore wall surface charge, low electrolyte molarities, a high voltage, and a slow fluid flow through the pore.

An inhomogeneous ion distribution in the pore will also affect the electric potential but the effect for a NP200 is marginal even for low ion molarity, where the 


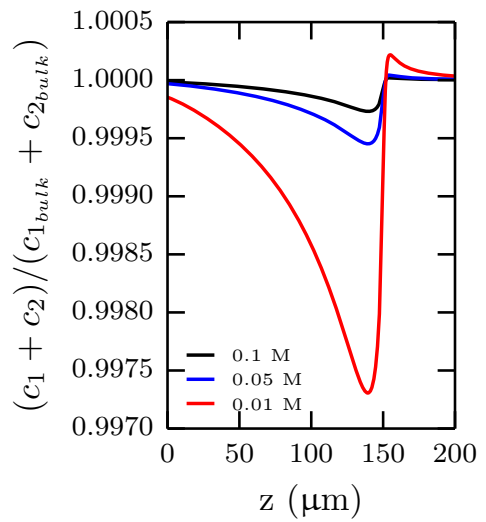

(a)

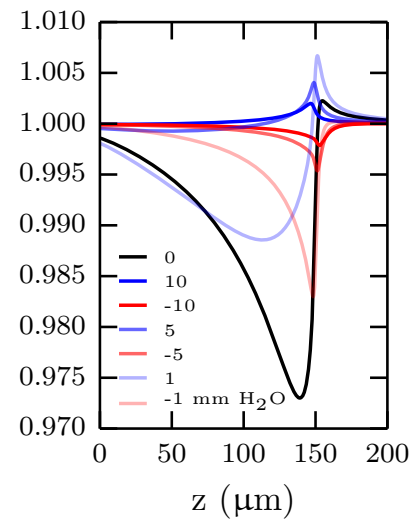

(b)

Figure 3.40: The ion concentration at the symmetry axis of a NP2000 pore with $\sigma_{\text {wall }}=-0.0149 \mathrm{C} \mathrm{m}^{-2}$ for (a) $V_{0}=0.1 \mathrm{~V}, P_{0}=0 \mathrm{~mm} \mathrm{H}_{2} \mathrm{O}$ and (b) $V_{0}=1 \mathrm{~V}$ and $c_{0_{i}}=10 \mathrm{mM}$. Molarity and pressure are varied in (a) and (b) according to the legend.

Table 3.4: The current in nA through a NP200 pore $\left(\sigma_{\text {wall }}=-0.0149 \mathrm{C} \mathrm{m}^{-2}\right)$ calculated with the FEM-ion model and a model assuming homogeneous conductivity $(0.86 \Omega \mathrm{m}) . \sigma_{\text {wall }}=-0.0423 \mathrm{C} \mathrm{m}^{-2}$ for the last row marked with $*$.

\begin{tabular}{cccc}
\hline$c(\mathrm{mM})$ & $1 \mathrm{~V}$ & $-1 \mathrm{~V}$ & $\begin{array}{c}\text { homoge. } \\
\text { model }\end{array}$ \\
\hline 10 & 14.41 & -20.19 & $+/-13.13$ \\
50 & 81.78 & -88.08 & $+/-65.63$ \\
100 & 166.51 & -172.86 & $+/-131.25$ \\
$10^{*}$ & 11.87 & -25.2 & $+/-13.13$ \\
\hline
\end{tabular}

effect is expected to be most pronounced (Fig. 3.41 (a)). The electric potential as a function of radial position one $\mu \mathrm{m}$ inside the pore, where the cross section is roughly an equipotential surface, drops close to the surface by the value $\zeta_{\text {wall }}$ (Fig. 3.41 (b)). The electric field gradient in the double layer can be estimated by dividing the $\zeta$-potential by the Debye length, leading to values $\sim 1 \times 10^{7} \mathrm{~V} \mathrm{~m}^{-1}$, several orders of magnitude higher than the maximal field at the pore orifice. The ion distribution and potential decay close to the charged surfaces is therefore unaffected by the external voltage for the range of voltages used in TRPS.

The ionic current can be calculated from the total ionic flux at the outlet. The values for various molarities and voltages in a NP200 pore are summarised in Tab. 3.4. It shows that contrary to the homogeneous conductivity model where the current is independent of the bias of the applied voltage, current rectification can be explained with the FEM-ion model. The effect is most pronounced for low values of $c_{0_{i}}$. The current calculated with the two models should agree at high mo- 


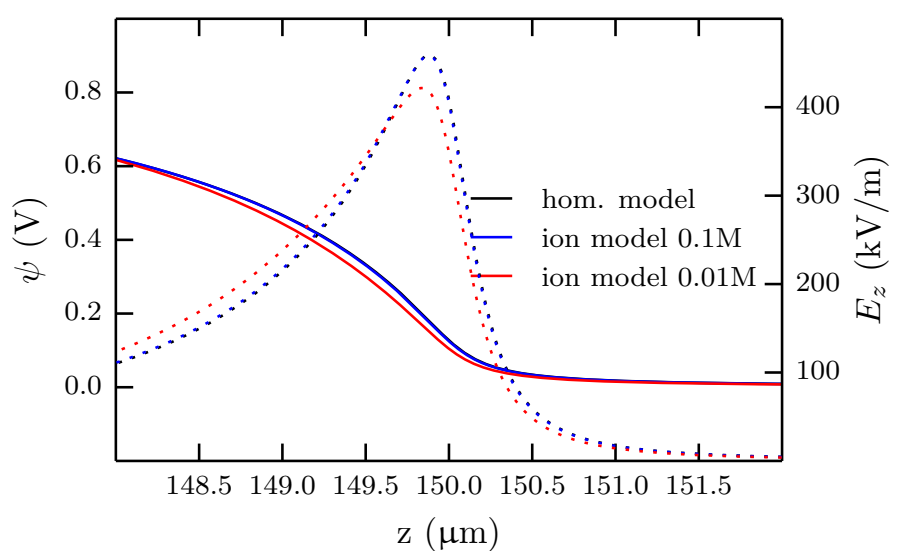

(a)

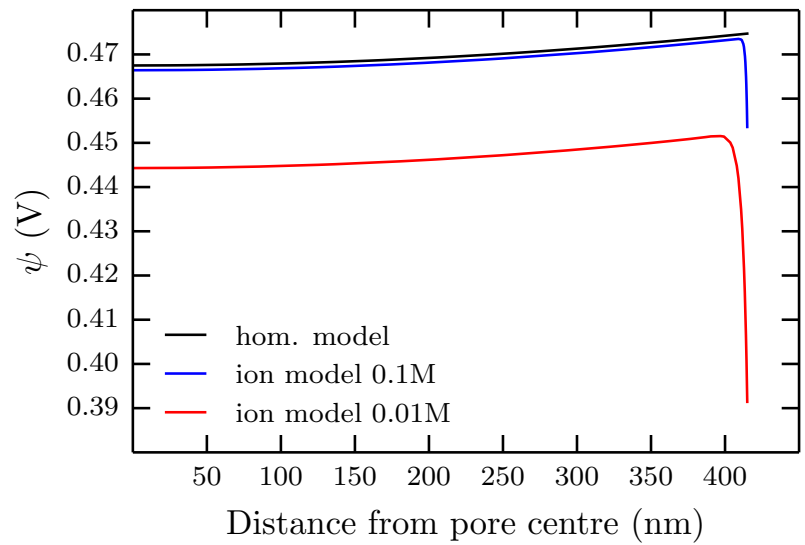

(b)

Figure 3.41: (a) The electric potential (-) and z-component of the electric field (- -) along the symmetry axis of a NP200 pore calculated assuming homogeneous conductivity and for the FEM-ion model for two different molarities $\left(\sigma_{\text {wall }}=\right.$ $-0.0149 \mathrm{C} \mathrm{m}^{-2}$ ). (b) The electric potential as a function of radial position at the plane $z=149 \mu \mathrm{m}$, which is $1 \mu \mathrm{m}$ inside the pore.

larities, which is approximately the case taking the uncertainty of the conductivity into account.

Addition of a Particle When a charged particle approaches the pore it will alter the ion distribution within the channel and can even lead to a current increase. This at first counter-intuitive effect can be understood by taking the ion distribution into account. The addition of a particle with higher charge than the pore wall will result in an increase of the ion concentration and therefore conductivity close to the pore orifice (Fig. 3.42). The current as a function of the particle position for three different molarities is shown in Fig. 3.43. At $(100 \mathrm{mM})$ the surface charges are shielded strongly enough so that no conductive peak is observed. However the pulse height is reduced by a factor of two compared to the calculation using the homogeneous conductivity model. This effect has to be kept in mind when 


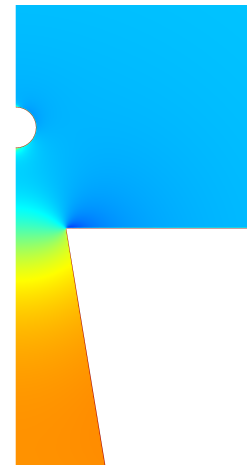

(a)

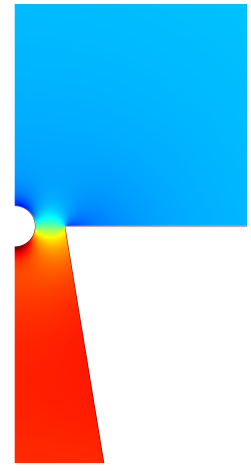

(b)
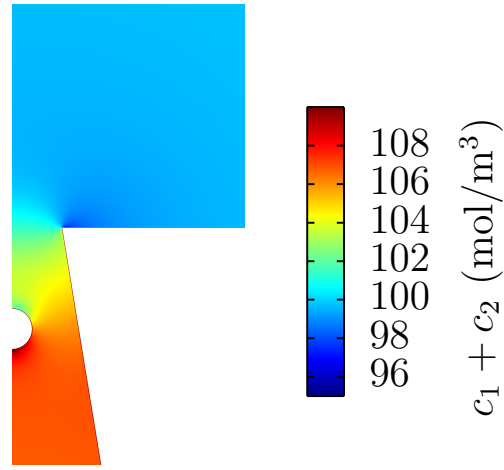

(c)

Figure 3.42: The ion concentration for three positions of a negatively charged particle in NP200 pore $\left(\sigma_{\text {wall }}=-0.0149 \mathrm{C} \mathrm{m}^{-2}, \sigma_{\text {part }}=-0.0231 \mathrm{C} \mathrm{m}^{-2}, c_{0_{i}}=50 \mathrm{mM}\right)$.

the pulse heights measured in TRPS are interpreted. For an ion concentration of $50 \mathrm{mM}$ the conductivity and the current increase when the particle approaches the pore. However, as the particle moves deeper into the pore the exclusion of conductive electrolyte becomes more significant and the current decreases again. This results in a so-called bi-phasic pulse and has been observed in various pore systems $[134,141,164]$.

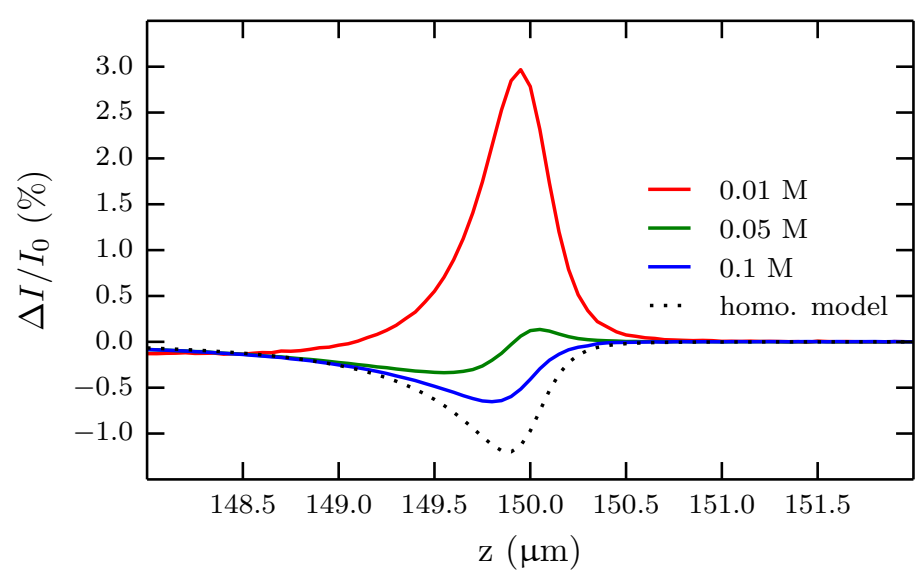

Figure 3.43: The relative current pulses for a negatively charged particle moving through an NP200 pore for different ion concentrations compared to the homogeneous conductivity model.

For an even lower molarity $(10 \mathrm{mM})$, the peak even becomes a purely conductive pulse as the increasing conductivity dominates the effect of electrolyte exclusion. For a larger pore the ion distribution is much more homogeneous so that current rectification and bi-phasic pulses are usually not observed. The effect of current rectification and the exact height and proportions of the conducting and resistive parts of a bi-phasic pulse depend on the applied voltage, the ion molarity and the surface charge of the wall and particles. A fluid flow through the pore flow will 
affect the ion distribution and therefore the exact pulse shape. As the flow of ions due to pressure driven and electroosmotic transport is not taken into account in the model presented here, the simulation will not accurately reflect shape and height of the pulses. However, the study is still valuable for making qualitative predictions.

\subsubsection{Particle Transport using a FEM-ALE-Ion Model}

A transport model that takes the non-zero particle size and the ion distribution into account can be developed analogous to Sec. 3.4.5 by coupling Eqs. 3.64, 3.66, 3.65 and 3.67 and calculating the surface forces on the particle. The model is called FEM-ALE ion model in the following. The hydrodynamic and electrokinetic forces on the particle can then be calculated by integrating the mechanical stress tensor (Eq. 3.59) and the Maxwell stress tensors (Eq. 3.63) over the particle surface. In this way, electroosmosis and electrophoresis are modelled from a microscopic level, resulting from a force on the EDL, transmitting the momentum to the rest of the fluid via viscous coupling [167].

Such a simulation is computationally very expensive and a simulation for a conical pore geometry as used in TRPS was not possible. However, the movement of a $100 \mathrm{~nm}$ particle in a cylindrical channel with a diameter of $500 \mathrm{~nm}$ and a length of $10 \mu \mathrm{m}$ could be simulated. The parameters were $P_{0}=100 \mathrm{~mm} \mathrm{H}_{2} \mathrm{O}, V_{0}=1 \mathrm{~V}$,

$\sigma_{\text {wall }}=-0.0149 \mathrm{C} \mathrm{m}^{-2}, \sigma_{\text {part }}=-0.0231 \mathrm{C} \mathrm{m}^{-2}$ and $c_{0_{i}}=10,50$ and $100 \mathrm{mM}$. The results were compared to the FEM-ALE model (Sec. 3.4.5) assuming homogeneous conductivity. It shows that the distances travelled by an initially resting particle in $1 \mathrm{~s}$ are within $10 \%$ for both models when the molarity is $50 \mathrm{mM}$ and $100 \mathrm{mM}$. For low molarity $(10 \mathrm{mM})$ the results are significantly different and the particle even moves in opposite direction for both models, which indicates that the ion distribution can have a significant influence on the movement of the particle for dimensions much higher than the Debye length. The FEM-ALE ion model models the movement and the resistive pulse with the highest physical accuracy of all models developed. It could be of benefit to TRPS in predicting charge effects like current rectification and bi-phasic pulses with the correct treatment of all transport mechanisms.

Electroosmotic Transport The tangential electric field at a charged surface gives rise to the electroosmotic flow, which can be modelled in the FEM-ion model using the volume force in the Navier-Stokes equation (Eq. 3.68). In that way the elecctroosmotic velocity in a channel of $500 \mathrm{~nm}$ diameter and $10 \mu \mathrm{m}$ length were calculated and compared to the $\zeta_{\text {wall }}$ formulation (Eq. 3.36). The resulting velocities show a good agreement (Tab. 3.5), which acts also as an indirect demonstration that equalising the zeta potential with the surface potential is appropriate for relatively low surface charges and high molarities and therefore shielding. For higher surface 


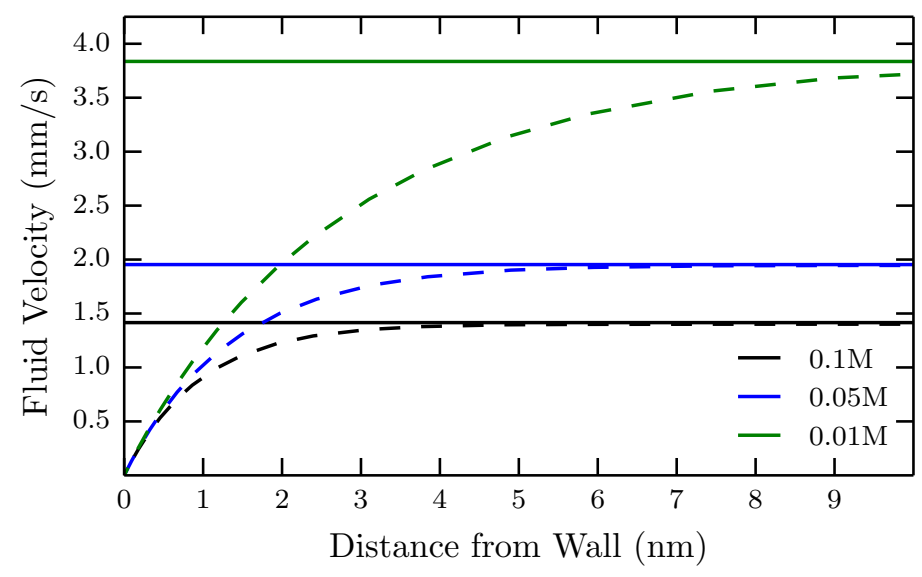

Figure 3.44: The electroosmotic flow in a cylindrical channel $(500 \mathrm{~mm}$ diameter, $\sigma_{\text {wall }}=-0.0149 \mathrm{C} \mathrm{m}^{-2}$ ) calculated with the FEM-ion model (- -) and the using the corresponding $\zeta_{\text {wall }}$ with Eq. $3.36(-)$ for different molarities.

charged densities this approach breaks down as the effect of the Stern layer will become more pronounced.

$$
\boldsymbol{F}=e N_{A} \sum_{i} z_{i} c_{i} \boldsymbol{E}
$$

Table 3.5: The electroosmotic velocities in a channel $\left(\sigma_{\text {wall }}=-0.0149 \mathrm{C} \mathrm{m}^{-2}, 500 \mathrm{~nm}\right.$ diameter, $10 \mu \mathrm{m}$ length) and an applied voltage of $1 \mathrm{~V}$ derived with the $\zeta_{\text {wall }}$ and FEM-ion approaches. $\sigma_{\text {wall }}=-0.0423 \mathrm{C} \mathrm{m}^{-2}$ for the last row marked with $*$. The values for $\zeta_{\text {wall }}$ can be obtained from Tab. 3.3.

\begin{tabular}{ccc}
\hline$c_{K C l}(\mathrm{mM})$ & $\begin{array}{c}v_{\text {eo }}\left(\mathrm{mm} \mathrm{s}^{-1}\right) \\
\zeta_{\text {wall }} \text {-model }\end{array}$ & $\begin{array}{c}v_{e o}\left(\mathrm{~mm} \mathrm{~s}^{-1}\right) \\
\text { ion-model }\end{array}$ \\
\hline 10 & 3.83 & 3.88 \\
50 & 1.95 & 1.96 \\
100 & 1.41 & 1.42 \\
$10^{*}$ & 7.22 & 6.27 \\
\hline
\end{tabular}

\subsubsection{The Mechanical Eigenfrequencies of a Pre-stretched Membrane}

One distinctive feature of TRPS is its ability to tune the pore size by applying mechanical stress. The change in the pore dimension has been studied empirically $[80,168]$. The exact relationship between the macroscopic stretch and the "micro- 
scopic" change in pore size is a classical structural mechanics problem which can be solved with the FEM method [169, 170].

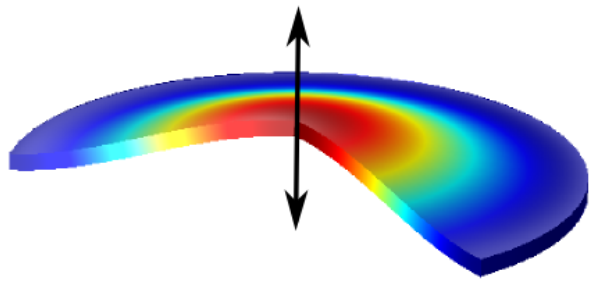

(a)

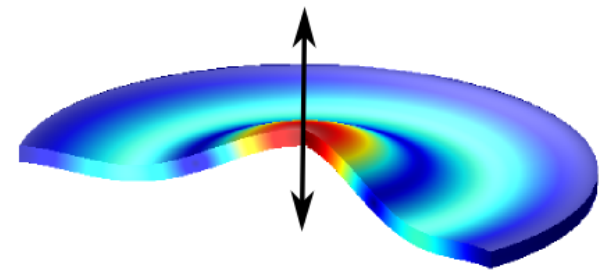

(b)

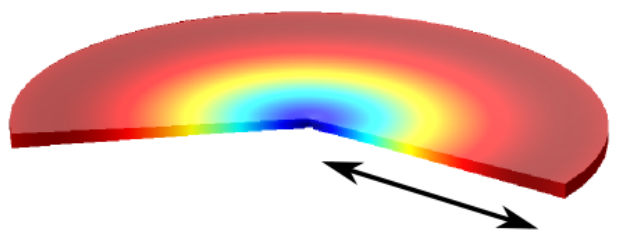

(c)

Figure 3.45: The Eigenfrequencies of a pre-stretched membrane (radius $2.5 \mathrm{~mm}$, thickness $150 \mu \mathrm{m}$ ) with a NP2000 pore in the centre at a stretch of $10 \%$. The values are $2591 \mathrm{~Hz}, 9779 \mathrm{~Hz}$ and $32054 \mathrm{~Hz}$ for the principal modes shown in (a), (b) and (c).

Here, the FEM method was used to determine the mechanical Eigenfrequencies of the pore membrane as it was suspected to relate to the low-frequency $(\sim 47 \mathrm{~Hz})$ noise that was observed in the coordination experiment when the laser was focussed onto the pore orifice. The Eigenfrequency problem of a prestretched membrane can be solved with the FEM method. COMSOL's "Solid Mechanics" module was used to set up the simulation. A linear elastic material model was used to model the stress-strain behaviour of polyurethane membrane (Young's modulus $G=51.7 \mathrm{MPa}$, Poisson ratio $\nu=0.49$ [170]) with the dimensions of the central septum of a TRPS membrane. The medium around the membrane has no influence on the resulting Eigenfrequencies. To avoid translational movement a boundary constraint in the axial direction for one point in the centre of the membrane has to be set. A mechanical stress was applied to reach a final stretch of $10 \%$. An Eigenfrequcney study of the pre-stretched membrane was then conducted. As expected, the frequencies of the first Eigenmodes of the membrane vibration are much higher than the observed noise and a mechanical vibration of the pore septum can be excluded as a reason for the observed noise. 


\subsection{Conclusion}

In this chapter several FEM models have been developed, applied to various problems connected to the TRPS technique, and compared to semi-analytic models, which are conventionally used in TRPS. The most important findings are summarized in the following.

Although the semi-analytical model neglects the radial components of the electric field, it is capable of accurately predicting (within 1\%) the total current and flow through a conical pore as well as the approximate potential shape and on-axis electric field. However, the FEM simulations clearly show that the electric field is not constant over the pore cross section and has a significant radial component at the pore edges. The axial component rises to twice its on-axis value at the pore edge, where the radial component becomes comparable to the axial component. The introduction of a particle of similar size alters the electric field distribution inside the pore significantly.

The results of the semi-analytic and FEM models for predicting the resistive pulse size for an insulating particle in a conical pore are in good agreement. The values derived from Maxwell and Deblois et al.'s expressions lead to systematic errors but can be used to approximately estimate the particle size. Maxwell's method gives slightly better results than Deblois et al.'s but overestimates the particle size. However, as both models do not account for off-axis particles, which result in larger pulse heights than on-axis particles and represent the majority of trajectories, the models will be closer to the real value of the resistive pulse. This explains their relative success in predicting the particle size in TRPS. As the pore geometry is usually not known precisely the uncertainty in the determination of the small pore radius is typically larger that the uncertainty caused by the model. The FEM model shows that the resistive pulse height is not exactly proportional to the volume of the particle, which is important when calibration particles are used.

The total fluid flow through a conical pore can be predicted with the semianalytic model in good agreement with the FEM-models. Again, the exact flow profile is more complex and entrance effects are observed in the FEM simulation. The pressure-driven flow profile close to the orifice is not parabolic. The electroosmotic flow is non-uniform at the pore orifice but develops into a plug flow a few $\mu \mathrm{m}$ inside the pore. The presence of a particle alters the flow field to a minor extent. Although slight hydrodynamic focussing can be seen at high pressure it is not expected to play a significant role in TRPS.

Off-axis trajectories lead to a double peak in the pulse distribution. A focussing due to electrophoresis is prominent in small pores, but dielectrophoresis does not play a role for the parameters in TRPS. The semi-analytic and FEM-point particle 
models overestimate electrophoretic transport.

The ion distribution in the pore for low molarity is not uniform and can lead to current rectification or bi-phasic pulses. These can be predicted with the FEM-ion model. Adding the particle dynamics to the model results in a model very close to the physical reality and would help to better understand the fundamentals of the technique. However, it comes at high computational cost and could not be tested in the present work.

Several of the results proved useful for the interpretation of the experimental data. FEM-modelling has not been implemented in TRPS previously and will be beneficial for a thorough understanding of the technique and particle analysis beyond this work. 


\section{Chapter 4}

\section{Materials and Methods}

The experiments were conducted by combining commercial equipment, namely the qNano (Izon Science Ltd.) and the Labram (Horiba Jobin Yvon). Both devices had to be adapted for the specific use, with the restriction that no permanent alterations to the spectrometer could be done as it is operated by different users on a day to day basis. In this chapter, the developed experimental techniques are presented in detail along with a discussion of several encountered effects, e.g. the laser-induced noise, that are relevant to the method. General topics like the measurement of the fluorescent cross section of a turbid sample or the confocality of a microscope system are specific to the setup but are explained in general terms so that they can be easily adopted to similar systems. The aim is that the reader understands the necessary physical concepts and has enough information to potentially carry out similar experiments her/himself. A model to predict the optical signals in the coordination experiments is presented at the end of this chapter, followed by a conceptual explanation of the custom-made software that was used to find, correlate and spectrally identify the events in the recorded data.

\subsection{Izon's qNano}

For the TRPS measurements the qNano was used throughout the experiments (Fig. 4.1 (a)). It has proven useful to measure synthetic and biological particles from the $\mu \mathrm{m}$-scale down to $\sim 50 \mathrm{~nm}$ and has been described in detail in several publications $[38,40,42]$ and two recent reviews [49, 50]. A cross-shaped polyurethane membrane specimen containing a stretchable pore in its centre is sandwiched between the upper and lower half of the fluid cell of the device (Fig. 4.1 (b)). As a result of the fabrication method, the pores are conically shaped. In experiments, the small-side pore aperture is facing the upper half of the fluid cell. Both halves of the cell are filled with electrolyte solution, with the top half of the fluid cell also containing the analyte particles. $\mathrm{Ag} / \mathrm{AgCl}$ electrodes in the fluid cell are used to apply a 


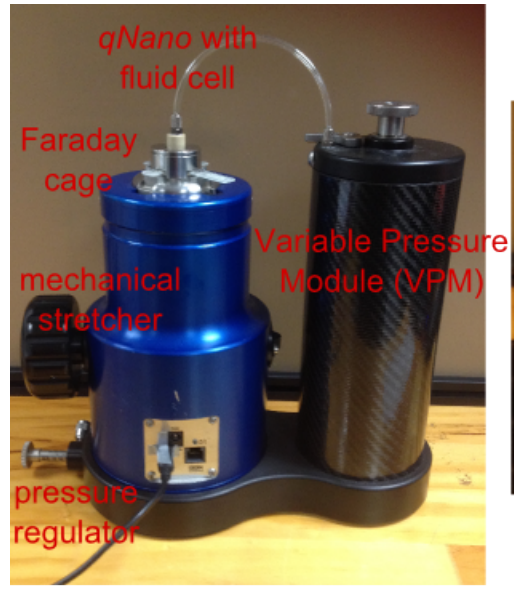

(a)

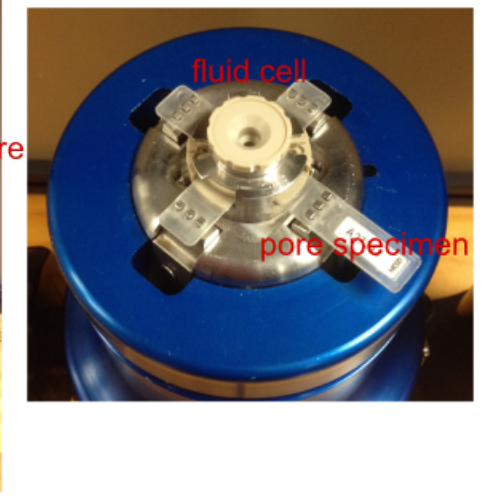

(b)

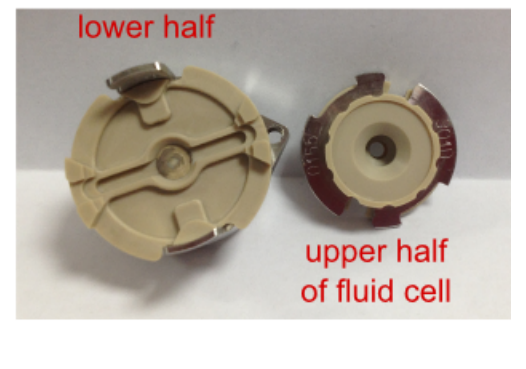

(c)

Figure 4.1: (a) The original resistive pulse device as purchased from Izon Science Ltd. including the pressure control module. (b) A pore specimen sandwiched between the two halves of the fluid cell, which is shown in detail in (c).

constant electric potential and to measure the resulting ionic current. The particles are driven through the pore by a combination of hydrodynamic, electroosmotic and electrophoretic transport.

The distinctive feature of TRPS is that the pore dimension can be dynamically adjusted by stretching or relaxing the membrane specimen. One advantage of this feature is that the pore can be actuated during an experiment in order to optimize the signal to noise ratio for a specific analyte so that a larger range of particle sizes can be measured with the same pore specimen compared to fixed-size pores. The flexibility of the pore size also enables the study of size related effects and can potentially be used to trap or gate particles. Additionally, clogging of the pore, a common problem in RPS devices and often the end of the lifetime of a pore specimen, can be removed by stretching the pore and applying hydrostatic pressure to flush out the particles.

The setup of the system is simple and fast. The membrane is placed on teeth like structures situated on four opposite jaws. A mechanical stress is applied in the horizontal plane and the groove in the lower half of the fluid cell is filled with electrolyte solution. Usually pH-buffered solutions with a $\mathrm{KCl}$ concentration between $10 \mathrm{mM}$ and $100 \mathrm{mM}$ are used in TRPS. After the upper half of the flow cell is put in place, the solution containing the particles is applied to the upper fluid compartment. A total amount of only $\sim 120 \mu \mathrm{L}$ of the sample is needed, with $80 \mu \mathrm{L}$ in the lower and $40 \mu \mathrm{L}$ in the upper half of the fluid cell. The Variable Pressure Module (VPM) is attached to the upper part of the fluid cell and by pushing and pulling a lever the pressure can be controlled with an accuracy equivalent to $1 \mathrm{~mm} \mathrm{H}_{2} \mathrm{O}(9.81 \mathrm{~Pa})$. The VPM is set in such a way to achieve the desired particle direction of motion and velocity. The intrinsic pressure head due to the liquid contained in the upper 
fluid cell is equivalent to $4.7 \mathrm{~mm} \mathrm{H}_{2} \mathrm{O}$ [38], which has to be taken into account when absolute pressures are of interest. The relative magnitude of the transport mechanisms (pressure-driven, electrophoresis and electroosmotic) is mainly dependent on the pore size (compare Sec. 3.3). The resulting current is recorded with a $50 \mathrm{kHz}$ sampling frequency using the customised software (Izon Control Suite Version 2.2) and the particles are afterwards analysed through their current pulse magnitude, frequency and duration which are connected to particle size, concentration and charge.

\subsubsection{The Adapted Setups}

The $q$ Nano has proven well engineered and reliable in many situations and therefore it was attempted to leave the device mostly in its original state. However, as the optical access to the pore is blocked by the connection to the pressure control module, a different flow cell had to be used for the coordination experiments. In the course of this work two different setups and flow cell designs have been developed, which are shown in Figs. 4.2 and 4.4. While the first setup requires minimal alterations and can easily be attached to any spectrometer without much challenge, the second setup, which has significant alterations, shows better performance and sensitivity.

\subsubsection{The First Setup}

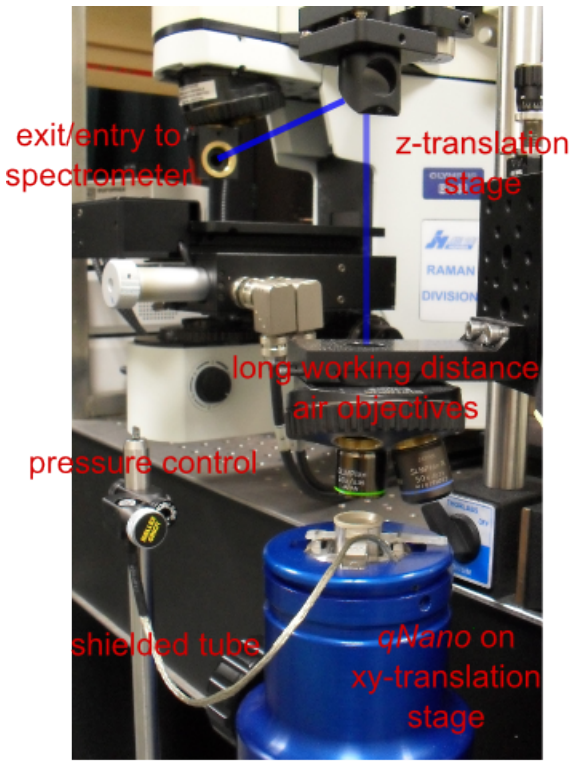

(a)

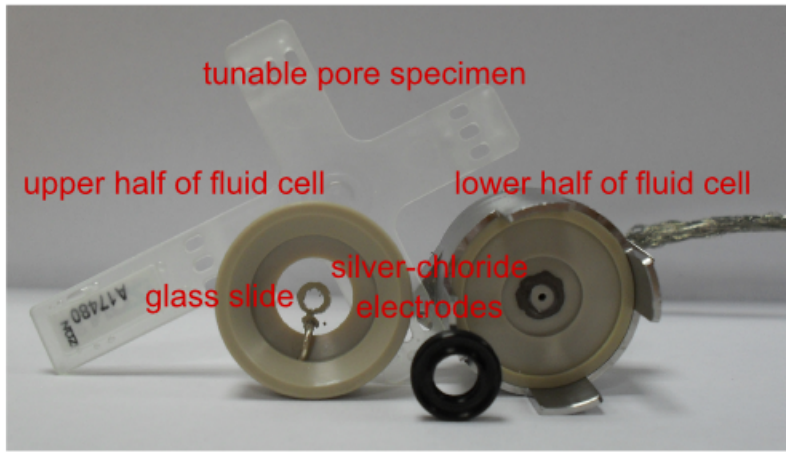

(b)

Figure 4.2: (a) The first setup, suitable for $\mu \mathrm{m}$-sized particles. (b) Dismantled fluid cell with tunable pore specimen. Surfaces that face upwards in the experiment are facing the camera. The rubber o-ring is placed in contact with the lower fluid cell, under the specimen.

In the first design a round standard cover glass $(16 \mathrm{~mm}$ diameter, $0.17 \mathrm{~mm}$ thick- 
ness) with a painted silver electrode (silver chloride paste) is used to allow a direct optical window to the pore orifice and provide electrical contact to the upper half of the fluid cell (Fig. 4.2 (b)). A drop of electrolyte solution containing the particles is placed in the $\sim 2 \mathrm{~mm}$ gap between the pore orifice and the glass slide. To control the hydrostatic pressure at the pore a tube was attached to the lower half of the fluid cell and filled with electrolyte. By simply moving the tube up and down along a post, the pressure can be adjusted in a way that the desired direction and speed of fluid and particles through the pore is achieved.

As the intrinsic pressure head is not exactly known for the new flow cells, the zero pressure point is found by using the fact that the current is dependent on the applied pressure. Figure 4.3 shows the change in current for a NP1000-rated pore when the pressure is changed from initially positive (higher hydrostatic pressure at the upper-side of the membrane) to negative. Around the highest positive curvature in the trace the event frequency reaches zero and the particles change their direction of travel at this pressure. Therefore it can be assumed that at this point the pressure across the membrane is almost zero and it can be used as a reference point. The reason for this pressure dependence of the current, which is also observable in the original device, has not yet been exactly clarified. However, as the simulations in Sec. 3.4.6 suggest, a connection to local conductivity changes due to ion accumulation and depletion regions close to the pore orifice, which are dependent on the fluid flow, is likely. A mechanical reason can be excluded as the hydrostatic pressure at the pore orifice is too small (the maximum is $200 \mathrm{~mm} \mathrm{H}_{2} \mathrm{O}$ using the $V P M)$ to significantly affect the size or shape of the pore. A streaming current, which develops in any channel with charged walls under the influence of a fluid flow, could be another possible explanation. However, the streaming current across a channel is proportional to the applied pressure, which is not observed here. Also an estimation of the achievable streaming current under parameters used in TRPS (maximum pressure of $150 \mathrm{~mm} \mathrm{H}_{2} \mathrm{O}, \zeta_{\text {wall }} \sim-20 \mathrm{mV}$ ) shows that the effect lies at least two orders of magnitude below the observed changes in current.

As the qNano does not fit on the microscope stage of the spectrometer used, it is placed on a small platform attached to the optical table. The laser beam is coupled out and the light collected back into the spectrometer using mirrors. An objective revolver mounted on a translation stage with $\mu \mathrm{m}$ precision holds the objectives, which are used for focussing the laser beam on the nanopore. The collection of the scattered light is done in backscattering geometry with the same objective as the excitation. The whole setup is shown in Figure 4.2 (a). The qNano is placed on two translation stages to allow for movement in the horizontal plane.

The limits of the setup are reached for particles below $\sim 1 \mu \mathrm{m}$ due to several reasons. First, the focussing and lateral manipulation becomes increasingly inac- 


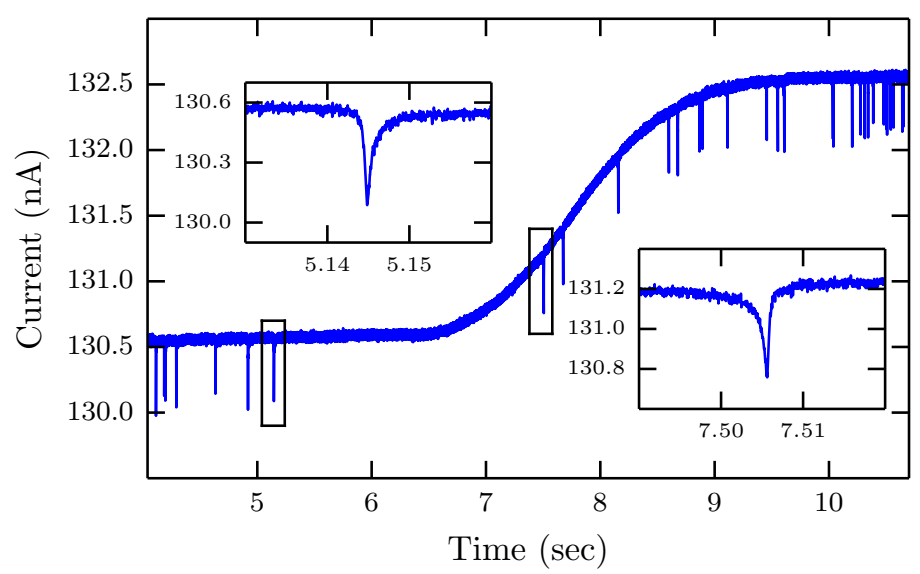

Figure 4.3: The current in a NP1000-rated pore at $16 \mathrm{mV}$ and $45 \mathrm{~mm}$ stretch as a function of the applied pressure. The direction of the particle movement can be deduced from the asymmetry of the resistive pulses. The pressure difference between the left and right current plateau is equivalent to only a few $\mathrm{mm}_{2} \mathrm{O}$.

curate at smaller length scales. Additionally, the flow cell design does not allow for immersion objectives with high numerical apertures as the minimal working distance is $\sim 5 \mathrm{~mm}$. Also, the relatively long distance between the dichroic mirror and the objective does not allow for a placement of lenses or lens systems to engineer the beam size at the desirable positions, i.e. closer to the objective. Finally, as the liquid drop put between pore orifice and glass slide is held up by surface tension, only a limited amount of surfactant in the electrolyte solution can be used. This can prevent a stable wetting of the pores, especially for pores at the lower size range used in TRPS.

\subsubsection{The Second Setup}

In the second setup (Fig. 4.4) all of the apparent drawbacks of the initial design could be addressed resulting in a more sensitive and stable device. The fluid cell was redesigned and mounted on a custom made stretcher, which is placed directly on the optical table, resulting in more accurate focussing and lateral positioning. The upper half of the fluid cell is open which allows for the use of immersion objectives with high numerical aperture. A hydrophobic film (Parafilm) is attached to the bottom of the upper half of the fluid cell so that leakage is prevented and the use of more surfactant to assist pore wetting is now possible. Additionally, the liquid can be exchanged without dissembling the fluid cell, which is a big advantage in practice. The upper electrode consists of a wire made of fine silver (Goodfellow Cambridge Ltd.) covered in a layer of silver chloride instead of the painted glass slide, which was prone to damage. To cover the silver wire with a chloride layer, the inner compartment of the fluid cell is filled with household strength bleach $(4.2 \%$ 


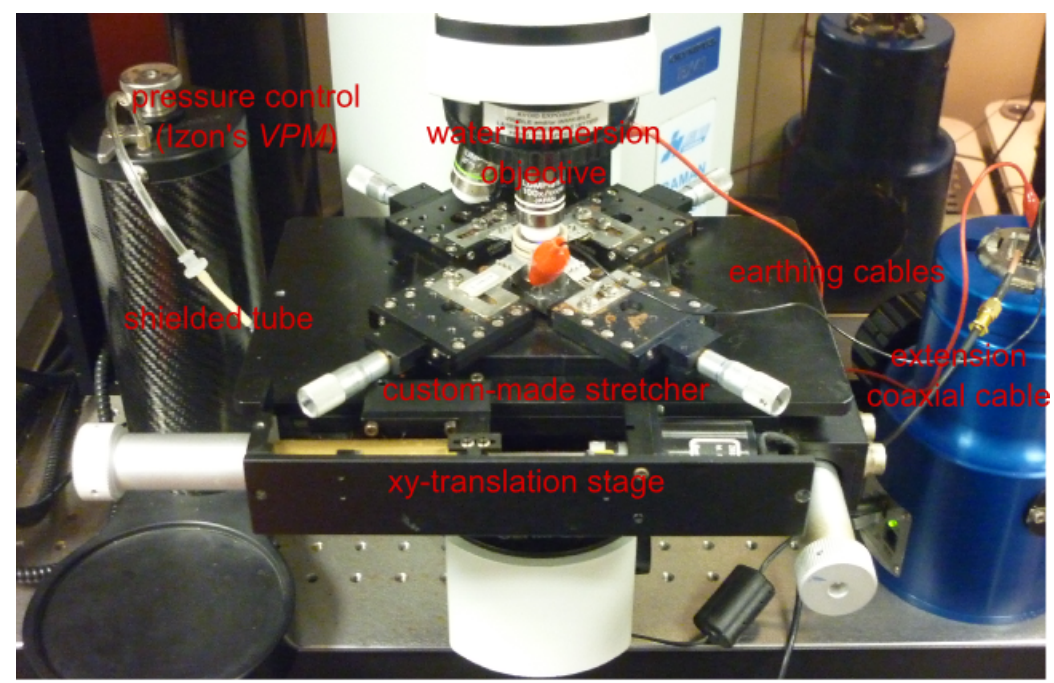

(a)

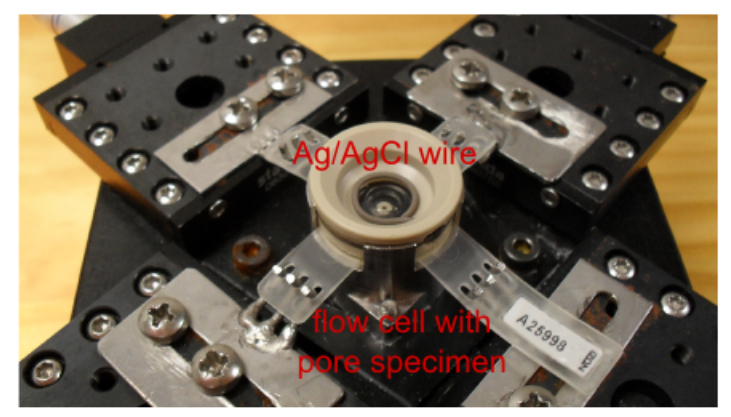

(b)

Figure 4.4: (a) The second setup, which has an improved sensitivity compared to the first design, and its flow cell in detail (b).

sodium hypochloride) and rinsed after $\sim 20$ min when a grey layer has developed. During this procedure it has to be taken care that the outer silver ring does not come into contact with the bleach to assure a good electric contact with the negative pole base of the fluid cell. The accuracy of the pressure control has been improved by using the VPM of the original device (see "pressure regulator" in Fig. 4.1 (a)).

\subsubsection{Setting up the Experiment}

It is important to prevent the occurrence of air bubbles in the lower fluid compartment, which leads to either a large noise or makes any measurement impossible as the bubbles will inevitably rise to the membrane and block the pore. In such a case, the fluid cell has to be dissembled, cleared from bubbles and reassembled again. To avoid this issue the fluid cell for both designs should be assembled as described in the following. First, a syringe filled with electrolyte is attached to the end of the tube which is connected to the lower fluid compartment, which is then filled with the liquid. It is advised not to add any surfactant to the solution used in the lower fluid cell, as a surfactant containing fluid is more likely to contain small air bubbles. 
It also has to be ensured that the cavity is completely filled (Fig. 4.5 (a)) before the O-ring, which should be pre-wetted, is put in place. Before the actual pore is used, a dummy pore, which is not used in the experiments any more as it can get damaged during this process, should be installed, establishing a firm seal with the O-ring at a high stretch. Then, by applying high negative and positive pressures alternatively with the syringe, remaining air in the chamber and under the O-ring will rise to the membrane, where it gets trapped (Fig. 4.5 (b)). After the dummy pore has been removed, the chamber is cleared from any bubbles, and the missing liquid is replaced so that a slight meniscus forms above the O-ring (Fig. 4.5 (c)). Then the measurement pore and the upper half of the fluid cell can be installed.

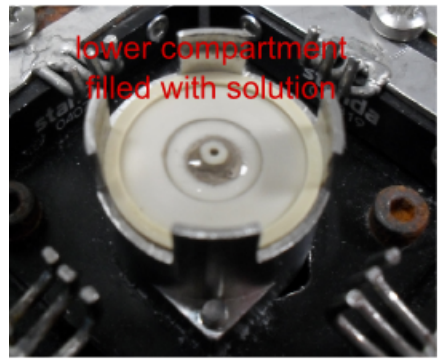

(a)

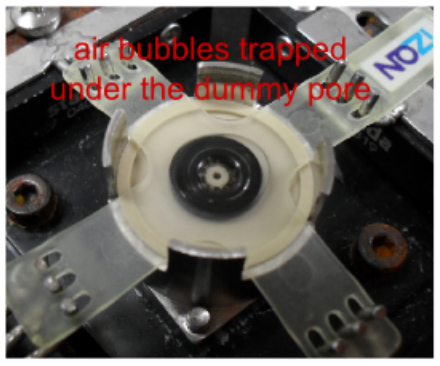

(b)

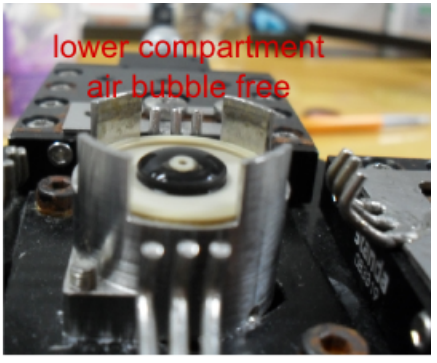

(c)

Figure 4.5: The assembly method for the adapted fluid cell to avoid the formation of air bubbles (described in the text).

In the case of the second fluid cell design, which is open at the top, the fluid in the upper compartment can contain more surfactant compared to the first design. The wetting of the pore can be assisted by applying a gentle negative pressure with the syringe so that surfactant containing fluid gets sucked into the pore. After the flow cell is successfully assembled, excess fluid should be removed from the part of the tube which is not surrounded by the metallic mesh to minimise electric noise. Then the end of the tube can be connected to the pressure control module.

The fully installed setups for both designs are shown in Figs. 4.2 (a) and 4.4 (a). In case of the second design the platform is placed onto the microscope stage and the coaxial extension and the earthing cables are connected to the qNano. A stable current should soon be achieved after switching on the voltage. The stretch is adjusted to achieve the desired baseline current and signal to noise ratio depending on the size of the sample particles. Due to the design of the claw like structure used to hold the cruciform in place in the customised stretcher, the stretch is defined as the closest distance between opposite holes of the cruciform. This is different to the original setup and the first setup, where the stretch is measured between the outer side of the teeth that hold the specimen in place. To transform these two definitions and compare the stretches Tab. 4.1 is used. Its values have been 
obtained by repeatedly measuring the distance between the outside of the metallic teeth and the corresponding distance between the inside of the opposite, centre holes. Although in normal operation, the stretch in $\mathrm{x}$ - and $\mathrm{y}$-directions are set to the same values in the second setup they could in principle be regulated independently to achieve an elliptical pore shape.

Table 4.1: The stretch as defined for the second setup and the equivalent values in the original device.

\begin{tabular}{cc}
\hline $\begin{array}{c}\text { Original Setup } \\
(\mathrm{mm})\end{array}$ & $\begin{array}{c}\text { Custom-made } \\
\text { Stretcher }(\mathrm{mm})\end{array}$ \\
\hline 44 & 37.48 \\
45 & 38.22 \\
46 & 38.91 \\
47 & 39.67 \\
48 & 40.37 \\
49 & 41.11 \\
50 & 41.82 \\
\hline
\end{tabular}

\subsubsection{The Electronic Noise}

The signal bandwidth and the current noise are two crucial parameters for the RPS technique. While a high enough bandwidth is necessary to resolve the details of a current pulse and obtain the correct pulse height [171], the signal noise determines the detection limit of the device, and has to be therefore kept as small as possible. Theoretical and applied studies on the noise in nano- and microscale pores and methods to reduce it can be found in the literature [172-174]. Here, only a comparison of the practical performance of the original and adapted setup will be given.

The original device has been optimized in its signal to noise ratio for a current of $\sim 120 \mathrm{nA}$ and, when used with a Faraday cage that encloses the flow cell (Fig. 4.1 (a)), is well shielded against external noise sources. The noise spectrum (Fig. 4.6 (a)) shows the typical $1 / f$ dependency often observed in nano- and micro-sized pores [175]. A RMS value of the noise of $\sim 10 \mathrm{pA}$ is considered acceptable and allows for a good quality measurement of particles down to the size of $\sim 1 / 5$ of the small pore orifice. Due to the adaptions made in the flow cell design, e.g. the tube filled with electrolyte attached to the lower fluid cell, an influence on the device performance is possible. Especially the second design, which has an extension cable to connect to the qNano and an additional earthing cable (Fig. 4.4 (a)), might be prone to an enhanced noise. 


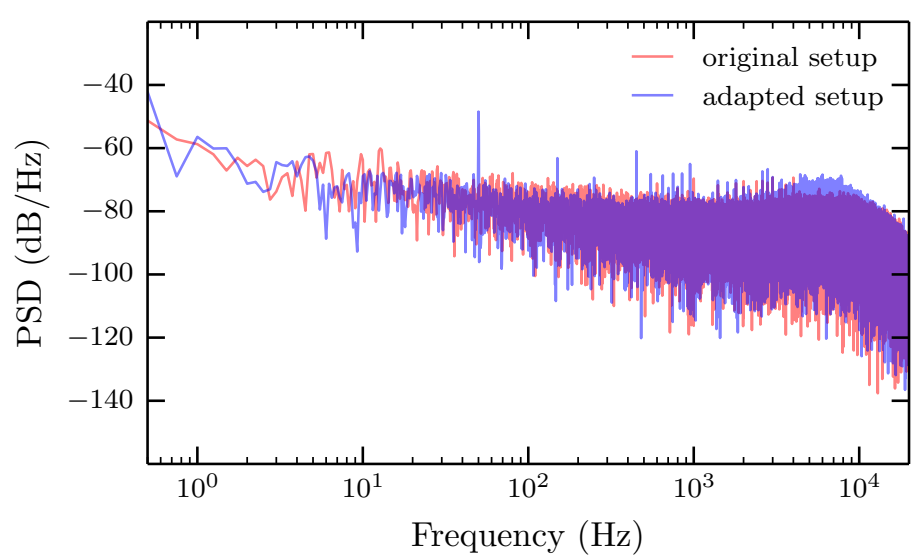

(a)

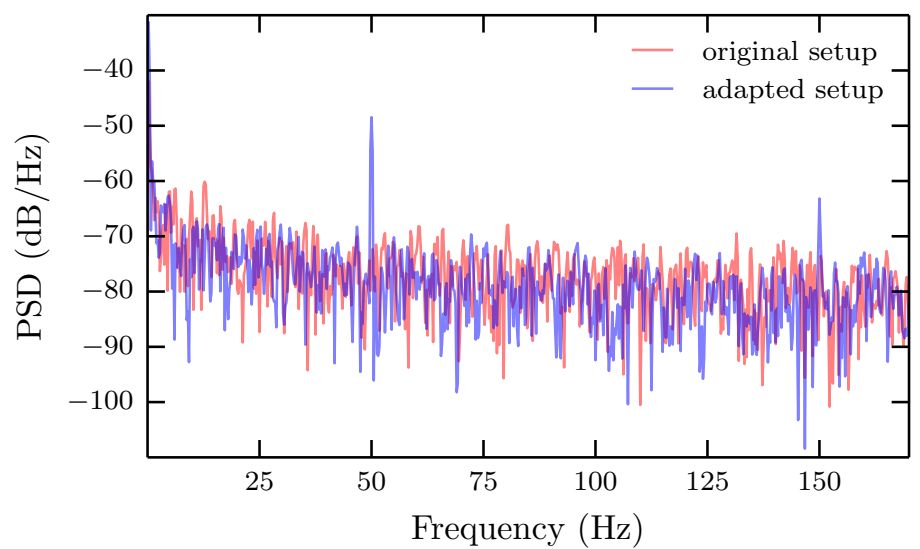

(b)

Figure 4.6: The power spectral density (PSD) for the original and adapted (second) setup using the same pore and settings (NP200, $1 \mathrm{~V}, 45 \mathrm{~mm} / 38.22 \mathrm{~mm}$ stretch, pressure of $10 \mathrm{~mm} \mathrm{H}_{2} \mathrm{O}$ ), showing the whole (a) and the low (b) frequency region in detail.

In practice, when the tube is shielded with a metallic mesh and the flow cell is properly earthed, both adapted setups show a similar current baseline and RMS noise compared to the original device with the identical pore specimen and the same stretch, pressure and voltage settings. The current trace of the original and the second setup using a NP 200-rated pore is shown in Fig. 4.7 (d). An analysis of the power spectral densities of the signals (Fig. 4.6) reveals a slightly higher noise level of the adapted setup at $50 \mathrm{~Hz}$ and its odd harmonic multiples. This is most likely due to the fact that a Faraday cage can not be conveniently used due to the necessity for easy optical access. Additionally, the noise level at certain high frequencies is enhanced, which, has not affected the experiments noticeably. In conclusion, although slightly higher noise levels are observable, the performance of the adapted device is comparable to the original setup. 


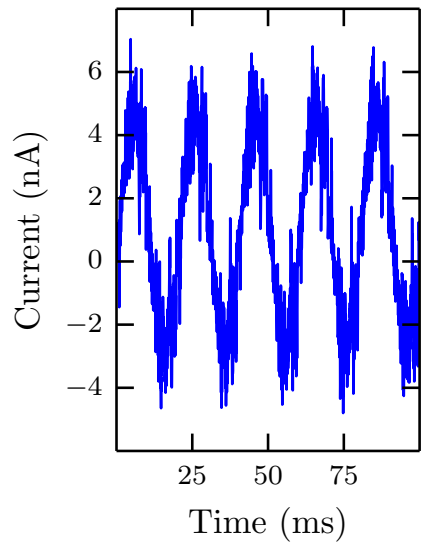

(a)

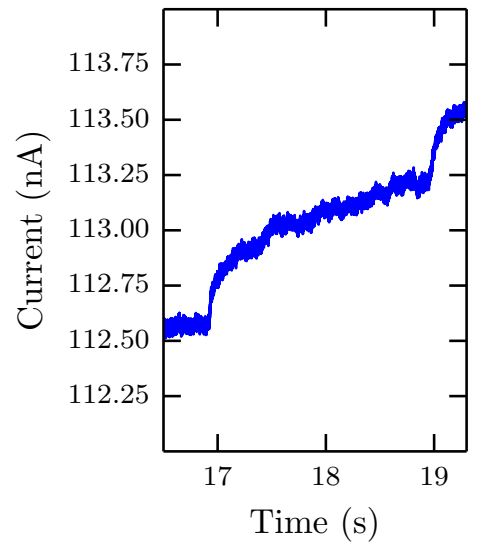

(c)

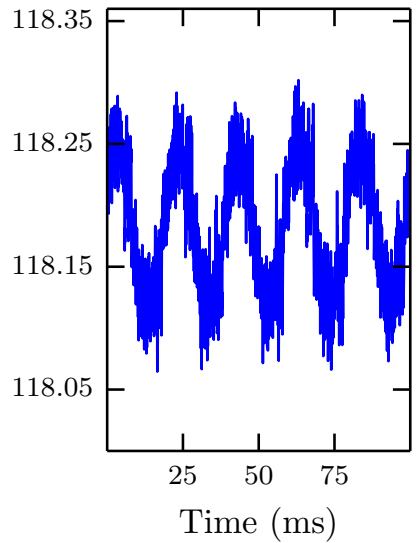

(b)

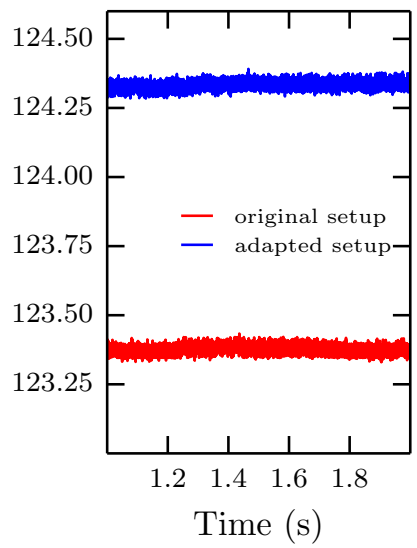

(d)

Figure 4.7: The noise in the second setup for a NP200-rated pore, $1 \mathrm{~V}$ and $38.22 \mathrm{~mm}$ stretch when the earthing cable is not connected (a), the electrolyte containing tube is not properly shielded (b), and an air bubble is trapped in the lower compartment close to the pore (c). (d) shows the current trace in comparison with the original setup under identical settings.

\subsubsection{Troubleshooting}

During the setup design and preliminary experiments very high noise levels in the current trace were encountered, which prevented a successful measurement. This could be attributed to several reasons. A strong $50 \mathrm{~Hz}$ noise is observable if either the earthing cable is not attached to the fluid cell or the tube filled with electrolyte is not properly covered by the shielding mesh (Figs. 4.7 (a) and (b)). An exposure of the tube even on the length of a few $\mathrm{mm}$ is enough to cause the noise. Therefore the excess liquid should be removed from the end of the tube before connecting it to the VPM. Also a contact of the connector of the coaxial extension cable with the housing of the qNano has to be avoided, as it will result in the same noise. A trace showing a very unsteady background current with a high noise level which is enhanced when the device is shaken has been observed in a newly assembled device. However, this is connected to an air bubble being trapped in the pore (Fig. 4.7 (c)). 
In such a case, the flow cell has to be dissembled, cleared from any bubbles and carefully reassembled again.

If the current with an applied voltage of $0 \mathrm{~V}$ deviates significantly from $0 \mathrm{nA}$ (below $\sim|5 \mathrm{nA}|$ is acceptable) or is drifting strongly over time, this indicates that the electrodes are degraded. With the silver-chloride layer used up on one of the electrodes, the electrode potentials are different and the current starts drifting. In such a case it is usually sufficient to renew the silver-chloride cover of the upper electrode. The remaining chloride layer should be removed using concentrated ammonium hydroxide before the silver wire is dipped into bleach till it appears grey. This is a common procedure in electrochemistry [176]. If it does not resolve the problem also the electrode of the lower half of the fluid cell has to be repainted with silver-chloride paste. This, however, occurs very rarely.

\subsubsection{The Laser-Induced Noise}

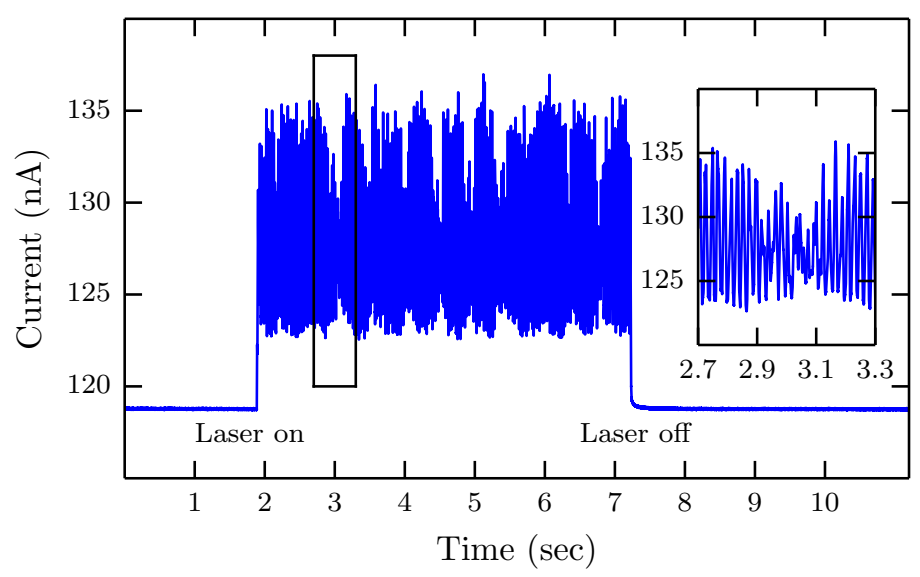

Figure 4.8: Focussing a laser (458 $\mathrm{nm}$ Argon-ion laser, $4.7 \mathrm{~mW}, \times 100$ water immersion objective with a focal length of $1.8 \mathrm{~mm}$ ) onto the pore orifice induces a large noise in a NP1000-rated pore $(0.14 \mathrm{~V}, 40 \mathrm{~mm}$ stretch). The region in the black box is shown in the inset at a higher time resolution.

When a laser is focussed onto the pore orifice a low-frequency noise appears in the current trace. Additionally, the current also shows a net increase (see Fig. 4.8). The noise disappears instantaneously when the laser is switched off or when the laser focus is misaligned laterally from the pore orifice by as little as $1 \mu \mathrm{m}$. An axial misalignment results in a reduction to a lesser extent. Both the net increase and the noise amplitude increase with the laser intensity, but no accurate dependency could reproducibly be observed. The very same pore, under the exact same voltage, pressure and stretch conditions illuminated with the same laser power can show noise levels of up to an order of magnitude difference, usually increasing with time. 


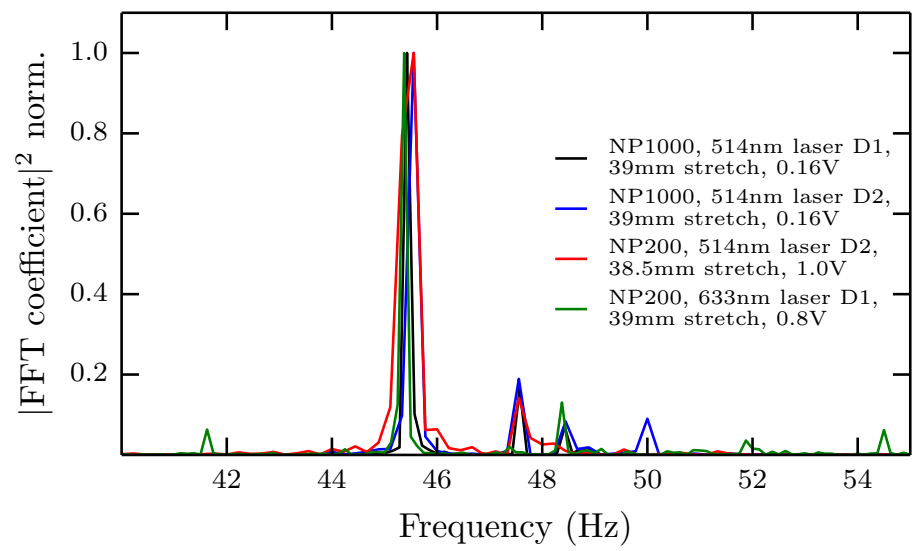

(a)

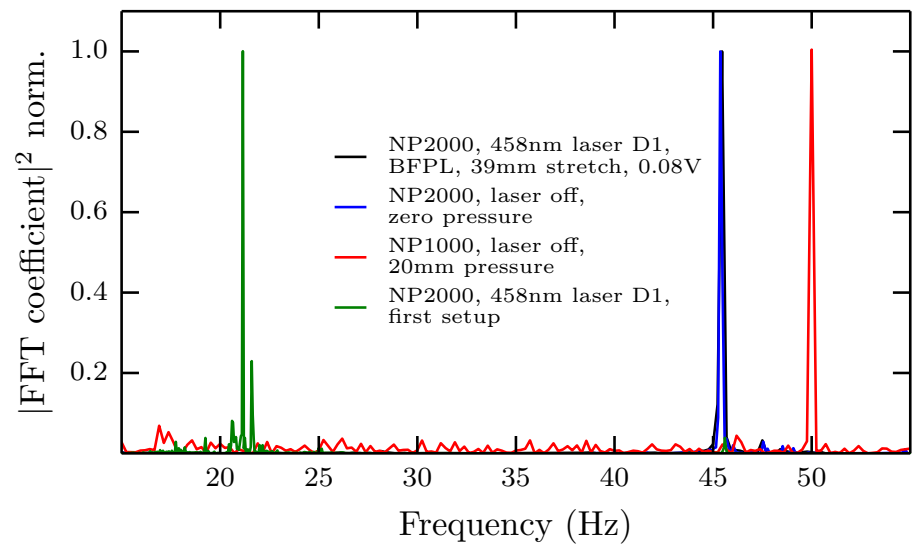

(b)

Figure 4.9: The squared Fourier components (normalised to its largest value) of the current signal under various experimental conditions (see legends). All but one (green line in (b)) of the current data traces were recorded with the second setup.

The underlying reason for this phenomenon was not obvious and intensive studies of the effect were carried out under a variety of parameters.

The frequency of this noise is not dependent on the wavelength of the laser $(458 \mathrm{~nm}, 514 \mathrm{~nm}$ Argon-ion and $633 \mathrm{~nm}$ He-Ne laser, with neutral density filters to regulate the intensity, refer to Tab. 4.2 for details), the pore size, the spot size, the applied voltage or hydrostatic pressure, the electrolyte strength, the electrolyte composition, the orientation or the stretch of the pore. The normalised Fourier components of the current signal for different experimental conditions are shown in Figs. 4.9 (a) and (b). For the second setup, the main frequency of the noise is at $\sim 45.5 \mathrm{~Hz}$, with several smaller peaks at slightly higher frequencies, leading to a characteristic beating in the signal trace. For a variety of experimental conditions the noise frequency stays constant within $0.2 \mathrm{~Hz}$. Although the noise is close to the frequency of the electric mains $(50 \mathrm{~Hz})$, the peaks are clearly separated and can be observed together if the laser-induced noise is small (Fig. 4.9 (a), blue line). For higher laser powers the peak at $45.5 \mathrm{~Hz}$ is dominating the mains peak, which itself 
is the largest peak in the signal spectrum if the laser is turned off (Fig. 4.9 (b), red line) and a non-zero hydrostatic pressure is applied. For the first setup, the peak of the induced noise appears at $\sim 22 \mathrm{~Hz}$, although a small peak is visible at $45.5 \mathrm{~Hz}$, about twice the dominant frequency.

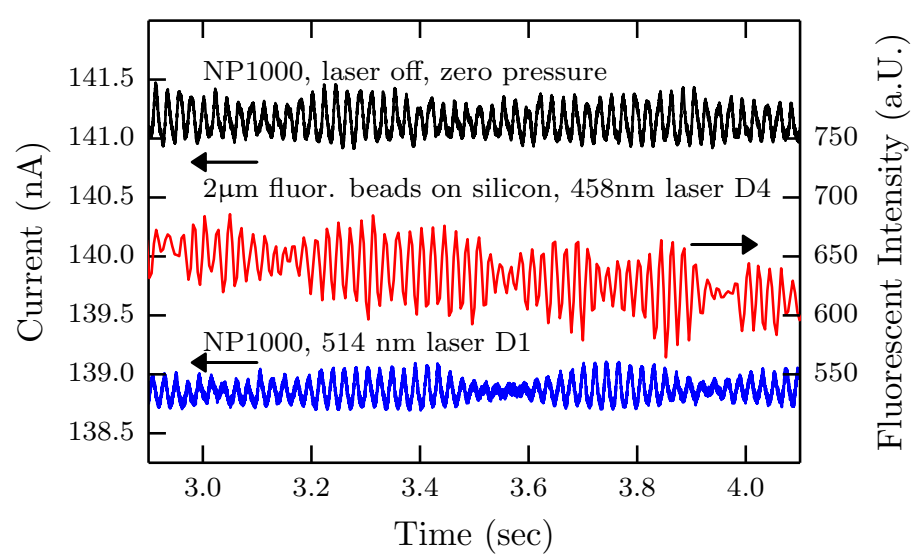

(a)

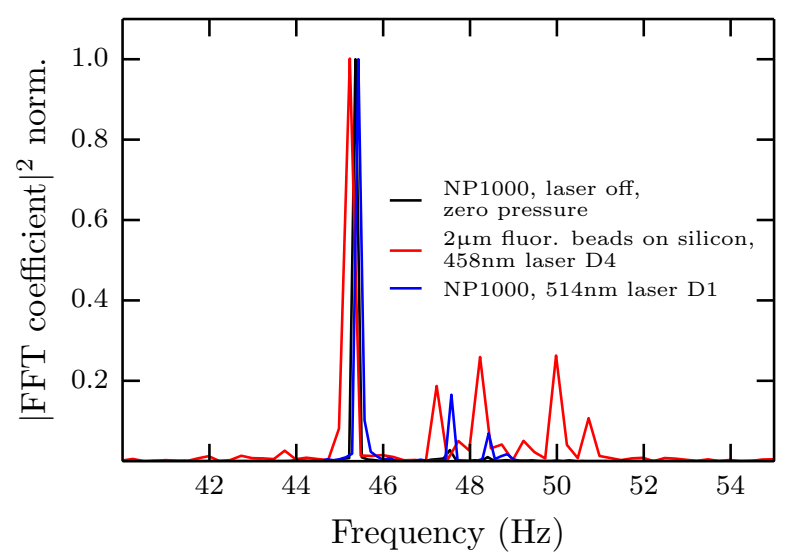

(b)

Figure 4.10: (a) The same low-frequency noise can be observed in the current trace when a laser is focussed on the pore orifice (blue) or when the hydrostatic pressure is carefully balanced to zero (black). It also appears in the trace of the optical signal measuring the fluorescences decay of an immobilized polystyrene bead (red). The spectral analysis of the signals are shown in (b).

Light-induced current enhancement and noise have both been observed by several groups before. Keyser et al. [177] found that local heating of the water due to an infra-red laser and the resulting increase of electrolyte conductivity was the reason for the current enhancement and they utilized the effect to determine the 3D topography of the laser focus. Li et al. [178] observed a laser induced noise in plasmonic nanopores and attributed the effect to a resonant coupling to surface plasmons. Smeets et al. [179] found a connection of the low frequency noise in solid state nanopores to surface charge fluctuations even without a laser present. In another paper [180] the same group attributed a similar noise to nanobubbles expanding 
under the illumination of an infrared laser.

Figure 4.11: Conductive peaks most likely due to the formation of nanobubbles in a NP1000-rated pore $(0.12 \mathrm{~V}, 39 \mathrm{~mm}$ stretch, $100 \mathrm{~mm}$ applied pressure). The laser $(514 \mathrm{~nm}, D 0.6)$ was focussed by a $\times 100$ water immersion objective with the use of a $500 \mathrm{~mm}$ BFPL lens.

For the laser wavelengths used in this work the absorption coefficient of water is at least three orders of magnitude smaller than in the infra-red. Additionally the laser power is much lower than the one reported by $[177,180]$, so that a substantial heating effect of the electrolyte can be excluded. Also, the laser stability was measured and showed no noticeable noise pattern. Although the absorbance of the membrane material in the range of the visible light is small (Fig. 4.28), the material can be damaged when the laser is operated at maximum power and focused with a short focal objective lens (Fig. 4.27). Therefore an interaction with the pore material will occur and local heating(expansion)-cooling(contraction) cycles with a net increase of the pore size could be the reason for the noise. However, a frequency dependence on the stretch, pore size or spot size should be observed in such a case. A possible light-induced charge generation is also an unlikely candidate as a dependency on the laser wavelength should then be observed.

The consistency of the noise frequency indicates a coupling to a mechanical Eigenfrequency of the system. The noise disappears immediately when the laser is slightly focussed away from the pore orifice so that a oscillation of the whole membrane can be excluded. Additionally, the fundamental axial and radial modes of a pre-stressed thermoplastic polyurethane membrane, which were simulated with the FEM method (see Sec. 3.4.7), show frequencies in the order of several kHz, much higher than the observed value.

Interestingly, with the laser turned off, the same characteristic noise can also be observed in the current trace when the hydrostatic pressure is carefully adjusted to zero (Fig. 4.10). Also the noise intensity is larger for smaller pores which are 
intrinsically more sensitive to pressure changes when no directed flow through the pore is established. The noise is enhanced in intensity when an air bubble is trapped under the pore. Additionally, the exact same noise pattern can be seen in the fluorescence decay of $\mu \mathrm{m}$ sized particles, which are attached to a silicon substrate. Even in a time resolved measurement of the Raman signal of silicon (see Sec. 4.2.5) the same peak in the power spectral density is observed (Fig. 4.24). All of these above observations lead to the conclusion that the noise originates from a mechanical vibration of the optical table and is not mainly caused by the illumination of the pore with a laser.

However, this conclusion leaves the question open as to why the current shows a net increase and why the noise is so strongly enhanced when the laser is focussed onto the pore orifice. A possible explanation could be the formation of micro- or nanobubbles either in the solution or at the membrane walls under laser exposure, which amplify the mechanical vibrations. This theory is supported by the observation of poly-disperse peaks in the current trace under high positive pressure conditions and a high laser intensity (Fig. 4.11). It can be excluded that these events originate from polystyrene beads as the specific pore this data was recorded from had never been used before and the device was newly assembled. The peaks are nonuniform in size and are all conductive, with the peak asymmetry indicating that the bubbles move from the upper- to the lower- side of the pore. The effect can only be observed at high laser intensities and high positive pressures, which are usually not used in the coordination experiments. The peaks can only be identified with the resolution shown in Fig. 4.11 with a freshly assembled pore, as the noise quickly $(\sim$ min) rises to a level where the peaks cannot be identified in the raw trace. Although a small net increase in the current is observable when the laser is switched on and the $45.5 \mathrm{~Hz}$ noise is visible in the frequency analysis of the trace, the noise level in this specific trace is relatively small. However, within a timespan of $\sim$ min under these conditions the current trace resembles the one shown in Fig. 4.8, leading to the conclusion that some of the bubbles might get trapped in the orifice and accumulate. This can also be seen optically as a white shining spot at the orifice.

The formation of bubbles in solid-state nanopores has been reported by Smeets [180]. Weber [181] studied laser-induced nanobubbles forming at the surface of metallic nanoparticles. An observation of nano- or microbubbles in polymer pores induced by visible laser light has not been reported before. The exact mechanism of the bubble formation, the net current increase, the noise enhancement and the conductive nature of the peaks caused by the bubbles could be investigated further. However it can be concluded that the noise originates from a mechanical Eigenfrequency of the optical table. Interestingly, by placing the device on either the optical table (first setup) or the microscope stage (second setup) different frequencies are 
dominant in the noise spectrum. If the device is placed on the benchtop next to the optical table several peaks between $10 \mathrm{~Hz}$ and $20 \mathrm{~Hz}$ can be observed. At zero applied pressure these can also be seen when the original setup is used, but are much smaller as the device is heavier and more stable than the custom-made setup. The use of an actively damped optical table would be a simple but expensive method to reduce the noise for future experiments. Although the observed noise is undesired for the specific purpose of the work, the measurement of small mechanical vibrations could be an atypical but additional application for the RPS technique.

\subsubsection{Filtering the Signal}

The laser-induced noise is an undesired effect. However, it has proven useful to align the laser spot with the pore orifice. Especially for small pores the alignment can be quite difficult optically, so that the noise can be a helpful indicator, although this method should not be used extensively as it can lead to a bubble formation in the pore. The removal of the noise in a post processing step is relatively simple as the time scales of the noise and the resistive pulse signal are usually about two orders of magnitude apart. Therefore the influence of the filtering step on the actual resistive pulses is minimal. The filtering can be done with an adequately designed Finite Impulse Response (FIR) filter. An introduction to filter design can be found in Saramaki's book [182].

For the signals used here a cut-off frequency of $75 \mathrm{~Hz}$ with a "Kaiser" window of $15 \mathrm{~Hz}$ width has proven most successful. The method is very fast and leads to good results for most current traces. However, for slow particles and therefore long events (experiment D1 in Sec. 6.2) the filtering leads to an "overshoot" artefact at the beginning and the end of each resistive pulse due to the proximity of the frequency components of the noise and the pulse signal. A similar observation has been made by Garze-Licudine et al. [45] who employed a high-pass filter to remove DC content and a low-frequency drift from the raw current trace recorded with the same resistive pulse device. A comparison of the raw trace of a "slow" event and the filtered signals using different cut-off frequencies is shown in Fig. 4.12 (a). If the cut-off is too low, the noise is still visible to some extent in the filtered signal while a higher cut-off frequency successfully removes the noise but leads to a more enhanced "overshoot". This makes the next post-processing step, the event search algorithm, more difficult and prone to errors.

To address the "overshoot" problem a wavelet based filter method can be used instead of the FIR method. Recently the application of wavelets to filter resistive pulse signals has gained increasing attention as the technique is pushed to its limits in the detection of single molecules [183-185]. A general introduction to wavelets can be found in the literature $[186,187]$. In brief, a wavelet transform decomposes the 


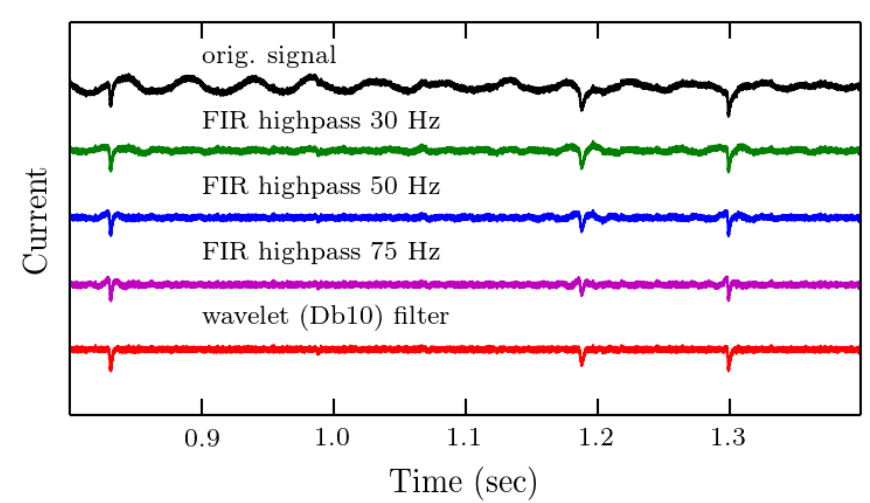

(a)

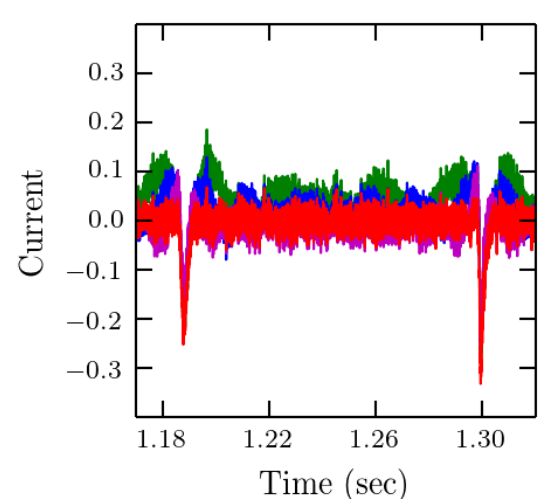

(b)

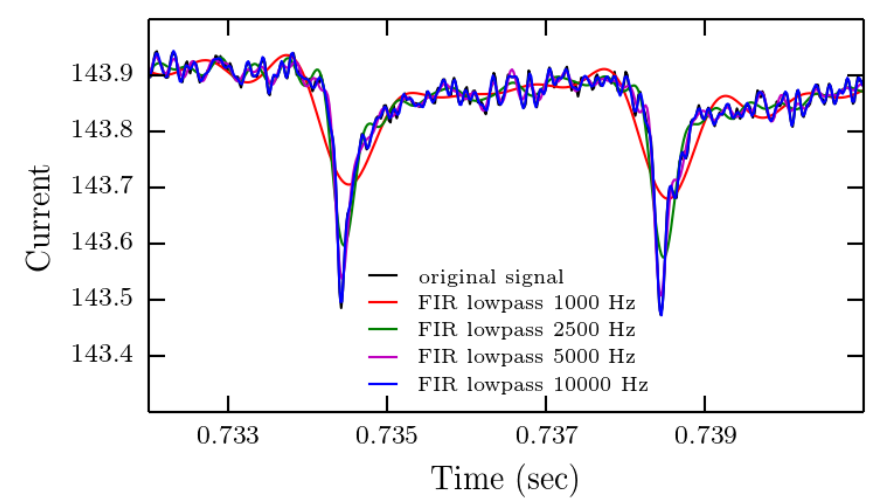

(c)

Figure 4.12: (a) The original, noisy signal and the filtered signal using a FIR filter with various cut-off frequencies and a wavelet based filter method. (b) The filtered signals in detail. (c) Lowpass-filtered RPS signals with different cut-off frequencies.

original signal into levels which contain information about the different frequency components. The highest frequency, usually high-frequency noise, is contained in the lowest level, which is connected to the smallest scale of the wavelet. Compared to a Fourier analysis, which decomposes the signal into infinite sine functions, wavelets have the appearance of finite "sinusoids". Therefore wavelets are better suited to decompose signals with highly localised events, like the current trace of a resistive pulse device. Many types of wavelets, called the mother wavelets, have been designed, some of them for specific functions, e.g. image compression. Each has its advantages and disadvantages for a given signal and has to be chosen on a case-by-case basis to fit the purpose.

Figure 4.13 shows the example of a noisy signal and its decomposition into 12 levels using MATLAB's wavelet toolbox and Doubechie's "DB10" wavelet. It reveals that most components of the resistive pulse signal are contained between the 7 th and the 10th decomposition level. However, there is an overlap of the frequency region of the peaks and the background noise. Therefore a modification of the wavelet and scaling coefficients by either using hard or soft thresholding before the signal is 


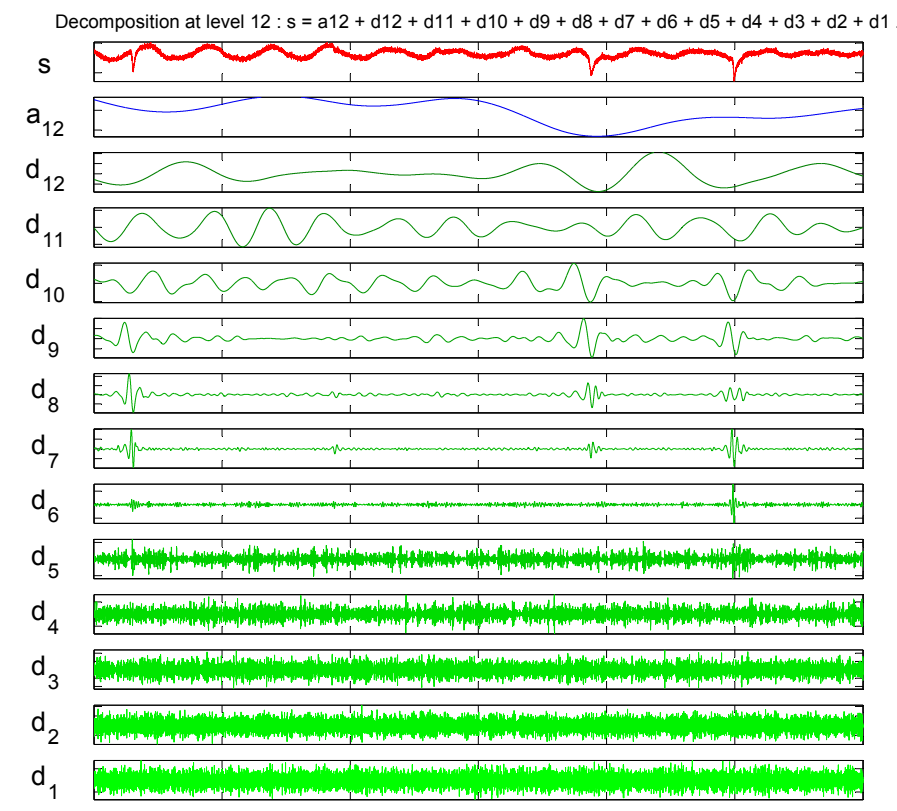

Figure 4.13: The wavelet decomposition of a noisy current trace, with three translocation events.

reconstructed will lead to a distortion of the pulses or an unsatisfying suppression of the background noise, equivalent to the Fourier method. The general problem that the signal and the background have overlapping frequency regions is encountered in many applications of signal processing. To extract Raman intensities superimposed by a strong fluorescence background Galloway et al. [188] developed an algorithm using wavelets. In brief, the method iteratively decomposes and modifies the original signal until the background is only contained within the final set of approximation coefficients and can be removed. The method has been implemented in a MATLABbased code called "COBRA", which is freely available from the group's website [189].

The software was successfully used in this work to remove the low-frequency noise in current traces containing "slow" events. With the appropriate parameters (scale level of 8, 10 iterations for the given example, "DB10" wavelet) the "overshoot" problem does not occur any more (Fig. 4.12 (a)) and the filtered signal shows a straight baseline compared to the FIR filtered signal. One drawback is that the wavelet algorithm is computationally approximately ten times more expensive than the FIR method. The filtering of a $10 \mathrm{~s}$ long current trace takes about $100 \mathrm{~s}$ on an average $\mathrm{PC}$.

It has to be remarked here that although it is possible to remove the high frequency noise in a similar post-processing step, this should be avoided as it can significantly alter the shape of the resistive pulse. It is analogous to under-sampling during the data acquisition and can result in an underestimation of the pulse peak height [167], especially for fast moving particles. An example is shown in Fig. 4.12 
(b), where a upper cut-off frequency of $5000 \mathrm{~Hz}$ leads to underestimation of the pulse height by more than $10 \%$.

\subsection{Horiba's LabRAM}

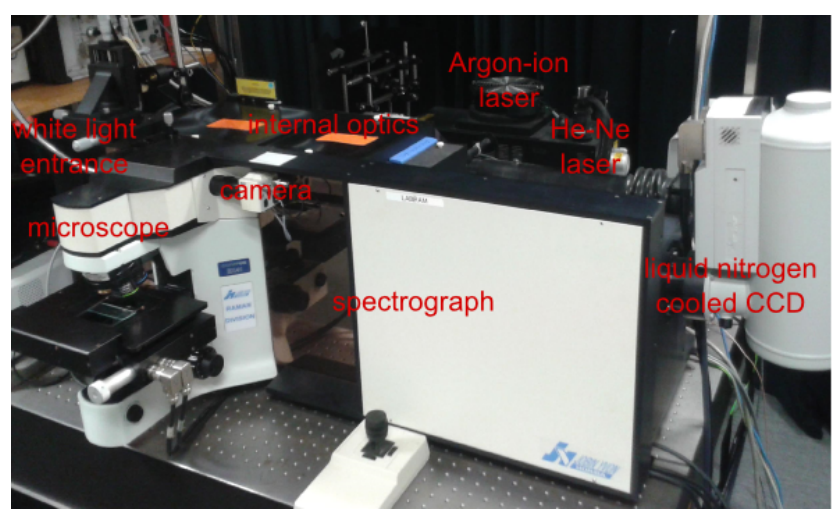

Figure 4.14: A photograph of the LabRAM and some of its components. The spectrograph is contained within a lightproof box behind the white panel.

A LabRAM Raman spectrometer (Fig. 4.14) from Horiba Jobin-Yvon was used to conduct the optical measurements. It is suitable for micro measurements as it is attached to a Olympus BX41 Microscope, which is equipped with an objective revolver and a CCD camera for sample viewing. The sample position can be adjusted with sub-micrometer precision through a motorized xy-translation stage. The system is connected to a He-Ne laser and a tunable Argon-ion laser. Figure 4.15 shows a schematic (a) and a photograph (b) of the internal optics. A crucial element of the setup is the dichroic mirror that reflects the laser light to the objective but transmits the collected, wavelength-shifted light originating from the sample. Two types of filters are common. Edge filters block the light of the excitation laser wavelength and are used for the fluorescence measurements. Notch filters have a transmission in the band stop region of less than $10^{-6}$, but allow light outside this region to pass with a transmission of typically $\sim 90 \%$, having the advantage that the anti-Stokes line in a Raman spectrum can also be observed. Crucial for the correct function of the edge or notch filter is its tilt as the transmission is very sensitive to the angle of incidence. The angle is adjusted using spacers of various thickness to achieve the desired transmission function.

The laser light is focussed onto the sample and the scattered light is collected in the backscattering geometry with the same objective. After it passes the dichroic filter it is focussed onto a square pinhole with an adjustable size by a lens with a focal length of $f_{p h l}=40 \mathrm{~mm}$. The pinhole blocks out stray light, allows for "confocal" imaging and also acts as the entrance slit of the spectrometer. Details 


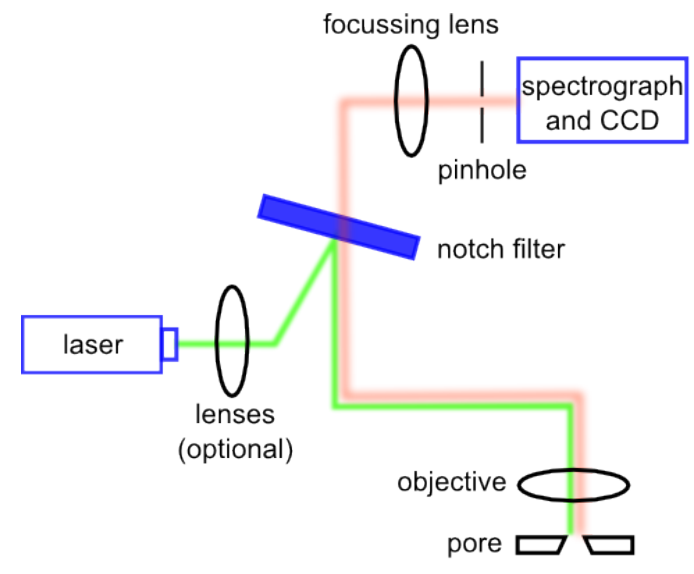

(a)

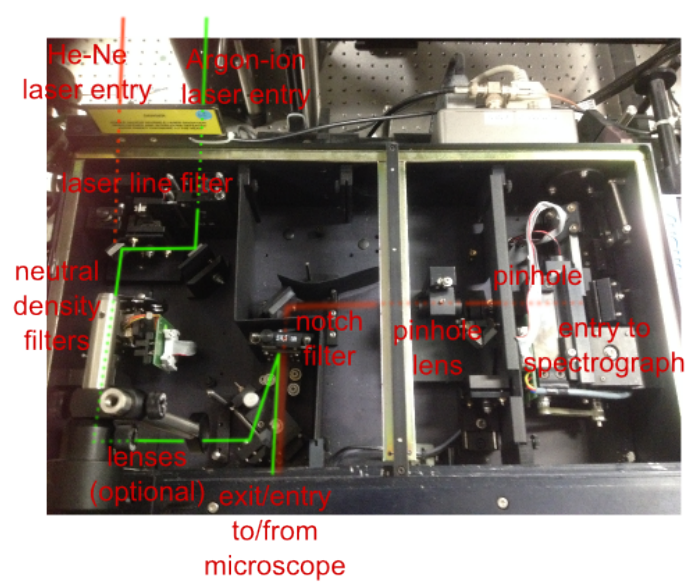

(b)

Figure 4.15: The excitation and collection beam paths in the LabRAM as a simplified illustration (a) and a photograph (b). The external laser enters through two ports, by using a mirror the can be switched. The laser then subsequently passes a bandpass filter that blocks out-of-line radiation and a neutral density filter to adjust its intensity. A back focal plane lens or a beam expander system (see Chap. 5) can be used to engineer the beam size before it hits a dichroic mirror and get reflected to the microscope objective which focusses the beam on to the sample. The scattered light is collected with the same objective, transmitted and focussed onto a pinhole before it enters the spectrograph, which disperses it into spectral components.

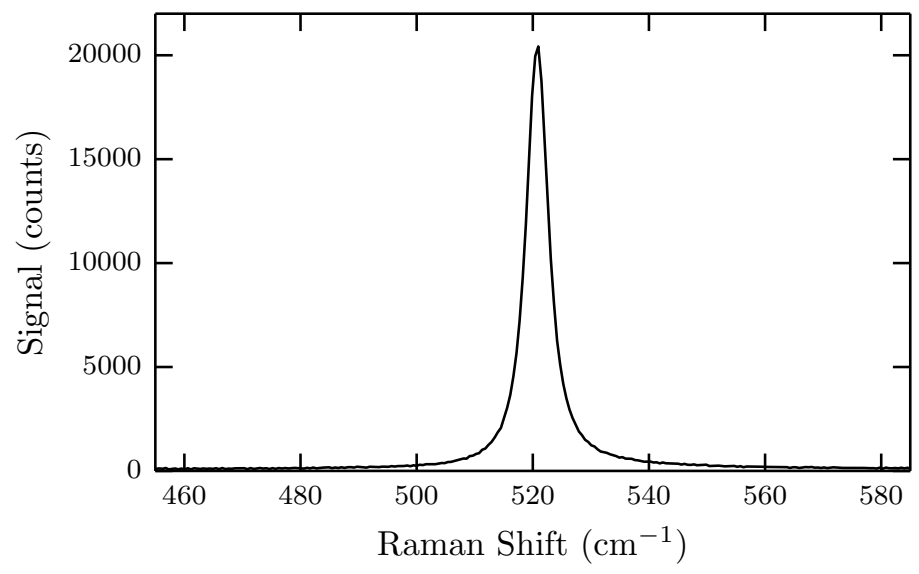

Figure 4.16: The $520.5 \mathrm{~cm}^{-1}$ Raman line of a flat silicon sample used to calibrate the spectrometer (1s integration time, $\times 100$ Olympus water immersion objective, $514 \mathrm{~nm}$ Argon-ion laser with $17.8 \mathrm{~mW}$ laser power at the sample).

of the confocality of the system are relevant to the coordination experiments and are discussed in detail in Sec. 4.2.3. After the pinhole, the light is redirected by several mirrors before it is collimated by a concave mirrors onto a diffraction grating, which disperses the light. Blazed, holographic gratings with 300 grooves $/ \mathrm{mm}$, 600 grooves/mm, 1800 grooves/mm and 2400 grooves/mm are available. A grating with a higher groove density has a higher resolution but also offers a narrower 
spectral window so that for fluorescence measurements usually the grating with the lowest density is used. The dispersed light is then focussed with a second concave mirror (Czerny-Turner Configuration, focal length of the spectrograph $f_{\text {spec }}=$ $800 \mathrm{~mm}$ ) onto a liquid nitrogen cooled CCD with $1024 \times 256$ pixels. The spectral information is contained in the $\mathrm{x}$-direction of the chip. The intensity of the pixels in $y$-direction is summed up to obtain the signal. Hereby the region of integration is chosen in order to minimise the intrinsic and the readout noise. The electronic gain of the CCD can also be adjusted. Throughout the experiments it was set in a way that one count corresponds to two incident photons. The minimal time resolution of the CCD is $4.25 \mathrm{~ms}$. The $520.5 \mathrm{~cm}^{-1}$ Raman line of a silicon sample (Fig. 4.16) is used to calibrate the spectrometer. The control of the measurement parameters, e.g. integration time, grating position, pinhole size, etc. and the display of the data is done using software (Labspec 5.74.29 beta) provided by the manufacturer. The spectra are saved and displayed as a intensity versus the Raman shift, which is consequently translated into the wavelength of the spectrum.

\subsubsection{Lasers, Objectives and Lenses}

The $458 \mathrm{~nm}$ and $514 \mathrm{~nm}$ lines of a tunable Argon-ion laser (Melles-Griot 543-APA01) and the $633 \mathrm{~nm}$ line of a He-Ne laser (Melles-Griot) were available. The powers at the laser output and at the sample were measured with a power meter (Thorlabs T-100) and are summarised in Tab. 4.2 along with the beam parameters. It is apparent that the losses due to the reflections at up to eight mirrors and the transmission through the objective are quite significant. For the regulation of the power, neutral density filters with an attenuation $D=0.3,0.6,1,2,3$, and 4 corresponding to a theoretical transmittance of $10^{-D}=1 / 2,1 / 41 / 10,1 / 100,1 / 1000$, and $1 / 10000$ of the maximum power are available. While the real transmittance, measured with the power meter, agrees very well with these numbers for the first four filters, the values for the last two filters are 1/600 and 1/6500 respectively, which deviates significantly from their nominal transmissions. For the first setup the values are slightly smaller than those given in Tab. 4.2 as more mirrors have to be used to guide the laser onto the sample.

Throughout the work infinity-corrected Olympus objectives were used. Due to the flow cell design and the limited accuracy in lateral and axial focussing, only Long Working Distances (LWD) air objectives with a maximum magnification of $\times 50$ can be used in the first setup. For the second setup, water immersion objectives with a higher numerical aperture and magnification of up to $\times 100$ are used. The parameters of the used objectives are summarized in Tab. 4.3. Achromatic, spherical lenses with various focal lengths purchased from Thorlabs were used to engineer the laser spot 
Table 4.2: The output powers and the beam parameters of the lasers used for the experiments with no neutral density filter present. $w_{0}$ and $\theta_{0}$ are the waist and the divergence of the beam. The powers at the sample were measured for two Olympus water immersion objectives.

\begin{tabular}{cccccc}
\hline $\begin{array}{c}\text { Laser } \\
\text { Type }\end{array}$ & $\begin{array}{c}\text { Wavelength } \\
(\mathrm{nm})\end{array}$ & $\begin{array}{c}\text { Power at } \\
\text { the Laser } \\
\text { Exit }(\mathrm{mW})\end{array}$ & $\begin{array}{c}\text { Power at the } \\
\text { Sample } \\
(\mathrm{x} 20 / \mathrm{x} 100)(\mathrm{mW})\end{array}$ & $\begin{array}{c}w_{0} \\
(\mu \mathrm{m})\end{array}$ & $\begin{array}{c}\theta_{0} \\
(\mathrm{mrad})\end{array}$ \\
\hline Argon-ion & 458 & 20.5 & $5.8 / 4.7$ & 360 & 0.5 \\
Argon-ion & 514 & 78.1 & $22.8 / 17.8$ & 360 & 0.5 \\
HeNe & 633 & 15.33 & $6.89 / 5.35$ & 350 & 0.64 \\
\hline
\end{tabular}

size.

Table 4.3: Specifications of the used objectives (water immersion $=$ water imm., Long Working Distance Objective = LWD). Throughout this work infinity-corrected objectives were used.

\begin{tabular}{cccc}
\hline Magnification & $\begin{array}{c}\text { Focal Length } \\
(\mathrm{mm})\end{array}$ & $\begin{array}{c}\text { Numerical } \\
\text { Aperture }\end{array}$ & Comment \\
\hline 10 & 18 & 0.2 & air, LWD \\
20 & 9 & 0.35 & air, LWD \\
50 & 3.6 & 0.45 & air, LWD \\
20 & 9 & 0.5 & water imm. \\
100 & 1.8 & 1 & water imm. \\
\hline
\end{tabular}

\subsubsection{Aligning the System}

Very accurate alignment of the optical components is crucial to the success of the coordination experiments. To achieve a maximum signal from the particles traversing the pore and prevent the detection of false positive events both the excitation and the collection have to be aligned with the centre of the pore orifice. Figure 4.17 shows a schematic of the excitation and collection beam paths in an aligned setup. Using a Back Focal Plane Lens (BFPL) or several lenses placed at the appropriate distance before the objective the laser is focussed on the pore orifice. The role of the BFPL is first to enlarge the beam so it illuminates the whole pore orifice and second to make the laser focus coincide with the objective focal plane (compare Sec. 5.4.1). The signal is collected with the same objective and then refocussed onto the pinhole. To locate and correctly focus the sample, i.e. the pore orifice, a CCD camera is used prior to the measurement. By temporarily lowering two semi-transparent mirrors 


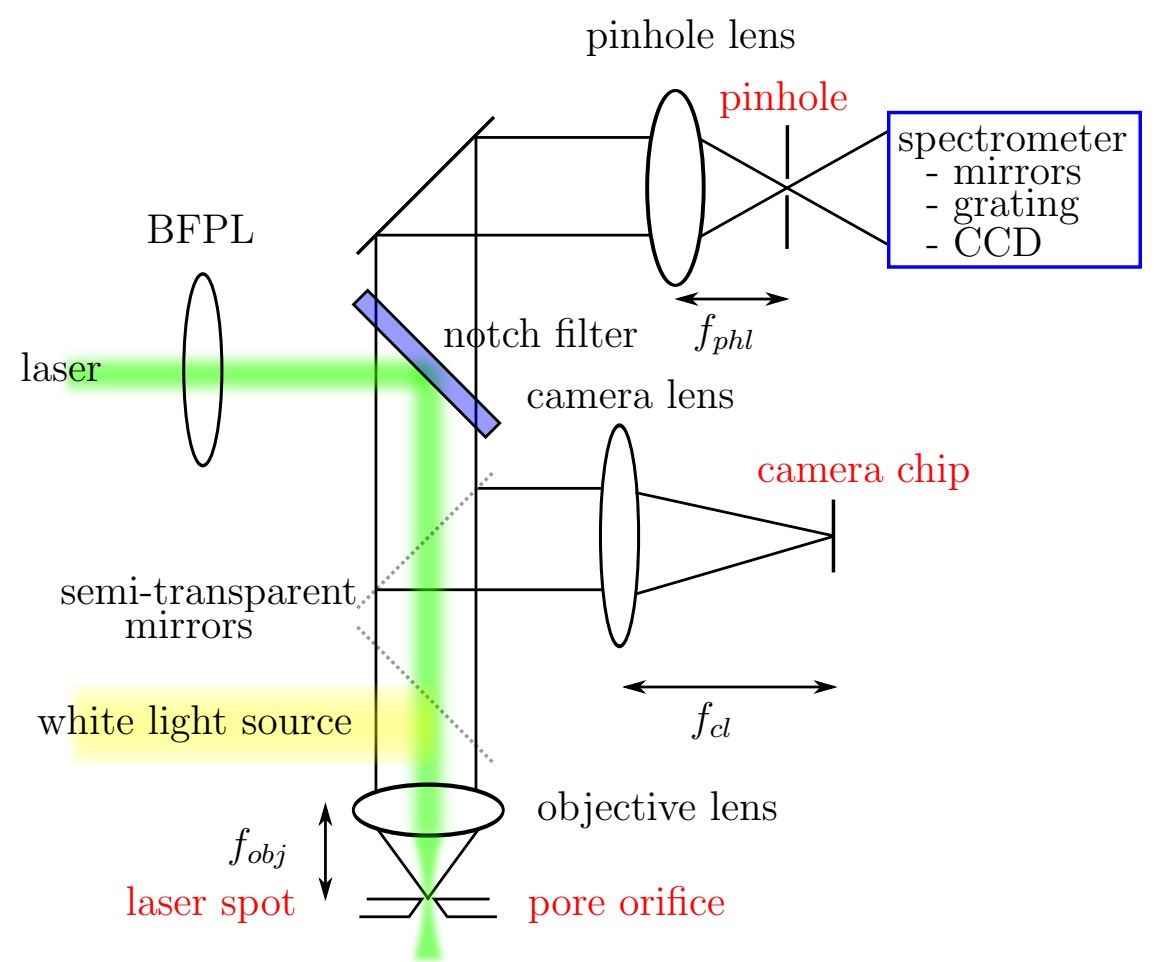

Figure 4.17: A schematic layout of an aligned system. Components in red have to be conjugated to each other in order to achieve an optimized detection.

into the beam path the sample is illuminated with white light and the scattered light is redirected to another lens, that focusses the image on the CCD chip of the camera, where the image is created. In a well aligned system, the sample is placed at the focal plane of the objective and the beam paths between the objective lens and both the pinhole and the camera lenses are parallel. That way the pore orifice and the laser focus are conjugated to the planes of the pinhole and the camera chip. An object in the focus of the objective will therefore form an image in these planes. The magnifications of the images formed at the camera and the pinhole then becomes $f_{c l} / f_{o b j}$ and $f_{p h l} / f_{o b j}$ respectively.

\subsubsection{Aligning the Camera Lens}

As a start of the alignment procedure it has to be assured that the camera chip is exactly positioned at the focal plane of the camera lens. If the lens is a distance $\Delta_{c l}$ too close or too far away from the CCD, the pore will appear in focus at a certain distance $\Delta_{o b j}$ from the objective focus, leading to an erroneous collection. With $L$ the separation between the two lenses, a simple calculation using geometric optics yields 


$$
\Delta_{o b j}=f_{o b j}\left(\frac{1}{1-\frac{f_{o b j}}{L-f_{c l}^{2} / \Delta_{c l}}}-1\right) \sim \Delta_{c l} \frac{f_{o b j}^{2}}{f_{c l}^{2}}
$$

If $L<<f_{c l}^{2} / \Delta_{c l}$, the approximation can be used, which makes the equation independent of $L$.

To illustrate the importance of an exact alignment an example relevant to the specific setup is discussed in the following. For $f_{c l}=100 \mathrm{~mm}, L=300 \mathrm{~mm}$ and assuming that the camera lens is $5 \mathrm{~mm}$ ( $5 \%$ of its focal length) too far away from the chip the image of the pore will appear to be in focus at a distances $1.90 \mu \mathrm{m} / 47.40 \mu \mathrm{m}$ closer to the objective focal plane of a $\times 100 / \times 20$ Olympus objective $\left(f_{o b j}=1.8 \mathrm{~mm} / 9 \mathrm{~mm}\right)$. Although the offsets appear relatively small, they have an effect on the collection efficiency as the image of the sample is consequently formed $0.69 \mathrm{~mm}$ (for both objectives, the distance from the objective to the pinhole lens is $650 \mathrm{~mm}, f_{p h l}=40 \mathrm{~mm}$ ) beyond the pinhole in this case, leading to a reduced collection efficiency from the sample plane. Additionally, in case no BFPL is used (usually for the smallest rated pores) the beam width of the focussed laser at the pore orifice will be significantly larger than expected, which will cause an increase in the detection of particles that are close to the pore orifice but are not passing through it, so called "false positive" events.

To verify or align the correct position of the camera lens the fact that $\Delta_{o b j}$ is dependant on $f_{o b j}^{2}$ can be exploited. By using two or more objectives with different focal length (from the same manufacturer) and measuring how far the foci have to be tuned using the fine adjustment knob of the microscope table, $\Delta_{c l}$ can be determined and the lens position changed accordingly. For a well aligned system the focus should stay constant within a few $\mu \mathrm{m}$ when the objectives are changed (Fig. 4.18 (a)).

\subsubsection{Aligning the Pinhole Lens}

After the camera lens has been aligned the pinhole lens has to be positioned so that the pinhole and the objective focal plane are conjugated. This is done by focussing first on a flat, reflective sample, using the white light source. Then the pinhole is illuminated from the back (the $L a b R A M$ has an in-built diode for this purpose) and its reflected image is observed with the camera. The correct position of the pinhole lens is found when the edges of the pinhole appear sharp at the same focus as the sample (depending on the used objective the pinhole needs to be adjusted in size) and the image has the approximate size as expected from the calculated magnification (Fig. 4.18 (b)). 


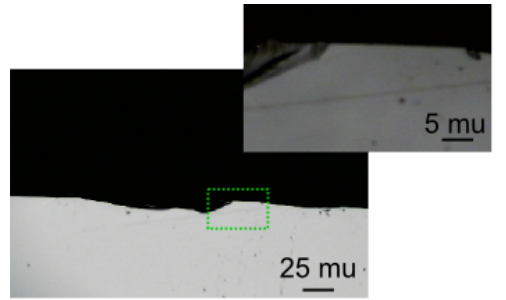

(a)

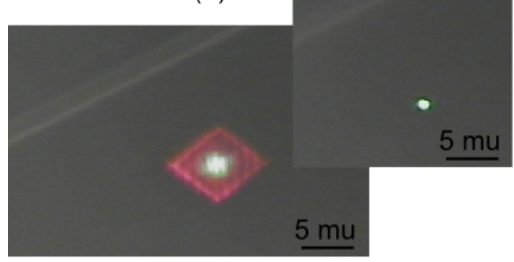

(c)

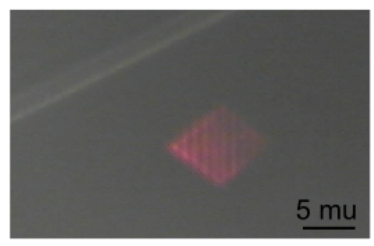

(b)

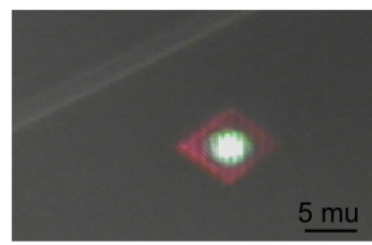

(d)

Figure 4.18: (a) The edge of a silicon sample as viewed with a $\times 20$ and $\times 100$ (inset) Olympus objective. If the camera lens is properly adjusted no refocussing is needed when the objectives are switched. (b) A feature of the silicon sample and the pinhole (diagonal of $200 \mu \mathrm{m}$ ) are brought to the same focus through aligning the pinhole lens. The size of the pinhole in the image is $\sim 9 \mu \mathrm{m}$ as expected from the magnification $\left(f_{p h l} / f_{o b j}=40 \mathrm{~mm} / 1.8 \mathrm{~mm}=22.22\right)$. (c) The laser is aligned with the centre of the pinhole. Note that the actual "laser focus" is beyond the focus of the white light (inset) inherent to the focussing properties of a Gaussian beam as discussed in Sec. 5.3. (d) "Laser focus" and "white light focus" coincide after the installation and alignment of a BFPL (500 $\mathrm{mm}$ focal length). The laser spot is now visibly larger.

\subsubsection{Aligning the Laser with the Pinhole Centre}

The centre of the pinhole is then the reference point the incoming laser has to be aligned to. This is done by iteratively adjusting a pair of mirrors with the "walking the beam" method until the laser is at the right position (Fig. 4.18 (c)) and shows a symmetric focus-defocus behaviour. A back focal plane lens or a tuneable beam expander system which are used to engineer the spot size (Chap. 5) can then be added in the beam path and aligned with the pinhole centre in a subsequent step. Hereby, the lenses have to be attached on a mount that allows for four independent degrees of freedom in order to enable a correct alignment. A combination of two translation stages and two tilt stages has proven successful in the setup used for this work (see Fig. 5.15). After the lenses are installed a visibly larger laser spot that is also conjugated with the objective focal plane can be seen in the camera (Fig. 4.18 $(\mathrm{d}))$.

As the camera and the pinhole lens can be securely locked in place, their alignment has to be done only once. However, the correct alignment should be verified every now and then as the normal use of the spectrometer can lead to small changes over time. The notch filter is a moveable part of the setup and is often changed, 
leading a slightly different reflection angle of the incoming beam. Therefore the alignment of the laser and the lenses used to engineer the spot size has to be done at the start of each series of experiments to ensure an optimal sample excitation.

\subsubsection{Finding the Pore}

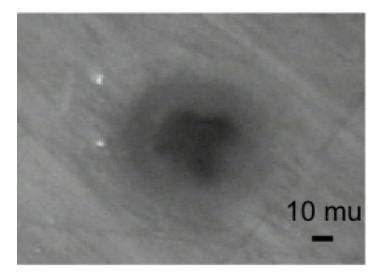

(a)

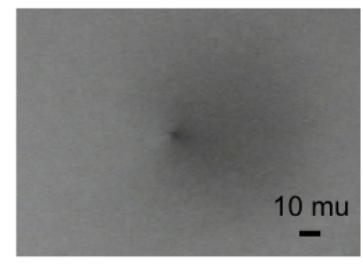

(b)

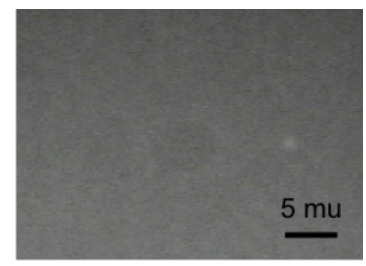

(c)

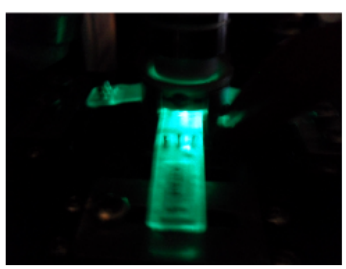

(d)

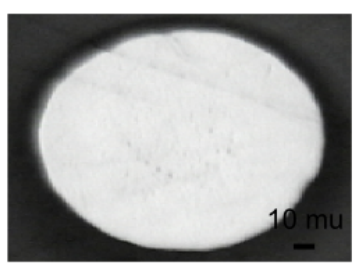

(e)

Figure 4.19: The large (a) and small (b) opening of a NP400-rated pore imaged with a $\times 20$ water immersion objective focussing on the upper/lower side of the membrane respectively. (c) The small opening of a NP1000-rated pore using a $\times 100$ objective. (d) A high light scattering in the arms of the cruciform can be seen when the laser is focussed on the orifice. (e) An air bubble trapped on the trans side of the membrane.

Finding a tiny hole of a size of down to $500 \mathrm{~nm}$ diameter in a nearly transparent membrane is a non-trivial task. However, due to the conical geometry of the pore and its large orifice with a diameter of $\sim 50 \mu \mathrm{m}$, it can be spotted as a dark hole with a visible outline (see Fig. 4.19 (a)). The pore is first located using a low magnifying objective focussed on the lower side of the membrane. When focussing on the upper side, the small pore orifice will become visible as a dark dot (see Fig. 4.19 (b)). Switching to the high magnifying objective intended to be uses for the experiment then just requires some minor lateral corrections to achieve a good alignment with the pore centre. The orifice is low in contrast (see Fig. 4.19 (c)), so that an exact location can be a challenge, especially for the smallest rated pores.

A high scattering of the laser light into the cruciform arm also indicates that the laser is located at the pore centre Fig. 4.19 (d). Additionally, it also appears that the laser-induced noise in the current trace becomes maximal when a good alignment is achieved. However this method should be used carefully as the membrane can be damaged at high laser powers and air bubbles that are formed at the laser focus can become trapped in the orifice. If a white haze can be seen around the pore, an air bubble has either been introduced during the assembly of the fluid cell or 
through the laser exposure (see Fig. 4.19 (e)). As air bubbles prevent a stable electrical recording, the flow cell has to be disassembled, cleared from any bubbles and reassembled again in such a case.

\subsubsection{The Detection Efficiency Profile of the Setup}

Apart from the excitation profile, which is determined by the laser beam intensity $I(\rho, z)$ and can be engineered using the methods discussed in Chap. 5, the optical signal is also determined by the lateral and axial detection efficiencies, which are specific to the optical setup. An accurate characterisation of these efficiencies is required to choose the most suitable parameters, e.g. the objective and pinhole size, for the coordination experiments.

In the setup used, the light is first collected by an objective and then refocussed onto a pinhole (compare Fig. 4.15 (a)). At correct alignment, the plane of the pinhole is conjugated with the focal plane of the objective and the magnification $M$ of the image formed at the aperture is given by $f_{l e n s_{p h}} / f_{o b j}$. The parts of the image that lie within the aperture are detected and parts outside will be blocked, determining the lateral detection efficiency. The pinhole is a square aperture with an edge length $2 L$ and the detection efficiency $\eta$ can be written as [190]

$$
\eta(x, y, z)= \begin{cases}\eta_{\perp}(z) & \text { for }-L / M \leq x, y \leq L / M \\ 0 & \text { otherwise }\end{cases}
$$

Hereby any diffraction effects at the pinhole are neglected. This assumption is reasonable as for the smallest usable pinhole (diagonal of $35 \mu \mathrm{m}$ ), the angle $\theta$ for the first order diffraction minimum $(2 L \sin \theta=\lambda)$ is $\sim 1.2^{\circ}$, which is still within the acceptance angle of the spectrometer and will therefore contribute to the signal. Note that in the used software the pinhole diagonal $2 \sqrt{2} L$ is defined, which is also used to describe the size of the pinhole in the following.

The axial detection efficiency $\eta_{\perp}(z)$ depends on the optical setup, i.e. the focal lengths $f_{o b j}, f_{l^{e n s_{p h}}}$ and the size of the pinhole. In general it will reach its maximum at the focal plane of the objective and will decrease from there in a bell-shaped manner, with the Full Width Half Maximum (FWHM) of the curve defined as the confocal depth. The profile can experimentally be obtained by measuring the Raman or fluorescence signal originating from a flat thin sample as a function of the distance $z$ from the focal plane. However, assuming that this profile represents the axial detection efficiency $\eta_{\perp}(z)$ can be misleading as remarked by [190] in the supplementary information. Due to the Gaussian beam profile of the laser, the size of the illuminated area on the sample is dependent on $z$ itself. By scanning $z$, part of 
the image of the beam can be cut out laterally by the pinhole so that a bell-shaped response (due to the Gaussian-shift of the focussed laser the shape will actually be asymmetric) can even be achieved for a constant $\eta_{\perp}(z)$. Therefore, when measuring $\eta_{\perp}(z)$ with this method, it has to be ensured that the pinhole is sufficiently open so that the effect of the lateral efficiency can be neglected. For smaller pinholes the effect can be corrected in a post-processing step (function $g$ in [190]), which requires an accurate knowledge of the beam parameters. Alternatively the beam can be enlarged with the techniques described in Sec. 5.4 to ensure a constant illumination and therefore a deconvolution from the lateral efficiency for the scanned range of $z$. This method was chosen to measure $\eta_{\perp}(z)$ in the $L a b R A M$ system for the two water immersion objectives used in the coordination experiments $(\times 100$ and $\times 20$ from Olympus) and for several pinhole sizes.

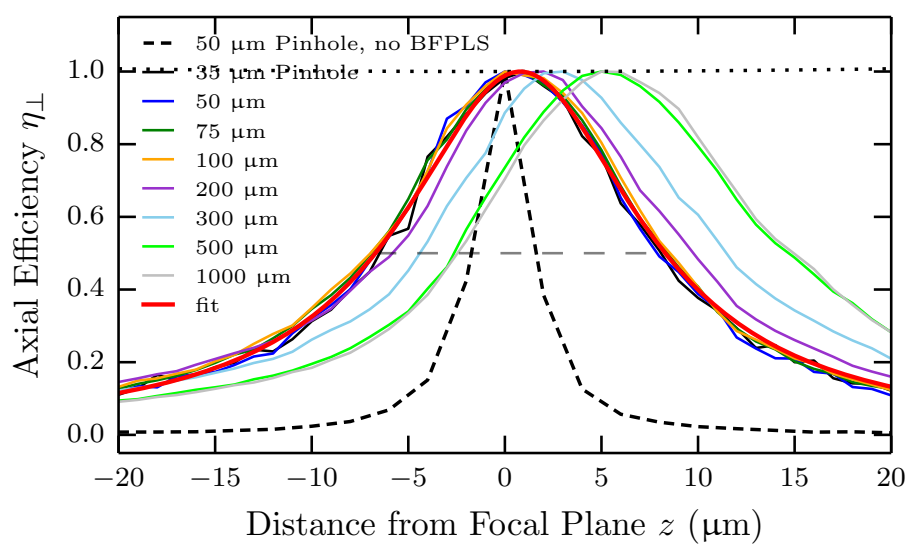

(a)

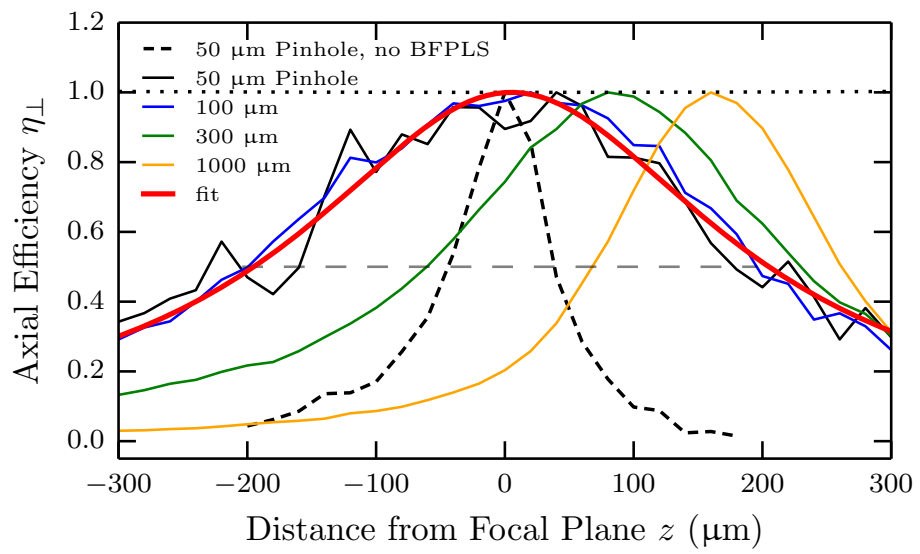

(b)

Figure 4.20: The axial detection efficiency for a $\times 100$ (a) and $\times 20$ (b) water immersion objective in the LabRAM setup for several pinhole diagonals. The $\cdot \cdot$ horizontal lines show the beam profiles used for the measurements normalized by their waist. The FWHM of the axial detection efficiency is indicated by the - - horizontal line. The - - curve in (a) and (b), showing a significantly narrower profile, are the results obtained when no additional lenses are used to enlarge the beam. 
A combination of lenses (tuneable beam expander system with $-18 \mathrm{~mm}$ and $50 \mathrm{~mm}$, see Sec. 5.5) was used to create a low divergent beam with a width that is almost constant for the scanned region (see the dotted horizontal lines in Figs. 4.20 (a) and (b)). The short penetration depth (below $1 \mu \mathrm{m}$ ) of light into silicon, which is used as a sample, ensures that the signal originates from the surface. The intensity of the $520.5 \mathrm{~cm}^{-1}$ Raman peak is determined by fitting a pseudo-Voigt profile, a linear combination of a Gaussian and a Lorentzian curve, to the measured spectrum. By scanning $z$ in both directions, with a negative value of $z$ meaning that the sample is closer than $f_{o b j}$ to the objective, the axial detection efficiency profile is obtained. The results are plotted in Figs. 4.20 (a) and (b), showing that the confocal depth is $\sim 15 \mu \mathrm{m}$ for the $\times 100$ and $\sim 400 \mu \mathrm{m}$ for the $\times 20$ objective. These values are nearly independent of the pinhole dimension, implying that the smallest available pinhole size of $35 \mu \mathrm{m}$ is still too large to efficiently reduce light from areas above and below the focal plane of the objective to reach the detector and work in a "truly" confocal regime. A further reduction of the pinhole size, where a smaller FWHM can be expected, is not possible with the specific mechanism. The detection profiles can be fitted very well with a Lorentzian function (red line in Figs. 4.20 (a) and (b)), although a physical reason for that specific form is not obvious.

In order to demonstrate the effect of the convolution between lateral and axial detection efficiency the measurement is repeated for a divergent laser beam (no BFPL). The resulting curves show a much narrower FWHM (3.5 $\mu \mathrm{m}$ and $84 \mu \mathrm{m})$. However, this is attributed to the broadening of the beam width in the $z$ direction and subsequent blocking of parts of the signal by the pinhole due to the lateral efficiency. The values obtained this way do not correspond to the axial detection efficiency as discussed above, which shows that the results obtained with the z-scan technique have to be interpreted very carefully. Many reports in literature and by manufacturers neglect this difference and may not operate in a truly confocal regime as they claim.

The asymmetry of the profiles and the shift of the highest efficiency to positive values of $z$ for a pinhole size larger than $200 \mu \mathrm{m}$ is apparent in Figs. 4.20 (a) and (b). This can qualitatively be understood by calculating the position and magnification of the images formed by the objective and pinhole lens as a function of $z$. For a given distance between the two lenses lens (650 $\mathrm{mm}$ in the LabRAM), the amount of signal that passes through the pinhole can be higher for a value of $z$ larger than $f_{o b j}$ due the location and size of the intermediate image formed by the objective, even though no actual image is formed at the pinhole in this case. The exact quantitative relationship of the light intensity through the pinhole as a function of the sample's distance from the focal plane of the objective could be obtained using ray tracing. However, this is beyond the scope of this work. 


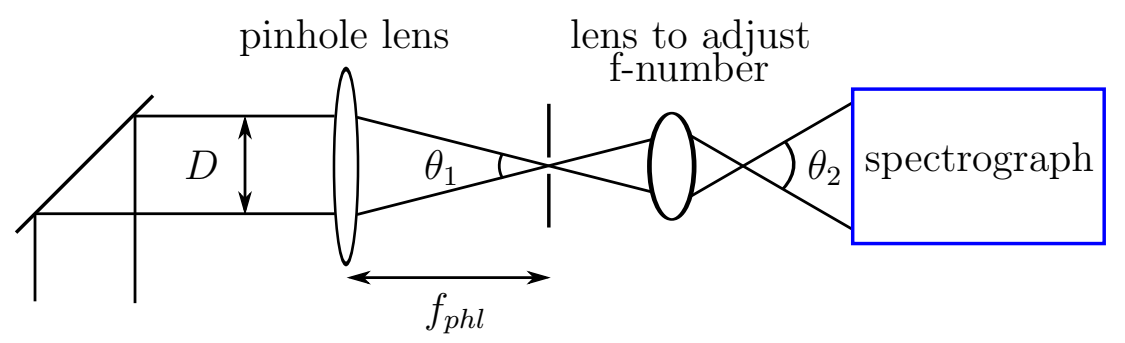

Figure 4.21: An illustration of the proposed setup that would allow for a better adjustment of axial and lateral detection efficiency to suit the requirements in the coordination experiments. A pinhole lens with a longer focal length results in a higher magnification of the image. An additional lens after the pinhole is used to match the spectrometer's f-number.

In conclusion, to suppress signals originating beyond the focal plane from reaching the detector and achieve a symmetric detection profile around the objective focal plane, the pinhole should be closed to $100 \mu \mathrm{m}$ in our system. The confocal depth for the $\times 20$ and $\times 100$ objectives are $15 \mu \mathrm{m}$ and $400 \mu \mathrm{m}$ respectively. A further gain in confocality would be expected at a smaller pinhole dimension, which is not reachable with the specific design, limiting the function of the pinhole. Additionally, due to the relatively low magnification $M$ (22.22 for the $\times 100$ and 4.44 for the $\times 20$ objective), the pinhole, even at the smallest possible setting, is not able to limit the lateral detection to an extent that would be desired for the coordination experiments. For example, for a NP200-rated pore (diameter of $\sim 500 \mathrm{~nm}$ ), the pinhole would have to be closed to $16 \mu \mathrm{m}$ to efficiently suppress signals from particles that are outside the pore orifice. In this case, to minimise the signals causing "false positive" events, the use of a highly divergent laser beam is of advantage, meaning that a compromise between uniform pore illumination and the selective detection of only translocating particles has to be made.

In this context, an improvement of the optical setup is proposed, which could not be implemented in reality as no substantial alterations to the system, which is shared between several users, could be made. By replacing the pinhole lens with one of a longer focal length, obtaining a higher magnification, both the axial and lateral detection efficiency could be better adjusted to fit the specific purpose. However, an additional lens has to be placed after the pinhole (see Fig. 4.21) to compensate for the change of the f-number, which has to match the spectrometer's $(f /$ LabRAM $\sim 5)$ as closely as possible [191]. This is important to ensure an optimal signal collection and a high spectral resolution. Overfilling or underfilling should be avoided and leads either to a loss of signal or a decrease of resolution. 


\subsubsection{The Scattering Volume of an Objective}

For many experiments, e.g. the diffusion-based measurement of fluorescent or Ramanactive particles in solution, it is important to calculate the size of the probed volume. When using a laser for excitation, the corresponding profile will follow the properties of a Gaussian beam. With the knowledge of its waist $w_{0}$, an effective area $A_{e f f}=\pi w_{0}^{2} / 2$ [190] can be defined. It is the imaginary area which, illuminated with the constant power density $I_{0}$ (see Eq. 5.5), has an overall power equal to the one of the laser. For the collection profile, which can be measured as described above and may be represented by a Lorentzian function, an effective height $H_{\text {eff }}$ can be introduced [192]

$$
H_{e f f}=\int_{-\infty}^{+\infty} \eta_{\perp}(z) / \eta_{0} d z
$$

It describes the length from which the same signal would be observed if the collection efficiency was uniform along $z$ and equal to its maximal value $\eta_{0}$. It can be calculated by either a summation of the values in the measured efficiency or an integration over the fitted (Lorentzian) shape (compare Fig. 4.20). $A_{\text {eff }}$ and $H_{\text {eff }}$ together define the effective scattering volume $V_{e f f}=A_{\text {eff }} H_{\text {eff }}$, represented by a cylinder. It can be used to calculate the average particle number $N=c V_{\text {eff }}$ probed in an experiment and predict the expected signal. In our system, the effective scattering volume is $5.29{\mu \mathrm{m}^{3}}^{3}\left(A_{\text {eff }}=0.22 \mu \mathrm{m}^{2}, H_{\text {eff }}=23.56 \mu^{3}\right)$ for the $\times 100$ and $3544 \mu^{3}$ for the $\times 20$ objective $\left(A_{e f f}=5.64 \mu^{3}, H_{e f f}=628.32 \mu^{3}\right)$.

\subsubsection{The Total Detection Efficiency of the Spectrometer}

In order to estimate the sensitivity of the optical measurement and predict the minimum fluorescent or Raman cross section a potential sample must have to be detectable with the developed technique, the overall detection efficiency $\eta_{\text {total }}$ of the optical setup has to be determined. Here it is defined by the ratio of the number of photons detected on the CCD chip $P_{\text {Raman } C C D}$ to the number collected by the objective lens $P_{\text {Raman } o b j}$. It can be obtained by measuring the signal from a reference sample of known cross section $d \sigma / d \Omega$ and concentration $c_{0}$. With $N$ the average number of molecules in the effective scattering volume $V_{\text {eff }}, \Delta \Omega$ the solid angle detected by the objective $(\Delta \Omega=2 \pi(1-\cos \theta)$, with the numerical aperture of the objective $N A=n \sin (\theta)), P_{0}$ the total power of the laser at the sample and the effective area $A_{e f f}$ and height $H_{\text {eff }}$ of the objective, the Raman intensity $P_{R a m a n} n_{o b j}$ collected by the objective is given by 


$$
P_{\text {Raman }_{o b j}}=\frac{d \sigma}{d \Omega} N \Delta \Omega \frac{P_{0}}{A_{e f f}}=\frac{d \sigma}{d \Omega} c_{0} \Delta \Omega P_{0} H_{e f f} .
$$

This value can then be converted into the number of collected photons per second (energy per photon $\sim 2.64 \mathrm{eV}$ at a wavelength of $458 \mathrm{~nm}$ ) and compared to the total number of photons detected on the CCD chip within the spectral region of the sample mode to obtain $\eta_{\text {total }}$.

Here, the molecule 2-bromo-2-methylpropane (2B2MP) with a Raman cross section of $2.05 \times 10^{-29} \mathrm{~cm}^{2} \mathrm{sr}^{-1}$ of the $516 \mathrm{~cm}^{-1}$ mode at a wavelength of $458 \mathrm{~nm}$ [193] and a concentration of $8.76 \mathrm{M}$ was used as a sample. With the numerical aperture $N A$ of 1 ( $\times 100$ Olympus water immersion objective, $\left.H_{\text {eff }}=23.56 \mu \mathrm{m}\right)$ and an electronic gain setting of 2 (one count per two incident photons) the total detection efficiency was measured to be $3.7 \%$ for the spectrometer grating with 300 grooves $/ \mathrm{mm}$ and $2.5 \%$ for the grating with 1800 grooves $/ \mathrm{mm}$. These at first surprisingly low values can be understood when the total losses due to the transmittance of the objective, the notch filter and the pinhole lens, the reflectivity on six mirrors (less than $90 \%$ each), the efficiency of the grating, and finally the quantum efficiency of the CCD chip itself and its fill factor (ratio of light sensitive area versus total area of a pixel) are taken into account. The efficiencies depend on the wavelength used and given that the specific setup is optimised for UV-Raman measurements, an increase of the efficiency in the visible region through optimization of the optical components would be possible.

\subsubsection{The Time-Step Calibration in the $\operatorname{LabRAM}$}

After conducting the first coordination experiments, it was observed that most of the resistive peaks had corresponding optical peaks but the time scale appeared to be shifted and dilated. The times given by the software (Labspec 5.74 .29 beta) do not correspond to the real times when the " $1 \mathrm{MHz}$ ultrafast" detector mode was used (Fig. 4.22). As no further information from the manufacturer could be sourced, the time shift $t_{i n}$ and the dilation factor $r$ had to be quantified for different time settings in order to correlate electric and optical signals. The findings in this section are specific to the equipment used.

The notations used in the following are:

- $\Delta t \ldots$ time step between measurements

- $\tau$... integration time during which the signal is acquired 
- $t_{\text {in }}$... delay time from starting the measurement and the acquisition of the first spectra

- $\Delta t^{\prime} \ldots$ time step between measurement as set in the software (in the " $1 \mathrm{MHz}$ ultrafast" mode the condition $\Delta t^{\prime} \geq \tau^{\prime}+12 \mathrm{~ms}$ has to be fulfilled)

- $\tau^{\prime} \ldots$ integration time as set in the software

- $N$.. number of spectra taken

- $T$... total time of the measurement

- $T^{\prime} \ldots$ total time of the measurement in the output file

Specified in the software and given in the the output file:

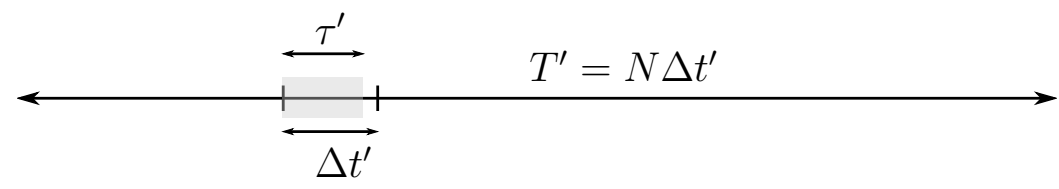

What happens in reality:

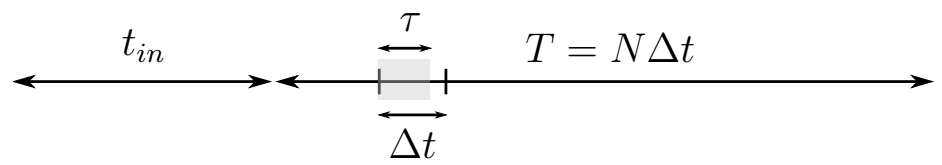

Figure 4.22: Illustration of the time step mismatch in the LabRAM. The time scale in the output file of the software is shifted and dilated relative to the real time.

To determine the dilation factor $r$, which relates $\Delta t$ to $\Delta t^{\prime}$ through $\Delta t=r \Delta t^{\prime}$, and the time shift $t_{i n}$, the following method is used. First, the laser is focussed on a sample with a reasonably stable signal, here the $520.5 \mathrm{~cm}^{-1}$ Raman line of a silicon sample was used, and the time step $\Delta t^{\prime}$, the integration time $\tau^{\prime}$ and the total measuring time $T^{\prime}=N \Delta t^{\prime}$ are defined in the software. Then the timer of a stopwatch is started along with the beginning of the measurement. After a certain time, the laser is turned off and the timer is stopped. The time $t_{\text {off }}$ on the stopwatch is noted and compared to the time $t_{o f f}^{\prime}$ in the output file when the signal drops to the background level. This procedure is repeated several times to obtain a plot similar to Fig. 4.23. By fitting a linear function to the data the values for $r$ and $t_{\text {in }}$ can be obtained. Alternatively, a mechanical chopper with a known frequency could be used instead of the stopwatch method. The method would be faster as many on-off cycles could be measured within only one time series. However, $t_{\text {in }}$ could not be obtained that way.

The results, which are summarised in Tab. 4.4, show that the dilation factor is dependent on the time step. Additionally a slight dependence on the binning factor 


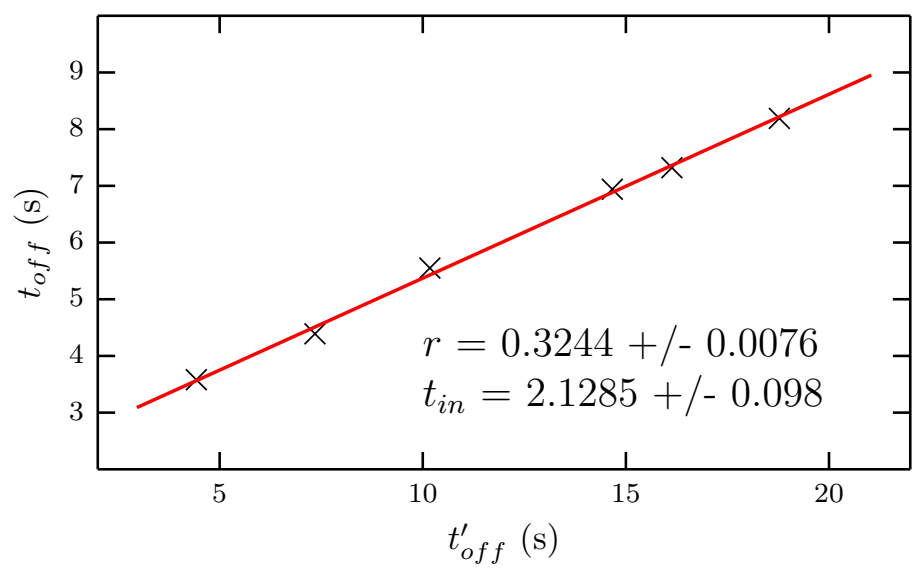

Figure 4.23: The "real" time as a function of the "Labspec" time for a time step $\Delta t^{\prime}=13.1 \mathrm{~ms}$ and an integration time $\tau^{\prime}=1 \mathrm{~ms}$.

(along the x-direction of the chip), and the integration area (along the $\mathrm{y}$-direction) is observed. For the minimal time step that is allowed by the software $\left(\Delta t^{\prime}=13.1 \mathrm{~ms}\right.$ for $\tau^{\prime}=1 \mathrm{~ms}$ ), $r$ is 0.3244 , leading to an actual time step between measurements of $4.25 \mathrm{~ms}$. This sets the highest time resolution of the optical detection and corresponds to a sampling frequency of $235 \mathrm{~Hz}$. After a closer look at the results the difference $\Delta t^{\prime}-\Delta t$ seems to be a constant value of $c \sim(8.86 \mathrm{~ms}$ ) (in the $1 \mathrm{MHz}$ "ultrafast" mode). With that and the definition of $r$, the relationship between $r$ and $\Delta t^{\prime}$ can be found to be

$$
r=1-\frac{c}{\Delta t^{\prime}}
$$

so that the dilation factors for other time settings are predictable. The time shift follows no obvious rule and cannot be understood without further information about the software and the chip architecture.

The integration time $\tau$, during which the CCD chip is sensitive to incoming photons and the signal is acquired, is in general different to the time step $\Delta t$ between measurements. It can be obtained by comparing the signal strength collected during one time step (for accuracy the sum of the spectra of several thousand time steps was used) to the signal measured in $1 \mathrm{~s}$. The intensities of the silicon Raman line are then obtained by fitting a pseudo-Voigt profile to the measured spectra. The analysis shows that the measured integration time $\tau$ is slightly (up to 5\%) larger than the actual time step. This is physically not possible and might be caused by the CCD cleaning step between measurement, which probably does not erase all charges. However, it can be concluded that the "dark" time, i.e. the time when the CCD chip is not responding to incident light, is not a substantial proportion of the 
Table 4.4: The time steps $\Delta t^{\prime}$ and the integration time $\tau^{\prime}$ as set in the software and the actual values as found with the method described in the text. The real integration time $\tau$, using a binning of 8 pixels, could not be obtained due to the low resolution of the spectra and a corresponding large error in the signal intensity.

\begin{tabular}{ccccccc}
\hline$\Delta t^{\prime}(\mathrm{ms})$ & $\tau^{\prime}(\mathrm{ms})$ & $\mathrm{r}$ & $t_{\text {in }}(\mathrm{s})$ & $\Delta t(\mathrm{~ms})$ & $\begin{array}{c}\Delta t^{\prime}-\Delta t \\
(\mathrm{~ms})\end{array}$ & $\tau(\mathrm{ms})$ \\
\hline 32 & 20 & 0.7231 & 2.0520 & 23.14 & 8.86 & 23.79 \\
22 & 10 & 0.5956 & 2.1241 & 13.10 & 8.90 & 13.20 \\
17 & 5 & 0.4815 & 2.0687 & 8.19 & 8.81 & 8.25 \\
13.1 & 1 & 0.3244 & 2.1285 & 4.25 & 8.85 & 4.26 \\
$13.1(100-160)$ & 1 & 0.3168 & 2.1718 & 4.15 & 8.95 & 4.35 \\
$13.1($ Bin 8$)$ & 1 & 0.3092 & 1.8653 & 4.18 & 8.92 & - \\
\hline
\end{tabular}

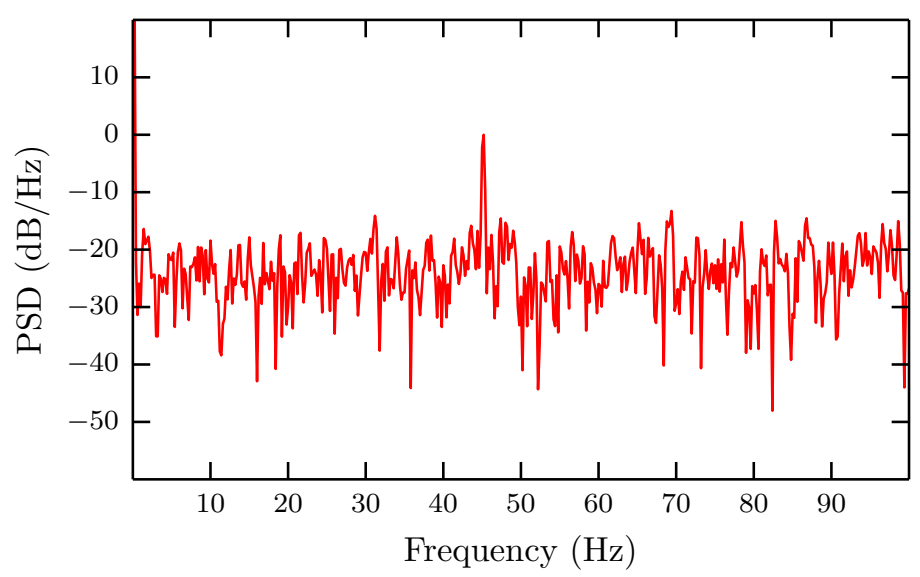

Figure 4.24: The Power Spectral Density (PSD) of the time-dependent intensity signal of the $520.5 \mathrm{~cm}^{-1}$ Raman line of silicon. The peak is at the very same position $45.5 \mathrm{~Hz}$ as found in the laser-induced noise observed in the current trace.

time step and is therefore not expected to play a significant role for the coordination experiments. Further considerations of the effect the "dark time" can have on the optical signal of a particle moving through the pore are discussed in Sec. 4.4.

Interestingly, a spectral analysis of the time-dependent Raman intensity signal (Fig. 4.24) reveals a peak at $45.5 \mathrm{~Hz}$. This is the very same frequency of the noise that is found in the current trace when the pore orifice is illuminated with a laser or the hydrostatic pressure across the membrane is adjusted to zero. This finding supports the theory that the origin of the noise is connected to a mechanical vibration of the system. 


\subsubsection{Noise of the CCD}

The noise in a CCD chip is a combination of photon noise, the inherent statistical fluctuation of the incoming photon flux, dark noise, the thermal generation of electrons within the CCD, and electronic readout noise, which results from both the transformation of charge carriers into a voltage signal and the subsequent analogto-digital conversion. In state of the art liquid nitrogen cooled CCDs used in spectrometers and with exposure times of several seconds the noise is usually photon dominated, with the RMS of the dark and readout noise in the order of only a few electrons per bin. However, for a high sampling frequency the readout noise can become dominant and then sets the limit for the device's performance.

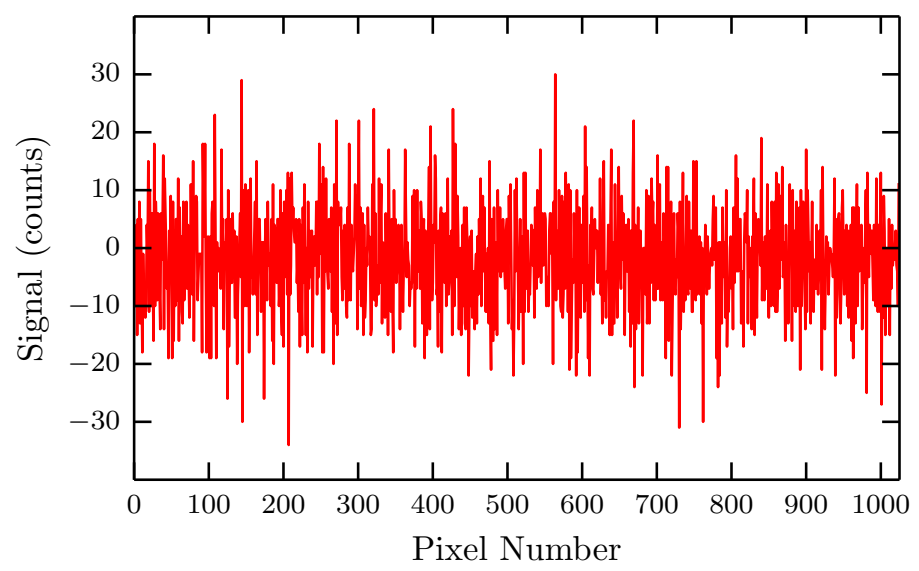

Figure 4.25: The pixel-resolved counts in the CCD for a real time step of $4.25 \mathrm{~ms}$ in the " $1 \mathrm{MHz}$ ultrafast" mode under "dark" conditions (integration of the pixels in y-direction from 1-256, gain setting $=2$, i.e. two incident photons correspond to one count).

In order to resolve events of single particles travelling through a nano- or micronscale pore the CCD has to be used at its highest time-resolution. Figure 4.25 shows the counts of each pixel column in the x-direction of the CCD. The noise is readout limited and has a typical RMS value of $\sim 10$ counts per pixel, setting the ultimate detection limit of the specific setup. A limitation of the integration area of the chip in the y-direction shows no reduction in the noise, indicating that dark noise plays a minor role. In the coordination experiments, with the laser focussed on the pore orifice, stray-light and photons originating from the membrane material will add to a background noise. However, their contribution is negligible compared to the bare readout noise and could also be removed using calibration techniques and frame comparison. To enhance the signal-to-noise ratio binning can be used, which comes with the disadvantage of a reduced spectral resolution. Recent and future development in the detector technology (ICCD, EMCCD, CMOS sensors) could improve the sensitivity of the technique further. 


\subsection{Pores, Solutions and Particles}

\subsubsection{Pores}

Izon Science offers tuneable pores for the detection of particles in the range from $40 \mathrm{~nm}$ to $200 \mu \mathrm{m}$. Each pore is characterised after fabrication based on its baseline current and given a rating of the form "NPx". Hereby x stands for the particle diameter in $\mathrm{nm}$ which the pore is best suitable to detect. As the pore size can be tuned by applying a mechanical stress, each pore can be used for a range of particle sizes. For each pore rating a particle concentration is recommended. A significantly higher concentration will increase the likelihood that two or more particles are present in the pore's sensing area in close succession and individual events can then not be resolved any more. A much lower concentration on the other hand results in sparse translocation events and a low throughput. Table 4.5 shows the particle ranges and concentrations recommended for the pores used in the experiments presented in this work. As each polyurethane membrane shows stress-softening (Mullins effect [194]) when initially stretched, they were preconditioned before first use. Willmott et al. [170] showed that ten stretch-relax cycles are sufficient to achieve a reproducible stretch-strain relationship.

Table 4.5: The different pore ratings used in this work with their target particle range and recommended particle concentration.

\begin{tabular}{ccc}
\hline Rating & $\begin{array}{c}\text { Particle Range } \\
(\mathrm{nm})\end{array}$ & $\begin{array}{c}\text { Recommended } \\
\text { Concentration } \\
\left(1 \times 10^{6} \text { particles } / \mathrm{mL}\right)\end{array}$ \\
\hline NP2000 & $1000-4000$ & 5 \\
NP1000 & $500-2000$ & 50 \\
NP400 & $200-800$ & 500 \\
NP200 & $100-400$ & 1000 \\
\hline
\end{tabular}

\subsubsection{Determining the Pore Dimensions}

The pores are fabricated by puncturing a polyurethane membrane with a chemically etched tungsten needle, resulting in a conical shape of the pores. While the large pore radius $b$ and the thickness $l$ of the membrane are in the range of $25 \mu \mathrm{m}$ and $150 \mu \mathrm{m}$ for all pore ratings, the size of the small pores radius $a$ varies greatly between the ratings. An approximate determination of the small pore diameter is needed to estimate the required size of the laser beam used for the coordination experiments. This can be done by calculating the small pore opening from the baseline current using 
Eq. 3.3 and Ohm's law. However, as the pores are not strictly conical this method is prone to errors. The pore dimensions can also be obtained optically. Figure 4.26 shows pictures of the small and large pore openings of various specimens using the microscope camera of the LabRAM. Previous work [34] showed that the pores are not perfectly round in cross section. Therefore, the characteristic dimension for the pore radii is chosen to be the hydraulic radius, equal to $2 A / P$, where $A$ is the cross sectional area and $P$ the perimeter of the outline of the pore opening.

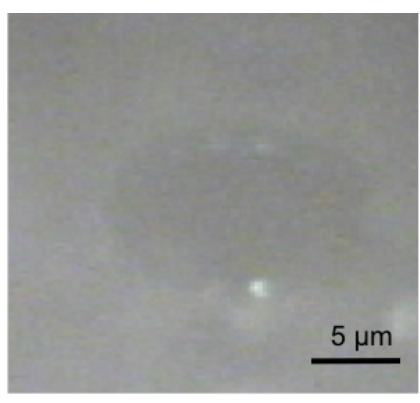

(a)

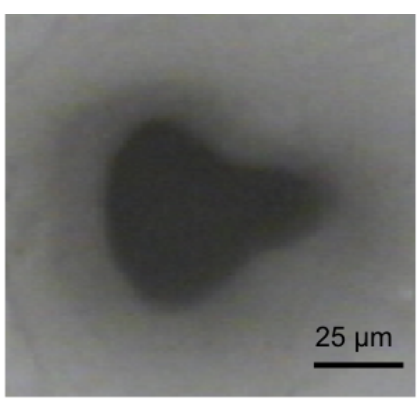

(b)

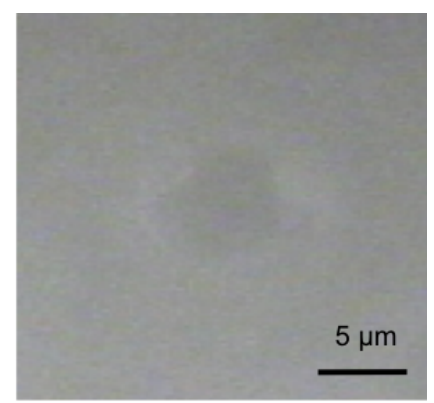

(c)

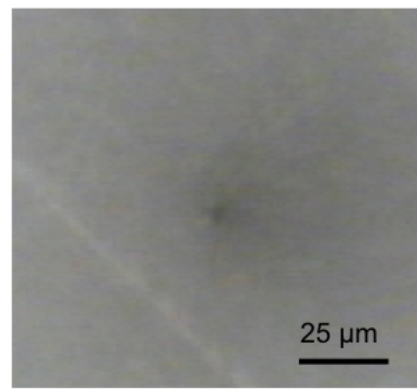

(d)

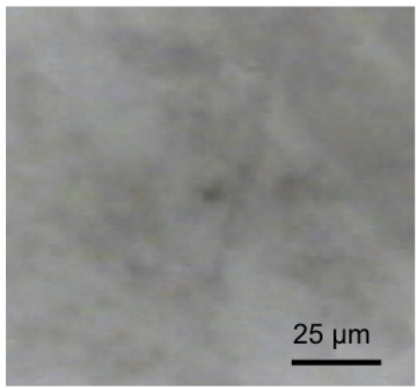

(e)

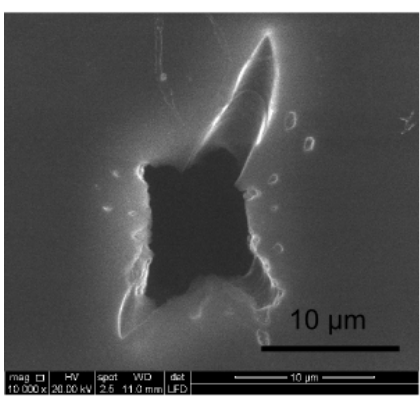

(f)

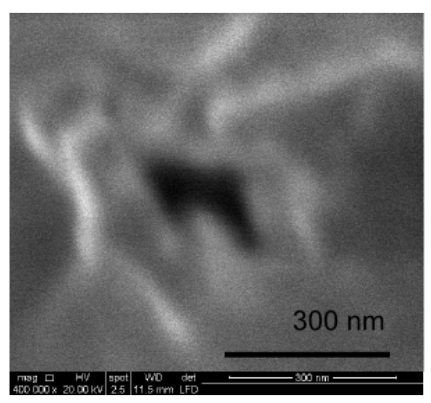

(g)

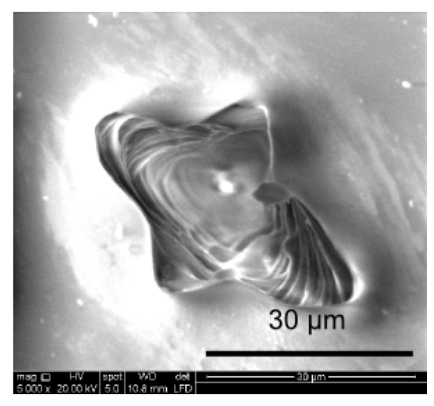

(h)

Figure 4.26: Optical images of the (a) small and (b) large pore opening of a NP2000 using the camera of the LabRAM. The small pore openings of a (c) NP1000 (d) NP400 and (e) NP200 pore (all at a stretch of $39 \mathrm{~mm}$, second setup) is shown in (c), (d) and (e) respectively. SEM pictures of the small and large pore opening of a NP200 pore are shown in (g) and (h) respectively. (f) The small pore opening of a NP2000 pore (different specimen from (a).

The use of the hydraulic radius can be prone to errors if the outline of the pore orifice is ragged, which leads to an overestimation of the perimeter and consequently 
an underestimation of the pore radius. In such a case it is more suitable to derive the radius from the cross section alone. For the used pores this was not an issue and the two methods lead to similar values (within 1\%). The largest factor of uncertainty is the determination of the correct focus as the pore appears to have different sizes when the focus changes even as little as $1 \mu \mathrm{m}$. The optical quantification of the small pore opening is only successful for pores with a rating higher than NP1000 in the specific setup. Below that, a reliable sizing is not possible due to the poor contrast of the pores and the inherent resolution limit. The radius can then be determined indirectly via Eq. 3.3 and the optically measured values for $b$ and $l$. To measure $b$ the pores are installed upside down from their usual orientation. The thickness of the pore can be derived by focussing on distinct feature on both sides of the membrane and noting the difference $\Delta$ that the $\mu \mathrm{m}$ adjustment knob has to be turned. As the refractive index of polyurethane $\left(n_{2}=1.5\right)$ is different from that of the surrounding medium $\left(n_{1}=1 / 1.33\right.$ for air/water $) \Delta$ has to be multiplied by $n_{2} / n_{1}$ to obtain the correct value for the pore length. Scanning Electron Microscopy (SEM) could also be employed to quantify the pore size. Figures 4.26 (f), (g) and (h) show SEM pictures (Fei Quanta FEG 250, low vacuum mode for uncoated samples) of various pore specimens revealing that the pore shape can significantly deviate from a circular geometry. A determination of the pore size using these SEM pictures was not used here as the stretch could not be set to the same values as used for the measurements.

\subsubsection{The Optical Properties of the Membrane Material}

The membranes used for the TRPS pores are made by injection-moulding of a polyether-based thermoplastic polyurethane (Elastollan ${ }^{\circledR} 1160 \mathrm{D}, \mathrm{BASF}$ ), which is a mixture of long and short chain polymers in order achieve its elastomeric properties. Polyurethane has no strong absorption bands in the visible region. However, during the course of this work it has been observed that the membrane can be irreversibly damaged using the $458 \mathrm{~nm}$ and $514 \mathrm{~nm}$ lines of the argon laser at high power densities (Fig. 4.27), so that an interaction with the light of these wavelengths has to occur to some extent. The effect could only be observed at the highest available power densities, i.e. using full power (see Tab. 4.2 for the nominal powers) and an objective that focuses the laser intensity to a small spot $(\times 50$ or $\times 100$ Olympus objectives $)$. The resulting holes are only a few $\mu \mathrm{m}$ in depth, as the laser intensity decreases quickly due to the high divergence of the focussed beam. The diameter of the holes increases with the time the material is exposed to the laser illumination, reaching its final size within a time scale of $\sim$ min. Images of some of the holes obtained using the optical microscope of the LabRAM (Figs. 4.27 (a) and (b)) and a SEM (Figs. 4.27 (c) and (d)) show a well-defined circular shape, with the smallest hole diameter of $\sim 300 \mathrm{~nm}$. 


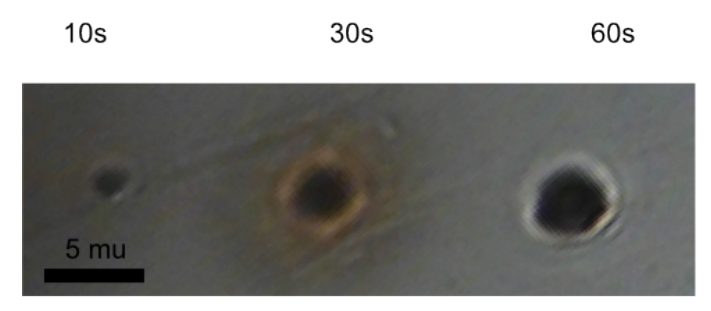

(a)

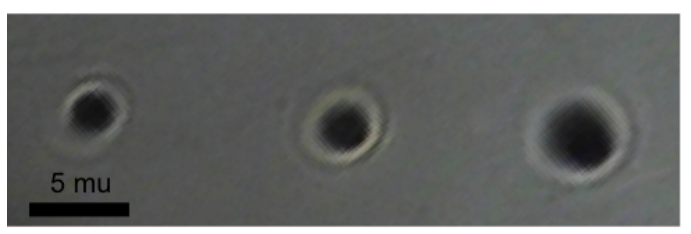

(b)

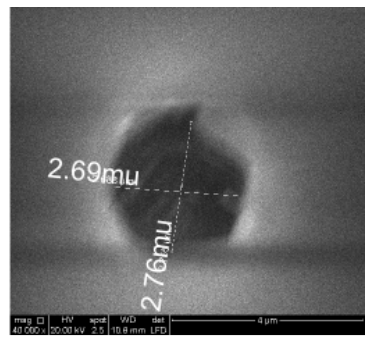

(c)

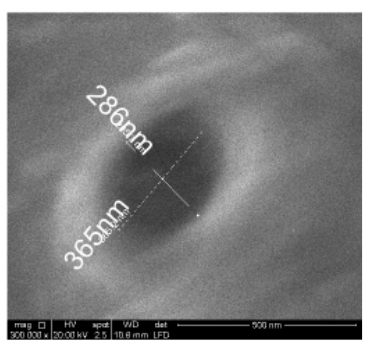

(d)

Figure 4.27: Optical images of holes in the membrane material caused by the illumination of the (a) $458 \mathrm{~nm}$, (b) $514 \mathrm{~nm}$ line of a tunable Ar-ion laser at high power density $(D 0, \times 50$ Olympus air objective). The labelled exposure time has an influence on the hole size. (c) SEM image of the middle hole in (a) (notice the discolouration of the surrounding pore material due to the exposure of the electron beam in (a)). (d) A hole with a considerably smaller diameter is formed by using a $\times 100$ water immersion objective.

The interaction of the material with laser light could possibly be utilized to fabricate well-defined circular shaped pores for TRPS. Pores fabricated with the conventional method using a tungsten needle show a less regular shape, especially for the smallest rated pores (Fig. $4.26(\mathrm{~g})$ ). As the typical power densities used in the coordination experiments are at least two orders of magnitude smaller, damage to the material around the pore can be excluded. No visible damage to the membrane could be observed when the He-Ne laser, which has a comparable output power to the Argon-ion laser, was used. This suggests that the absorption of the material is decreasing with higher wavelength.

To quantify the absorption of the material a setup using an integrating sphere was used. Measuring the absorption in a UV-Vis transmission setup leads to unsatisfactory results as the absorption is highly dependent on the exact sample position of the specimen due to lensing effects. Additionally, scattering contributes significantly to the extinction, leading to erroneous absorption values. Both problems could be addressed by using the same integrating sphere setup, which was also used to quantify the absorption cross section of fluorescent polystyrene beads in solution (see Sec. 4.3.3, details of the setup are described in [195]). For the measurement the central septum of a specimen was cut out of the cruciform, placed onto a sample holder and inserted into the sphere. The measured absorbance $A$ as a function of the 


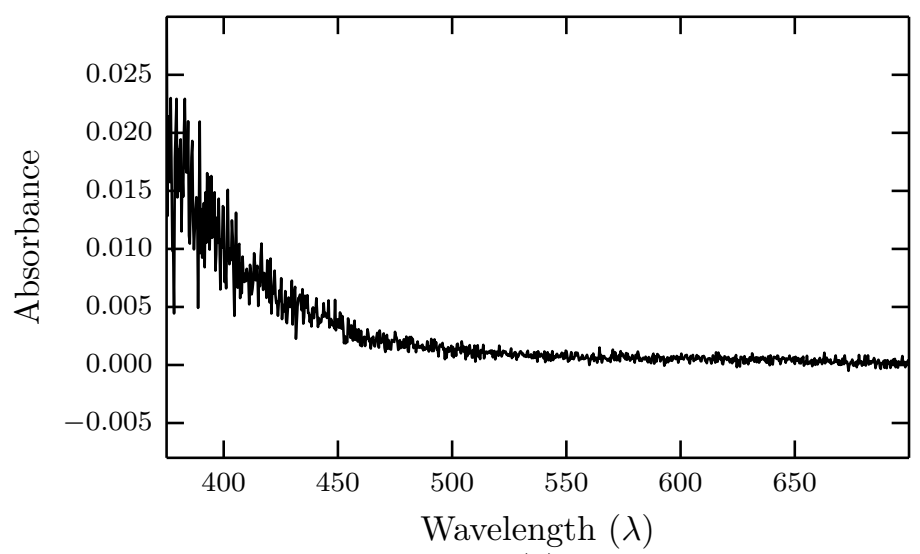

(a)

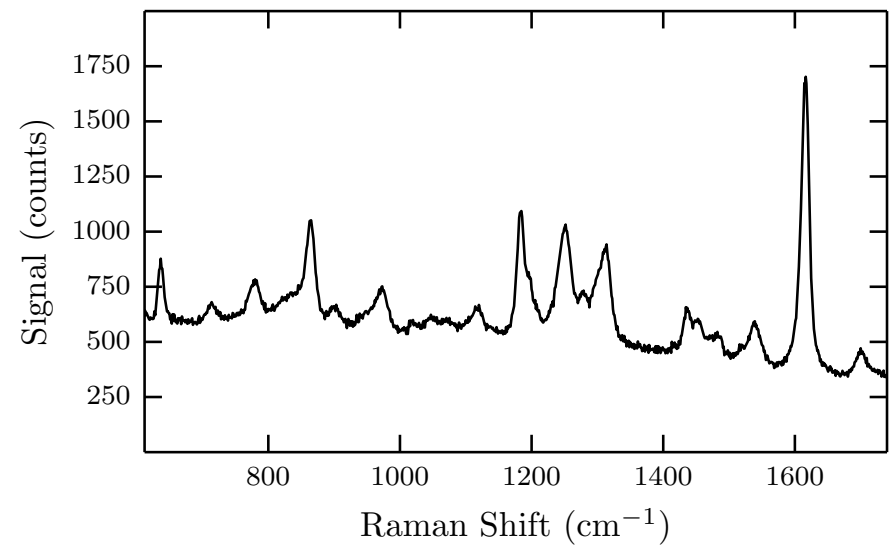

(b)

Figure 4.28: (a) The absorbance of the polyurethane membrane as measured in an integrating sphere (details in the text). (b) The Raman spectrum of the material using a He-Ne laser $(D 1)$, a $\times 100$ water immersion objective and an integration time of $10 \mathrm{~s}$.

wavelength is plotted in Fig. 4.28 (a). Polymers usually have absorption bands in the ultra-violet, and the observed curve appears to be the tail of such an absorption, decreasing with increasing wavelength. The absorption coefficient $\alpha$ can be estimated as follows. With an average number of reflections (sphere multiplier $M$ ) of 80 , a mean path length of $5 \mathrm{~cm}$ and a sample to sphere volume fraction $V_{\text {sample }} / V_{\text {sphere }}$ of $8 \times 10^{-5}$ the path length $L$ through the sample for the specific setup is $0.032 \mathrm{~cm}$. At $458 \mathrm{~nm}$ the absorbance $A=\alpha L$ was measured to be 0.0025 , resulting in a value for $\alpha$ of $0.08 \mathrm{~cm}^{-1}$. This means that for example a $1 \mathrm{~cm}$ thick sample of the material would absorb about $1-10^{-\alpha}=15 \%$ of the incoming light.

The absorption can now be used to estimate the deposited heat and the resulting temperature rise in the material when the laser is focussed onto the membrane. Without taking heat conduction within the material into account, a simple approach that incorporates the laser power, the volume of the heated material $\left(A_{\text {eff }} \times 2 z_{R}\right)$, the specific heat capacity and the density of polyurethane, yields that a local increase 
of the temperature of several hundred ${ }^{\circ} \mathrm{C}$ is possible. Due to high measurement uncertainties $(\sim 20 \%)$ and several simplifications, this estimate is far from precise. However, it indicates that the laser can cause local melting of the material. While the smaller absorption at $514 \mathrm{~nm}$ compared to $458 \mathrm{~nm}$ is compensated by the higher laser output at this wavelength, the absorption at $633 \mathrm{~nm}$ is too small to result in any visible damage of the material when the He-Ne laser is used.

The pore material also shows several distinct Raman peaks above a broad fluorescent background in the region from $600 \mathrm{~cm}^{-1}$ to $1800 \mathrm{~cm}^{-1}$ (Fig. 4.28). The signal is reasonably strong, but, under conditions used for the coordination experiments, i.e. laser intensity, focus on the orifice, integration time, it will be dominated by the readout noise of the CCD. It is therefore not expected to interfere with the spectral detection of translocating particles.

\subsubsection{Solutions}

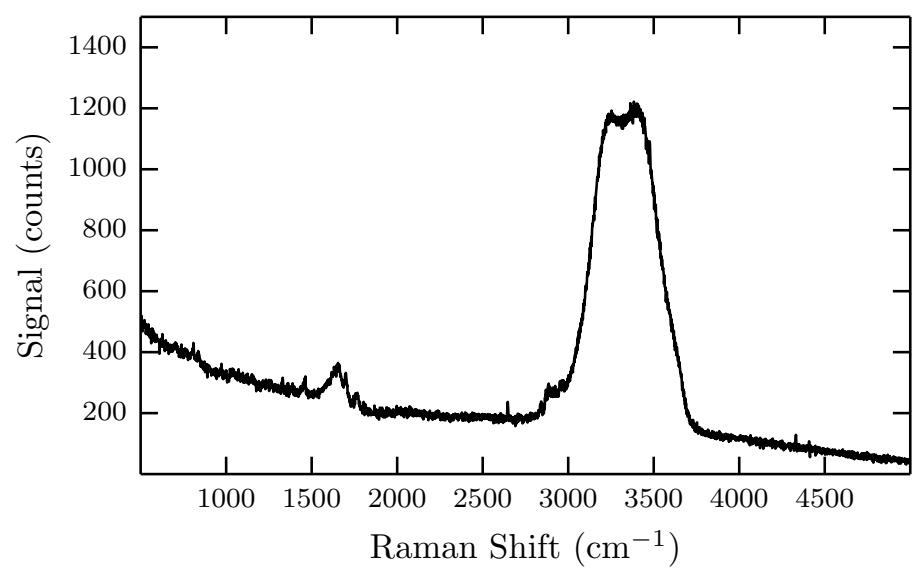

Figure 4.29: The Raman spectra of SEB (composition as described in the text) using 10 s integration time and the He-Ne laser.

Unless specifically noted, the particles were suspended in an ionic solution, often referred to as Standard Electrolyte Buffer (SEB) in TRPS literature. It contains $0.1 \mathrm{M} \mathrm{KCl}, 3 \mathrm{mM}$ ethylenediaminetetraacetic acid (EDTA) as chelating agent, and is buffered with tris(hydroxymethyl)aminomethane (Tris buffer). The components are dispersed in deionised water $(18.2 \mathrm{M} \Omega \mathrm{cm})$ and the $\mathrm{pH}$ is adjusted to 8 using $\mathrm{HCl}$ and $\mathrm{KOH}$. The solution is then filtered through a $0.2 \mu \mathrm{m}$ filter (Millipore). A small amount $(\sim 0.01 \%)$ of surfactant (Triton X-100) is added to increase pore wetting. To minimise the risk of air bubbles, the solution for the lower compartment of the adapted fluid cells is used without surfactant.

For the resistivity $\rho$ of the solution $0.86 \Omega \mathrm{m}$ is assumed, which is the value for a $0.1 \mathrm{M} \mathrm{KCl}$ solution at room temperature (293 K) [92]. The additional components 
have proven to only marginally affect the resistivity [196]. The solution does not show any distinct spectral features other than a broad peak between $3100 \mathrm{~cm}^{-1}$ to $3600 \mathrm{~cm}^{-1}$, originating from the $\mathrm{O}-\mathrm{H}$ stretching modes of water (Fig. 4.29). However, the signal is too weak to be observable at the time resolution and laser intensities as used for the coordination experiments.

\subsubsection{Particles}

Polystyrene particles of various sizes and fluorescent labels were purchased from Sigma-Aldrich and Bangs Laboratories. All particles have carboxyl-groups on their surface and carry, due to the $\mathrm{pH}$ of the solution, a negative charge. The fluorescent dyes, which are given names by the supplier but often correspond to a well known dye, are embedded in the polystyrene matrix. The as-received particle solutions were diluted with electrolyte to a concentration suitable for the specific pore size and sonicated for about 5 minutes prior to the experiments. The sizes, absorption and emission maxima, along with the undiluted concentration of the particles used are summarized in Tab. 4.6.

Table 4.6: The nominal particle diameters, undiluted concentrations and the absorption and emission maxima of the embedded dye as given by the supplier.

\begin{tabular}{|c|c|c|c|c|c|}
\hline Supplier & Dye & $\begin{array}{c}\text { Particle } \\
\text { Diameter } \\
\quad(\mu \mathrm{m})\end{array}$ & $\begin{array}{c}\text { Conc. } \\
\text { undiluted } \\
\left(10^{9} / \mathrm{mL}\right)\end{array}$ & $\begin{array}{l}\text { Absor. } \\
\text { Max. } \\
(\mathrm{nm})\end{array}$ & $\begin{array}{c}\text { Emiss. } \\
\text { Max. } \\
(\mathrm{nm})\end{array}$ \\
\hline Sigma-Ald. & Yellow-Green & $2 / 1.9$ & $5.71 / 6.66$ & 470 & 505 \\
\hline Sigma-Ald. & Red & 0.50 & 365 & 575 & 610 \\
\hline Bangs Lab. & $\begin{array}{c}\text { Suncoast- } \\
\text { Yellow }\end{array}$ & 0.97 & 200 & 540 & 600 \\
\hline Bangs Lab. & Envy-Green & 0.97 & 200 & 525 & 565 \\
\hline Bangs Lab. & $\begin{array}{c}\text { Dragon- } \\
\text { Green }\end{array}$ & 0.22 & 1717 & 480 & 520 \\
\hline Bangs Lab. & Surf-Green & 0.19 & 2665 & 470 & 525 \\
\hline
\end{tabular}

\subsubsection{The Stability of Carboxylated Polystyrene Particles in Solution}

The stability of the suspended particles is essential to RPS and the developed coordination method. During the course of this work, several instances of particle aggregates were encountered, which led to a more frequent blocking of the pore or even prevented any successful measurement. The stability of a colloidal solution is an important issue for many fields in science (e.g. nanoparticle research, food 
science) and is quantitatively described by the theory of Derjaguin, Landau, Verwey and Overbeek (DLVO theory) [100]. The main forces between charged colloids in solution, which in general contains additional ionic species, are the strong, but short-ranged Van der Waals attraction and the long-range, electrostatic repulsion. The resulting DLVO potential is a function of the distance $r$ between the centre of the particles and is given by the sum of the Van der Waals potential $U_{V d W}$ and the screened Coulomb $U_{\text {Coul }}$ potential

$$
\begin{aligned}
U(r)=U_{V d W}(r)+U_{\text {Coul }}(r)=\frac{-A_{H c}}{6}\left(\frac{2 a^{2}}{r^{2}-4 a^{2}}\right. & \left.+\frac{2 a^{2}}{r^{2}}+\ln \left(1-\frac{4 a^{2}}{r^{2}}\right)\right) \\
& +Z_{\text {eff }}^{2} \frac{\lambda_{B} \exp (-\kappa(r-2 a))}{r} .
\end{aligned}
$$

Here, $a$ is the radius, $Z_{\text {eff }}=Z /(1+\kappa a)$ the effective charge and $Z$ the bare charge of the particle. $\lambda_{B}=e^{2} /\left(4 \pi \epsilon_{0} \epsilon_{r} k_{B} T\right)$ is the Bjerrum length and represents the separation at which the electrostatic interaction between two elementary charges is comparable to their thermal energy. $\kappa$ is the inverse of the Debye screening length $\lambda$ (see Eq. 3.32) and is related to the Bjerrum length through the expression $\kappa=\sqrt{4 \pi \lambda_{B} N_{A} \sum_{i} z_{i}^{2} c_{i}}$. The Hamaker constant $A_{H c}$ quantifies the strength of the Van der Waals force and depends both on the solution and the material of the colloids.

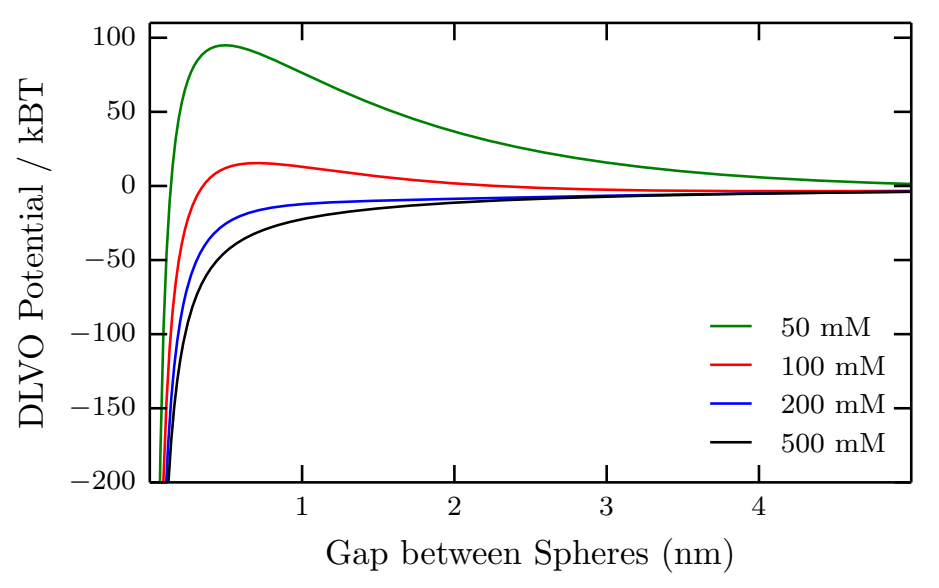

Figure 4.30: The DLVO potential between two negatively charged $\left(-0.0231 \mathrm{C} \mathrm{m}^{-2}\right)$ polystyrene particles with a radius of $100 \mathrm{~nm}$ at room temperature $(293 \mathrm{~K})$ for different concentrations of $\mathrm{KCl}$ (see legend) calculated with Eq. 4.6.

Figure 4.30 shows the DLVO potential between two negatively charged polystyrene spheres with a radius of $100 \mathrm{~nm}$ in a solution containing different concentrations of $\mathrm{KCl}$. The charge number $Z$ was derived from the zeta potential $(-30 \mathrm{mV})$ as explained in Sec. 3.3. The Hamaker constant of polystyrene in water is $1.3 \times 10^{-20} \mathrm{~J}$ 
[197]. The global minimum of the potential for all concentrations is at $r=0$, i.e. the state of aggregation, so that the suspended particles are, like any colloidal solution, thermodynamically unstable. However, as the system has to overcome an energy barrier with a transition rate proportional to the corresponding Boltzmann factor, a colloidal solution can be stable, or correctly metastable, for a very long time. A solution is considered metastable if the potential barrier is higher than $\sim 15-20$ $k_{B} T$ [124]. For lower values, the aggregation of particles will occur rather quickly.

This is in qualitative agreement with the observations made in the qNano with polystyrene beads (radius $110 \mathrm{~nm}$, "Dragon-Green" fluorescent dye) suspended in water with increasing concentrations of $\mathrm{KCl}$. The resistive pulse frequency decreases with increasing ion concentration and pore blockage frequently occurs at $200 \mathrm{mM}$. No events are observed at $500 \mathrm{mM} \mathrm{KCl}$, indicating that the particles are fully aggregated. Diffusion-based experiments with the same solutions in the LabRAM show the same trend. While many, short-pulsed events are observed at low ion concentrations, isolated, long events are dominant at high $\mathrm{KCl}$ content. The energy barrier at $100 \mathrm{mM}$, the default concentration of the standard buffer used for TRPS, is $\sim 15$ $k_{B} T$, just within the definition of a metastable system. An increase of $10 \%$ of ions reduces the barrier to $9 k_{B} T$, indicating that the transition from a metastable to an unstable colloidal system can occur within a small range in the ion concentration. Due to this and possible variations in the charge density of the beads used and the $\mathrm{pH}$ of the solution, the $\mathrm{KCl}$ concentration was reduced to $50 \mathrm{mM}$ for the particles with diameter $\sim 100 \mathrm{~nm}$ in order to assure that single particles are predominantly in the solution. The energy barrier increases with the particle size and a $100 \mathrm{mM}$ $\mathrm{KCl}$ concentration can be used for all the larger particles used in the experiments. Another reason for irreversible aggregation of polystyrene particles is freezing, which can occur during transport or storing. This may have happened to several purchased particle batches, which, as a consequence, became unusable.

In summary, before conducting TRPS or coordination experiments, consideration towards the particle stability has to be made. The ion concentration may have to be adjusted to keep the colloids dispersed in the solution, especially for small and weakly charged particles. Although in this section only qualitative results were reported, the qNano could be used to study the stability of colloidal solutions, e.g. the aggregation dynamics over time and its relationship with the ion concentration. The device would be very well suited for this application due to the particle-byparticle detection and the adjustable detection range to account for the formation of aggregates. 


\subsubsection{Optical Properties of the Samples}

The spheres used in the experiments are made of polystyrene and have a fluorescent dye embedded in their matrix. Polystyrene itself has no absorption bands in the visible region and appears therefore transparent. Its refractive index of 1.60 is significantly higher than that of the surrounding aqueous medium (refractive index of 1.33), resulting in significant scattering of the incident light. As the particle dimensions are comparable to the wavelength of visible light the scattering is roughly wavelength independent. The scattering cross section, its angular dependency and the resulting force on the particles can be obtained from Mie-theory (see Sec. 3.3).

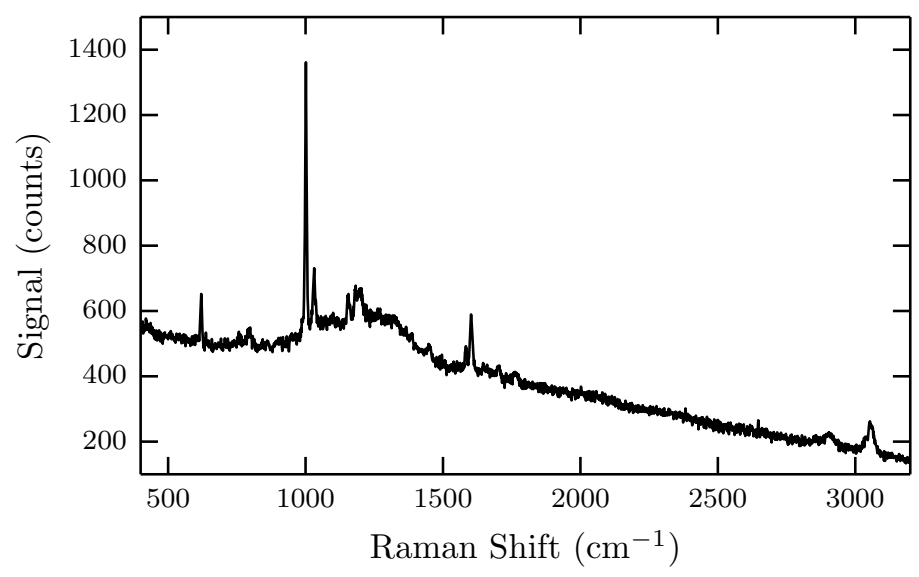

Figure 4.31: The Raman spectra of a polystyrene bead ( $2 \mu \mathrm{m}$ diameter, no fluorescent dye, purchased from Polysciences) immobilised on a glass slide using a $10 \mathrm{~s}$ integration time and the He-Ne laser with a $D 1$ filter.

Polystyrene has Raman active modes and shows a distinct spectrum (Fig. 4.31). However, the signal is relatively weak and, in the case of the sample beads, is dominated by the signal of the fluorescent dyes embedded in the bead, so that the Raman peaks are not observable in the coordination experiments. The maxima of the absorption and emission spectra of the various dyes are all in the visible region and are provided by the suppliers. In case of the particles purchased from Bangs Lab. also the shape of the spectra are published [198] (see Fig. 4.32 (a) for the Dragon-Green dye). However, as a slight change of the spectra is expected for dyes embedded in the polystyrene matrix and also no information about the absorption or fluorescence cross sections per bead is given by the suppliers, additional experiments are required. A quantification is also necessary in order to estimate the sensitivity of the developed coordination technique. The measurements are demonstrated here for several fluorescently-labelled polystyrene beads but can be employed in the same way for a general sample whose absorption and emission spectra may not be known. In such cases the absorption and emission profile should be determined prior to the 
conduction of coordination experiments in order to select the appropriate parameters for the setup, e.g. the appropriate laser, grating etc.

The Absorption Cross Section per Particle Making use of the Lambert-Beer law, the UV-Vis spectrometer is a widely used tool to measure the absorption of an analyte in solution. It is most commonly used in the transmission setup, where it actually measures the extinction, the sum of absorbed and scattered light, of a sample. While for molecular samples scattering is negligible compared to absorption, it can be highly dominant when the solution contains larger particles with significant scattering cross sections. In such a case, calculating the absorption coefficient from the transmitted light will lead to erroneous results. To overcome this problem and directly measure the absorbance of a turbid solution, which is often encountered in biological samples, a setup where the sample is located inside an integrating sphere can be employed [199]. Hereby, the influence of scattering is eliminated as the scattered light is diffusely reflected by the surface of the sphere, so that the absorption instead of the extinction is measured. The specific setup used was originally developed to measure the absorption of molecules attached to silver nanoparticles and has been described in detail previously [195]. With the detected spectral intensities $I_{\text {sample }}(\lambda)$ and $I_{\text {ref }}(\lambda)$ of the solutions with and without (here pure water) particles present, the absorbance $A$ of the sample is given by the expression

$$
A(\lambda)=-\log \left(\frac{I_{\text {sample }}(\lambda)}{I_{\text {ref }}(\lambda)}\right)=\epsilon(\lambda) c_{m} L_{\text {eff }}
$$

Therefore the decadic molar absorption coefficient $\epsilon\left[\mathrm{cm}^{-1} \mathrm{M}^{-1}\right]$ can be derived from the absorbance, the molar concentration $c_{m}$ of the analyte and the effective path length $L_{\text {eff }}$ that the light travels through the sample $\left(L_{\text {eff }}=1.8 \mathrm{~cm}\right.$ for the specific setup). $\epsilon$ relates to the absorption cross section $\sigma_{a b s}\left[\mathrm{~cm}^{-2}\right]$ via the relation $\epsilon=$ $N_{A} \sigma_{a b s} / \ln (10)$, with $N_{A}$ Avogadro's number, so that the absorption cross section becomes

$$
\sigma_{a b}=3.82 \times 10^{-21} \frac{A}{c_{m} L_{e f f}}
$$

The absorbance of an aqueous solution with "Dragon-Green"-labelled beads is shown in Fig. 4.32 (b). The unstable curve below $450 \mathrm{~nm}$ is due to the low intensity of the lamp used and therefore there is a high measurement uncertainty in this spectral region. The peak in the absorption agrees very well with the value given by the supplier and indicates that both the $458 \mathrm{~nm}$ and the $514 \mathrm{~nm}$ line of the argon ion 


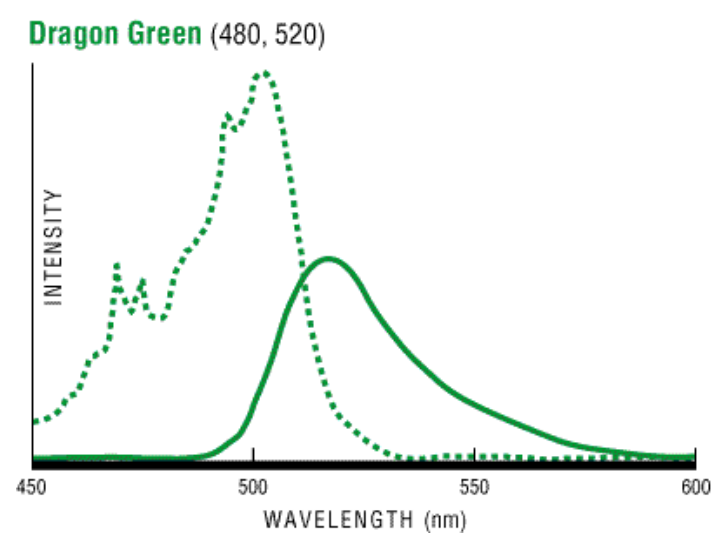

(a)

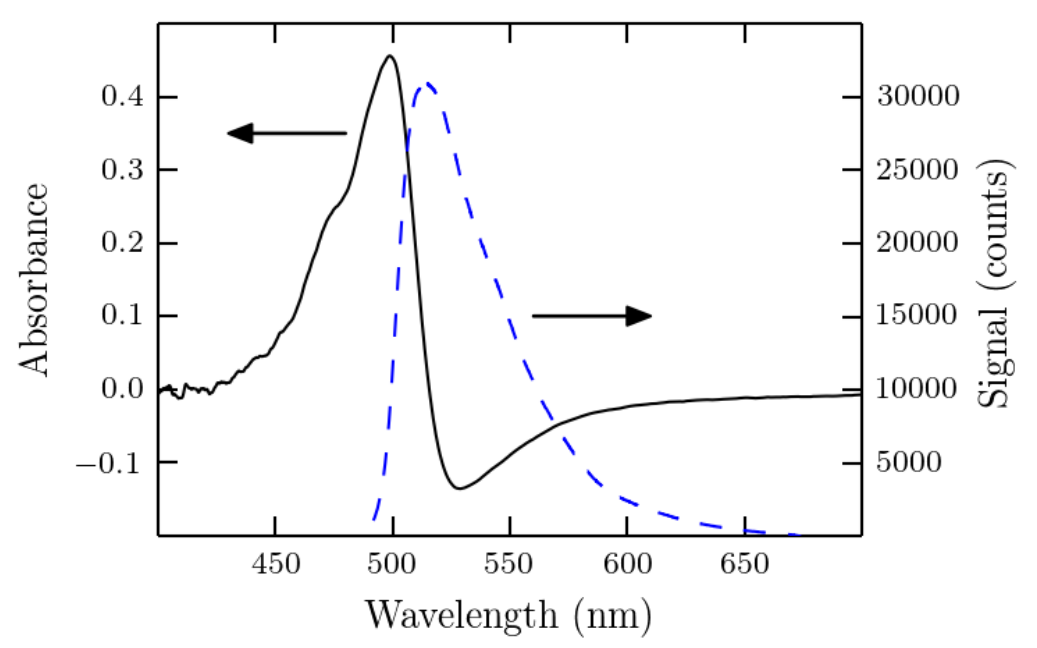

(b)

Figure 4.32: (a) The absorption $(\cdots)$ and emission $(-)$ spectra for the "DragonGreen" dye as given by the supplier. (b) The absorbance (-, black) of the fluorescently-labelled particles $\left(220 \mathrm{~nm}\right.$ diameter) in aqueous solution $\left(c_{m}=\right.$ $0.14 \mathrm{nM})$ as measured in an integrating sphere. The - - line shows the fluorescence profile as obtained in the LabRAM.

laser can be used to excite the dye. The negative reading above $515 \mathrm{~nm}$ is due to the fluorescence of the beads. Due to the overlap of absorption and emission profiles the peak in the fluorescence is shifted to a higher wavelength than given by the supplier (Fig. 4.32 (a)). A measurement of the fluorescence profile obtained in the LabRAM and used to quantify the fluorescence cross section of a particle, shows very good agreement with the corresponding spectrum given by the supplier.

With Eq. 4.8 the absorption cross section per bead at a wavelength of $458 \mathrm{~nm}$, which is consequently used in the coordination experiments, was derived to be $1.52 \times 10^{-12} \mathrm{~cm}^{2}$ for a "Dragon-Green"-labelled particle and $2.32 \times 10^{-12} \mathrm{~cm}^{2}$ for a "Surf-Green" bead. An accurate quantification of the cross section at $458 \mathrm{~nm}$ is possible as absorption and emission profile are sufficiently separate, while this is not the case at $514 \mathrm{~nm}$ where cross-talk correction has to be considered. The concentra- 
tion $c_{m}$ was derived from the original concentration given by the supplier and the dilution. The uncertainty of the measurement is mainly given by this dilution step and is estimated to be $\sim 10 \%$. For an unknown sample, the concentration could also be obtained using the qNano.

Apart from enabling the experimentalist to choose the right parameter for the following coordination experiments, e.g. the laser line, the quantification of the absorption cross section can also be used to roughly estimate the amount of dyes embedded in one bead. With a typical cross section of the order of $1 \times 10^{-16} \mathrm{~cm}^{2}$ per dye $\sim 10000$ dye molecules are present per bead with a $200 \mathrm{~nm}$ diameter. The amount of dye and therefore the optical signal is expected to scale with the volume of the bead, meaning that a $2 \mu \mathrm{m}$ bead will exhibit a 1000 times stronger signal.

The Fluorescence Cross Section per Particle The absolute fluorescent cross section per particle is of interest in order to predict the detection limit of the technique and to be able to model the optical signals. Using filters and cross-talk correction algorithms, the total fluorescence cross section could potentially be derived from UV-Vis measurements using an integrating sphere as shown above (see Fig. $4.32(\mathrm{~b})$ ). However, here a different approach was chosen. The fluorescence profile was measured using the LabRAM and compared to the signal of a dye with known cross section $d \sigma_{r e f} / d \Omega$. The total fluorescence cross section of the sample $\sigma_{\text {sample }}$ can then be derived from the number of counts, integrated over the entire spectral range, of the sample spectrum counts sample, the counts of the reference counts $_{\text {ref }}$, the respective laser intensities $P_{\text {sample }}$ and $P_{r e f}$, and the concentrations of the sample $c_{\text {sample }}$ and the reference $c_{r e f}$ with the relation [190]

$$
\sigma_{\text {sample }}=4 \pi \frac{d \sigma_{\text {ref }}}{d \Omega} \frac{\text { counts } s_{\text {sample }}}{\text { counts }_{\text {ref }}} \frac{P_{\text {ref }}}{P_{\text {sample }}} \frac{c_{\text {ref }}}{c_{\text {sample }}} .
$$

It has to be ensured that the same parameters, i.e. objective, pinhole size, etc. are used for both measurements. The factor $4 \pi$ in the above equation is justified by the random orientation of the beads and the dyes within so that the polarization ratio $\rho=I_{\perp} / I_{\|}$is equal to 1 and the general expression for the fluorescence cross section $\sigma=\frac{8 \pi}{3} \frac{1+2 \rho}{1+\rho} \frac{d \sigma}{d \Omega}$ becomes $4 \pi \frac{d \sigma}{d \Omega}[124]$.

As an example the quantification of the fluorescence cross section per bead of $2 \mu \mathrm{m}$ polystyrene particles labelled with "Yellow-Green" is demonstrated. The spectrum of an aqueous solution of the beads is shown in Fig. 4.33 along with the spectrum of 2-Bromo-2-methylpropane (2B2MP), which was chosen as a reference standard. The cross section of the $516 \mathrm{~cm}^{-1}$ Raman mode of $2 \mathrm{~B} 2 \mathrm{MP}$ is $2.05 \times 10^{-29} \mathrm{~cm}^{-2} \mathrm{sr}^{-1}$ at an excitation wavelength of $458 \mathrm{~nm}$ [193]. Compared to 


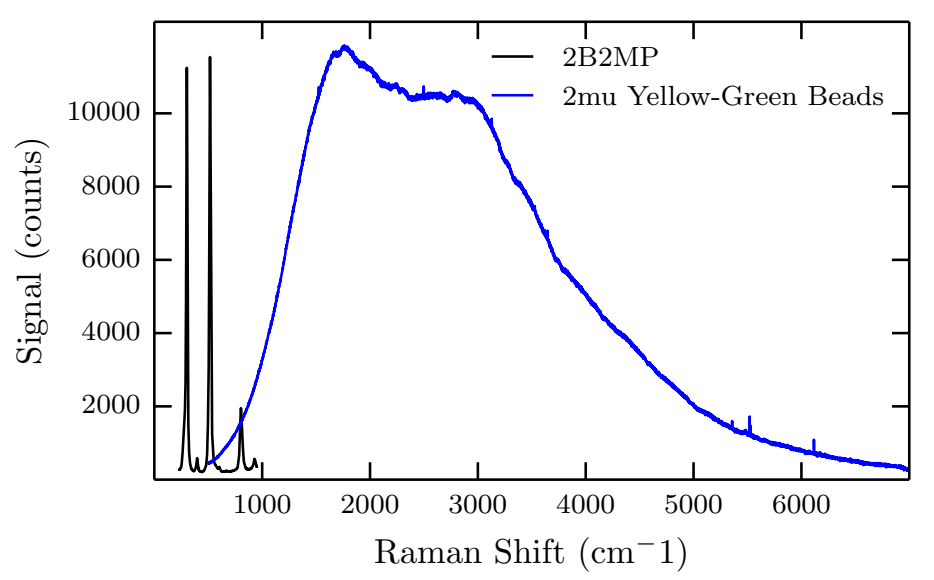

Figure 4.33: The spectra of 2B2MP (black) $\left(c_{r e f}=8.76 \mathrm{M}\right)$ and "Yellow-Green" fluorescently-labelled polystyrene beads (blue) $\left(c_{\text {sample }}=2.21 \times 10^{-4} \mathrm{nM}\right.$ in aqueous solution). The $458 \mathrm{~nm}$ line of an Argon-ion laser at full power and D2, a $\times 20$ water immersion objective and a 1800 grooves/mm grating were used.

the width of a Raman mode, the fluorescence profile is much broader so that the full spectrum has to be joined together from separate windows in a post-processing step. When calculating the overall intensity of the fluorescence peak it has to be assured that the intensities are renormalised according to the non-uniform step size of the wavenumbers.

To allow for an accurate measurement, the average number of beads in the scattering volume has to be sufficiently high, otherwise the signals will fluctuate heavily. Especially for particles in the um-scale, this implies using concentrations where the solution is visibly turbid. Both the incoming laser beam and the signal originating from the scattering volume will therefore be attenuated due to extinction following Lambert-Beer's law. The signal does not increase linearly with the bead concentration and uncorrected use of the integrated fluorescence intensity will lead to erroneous results in determining $\sigma_{\text {sample }}$. To account for this effect, the fluorescence intensity has to be measured at several concentrations $c_{\text {sample }}$ and fitted to the relation

$$
I\left(c_{\text {sample }}\right)=A c_{\text {sample }} 10^{-\kappa c_{\text {sample }}}=A c_{\text {sample }} 10^{-\epsilon 2 L_{w d} c_{\text {sample }}},
$$

using the parameters $A$ and $\kappa$. Here, $A$ is a scaling constant, and $\kappa$ the attenuation coefficient. With the knowledge of the distance $L_{w d}$ that the light travels in the solution (for immersion objectives it is the distance from the objective surface to the focus, or working distance), $\kappa$ can be translated into the decadic molar extinction coefficient $\epsilon$. The parameter $A$ is used to calculate the correction factor $x_{\text {corr }}\left(c_{\text {sample }}\right)=A c_{\text {sample }} / I\left(c_{\text {sample }}\right)$, which the detected fluorescence intensities have 
to be multiplied by to account for the extinction.

Figure 4.34 shows the fluorescence intensities for $2 \mu \mathrm{m}$ polystyrene particles labelled with "Yellow-Green" dyes. The fitted curve using Eq. 4.10 increases linearly for small concentrations, reaches a maximum for a concentration $c_{\text {sample } \max }=\frac{1}{\ln (10) \kappa}$, and then decreases due to the rising extinction of the turbid solution. As the measurement uncertainty varies largely with the particle concentration, the fit is weighted by the standard error of the mean. For this specific experiment $A=$ $8.47 \times 10^{8} \mathrm{nM}^{-1}$, which results in a correction factor $x_{c o r r}=56.71$ for the highest used concentration $\left(2.21 \times 10^{-4} \mathrm{nM}, 1 / 50\right.$ dilution $)$. For very low concentrations the factor will reach $\sim 1$ so that no correction step is necessary. However, the corresponding measurement uncertainty is very high due to large fluctuations of the number of particles in the scattering volume. The cross section $\sigma_{\text {sample }}$ using Eq. 4.9 and the correction factor is $3.26 \times 10^{-9} \mathrm{~cm}^{2}$ per bead.

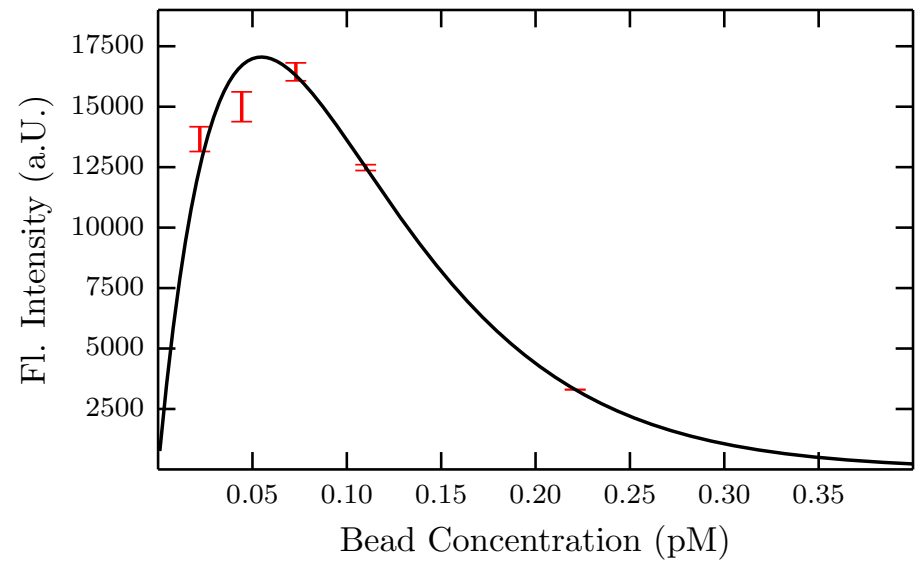

Figure 4.34: The total fluorescence intensity and the measurement uncertainty for five concentrations of $2 \mu \mathrm{m}$ polystyrene particles labelled with "Yellow-Green" dyes. Each datapoint is the average of 100 acquisitions with an integration time of $1 \mathrm{~s}$. The solid line is the curve fitted to the model described by Eq. 4.10.

With $\kappa=7.94 \times 10^{3} \mathrm{nM}^{-1}$ and the objective working distance $L_{w d}$, the decadic molar extinction coefficient can be calculated. It can subsequently be used to determine the amount of signal that will be lost due to extinction. Here, for the corresponding pore rating (NP2000), a dilution of the original particle solution of $\sim$ 1000 is necessary to reach the recommended concentration of $1.1 \times 10^{-5} \mathrm{nM}$. With a $\times 20\left(L_{w d}=3.3 \mathrm{~mm}\right)$ water immersion objective the transmission is then $82 \%$, while for a $\times 100$ objective $(1.5 \mathrm{~mm})$ it is $91 \%$. This shows that, although the loss of signal is small, it is relevant if the exact signal strength is of interest.

The fluorescence quantum yield $Q$, i.e. how many absorbed photons are converted into fluorescence, is defined by the ratio 


$$
Q=\frac{\sigma_{f l}}{\sigma_{a b}}
$$

The values for several particles are summarized in Tab. 4.7, showing that while the "Surf-Green" dye has a higher absorption cross section, its quantum yield is significantly lower, resulting in a lower fluorescence cross section compared to the "Dragon-Green" labelled bead.

Table 4.7: The absorption cross section $\sigma_{a b s}$, fluorescence cross section $\sigma_{f l}$ and the quantum yield per bead for various fluorescently-labelled particles obtained by the methods described above.

\begin{tabular}{ccccc}
\hline Supplier & Dye & $\begin{array}{c}\sigma_{a b s} \\
\left(10^{-12} \mathrm{~cm}^{-2}\right)\end{array}$ & $\begin{array}{c}\sigma_{f l} \\
\left(10^{-12} \mathrm{~cm}^{-2}\right)\end{array}$ & $\mathrm{Q}$ \\
\hline Bangs Lab. & Surf-Green & 2.32 & 0.581 & $25 \%$ \\
Bangs Lab. & $\begin{array}{c}\text { Dragon- } \\
\text { Green }\end{array}$ & 1.52 & 1.21 & $80 \%$ \\
Sigma Ald. & Yellow-Green & - & 3260 & - \\
\hline
\end{tabular}

Photo-bleaching Photo-bleaching is important if the temporal dynamics are captured in an experiment. Most dyes exhibit photo-bleaching, which involves an often irreversible, so-called inter-system crossing into a triplet state [200] and therefore blocks the usual radiative pathway, i.e. the molecules stops fluorescing. For an ensemble of molecules the emission intensity $I(t)$ will decay in time with the photobleaching rate $\Gamma_{B}$ following the relation

$$
I(t)=I(t=0) \exp ^{-\Gamma_{B} t}
$$

In the absence of saturation, which only plays a role at much higher laser intensities than used in this work [124], $\Gamma_{B}$ increases linearly with the power of the illumination. It is therefore relevant to the coordination technique as the sample's fluorescence could be reduced significantly if the laser power is too high.

$\Gamma_{B}$ can be quantified by measuring the autocorrelation function of the fluorescence intensity (Fluorescence Correlation Spectroscopy (FCS)). The analysis is complex as particles which have been bleached are replenished by new beads that diffuse into the scattering volume. However, an exact quantification is not necessary here and for particles in the order of several hundred nm and larger, which have relatively slow diffusion velocities, a simple test can show if photo-bleaching is relevant for a 


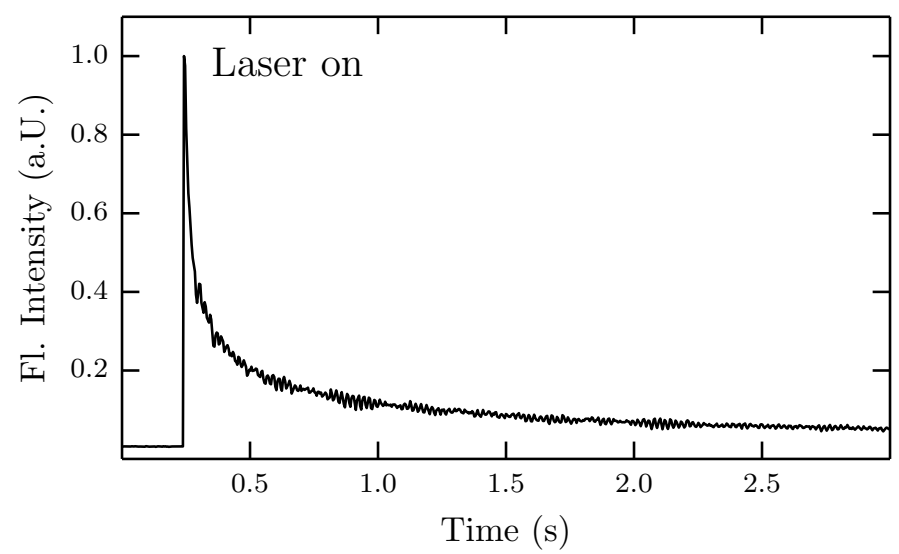

(a)

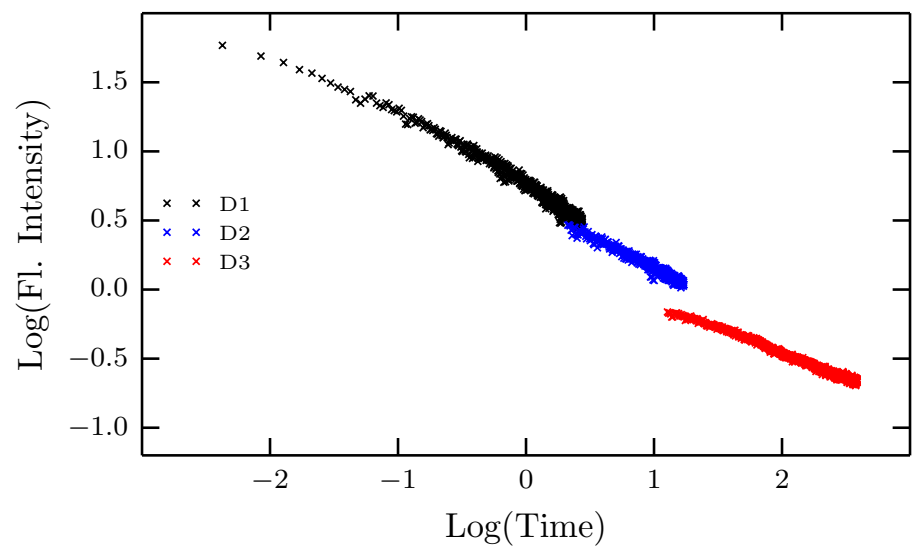

(b)

Figure 4.35: (a) The fluorescence decay of a single $2 \mu \mathrm{m}$ "Yellow-Green"-labelled bead immobilized on silicon and excited with a laser $(458 \mathrm{~nm}$ line, beam waist of $1.50 \mu \mathrm{m}$ ) with $0.47 \mathrm{~mW}(D 1)$ total power, corresponding to a maximal power density of $1.33 \times 10^{4} \mathrm{~W} \mathrm{~cm}^{-2}$ (b) The fluorescence intensity over time for three choices (see label) of neutral density filters in a log-log plot. Intensities and times are renormalized according to the filter transmission.

certain choice of spot size and laser intensity. In a time-dependent measurement, if the fluorescence intensity is approximately stable after switching the laser on it can be assumed that the particles do not bleach significantly. It has to be ensured that the average number of particles in the scattering volume is sufficient so that fluctuations are not relevant.

For particles that are large enough to observe with the microscope and settle due to gravity in a reasonable time, the fluorescence decay can be measured directly by focussing on a single bead. Figure 4.35 shows such a measurement for $1 \mu \mathrm{m}$ beads immobilised on silicon. From (a) a value of $\Gamma_{B}^{-1} \sim 0.5 \mathrm{~s}$ can be extracted for a power density of $1.33 \times 10^{4} \mathrm{~W} \mathrm{~cm}^{-2}$. Using various neutral density filters the study can be extended to intensity levels over several orders of magnitude. The data segments shown in Fig. 4.35 (b) should join together smoothly and have the same slope in the 
chosen presentation (log-log plot), determined by the decay rate. Here, mainly due to variations in the lateral and axial focussing, this is not exactly the case. However, it can be concluded that in a typical coordination experiments, where the maximal duration a particle spends in the region close to objective focus is in the order of tens of ms, photo-bleaching will not play a role when a neutral density filter $D 2$ or higher is used. For smaller particles, where higher laser powers densities are used, bleaching could occur. In this case the usually desirable uniform illumination with a low beam divergence might be disadvantageous, as particles can then be bleached before entering the scattering volume.

\subsection{Modelling of the Optical Signals}

The modelling of the transient current pulses caused by insulating spherical particles traversing a conical pore has been studied in detail in Chap. 3. The aim of this section is to conduct an analogous study of the time dependent optical signals. This can help to design the experimental setup as the success of the coordination technique depends on many parameters, e.g. the choice of the objective, laser spot, pinhole size, applied pressure, voltage, etc.

The method of the model is developed in the following, starting with the excitation profile $\operatorname{Exc}(z, r)$ of the laser beam. $\operatorname{Exc}(z, r)$ is determined by the beam parameters (Tab. 4.2), additional lenses that are used to shape the beam and the focal length of the objective (refer to Chap. 5 for details). It is given by

$$
\operatorname{Exc}(z, r)=\frac{2 P \times 10^{-D}}{\pi w^{2}(z)} \exp \frac{-2 r^{2}}{w^{2}(z)}
$$

with $P$ the total power of the laser (measured after the objective), $D$ the attenuation of the neutral density filter used, and $w(z)$ the width of the laser beam determined by Eq. 5.1. The sample's cross section, fluorescent or Raman in nature, then determines the response to the excitation. The photons, emitted in all spatial directions, are only partly collected by the objective. This is taken into account by the solid angle for the detection $\Delta \Omega_{\text {det }}=2 \pi\left(1-\cos \theta_{\text {det }}\right)$, which can be calculated from the numerical aperture $N A$ of the objective and the refractive index of the medium $n_{m}$ via the relation $N A=n_{m} \sin \theta_{\text {det }}$. Additionally, the detection efficiency profile $\operatorname{Det}(x, y, z)$, which is dependent on the choice of objective and pinhole size, as discussed in Sec. 4.2.3, has to be considered. $\operatorname{Det}(x, y, z)$ is 1 at the centre of the objective focus and decreases in a Lorentzian shaped manner in the axial direction. The detection profile in the lateral direction can be modelled by a step function given by Eq. 4.2. The overall detection efficiency profile is then given by 


$$
\operatorname{Det}(x, y, z)=\eta(x, y, z) \times \frac{\gamma_{\text {det }}^{2} / 4}{z^{2}+\gamma_{\text {det }}^{2} / 4}
$$

with $\gamma$ the full width at half maximum (FWHM) of the profile that has to be determined experimentally for the specific choice of objective and pinhole size (see Sec. 4.2.3). The total detection efficiency, which originates from losses in the detection path, is taken into account by the factor $\eta_{\text {total }}$ (Sec. 4.2.4). The incident power on the CCD can be converted into a number of photons, which is then translated into the total counts per second, with the knowledge of the electronic gain $g$. Multiplying this number with the integration time $\tau$ yields the total counts $S_{\text {opt }}(t)$ registered by the CCD and is given by

$$
S_{\text {opt }}(t)=\operatorname{Exc}\left(z(t),(r(t)) \times \sigma_{f l} \frac{\Omega}{4 \pi} \times \operatorname{Det}(x(t), y(t), z(t)) \times \eta_{\text {total }} \frac{\lambda_{\text {laser }}}{h c} g \Delta \tau\right.
$$

Here, a uniform angular distribution of the emitted radiation is implicitly assumed and polarization effects are neglected. Additionally, photo-bleaching or saturation are not expected to play a role, which is justified at a sufficiently low laser power. The total number of counts calculated by $S_{\text {opt }}(t)$ is spread over a certain area on the CCD, and depends on the nature of the emission. While a fluorescence peak, depending on the used grating, can cover the entire chip, a Raman line is typically only a few pixels broad. The signal is dependent on the particle position $(x(t), y(t), z(t))$, which can be obtained by the transport models discussed in Chap. 3. As even for the highest time resolution of the CCD the position during the integration time changes significantly, the multiplication with $\tau$ in Eq. 4.15 is actually implemented in the model as a numerical integration using Simpson's formula.

The calculated current and optical signals from two typical coordination experiments are shown in Fig. 4.36. While (a) represents an experiment at the largest size range used in this work, (b) is at the smaller range of samples. The semi-analytic model was used to determine the particle trajectories. As the particle is assumed to be condensed to a point at the pore centre and is therefore excited with the maximal laser power, the signals will generally exhibit slightly smaller values in reality. Assuming a triangle shape of the fluorescence and a width of the profile that covers the whole 1024 lateral pixels of the CCD, the maximum number of counts in the recorded spectrum (two times the integrated intensity divided by 1024) will be several thousand for the large particles and several hundred for the small particles shown in Figs. 4.36 (a) and (b) (the figures show the integrated intensities). The signals originating from particles going through the pore on an off-axis trajectory will 


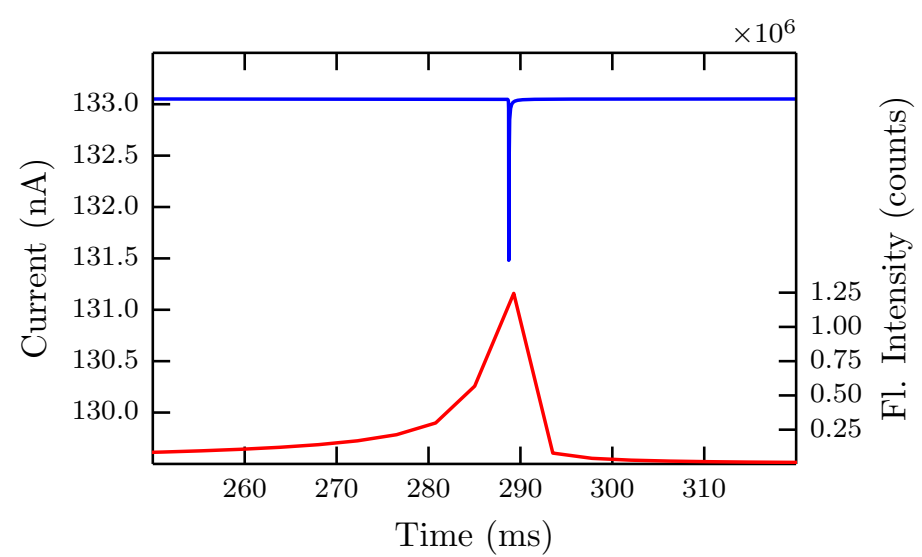

(a)

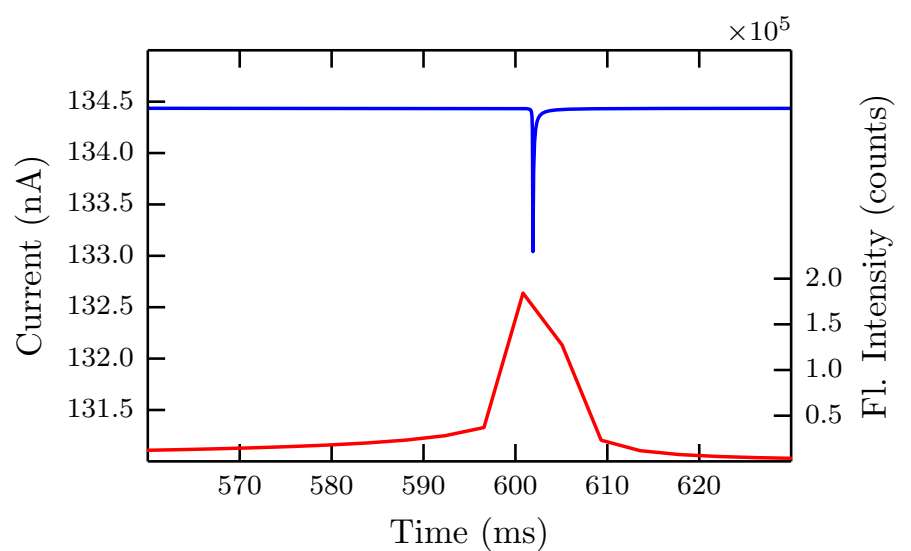

(b)

Figure 4.36: (a) The calculated current and optical signals of a $2 \mu \mathrm{m}$ fluorescentlylabelled ("Yellow-Green") polystyrene bead traversing a NP2000 pore on-axis (small pore radius of $2.5 \mu \mathrm{m}$ ) at an applied voltage of $0.1 \mathrm{~V}$ and a hydrostatic pressure of $100 \mathrm{~mm} \mathrm{H} \mathrm{H}_{2} \mathrm{O}$. The $458 \mathrm{~nm}$ line of an Argon-ion laser $(0.047 \mathrm{~mW}$ total power at the sample) is used to excite the bead. The beam is focussed onto the pore orifice using a $\times 100$ objective and additional lenses, resulting in a beam waist of $5.4 \mu \mathrm{m}$. (b) Simulation of the signals of a $220 \mathrm{~nm}$, fluorescent ("Dragon-Green") bead passing through a NP200 pore on-axis with an applied voltage of $1 \mathrm{~V}$ and a hydrostatic pressure of $10 \mathrm{~mm} \mathrm{H}_{2} \mathrm{O}$ using a $\times 100$ objective (beam waist $0.38 \mu \mathrm{m}$ ). The trajectories for both (a) and (b) were obtained using the semi-analytic model.

be smaller, determined by the profile of the laser beam. If the beam is sufficiently large, the pinhole will influence the signal by cutting out any signal originating from parts of the trajectory outside the lateral detection. The comparison of signals from on- and off-axis particles using the FEM point particle model is demonstrated on the basis of an actual coordination experiment in Sec. 7.2, and shows that the predicted intensities are in good agreement with the ones experimentally measured.

It is obvious from Fig. 4.36 that the optical signals are significantly longer in duration that the electric signals. This is because the "sensing zone" of the optical detection, the convolution of the excitation and detection profiles, is extended to a 
larger volume compared to TRPS, where the pulses are generated within a few $\mu \mathrm{m}$ around the pore orifice. This explains the occurrence of, "multi events", where two or more resistive pulses only have one optical counterpart.

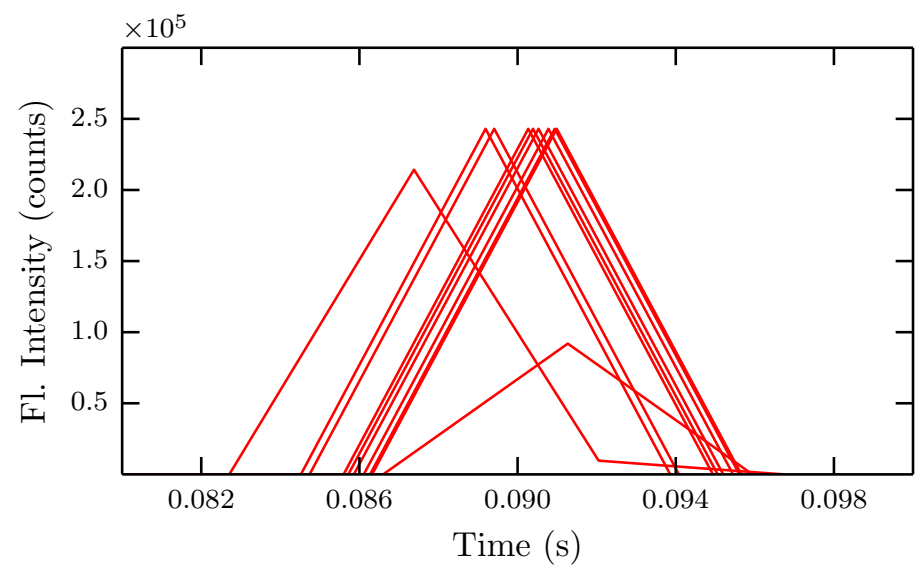

(a)

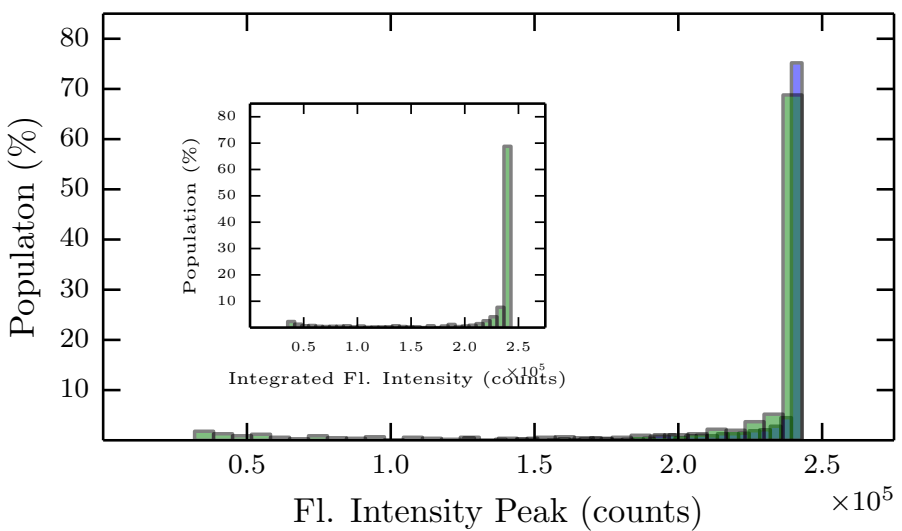

(b)

Figure 4.37: The optical signal of a $2 \mu \mathrm{m}$ fluorescently-labelled particle approaching the pore at the edge (the trajectory was derived using the FEM point particle model) and otherwise identical parameters as in Fig. 4.36 (a) for 10 different start times (1 ms apart) of the signal allocation. (b) shows the resulting peak distribution of 1000 such events and randomly generated start times with (green) and without (blue) the consideration of CCD "dark" times. The inset shows the distribution of the signals, which were integrated over their pulse duration.

The fact that the peaks are not exactly matching is due to the relatively low time resolution of the optical measurement. The particle position at the start of a time frame during which the signal is allocated will influence the height of the signal peak. This has been implemented in the simulation and is shown in Fig. 4.37. A variation in the integration start times shows that the signals vary in height for identical experimental parameters. However, as the signal is continuously integrated, the area under the peaks is identical. This changes when the "dark" time of the CCD, the time between measurements when the CCD is unresponsive to incoming photons, is taken into account. The signal will become partly undetected or potentially, for fast 
moving particles, fully undetected by the CCD. However, even with the conservative assumption of a "dark" time of $10 \%$ of the integration time $(4.25 \mathrm{~ms})$ in combination with a particle moving at an upper speed typical for TRPS and with the time for the signal allocation being further reduced to a small fraction of its trajectory due to a small pinhole size, the distribution of the simulated signals shows only a slight trend toward a less intense signal. The effect can therefore be excluded under the experimental conditions used.

The simulation is helpful for choosing the most suitable parameters for a specific coordination experiment. For example the use of an objective with lower focal length in (a) would result in a significantly longer pulse, increasing the likelihood of "multi events". The simulation can be used to predict the theoretical detection limit of the setup, which is determined by the noise of the CCD. Also the potential for applying the technique to the inherently much weaker Raman spectroscopy and its derivative SERS can be estimated. Using the semi-analytic model for the particle transport and the optical model presented in this section the signal caused by Rhodamin6G molecules, each with a resonant cross section of the $1510 \mathrm{~cm}^{-1}$-mode of $149 \times 10^{-26} \mathrm{~cm}^{2} \mathrm{sr}^{-1}$ [201] at an excitation of $532 \mathrm{~nm}$ ), is predicted to be measurable if 1000 molecules are attached to the surface of a dimer of two silver colloids (diameter of $60 \mu \mathrm{m}$ each), which provides an average enhancement factor of $10^{6}$ [124]. The model predicts a signal of 150 counts for the peak of the $1510 \mathrm{~cm}^{-1}$-mode (the peak width on the CCD using a grating with 1800 grooves $/ \mathrm{mm}$ is $\sim 20$ pixels) if the following setup is used:

- The $532 \mathrm{~nm}$ line of the Ar-ion laser and a neutral density filter D1 with a total of power of $0.2 \mathrm{~mW}$ results in a sufficient signal and a laser-induced noise level that is low enough not to disrupt the measurement.

- The $\times 100$ objective in combination with a back focal plane lens with a focal length of $500 \mathrm{~mm}$ (see Chap. 5) is used to achieve a uniform (beam waist of $1.50 \mathrm{\mu m}$ ), slowly divergent beam throughout the optical detection region. The configuration also reduces the power density at the pore orifice in order to further decrease the noise level and formation of air bubbles.

- The translocation of the dimer through a NP100 pore (radius of the small pore orifice is assumed to be $150 \mathrm{~nm}$ ) at an applied voltage of $1.5 \mathrm{~V}$ results in a relative pulse height of $0.12 \%$, which is measurable. To prevent further agglomeration of the dimers the molarity of the electrolyte has to be reduced to $10 \mathrm{mM} \mathrm{KCl}$. This, the small pore size and the high voltage increases the influence of the particle and membrane charge on the resistive pulse, which is not taken into account in the semi-analytic transport model. The particle 
is also assumed to travel through the centre of the pore. Off-axis trajectories result in smaller, but due to the relatively large beam to pore ratio, still measurable signals.

- A pressure of $-20 \mathrm{~mm} \mathrm{H}_{2} \mathrm{O}$ is applied to balance the electrokinetic effects and achieve a slow, downward velocity of the dimer of $5 \mathrm{~mm} \mathrm{~s}^{-1}$ at the pore orifice. The dimer stays therefore longer than one time step of the optical measurement in the detection volume, allowing for the accumulation of the signal.

- A integration time of $20 \mathrm{~ms}$ is used to increase the signal per time step. Additionally a lower time resolution results in a lower readout noise.

- Due to the longer integration time, the FWHM of the optical detection has to be reduced to $5 \mu \mathrm{m}$ in the axial direction to suppress signals from particles travelling close to each other. However, the detection height has to be kept sufficiently large to allowing for a long enough part of the trajectory to contribute to the signal.

The detection would be challenging in many ways and the experiential implementation was not attempted here as several requirements, including the use of the desired laser line and the reduced optical detection in the axial direction (Sec. 4.2.3), cannot be achieved using our setup without additional modifications. However, this example should illustrate how the model can be useful to assist in the design of an experiment and to predict the influence of the various experimental parameters on the signals. The most obvious way to increase signal might be to use a higher laser power, which is problematic as this results in a larger noise, the creation of bubbles and undesired interactions with the pore material and should therefore be avoided. The maximal usable laser power is therefore one of the main limiting factors of the technique.

\subsection{Methods and Algorithms for the Data Anal- ysis}

The data analysis of the TRPS and optical traces is an essential part of the technique. The raw traces have to be separately post-processed, searched for events, and then correlated in time so that the information about the analyte obtained by the two measurements can be combined. Apart from the event search and correlation algorithms, a filtering step and the analysis of the spectral information has been implemented in the programming language Python. 


\subsubsection{Removing the Laser-induced Noise}

Before the current trace can be searched for events, the low frequency noise due to the laser exposure has to be removed. This is only necessary for high laser intensities and the step could be omitted for most data presented in Chap. 7. For the experiments presented in Chap. 6 and the smallest particles in Chap. 7, an impulse response highpass filter and an algorithm using wavelets developed by Galloway et al. [188] were used. For further details of the noise removal step the reader is referred to Sec. 4.1.2. Although the noise is also observable in the optical trace, it is mostly not large enough to necessitate filtering the signal. Additional artefacts of a filtering step are also expected due to the close proximity of the frequency of the optical data acquisition and the noise.

\subsubsection{The Event Finding Algorithms}

From the size and shape of an RPS event a wide range of information about the sample can potentially be extracted. An efficient and fail-safe event finding algorithm is therefore essential and also has a large influence on the interpretation of the acquired data [202]. Custom made algorithms for both the current and optical data were implemented separately.

\subsubsection{Electrical Trace}

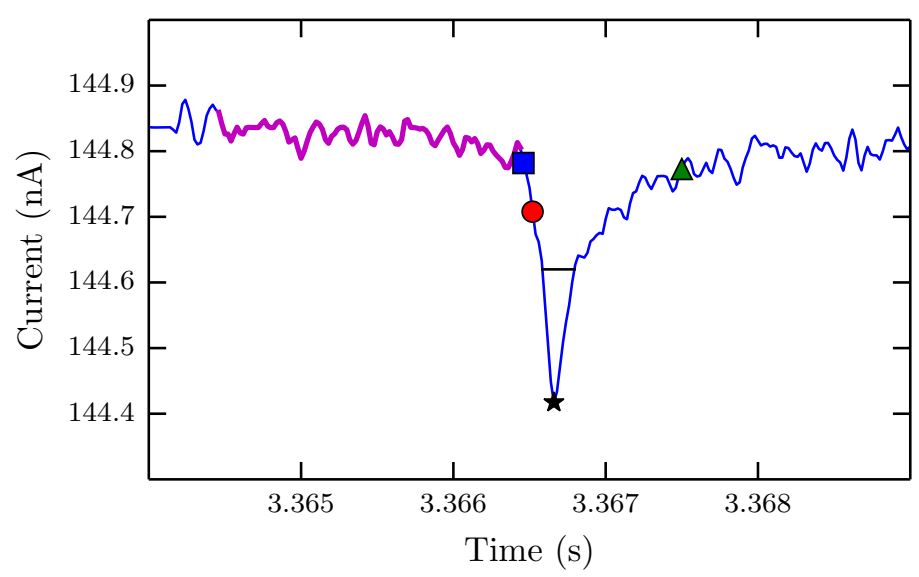

Figure 4.38: A transient current pulse caused by a $0.97 \mu \mathrm{m}$ particle. The markers show the start (blue square), the end (green triangle) and peak (black star) of the pulse. The horizontal line indicates the FWHM of the event. The magenta coloured part of the trace shows the window that is used to calculate the average current. The threshold that triggers the algorithm to recognise an event, is set to $0.1 \mathrm{nA}$, and is represented by the datapoint marked with the red circle.

Although the software of the qNano provides an event finding algorithm, an in- 
house solution was developed to accustom better for the specific purpose and allow for control over the search parameters, e.g. threshold, window size. The implemented algorithm is used in many RPS techniques and has been described by Pedone et al. [203]. A moving window of 100 data points was used to calculate the average current. The pulse threshold is typically set to $0.1 \mathrm{nA}$ below this average. If the current falls below the threshold a translocation is identified. The starting point of the event is found by tracing back to the first datapoint that is within three standard deviations below the average. In this procedure the average is updated so that data points which lie within an event do not contribute to the average. For particles that travel upwards the trace is analysed in reverse order. The slow onset of the resistive pulses would otherwise lead to complications as the average will adjust to the pulse, especially for slow particles. The endpoint of the event is defined by the first datapoint that rises to a value within three standard deviations of the background current. Every event is characterised and identified by its start time, end time, the time at the peak of the pulse, the peak height relative to the average and the FWHM of the event. Figure 4.38 shows an example of a resistive pulse and the characteristic points.

\subsubsection{Optical Trace}

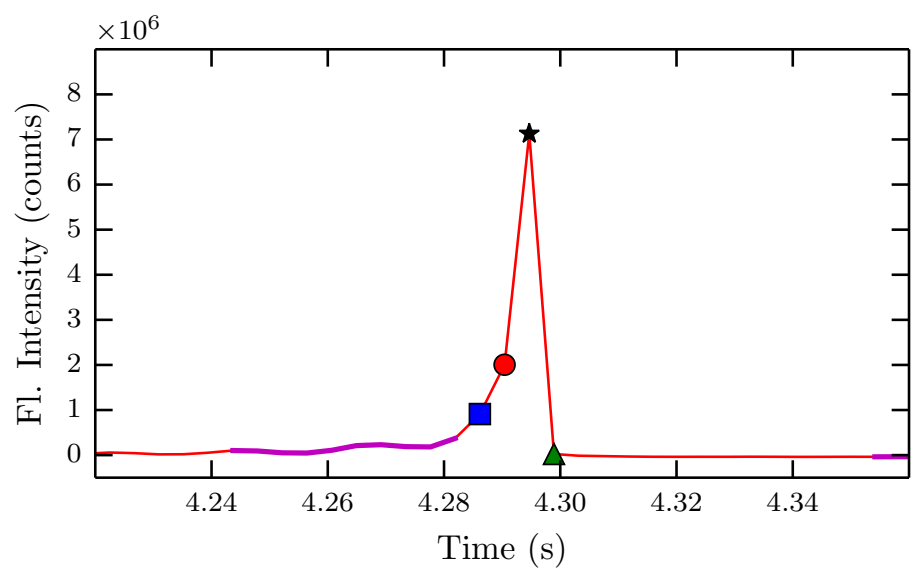

Figure 4.39: A peak in the optical trace caused by a $0.97 \mu \mathrm{m}$ fluorescent particle. The markers show the start (blue square), the end (green triangle) and peak (black star) of the pulse. The magenta coloured part of the trace shows the window that is used to calculate the average current. The threshold that triggers the algorithm to recognise an event is represented by the datapoint marked with the red circle.

The first post-processing step for the optical data is to integrate the measured spectra to obtain the fluorescence intensity as a function of time. This is done by summing up all the counts within the fluorescence peak. Events are then identified and characterised in the same way as with the current data, although the search 
parameters have to be adjusted to account for the low time resolution and the upward spikes. The moving window is 10 data points long and the pulse threshold often has to be adjusted due to varying background intensities. Particles that get attached to the membrane close to the pore orifice are problematic, as they suddenly increase the background intensity. Each identified event is characterised and identified by its start time, end time, the time at the peak, the peak height and the intensity integrated over the event duration. As the peak only consists of a few data points the definition of a FWHM is not reasonable. Figure 4.39 shows the specific points of a typical event.

\subsubsection{Aligning the Measurements}

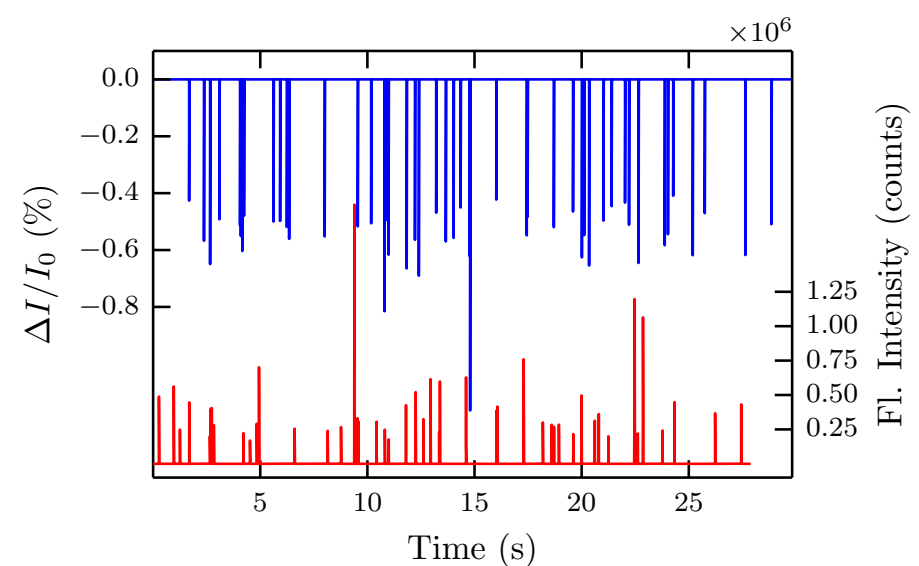

(a)

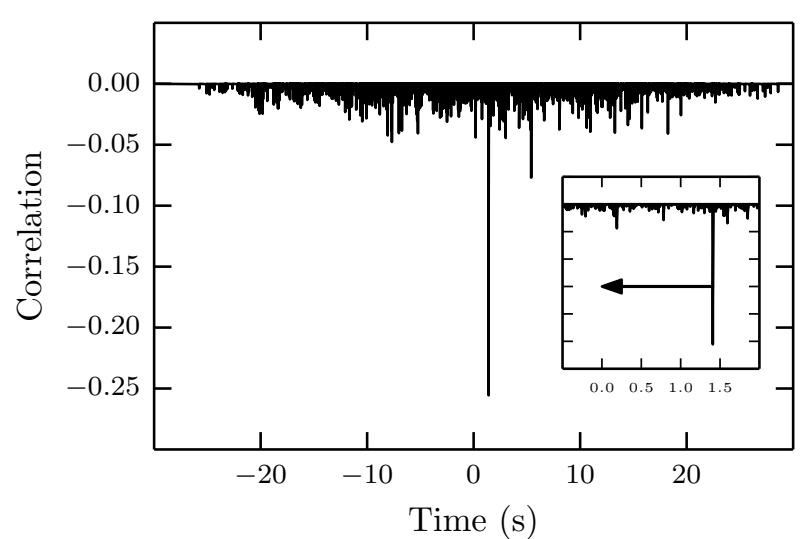

(b)

Figure 4.40: The electrical and optical traces of a coordination experiment using $2 \mu \mathrm{m}$ "Yellow-Green" beads. The values between events were set to zero in both traces. The corresponding correlation function is shown in (b) and indicates that the traces have to be shifted by $1.41 \mathrm{~s}$ relative to each other, which can be seen in the magnified inset.

As the electrical and optical measurements are recorded separately, a method to align the traces has to be found. Several methods were tested and can be employed. 
First, the traces were searched by hand for two pairs of corresponding events, reasonably spaced apart from each other, and their times were noted. The time shift, and also the time dilation factor with which the optical trace is consequently multiplied, can then be calculated. This method has the advantage that the dilation factor, discussed in Sec. 4.2.5, does not have to be explicitly known to correlate the traces. However, the method is time-consuming and is therefore not suitable for routine use. Alternatively, in case the laser induced noise is visible upon laser illumination, "marker events" can be introduced by switching the laser on close to the beginning and off at the end of each measurement. The identification of the on/off times can be automated and the method has the advantage that additional information about the actual time delay between optical and electrical pulses can be obtained. However it decreases the usable part of each trace.

A third method utilizing the correlation function between the traces was routinely used for aligning the traces. Using the Python function "conv", the correlation function is calculated between the electrical and the optical traces, which is multiplied beforehand with the corresponding dilation factor $r$ (see Sec. 4.2.5). The time shift can be deduced from the minimum of this function (see Fig. 4.40 (b)). As the noise between detected events makes the location of the minimum more difficult, the correlation function is calculated after the events were identified in each trace individually and the signals outside the event durations were set to zero. Figures 4.41 (a) and (b) show modified traces and the resulting correlation function. The approach could also be used to obtain both the time shift and the dilation factor if a fitting routine is implemented that maximises the correlation function for a variation of the dilation factor.

\subsubsection{Finding the Matching Events}

Another algorithm searches for matching events by checking for each current event if its peak time is between the start and the end of any optical event. If a corresponding optical event is found the information of both measurements is combined and the search is carried out for the next current event. Due to the relatively low optical time resolution it can occur that two or more current events have the same corresponding optical events. These events along with the unmatched events in both traces are located, flagged (Fig. 4.41), and excluded from further analysis. The information of the matching events is joined and statistically analysed using scatter plots and histograms for visualisation. For the bin size of the histograms the square root of the number of events is used. To quantify the correlation between the data the Pearson correlation coefficient is used [204]. 


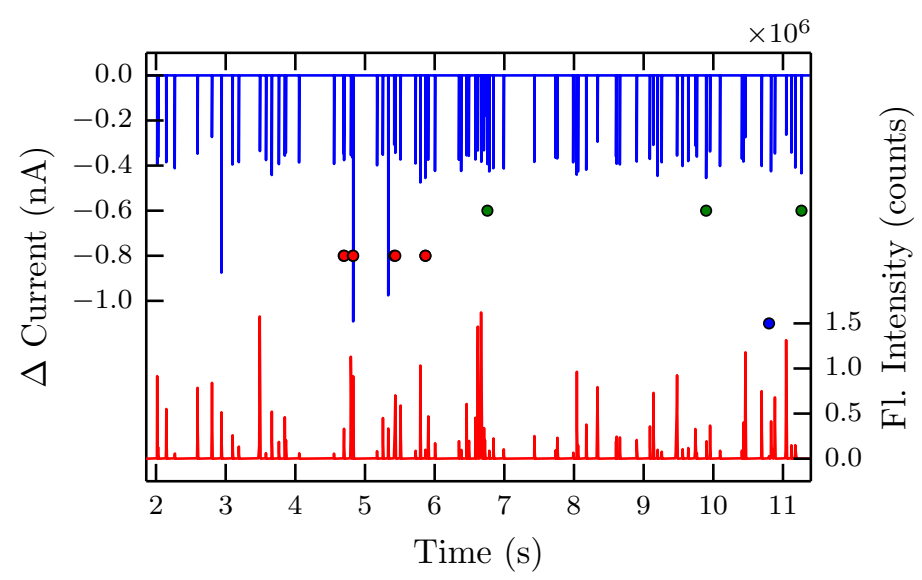

Figure 4.41: The electrical and optical traces of a coordination experiment using $2 \mu \mathrm{m}$ "Yellow-Green" beads after alignment and the identification of matching event. Red dots indicate multi events, blue dots unmatched current and green dots unmatched optical events.

\subsubsection{Spectral Analysis of the Detected Events}

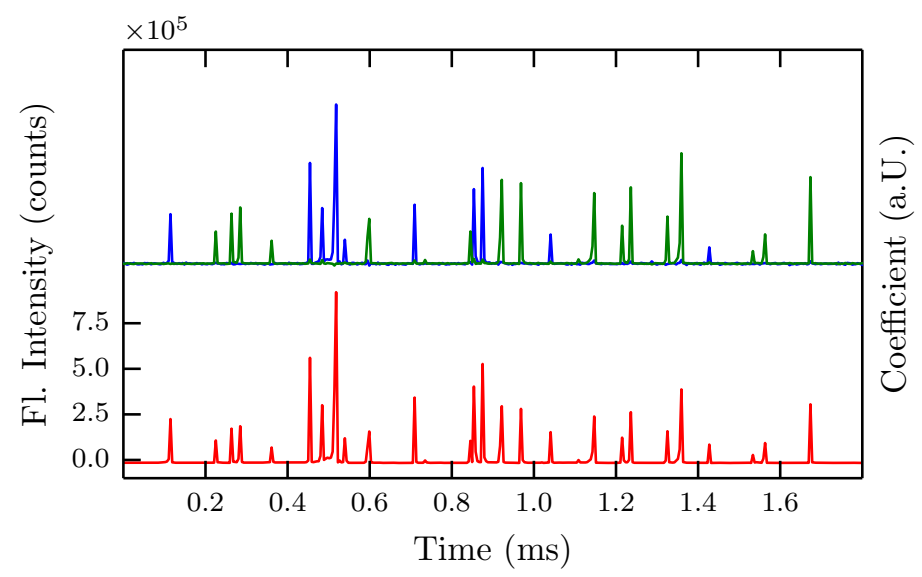

Figure 4.42: A linear least-squared fitting analysis of the spectra detected in a coordination experiment using a mixture of two sets of particles with different fluorescentlabels and the same diameter of $0.97 \mu \mathrm{m}$. The fluorescence intensity trace is red, the values for the coefficients of the two reference spectra for each detected event is in green/blue.

The spectral information is one of the distinct features of the technique and the analysis of the captured spectra is therefore important. If the reference spectra of the used "pure" components of the sample are known, a linear least squares analysis can be used to identify the particle species for each event. The method finds the least squares solution to the linear matrix equation $A x=B$, with $A$ the coefficient matrix, that contains the known reference spectra. Additionally a background spectrum of the illuminated pore without any particles present should 
be used if the optical events are small. Figure 4.42 (a) shows the spectral coefficients for the detected events using the Python function "lstsq". The coefficients reveal that events are mostly pure events with little spectral overlap. In this context it shall be noted that Principal Component Analysis (PCA) is a powerful tool [205] that can be used to identify the main components of a series of data. This can be applied to the spectra of the identified events and, assuming that mostly pure spectra are measured, the methods finds the components of the sample. The PCA algorithm can be implemented with the Python function "svd".

\subsection{Conclusion}

In this chapter the developed experimental setups that were used to conduct the coordination experiments presented in the following two chapters were introduced. Hereby a detailed understanding of both the electrical and optical apparatus was necessary for the success of the technique. Although the laser-induced noise was discussed in sufficient detail for this work, some questions of this potentially interesting effect could still be further investigated. The absorbance and fluorescence cross sections of some samples used were quantified in order to be able to make a statement about the sensitivity of the method. To assist the experimental design, a model to predict the optical signals, that partly uses results from Chap. 3, was developed. In the last section of this chapter, the methods and algorithms used for the data processing, which were developed in-house and are an integral part of the technique, were described. 
4.6. Conclusion 


\section{Chapter 5}

\section{Engineering the Size of a Gaussian Beam}

\subsection{Introduction}

With the implementation of microscopic methods in laser spectroscopies, sub- $\mu \mathrm{m}$ spatial resolution is nowadays routinely achieved [206]. A diffraction limited spot size is created by expanding the laser beam so that it fills the back aperture of an objective with a high numerical aperture [207]. Because of the high collection efficiency of such objectives these are also used even when a high spatial resolution is not necessary and a larger beam size is actually more suitable for the measurement. Typical situations are when a small power-density is desired to avoid bleaching or damaging the sample $[208,209]$ without reducing the overall signal, to ensure an uniform excitation profile in the detected area [209] or to spatially average over a large sample region without the need for scanning techniques. Some other spectroscopic methods require a small beam divergence associated with a large beam size and these include the excitation of surface plasmon polaritons [210, 211] or angle-dependent spectroscopy [212].

To achieve a large beam size one or more additional lenses have to be introduced in the optical train before the objective. Simple ray optics are often used to calculate the beam transformation of a laser beam. The prediction of the resulting beam sizes and the position of the beam waists therefore appears to be a trivial task. However, this treatment can lead to erroneous results and even the most simple case, the focussing of a laser beam by a single lens (Fig. 5.1) leads to discrepancies from the expected behaviour. During the course of this work and in an attempt to design the spot sizes to match the cross section of the TRPS pores, the shortcomings of the simplified treatment were discovered and Gaussian beam optics were used instead. In this chapter it will be shown what pitfalls can occur in 
a typical laboratory setup when not using the appropriate formalism. As a start the properties of a Gaussian beam and its peculiar transformation using a simple lens will be introduced. Then, methods to engineer the spot size using examples relevant to the coordination technique are presented and discussed. In the last section the beam sizes for two objectives in combination with various additional lenses in order to engineer the beam size are measured and compared to the theoretical values.

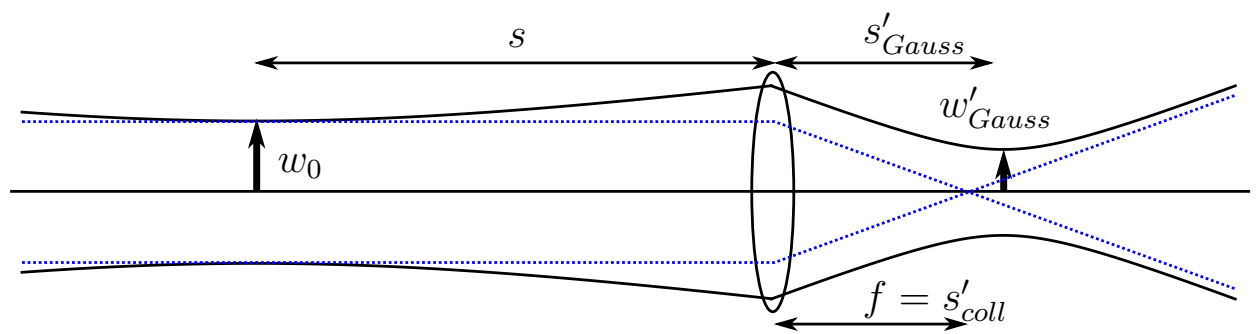

Figure 5.1: A schematic illustration of the transformation of a laser beam with the input beam waist $w_{0}$ (distance to lens $s$ ) through a lens with focal length $f$ using Gaussian beam optics (- black) and a commonly used simplification, collimated ray optics (.. blue).

\subsection{The Properties of a Gaussian Beam}

Here, the main parameters defining a Gaussian beam are recalled briefly, the reader is referred to [213] for further information. The shape of a laser beam is the result of its formation in a cavity like structure. For stable resonators, the transverse Eigenmodes of the cavity can be closely described by Hermite-Gaussian functions, denoted by $\mathrm{TEM}_{\mathrm{nm}}$, which are solutions of the governing Helmholtz equation in paraxial approximation. The lowest order, or fundamental, mode is called $\mathrm{TEM}_{00}$ and has a circularly symmetric Gaussian intensity profile in any plane transverse to the propagation direction. The beam begins as a "perfect" plane wave of finite width at the beam waist, which is located at the output mirror of the laser if it is flat. From there diffraction causes the light waves to spread transversely as they propagate. The width $w$ of the beam, which is defined as the radius at which the beam intensity has fallen to $1 / e^{2} \approx 13.5 \%$ of its peak intensity at the centre, has the following dependence on the axial distance $z$ :

$$
w(z)=w_{0}\left(1+\left(\frac{z}{z_{R}}\right)^{2}\right)^{1 / 2}
$$

with $w_{0}$ the width at the beam waist, positioned in the plane $z=0$.

An illustration of the beam parameters is shown in Fig. 5.2. The waist diameter 


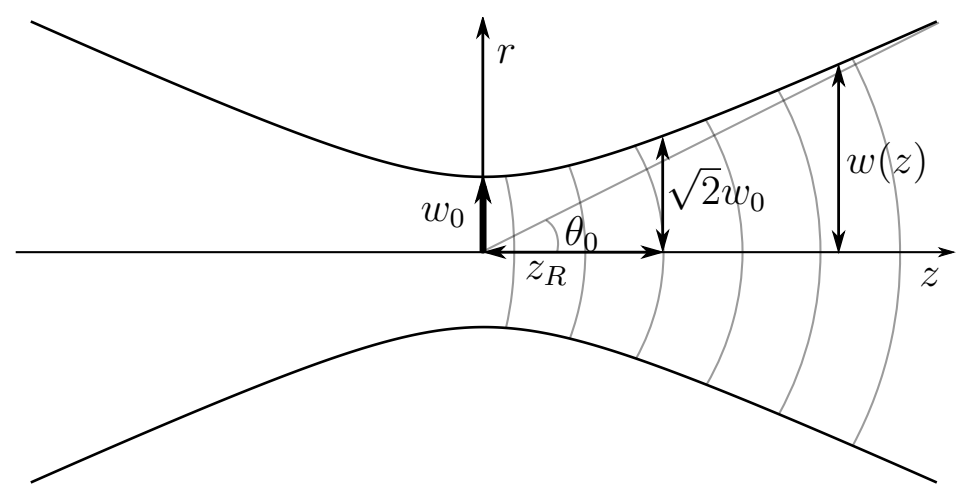

Figure 5.2: The beam waist $w_{0}$, the beam width $w(z)$, the beam divergence $\theta_{0}$ and the Rayleigh range $z_{R}$ of a Gaussian beam. The wavefronts are indicated with grey lines.

$2 w_{0}$ is usually referred to as the spot size. The parameter $z_{R}$ is known as the Rayleigh range, which is half of depth of focus, and is defined as the distance over which the beam width spreads by a factor of $\sqrt{2}$. $z_{R}$ is determined by the laser wavelength $\lambda$ and $w_{0}$ through the relation

$$
z_{R}=\frac{\pi w_{0}^{2}}{\lambda}
$$

The radius of curvature of the wave fronts is given by

$$
R(z)=z\left(1+\left(\frac{z_{R}}{z}\right)^{2}\right)
$$

In the near field $\left(z<<z_{R}\right), R \approx z_{R}^{2} / z \rightarrow \infty$ for $z \rightarrow 0$, so that the beam resembles a plane wave at the beam waist. The wave fronts then gradually curve, with the radius reaching its minimum $\left(R=2 z_{R}\right)$ at $z=z_{R}$. In the far field $\left(z>>z_{R}\right)$, $R \approx z$, and the wave fronts approximate spherical waves originating from a point source centred at the waist. Simultaneously $w(z)$ asymptotically approaches the value $w_{0} z / z_{R}$ for large $z$, defining a cone with half-angle $\theta_{0}$. Hence, in the far field, the half-angle of the divergence is given by

$$
\theta_{0}=\frac{w}{z}=\frac{\lambda}{\pi w_{0}}
$$

The divergence is inversely proportional to the waist, so that in order to achieve a highly directional beam, a large beam waist is required. One can see from the above expressions that $w_{0}$ and $\lambda$ determine all the beam properties. More generally, the knowledge of two quantities, e.g. $w\left(z_{1}\right), w\left(z_{2}\right) ; R\left(z_{1}\right), R\left(z_{2}\right)$; or $w\left(z_{1}\right), \theta_{0}$; is enough 
to determine all the beam properties and the waist position of a Gaussian beam.

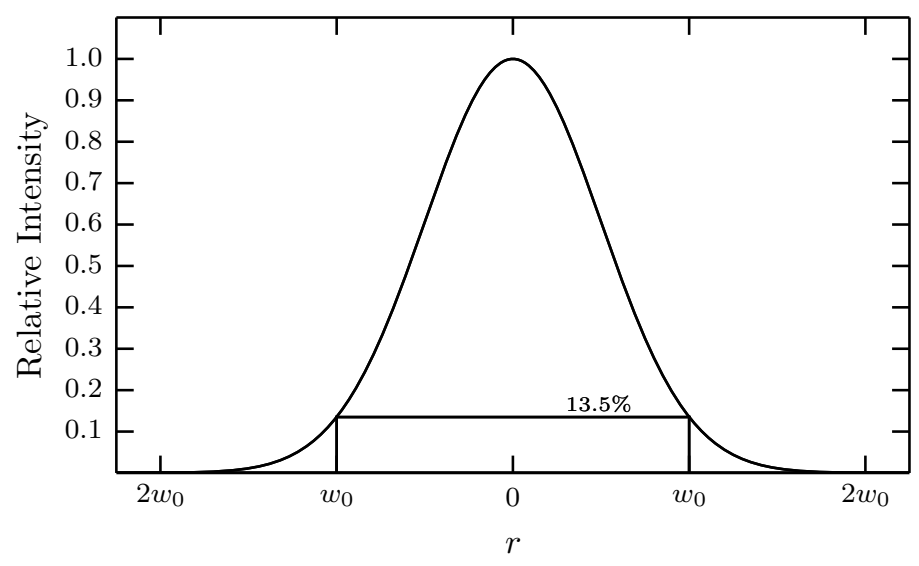

Figure 5.3: The intensity profile of a Gaussian beam as a function of the radial distance $r$ from the beam centre. $w_{0}$, the distance at which the beam intensity has fallen to $1 / e^{2} \approx 13.5 \%$ of the maximal intensity at the centre is defined as the waist of the beam.

The optical intensity $I(r, z)$ is a function of the axial and radial distances $z$ and $r$, with $z=0$ at the beam waist and $r=0$ at the beam centre

$$
I(r, z)=I_{0}\left(\frac{w_{0}}{w(z)}\right)^{2} \exp \left(-\frac{2 r^{2}}{w^{2}(z)}\right)
$$

In the radial direction the intensity has a Gaussian profile (see Fig. 5.3). In the axial direction, the intensity on the beam axis follows the expression

$$
I(0, z)=\frac{I_{0}}{1+\left(z / z_{R}\right)^{2}}
$$

and reaches half its peak value at a distance $z_{R}$ from the beam waist. Usually, the output power of the laser is known. The total optical power $P$ carried by the beam is given by

$$
P=\int_{0}^{\infty} I(r, z) 2 \pi r d r=\frac{I_{0} \pi w_{0}^{2}}{2}
$$

which can be used to rewrite the intensity $I(r, z)$ in terms of $P$

$$
I(r, z)=\frac{2 P}{\pi w^{2}(z)} \exp \left(-\frac{2 r^{2}}{w^{2}(z)}\right)
$$


The power within a circle of radius $w$ contains approximately $86 \%$ of the total power. $99 \%$ is contained within a circle of radius $1.5 w$.

\subsection{Focussing of Gaussian Beams}

\subsubsection{Gaussian Beam Optics vs. Geometric Optics}

In a laboratory experiment it is often necessary to design a lens system that creates a beam that has a certain diameter and waist location. To do so one has to follow the properties of the Gaussian beam throughout its transformation by each lens. The behaviour of a Gaussian beam that is transformed by a lens can be significantly different from what would be expected using geometric optics, defining the input beam waist as the object. An example that illustrates this difference is that of a laser beam with its waist located at the front focal plane of a convex lens, in which case the waist of the emerging beam is at the back focal plane of the lens. Following geometric optics one would expect to get a collimated beam with the image (waist) at infinity. Another descriptive example is that of a lens placed at the waist of a Gaussian beam. While from geometric optics one would assume the beam to be focused at the lens' focal plane (focal length $f$ ), the accurate calculation [214] shows that the actual waist position is at $s^{\prime}=f /\left(1+\left(f / z_{R}\right)^{2}\right)$. The effect that a lens appears to have a shorter focal length for Gaussian beams is called Gaussian shift [215]. In order to achieve the desired results, familiar concepts using geometric optics can therefore not be applied for Gaussian beams.

\subsubsection{Self's Formalism for Focussing a Gaussian Beam}

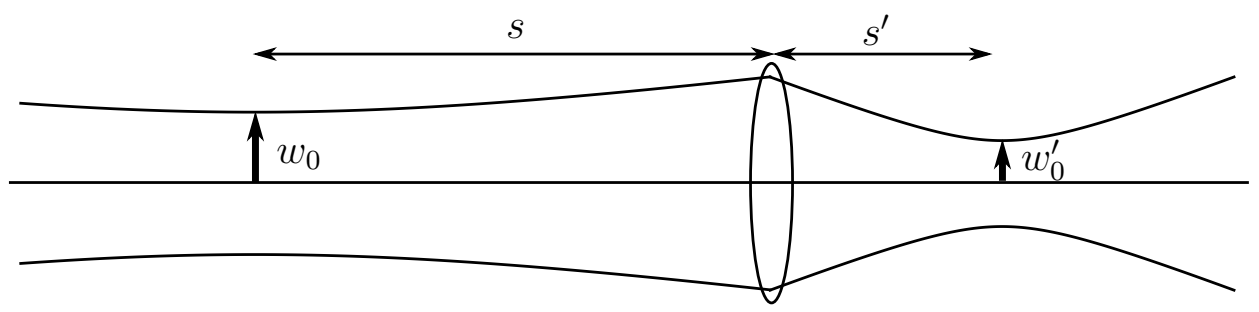

Figure 5.4: The transmission of a Gaussian beam through a convex lens with the notation as used in the main text.

The mathematical description of how Gaussian beams transform in the optical train of lenses and mirrors can be done using general matrix algebra [213, 216]. A more approachable method was developed by Self [214] by calculating the waist position and the Rayleigh range of the transformed Gaussian beam after each optical component. This can be done using a formula analogous to the usual lens-maker's 
equation used in geometric optics. Hereby the waist of the incoming beam represents the object at a distance $s$ from the lens. The image distance $s^{\prime}$ is the distance from the lens to the waist of the transformed beam, which represents the image (Fig. 5.4). Exploiting the fact that for a thin lens, the beam radius is left unchanged at the lens surfaces while the radius of curvature is altered by an factor $1 / f$, the modified lens formula for a Gaussian beam is obtained,

$$
\frac{1}{f}=\frac{1}{s+z_{R}^{2} /(s-f)}+\frac{1}{s^{\prime}}
$$

with the usual convention of positive $f$ values for converging and negative $f$ values for diverging lenses. Expressing $s^{\prime} / f$ as a function of $s / f$, this can be rewritten as

$$
\frac{s^{\prime}}{f}=1+\frac{s / f-1}{(s / f-1)^{2}+\left(z_{R} / f\right)^{2}},
$$

whereas its geometric optics equivalent is

$$
\frac{s^{\prime}}{f}=1+\frac{1}{s / f-1}
$$

Expressions 5.10 and 5.11 are plotted in Fig. 5.5 (a) with $z_{R} / f$ as parameter. All curves with $z_{R} / f \neq 0$ go through the point $s / f=s^{\prime} / f=1$, which corresponds to the case where the waist of the incoming beam lies at the front focal plane and the waist of the emerging beam at the back focal plane of the lens. The pole that appears in geometric optics (branch with $z_{R} / f=0$ ) has been removed by the term $\left(z_{R} / f\right)^{2}$, which means that the image distance cannot become infinite. There is a maximum and minimum image distance for Gaussian beams, with the maximum for an object at $s=f+z_{R}$ and the minimum for $s=f-z_{R}$. This is different from the geometric optics case where the maximum image distance occurs when the object is at the focal point of the lens. In contrast to geometric optics, where there is a minimum object to image distance of $s+s^{\prime}=4 f$ for $s=s^{\prime}=2 f$ when the object and image are real, there is no minimum object to image distance for Gaussian beams.

The magnification of the emerging beam waist relative to the incident beam waist is given by

$$
m=\frac{w_{0}^{\prime}}{w_{0}}=\frac{1}{\sqrt{(1-(s / f))^{2}+\left(z_{R} / f\right)^{2}}},
$$




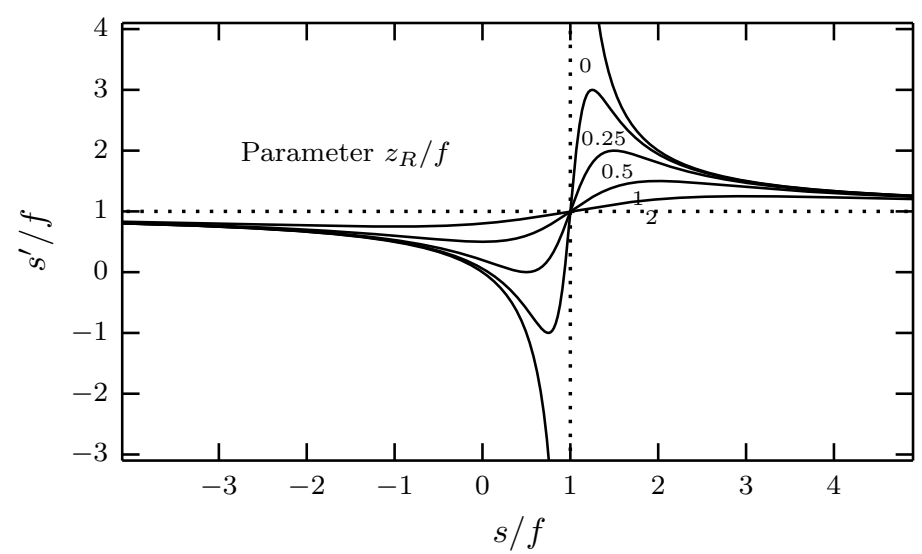

(a)

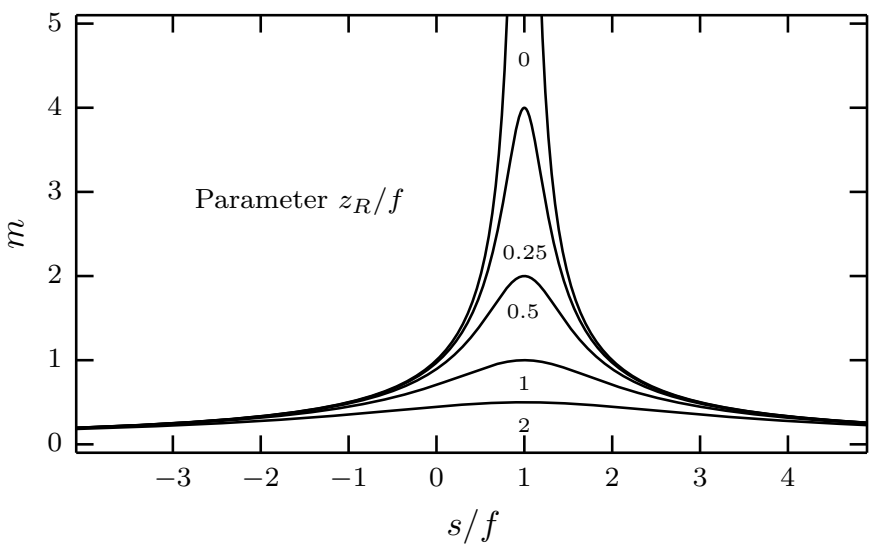

(b)

Figure 5.5: (a) Plot of the lens equation for Gaussian beams (Eq. 5.10) with the normalized Rayleigh range of the input beam as the variable parameter. The case $z_{R} / f=0$ represents the geometric optics equivalent (Eq. 5.11). (b) The magnification of a Gaussian beam's waist plotted as a function of the normalized objective distance.

and is plotted in Fig. 5.5 (b) as a function of the parameter $z_{R} / f$. Due to the term $\left(z_{R} / f\right)^{2}$ the pole that appears in the geometric optics case $\left(z_{R} / f=0\right)$ for an object placed at the front focal plane of the lens $(s=f)$ vanishes and the magnification has a maximum of $f / z_{R}$ instead.

As in geometric optics in a system comprising only lenses, the product of the image size (waist size), the ray angle (divergence) and the refractive index is a constant throughout the whole optical train. This is also known as the optical invariant or Lagrange invariant and can be written in the form

$$
\theta_{0} w_{0} n_{0}=\theta_{0}^{\prime} w_{0}^{\prime} n_{0}^{\prime}
$$

in the case of a single lens. Therefore, focussing the beam to a smaller spot increases the divergence accordingly. Fully collimated Gaussian beams using beam expander 
optics can never be achieved due to the intrinsic divergence of a light beam caused by diffraction. Using Eq. 5.13 the divergence of the focussed beam is equal to

$$
\theta_{0}^{\prime}=\frac{\theta_{0}}{m}
$$

and the Rayleigh range is given by

$$
z_{R}^{\prime}=\frac{\pi w_{0}^{\prime 2}}{\lambda}=m^{2} z_{R}
$$

With Eqs. 5.9, 5.12, 5.14 and 5.15 the properties of the emerging Gaussian beam are fully described. It has to be mentioned that Self's method, like all other calculations using the paraxial beam approximation including the model of the Gaussian beam, is only valid if the wave fronts are tilted by less than about $30^{\circ}$ from the direction of propagation. This corresponds to a beam width $\approx 2 \lambda / 3$. If smaller beam widths occur somewhere in the optical train, the paraxial approximation is no longer valid and the Helmholtz equation has to be solved directly.

It is also assumed that the lens does not significantly aperture the beam. If aperturing occurs, a beam intensity different from a Gaussian profile is the result and the spot position is different from the one calculated in Eq. 5.9. In the extreme case of a strongly aperturing lens so that the beam at the lens approaches uniform laser intensity, the image will show the usual Airy diffraction pattern and the image position will be where it is expected from geometric optics. As a general rule of thumb, for the transformed beam to keep its Gaussian profile, the diameter of any aperture in the optical path has to be bigger than 1.5 times the beam diameter $2 w(z)$ at the aperture.

\subsubsection{A Fast Method to Calculate the Spot Size}

In a practical laboratory situation, one often works with a single objective lens with a short focal length up to several meters away from the laser exit. In this case, a simple equation for the spot size can be derived for the case $s=0$. According to Eq. 5.12 the waist of the focussed beam is then given by $w_{0}^{\prime}=w_{0} / \sqrt{\left(1+\left(z_{R} / f\right)^{2}\right.}$. Under the assumption that the Rayleigh range of the incident beam is much longer than the focal length $\left(z_{R} / f\right.$ large $)$ this can be approximated to $w_{0}^{\prime} \approx f \lambda /\left(\pi w_{0}\right)=\theta_{0} f$. The waist for the general case of an arbitrary laser to lens distance $s$ can then be calculated using the beam width $w=w(s)$ (Eq. 5.1) instead of the waist $w_{0}$, so that $w_{0}^{\prime}$ for the focussed beam becomes 


$$
w_{0}^{\prime}=\frac{f \lambda}{\pi w}
$$

The same result can be derived using geometric optics and the assumption that the laser is focussed close to the focal point of the lens, which is fulfilled for a large value of $z_{R} / f$. Then $\theta_{0}^{\prime} \approx w$ (at lens) $/ f$ and equating this with the expression $\theta_{0}^{\prime}=\lambda /\left(\pi w_{0}^{\prime}\right)$ gives the same expression for $w_{0}^{\prime}$ as above. In technical papers and data sheets Eq. 5.16 is more often used in the form

$$
D_{0}^{\prime}=\frac{4 f \lambda}{\pi D}=\frac{1.27 f \lambda}{D}
$$

with $D$ the incident beam size at the lens aperture and $D_{0}^{\prime}=2 w_{0}^{\prime}$ the focussed spot size. Although the waist position $s^{\prime}$ of the transformed beam can be closely approximated by $s^{\prime}=f /\left(1+\left(f / z_{R}\right)^{2}\right) \approx f$ in this case, the actual spot size at the objective focus can be much larger due to the high divergence of the focussed beam when using an objective with a short focal length. This has to be considered if the same objective is used to collect the signal and the size of the illuminated sample area is of relevance. An illustrative example for this scenario is given in the next section.

\subsubsection{Real Gaussian Beams}

In reality, we do not deal with truly Gaussian laser beams. Although a typical HeNe laser operating in $\mathrm{TEM}_{00}$ mode is a close approximation, the usual laser output contains some components of higher-order Eigenmodes $\mathrm{TEM}_{\mathrm{nm}}$, which propagate differently to a Gaussian beam. To account for this issue, the concept of the beam quality factor $M^{2}$ has been introduced. Based on the fact that for any given laser mode the product of the beam waist and the far-field divergence, called beam product in the following, is constant as the beam propagates through the optical train, $M^{2}$ is given by the ratio

$$
M^{2}=\frac{w_{0_{\text {real }}} \theta_{0_{\text {real }}}}{w_{0} \theta_{0}}
$$

where $w_{0_{\text {real }}}$ and $\theta_{0_{\text {real }}}$ are the beam waist and the far field divergence of the real beam and $w_{0}$ and $\theta_{0}$ are the parameters of an ideal Gaussian beam of the same wavelength. For a true Gaussian beam $M^{2}$ becomes 1 and the beam product is $\lambda / \pi$. For a multi mode beam $M^{2}>1$ and the beam waist and the divergence are each by 


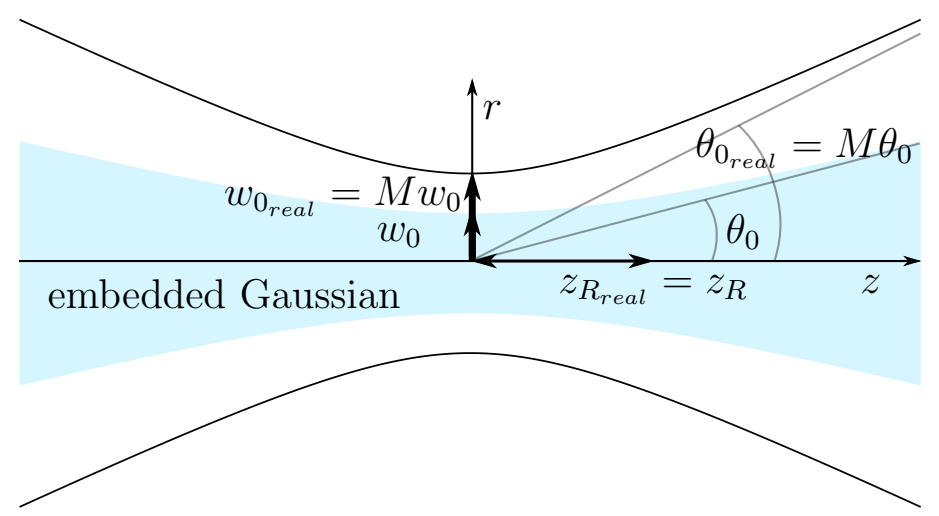

Figure 5.6: The modified beam waist and beam divergence of a real Gaussian beam and its embedded "perfect" Gaussian (shaded blue).

a factor of $M$ larger than those of the "embedded" ideal Gaussian (Fig. 5.6), while the intensity is smaller by a factor of $1 / M^{2}$. The wave front curvature of real and ideal Gaussian beams remain the same. The value of the Rayleigh range, which is defined by

$$
z_{R R}=\frac{\pi w_{0 R}^{2}}{M^{2} \lambda}=z_{R}
$$

also keeps its value. Thus, all of the formalism discussed above can still be used for the propagation and transformation of a real laser beam. The only notable difference is that the beam width throughout the whole optical train is larger by a factor of $M$. Consequently, the minimum focussed spot size of a real laser beam is $M$ times larger than the one of the embedded Gaussian beam. $M^{2}$ is usually between 1 and 1.1 for He-Ne laser, in the range of 1.1 to 1.7 for ion gas lasers, and can reach values as high as 10 for high energy multi mode lasers.

\subsubsection{The Beam Width at the Focal Plane of the Objective}

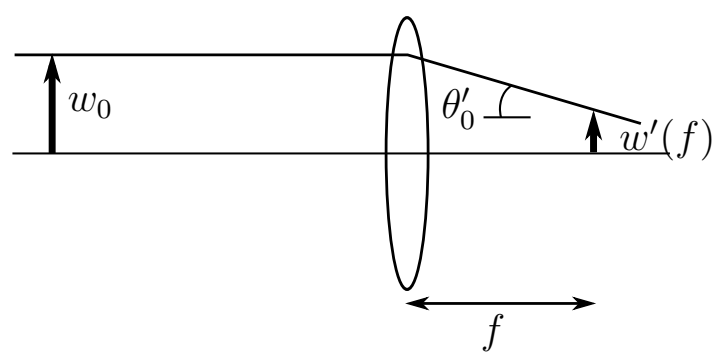

Figure 5.7: Illustration to derive the width of a Gaussian beam at the focal plane of a lens.

By explicitly calculating $w^{\prime}(f)$ using Eqs. 5.1, 5.9 and 5.12 one can find the simple expression 


$$
w^{\prime}(f)=f \theta_{0},
$$

which means that the beam width at the focal plane of a lens is independent of any other parameters like $w_{0}, s$ or $\lambda$. This at first surprising result can be understood in the limit of ray optics by a simple illustration (Fig. 5.7). For $w_{0}>>w^{\prime}(f)$ one gets $\theta_{0}^{\prime}=w_{0} / f$ and with the optical invariant the above relation for $w^{\prime}(f)$ follows. The same result can also be obtained using Eq. 5.16 by replacing $w$ with $w_{0}$. Throughout many technical notes and datasheets it can be found that the simple expression $f \theta$, which describes the beam width $w^{\prime}(f)$ at the focal plane of a lens, is defined as the actual beam waist $w_{0}^{\prime}$. This is not the case as in general $w^{\prime}(f) \neq w_{0}^{\prime}$ and can lead, especially for highly focussed beams, to large discrepancies. Several examples are shown in Tab. 5.1.

\subsubsection{Modifications to Self's Formalism for Immersion Ob- jectives}

Self derived his equations assuming that the medium on each side of the lens is vacuum or air with a refractive index of 1 . In many techniques, especially in the life sciences, oil or water immersion objectives are used and the refractive index of the medium on right side of the lens in Fig. 5.4 is then different from 1. Self's formalism, however, is still valid and the equations only have to be slightly modified. For their derivation the general lens equation must be used,

$$
n_{1} / s+n_{2} / s^{\prime}=n_{1} / f_{1}=n_{2} / f_{2}
$$

with $n_{1}, n_{2}$ the refractive indices of the media and $f_{1}, f_{2}$ the corresponding focal lengths. In the following, the reasonable assumption is made that the laser is surrounded by air so that $n_{1}=1$. For the focal length of the lens its value in air $f_{1}=f$ is used. This is also the number usually noted in the datasheets of immersion objectives as their magnification is defined relative to a tube lens with a known focal length in air (for Olympus $180 \mathrm{~mm}$ ). The focal length of the lens on the side with the immersion medium is then $f n_{2}$.

The position $s^{\prime}$ of the focussed beam waist can simply be calculated by multiplying the value obtained from Eq. 5.10 with the refractive index $n_{2}$ of the medium. The equation for the magnification (Eq. 5.12) remains unchanged, so that the beam waist focussed by an air and an immersion objective with equal focal length $f$ are exactly the same. Due to the invariance of the beam product (Eq. 5.13), the di- 
vergence has to decrease and consequently the Rayleigh length must increase by a factor of $n_{2}$. The profile of a Gaussian beam in an immersion medium $\left(n_{2}>1\right)$ appears therefore more elongated than in air. The beam width at the focal plane $w^{\prime}(f)$ of the immersion objective remains unchanged, as the lower divergence is compensated by the larger absolute distance between beam waist and focal plane of the lens. The same approach applies to a beam focussed through a layer of glass or water using an air objective, as in experiments conducted with the first developed setup (Chap. 6). The beam has the same waist but is shifted further into the medium as the divergence is decreased in the part of the beam path in the medium with the higher refractive index [217]. This is again a direct consequence of the invariance of the beam product.

\subsubsection{Spot Sizes and Gaussian Shifts in a Laboratory Situ- ation}

To illustrate the concepts described above and point out the possible pitfalls when focussing Gaussian beams, a realistic laboratory situation is discussed. The beam sizes and waist positions of a laser beam focussed by lenses of various focal lengths are calculated using Self's method (Eqs. 5.10 and 5.12) and compared with the results obtained with geometric optics and its amplification, collimated ray optics. Equation 5.10 should be used instead of Eq. 5.9 to calculate the waist position $s^{\prime}$ since it does not show the singularity for $s=f$.

Figure 5.8 shows the beam profiles obtained by focussing a laser with lenses of $18 \mathrm{~mm}, 9 \mathrm{~mm}, 3.6 \mathrm{~mm}$ and $1.8 \mathrm{~mm}$ focal length, corresponding to a $\times 10, \times 20$, $\times 50$ and $\times 100$ magnification for Olympus objectives. The laser and the lens are positioned in the negative half space of the vertical axis. For the refractive index on both sides of the lens a value of 1 is assumed. The distance $s$ between the laser exit, where the waist is assumed to be, and the lens is $1560 \mathrm{~mm}$. The original beam is produced by an Ar-ion laser with $\lambda=514 \mathrm{~nm}, w_{0}=360 \mu \mathrm{m}$ and $\theta=0.5 \mathrm{mrad}$, resulting in a beam quality factor $M^{2}=1.23$ and a Rayleigh range of $z_{R}=720 \mathrm{~mm}$. In the usual geometric optics equations the size of the input beam waist with its distance from the lens are defined as the object size and the object distance $s$.

The obtained values for the focussed waist and its position are larger and further away from the focal plane when using the usual equations for geometric optics (Fig. 5.8). This effect is called Gaussian shift as the lens appears to have a shorter focal length for Gaussian beams. The fact that the Gaussian beam is focussed a distance away from the focal plane of the lens is often mistakenly interpreted as the Gaussian shift.

Approximating the laser as a collimated beam is a common laboratory approach 


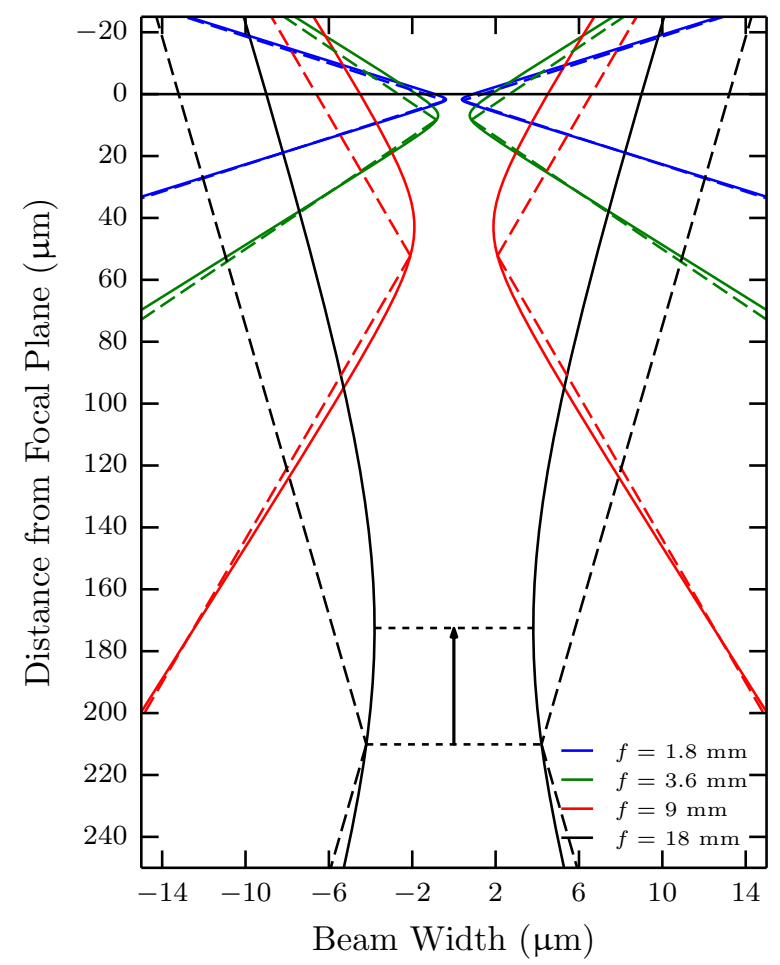

Figure 5.8: Beam profiles of a laser beam focussed by lenses with various focal lengths (see legend). The - lines are the profiles obtained using Self's method, and the - - lines using the geometric optics approach. The horizontal black line indicates the focal plane of the lens and the arrow illustrates the Gaussian shift. Note that the vertical axis is compressed compared to the horizontal axis.

Table 5.1: Properties for a laser beam $\left(\lambda=458 \mathrm{~nm}, w_{0}=360 \mu \mathrm{m}, \theta_{0}=0.5 \mathrm{mrad}\right.$, resulting in a $z_{R}=720 \mathrm{~mm}$ ) focussed by lenses with various focal lengths $f$. Results are calculated using Self's approach (Eqs. 5.10 and 5.12), except for the approximate beam waist calculated with Eq. 5.16. The laser to lens distance $s$ is $1560 \mathrm{~mm}$ for the first six rows, and $50 \mathrm{~mm}$ for the last row, labelled *.

\begin{tabular}{ccccc}
\hline$f(\mathrm{~mm})$ & $w_{0}^{\prime}(\mu \mathrm{m})$ & $\begin{array}{c}w_{0}^{\prime} \text { approx. } \\
(\mu \mathrm{m})\end{array}$ & $s^{\prime}-f$ & $w^{\prime}(f)(\mu \mathrm{m})$ \\
\hline 1.8 & 0.38 & 0.38 & $1.71 \mu \mathrm{m}$ & 0.90 \\
3.6 & 0.76 & 0.75 & $6.9 \mu \mathrm{m}$ & 1.80 \\
9 & 1.89 & 1.89 & $43.0 \mu \mathrm{m}$ & 4.5 \\
18 & 3.81 & 3.77 & $172.5 \mu \mathrm{m}$ & 9.0 \\
300 & 74.42 & 62.86 & $53.85 \mathrm{~mm}$ & 150 \\
1000 & 394.7 & 209.5 & $673.1 \mathrm{~mm}$ & 500 \\
$9^{*}$ & 4.49 & 4.49 & $6.4 \mu \mathrm{m}$ & 4.5 \\
\hline
\end{tabular}

and makes its transformation through a lens and lens combinations, e.g. beam expanders, very simple. The position of the focussed beam waist is then assumed to be equal the focal plane of the lens and the beam waist can be calculated using 
Eq. 5.16. Table 5.1 summarizes the results for the beam waist obtained with the simplified equation along with the exact calculation using Eq. 5.12 for lenses with various focal lengths. The approximated results are satisfying for large values of $z_{R} / f$, but Eq. 5.16 starts to fail when $f$ is of the same order as $z_{R}$.

As the distance $s^{\prime}-f$ is also below $1 \%$ of the focal length of the lens for large values of $z_{R} / f$, the approach appears to be appropriate for a lens with a short focal length, i.e. a typical objective. However, as the divergence $\theta_{0}^{\prime}$ increases with decreasing beam waist, even the small difference $s^{\prime}-f$ can result in a large difference of the size of the beam waist and the beam width at the focal plane. For the objective used in our system $w^{\prime}(f)$ is $\sim 2.4$ times larger than $w_{0}^{\prime}$, resulting in a power density $\sim 5.7$ times smaller than at the waist position. This has to be considered in experiments where the excitation spot size and absolute intensity at the surface of the sample used are of relevance and especially when the same objective is used for the signal collection (backscattering configuration). $w^{\prime}(f)$ approaches $w_{0}^{\prime}$ for large $z_{R} / f$ and a sufficiently small $s$, a situation which is not likely to happen in a laboratory situation.

The assumption that the laser is a collimated beam is also conveniently used to determine the focal length of a lens by simply measuring the distance between the lens and the minimal focussed beam width. The method is accurate when the lens is placed at the beam waist of the laser (compare Eq. 5.10), which is usually equivalent with the laser exit. However, this is often not possible due to spatial constraints and the lens has to be placed a distance away from the beam waist so that the obtained focal length can be significantly different from the actual one. For example, placing a lens with a focal length of $400 \mathrm{~mm}$ a distance of $1000 \mathrm{~mm}$ away from the beam waist of the Ar-ion laser results in a focussed spot $509 \mathrm{~mm}$ away from the lens. This will probably lead the experimentalist to assume the lens has a focal length of $500 \mathrm{~mm}$ instead of the actual $400 \mathrm{~mm}$.

In a typical microscopy-based spectrometer setup the sample is viewed with an inbuilt camera. Hereby, the image is created by a lens that refocusses the light collected by the objective onto a CCD, positioned at the focal plane of the camera lens (Sec. 4.2). If the sample does not show distinct features, e.g. is highly reflective, the experimentalist often chooses the sample position where the laser spot appears at a minimum as the focus. This approach can be problematic as the so determined position does not coincide with either the focal plane of the objective (as discussed above) or, more surprisingly, with the position of the beam waist of the focussed laser if the sample is mostly specular reflective. It can be calculated with Self's formalism that the image of the laser spot in the camera is minimal when the sample is positioned at half the distance between the focal plane of the objective and the actual laser waist. This is counter-intuitive at first and when measuring the distance 
between the focus at white light illumination and the minimum of the laser spot size, the resulting values are halved from what is expected with Eq. 5.10. Using a diffusively reflecting sample, the reflected light can be understood as a superimposition of point sources for which geometric optics can be used. The position of the smallest laser spot then coincides with the white light focus.

As a conclusion of this section it shall be noted that the properties of a focussed Gaussian beam should generally be calculated using Eqs. 5.9, 5.12, 5.14 and 5.15 and one has to be aware that the often intuitive applied rules of geometric optics do not yield the correct results in many cases.

\subsection{Engineering the Spot Size}

The spot size for a laser focussed by a lens is determined by the laser properties $w_{0}$ and $\theta_{0}$, the focal length $f$ and the laser to lens distance $s$. In many applications, $w_{0}, \theta_{0}$ and $s$ are fixed, and also the choice of $f$ is limited as the experimentalist often uses objective lenses with high numerical apertures and short focal lengths to optimise the signal collection. To be able to engineer the spot size to a desired value in such a situation, further lenses have to be introduced between the laser and the objective lens. Smaller spot sizes are often needed to get high power densities and high resolution and can be achieved by expanding the beam before the objective lens. To keep the Gaussian profile, the radius of the lens aperture should be at least 1.5 times larger than the width of the beam at the lens. The method is commonly used and will not be discussed any further in this context. Other applications, including the coordination experiments, require a uniform illumination across the sample and therefore a large spot size with a small divergence. If no additional optics are used, the largest possible beam waist of $f \theta_{0}$ is reached when the laser waist coincides with the focal plane of the objective. In practical situations this is not feasible as the distance $s$ from the laser exit to the objective is usually fixed at much larger distances. Furthermore, the gain in the spot size is limited this way. However, when another lens with a suitable focal length is placed at the correct position between the laser exit and the objective lens, larger spot sizes can be achieved.

\subsubsection{Back Focal Plane Lens}

One intuitive way to achieve a larger beam size is to put an additional lens in the optical train so that it focusses the incident laser beam on to the back focal plane of the objective [210]. This is illustrated in Fig. 5.9. The lens $L_{1}$ is referred to as the Back Focal Plane Lens (BFPL). It images the original beam on to the back focal plane of the second lens $L_{2}$, which then subsequently creates a beam with a waist 


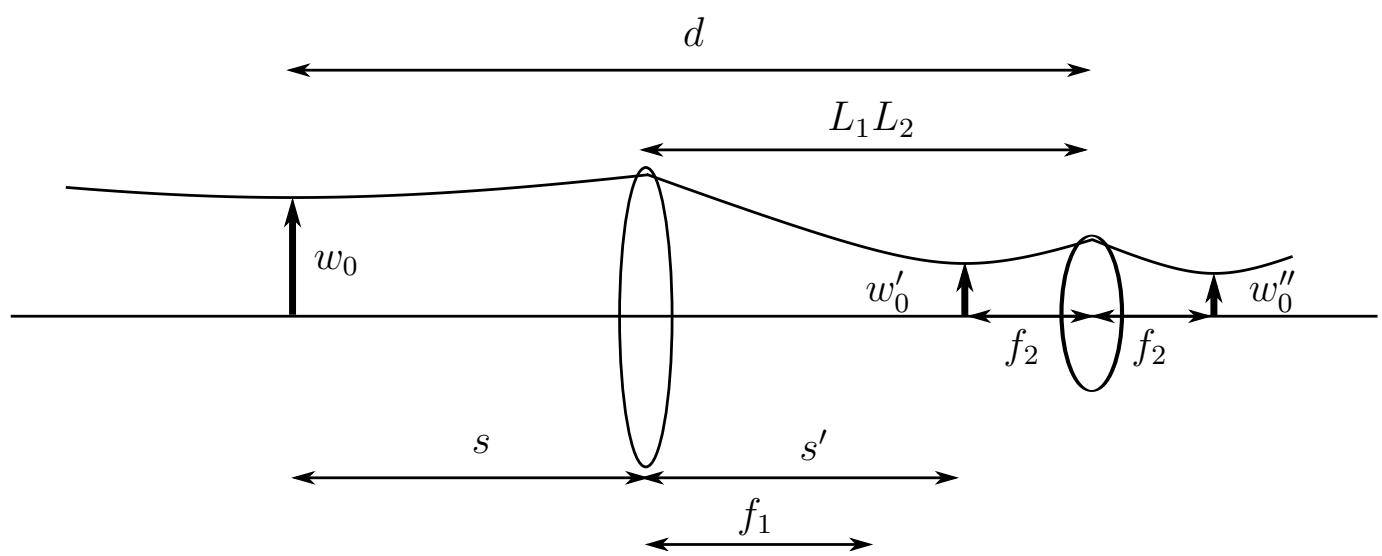

Figure 5.9: The left-hand lens $L_{1}$ focusses the original laser beam onto the back focal plane of the objective lens $L_{2}$ to magnify the beam spot size $w_{0}^{\prime \prime}$, which is identical to $w^{\prime \prime}\left(f_{2}\right)$ in this case. The total length between laser exit and the objective lens is $d$, and the distance between the two lenses is $L_{1} L_{2}$.

$w_{0}^{\prime \prime}$ at its own front focal plane. The setup is similar to that of a reversed Keplerian beam expander, as the focal length of the objective lens is usually smaller than that of the BFPL. Intuitively, the correct position for the BFPL is at a distance $f_{1}+f_{2}$ away from the objective lens. However, as a Gaussian beam cannot be seen as truly collimated, the actual value for $L_{1} L_{2}$ that fulfils the condition $L_{1} L_{2}=s^{\prime}+f_{2}$ for a fixed total path length $d=s+s^{\prime}+f_{2}$ differs from the one expected.

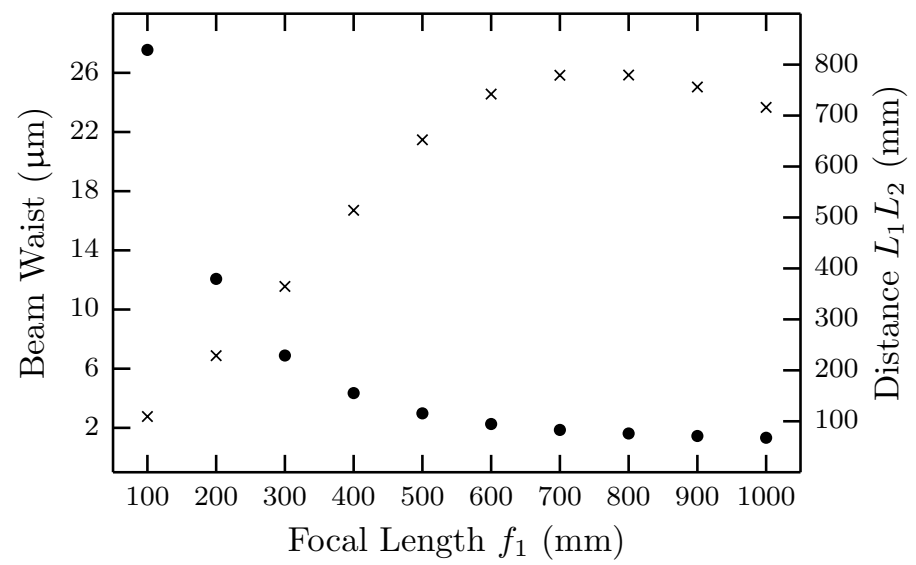

Figure 5.10: The required distance $L_{1} L_{2}(\times)$ between a BFPL with focal length $f_{1}$ and an objective lens $L_{2}\left(f_{2}=3.6 \mathrm{~mm}\right)$ to achieve the situation depicted in Fig. 5.9, and the corresponding beam waist $(\bullet)$.

In Fig. 5.10 the predicted values for $L_{1} L_{2}$ and the resulting beam waists $w_{0}^{\prime \prime}$ are plotted as a function of the focal length of the BFPL. The total path length $d=$ $1560 \mathrm{~mm}$ and the focal length of the objective lens $f_{2}=3.6 \mathrm{~mm}$ are left constant. For example, when a BFPL with $f_{1}=100 \mathrm{~mm}$ is positioned $109.37 \mathrm{~mm}$ away from the objective lens a beam waist $w_{0}^{\prime \prime}$ of $27.55 \mu \mathrm{m}$ is achieved. The gain in size compared 
to using the objective lens alone $\left(w_{0}^{\prime}=0.76 \mu \mathrm{m}\right)$, is $\approx 36.25$ fold. Compared to the beam width at the focal plane $(1.8 \mu \mathrm{m})$ the gain is $\approx 15.31$. With the collimated beam approach the position between the lenses is calculated to be $103.6 \mathrm{~mm}$ and a size of the focussed beam of $f_{1} / f_{2} \times w_{0}=12.96 \mu \mathrm{m}$ is expected. Positioning the BFPL at this "wrong" position leads, interestingly, to the exact value for the beam waist of $12.96 \mu \mathrm{m}$. However, the width at the objective focus is $27.64 \mu \mathrm{m}$ due to twice as large divergence compared to the "correct" position and the waist is located only $1.84 \mathrm{~mm}$ from the lens. This shows that although the size at the focal plane is correct, the focussed beam waist is neither at the correct position nor has the desired minimal divergence.

The achievable magnification of the beam decreases quickly with increasing $f_{1}$, reaching a gain of only $\approx 1.75(1.33 \mu \mathrm{m})$ for a BFPL with $1000 \mathrm{~mm}$ focal length. The relative difference of the optimal position and the one predicted with the beam expander formalism $\left(L_{1} L_{2}=f_{1}+f_{2}\right)$ is increasing with $f_{1}$, e.g. for $f_{1}=500 \mathrm{~mm}$, the BFPL has to be placed $652 \mathrm{~mm}$ away from the objective lens, resulting in a value for the beam waist of $2.98 \mu \mathrm{m}$.

The accuracy with which the experimentalist has to position the BFPL to achieve the largest spot size is high for small values of $f_{1}$ and decreases with increasing $f_{1}$. For example, the position of a $100 \mathrm{~mm}$ BFPL must be accurate within $1.5 \mathrm{~mm}$ on either side of the calculated value in order to get a $10 \%$ tolerance in the beam size. For a $500 \mathrm{~mm}$ lens the tolerance in position increases to $95 \mathrm{~mm}$.

Experimentally the correct position for the BFPL can be found by observing the beam at some distance after $L_{2}$ where the laser spot width can easily be viewed by eye. The distance should be at least ten times the focal length of the objective lens. Making use of the invariance of the beam product, the required position of the BFPL can be found where the spot size is minimal. This way, one will achieve the situation where the peak is as shown in Fig. 5.11 (a). This is not exactly at $s^{\prime}=f$ but is usually close enough for most purposes. To achieve exact coincidence one can calculate the difference and adjust the distances by moving further (from $624 \mathrm{~mm}$ to $652 \mathrm{~mm}$ in this example). By simply observing the spot at the focal point, using for example the camera feature of the spectrometer, one would not be able to find the correct position of the BFPL as the size of the beam width at the focal plane depends linear on the distance between the lenses close to the critical position (Fig. 5.11 (b)).

Although large spot sizes can be achieved with a BFPL, in some situations even larger spots are required or the positioning of the BFPL is restricted in a way (e.g. a beam splitter is in the optical path), so that only lenses with long focal lengths can be used. In these cases, a beam expander can be placed in front of the BFPL to achieve the desired spot size, which will be discussed in the next section. 


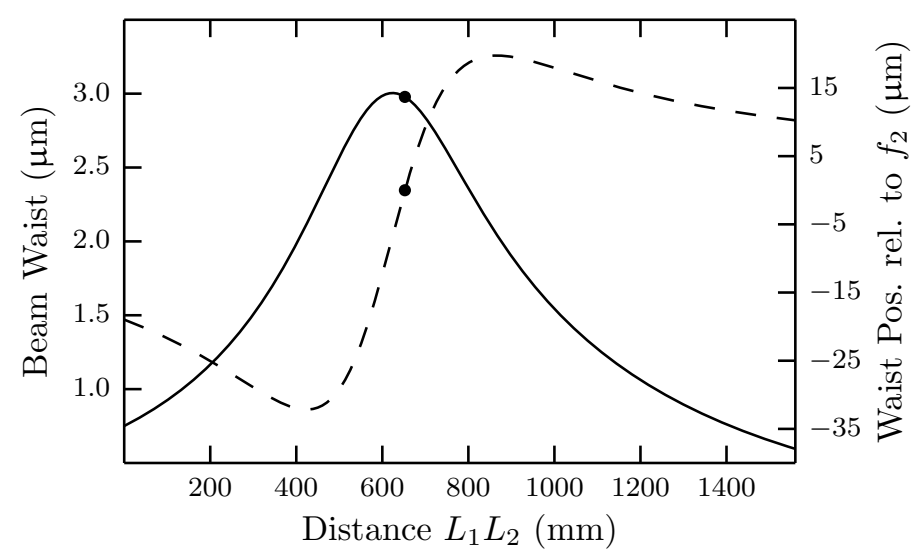

(a)

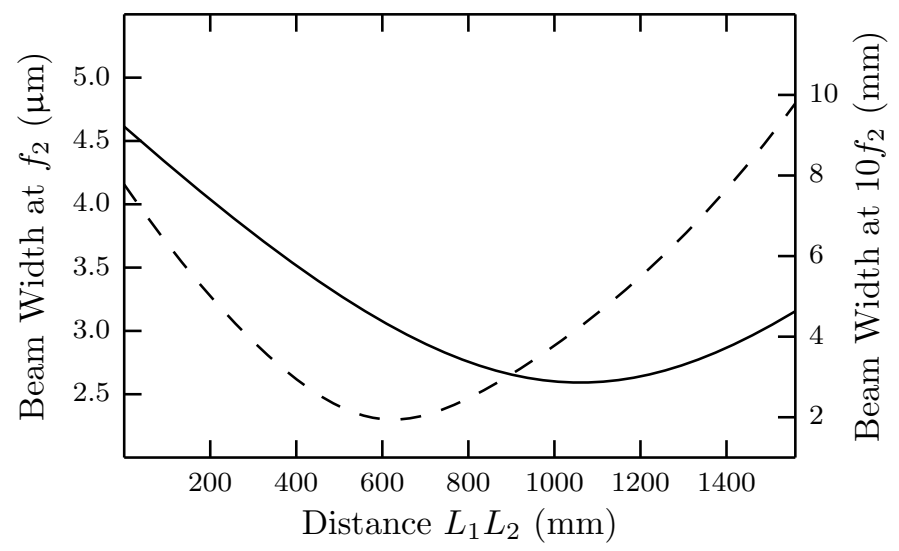

(b)

Figure 5.11: (a) The size of the beam waist (-) and its position (- -) as a function of the lens distance when a BFPL with a focal length of $500 \mathrm{~mm}$ and an objective lens with $3.6 \mathrm{~mm}$ is used. Negative values indicate that the beam waist is located closer to the lens than its focal plane. Note that in order to achieve the situation depicted in Fig. 5.9, the required distance (marked with •) is not exactly equivalent to the maximum beam waist, which is achieved at a slightly shorter distance. (b) The beam width at the objective focus (-) and at a distance $10 f_{2}\left(-\right.$ - $^{-}$from the objective lens.

We note that if the condition that the spot size is coinciding with the objective plane can be neglected and simply a large beam width is required at the objective plane, a lens with a shorter focal length than the BFPL otherwise used, called a field lens (FL) in the following, can be used. The laser will then be focussed beyond the optical plane to a small spot but, due to a high divergence, the beam is enlarged at the objective focus. Figure 5.12 shows the beam sizes which can be achieved that way for field lenses with various focal lengths under the assumption that the position of the FL is constrained. This method is commonly used for solid surfaces where only a large beam width (but not a small divergence or parallel wavefronts at the sample) is required. An even simpler method to achieve large beams for solid 


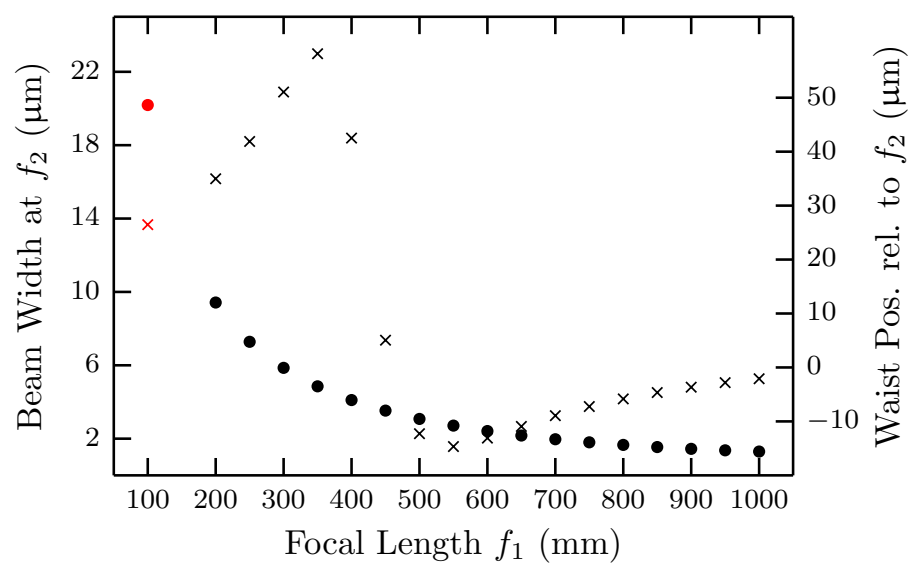

Figure 5.12: The beam width $(\bullet)$ at the objective focal plane and the position $(\times)$ of the beam waist relative to it as a function of the focal length of the BFPL. The distance between the lenses is fixed at $600 \mathrm{~mm}$ and the focal length of the objective lens is $3.6 \mathrm{~mm}$. The red coloured markers indicate that the condition for using the paraxial assumption is no longer valid.

surfaces is to focus beyond the sample plane, which has a negative influence on the collection efficiency.

\subsubsection{Beam Expander as Back Focal Plane Lens}

Placing a beam expander before the BFPL in the optical train results in a smaller spot at the back focal plane of the objective lens, and therefore a larger beam waist at its front focal plane. Both commonly used types of beam expanders can be used for this purpose. The Keplerian expander consisting of two convergent lenses has the advantage that the beam can be cleaned by positioning a pinhole between the lenses. The advantage of the Galilean expander, with one divergent (focal length $\left.f_{\text {exp } 1}\right)$ and one convergent lens $\left(f_{\exp 2}\right)$, is its more compact size. In both cases the distance between the lenses is close to $f_{\text {exp } 1}+f_{\exp 2}$ (again, the correct calculation gives slightly different values) and the magnification is roughly the ratio of the focal lengths $f_{\exp 2} /\left|f_{\exp 1}\right|$.

To reduce the optical elements the second, convergent lens of the beam expander and the BFPL (focal length $f_{B F P L}$ ) can be combined into one convergent lens with the new focal length $f=\left(f_{\exp 2} f_{B F P L}\right) /\left(f_{\exp 2}+f_{B F P L}\right)$, so that the setup becomes as illustrated in Fig. 5.13. The ideal distance $L_{1} L_{2}$ to fulfil the condition $L_{2} L_{3}=s^{\prime}+f_{3}$ can then be calculated with a simple optimization algorithm for a given value of $d$. Apart from producing large beam sizes, the same setup can also be used to achieve smaller spot sizes than by using a single objective lens, showing the versatility of the technique.

As an example the required distance $L_{1} L_{2}$ for a system consisting of three lenses 


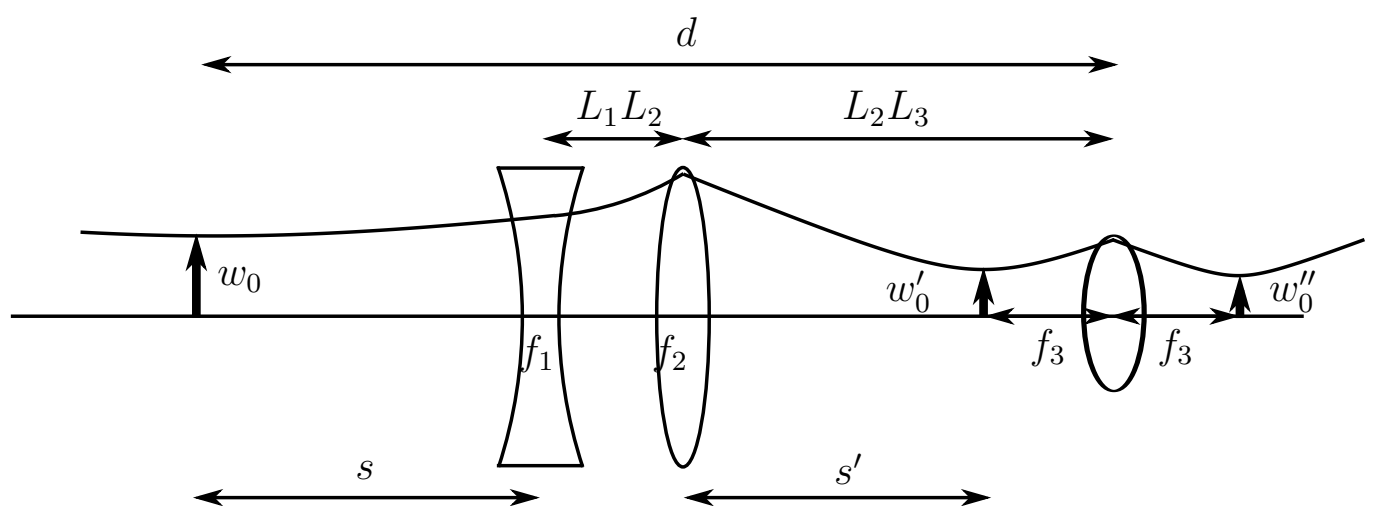

Figure 5.13: A combination of two lenses $L_{1}$ and $L_{2}$ is used to expand the beam and focus it on to the back focal plane of lens $L_{3}$, resulting in a large beam size at its front focal plane.

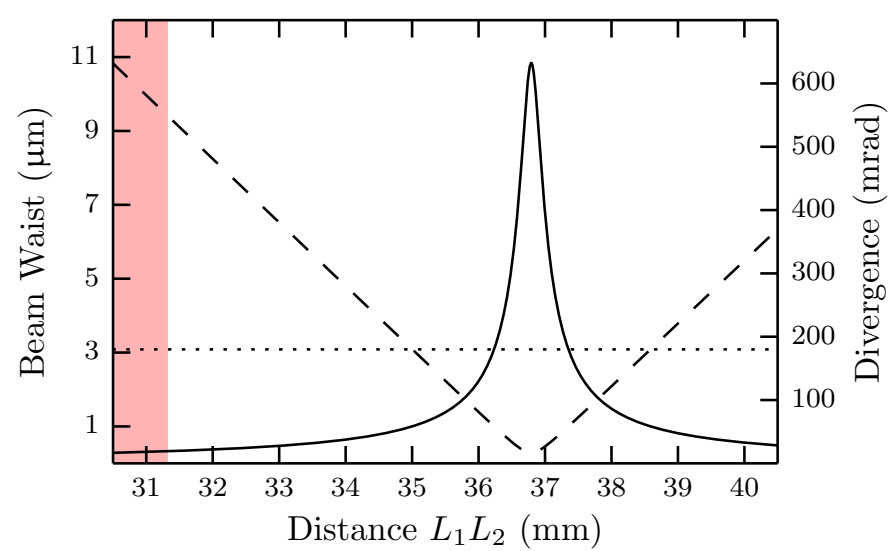

(a)

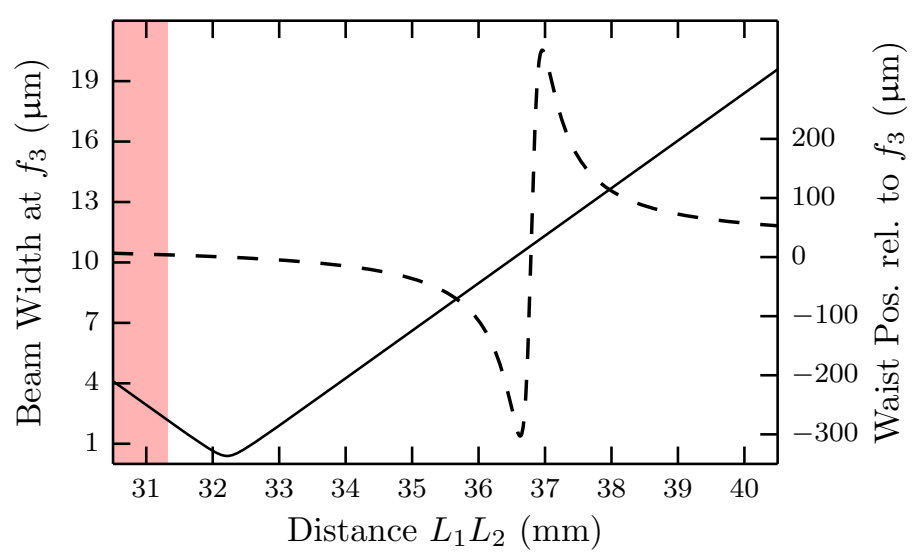

(b)

Figure 5.14: (a) The beam waist (-) and its divergence (- -) as a function of the distance between the lenses with the parameters described in the main text. The horizontal dotted line is the constant product of the waist and the divergence. (b) The beam width at the focal plane (-) and the distance of the beam waist (- -) to the focal plane as a function of $L_{1} L_{2}$. Shaded red areas indicate that the paraxial assumption is no longer valid. 
with $f_{1}=-18 \mathrm{~mm}, f_{2}=+50 \mathrm{~mm}, f_{3}=3.6 \mathrm{~mm}$, a total path length $d=1560 \mathrm{~mm}$ and $L_{2} L_{3}=600 \mathrm{~mm}$ is calculated. For $L_{1} L_{2}=36.79 \mathrm{~mm}$, the beam waist will reach its maximum of $10.84 \mu \mathrm{m}$ and be positioned exactly at the objective focus. The increase in the beam waist compared to using just a single objective lens $\left(w_{0}^{\prime}=\right.$ $0.76 \mu \mathrm{m}$ ) is $\approx 14.3$ fold. Figure 5.14 (a) shows the beam waist, the divergence, and the beam product as a function of the distance between the lenses $L_{1}$ and $L_{2}$. While the maximum spot size is very sensitive to the distance $L_{1} L_{2}$ (to achieve a $10 \%$ tolerance the positioning accuracy must be within $70 \mu \mathrm{m}$ on either side), the beam width at the focal plane is linearly dependent on $L_{1} L_{2}$ (Fig. 5.14 (b)), making the system easy to use in practice. Experimentally the position for the maximal spot size can be found by observing the beam about ten times $f_{3}$ away and finding the minimal beam size.

The system also has the advantage that it can be quickly changed to obtain a smaller beam size than achievable with the single objective lens alone. If the distance between the lenses is changed to $32.22 \mathrm{~mm}$ (again, not exactly what is predicted from the usual beam expander theory), the beam waist is $0.39 \mu \mathrm{m}$, about half of the standard size of $0.76 \mu \mathrm{m}$. In Fig. 5.14 (b) it can be seen that there are two positions where the beam waist is exactly located at the focal plan of the objective. One is where the spot size becomes maximal, the other where the spot size becomes minimal. Between these two points the beam width at the focal/sample plane shows a linear characteristic so that it can be continuously tuned from approximately 0.5 to 14 times the size that can be obtained using the objective lens alone.

\subsubsection{Beam Size Engineering for the Coordination Tech- nique}

The sizes of pores used in the coordination technique encompass an order of magnitude. Also, an objective with a high numerical aperture and magnification is required to maximise the signal and reduce the occurrence of multi events (Sec. 7.2.3). Therefore, the methods developed above were implemented experimentally to engineer the beam size and to simulate the optical signals expected from fluorescently-labelled particles (Sec. 7.2).

Figures 5.15 (a) and (b) shows the installation of the BFPL and the beam expander system in the LabRAM. The setup has to allow for enough degrees of freedom to correctly align the lenses. In our case three translation and two tilt stages are iteratively adjusted. The distance between the two lenses in the beam expander system can be adjusted with $\mu \mathrm{m}$ precision. Due to the spatial limitation in the beam path of the LabRAM, the distance between the objective lens and any additional lens has to be larger than $600 \mathrm{~mm}$. This allows only for a BFPL with a minimum 
focal length of $500 \mathrm{~mm}$, which limits the maximal achievable beam waist with a BFPL. To obtain larger sizes a beam expander system with a lens combination of $f_{1}=-18 \mathrm{~mm}$ and $f_{2}=+50 \mathrm{~mm}$ was chosen. The lens $L_{2}$ and the BFPL can be combined to a single convergent lens with a theoretical focal length of $45.45 \mathrm{~mm}$, meaning that the lens $L_{2}$ can still be used.

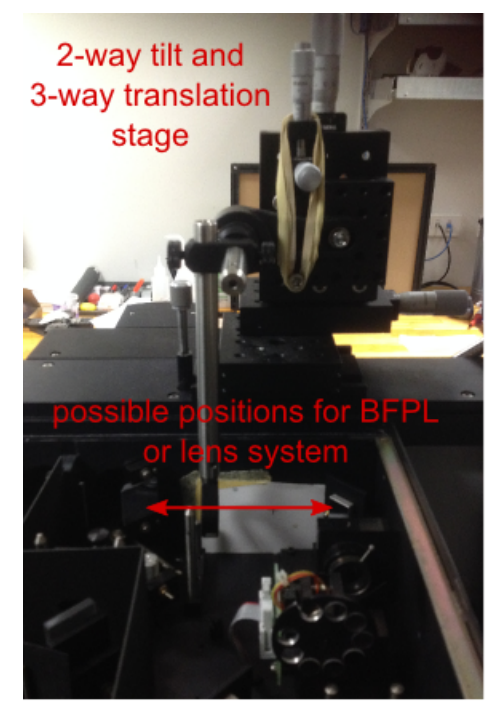

(a)

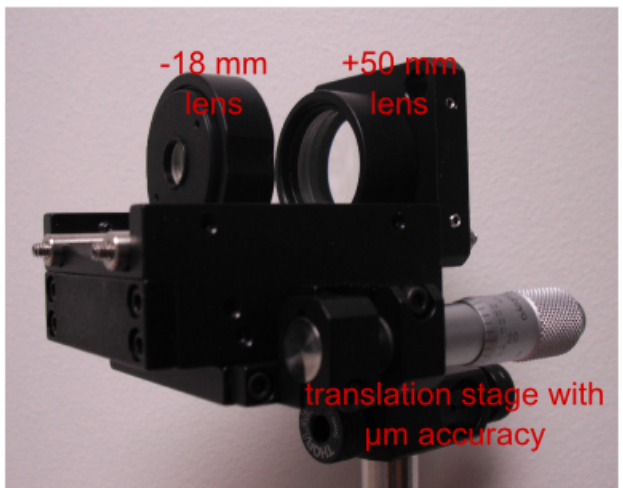

(b)

Figure 5.15: (a) Installation of a BFPL in the LabRAM system. The available space for the system is limited to $\sim 15 \mathrm{~mm}$ of the optical beam path. (b) The two lenses used in the beam expander system are mounted in a contraption to tune the distance between them with $\mu \mathrm{m}$ precision.

In Fig. 5.16 four predicted beam profiles using a single water immersion objective with a focal length by itself, in combination with a BFPL, a FL, and a beam expander lens combination are illustrated along with the size and shape of a TRPS pore. The focal plane of the objective is equivalent to the horizontal broken lines. While the resulting beam width at the focal plane using the objective lens alone or in combination with a BFPL is smaller than the radius of the pore orifice, it is larger for the FL and the beam expander system. A beam profile that is axially symmetric around the pore orifice is achieved with the BFPL and the beam expander system. The FL, which is easier to install and to align than the beam expander system, produces a highly divergent beam with its waist far below the pore orifice. The most uniform illumination of the pore orifice can be achieved with the beam expander system. As the outline represents the beam width at which the intensity has fallen to $\approx 13.5 \%$ of the central intensity, uniform optical signals are not expected for any of these systems and significantly larger beam sizes would be required.

Larger beam sizes can be achieved with a higher ratio of $f_{2} /\left|f_{1}\right|$ or when the distance $L_{2} L_{3}$ is reduced. This is either difficult to implement due to alignment reasons or not feasible due to the space constriction. Figure 5.17 shows the required 


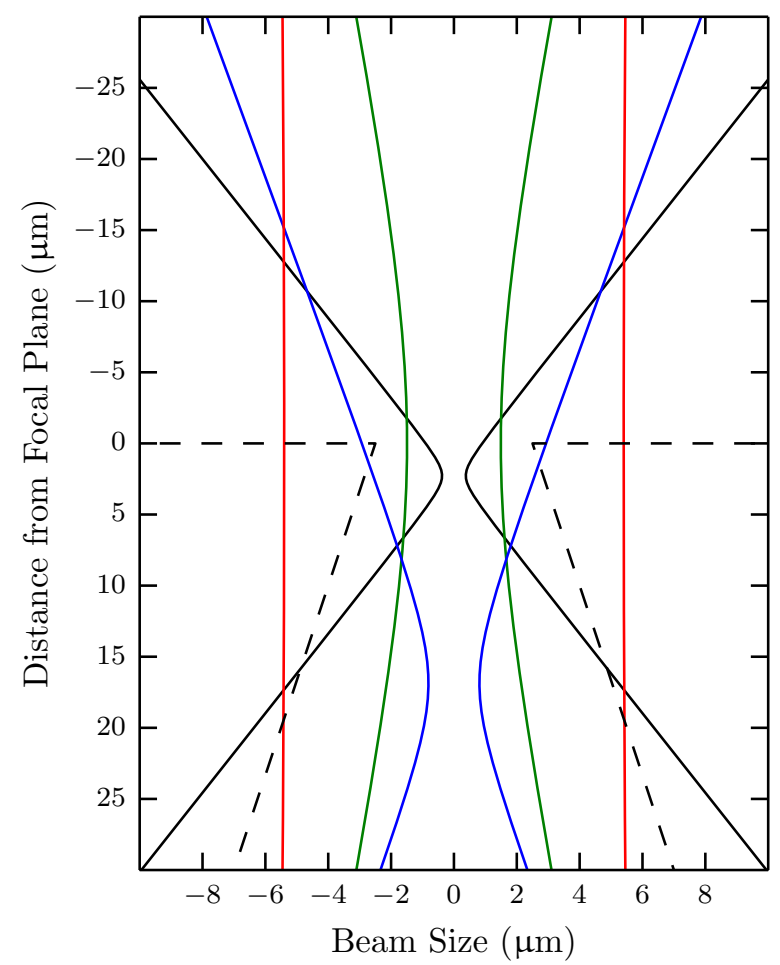

Figure 5.16: Comparison of the beam profiles using a single water immersion objective lens (black line) with a focal length (in air) of $1.8 \mathrm{~mm}$, an additional BFPL (green line) with a focal length of $500 \mathrm{~mm}$ (distance between the BFPL and the objective lens $\left.L_{1} L_{2}=651 \mathrm{~mm}\right)$, a FL with $300 \mathrm{~mm}$ focal length $\left(L_{1} L_{2}=600 \mathrm{~mm}\right)$ and a combination of two lenses with $f_{1}=-18 \mathrm{~mm}$ and $f_{2}=+50 \mathrm{~mm}\left(L_{1} L_{2}=\right.$ $\left.36.77 \mathrm{~mm}, L_{2} L_{3}=600 \mathrm{~mm}\right)$. The - - lines indicate the typical size and shape of the pores used for the tunable resistive pulse sensing experiments at the $\mu \mathrm{m}$-scale.

distances $L_{1} L_{2}$ between the standard lens combination and the resulting beam waists as a function of $L_{2} L_{3}$, assuming there is no restriction in the position of the lenses. A beam waist of over $160 \mu \mathrm{m}$ can be achieved when the system is positioned $100 \mathrm{~mm}$ from the lens. Caution has to be taken when the beam expander system is used so that the beam is not apertured by any element of the optical setup, e.g. the dichroic filter. For high ratios of $f_{2} /\left|f_{1}\right|$, it has to be ensured that the paraxial approximation is valid throughout the optical train, meaning that the smallest spot size must be larger than $\approx 2 \lambda / 3$. The big advantage of the beam expander system is that the beam width at the focal plane of the objective can be continuously tuned by simply changing the distance between the two lenses. Although the condition that the beam waist is equal to the focal plane is not fulfilled for most of the distances (Fig. 5.14), the method can still be used to engineer the illumination for the wide range of pore sizes used in TRPS with simple optical components. 


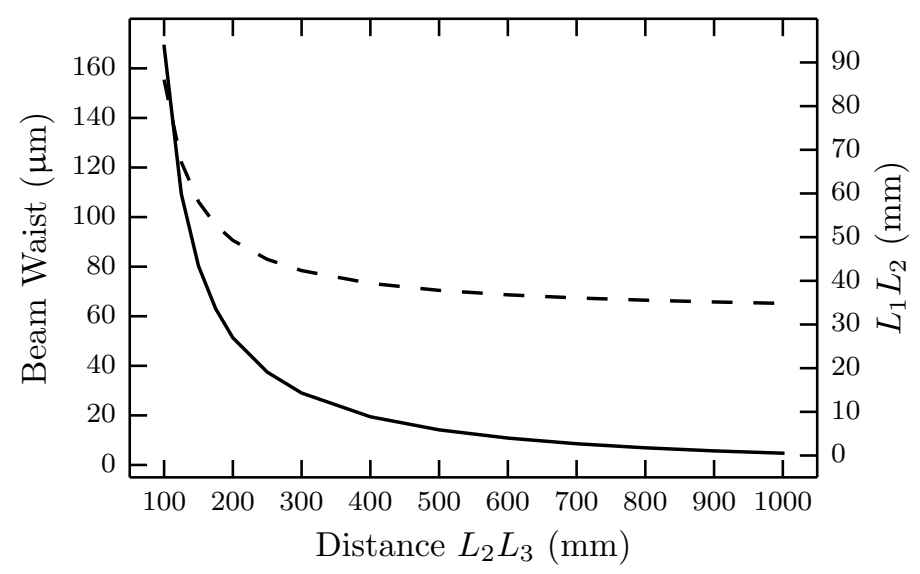

Figure 5.17: The beam waist (-) and the required distance (- -) between two lenses $L_{1}\left(f_{1}=-18 \mathrm{~mm}\right)$ and $L_{2}\left(f_{2}=+50 \mathrm{~mm}\right)$ of the beam expander system as a function of the distance $L_{2} L_{3}$ to the objective lens in order to to achieve the situation depicted in Fig. 5.13.

\subsection{Experimental Verification}

Experimentally the width of a laser beam can be measured with the "scanning-knife method" (or "edge-scan" technique) [218]. The laser beam is scanned laterally over a sharp edge of a uniform, strongly absorbing sample. The fluorescence or Raman intensity, originating from the sample surface, is registered and fitted with Eq. 5.22 to obtain the beam width. With the edge of the sample at $x_{0}$ and assuming a scan in $x$-direction the intensity $I(x)$ of the signal is given by

Table 5.2: The calculated and measured beam width at the focal plane $w(f)$ of an $\times 10$ air and a $\times 20$ water immersion objective in combination with various lenses positioned at a distance $\sim 600 \mathrm{~mm}$ from the objective lenses. The $514 \mathrm{~nm}$ line of an Ar-ion laser was used.

\begin{tabular}{ccc}
\hline Objective-Lens Comb. & $w(f)_{\text {calc }}(\mu \mathrm{m})$ & $w(f)_{\text {meas }}(\mu \mathrm{m})$ \\
\hline$\times 10$ & 9.00 & 9.32 \\
$\times 10$ BFPL500 mm & 15.38 & 14.74 \\
$\times 10$ FL300 mm & 29.30 & 25.83 \\
$\times 10$ BFPL-18/ $+50 \mathrm{~mm}$ & 55.64 & 58.31 \\
\hline$\times 20$ & 4.5 & 4.62 \\
$\times 20$ BFPL500 mm & 7.69 & 6.03 \\
$\times 20$ FL300 $\mathrm{mm}$ & 14.65 & 12.16 \\
$\times 20$ BFPL-18/ $+50 \mathrm{~mm}$ & 27.63 & 27.09 \\
\hline
\end{tabular}




$$
I(x)=\frac{I_{0}}{2}\left(1+\operatorname{erf}\left(\frac{\sqrt{2}\left(x_{0}-x\right)}{w}\right)\right)
$$

with erf the error function. It has to be ensured that any pinhole or slits used in the detection optics are sufficiently open to not aperture the collection path. The method is simple and easy to implement but its accuracy decreases for small spots due to limitations in the positioning and additional diffraction effects. It can therefore not be used for beam widths smaller than $2 \mu \mathrm{m}$ [190]. As the width using the $\times 50$ or $\times 100$ objectives used for most of the calculations above is expected to be too small to allow an accurate measurement with this method, the beam widths at the focal plane of two objectives with low magnification $(\times 10$ and $\times 20$ immersion) in combination with various lenses were measured.

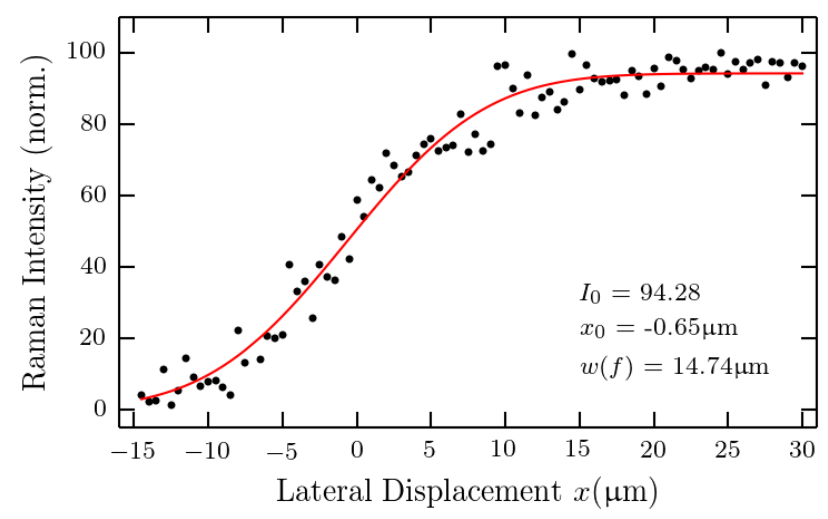

(a)

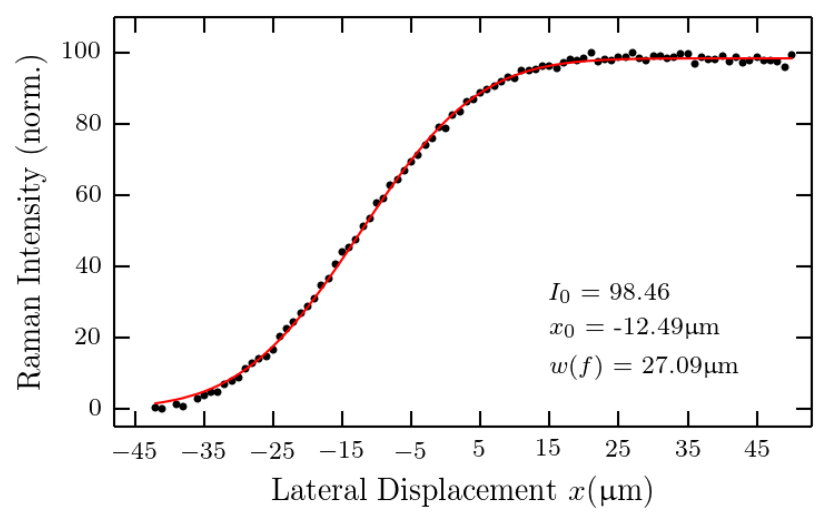

(b)

Figure 5.18: The Raman intensities of the $520.5 \mathrm{~cm}^{-1}$ mode of a silicon sample using the edge-scan technique and a (a) $\times 10$ objective with a BFPL with a focal length $500 \mathrm{~mm}$ and a (b) $\times 20$ water immersion objective with a $-18 \mathrm{~mm} / 50 \mathrm{~mm}$ beam expander system. The red line is the fit obtained with Eq. 5.22. The parameters of the fit are given in the plots.

Figures 5.18 (a) and (b) show the data for two of these measurements. The uncertainty is higher for the $\times 10$ objective due to the lower signal and a higher back- 
ground. The results for all objective-lens combinations are summarized in Tab. 3.5 along with the theoretical values using Self's approach. Experimental and calculated values are in good agreement, with the relative difference decreasing with larger beam size. This is expected as the measurement uncertainties are higher for small beams due to accuracy of the focussing and the method itself, which relies on a sharp edge. The method can also be used to obtain the beam width at any given axial position of the beam as long as the axial and lateral collection efficiencies for the optical detection permit this. In that way the beam parameters $w_{0}, \theta_{0}$ of the original laser beam can be deduced from measurements at two points. This was not applied here as the size of the beam width at the focal plane was of most interest and the laser was well characterised.

\subsection{Conclusion}

In this chapter Self's method was used to calculate the beam profile of a Gaussian beam that is being transformed by one or more lenses. It was shown that the usual concepts using geometric optics and its simplification, collimated ray optics, can lead to large discrepancies. The fact that a Gaussian beam is generally not exactly focussed at the focal plane of a lens is an often neglected fact in practice, which can lead to significant mistakes when considering the illuminated area and power density. Ways to engineer the beam in a typical laboratory situation with spatial constraints have been presented. The introduction of a back focal plane lens delivers good results. The required positions deviate from those intuitively expected. The maximal achievable beam size is limited with a BFPL alone. Using a tuneable beam expander consisting of a divergent and a convergent lens extends the range of beam sizes further. The advantage of the system is also that the width at the focal plane of the objective can be continuously tuned over a wide range of beam sizes. Experiments have shown good agreement with the predicted values for a range of various combinations of lenses and objectives. Theoretical and experimental tools developed here were implemented in the prediction of the optical signals and to engineer the beam size in the experimental parts of this thesis (Chaps. 6 and 7). Although the underlying theory used in this chapter has been long known, applied work in this form has not been published to the best of our knowledge and should be of benefit for a vast variety of experimental configurations. A manuscript presenting the results of this chapter has been published recently [219]. 


\section{Chapter 6}

\section{Detection of Microparticles - First Setup}

In this chapter, results from a series of coordinated electrical and optical measurements of micron-scale fluorescent particles using the first setup are presented and discussed. At a sufficiently low flow rate through the pore, controlled by the applied hydrostatic pressure, a matching rate of more than $90 \%$ between the detected fluorescence and RPS events could be achieved. Parts of this work have been published [220]. The setup requires minimal modifications to the qNano and is compatible with any microscope spectrometer system. It can be easily implemented and is therefore suitable for a temporary setup. However, for several reasons discussed at the end of this chapter, the detection is limited to the $\mu \mathrm{m}$-range. To improve the sensitivity of the technique a second setup, which requires more substantial modifications to both devices, was developed as a consequence. Its results are subject of the next chapter.

\subsection{Experimental Details}

For a basic description of the setup the reader is referred to Sec. 4.1.1. Here, only specific details relevant to the measurements presented in this chapter are given. The results were obtained in a series of experiments conducted with the very same pore and particle solution.

\subsection{1 qNano and Pore Specimen}

A pore specimen with the manufacturer's label NP2000, rated as most suitable for measurements of $1 \mu \mathrm{m}$ to $4 \mu \mathrm{m}$ particles, was used. To minimize the blocking probability of the pore the stretch between opposite jaws of the qNano was set to $48 \mathrm{~mm}$. An electric potential of $V=0.1 \mathrm{~V}$ was applied across the membrane, resulting in a baseline current of $\sim 150 \mathrm{nA}$. 


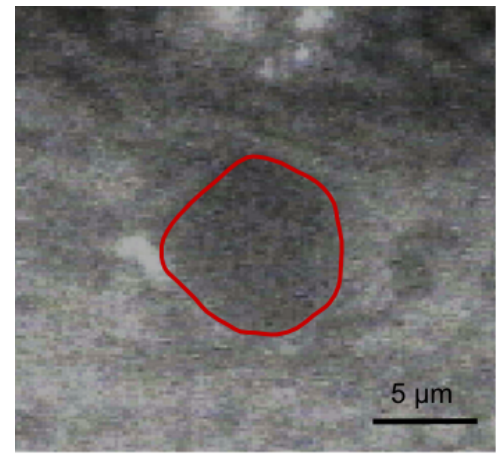

(a)

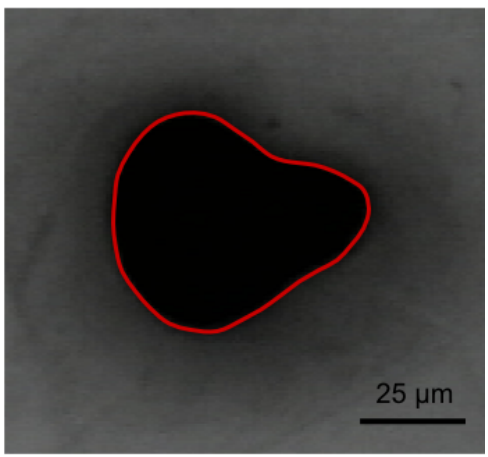

(b)

Figure 6.1: Optical image of the (a) small and (b) large pore opening of the pore specimen used. The red line shows the outline of the orifices used to determine the hydraulic radii.

In order to estimate the size of the laser spot required to illuminate the pore and to derive the particle size and concentration from the current peak height and event frequency via Eqs. 6.1 and 6.2, the dimensions of the pore had to be determined. Previous work [34] showed that the pores are not perfectly round in cross section. Therefore, the characteristic dimension for the pore radii is chosen to be the hydraulic radius, equal to $2 A / P$, where $A$ is the cross sectional area and $P$ the perimeter. Optical images of both pore apertures were taken using the microscope and the camera feature of the LabRAM. The images were then processed with the software Inkscape to obtain $A$ and $P$. The radii $a$ and $b$ (see Figs. 6.1 (a) and (b)) of the small and large pore openings at $48 \mathrm{~mm}$ stretch were deduced to be $4.1 \mu \mathrm{m}$ and $24.5 \mu \mathrm{m}$, with estimated measurement uncertainties of $10 \%$ and $5 \%$ respectively. The pore length $L$, which is equivalent to the membrane thickness, was measured by bringing distinct features of the upper and lower side of the membrane surface into focus and measuring the difference in the position of the translation stage. $L$ was measured to be $180 \mu \mathrm{m}$ for the pore specimen used, with an estimated uncertainty of $5 \%$.

\subsubsection{Optical Setup}

The use of the $458 \mathrm{~nm}$ line of an Ar-ion laser and a neutral density filter with an attenuation $D=1$ resulted in a total power at the sample of $0.4 \mathrm{~mW}$. The laser was focussed with a $\times 20$ long working distance objective from Olympus with a focal length of $9 \mathrm{~mm}$ and a numerical aperture of 0.35 . A $1000 \mathrm{~mm}$ back focal plane lens was used to enlarge the laser spot and achieve a more uniform, less divergent beam at the focal point of the objective. With the formalism presented in Sec. 5.3.2, the laser parameters (Tab. 4.2), and a total distance from the laser exit to the objective of $2500 \mathrm{~mm}$ (the distance between BFPL and objective is $1200 \mathrm{~mm}$ ), 
the beam waist was derived to be $3.40 \mu \mathrm{m}$, positioned $16.4 \mu \mathrm{m}$ above the objective focal plane. The beam spot at the focal plane of the objective, which is equivalent to the axial position of the small pore orifice, was $7.02 \mu \mathrm{m}$. Illumination across the pore diameter $(8.2 \mu \mathrm{m})$ was therefore not uniform but broad enough to detect the traversing particles. A variation in the optical signal depending on the actual trajectory of a particle through the pore is expected as the laser intensity at the width $w$ is $1 / e^{2}$ or about $14 \%$ of the intensity at the centre. A diffraction grating with 300 grooves $/ \mathrm{mm}$ was used to disperse the collected light before it was detected by the nitrogen cooled CCD.

The alignment of the laser with the centre of the pore was achieved using the microscope camera. Additionally, the low-frequency noise in the electrical trace caused by the laser illumination, discussed in detail in Sec. 4.5.1, was utilized for a more accurate alignment. A sudden increase in the current of up to $10 \%$ of the baseline current modulated with a low-frequency oscillations was observed when the laser shines on the pore orifice (Fig. 6.2 (a)). The amplitude of the noise is very sensitive to the laser position with the best alignment achieved at a maximized noise. An analysis of the frequency spectrum of the noise shows two peaks with frequencies of $20.5 \mathrm{~Hz}$ and $21.5 \mathrm{~Hz}$ (Fig. 6.2 (b)). The close proximity of the peaks leads to beating, which was observed at the frequency of $1 \mathrm{~Hz}$. The noise was filtered using a Finite Impulse Response (FIR) highpass filter algorithm with a cutoff frequency of $75 \mathrm{~Hz}$ and a Kaiser window of $20 \mathrm{~Hz}$ width.

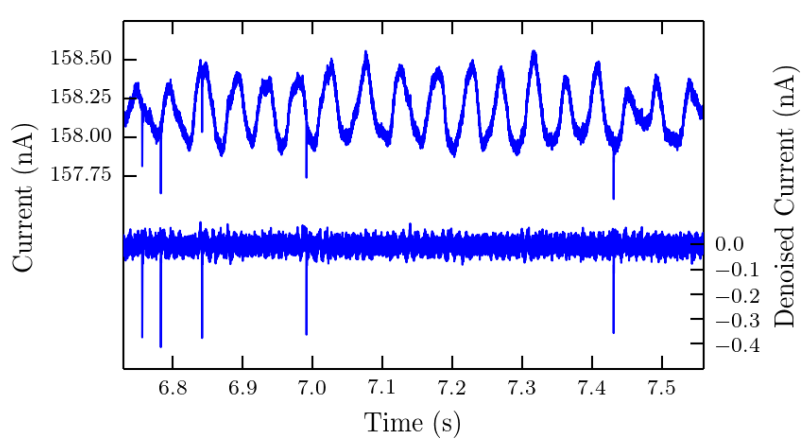

(a)

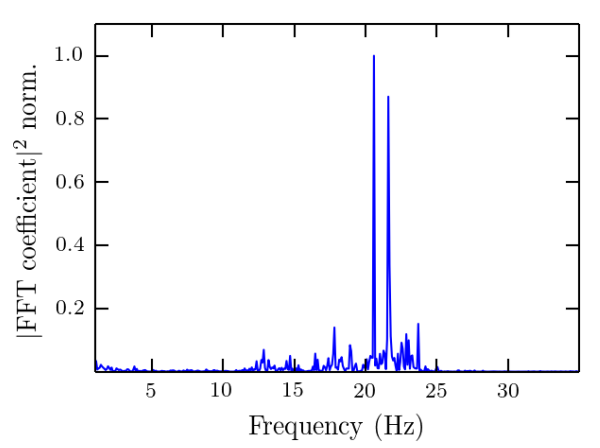

(b)

Figure 6.2: (a) The upper and lower line show part of the current trace (experiment D3) with several translocation events prior to and after digital filtering. (b) A frequency analysis of the raw current trace reveals the dominant frequencies of the noise.

\subsubsection{Sample}

The particles used in experiments are carboxylated polystyrene beads with a nominal diameter of $1.9 \mathrm{\mu m}$ purchased from Sigma-Aldrich. Fluorescent dyes with the manufacturer label "Yellow-Green" (YG) are embedded in the polymer matrix. The 
maxima of absorption and emission are at $470 \mathrm{~nm}$ at $505 \mathrm{~nm}$, stated by the supplier. The as-received solution was diluted with standard electrolyte buffer (SEB, see Sec. 4.3.2) by a factor of 5000 to a concentration of $\sim 1.33 \times 10^{6}$ particles $/ \mathrm{mL}$ and sonicated for several minutes prior to experiments. With the method described in Sec. 4.3.3 the total fluorescence cross-section of a single bead was measured to be $3.17 \times 10^{-9} \mathrm{~cm}^{2}$. Taking a typical cross-section of a fluorescent molecule, the estimated number of molecules per $2 \mu \mathrm{m} \mathrm{YG} \mathrm{bead} \mathrm{is} \mathrm{in} \mathrm{the} \mathrm{order} \mathrm{of} \sim 10^{7}$. Although this should only be understood as a rough estimate, it is, under the assumption of volumetric scaling, in agreement with the numbers obtained from the absorbance measurement presented in the last section using $220 \mathrm{~nm}$ particles.

\subsubsection{Control of the Particle Transport}

Both pore and particles are negatively charged, giving rise to electroosmotic and electrophoretic components of the particle transport through the pore. However, pressure-driven transport is expected to dominate at the $\mu \mathrm{m}$-scale and electrokinetic effects can be neglected (see Sec. 3.3). This makes the applied pressure the only crucial variable determining the fluid and therefore particle velocity through a pore with fixed dimensions. The simple pressure system used, consisting of a tube filled with electrolyte solution that is free to move in axial direction, was capable of controlling the pressure sufficiently precisely. All experiments presented here were conducted at hydrostatic pressures between $10 \mathrm{~mm}$ and $100 \mathrm{~mm} \mathrm{H}_{2} \mathrm{O}\left(1 \mathrm{~mm} \mathrm{H} \mathrm{H}_{2} \mathrm{O}=\right.$ $9.81 \mathrm{~Pa}$ ) across the membrane. Compared to the TRPS signals, which are recorded at a sampling rate of $50 \mathrm{kHz}$, the optical detection is much slower with a time resolution of $4.25 \mathrm{~ms}$ between two spectra. Therefore particles have to traverse the pore at a speed and frequency sufficiently low so that individual beads are optically distinguishable.

\subsection{Results and Discussion}

In the following section the data for five demonstrative experiments, conducted at different hydrostatic pressures but otherwise exactly the same parameters, are presented and discussed. Each experiment is identified by its label (D for downward, $\mathrm{U}$ for upward moving particles). The applied pressures and the numbers of identified events for each experiment are summarized in Tab. 6.1. The pressure values are known to an uncertainty of $\pm 1 \mathrm{~mm} \mathrm{H}_{2} \mathrm{O}$ for experiments D1 and D3. Experiment U1 was conducted at a negative pressure in order to drive particles upwards through the pore at a medium speed, and the pressure for D2 was set near the middle of the two known points. The pressure in experiment D4 was chosen to be $\sim 150 \mathrm{~mm} \mathrm{H}_{2} \mathrm{O}$, 
which resulted in blocking of the pore and premature termination of the current recording. The recorded time for each experiment is $9.686 \mathrm{~s}$, determined by the CCD on-board memory capacity, except for D4 (6.512 s).

Table 6.1: Details of five experiments with coordinated resistive pulse and optical measurements. Numbers of events which show no corresponding events in the other trace are given in brackets. The Wilson score [221] is a lower bound for the matching percentage at $97.5 \%$ confidence, assuming a binomial distribution. The average fluorescent intensity $F I$ is given in counts $\times 10^{5}$ with its relative standard deviation as a measure of signal uniformity.

\begin{tabular}{cc|cccc|cc}
\hline \multirow{2}{*}{ Exp. } & $\begin{array}{c}\text { P } \\
\left(\mathrm{mm} \mathrm{H}_{2} \mathrm{O}\right)\end{array}$ & TRPS & Optical & Matching & Double & Score & $F$ \\
\hline D1 & $10 \pm 1$ & $8(0)$ & $8(0)$ & $8(100 \%)$ & 0 & $76 \%$ & $21.60(36 \%)$ \\
U1 & $\sim-30$ & $22(0)$ & $21(0)$ & $20(91 \%)$ & 1 & $78 \%$ & $8.79(71 \%)$ \\
D2 & $\sim 70$ & $58(4)$ & $54(0)$ & $54(93 \%)$ & 0 & $87 \%$ & $8.76(86 \%)$ \\
D3 & $100 \pm 1$ & $82(3)$ & $76(1)$ & $71(87 \%)$ & 4 & $82 \%$ & $4.26(93 \%)$ \\
D4 & $\sim 150$ & $83(4)$ & $71(0)$ & $63(76 \%)$ & 8 & $70 \%$ & $5.15(107 \%)$ \\
\hline
\end{tabular}

Figures 6.4 (a)-(e) show the coordinated electrical and fluorescent intensity traces of the experiments. While 82 translocations can be detected in the current at high pressure $\left(100 \mathrm{~mm} \pm 1 \mathrm{~mm} \mathrm{H}_{2} \mathrm{O}\right)$, only 8 events are observed in the same time at low pressure $\left(10 \mathrm{~mm} \pm 1 \mathrm{~mm} \mathrm{H}_{2} \mathrm{O}\right)$. This approximate ratio of events (10:1) is expected as the volumetric flow rate is directly proportional to the applied pressure. The event rates for experiments U1, D2 and D4 are also consistent with the approximate applied pressures. Compared to a solution based method that relies on diffusion (diffusion constant $D$ of $0.23 \mu \mathrm{m}^{2} \mathrm{~s}^{-1}$, concentration $\sim 0.01$ particles / detection volume) a much higher throughput of more than 10 particles $\mathrm{s}^{-1}$ can be achieved in our setup. Figure 6.3 shows the optical trace for a typical experiment at this length scale using freely-diffusing particles, resulting in approximately only one event in $5 \mathrm{~s}$.

Figure 6.5 shows the spectrum and its time evolution for a particle moving through the pore at low pressure $\left(10 \mathrm{~mm} \mathrm{H}_{2} \mathrm{O}\right.$, experiment D1). A rise of the measured intensity to its peak height when the particle is at or close to the pore orifice can be observed. As the particle moves through the pore and out of the laser spot and the detection region, the signal decreases again towards a constant background level. The spectral shape stays constant in this process and single spectrum can be superimposed by multiplication. The maximum of the emission profile is consistent with the value stated by the supplier $(505 \mathrm{~nm})$.

While a resistive pulse in the current trace consists, depending on the particle speed, of up to a hundred data points covering a time span of $2 \mathrm{~ms}$, a corresponding 


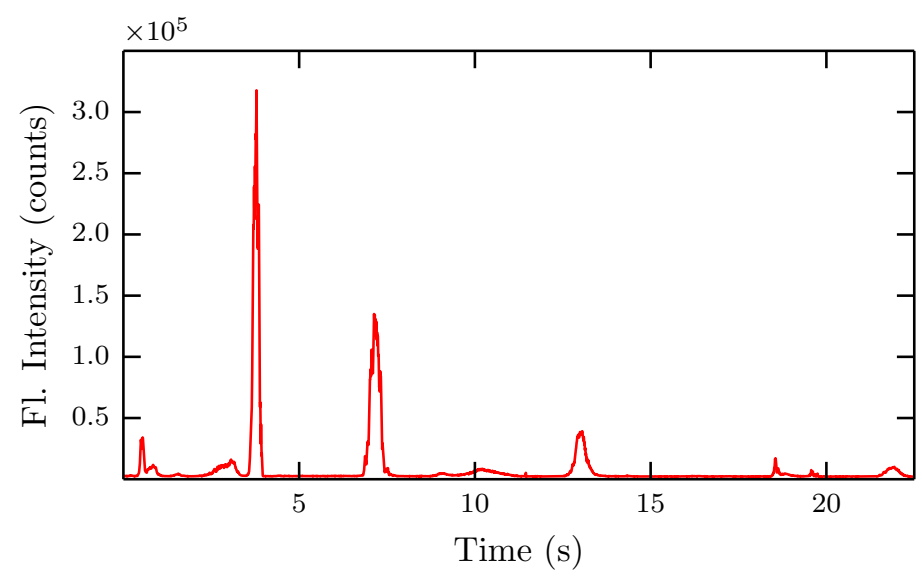

Figure 6.3: The time dependent fluorescence intensity of a solution with freelydiffusing $2 \mu \mathrm{m}$ particles with a concentration of $1.14 \times 10^{6}$ particles $/ \mathrm{mL}(D 2$, $\times 20$ BFPL500).

event in the optical signal trace is between one and five data points $(21.5 \mathrm{~ms})$ long. This is due to the difference in time resolution of the two techniques $(50 \mathrm{kHz}$ in the electrical and $235 \mathrm{~Hz}$ in the optical detection). The longer total time span of an optical event can be explained by the larger optical "sensing zone" compared to the region where the TRPS device is sensitive to a translocating particle. Simulations of both signals show that while an on-axis particle is still detectable optically within $\sim 150 \mu \mathrm{m}$ on either side from the pore orifice, the current is indistinguishable from the background value when the bead is just $\sim 20 \mu \mathrm{m}$ inside the pore. To illustrate the effect of the fluid velocity on the pulse shapes, three events representing slow (top), fast (middle), and a "double event" (bottom) are plotted in Fig. 6.6. It is clear that the width and therefore the number of data points used to sample each event corresponds to the velocity of the particle translocation. Two particles moving through the pore in close succession cannot be resolved in the fluorescent trace, and this is misinterpreted as one long event, called a "double event" in the following.

The average fluorescent intensity, which is listed in Tab. 6.1, is decreasing with increasing pressure and therefore particle speed. This can be explained by the shorter time period a particle that travels at a higher speed spends in the most sensitive region for the optical detection, which is close to the pore orifice. Note that the unit of the fluorescent intensity was changed from an arbitrary unit used in [220] to the total number of counts integrated over the CCD.

Table 6.1 summarizes the identified events in the 5 experiments. Along with the number of events in each individual trace, the number of matching events is given. A matching event is represented by exactly one pulse in each of optical and current traces. The matching rate is defined as the number of matching events divided by the amount of events in the resistive signal. Therefore the matching rate can still 


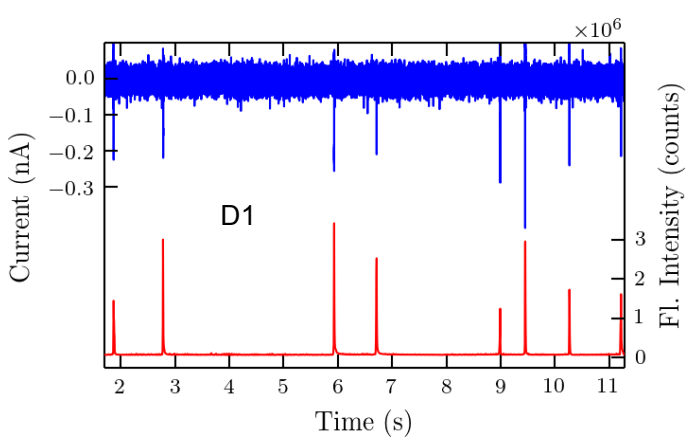

(a)

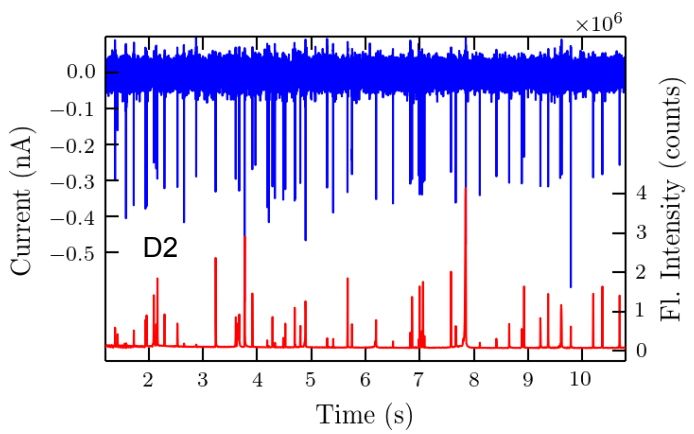

(c)

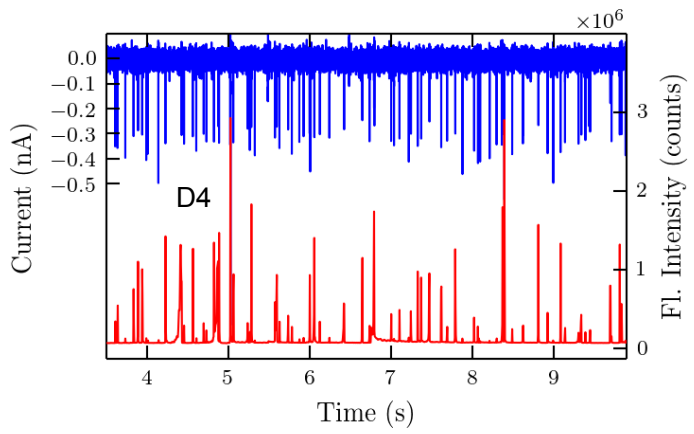

(e)

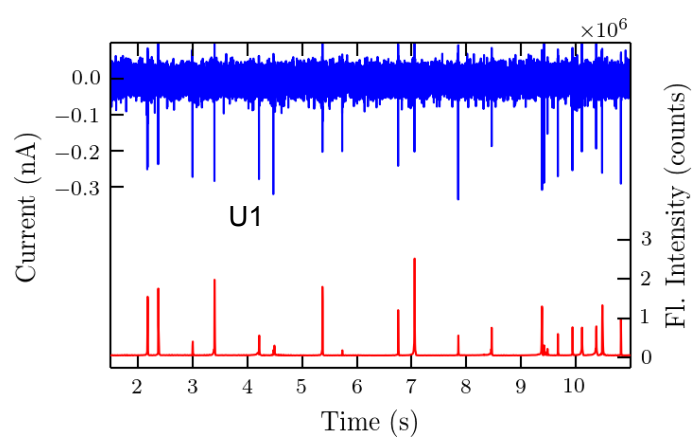

(b)

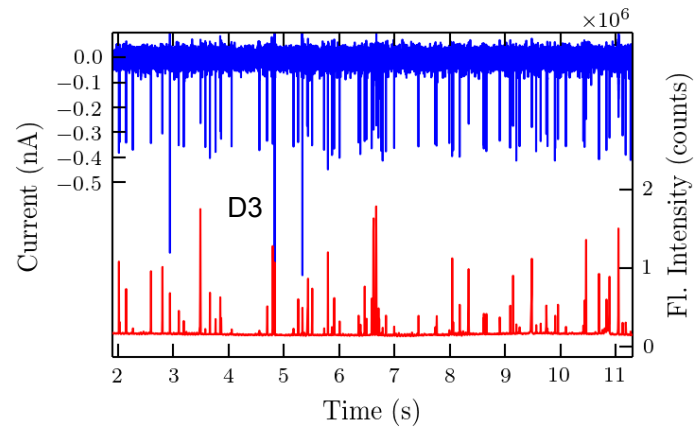

(d)

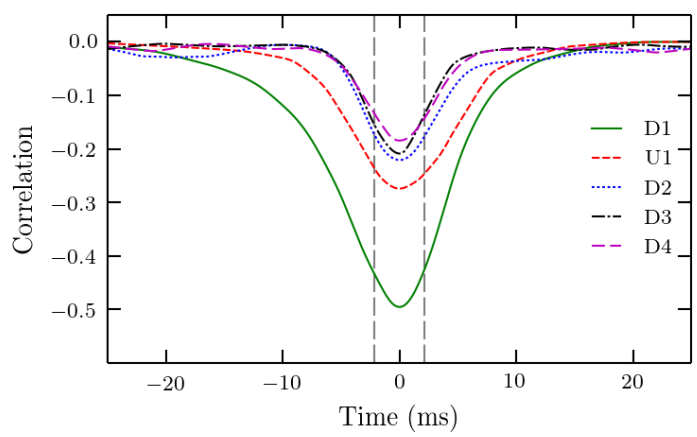

(f)

Figure 6.4: (a)-(e) Current (blue, upper) and fluorescence intensity (red, lower) traces for five experiments with increasing total hydrostatic pressure (see Tab. 6.1). (f) The correlation functions of the experiments. The sampling rate in the optical measurement of $4.25 \mathrm{~ms}$ is indicated with vertical dotted lines.

be $100 \%$ when all resistive events find a partner but some optical events find no corresponding partner. However, that does not occur here as the number of events observed in the current trace is always larger than in the optical trace, especially at high pressures. This is to some extent due to the effect that particles passing through the pore in close succession cannot be distinguished as different events in the optical trace. Some other events are not picked up by the event finding algorithm because their intensities are too small, but can be seen in the raw trace. Only one instance of a measurable optical signal that did not produce a resistive pulse is observed throughout all five experiments, showing "false positive" events caused by particles that are close to the pore but are not traversing it plays practically no role. There is a clear increase of double events and undetected events and therefore 


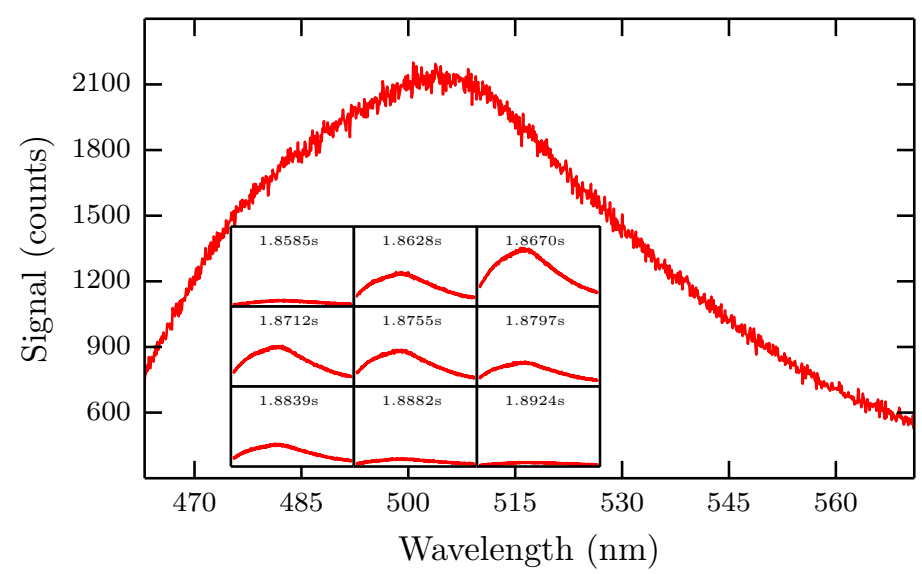

Figure 6.5: Fluorescence spectrum of a particle traversing the pore at low speed (experiment D1). The inset shows the signal intensity and the recorded time for 9 consecutive frames.
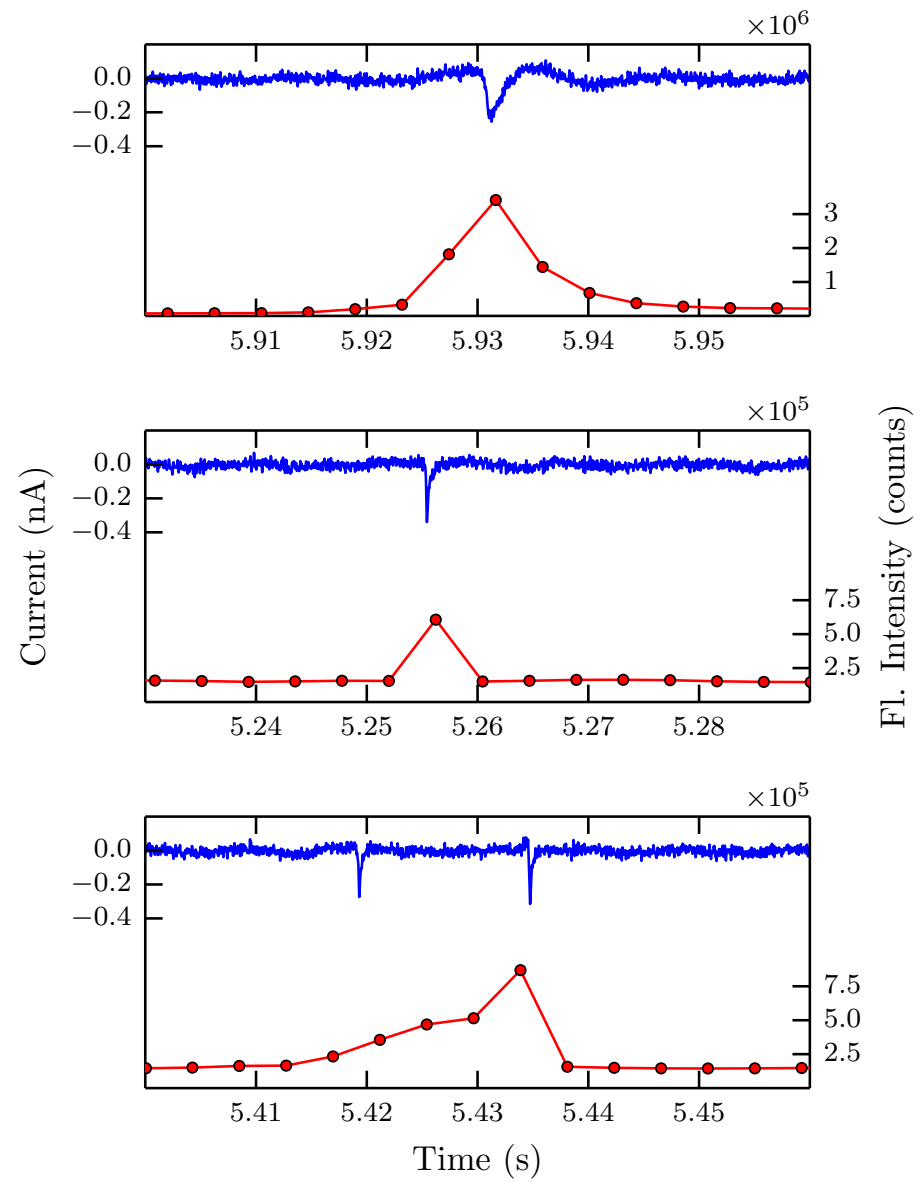

Figure 6.6: Typical events from experiments D1 (low-pressure, top) and D3 (highpressure, middle), and a "double event" (bottom) as described in the text.

a decrease of the matching rate as the pressure is increased. Depending on the particle concentration, the pressure can be adjusted to optimise the event frequency 
and therefore the matching rate of the correlated detection in our setup. The rate at which single resistive and fluorescence pulses are matched is recorded in Tab. 6.1, along with a lower bound at $97.5 \%$ confidence, assuming a binomial distribution for matched and unmatched events. Overall, the matching rate is very likely to be better than $83 \%$ in all experiments.

The correlation functions between the electrical and optical traces shown in Fig. 6.4 (f) were calculated using the Python function "conv" and were used to align the traces. The width of each correlation peak is related to the event width of the optical measurement and is limited by the sampling rate of $4.25 \mathrm{~ms}$. The peak height decreases with increasing pressure, which reflects the smaller matching rate and the fact that an increase in the number of events leads to more closely-spaced events.

The detected events in each experiment can be further statistically analysed. Here, experiment D3 is taken as an example as it shows a sufficient number of events in both traces and a high matching rate. The current peak heights (Fig. 6.7 (a)) and consequently the calculated particle sizes derived with Eq. 6.1 show a narrow distribution around the nominal diameter of $1.9 \mu \mathrm{m}$. As the current peak height is proportional to the particle volume [36], the three events which have approximately double the typical current peak height are most probably due to agglomeration of two particles.

The variation of the peak heights in the fluorescence trace is much greater (Fig. 6.7 (c)). The beads show a reasonably uniform fluorescence intensity (the relative standard deviation of the intensity for a similar particle set was determined to be $7.9 \%$, see Sec. 7.2.1) when immobilized on a silicon wafer and measured one by one, so the peak height variation cannot simply be explained by variations in the intrinsic fluorescence. The reason for the non-uniform fluorescence peak sizes is that the particles move through the pore on different paths. Some beads travel close to the centre, where the laser intensity is high, whereas it is smaller for beads travelling close to the pore walls. With the beam width of $3.51 \mu \mathrm{m}$, a bead travelling at the very edge of the pore is exposed to a laser intensity of about $20 \%$ of the intensity experienced by beads travelling through the middle. This value drops to $6 \%$ under the assumption that the laser centre is $1 \mu \mathrm{m}$ off-centre, which is a plausible value given the accuracy of the alignment using translation stages and the mechanical stability of the system. The distribution shows peak heights from $1.5 \times 10^{4}$ to $1.5 \times 10^{6}$ counts with the majority at the small end of the distribution, suggesting that most particles travel close to the pore walls.

To achieve a bigger spot size and therefore a more uniform illumination of the pore orifice either a BFPL with a shorter focal length or an objective with a longer focal length can be used. Implementation of the former is not feasible in our current 
setup as excitation and collection pathways are partly parallel an additional lens can only be used in the position before the notch filter, requiring a focal length of at least $1000 \mathrm{~mm}$. The latter leads to an increase in the axial detection height and therefore an increase in false positive optical events. Using the two lens system as presented in Sec. 5.4.2 could be a solution to the problem but has not been developed at the time of the conduction of these experiments.

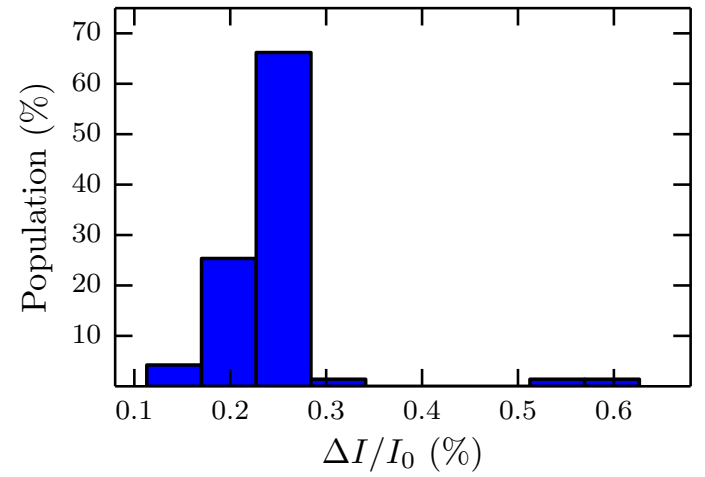

(a)

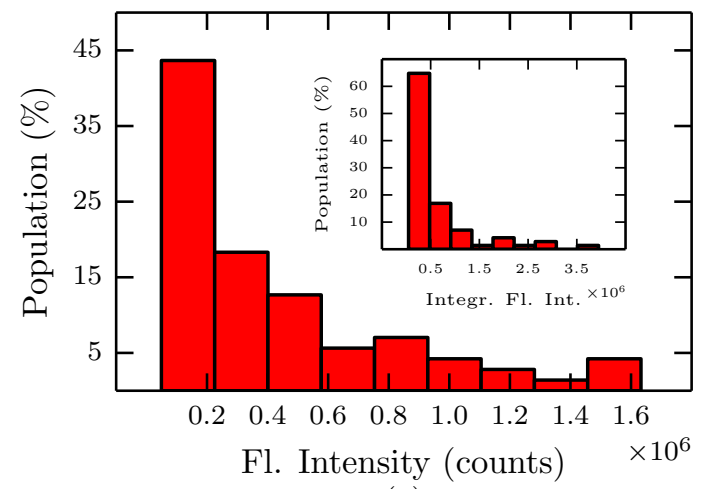

(c)

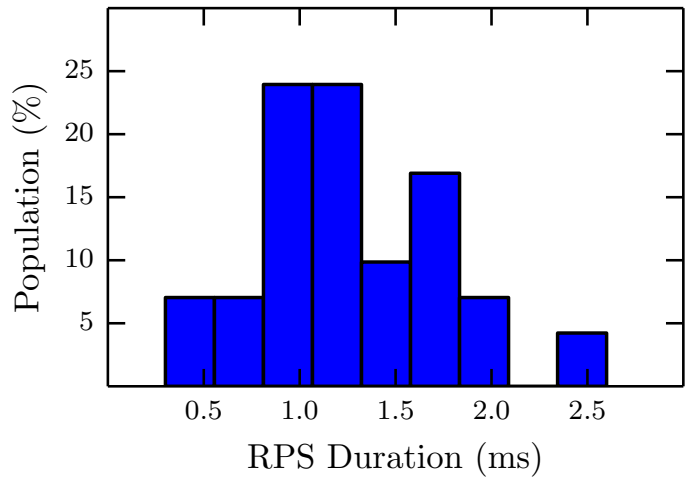

(b)

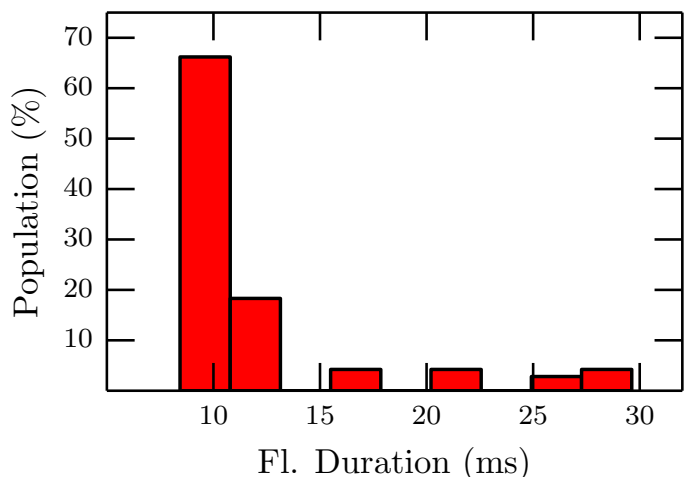

(d)

Figure 6.7: The distribution of (a) the resistive pulse heights, (b) the resistive pulse durations, (c) the fluorescence intensities and (d) the durations of the optical events for experiment D3. The inset in (c) shows the fluorescent intensity integrated over the duration of the optical events. Double events and events that find no corresponding partner have been excluded from the analysis.

The broad fluorescence peak distribution could also be explained by detection of multiple particles. However, this possibility can be excluded as the fluorescence signal usually falls to the background level between peaks, unless in the case of the double events discussed below. Furthermore, there is only one instance of a measurable optical signal that did not produce a resistive pulse. Many more such events would be expected if two or more particles were detected optically at the same time. Another possible explanation for the high variation in fluorescent peak heights could be the low sampling period compared with the relatively high particle velocity of up to $20 \mathrm{~mm} \mathrm{~s}^{-1}$ at the pore orifice. Undersampling can lead to an underestimation 
of peak heights as discussed in [167]. In our setup, the acquisition of the optical signal is continuous and each spectrum represents the counts in the sampling window. If undersampling is a factor, the integrated intensity over each fluorescence peak should be consistent, which is not the case (Fig. 6.7 (c) inset). Simulations of the expected optical signal with the experimental parameters, analogous to the ones in Sec. 4.4, also show that the low time resolution, even under consideration of the "dark" time of the CCD (the time proportion when the CCD is reading out the signal and is therefore insensitive to detection), cannot lead to the high signal variation observed.

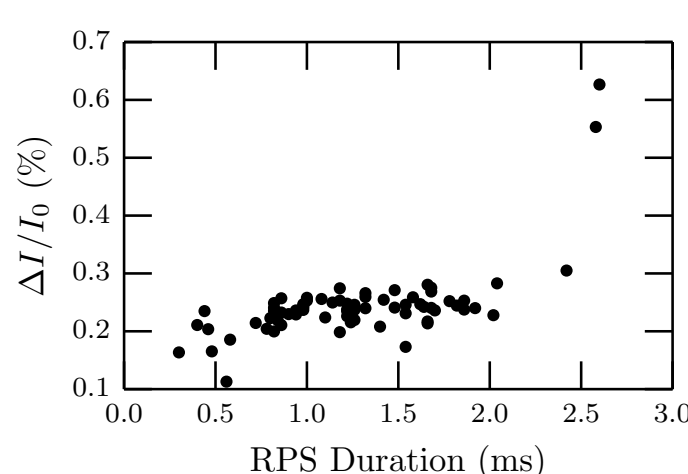

(a)

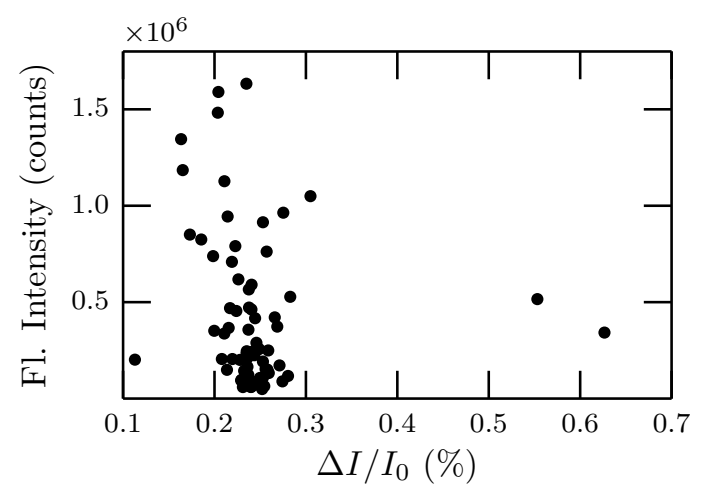

(c)

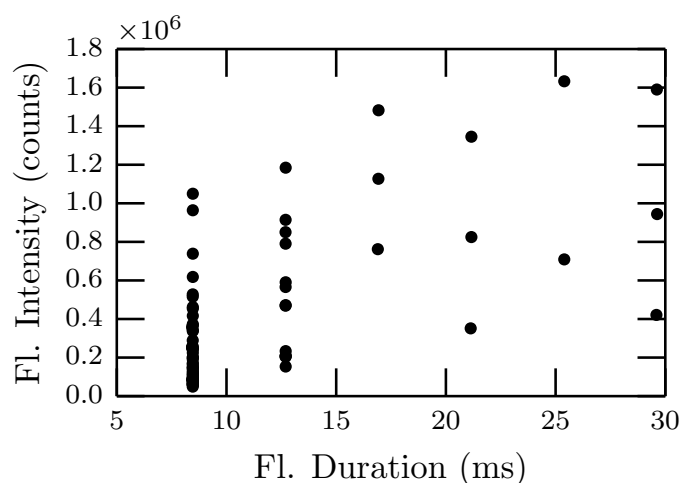

(b)

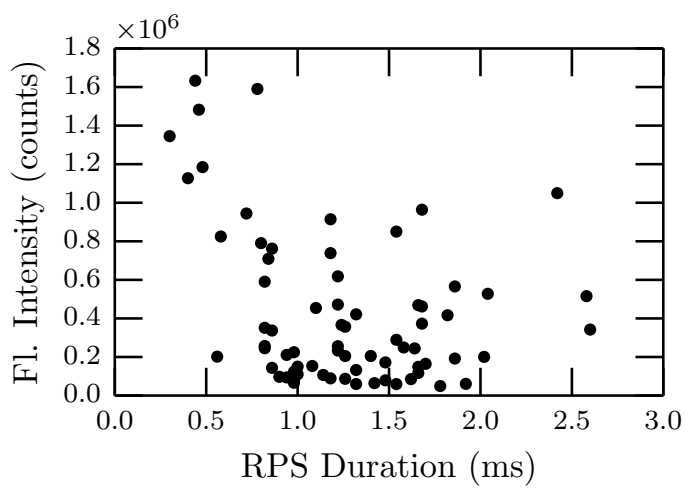

(d)

Figure 6.8: (a) The correlation between the resistive pulse durations and the resistive peak heights (Pearson coefficient: 0.62). Scatter plots of the measured fluorescence intensities with (b) the durations (0.64) of the optical events (c) the resistive peak heights $(-0.14)$ and $(d)$ the resistive pulse durations $(-0.34)$ for the experiment D3.

The duration of the current pulses, determined by the particle velocity, has a relatively broad distribution (Fig. 6.7 (b)). As shown in the FEM simulations in Sec. 3.4.4, the flow profile close to the pore orifice is highly non-uniform and becomes a parabolic profile several $\mu \mathrm{m}$ inside the pore. Particles travelling on offaxis trajectories will therefore generate longer current pulses with a higher duration. The distribution of the durations of the fluorescent signals in Fig. 6.7 (d) shows that a majority of the detected events are in the lowest bin around $9 \mathrm{~ms}$. This means that those events consist of only one datapoint above the background level. 
Only a minority of events are five or even six datapoints long. These events are caused by particles that travel very close to the pore's symmetry axis where the laser power, due to its relatively low divergence (the beam diameter reaches twice its width at a distance of $111 \mu \mathrm{m}$ away from its waist), is sufficiently high to excite the beads even a few hundred $\mu \mathrm{m}$ beyond the pore entrance. At the hydrostatic pressure of $100 \mathrm{~mm} \mathrm{H}_{2} \mathrm{O}$ an on-axis particle will traverse the pore within $10 \mathrm{~ms}$, or approximately two datapoints. The remaining datapoints for the "long" events are allocated when the particles are slowly approaching the pore close to its axis. Such events are naturally quite rare as most particles approach the pore off-axis (see the flow distribution discussed in Sec. 3.4) so that the optical signals are much shorter due to the rapidly falling laser intensity in the radial direction. Simulations of the optical signals using on and off-axis particle trajectories and pore geometry similar to the one used here are presented in the next chapter (Sec. 7.2) and support this claim, showing that the particle trajectory is the decisive factor determining the signal height.

Figure 6.8 shows several scatter plots of the electrical and optical characteristics of the detected events. In order to determine potential correlations between those, the Pearson coefficient [222], which measures the linear correlation between two variables, was calculated for each of the plots. Hereby a value of 1 implies a positive, -1 a negative and 0 no correlation at all.

A strong positive correlation of 0.62 is found between the durations and the heights of the resistive peaks (Fig. 6.8 (a)). This can be explained with the slow fluid velocity but high electric field and therefore current density at the pore edge (compare Secs. 3.4.4 and 3.4.2). A particle that travels close to the pore walls causes a long and large resistive pulse. The correlation between the durations and the heights of the fluorescent signals (Fig. 6.8 (b)) is also positive (Pearson coefficient of 0.64 ). This is due to the fact that particles travelling close to the pore centre are exposed to a higher laser intensity for a longer period of time. A large optical signal is therefore expected to have a short and small corresponding resistive pulse, resulting in a negative correlation between the two measurements.

This is also observed in the correlation between the fluorescent intensities and the durations of the resistive pulses (Fig. 6.8 (d)), which has a value of -0.34 and confirms that off-axis particles travel at a slower velocity and closer to the pore walls, where the laser intensity and therefore the detected optical signal is lower. The correlation between resistive pulse heights and the fluorescence intensities (Fig. 6.8 (c)) is also negative (-0.14). Interestingly, the two events which originate from particle dimers (the third such event had to be excluded as it was part of a double event) do not result in a significantly higher optical signal. Both values for the cross correlation between the electrical and optical signals are not very high, indicating only a weak 
negative correlation. This is most likely due to a slight misalignment of the laser. Additionally, photo-bleaching could play a role in the experiments presented in this chapter as the laser power density used was comparable to values that trigger photobleaching in immobilized beads (compare Sec. 4.3.3). Photo-bleaching would mostly affect on-axis particles and would therefore result in a weaker correlation.

Although the variation in the optical signals was originally not desired, it appeared to be beneficial as additional qualitative information about the particles could be obtained through it. The observations also lead to the simulations of particle trajectories using FEM and implementing the ALE method to determine particle-field interactions. One finding that resulted from these investigations was that the current pulse depends on the trajectory of the particle and causes intrinsic broadening of the current peaks, both in size and duration.

Using the pore-resistance model described in [80], the particle diameter can be calculated for each event from the observed current peak magnitude $\Delta I$ with the expression

$$
d=\sqrt[3]{\frac{4 \pi a^{4}}{\rho}\left(\frac{V}{I_{0}-\Delta I}-\frac{V}{I_{0}}\right)} .
$$

$I_{0}$ is the background current when there is no particle in the pore and $\rho$ is the resistivity of the electrolyte $(0.86 \Omega \mathrm{m}$ for a $0.1 \mathrm{M} \mathrm{KCl}$ solution, from [92]). With the pressure-flow model developed in [38], the fluid flow through the pore and therefore the translocation speed of the particles and their event frequency are linearly dependent on the applied pressure. The flow rate $Q\left([Q]=\mathrm{m}^{3} \mathrm{~s}^{-1}\right)$ through a conical shaped pore can be evaluated using [40]

$$
Q=\frac{P}{\eta}\left(\frac{a^{3} b^{3}}{\frac{3}{2}\left(a^{3}+b^{3}\right)+\frac{8 L}{3 \pi}\left(a^{2}+a b+b^{2}\right),}\right),
$$

where $P$ is the hydrostatic pressure across the pore, $\eta$ the viscosity of the electrolyte (1.002 $\mathrm{mPas}$ for $\mathrm{H}_{2} \mathrm{O}$ at $293 \mathrm{~K}$ [223]) and $L$ the pore length. $L$ is equivalent to the membrane thickness and is dependent on the applied stretch. The particle concentration $c\left([c]=\right.$ particles $\left./ \mathrm{m}^{3}\right)$ can then be calculated from the particle count $J\left([J]=\right.$ particles $\left.\mathrm{s}^{-1}\right)$ with the relation $c=J / Q$.

For each experiment, the average particle size and the particle concentration are calculated using Eqs. 6.1 and 6.2 and are summarised in Tab. 6.2. The values for the particle diameter agree very well with the nominal value of $1.9 \mu \mathrm{m}$ claimed by the supplier. The nominal concentration deduced from the supplier information and the 
Table 6.2: The mean diameter $d_{\text {calc }}$ and the concentration $c_{\text {calc }}$ are calculated with Eqs. 6.1 and 6.2 respectively. Diameter uncertainties in brackets represent the relative standard deviation. The concentration is given in $10^{6}$ particles $/ \mathrm{mL}$.

\begin{tabular}{ccc}
\hline Exp. & $d_{\text {calc }}(\mu \mathrm{m})$ & $c_{\text {calc }}$ \\
\hline D1 & $1.85(8.5 \%)$ & 1.10 \\
U1 & $1.88(5.2 \%)$ & 1.01 \\
D2 & $1.93(7.8 \%)$ & 1.14 \\
D3 & $1.91(8.5 \%)$ & 1.12 \\
D4 & $1.86(9.2 \%)$ & 1.13 \\
\hline
\end{tabular}

dilution of 1:5000 is $1.33 \times 10^{6}$ particles $\mathrm{mL}^{-1}$, slightly higher than the values derived from the particle count. Taking into account uncertainties due to the dilution and the pore dimensions, the values are in reasonable agreement. Using the rules for the propagation of uncertainties, the dominant uncertainty is that of the small pore diameter (10\%), suggesting an absolute uncertainty of at least $13 \%$ in the size and $30 \%$ in the concentration measurements. Uncertainties in the other pore dimensions and the resistivity and viscosity do not contribute significantly to the overall error.

Taking into account that Eq. 6.1 (Maxwell's approach, compare Sec. 3.4.3.1) is derived for an on-axis particle in a cylindrical pore, the agreement of the measured and nominal particle diameter is surprising at first. It has been shown that the model underestimates the particle size (see Fig. 3.14 and Tab. 3.1) for a conical pore. However, as most particles travel on off-axis trajectories, resulting in larger pulses compared to an on-axis particle, the average pulse height is actually close to Maxwell's prediction. In that way the effects of two simplifications compensate each other. The results show that a good estimation of the particle size and concentration using an optical determination of the pore dimensions and simple resistive and flow models is possible. For pores in the sub- $\mu \mathrm{m}$ range this method should not be used as the uncertainty in determining the small pore dimension will be significantly larger. The usual, more accurate, method for TRPS measurements is to use calibration beads of known size [36] or concentration [34].

\subsection{Further Discussion and Possible Improvements of the System}

The experiments show a good matching rate, however the fact that the "sensing zone" of the optical detection is relatively large can result in an unacceptable amount of double events. The success of the experiments presented above is partially also 


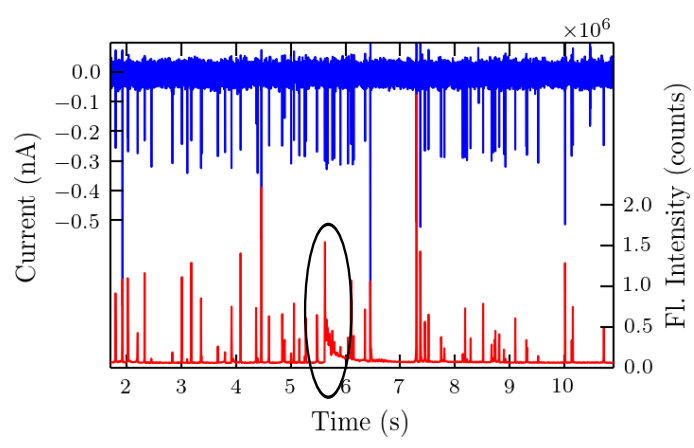

(a)

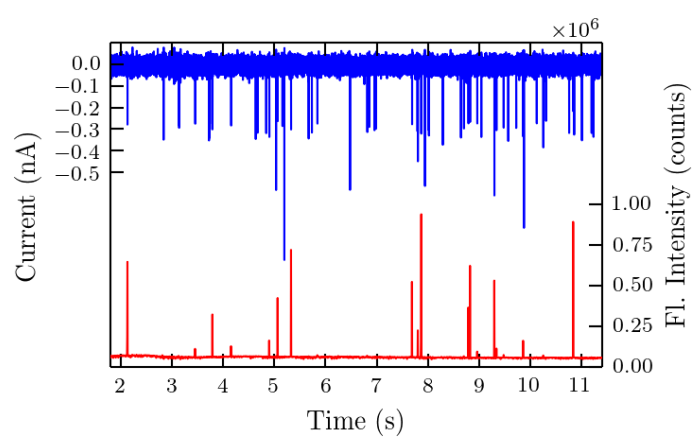

(b)

Figure 6.9: Current (blue, upper) and fluorescence intensity (red, lower) traces for an experiment (a) showing a multi event and (b) using a $\times 50$ objective.

dependent on the high dilution used. The dilution was five times as large as recommended for this kind of pore as otherwise too many double events would occur. This limits the usability and accuracy of the technique significantly as usually a high throughput is desirable. Figure 6.9 (a) shows a "multi event" in an experiment with upwards moving particles. The event is probably caused by a particle travelling onaxis and causing a very long optical signal of at least 20 data points and considerable peak height so that five following events are mostly hidden in its tail. This is due to the relatively low divergence of the laser beam and the large axial detection profile. Both are the reason for the large optical "sensing zone" so that many double or even multi events will be detected at a high particle concentration. In this context it shall be mentioned that the effective height for the axial detection $H_{\text {eff }}$ quoted in [220] was obtained wrongly as $250 \mu \mathrm{m}$. As discussed in Sec. 4.2.3, the laser intensity was not sufficiently uniform, so the results of the scanning method were misinterpreted. The correct effective height will be even higher than the value of $632 \mu \mathrm{m}$, obtained with a $\times 20$ immersion objective, as the use of a glass slide enlarges the axial focus $[224,225]$. However this detail does not affect the interpretation of the results as these were qualitative in nature.

To get a higher throughput and reduce the number of double or multi events, the sensing zone of the optical detection has to be reduced. This can be achieved by either designing a more divergent laser beam with a rapidly falling intensity or a smaller axial detection efficiency. Both can be achieved by using an objective with a shorter focal length. With a $\times 50$ long working distance objective a much narrower detection was achieved and no multi events were detected in the optical trace (Fig. 6.9 (b)). However many particles could not be detected due to the smaller laser beam width, which is a direct consequence of the laser beam product being constant. Therefore the non-uniformity of the optical signals increases and prevents the detection of particles travelling near the pore edges. To compensate for this effect and increase the spot size again the use of additional lenses to engineer the 
beam is necessary. As excitation and collection pathways are partly parallel and a field lens can be used only in the position before the notch filter, the choice of lenses is limited to a focal length larger than $1000 \mathrm{~mm}$ in the specific setup, which is not sufficient to obtain the desired beam width. The use of a more complex tuneable beam expander system consisting of a divergent and a convergent lens could be a solution to this problem in future.

Another drawback of the system and a limiting factor for the detection of smaller particles is its mechanical stability. The lateral positioning is difficult as the qNano, having a considerable weight, has to be placed on translation stages and cannot be firmly clamped to the platform, resulting in enhanced vibrations. Additionally it has proven to be difficult to locate and accurately focus on the pore orifice using highly magnifying objectives. While at the $\mu \mathrm{m}$-scale this is not a major problem and particles can still be resolved with sufficient accuracy, it would definitely play a role for smaller pores and particles. Although $500 \mathrm{~nm}$ particles could be detected with this specific setup, the results were not consistent enough to be presented.

A further closure of the pinhole in the collection path which was $1000 \mu \mathrm{m}$ throughout the experiments above is not expected to play a role. A pinhole lens with a longer focal distance should be installed to independently regulate the detection area and height. In that way objectives with longer focal length could be used, resulting in a larger beam width and only requiring a simple lens for beam spot engineering, reducing the complexity of the setup.

Due to the relatively high laser power the noise is an issue in this experiment. It will be shown later that with the second setup sufficiently large optical signals could be achieved at lower laser powers. The possible reduction of signal due to photo-bleaching was not considered for the experiments presented here. The laserinduced noise was relatively high, but could be eliminated in the current traces with a FIR filter. As shown in Sec. 4.1.2 this can lead to an overshoot problem when the frequency regions of noise and pulses are too close. However as the frequency is lower than in the second setup the filtering is relatively successful. The use of the wavelet filter avoids the overshoot problem altogether.

The fluorescent beads we are using are bright compared to biological samples. To increase the optical signal when smaller or less intense samples are measured, an objective with a higher numerical aperture and/or a higher laser power has to be used. There is also possible improvement in the efficiency of the spectrometer, which was measured to be $\sim 3 \%$. That means that only one in 30 photons collected by the objective is detected on the CCD. The rest are lost mainly due to imperfect mirrors, the grating and the quantum efficiency of the CCD. 


\subsection{Conclusion}

In this proof of principle study we have demonstrated the coordinated detection of colloidal microparticles using two complementary techniques. Tunable resistive pulse sensing data were used to measure particle size and particle concentration, while optical measurements add full spectral information on a particle-by-particle basis. The data show close correlation in time and by slowing the fluid and therefore event frequency, a matching rate in excess of $90 \%$ can be achieved. Ongoing work will focus on experimental development in order to extend detection to smaller particles. The technique can also be exploited to study particles with different emission spectra but otherwise identical properties, suggesting possible applications in the biosciences. With improved optical detector efficiency, Surface-Enhanced Raman Spectroscopy (SERS) would become another candidate for optical coordination with TRPS. 
6.4. Conclusion 


\section{Chapter 7}

\section{Coordinated Detection using the Second Setup}

In order to address the problems and limitations experienced in the first setup, a second, improved setup was designed. It has been described in Sec. 4.1.1, which the reader is referred to. Here, only specific details about the conducted experiments are given. The chapter is structured into five sections. After a short introduction that shows a series of frequently encountered signals, it starts with comparison of the performance of the two setups on the basis of results from experiments conducted with similar particles and experimental parameters as presented in the previous chapter. Additionally, by using improved optics, the experiments are refined and the influence of particle speed and the size of the laser beam on the signal and the matching rate are studied. In the next section, a mixture of $1 \mu \mathrm{m}$ polystyrene particles with two different fluorescent labels is used to demonstrate the capability of the technique to distinguish between samples of the same size but different optical emission spectra. Going further down in size and therefore posing additional challenges, the results of the coordinated detection of particles with a diameter of $500 \mathrm{~nm}$ and $200 \mathrm{~nm}$ are presented and discussed in the fourth and fifth sections. Simulations are introduced where needed in order to understand and interpret the results.

\subsection{Introduction}

During the course of collecting data for this chapter, several characteristic signals, mostly undesired and causing difficulties for either the detection or the event finding algorithms, could be observed on a regular basis and are pictured in Fig. 7.1. An optical event that spans over two or more resistive pulses, as shown in (a), is usually connected with a particle that approaches the pore orifice on axis, and can reduce the matching rate significantly. The signals shown in Figs. 7.1 (b), (c) and (d) 
indicate a particle that is blocking the pore, or attached to the surface near the pore entrance, either permanently (b), for a long time (c), or for a short time (d). While (c) and (d) may make a part of one experimental run unusable, (b) causes bigger problems, and often the pore has to be cleaned thoroughly as a consequence. The laser induced noise, which was the subject of Sec. 4.1.2 can also prevent a successful measurement. (f) pictures a situation where a large optical event causes a series of small pre-events which do not correspond to an actual translocation. These preevents are responsible for the majority of unmatched optical events in the traces presented in this chapter. It shall also be mentioned that large, optical events in (a) and (f) usually correspond to a small resistive pulse. The reason for that will be explained in the next section.

\subsection{Coordinated Detection of $2 \mu \mathrm{m}$ Particles}

\subsubsection{Experimental Details}

The number of detected events and therefore the length of the recorded trace is of statistical importance for the technique. At the full spectral resolution and a time step of $4.25 \mathrm{~ms}$ the recorded time is limited to $\sim 10 \mathrm{~s}$ due to the limited on-chip CCD memory. To extend this time, binning can be used, which reduces the spectral resolution of the measurement but still delivers acceptable results for fluorescent particles. Most spectra taken in this chapter are binned by a factor of 8 and traces with an overall time of $27.8 \mathrm{~s}$ are recorded. If the optical measurement is recorded with maximum spectral resolution the time is reduced to $9.7 \mathrm{~s}$, which is noted in the last column of the tables that summarize the results.

In the following the influence of the beam sizes on the detected signals is studied. Various back focal plane lenses and lens combinations were used to engineer the beam spot for both low and high magnification objectives $(\times 20$ and $\times 100$ water immersion). The resulting waists, the beam widths at the objective focal plane, and the Rayleigh ranges are summarized in Tab. 7.1. As these are frequently used throughout the chapter the combination of, for example, a $\times 20$ objective with a back focal plane lens with a focal length of $500 \mathrm{~mm}$ is abbreviated as $\times 20$ BFPL500.

For all traces presented in this section the same pore specimen was used. The stretch was set to $37.5 \mathrm{~mm}$ unless otherwise noted. The hydraulic radii of the small and large pore openings (Fig. 7.2) were determined optically to be $3.4 \mu \mathrm{m}$ and $13.4 \mu \mathrm{m}$ respectively. The membrane thickness is $175 \mu \mathrm{m}$. The pore was therefore slightly smaller than the one used in the previous chapter, resulting in larger relative resistive pulses and a lower fluid and particle flow at equal pressure. Although the stretch was lower, blocking of the pore occurred infrequently and also 


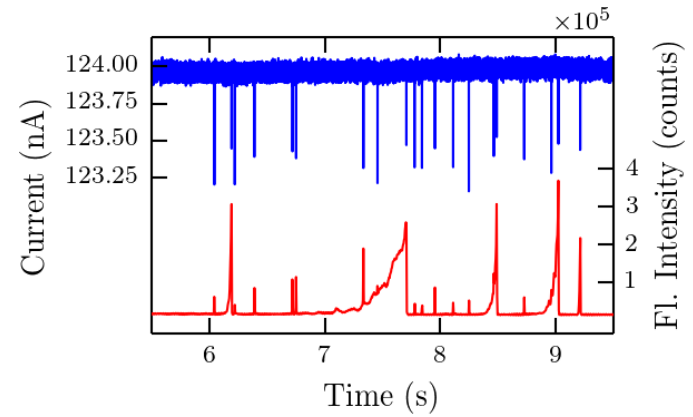

(a)

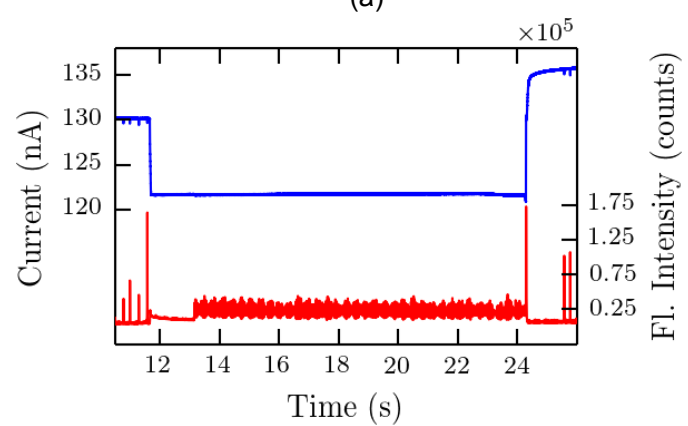

(c)

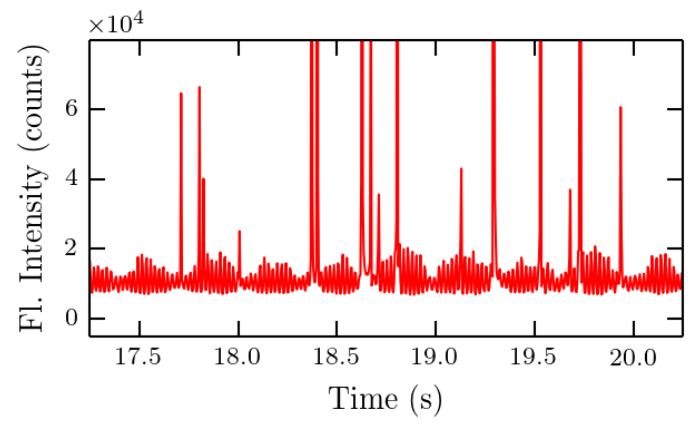

(e)

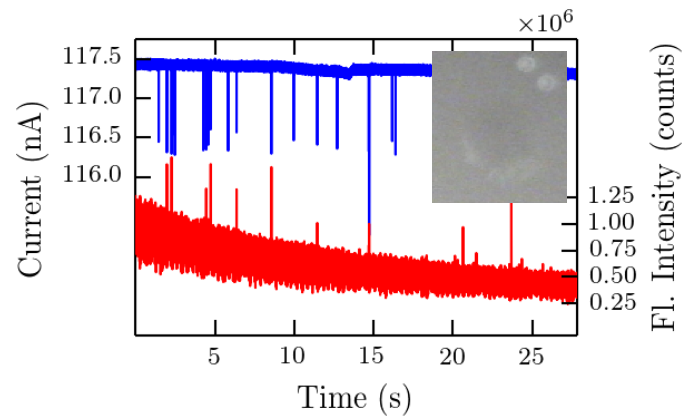

(b)

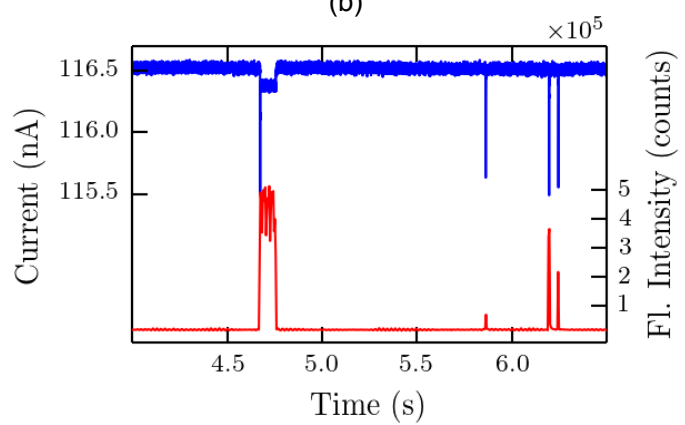

(d)

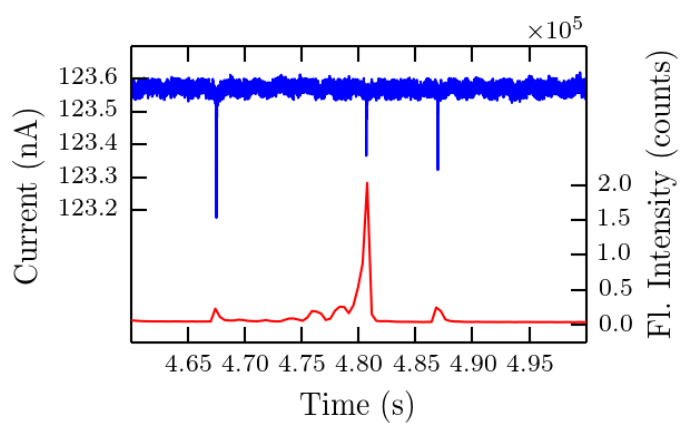

(f)

Figure 7.1: Artefacts observed using the second setup. (a) A long optical event that spans over several resistive pulses is caused by a $2 \mu \mathrm{m}$ particle. A particle is stuck (b) permanently (the inset shows the image of two particles attached close to the pore orifice), for (c) a long time or (d) a short time. (e) The laser induced low frequency noise as observed in a NP400 pore. (f) An optical event with small pre-events that do not correspond to any resistive pulse.

only temporarily so that a lot of data could be collected with the same pore specimen. This may be attributed to the design of the fluid cell (see Sec. 4.1.1) and the ability to use more surfactant in the second setup which increases the wetting of the pore. A voltage of $0.10 \mathrm{~V}$ was used unless otherwise noted.

The particles used in this section were purchased from Sigma-Aldrich and have similar specifications as the ones used in the last chapter. However, the beads are slightly larger according to the manufacturers specification (2 $\mu \mathrm{m}$ instead of $1.9 \mu \mathrm{m})$. Although a dye with the same label ("Yellow-Green") is used, the fluorescent profile appears slightly different (compare Figs. 7.3 and 6.5). In order to determine the uniformity of the fluorescence intensity of the beads, ten beads were measured 
Table 7.1: The beam waist $w_{0}$, its distance $\Delta$ from the objective focal plane, the beam width $w_{f}$ at the focal plane and the Rayleigh range $z_{R}$ of various water immersion objective BFPL combinations used in this chapter. The values were obtained with Self's formalism and the parameters from Tab. 4.2.

\begin{tabular}{cccccc}
\hline Objective & Lens $(\mathrm{es})$ & $w_{0}(\mu \mathrm{m})$ & $w_{f}(\mu \mathrm{m})$ & $\Delta(\mu \mathrm{m})$ & $z_{R}(\mu \mathrm{m})$ \\
\hline$\times 20$ & - & 1.89 & 4.50 & 57.14 & 26.53 \\
$\times 20$ & $500 \mathrm{~mm}$ & 7.42 & 7.69 & -109.89 & 407.25 \\
$\times 100$ & - & 0.38 & 0.90 & 2.28 & 1.05 \\
$\times 100$ & $500 \mathrm{~mm}$ & 1.50 & 1.54 & -3.98 & 16.52 \\
$\times 100$ & $300 \mathrm{~mm}$ & 0.81 & 2.93 & 16.87 & 4.86 \\
$\times 100$ & $-18 / 50 \mathrm{~mm}$ & 5.40 & 5.40 & 0 & 215.51 \\
\hline
\end{tabular}

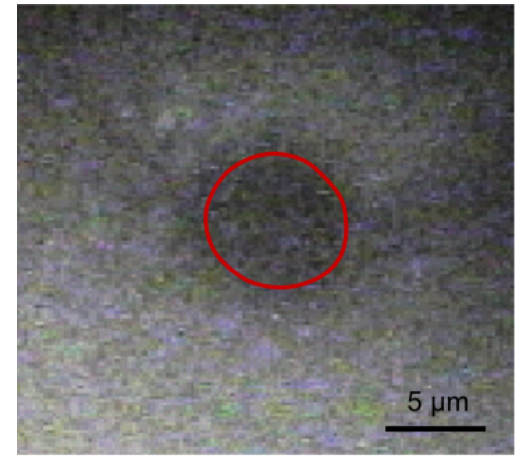

(a)

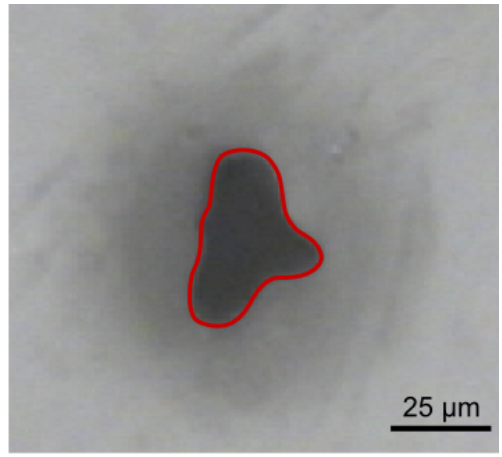

(b)

Figure 7.2: Optical images of the (a) small and (b) large openings of the pore specimen used in this section. The red line shows the outline of the orifices.

one-by-one after they have settled from solution onto a silicon chip. The relative standard deviation of the intensity was determined to be $7.9 \%$. The particles, with an undiluted concentration of $1.14 \times 10^{6}$ particles $/ \mathrm{mL}$ were suspended in the SEB buffer and sonicated prior to use. The concentrations used in each individual experiment are given along with other experimental parameters in Tab. 7.2.

Analogous to the experiments presented in the last chapter an objective with a low magnification $(20 \times)$ was used. However, due the design of the fluid cell, a water immersion with a slightly higher numerical aperture (0.5 compared to 0.35$)$ could be used instead of an air objective. The immersion objective was used alone and in combination with a lens $(\times 20$ BFPL500) to obtain a larger beam size. To ensure an axial collection profile that is symmetric and centred around the focal plane of the objective (Sec. 5.4.1), the diagonal of the square pinhole is chosen to be $100 \mu \mathrm{m}$. Therefore the lateral collection is given by a square with a side length of $16 \mu \mathrm{m}$ centred at the at the pore orifice. The FWHM of the axial detection efficiency, which was measured to be $400 \mu \mathrm{m}$ extends beyond the pore orifice. The $458 \mathrm{~nm}$-line 


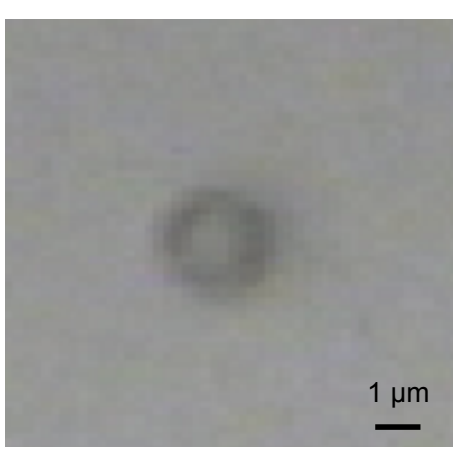

(a)

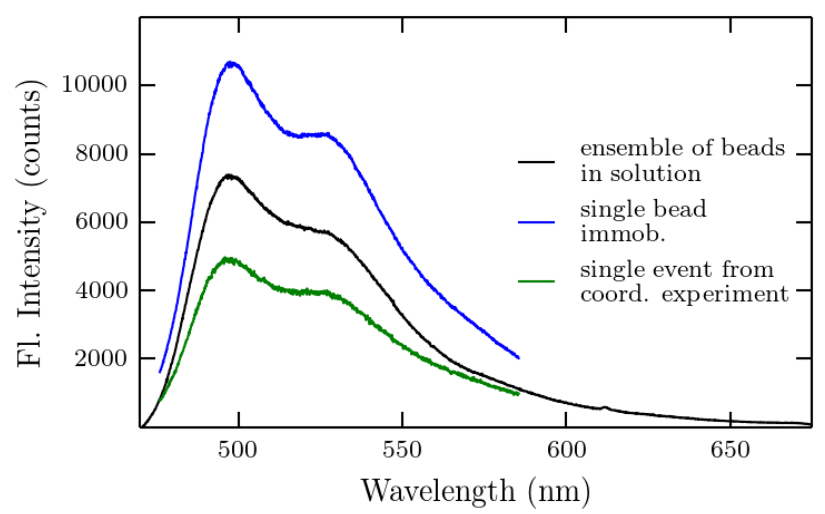

(b)

Figure 7.3: (a) A $2 \mu \mathrm{m}$ bead immobilized on a silicon wafer observed with a $\times 100$ objective. The corresponding spectra is shown in (b) and was measured using a neutral density filter $D 5$, the $\times 100 B F P L 500$, and an integration time of $1 \mathrm{~s}$. The spectrum from an ensemble of particles in solution $\left(1.14 \times 10^{8}\right.$ particles $/ \mathrm{mL}, \times 100$ BFPL500, $D 3,1$ s integration time) was measured using a multiple window to cover a broader spectral region. The spectrum of a translocating particle was detected in experiment I27.

of the Ar-ion laser with a neutral density filter $D 2$ was used, resulting in a total laser power at the sample of $0.058 \mathrm{~mW}$. The power is low enough that the laser induced noise was not observed in either the optical or current signal. Consequently the filtering step of the raw current data, which was necessary for the experiments in the last chapter, is obsolete.

\subsubsection{Results using a Low Magnifying Objective}

The results from seven coordination experiments obtained at two different applied pressures, two beam sizes and three particle concentrations, are summarized in Tab. 7.2. The number of "multi" events is defined as the sum of the number of current events that are within one optical event. For example, if three resistive pulse events are detected within the start and end time of the same optical event and five other current events within another optical event, the total number of multi events would be 8 . This is different to the last chapter where only double events were found and one double event consisted of two current events. Experiment "D4" in the last chapter would have 16 multi events with the new definition. 
Table 7.2: Experimental parameters and results of seven experiments using $2 \mu \mathrm{m}$ particles with the second setup, including the number of identified events and their matching rate at different applied pressures. Numbers of TRPS and optical events which show no corresponding events in the other trace are given in brackets. The Wilson score is a lower bound for the matching percentage at $97.5 \%$ confidence. The applied pressure $P$ was measured with an uncertainty of $1 \mathrm{~mm} \mathrm{H}_{2} \mathrm{O}$. The width of the laser beam $w_{f}$ is given at the focal plane of the objective. The used objective-BFPL combination can be deduced from Tab. 7.1. Mean diameter $d_{\text {calc }}$ and concentration $c_{\text {calc }}$ of particles are calculated with Eqs. 6.1 and 6.2. Diameter uncertainties represent the standard deviation. Nominal $\left(c_{\text {nom }}\right)$ and calculated $\left(c_{\text {calc }}\right)$ concentration are both given in $10^{6}$ particles $/ \mathrm{mL}$. The average fluorescent intensity $F I$ is given with its standard deviation. Parameters for specific experiments that differ from the values described in the text are specifically noted in the last column (here, experimental duration).

\begin{tabular}{|c|c|c|c|c|c|c|c|c|c|c|c|c|}
\hline Exp. & $\begin{array}{c}P \\
\left(\mathrm{~mm} \mathrm{H}_{2} \mathrm{O}\right)\end{array}$ & $\begin{array}{l}w_{f} \\
(\mu \mathrm{m})\end{array}$ & $c_{\text {nom }}$ & TRPS & $\begin{array}{l}\text { Number } \\
\text { Optical }\end{array}$ & $\begin{array}{l}\text { f Events } \\
\text { Matching }\end{array}$ & Multi & $\begin{array}{l}\text { Wilson } \\
\text { Score }\end{array}$ & $\begin{array}{c}F I \\
\times 10^{5} \text { counts }\end{array}$ & $d_{\text {calc }}(\mu \mathrm{m})$ & $c_{\text {calc }}$ & Note \\
\hline I1 & 100 & 7.69 & 1.14 & $96(1)$ & $95(2)$ & $91(95 \%)$ & 4 & $91 \%$ & $0.71(89 \%)$ & $2.05(7.9 \%)$ & 1.45 & \\
\hline $\mathrm{I} 2$ & 100 & 7.69 & 1.14 & $90(2)$ & $79(0)$ & $73(81 \%)$ & 15 & $76 \%$ & $0.77(84 \%)$ & $2.06(7.0 \%)$ & 1.36 & \\
\hline I3 & 100 & 4.50 & 0.76 & $66(0)$ & $64(1)$ & $61(93 \%)$ & 5 & $87 \%$ & $1.10(98 \%)$ & $2.16(5.9 \%)$ & 0.99 & \\
\hline $\mathrm{I} 4$ & 100 & 7.69 & 0.76 & $18(0)$ & $18(0)$ & $18(100 \%)$ & 0 & $87 \%$ & $0.49(52 \%)$ & $2.27(4.4 \%)$ & 0.78 & $9.7 \mathrm{~s}$ \\
\hline $\mathrm{I} 5$ & 50 & 7.69 & 1.14 & $41(2)$ & $38(0)$ & $37(90 \%)$ & 2 & $82 \%$ & $1.04(79 \%)$ & $2.16(7.2 \%)$ & 1.24 & \\
\hline I6 & 50 & 7.69 & 0.76 & $35(0)$ & $33(0)$ & $31(89 \%)$ & 4 & $80 \%$ & $1.30(53 \%)$ & $2.18(6.4 \%)$ & 1.06 & \\
\hline I7 & 50 & 4.50 & 0.57 & $16(0)$ & $16(0)$ & $16(100 \%)$ & 0 & $85 \%$ & $3.75(58 \%)$ & $2.26(8.0 \%)$ & 0.48 & \\
\hline
\end{tabular}


The matching rate and Wilson score are above $80 \%$ and $70 \%$ respectively for all experiments. The occurrence of multi events, which increase in frequency with a higher applied pressure, is the main reason for a reduction of these rates. A typical example is experiment I2 (Fig. 7.4), which shows three very long optical events containing five resistive pulses each, totalling 15 multi events, and therefore a matching rate of only $79 \%$. Throughout all the experiments only a few resistive and optical pulses do not have a corresponding partner. This occurs usually close to large multi events (compare Fig. 7.1 (f)) and is an issue that is connected to the event finding algorithm and not the detection limit in general. All multi and unmatched events are excluded from further analysis and are therefore not visible in the following histograms and scatter plots.

For the interpretation of the results, the matching events of each experiment are visualized in histograms and scatter plots. Here, the data of experiment I2 is shown in Figs. 7.5 and 7.6. The scatter plot between the duration and the magnitude of a current peak (Fig. 7.6 (a)) reveals a strong positive correlation with a Pearson coefficient of 0.73 . This indicates that particles that travel closer to the pore walls, where the fluid flow is smaller, cause a higher resistive pulse. This is in agreement with the results of the simulations conducted in Chap. 3 and can be explained by the non uniform distribution of the electric field across the pore. A particle travelling at the pore edges is therefore blocking more current and results in a larger resistive pulse. This also affects the intrinsic peak height distribution of a resistive pulse experiment (Fig. 7.5(a)). Likewise, the correlation between the duration and the height (Fig. 7.6) of the optical events is also positive (Pearson coefficient of 0.60), although for a different reason. Particles that travel closer to the centre are exposed to a higher laser intensity, assuming a perfect alignment of the laser with the centre of the pore orifice. Such a particle is also exposed to a higher laser intensity for longer as the Rayleigh range of the beam is much larger than its waist. Additionally, in the specific setup, the axial detection efficiency, which extends far beyond the pore orifice, does not limit the detection of such a particle even if its relatively far away and slowly approaches the pore. This can be seen in the characteristic slow onset of such events. However, as the flow distribution (see Sec. 3.4) favours off axis particles, there are relatively few large events in Figs. 7.5 (c) and (d). Both cross correlations between the peak height and the duration of the resistive pulse with the fluorescence intensity (Figs. 7.6 (c) and (d)) show the expected negative values (-0.26 and -0.28).

The total intensity of the fluorescence events decreases with increasing pressure. This is simply understandable with the shorter time a faster particle spends at the region close to the pore orifice, where the laser power is focussed. Simulations using the semi-analytic model and a pore of the dimension used in the experiments show that particles can reach velocities of up to $100 \mathrm{~mm} \mathrm{~s}^{-1}$ at the pore orifice and are 


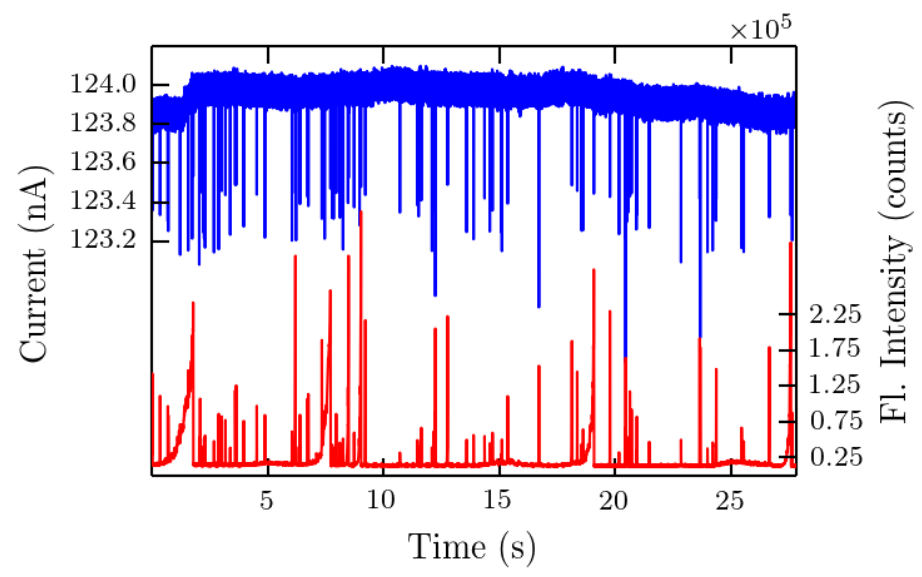

Figure 7.4: The coordinated current and optical traces for experiment I2.

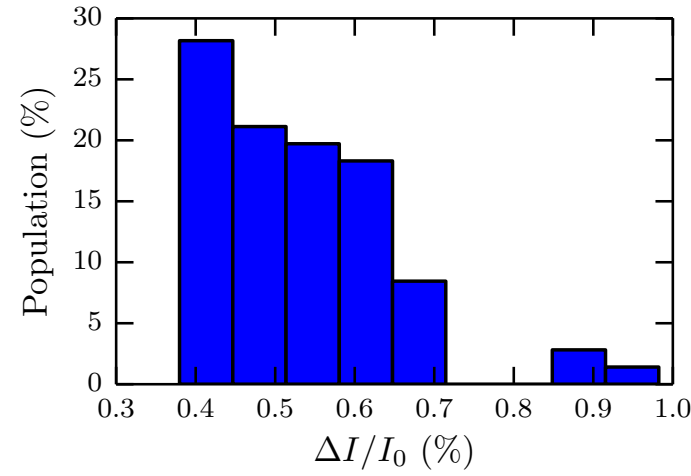

(a)

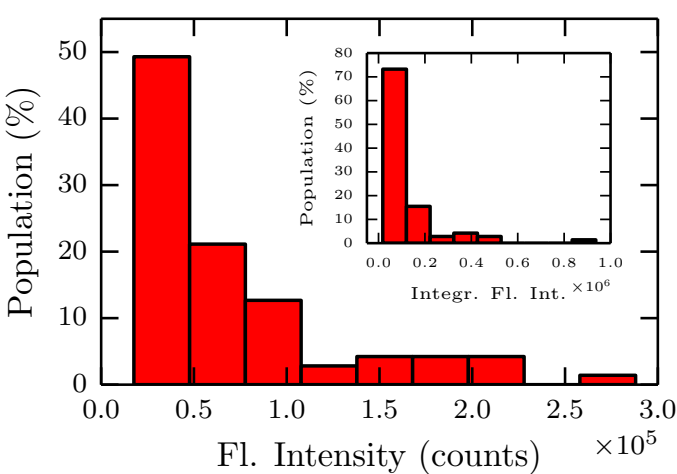

(c)

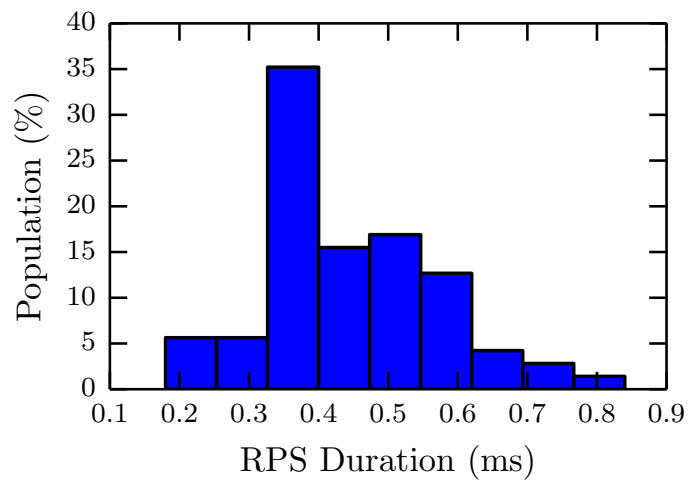

(b)

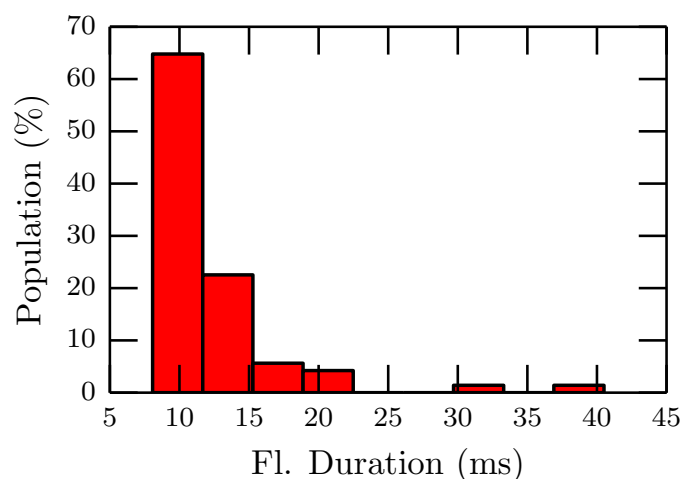

(d)

Figure 7.5: The distribution of (a) the resistive pulse heights, (b) the resistive pulse durations, (c) the fluorescence intensities and (d) the durations of the optical events for experiment I2. The inset in (c) shows the fluorescent intensity integrated over the duration of the optical events.

accelerated very quickly. As the hydrodynamic transport is the dominant transport mechanism at this length scale, a linear relationship between pressure and velocity and therefore an inverse relationship with the optical signal is expected. Although the amount of data is limited and the exact focussing will be slightly different for 


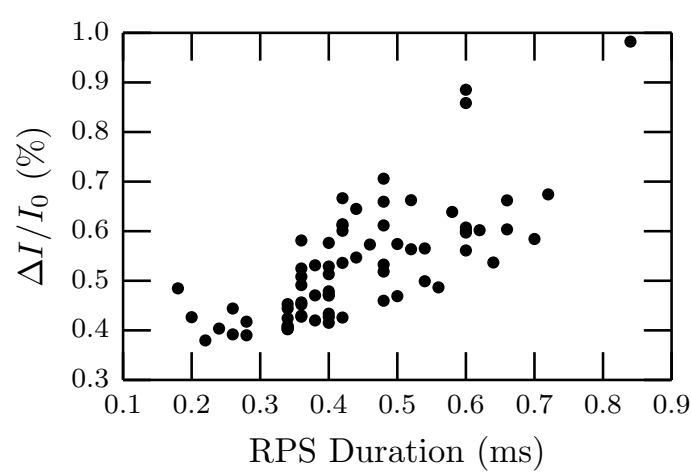

(a)

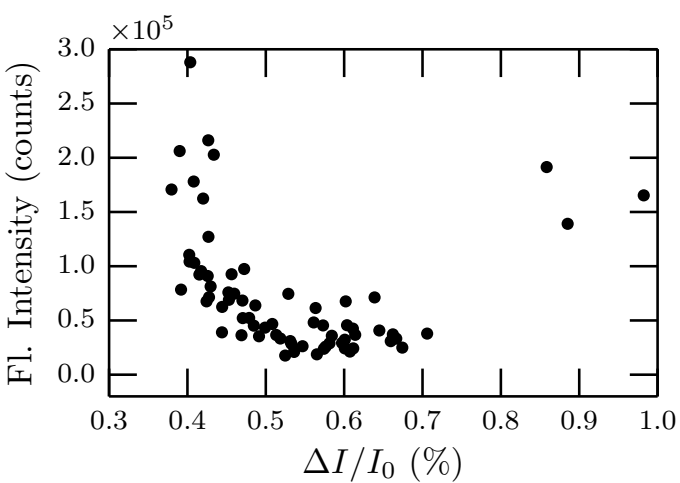

(c)

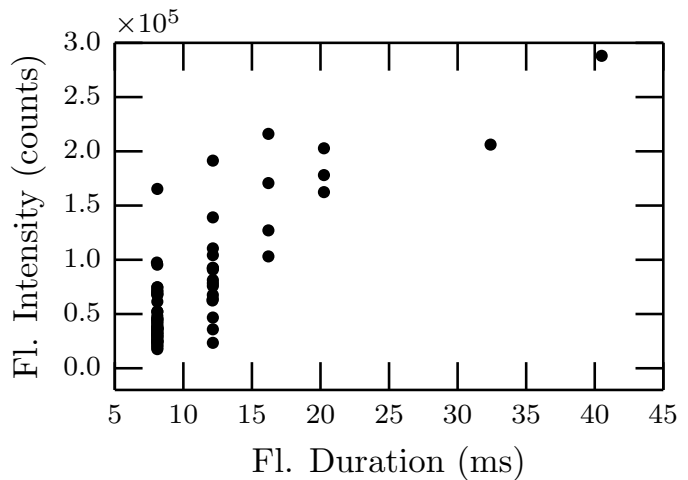

(b)

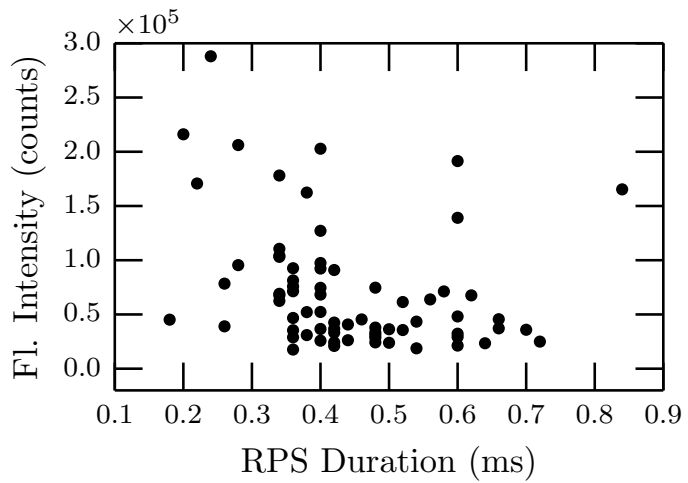

(d)

Figure 7.6: (a) The correlation between the resistive pulse durations and the resistive peak heights (Pearson coefficient: 0.73). Scatter plots of the measured fluorescence intensities with (b) the durations (0.60) of the optical events (c) the resistive peak heights $(-0.26)$ and $(\mathrm{d})$ the resistive pulse durations $(-0.28)$ for the experiment $\mathrm{I} 2$.

the different runs, a clear increase at the lower pressure is observable in Tab. 7.2. Additionally, a smaller spot also results in a higher fluorescence intensity, which can naively be explained with the higher power density at the sensing region. The actual signal intensity is a convolution of excitation profile, particle trajectory and the axial and radial detection efficiency. The idea of installing a BFPL in order to create a more uniform signal distribution is not successful. Moreover, the uniformity of the signals does not appear to follow any obvious rule connected to the either the beam size or the applied pressure. Reducing the pressure to increase the signal is the obvious choice, however, it comes with a decrease in throughput and therefore in the statistical significance of the acquired data.

In this context the difference with purely diffusion based detection of particles used should be mentioned. Figure 6.3 shows the time dependent optical signal using the same solution of particles and optical parameters as in experiments I1. Due to the relatively slow diffusion (the diffusion constant is $0.23 \mu \mathrm{m}^{2} \mathrm{~s}^{-1}$ for a $2 \mu \mathrm{m}$ particle) of $\mu \mathrm{m}$-scale particles, the detected particles per time is much lower compared to the coordination technique. 


\subsubsection{Comparison with the First Setup}

The matching rates in the second setup are slightly larger than for the first setup. However, the throughput of the experiments conducted with the first setup is approximately threefold larger due to a larger pore specimen. The second setup is easier and more accurate to align and focus, which may result in the overall higher matching rate. While the laser power used in the first setup is approximately seven times as high as the one used in the second setup, the signals at the same pressure are only four times higher. The remaining factor of two can be explained by the lower effective angle of detection in the first setup, which due to the design of its fluid cell, requires a glass slide (Sec. 4.1.1). Therefore light gets refracted at the glass-air interface and decreases the solid angle of the collection by approximately a factor of $n_{\text {water }}^{2} / n_{\text {air }}^{2}=1.33^{2} / 1^{2}=1.8$.

Both systems are capable of detecting fluorescently labelled $\mu$ m-scale polystyrene beads. While the advantage of the first setup is its minimal modifications compared to the original TRPS device and its fast setup, it is not able to detect sub-pm particles simply because it lacks the mechanical accuracy for the alignment and stability. In both systems, when a low magnification objective is used, the particle concentration should be reduced compared to an isolated TRPS experiment in order to achieve good matching rates. As the optical sensing region is extended far beyond the pore orifice the double and multi events will frequently occur at higher concentrations. A longer focal length of the pinhole lens as discussed in Sec.4.2.3 would result in a smaller detection volume and would mitigate this problem. As throughput is one of the important features of the TRPS technique, it is crucial to overcome this shortcoming by either using a pinhole that works in a "true" confocal regime or employing an objective with a higher magnification. In the next sections the experiments will be conducted exclusively with a $\times 100$ water immersion objective, which enables the detection of sub- $\mu \mathrm{m}$ particles.

\subsubsection{Using a Higher Magnifying Objective}

The reduction of the optical sensing region using a high magnification objective is favourable for several reasons. First, the collection can be aligned more accurately with the centre of the pore orifice. Then, as the solid angle of the collection scales with $N A^{2}$, the detected signal increases. As a consequence the excitation power can be reduced, which is favourable with regards to the noise issue. Also the problem of long multi events that reduce the matching rate is eliminated. Finally as the magnification with respect to the pinhole lens is increased the pinhole can now be used to laterally limit the detection to a square that includes the pore orifice but cuts out the signal from areas outside. In that way particles that get attached to the 
membrane in close proximity of the pore do not influence the detection. On the other hand, a shorter focal length of the objective leads to a smaller beam waist which can be significantly smaller than the pore orifice so that a concentrated illumination at the centre of the pore is created. However, due to the large divergence of a tightly focussed laser beam and its intrinsic offset relative to the objective focus, the spot is already more than doubled in diameter at the pore orifice and reaches a value of $0.9 \mu \mathrm{m}$. Also as seen from the results in Tab. 7.2 a smaller beam size does not automatically imply a less uniform or smaller signal as the signal is not collected from a single point but along the entire particle trajectory. A strongly focussed beam might even be an advantage in order to further limit the detection to the pore orifice, for example when smaller particles are measured. The high divergence ensures that the excitation power drops quickly away from the focus.

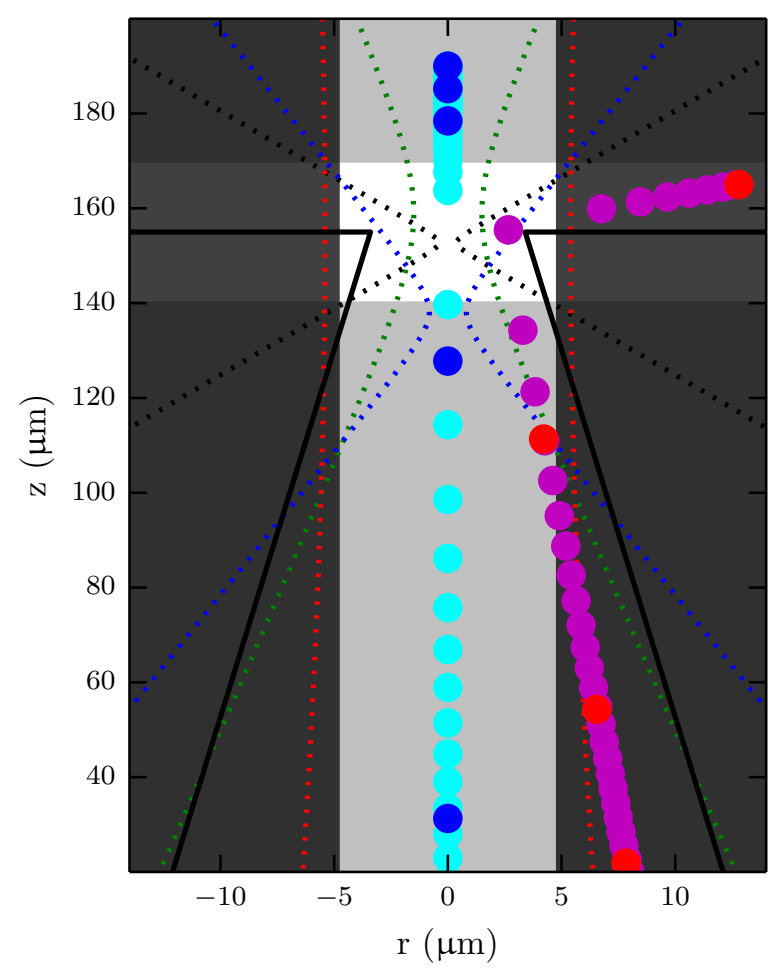

Figure 7.7: An illustration of the experiments using the $\times 100$ objective, including the pore outline (solid black line) and the various beam shapes (dotted lines). The optical detection region is indicated with differently shaded areas. The dark shaded areas are outside the lateral detection of the pinhole and any signal originating from there is suppressed. The light grey area is outside the FWHM of the axial detection region for the objective. Both white and light grey regions contribute to the signal, however, the detection efficiency falls off in a Lorentzian-shaped manner from the pore orifice. The magenta/red coloured spheres correspond to the particle positions of an off-axis trajectory at $10 \mathrm{~mm} / 100 \mathrm{~mm} \mathrm{H}_{2} \mathrm{O}$ applied hydrostatic pressure, one time frame $(4.25 \mathrm{~ms})$ of the optical detection apart. The blue and cyan beads illustrate an on-axis trajectory for the same pressures. Both trajectories were obtained using the FEM-point particle model described in Sec. 3.4.5. 
As the collection efficiency has improved due to the higher numerical aperture of the $\times 100$ objective, the power could be reduced by a factor of 10 (neutral density filter D3). The pinhole is set to $300 \mu \mathrm{m}$, which corresponds to a square with a side length of $9.7 \mu \mathrm{m}$ at the objective plane, only slightly larger than the pore diameter. An illustration of the experimental situation with two possible particle trajectories, that were obtained with the FEM-point particle model is shown in Fig. 7.7. The time resolved positions of the particles, spaced apart by the time-step of the optical detection, is marked for two hydrostatic pressures with the beads starting at a position outside the detection volume. The actual starting position of the particle in the FEM simulation is far beyond this point to ensure a negligible initial velocity. One can see from the illustration that off-axis particles are cut out laterally by the pinhole, so that especially for high pressures the particle spends less than half of one time-step in the sensing region. This results in a significantly smaller signal compared to on-axis particles even if a totally uniform illumination in the radial and axial directions was achievable. An influence of the CCD "dark time" can still be neglected as its duration is below $5 \%$ of one time step.

However, a more significant factor that influences the signal is the beam profile. As the dynamic range of a CCD is limited, a high uniformity of the signals could lead to the result that some particles saturate the detection while others are undetected. Therefore various lens combinations were tested for different pressures and particle concentrations and the effect on the signal uniformity was studied. For most experiments a $\times 100$ FL300 combination is used as it has a relatively large beam spot at the pore orifice and at the same time is easier to install and align compared to the $\times 100$ BFPL- $18 /+50$ system.

\subsubsection{Results}

The results from 26 experiments are summarized in Tab. 7.3 along with the experimental parameters. Below a pressure of $50 \mathrm{~mm} \mathrm{H}_{2} \mathrm{O}$ matching rates and Wilson scores above $90 \%$ are reached repeatedly. The signal increases with decreasing pressure as expected. Compared to the setup using the low magnification objective multi events still occur. However, these are not caused by on-axis particles that have a long signal onset. The multi events detected here are of the kind illustrated in Fig. 7.1 (c) and occur due to two or rarely three particles travelling in close succession through the pore so that they cannot be resolved due to the relatively low time resolution of the optical detection. The increase with pressure of such events is expected due to the higher particle velocity, as the capture of a particle into the pore is a classic arrival time Poisson process [4] with no memory effect. The difference in the number of multi events at the same parameters is therefore subject to an intrinsic variation. In addition to the multi events, several resistive pulses do not have a corresponding 
partner at the highest applied pressure. This is due to the decreasing optical signal at high particle velocities. There are very few undetected optical events. The ones that occur are of the form shown in Fig. 7.1(f) and are small "pre-events" shortly before a large event is detected. They have a small pulse height but cannot be distinguished by the event finding algorithm from a translocation event. A higher pore stretch (experiments I9 and I17) leads to a higher throughput and an increase in the signal variability due to a lower beam to pore ratio. 
Table 7.3: Experimental parameters and results (analogous to Tab. 7.2) of a series of experiments using the higher magnifying objective with $2 \mu \mathrm{m}$ beads. Parameters that differ from the values described in the text are noted in the last column (here, str. $=$ stretch, $V_{0}$, experimental duration)

\begin{tabular}{|c|c|c|c|c|c|c|c|c|c|c|c|c|}
\hline Exp. & $\begin{array}{c}P \\
(\mathrm{~mm})\end{array}$ & $\begin{array}{c}w_{f} \\
(\mu \mathrm{m})\end{array}$ & $c_{\text {nom }}$ & TRPS & $\begin{array}{l}\text { Number } \\
\text { Optical }\end{array}$ & $\begin{array}{l}\text { f Events } \\
\text { Matching }\end{array}$ & Multi & $\begin{array}{l}\text { Wilson } \\
\text { Score }\end{array}$ & $F I$ & $d_{\text {calc }}(\mu \mathrm{m})$ & $c_{\text {calc }}$ & Note \\
\hline I8 & 100 & 2.93 & 5.71 & $650(80)$ & $496(7)$ & $420(65 \%)$ & 150 & $63 \%$ & $0.95(115 \%)$ & $1.84(7.3 \%)$ & 9.78 & \\
\hline I9 & 100 & 2.93 & 1.14 & $282(19)$ & $258(16)$ & $220(78 \%)$ & 44 & $75 \%$ & $0.46(138 \%)$ & - & - & str. $39 \mathrm{~mm}, 0.06 \mathrm{~V}$ \\
\hline $\mathrm{I} 10$ & 100 & 2.93 & 1.14 & $109(6)$ & $96(0)$ & $89(82 \%)$ & 14 & $77 \%$ & $0.54(98 \%)$ & $2.08(7.0 \%)$ & 1.64 & \\
\hline $\mathrm{I} 11$ & 100 & 5.40 & 1.14 & $88(4)$ & $82(2)$ & $76(86 \%)$ & 8 & $81 \%$ & $1.64(92 \%)$ & $1.95(8.1 \%)$ & 1.33 & $D 2$ \\
\hline $\mathrm{I} 12$ & 100 & 1.54 & 1.14 & $82(2)$ & $78(0)$ & $76(93 \%)$ & 4 & $88 \%$ & $1.77(109 \%)$ & $2.09(7.0 \%)$ & 1.24 & \\
\hline $\mathrm{I} 13$ & 100 & 0.90 & 1.14 & $77(6)$ & $73(4)$ & $67(87 \%)$ & 4 & $81 \%$ & $3.66(79 \%)$ & $2.06(6.6 \%)$ & 1.16 & \\
\hline $\mathrm{I} 14$ & 100 & 2.93 & 0.76 & $58(1)$ & $59(1)$ & $58(100 \%)$ & 0 & $95 \%$ & $0.81(80 \%)$ & $2.13(6.1 \%)$ & 0.87 & \\
\hline $\mathrm{I} 15$ & 50 & 2.93 & 5.71 & $289(6)$ & $272(8)$ & $248(86 \%)$ & 35 & $83 \%$ & $1.61(114 \%)$ & $2.01(7.0 \%)$ & 8.70 & \\
\hline I16 & 50 & 2.93 & 5.71 & $235(1)$ & $218(0)$ & $204(87 \%)$ & 30 & $84 \%$ & $2.32(102 \%)$ & $2.00(6.3 \%)$ & 7.08 & \\
\hline I17 & 50 & 2.93 & 1.14 & $92(2)$ & $85(8)$ & $82(89 \%)$ & 2 & $84 \%$ & $1.21(128 \%)$ & - & - & str. $39 \mathrm{~mm}, 0.06 \mathrm{~V}$ \\
\hline $\mathrm{I} 18$ & 50 & 2.93 & 1.14 & $51(0)$ & $50(0)$ & $49(96 \%)$ & 2 & $90 \%$ & $1.61(91 \%)$ & $2.21(5.2 \%)$ & 1.54 & \\
\hline $\mathrm{I} 19$ & 50 & 5.40 & 1.14 & $48(0)$ & $48(0)$ & $48(100 \%)$ & 0 & $94 \%$ & $4.22(71 \%)$ & $2.09(6.4 \%)$ & 1.45 & $D 2$ \\
\hline $\mathrm{I} 20$ & 50 & 1.54 & 1.14 & $46(1)$ & $46(1)$ & $45(98 \%)$ & 0 & $92 \%$ & $2.12(89 \%)$ & $2.20(7.6 \%)$ & 1.38 & \\
\hline $\mathrm{I} 21$ & 50 & 0.90 & 1.14 & $40(1)$ & $40(1)$ & $39(98 \%)$ & 0 & $91 \%$ & $3.23(94 \%)$ & $2.10(6.2 \%)$ & 1.20 & \\
\hline $\mathrm{I} 22$ & 50 & 2.93 & 1.14 & $34(0)$ & $32(0)$ & $30(88 \%)$ & 4 & $79 \%$ & $1.63(90 \%)$ & $2.23(6.1 \%)$ & 1.03 & \\
\hline $\mathrm{I} 23$ & 50 & 2.93 & 0.57 & $17(0)$ & $17(0)$ & $17(100 \%)$ & 0 & $86 \%$ & $2.29(94 \%)$ & $2.24(5.7 \%)$ & 0.51 & \\
\hline $\mathrm{I} 24$ & 10 & 2.93 & 5.71 & $60(0)$ & $63(3)$ & $60(100 \%)$ & 0 & $95 \%$ & $5.19(99 \%)$ & $2.05(5.3 \%)$ & 9.04 & \\
\hline $\mathrm{I} 25$ & 10 & 2.93 & 5.71 & $37(0)$ & $37(0)$ & $37(100 \%)$ & 0 & $93 \%$ & $8.17(73 \%)$ & $2.04(5.2 \%)$ & 5.57 & \\
\hline $\mathrm{I} 26$ & 10 & 2.93 & 5.71 & $30(0)$ & $29(0)$ & $28(93 \%)$ & 2 & $83 \%$ & $9.07(70 \%)$ & $2.05(6.2 \%)$ & 4.51 & \\
\hline $\mathrm{I} 27$ & 10 & 2.93 & 5.71 & $20(0)$ & $20(0)$ & $20(100 \%)$ & 0 & $88 \%$ & $5.46(116 \%)$ & $2.08(6.0 \%)$ & 8.65 & $9.686 \mathrm{~s}$ \\
\hline $\mathrm{I} 28$ & -20 & 2.93 & 5.71 & $129(5)$ & $122(3)$ & $114(88 \%)$ & 10 & $84 \%$ & $2.94(77 \%)$ & $1.86(4.6 \%)$ & 3.89 & \\
\hline I29 & -50 & 2.93 & 1.14 & $60(0)$ & $61(1)$ & $60(100 \%)$ & 1 & $95 \%$ & $1.16(67 \%)$ & $2.02(3.6 \%)$ & 1.81 & \\
\hline $\mathrm{I} 30$ & -50 & 2.93 & 1.14 & $42(0)$ & $42(0)$ & $42(100 \%)$ & 0 & $94 \%$ & $3.59(37 \%)$ & $1.84(2.0 \%)$ & 1.26 & \\
\hline I31 & -50 & 2.93 & 1.14 & $36(0)$ & $35(0)$ & $34(94 \%)$ & 2 & $85 \%$ & $5.01(47 \%)$ & $1.89(6.3 \%)$ & 1.08 & \\
\hline $\mathrm{I} 32$ & -50 & 2.93 & 1.14 & $32(1)$ & $30(0)$ & $29(91 \%)$ & 2 & $81 \%$ & $1.30(50 \%)$ & $1.97(3.0 \%)$ & 0.97 & \\
\hline $\mathrm{I} 33$ & -100 & 2.93 & 0.57 & $31(0)$ & $31(0)$ & $31(100 \%)$ & 0 & $92 \%$ & $1.99(35 \%)$ & $1.69(4.3 \%)$ & 0.47 & \\
\hline
\end{tabular}




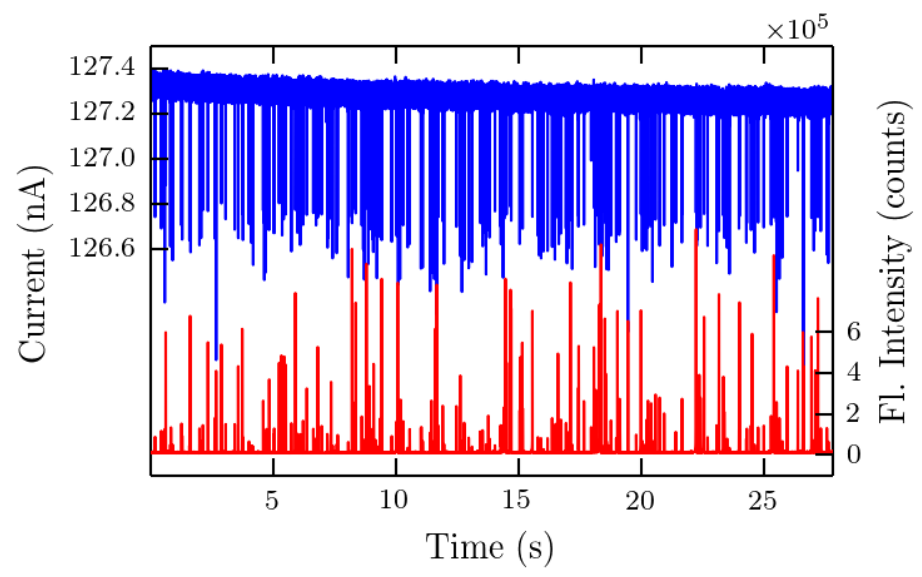

Figure 7.8: The current and optical traces for experiment I16.

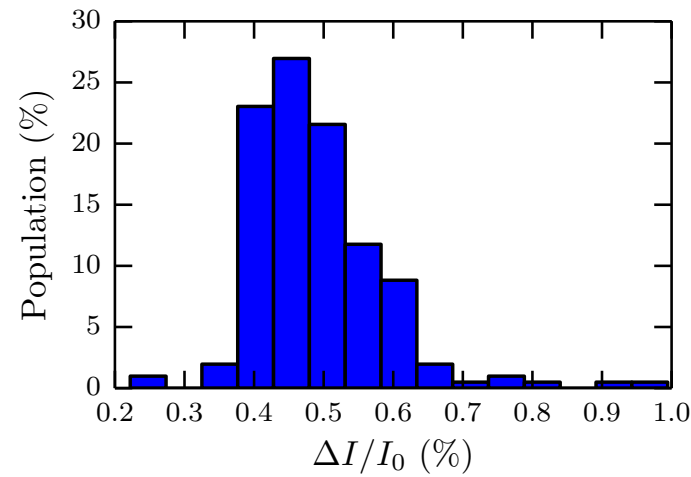

(a)

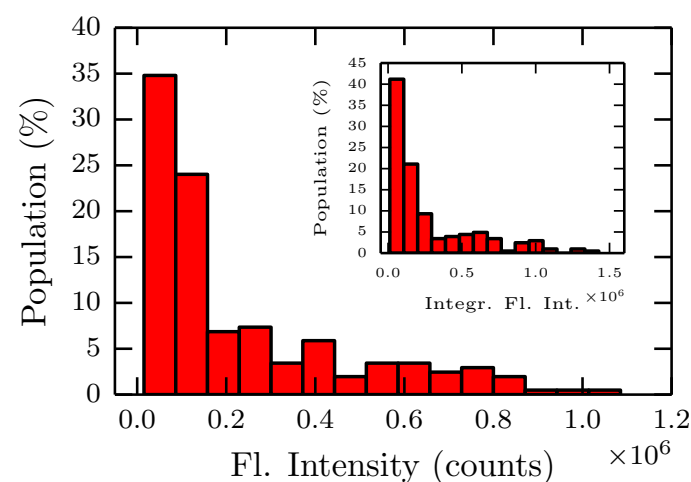

(c)

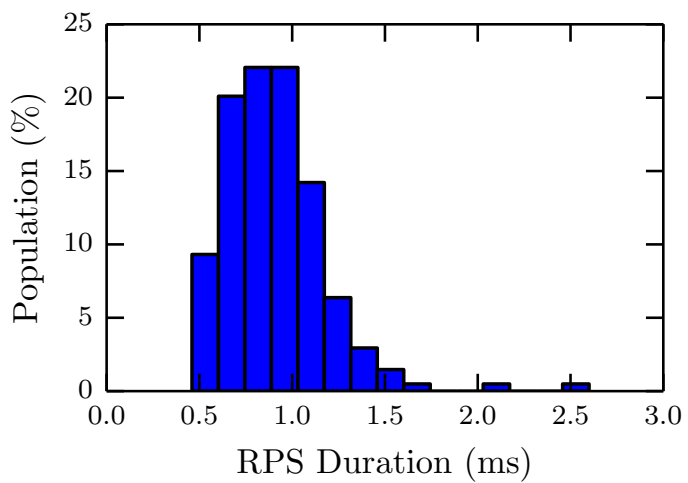

(b)

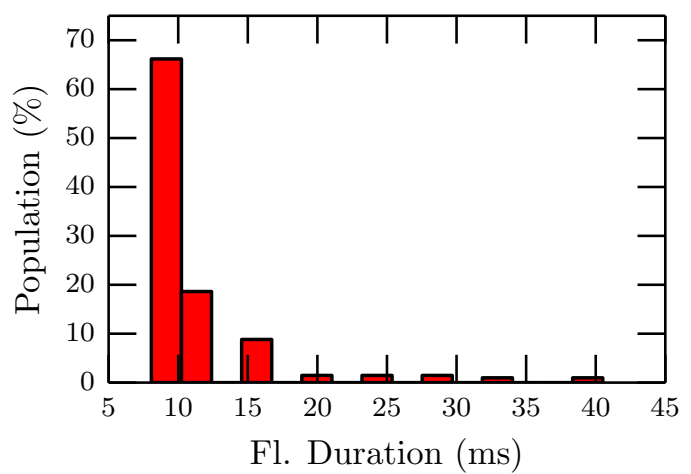

(d)

Figure 7.9: The distribution of (a) the resistive pulse heights, (b) the resistive pulse durations, (c) the fluorescence intensities and (d) the durations of the optical events for experiment I16. The inset in (c) shows the fluorescence intensities integrated over the duration of the optical events.

Figures 7.9 and 7.10 show the histograms and scatter plots of 204 matching events that were detected in the trace (Fig. 7.8) for experiment I16. The Pearson coefficients between the electrical and optical pulse heights with their corresponding pulse durations have values of 0.61 and 0.79 respectively and show the expected 


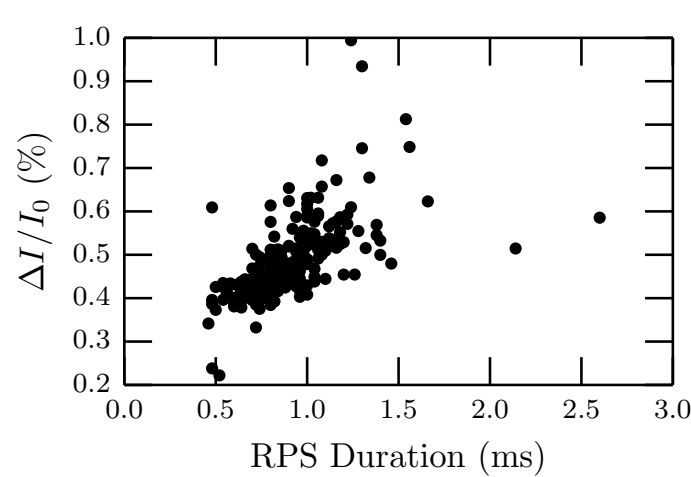

(a)

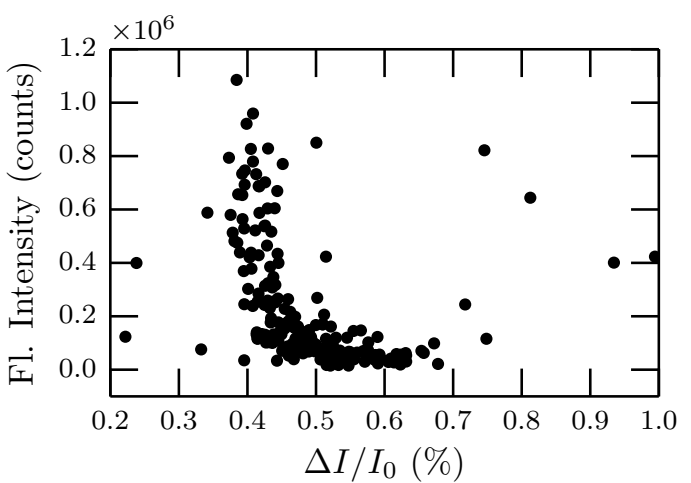

(c)

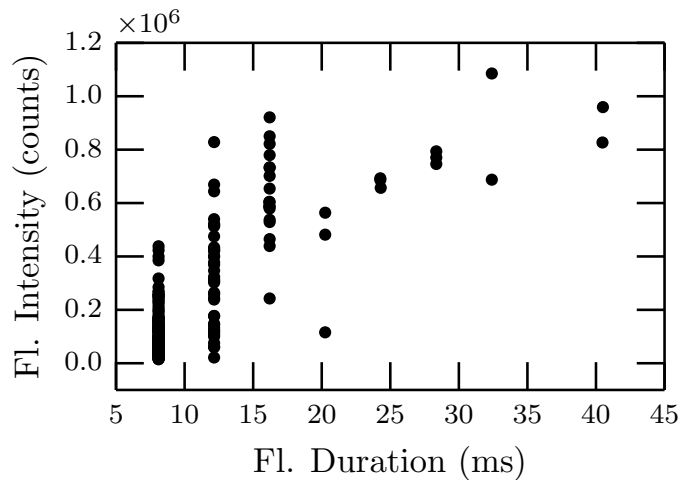

(b)

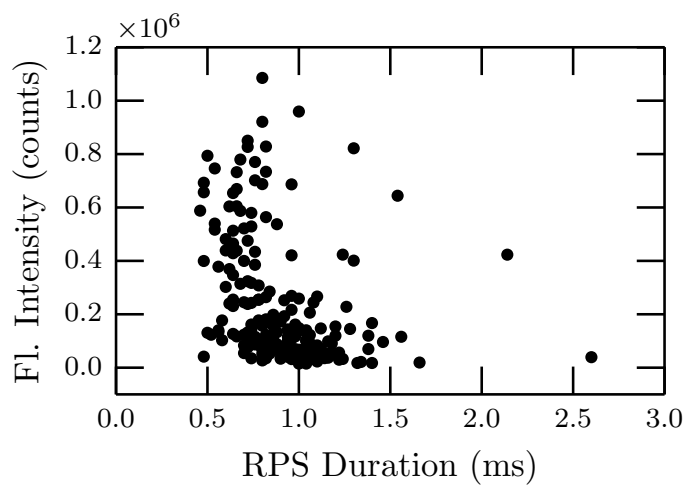

(d)

Figure 7.10: (a) The correlation between the resistive pulse durations and the resistive peak heights (Pearson coefficient: 0.61). Scatter plots of the measured fluorescence intensities with (b) the durations (0.79) of the optical events (c) the resistive peak heights $(-0.34)$ and $(d)$ the resistive pulse durations $(-0.33)$ for the experiment I16.

strongly positive correlations. The correlation between the resistive pulse heights (durations) with the fluorescence intensities has a value of -0.34 (-0.33), which confirms the trends seen in the previous section. The distribution of the fluorescence signals (Fig. 7.9 (c)) shows that most particles are travelling off-axis. Most optical events consist of only one datapoint, which due to the definition of the duration, corresponds to the peak in the distribution at $8.5 \mathrm{~ms}$ (Fig. 7.9 (d)). Long, on-axis axis events are very rare and span over a maximum of ten time steps of the optical detection, which is much shorter than the multi events seen in experiments using the low magnifying objective.

The expected optical signals for an on- and an off-axis particle at various pressures were derived with the model presented in Sec. 4.4 and are summarized in Tab. 7.4. The parameters for the simulation were identical to those in the experiment. The trajectories are obtained with the FEM-point particle (3D) model and represent one particle travelling through the centre and one travelling at the edge of the pore (compare Fig. 7.7). The absolute signals for an applied pressure of 
$50 \mathrm{~mm} \mathrm{H}_{2} \mathrm{O}$ agree very well with the bottom and top end of the signal distribution of experiment I16 (Fig. 7.9 (c)) and also predict the trend with the applied pressure correctly. This is remarkable as the model involves many parameters and, although only the two extreme cases of trajectories are considered, it shows that the model can be used design and optimise a coordination experiment.

Table 7.4: The simulated signals for different applied pressures using the model presented in Sec. 4.4. The parameters of the particle, pore and optical setup are identical to experiment I16 $(300 \mu \mathrm{m}$ pinhole, $\times 100 \mathrm{FL} 300$ system, filter D3). The total signals are listed for both a particle that moves through the pore on an on-axis trajectory and one that approaches the pore on a trajectory near the very edge of the pore (off-axis). The uniformity factor is the ratio between these two signals.

\begin{tabular}{cccc}
\hline$P\left(\mathrm{~mm} \mathrm{H}_{2} \mathrm{O}\right)$ & $\begin{array}{c}\text { On-Axis } \\
(\text { counts })\end{array}$ & $\begin{array}{c}\text { Off-Axis } \\
(\text { counts })\end{array}$ & $\begin{array}{c}\text { Uniformity } \\
\text { Factor }\end{array}$ \\
\hline 10 & $2.34 \times 10^{6}$ & $1.33 \times 10^{5}$ & 17.6 \\
50 & $6.92 \times 10^{5}$ & $2.98 \times 10^{4}$ & 23.2 \\
100 & $3.73 \times 10^{5}$ & $1.58 \times 10^{4}$ & 23.6 \\
\hline
\end{tabular}

Figure 7.11 shows the relationship between the average optical signals acquired in the experiments I1 to I33 and the applied pressure, itemized by the beam size. Although the variation can be high at the same pressure, most likely due to slight misalignments and uncertainties of the pressure measurement, the general trend that the signals increase at a lower absolute pressure can be confirmed. At any particular pressure, smaller spot sizes perform better. Significantly larger signals are acquired with the $\times 100$ objective compared to the $\times 20$ even if the values are corrected for its higher numerical aperture. These observations lead to the conclusion that a more tightly focussed beam is of advantage for the signal collection even if the beam waist is significantly smaller than the pore orifice.

The variation of the optical pulse heights is much larger than those of the resistive pulses. This is due to the non-uniform illumination profile of the beam and the fact that particles move through the pore on different trajectories. A high signal variation is undesired as the dynamic range of a CCD is limited and in an attempt to improve the uniformity of the signals several beam widths have been tested. Although a slightly more uniform distribution could be achieved with the largest beam size (uniformity factor of 10 for experiment II19 $\times 100$ BFPLs compared to a typical value of 20 for the $\times 100$ FL300 system at the same pressure), the results are still not ideal. Simulations using a beam waist of $15 \mu \mathrm{m}$, which could not be implemented in the used setup due to spatial and alignment reasons, show that uniformity factors of $1.13,1.81$, and 2.27 can theoretically be reached for applied pressures of 10,50 


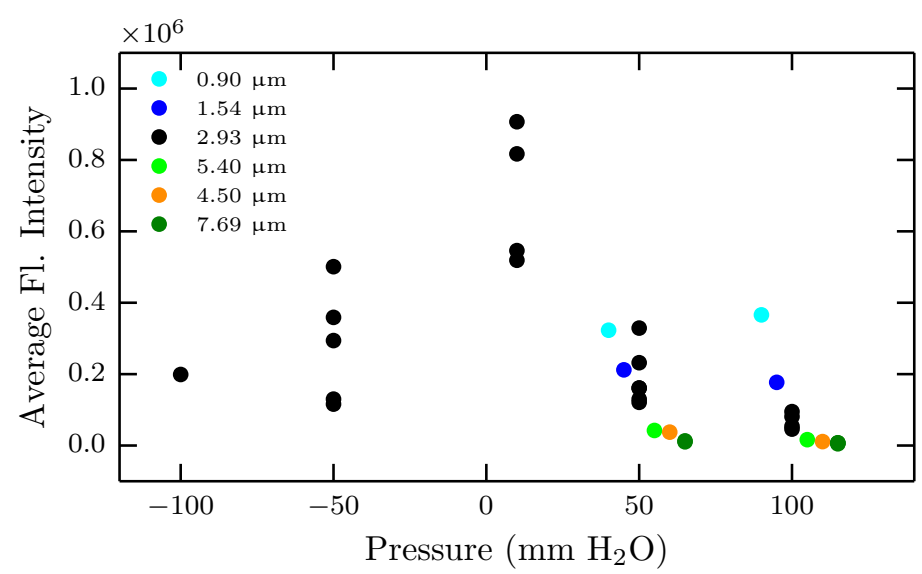

Figure 7.11: The average optical signals as listed in Tab. 7.2 and Tab. 7.3 as a function of the applied pressures. The color of the markers indicates the beam size (see legend). To avoid any overlap of the data points the values for the pressure were increased/decreased by $5 \mathrm{~mm} \mathrm{H}_{2} \mathrm{O}$ increments for different beam sizes. The value for $\times 20, \times 20$ BFPL20 and $\times 100$ BFPLs have been divided by 10 to correct for the higher laser power used in these experiments.

and $100 \mathrm{~mm} \mathrm{H}_{2} \mathrm{O}$ and otherwise identical experimental parameters. The simulations also show that even with a hypothetically perfectly uniform illumination, the peak height distribution will still have an intrinsic variation. This is because, especially for high velocities, particles which travel on different trajectories spend different periods of time in the detection region and parts of the signals are cut out laterally by the pinhole (see Fig. 7.7). A pinhole, however, is necessary when the illumination is significantly high outside the pore orifice, in order to suppress signals originating from particles that are approaching the pore or are attached to the membrane. As a conclusion, the use of the lens systems tested did not have the desired effect on the signal uniformity, as the achieved beam dimensions are still too small. Their use is, however, recommended for other reasons. Firstly, it has been shown in Sec. 4.3.1 that the beam created by the $\times 100$ objective can damage the pore at high laser powers. And secondly, the high power density of a tightly focussed beam also leads to an increase of the laser-induced noise. Therefore a highly magnifying objective should not be used without a BFPL, especially at high laser powers.

\subsubsection{Upwards Particles - Flow Focussing}

For particles that flow upwards through the pore, a consistently more uniform distribution could be achieved, indicated by the standard deviation in Tab. 7.3. Although the effect is small, it was investigated further as the current pulse distribution also exhibits a smaller standard deviation, and at the same time the average of the resistive pulse heights appears at a lower value. Additionally, by using the camera 
feature of the LabRAM and focussing on the pore orifice, particles can be visually observed when they approach the pore slowly before they are accelerated into the pore and disappear out of the camera focus. While at positive pressures, particles can be frequently observed approaching the pore at high angles, i.e. in the focal plane of the objective, upwards moving particles can only be seen as a short flash in the pore orifice before they disappear out of the focus. These observations indicate, although just qualitatively, that upwards moving particles are less likely to take extreme off-axis trajectories through the pore. It is therefore suggested that upwards moving particles are pushed towards the pore centre by a radial force and experience a flow focussing effect, which consequently leads to a lower resistivity pulse height and a narrower distribution. The effect cannot be observed for downwards moving particles (compare simulations of a similar pore in Sec. 3.4), as they are not subjected to a high and non-uniform flow field for a sufficient time period to experience a significant momentum inwards.

In this context it shall be mentioned that the particle direction can be easily deduced from the shape of the electrical and optical pulse. While downwards moving particles show a sharp onset and a slow offset of the pulse, upwards moving particles have the inverse characteristic. Optical pulses show the opposite behaviour, in cases consisting of more than one data point. This is because the optical detection region extends beyond the pore and a particle that approaches the pore can already be detected optically before it is quickly accelerated into the pore and moves out of the optical detection volume.

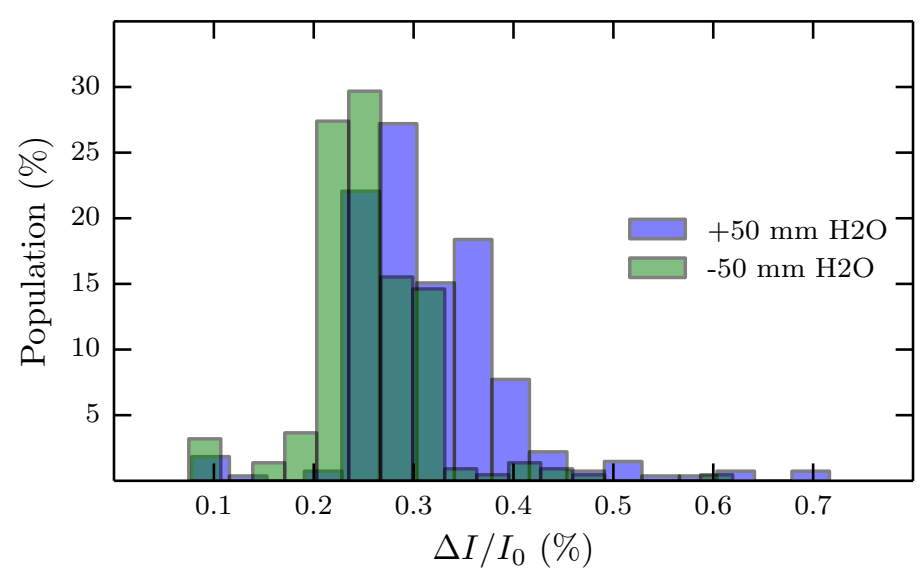

Figure 7.12: The resistive pulse distribution of $2 \mu \mathrm{m}$ particles at an applied pressure of $50 \mathrm{~mm} \mathrm{H}_{2} \mathrm{O}$ and $-50 \mathrm{~mm} \mathrm{H}_{2} \mathrm{O}$, using qNano device (a stretch of $45.80 \mu \mathrm{m}$, a voltage of $0.08 \mathrm{~V}$ and a concentration of $5.71 \times 10^{6}$ particles $/ \mathrm{mL}$ was used).

A flow focussing of upwards moving particles could also be observed using the original qNano device setup, with particles placed in both fluid cell compartments. Figure 7.12 shows the shift of the current pulse distribution to lower values for 
upwards moving particles when a pressure of the opposite sign but the same absolute value is applied. The average of the relative pulse heights of 272 events at positive pressure is $0.316 \%$ (with a standard deviation of $26 \%$ ). The value for the upwards moving particles (219 events) is $0.256 \%$ (25\%), which is significantly smaller and supports the observation made in the coordination experiments. However, it could not be confirmed that the distribution is more uniform for this specific measurement.

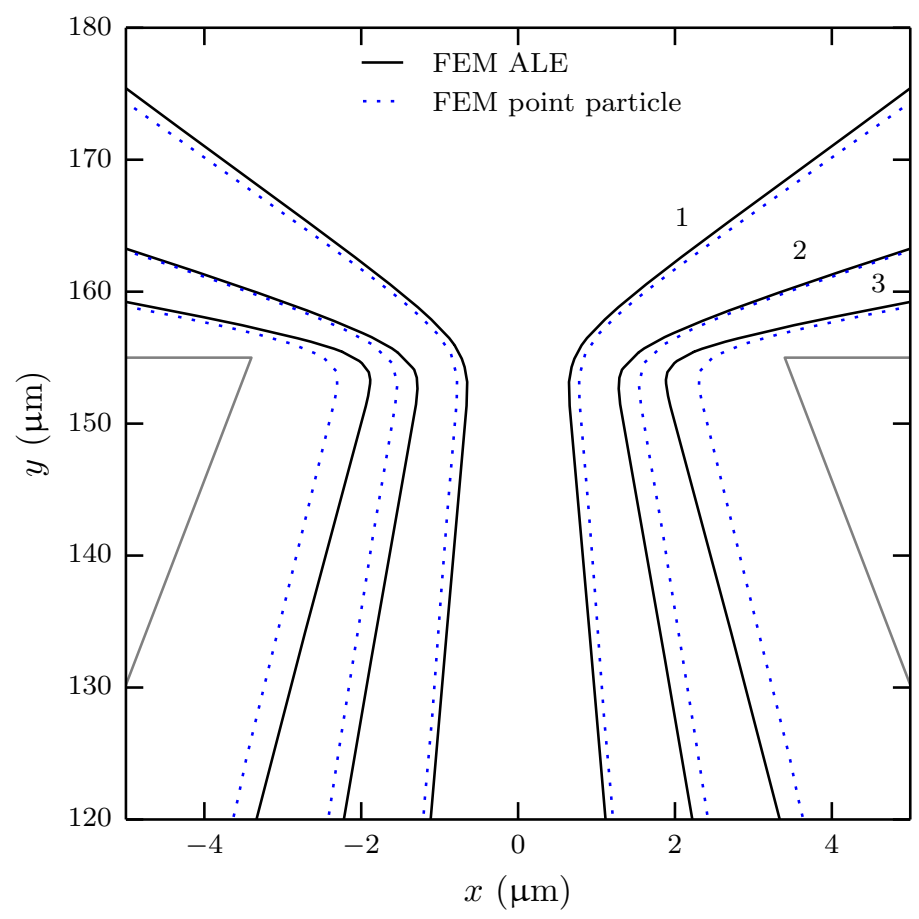

Figure 7.13: The trajectories of three upwards moving particles $(P=-50 \mathrm{~mm})$, initially positioned $3 \mu \mathrm{m}(1), 6 \mu \mathrm{m}(2)$ and $9 \mu \mathrm{m}$ (3) from the centre of the large pore opening, obtained with the FEM-ALE and the FEM-point particle model.

The effect was also studied in a simulation using the 2D FEM-ALE and FEMpoint particle models developed in Sec. 3.4. The dimensions of the pore and particles were the same as in the experiment. A pressure of $-50 \mathrm{~mm} \mathrm{H}_{2} \mathrm{O}$ was applied to the upper boundary of the model domain. The particles, which were initially placed at different positions at the large pore orifice, are pushed upwards through the pore. By comparing the results of the FEM-ALE with the FEM-point particle model, which does not account for the finite size of the particle and particle-field interactions, a slight focussing effect due to a radially acting force could be confirmed (Fig. 7.13). The effect is small and, as it is an inertial effect, is expected to decrease at smaller length scales and slower fluid velocity. However, as the beam intensity is maximal at the pore centre and the electric field inhomogeneity is concentrated at the pore edge, both the more uniform optical signals and the smaller resistive pulses can be understood. While flow focusing does not play a role for downwards particles (compare Sec. 3.4 using a similar pore geometry) due to the short time span they 
are in a high flow field, it seems that upwards moving $\mu \mathrm{m}$-scale particles feel a small radial force towards the centre of the pore. The flow-focussing effect is biggest near the edges, causing the distribution of the resistive pulses to shift to smaller values. This effect could be of use for designing pores so that a better measurement can be achieved. However, as the flow focussing effect scales with the Reynolds number, the effect will decrease with particle size and will not play a role at the nm-scale.

\subsubsection{Particle Sizing and Concentration Measurement}

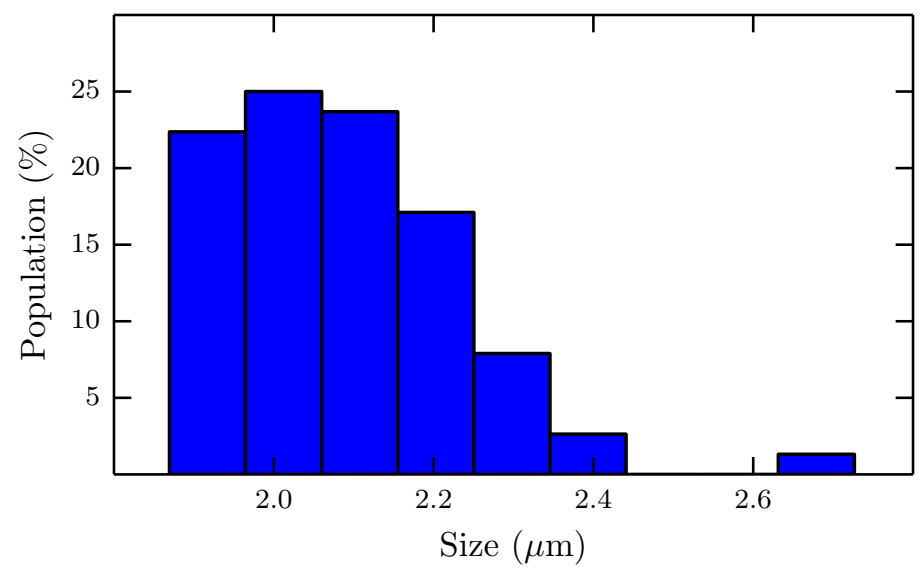

Figure 7.14: The distribution of the particle diameters derived from the resistive pulse heights measured in the experiment I12.

For each experiment, the average size of the particles and the bead concentration are calculated from the resistive pulse heights and the event frequencies using Eqs. 6.1, 6.2 and the optically determined dimensions for the pore (see Sec. 7.2.1). The results for the diameter and concentration, listed in Tab. 7.2 agree within $10 \%$ and $30 \%$ of the nominal values as stated by the supplier. The size distribution (Fig. 7.14) is centred around $2 \mu \mathrm{m}$ with a slight bias towards larger particles, which is typical for the technique due to off-axis particles (compare Sec. 3.4.5). The results depend strongly on the size of the small pore orifice, which is determined with an estimated uncertainty of $10 \%$. With the propagation of uncertainty this results in an error of $\sim 13 \%$ in size and $\sim 30 \%$ in concentration. However, it shows that with a simple optical measurement of the pore dimensions and a simple model of the resistive pulse height and fluid flow, a satisfactory result for the size and concentration can be obtained. The difference between the experimental runs can be attributed to small variations of the pore dimensions as the device was assembled several times during the collection of the data. Although special care was taken to apply the same stretch to the pore, even small changes to the pore size could have a large effect on the calculation of the size. This explains the higher deviations from the nominal 
values for experiment I8 to I33 (Tab. 7.3). For a more accurate measurement the calibration method developed by Izon [36] should be used.

\subsection{Co-ordinated Detection of a $1 \mu \mathrm{m}$ Particle Mix- ture}

A mixture of two particle sets with different fluorescent labels was used to demonstrate the capability of the technique to distinguish between samples of different optical emission. The particle sets (obtained from Bangs Lab.) both have a nominal diameter of $0.97 \mu \mathrm{m}$, and the undiluted concentration is $2.00 \times 10^{10}$ particles $/ \mathrm{mL}$. One particle set is labelled with a dye named "Suncoast-Yellow", the other with a dye named "Envy-Green", abbreviated SY and EG in the following. The absorption spectra of the dyes have a maximum close to the $514 \mathrm{~nm}$-line of the Ar-ion laser, which is used for excitation in the following experiments. With the use of the neutral density filter $D 3$, the total laser power at the sample is $0.0178 \mathrm{~mW}$. The $\times 100$ BFPL500 system and the $\times 100$ objective without additional lenses were used. The pinhole was set to $200 \mu \mathrm{m}$, which corresponds to a square with a side length of $6.4 \mu \mathrm{m}$ at the sample. A NP1000 rated pore was used with a stretch of $37.50 \mu \mathrm{m}$ and an applied voltage of $0.22 \mathrm{~V}$. A particle concentration of $4.01 \times 10^{6}$ particles $/ \mathrm{mL}$ for each species was chosen for the experiments summarized in Tab. 7.5.

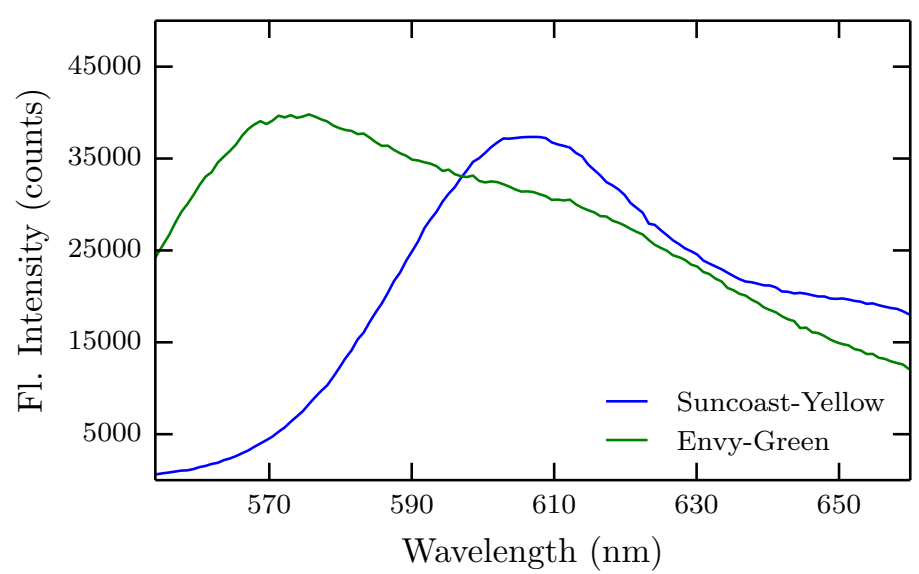

Figure 7.15: The spectra of a pure solution of "Suncoast-Yellow" and "Envy-Green" beads measured in immersion $\left(4.01 \times 10^{8}\right.$ particles $/ \mathrm{mL}, \times 20$ objective, integration time $1 \mathrm{~s}$, filter D3).

\subsubsection{Spectral Identification of the Species}

The experiments and the analysis are conducted in the same fashion as before. However an additional analysis step is introduced after the events have been detected 
and correlated with the event finding and correlation algorithm. A linear least squares algorithm (Python function "linalg") was employed to identify each event according to its spectrum. The spectra of pure solutions of SY and EG beads (Fig. 7.15) were used as a reference for the algorithm. Additionally a background spectrum of the illuminated pore without any particles present was recorded for an entire run and the averaged spectrum (integration time of $4.25 \mathrm{~ms}$ ) was used as third reference in the analysis. The algorithm gives three coefficients for each event, reflecting the contribution of each reference spectrum to the signal. According to the dominant coefficient each event can then be assigned to a particle species.

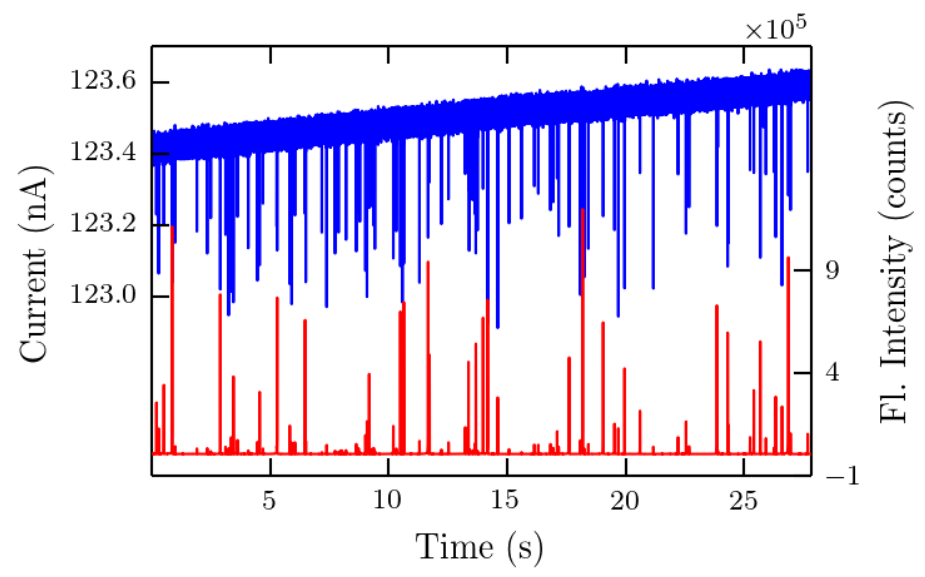

Figure 7.16: The current and optical traces for experiment II2.

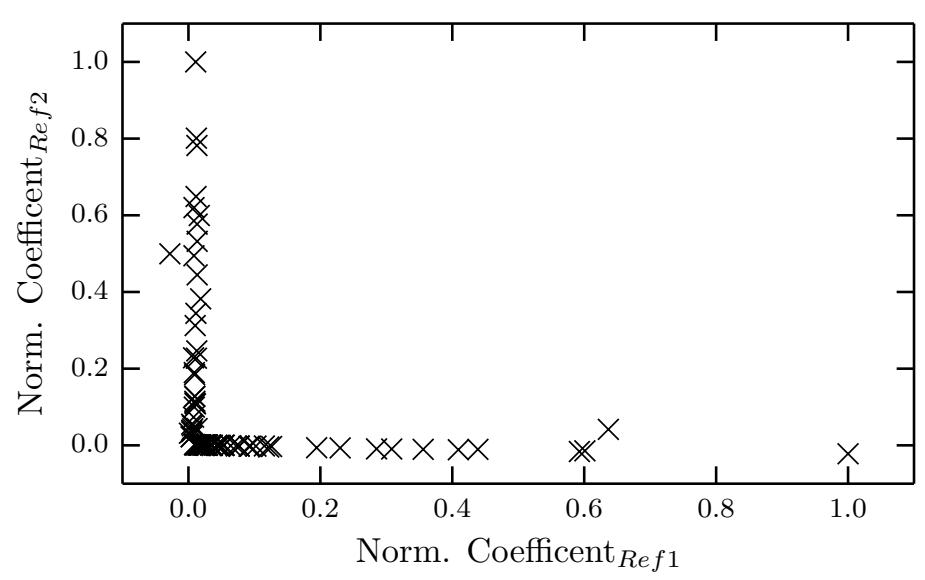

Figure 7.17: Scatter plot of the spectral coefficients ( $R e f_{1}$ corresponds to SY, $R e f_{2}$ to EG) for the matching events in experiment II2 using a linear least squares algorithm.

Figure 7.16 shows the current and optical trace of a representative experiment (II2). The spectra of the detected matching events were analysed at their maximum intensity and the coefficients for the SY and DG reference spectra are plotted in Fig. 7.17. It shows that the spectra of the detected events are almost pure with 


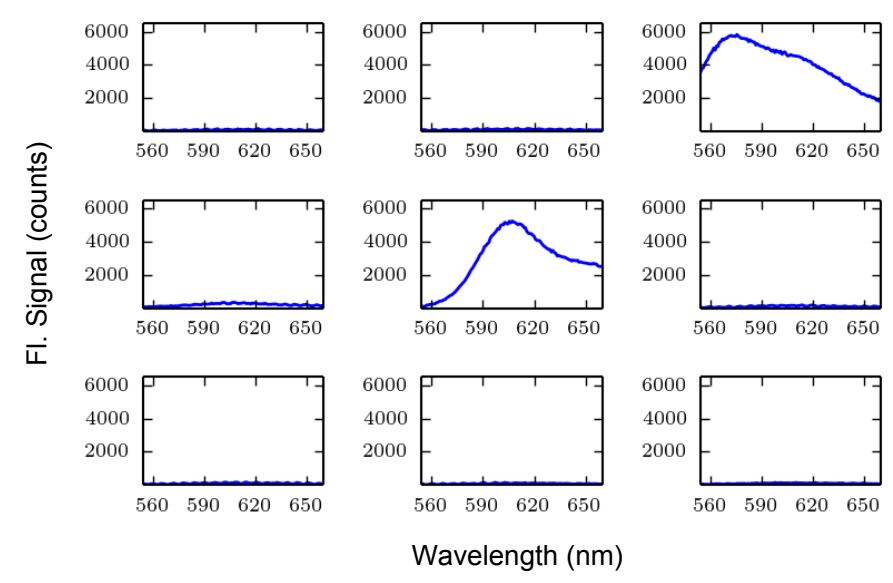

Figure 7.18: Nine consecutive time frames of experiment II13, showing the translocation of EG and SY particles in close succession without spectral overlap.

little overlap between the two components, proving that the particles are detected individually. This can also be seen in Fig. 7.18, which shows nine consecutive time frames of experiment II13. Although there is only one time frame between the detection of two differently labelled particles no spectral mixing is observed.

\subsubsection{Results}

The results of 11 coordination events are summarized in Tab. 7.5 and again show high matching rates and Wilson scores, increasing with decreasing pressure. The optical signals are higher for lower pressures and increase slightly when a smaller beam size is used. Neither the signal nor the detection of multi events changes significantly for larger pinhole settings. This indicates that a pinhole of $200 \mu \mathrm{m}$ does not significantly cut out signal from off-axis particles. This is expected as the laser beam waist mainly used is $1.50 \mu \mathrm{m}$ and therefore much smaller than the lateral detection region. The decrease of the laser power is also sufficient to suppress signals from particles close to the orifice, so that the pinhole's use is mainly to centre the axial detection around the pore orifice and reduce the FWHM of the axial detection to $15 \mu \mathrm{m}$. 
Table 7.5: Experimental parameters and results of a series of 11 experimental runs using mixed $0.97 \mu$ m particle sets with two different fluorescent labels (SY and EG). The columns are labelled analogous to Tab. 7.2. The standard deviations of the fluorescent intensity and the calculated particle sizes are of similar magnitude to in the experiments of the last two sections and are omitted in this table. Parameters for experiments that differ from the values described in the text are noted in the last column $($ here, $\mathrm{Ph}=$ pinhole, beam width, laser intensity, experimental duration).

\begin{tabular}{|c|c|c|c|c|c|c|c|c|c|c|c|c|c|c|c|}
\hline \multirow{2}{*}{ Exp. } & \multirow{2}{*}{$\begin{array}{c}P \\
(\mathrm{~mm})\end{array}$} & \multicolumn{6}{|c|}{ Number of Events } & \multirow{2}{*}{$\begin{array}{l}\text { Wilson } \\
\text { Score }\end{array}$} & \multirow{2}{*}{$F I_{S Y}$} & \multirow{2}{*}{$F I_{E G}$} & \multirow{2}{*}{\multicolumn{2}{|c|}{$\begin{array}{l}d_{\text {calc }_{S Y}} d_{\text {calc }_{E}} \\
(\mu \mathrm{m})(\mu \mathrm{m})\end{array}$}} & \multirow[b]{2}{*}{${ }^{H} c_{c a l}{ }_{S Y}$} & \multirow[b]{2}{*}{$c_{\text {calc }_{E G}}$} & \multirow{2}{*}{ Note } \\
\hline & & TRPS & Optical & Matching & Multi & SY & EG & & & & & & & & \\
\hline II1 & 100 & $105(9)$ & $105(9)$ & $96(91 \%)$ & 0 & 57 & 39 & $86 \%$ & 9.95 & 17.9 & 1.03 & 1.18 & 4.56 & 3.12 & $D 2$ \\
\hline II2 & 100 & $102(1)$ & $100(3)$ & $93(91 \%)$ & 8 & 58 & 35 & $86 \%$ & 1.32 & 3.51 & 1.03 & 1.20 & 4.64 & 2.79 & $w_{f}=0.90 \mu \mathrm{m}$ \\
\hline II 4 & 100 & $98(0)$ & $95(0)$ & $92(94 \%)$ & 6 & 54 & 38 & $90 \%$ & 1.38 & 2.70 & 1.06 & 1.19 & 4.31 & 3.04 & $\mathrm{Ph} 300 \mu \mathrm{m}$ \\
\hline II5 & 100 & $93(8)$ & $85(2)$ & $81(87 \%)$ & 4 & 41 & 40 & $82 \%$ & 1.12 & 2.22 & 1.11 & 1.24 & 3.27 & 3.19 & \\
\hline II6 & 100 & $84(0)$ & $82(2)$ & $76(91 \%)$ & 8 & 37 & 39 & $86 \%$ & 1.76 & 4.68 & 1.09 & 1.22 & 2.96 & 3.12 & $\mathrm{Ph} 500 \mu \mathrm{m}$ \\
\hline II8 & 50 & $52(0)$ & $46(2)$ & $42(87 \%)$ & 8 & 24 & 18 & $79 \%$ & 3.36 & 4.89 & 1.12 & 1.25 & 3.83 & 2.88 & $w_{f}=0.90 \mu \mathrm{m}$ \\
\hline II9 & 50 & $47(0)$ & $48(1)$ & $47(100 \%)$ & 0 & 20 & 37 & $94 \%$ & 3.91 & 4.62 & 1.08 & 1.27 & 3.19 & 4.31 & $w_{f}=0.90 \mu \mathrm{m}$ \\
\hline II10 & 50 & $43(1)$ & $42(1)$ & $40(93 \%)$ & 2 & 26 & 14 & $85 \%$ & 2.73 & 3.98 & 1.11 & 1.27 & 4.16 & 2.23 & \\
\hline II11 & 50 & $40(1)$ & $38(0)$ & $37(93 \%)$ & 2 & 18 & 19 & $84 \%$ & 2.89 & 5.27 & 1.13 & 1.28 & 2.88 & 3.04 & \\
\hline
\end{tabular}


The distributions of the resistive pulse heights and durations are broad and have unusual features for particles of the same nominal size. The histograms for a representative experiment are shown in Figs. 7.19 (a) and (b). After identifying the particle species and dividing up the distributions accordingly, it is revealed that the distributions of the resistive pulse heights and the pulse durations (Figs. 7.19 (c) and $(\mathrm{d}))$ of the two particle sets are different. For the specific experiment II2, the average of the pulse heights for the EG labelled beads is 1.56 times higher than for the SY beads. This value varies between 1.33 and 1.56 for the experiments II1 to II11 and corresponds, assuming a linear relationship between resistive pulse height and particle volume, to a size difference between $10 \%$ and $16 \%$. The values are reasonable as the particles, which are purchased from the same supplier but have a different dye embedded in their polystyrene matrix, are made in different production processes. Additional techniques like SEM could have been used to get certainty of this size variation, but were beyond the scope of this specific experiment.

Interestingly, while the distributions of the resistive pulses and also, to a lesser extent, the pulse durations show a clear separation of the two particle sets (Figs. 7.19 (c) and (d)), the distributions of the FWHM of the resistive pulses show almost no difference between species (Figs. 7.20 (a) and (b)). The reason for that might be that the FWHM is obtained from a small part of the resistive pulse and is therefore not so sensitive to small changes as the full pulse duration, making the pulse duration a better measure to distinguish between species than the FWHM.

The resistive pulse heights and the pulse durations (Fig. 7.19 (f)) show a strong positive correlation for both particle species. The distributions of fluorescence intensities of the two species are broader than the resistive pulse height distributions so that the intensity of a single event cannot be assigned to one of the species due to the significant overlap (Fig. 7.19 (e)). However, the average fluorescent signal is higher for the EG-labelled beads (Tab. 7.5). This agrees qualitatively with the intensities observed for an ensemble of beads measured in solution (Fig. 7.15) and is a consequence of the broader fluorescent profile of the EG beads.

As mentioned above, the difference in size of the two particle species is within the tolerance that can be expected from the manufacturing process. However, as the charges of the two particle sets are also different according to the supplier's data sheet, the effect is investigated further. The particle charge is inversely related to the average parking area per surface (carboxyl) group and is given to be $28.9 \AA^{2}$ for the SY and $7.9 \AA^{2}$ for the EG beads (datasheet provided by Bangs Lab. for each purchase). An effect of the particle charge on the resistive pulse size and shape has been shown experimentally by Weatherall and Willmott [134] for carboxylated polystyrene particles with a diameter of $200 \mathrm{~nm}$. It was discovered that the required condition for such charge effects is formation of a non-uniform ion distribution, also 


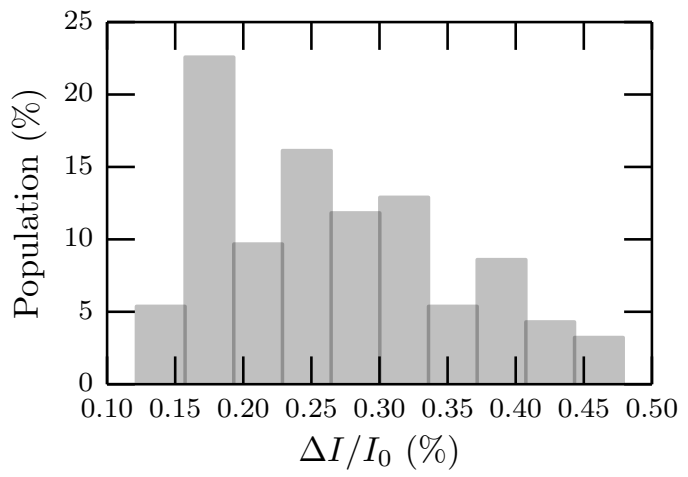

(a)

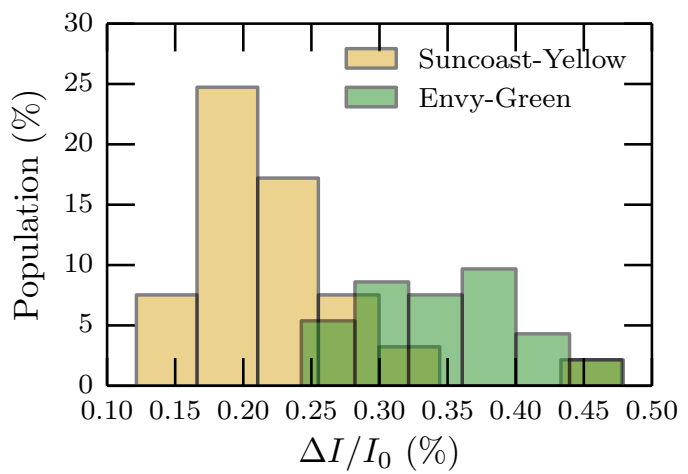

(c)

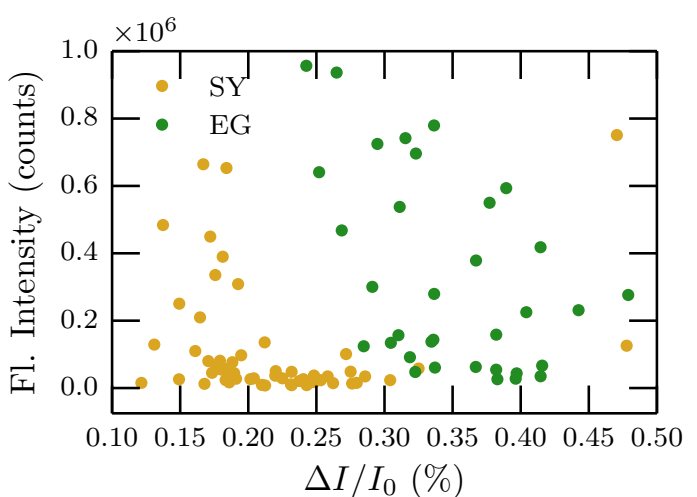

(e)

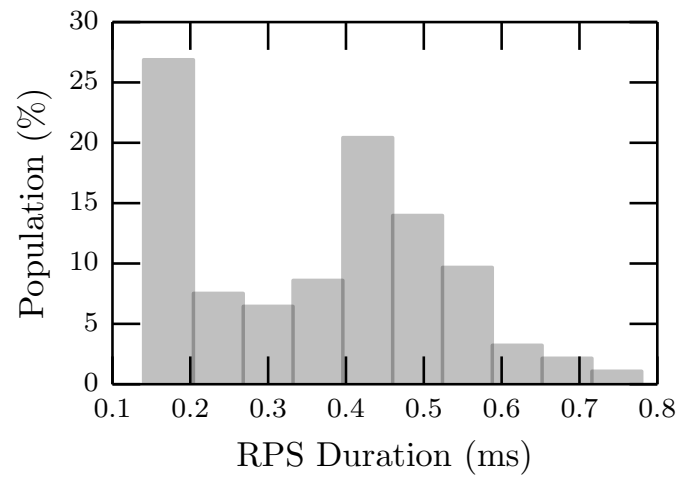

(b)

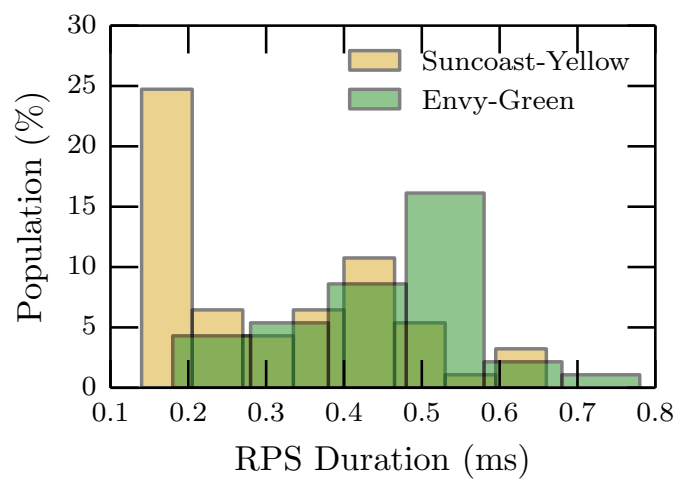

(d)

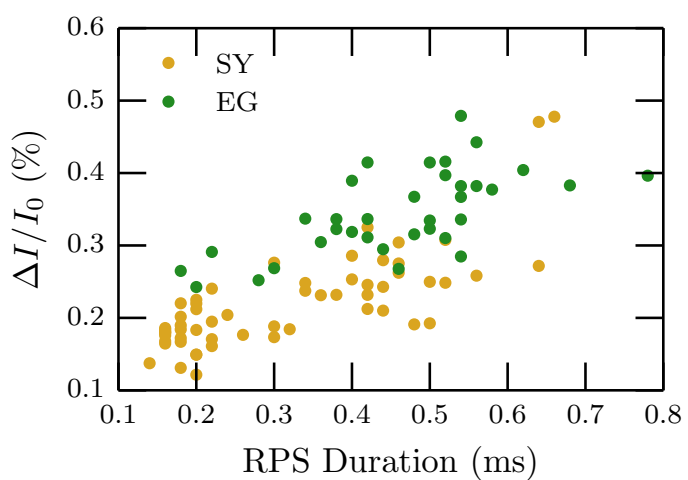

(f)

Figure 7.19: The distributions of the overall (a) resistive pulse heights and (b) the resistive pulse durations of the matching events detected in experiment II2. (c) and (d) show the corresponding plots following the identification of the particle species, the ratio $\Delta I_{E G} / \Delta I_{S Y}$ between the average pulse heights of the particle sets is 1.56. (e) The fluorescence intensities and the resistive pulse heights show a weak negative correlation (Pearson coefficient of -0.15 for the SY and -0.21 for the EG beads). The correlation between the resistive pulse durations and the pulse heights is shown in (f) and have a strong correlation (0.84 for the SY and 0.72 for the EG beads).

called charge polarisation in the following, with depletion and enrichment regions around the pore orifice. Although charge effects are expected to be smaller at the $\mu \mathrm{m}$-scale and at the used salt molarity of $100 \mathrm{mM}$, the almost four times higher charge density of the SY beads could potentially have an effect on the pulse heights. 
If charge effects are important, the difference between the pulse heights is expected to become more obvious at a lower salt molarity, which is used in the following experiments.

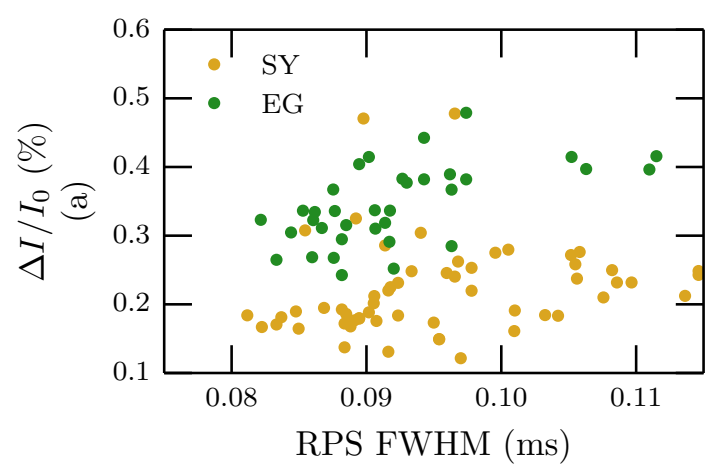

(a)

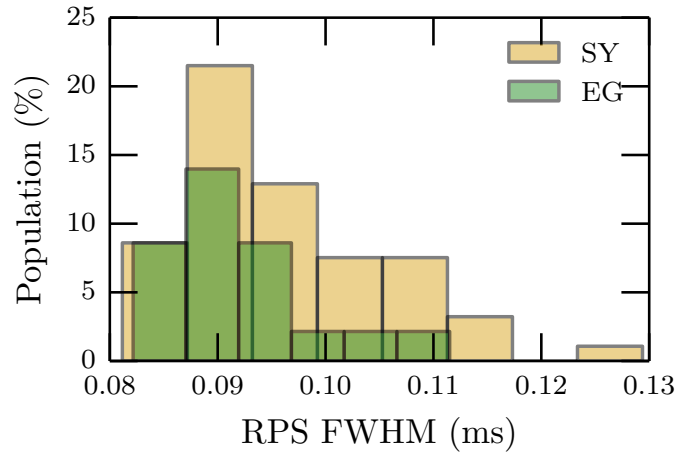

(b)

Figure 7.20: (a) Scatter plot between the FWHM of the resistive pulses and the pulse heights for experiment II2 (Pearson coefficient of 0.16 (SY) and 0.58 (EG)). (b) The distribution of the FWHM of the resistive pulses for the two species.

\subsubsection{Repetition at a Lower Molarity}

A buffer solution with a molarity of $10 \mathrm{mM} \mathrm{KCl}$ but otherwise identical composition to the SEB was used for experiments II13 to II24, which are summarized in Tab. 7.6. The particle concentration was chosen to be higher $\left(10.03 \times 10^{6}\right.$ particles $/ \mathrm{mL}$ of each species), so that a sufficient throughput could be achieved at a low pressure. A low pressure is essential in order to observe charge effects at this length scale, as it has been shown by [140] and in Sec. 3.4.6 that the fluid flow significantly disrupts the charge polarisation in the pore. A voltage of $1.5 \mathrm{~V}$ was applied to reach a background current of $\sim 120 \mathrm{nA}$. The higher voltage, compared to the experiments at $100 \mathrm{mM}$ molarity, also increases the charge polarisation.

The results of 13 experiments, summarised in Tab. 7.6, show lower matching rates and Wilson scores compared to the experiments conducted at higher molarity. This is, however, not connected to the salt concentration, but to the higher particle concentration used for the experiments I13 to I25, which leads to a greater number of multi events. The matching rate is again increasing with decreasing pressure. The signals are higher at lower hydrostatic pressures and particle velocities. The resistive pulse heights shift to slightly lower values and, more significantly, the ratio $\Delta I_{E G} / \Delta I_{S Y}$ of the average resistive pulse heights between the two species increases compared to the values at high salt molarity (1.70-1.99 (low molarity), 1.33-1.56 (high molarity), also see Figs. 7.19 (c) and 7.22 (a) and (c)).

This trend is also reproducible using the original setup of the qNano and similar 


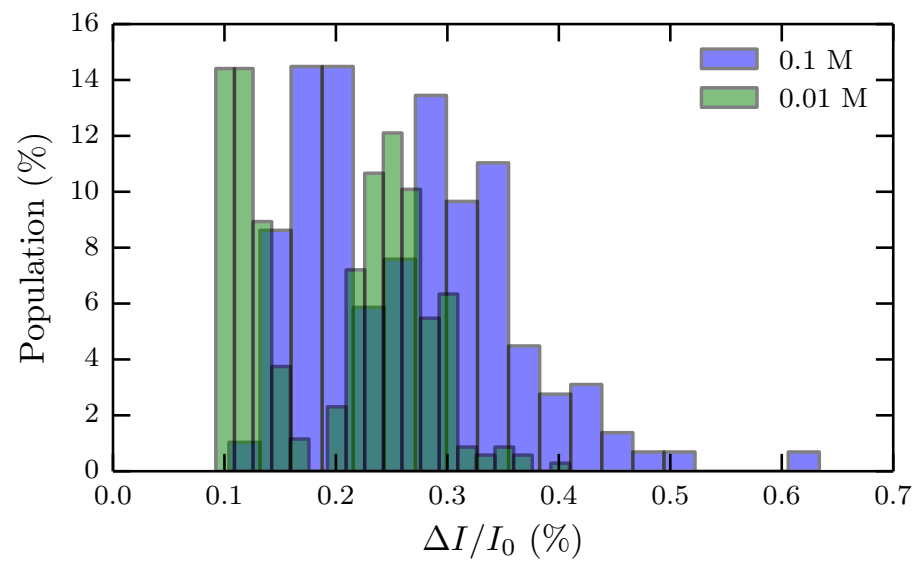

Figure 7.21: The distributions of the resistive pulse heights from two experiments using mixed particle sets and different salt molarities measured in the original qNano device. The distributions contain the data from 290 events at high and 347 events at low molarity. A stretch of $44 \mathrm{~mm}$, corresponding to a stretch of $37.50 \mathrm{~mm}$ in the adapted setup, a pressure of $50 \mathrm{~mm}$, and voltages of $0.18 \mathrm{~V}$ and $1.50 \mathrm{~V}$ were applied.

measurement parameters. While the qNano alone cannot distinguish between the two species, two peaks in the pulse distributions, corresponding to the SY and EG particle sets, are clearly visible in the pulse height distributions shown in Fig. 7.21. The peaks shift to lower values for the measurement at $10 \mathrm{mM} \mathrm{KCl}$ and while there is an overlap between the distributions at high molarity, no overlap is visible at low salt molarity. The adapted flow cell used for the experiment summarized in Tab. 7.5 and Tab. 7.6 had to be dissembled in order to change the electrolyte. Reassembling the device, even when done carefully to ensure a similar pore size, can lead to a change in pore geometry and could explain the change in the absolute pulse heights (compare Figs. 7.19 and 7.22). However, it does not explain the change in the ratio $\Delta I_{E G} /$ $\Delta I_{S Y}$. The results obtained with the original qNano in Fig. 7.21 were obtained by exchanging the electrolyte without changing the pore geometry, an advantage of the original setup. 


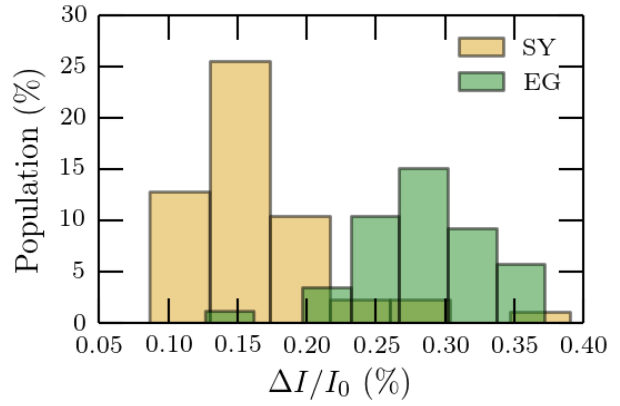

(a)

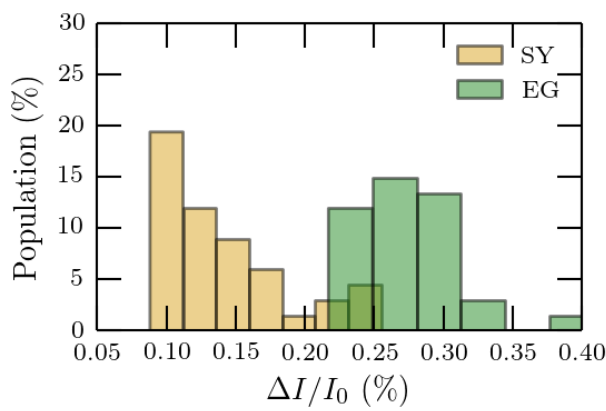

(c)

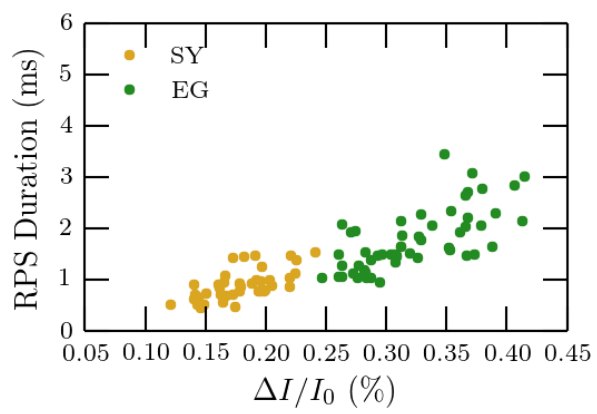

(e)

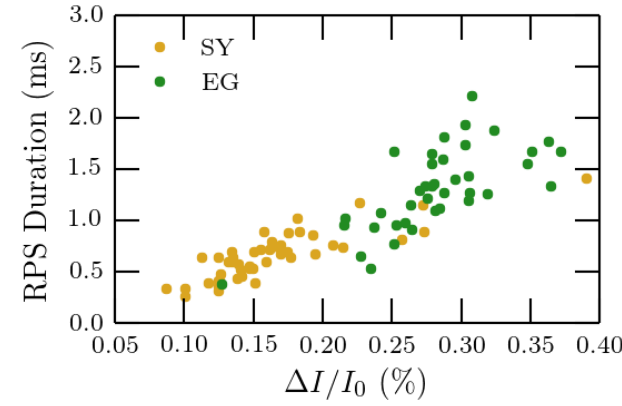

(b)

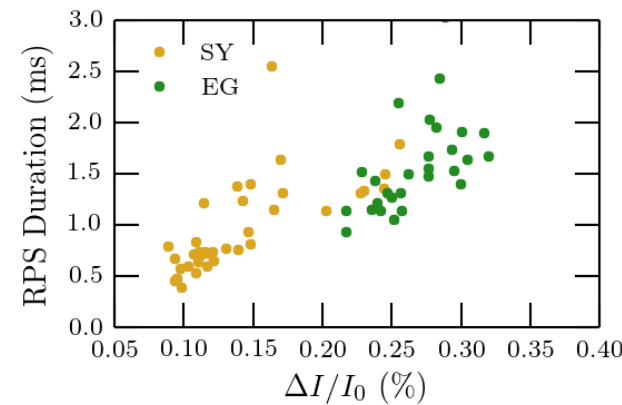

(d)

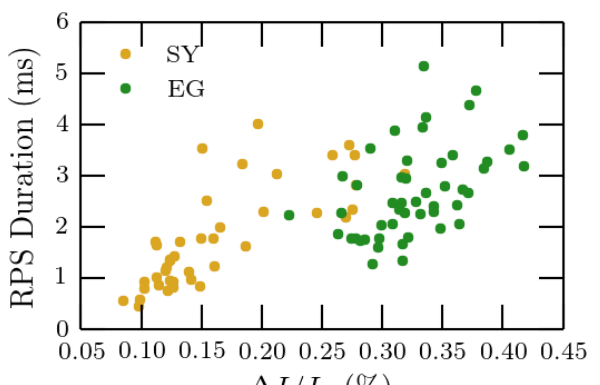

$\Delta I / I_{0}(\%)$

(f)

Figure 7.22: (a) The distribution of the resistive pulse heights and (b) the scatter plot of the pulse duration with the pulse heights for experiment II21, conducted at a voltage of $1.50 \mathrm{~V}$. (c) and (d) show the analogous plots for experiment II23 $(-1.50 \mathrm{~V})$. Both experiments were conducted at a pressure of $20 \mathrm{~mm} \mathrm{H}_{2} \mathrm{O}$. The ratio $\Delta I_{E G} / \Delta I_{S Y}$ is 1.72 for II21 and 1.95 for II23. The scatter plots of the pulse durations with the pulse heights for a pair of experiments at a pressure of $30 \mathrm{~mm}$ $\mathrm{H}_{2} \mathrm{O}$ conducted at $1.50 \mathrm{~V}$ (II15, pulse ratio 1.70 ) and $-1.50 \mathrm{~V}$ voltage (II16, pulse ratio 1.99) are shown in (e) and (f). 
Table 7.6: Experimental parameters and results of a series of experiments with a mix of $0.97 \mu \mathrm{m}$ polystyrene beads of two species at low salt molarity $(10 \mathrm{mM} \mathrm{KCl})$. The concentration per species is $10.03 \times 10^{6}$ particles $/ \mathrm{mL}$ and the absolute applied voltage is $1.50 \mathrm{~V}$ to compensate for the lower conductivity of the electrolyte. Experiments are conducted for both positive and negative voltage which is noted in the last column along with changes in the beam width, and the pinhole size if the parameters deviate from the usual values of $1.54 \mu \mathrm{m}$ and $200 \mu \mathrm{m}$ used in most experiments.

\begin{tabular}{|c|c|c|c|c|c|c|c|c|c|c|c|}
\hline \multirow{2}{*}{ Exp. } & \multirow{2}{*}{$\begin{array}{c}P \\
(\mathrm{~mm})\end{array}$} & \multicolumn{6}{|c|}{ Number of Events } & \multirow{2}{*}{$\begin{array}{l}\text { Wilson } \\
\text { Score }\end{array}$} & \multirow{2}{*}{$F I_{S Y}$} & \multirow{2}{*}{$F I_{E G}$} & \multirow{2}{*}{ Note } \\
\hline & & TRPS & Optical & Matching & Multi & SY & $\mathrm{EG}$ & & & & \\
\hline II12 & 50 & $185(14)$ & $161(3)$ & $146(79 \%)$ & 25 & 68 & 78 & $75 \%$ & 1.96 & 2.50 & \\
\hline II13 & 50 & $168(5)$ & $177(21)$ & $147(88 \%)$ & 16 & 71 & 76 & $84 \%$ & 2.15 & 3.06 & \\
\hline II14 & 40 & $129(0)$ & $130(5)$ & $121(94 \%)$ & 8 & 64 & 57 & $90 \%$ & 2.26 & 4.67 & \\
\hline II15 & 30 & $111(4)$ & $106(3)$ & $199(89 \%)$ & 8 & 44 & 55 & $86 \%$ & 3.13 & 6.50 & $w_{f}=0.90 \mu \mathrm{m}$ \\
\hline II16 & 30 & $100(0)$ & $96(1)$ & $90(90 \%)$ & 10 & 41 & 49 & $87 \%$ & 3.88 & 6.78 & $w_{f}=0.90 \mu \mathrm{m},-1.50 \mathrm{~V}$ \\
\hline II17 & 30 & $100(4)$ & $94(2)$ & $88(79 \%)$ & 8 & 48 & 40 & $73 \%$ & 3.46 & 6.64 & $-1.50 \mathrm{~V}, \mathrm{Ph} 300 \mu \mathrm{m}$ \\
\hline II18 & 30 & $96(4)$ & $90(3)$ & $82(85 \%)$ & 10 & 44 & 38 & $79 \%$ & 2.40 & 5.46 & \\
\hline II19 & 30 & $93(0)$ & $87(0)$ & $81(87 \%)$ & 12 & 50 & 31 & $82 \%$ & 4.04 & 6.07 & $\mathrm{Ph} 300 \mu \mathrm{m}$ \\
\hline II20 & 20 & $88(2)$ & $80(1)$ & $73(83 \%)$ & 13 & 42 & 31 & $77 \%$ & 3.52 & 6.18 & $w_{f}=0.90 \mu \mathrm{m}$ \\
\hline II 21 & 20 & $86(0)$ & $88(2)$ & $86(79 \%)$ & 0 & 47 & 39 & $73 \%$ & 2.72 & 7.27 & $\mathrm{Ph} 300 \mu \mathrm{m}$ \\
\hline II22 & 20 & $84(1)$ & $80(5)$ & $70(83 \%)$ & 13 & 29 & 41 & $77 \%$ & 3.95 & 4.91 & $-1.50 \mathrm{~V}$ \\
\hline II 23 & 20 & $74(1)$ & $73(3)$ & $67(91 \%)$ & 6 & 37 & 30 & $85 \%$ & 2.60 & 9.38 & $\mathrm{Ph} 300 \mu \mathrm{m},-1.50 \mathrm{~V}$ \\
\hline II 24 & 20 & $56(6)$ & $58(1)$ & $49(88 \%)$ & 6 & 24 & 25 & $81 \%$ & 2.73 & 4.68 & $-1.50 \mathrm{~V}$ \\
\hline
\end{tabular}




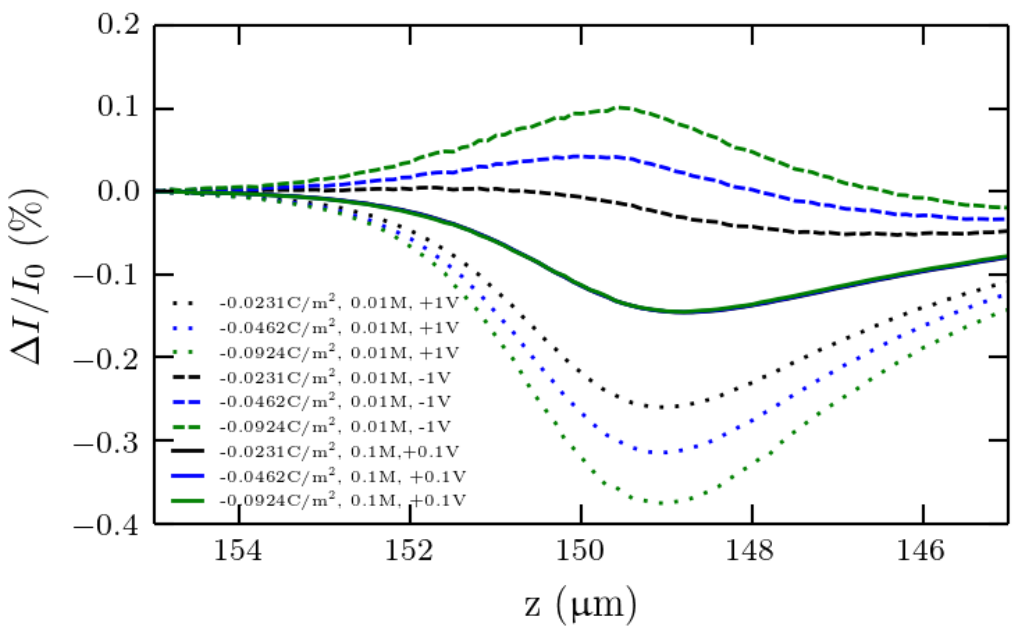

(a)

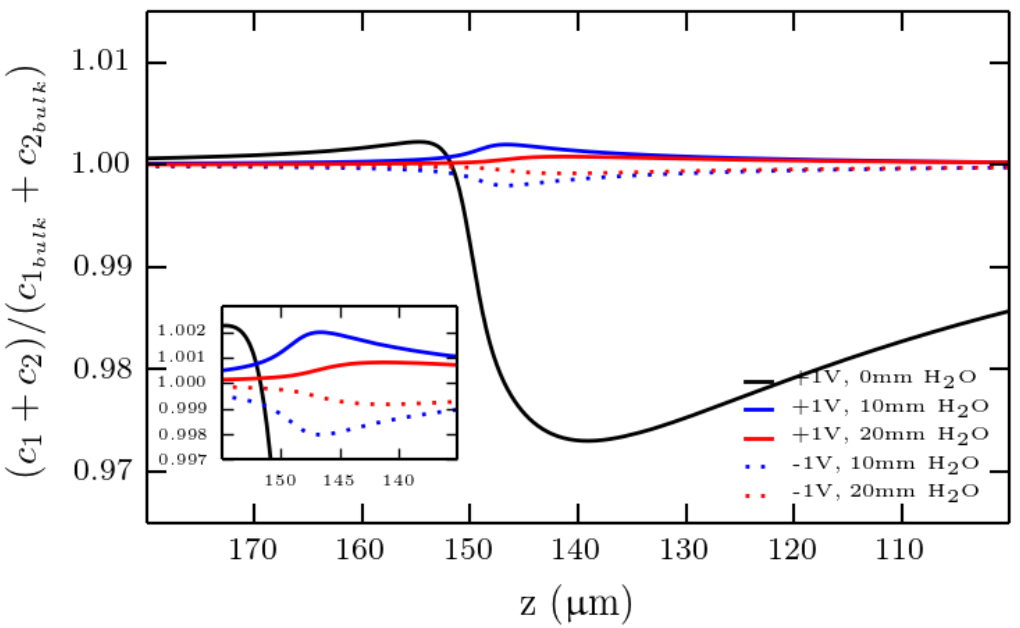

(b)

Figure 7.23: (a) The calculated resistive pulse caused by an insulating particle with $1 \mu \mathrm{m}$ diameter and different surface charge densities, salt molarities and applied voltages (see legend) as a function of its axial position (on-axis trajectory) in a conical pore (small pore opening $2.5 \mu \mathrm{m}$, large pore opening $25 \mu \mathrm{m}$, pore length $150 \mu \mathrm{m})$. The pore orifice is at a z-value of $150 \mu \mathrm{m}$. The charge density of the membrane is assumed to be $\sigma_{\text {wall }}=-0.0149 \mathrm{C} \mathrm{m}^{-2}$ and corresponds to $\zeta_{\text {wall }}=$ $-20 \mathrm{mV}$. (b) The ion concentration relative to the bulk concentration along the axis of the same pore as in (a) for $10 \mathrm{mM} \mathrm{KCl}$ and different pressures and voltages (see legend). The region close to the pore orifice is shown in the inset.

Although these observations indicate that the charge of the particles has an effect on the heights of the resistive pulses, it remains unclear if this is the main cause for the initially observed difference in the resistive pulse heights at high salt molarity. Using simulations developed in Sec. 3.4.6, evidence suggests that the differences observed are dominated by an actual size difference of the particle sets. The reasons for this are explained in the following.

Firstly, the simulations (Fig. 7.23 (a)) show that the particle charge has no effect on the resistive pulse at a salt molarity of $100 \mathrm{mM}$, the concentration conventionally 
used in TRPS. This was also concluded in [134], which used smaller particles with a diameter of $200 \mathrm{~nm}$, where charge effects are even more prominent.

Secondly, an increase in the resistive pulse height with increasing particle charge is expected in the usual configuration (positive voltage applied) at low salt molarity. In the experiments, both at high and low molarity, the opposite is observed and the SY beads, which are the higher charged particle set, show a consistently lower pulse height.

It has to be noted that the simulations were conducted without fluid flow through the pore. The current pulses in Fig. 7.23 (a) were obtained by executing the simulation for 100 particle positions with a spacing of $0.1 \mu \mathrm{m}$ between. A simulation that considers the fluid flow, the resulting particle movement (using the ALE method) and the ion distribution would reflect the physical reality more appropriately, but was not feasible with the computational resources available. However, the influence of the fluid flow on the ion distribution can be modelled without a particle present. Simulations of the on-axis ion distribution show that a pressure as low as $20 \mathrm{~mm}$ $\mathrm{H}_{2} \mathrm{O}$ can significantly disrupt the charge polarisation compared to a motionless fluid (Fig. 7.23 (b)), so that the depletion region disappears. Therefore the influence of the particle charge on the resistive pulse will be much smaller as pictured in Fig. 7.23 (a).

At low salt molarity, a negatively charged particle, driven downward through the pore at moderate pressures, causes a smaller resistive peak than expected if a purely volumetric exclusion of the electrolyte determines the pulse height. The resistive pulse decreases with increasing negative charge. This is consistent with the experiment as the absolute peaks become smaller and the ratio between the average pulse heights between the two, differently charged, particle species increases at low salt molarity (compare Figs. 7.19 (c), (f) with 7.22 (a), (b)). When the bias of the voltage is reversed, the pulse heights are reduced further and as the higher charged particles are more affected, an additional increase of the pulse ratios is observed.

This can be can be seen in Fig. 7.22, where the results from two experiments, which were conducted in immediate succession with no changes of pore geometry or pressure, are compared. The resistive pulse heights for both particle species shift to smaller values and, due the different charge of the particle sets, the ratio of the average pulse heights increases from 1.72 at a positive to 1.95 at a negative voltage bias. At a higher pressure $\left(30 \mathrm{~mm} \mathrm{H}_{2} \mathrm{O}\right)$ the absolute resistive pulses increase in height (II15, Fig. 7.22 (e)), which is due to the smaller charge polarisation observed for higher fluid flow, and after the voltage is reversed (II16, Fig. 7.22 (f)), they show the same trend as above, and the pulse ratios increase from 1.70 to 1.99 .

Apart from the change in the resistive pulse heights, the pulse durations also increase with a negative voltage bias. This can be explained by the increasing 
electrostatic interaction between pore and particle charges. Although the change in pulse durations can be observed for both pressures, the data set is not large enough to make a definite statement and the small relative increase could also be a consequence of a drift in the applied pressure.

It can be concluded that the initially observed difference in the resistive pulse at high salt molarity is attributed to an actual difference in size. A significant influence of the particle charge on the resistive pulse heights is not expected with the $\mathrm{KCl}$ concentration (100 mM) conventionally used for TRPS. At a salt molarity of $10 \mathrm{mM}$, low hydrostatic pressures and high voltages, charge effects become observable and could introduce a systematic error in the conventional determination of the particle size, when calibration particles with a significantly different charge are used. However, the influence of the particle charge on the resistive pulse heights could also be exploited to measure the charge. Apart from the standard procedure used in TRPS, which necessitates the comparison with a particle set of known zeta potential, a calibration free method could potentially be developed by making use of the pulse shifts at different molarities.

\subsubsection{Particle Sizing and Concentration Measurement}

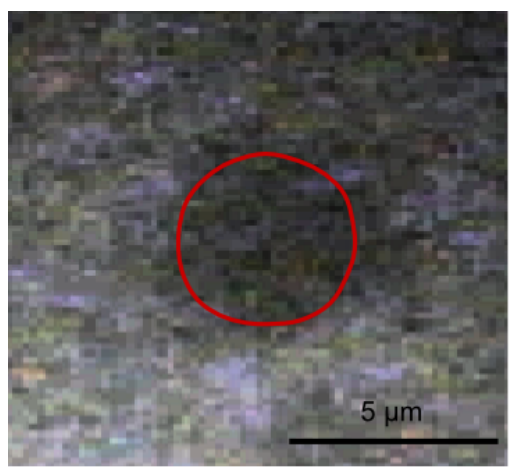

(a)

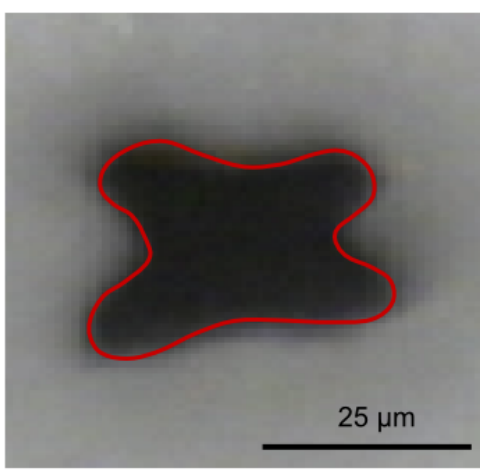

(b)

Figure 7.24: Optical images of the (a) small and (b) large pore openings of the pore specimen (NP1000) used in this section. The red line shows the outline of the orifices used to determine the hydraulic radii.

The small and large pore openings were determined optically to be $2.1 \mu \mathrm{m}$ and $10.5 \mu \mathrm{m}$ (Fig. 7.24). The thickness of the membrane is $186 \mu \mathrm{m}$. The calculated average diameters and the particle concentrations are listed in Tab. 7.5 for each experiment and both particle species. The values for the size for both particle sets are larger while the concentrations are lower (for most measurements) compared to the nominal values. This suggests that the small pore size was determined to be too large and a $10 \%$ smaller pore radius would lead to a better agreement with the nominal values. The discrepancy is a consequence of the measurement uncertainty 
of the optical sizing of the small pore opening. An accurate measurement is increasingly difficult for decreasing pore sizes as the values vary largely for only slight changes in the axial focus. This shows that although an approximate measurement of particle size and concentration can be obtained using an optical determination of the pore geometry, for a better accuracy the conventionally used method, which uses calibration particles of known size, should be used, especially for pores below a NP1000 rating.

\subsubsection{Determining the Species without Reference Spectra - Principal Component Analysis (PCA)}

If the spectra of the samples are unknown, a Principal Component Analysis (PCA) can be employed to find the main spectra and then identify the species in the collected data assuming that only events which involve just one particle type are captured. A comprehensive introduction to PCA can be found elsewhere [205, 226]. In brief, the method converts an $n$-dimensional dataset (here $\mathrm{n}=1024$, the number of pixels) into a set of linearly uncorrelated variables called principal components. It does this by solving the Eigenvalue problem of the covariance matrix with the resulting vectors forming an uncorrelated orthogonal basis set. A common PCA algorithm (Python function "svd") was employed to extract the dominating spectra from the data of the detected matching events here. The algorithm sorts the principal components in descending order of their Eigenvalues, so that the main spectra can be found in the first rows of the solution matrix. However, it is inherent to the method that also linear combinations of the underlying "pure" spectra can be identified as principal components. This was observed here and an additional step had to be undertaken to find the "real" principal components, which is demonstrated with the data of experiment II2 in the following.

The dash-dotted lines in Fig. 7.25 (b) show the first two principal components determined by the algorithm. The other components are orders of magnitude smaller and do not contain any useful information. Obviously the spectra do not match the reference spectra and even have negative values. The coefficients for the spectra of the matching events using a linear least square algorithm (compare Sec. 4.5.4) and the first two principal components as reference are orthogonal but are rotated compared to the desired direction, which is in the positive $\mathrm{x}$ - and y-axis (Fig. 7.25 (a)). By fitting linear functions through the data (red lines in Fig. 7.25 (a)), the rotation matrix can be found, which when applied to the first two principal components is used to extract the "real" principal components of the data. The so obtained spectra are in very good agreement with the measured reference spectra (Fig. 7.25 (b)). 


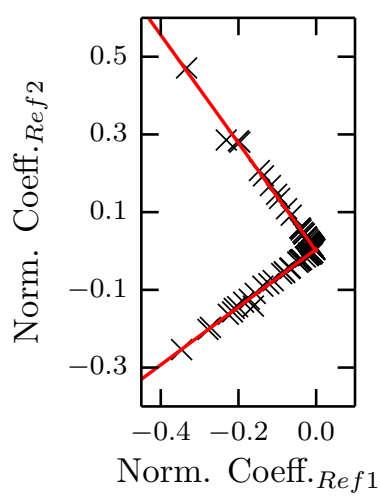

(a)

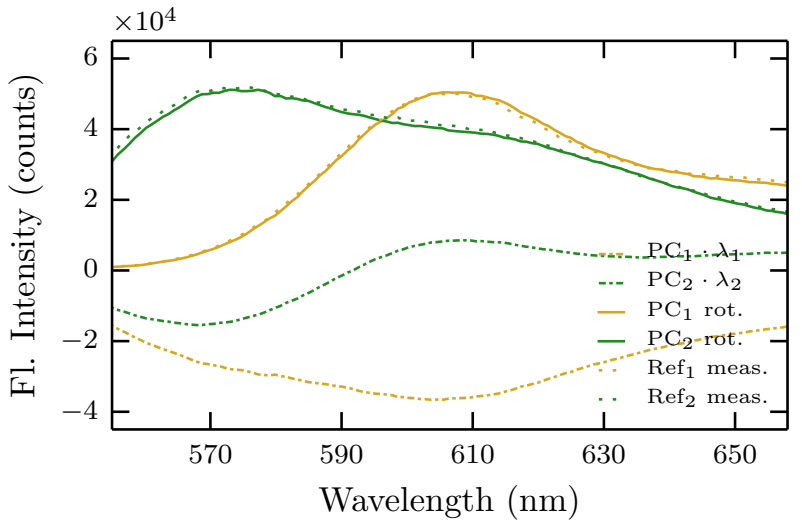

(b)

Figure 7.25: (a) Scatter plot of the coefficients for the detected events in experiment II2 using the first two principal components as a reference. (b) The measured reference spectra and the "original" and "real" first two principal components.

\subsection{Coordinated Detection of $500 \mathrm{~nm}$ Particles}

\subsubsection{Expected Problems for Sub- $\mu m$ Particles}

At length scales smaller than $1 \mu \mathrm{m}$ several additional phenomena are expected to become important. First, the amount of dye and therefore the optical signal scale with the volume of the particle. A decrease in the particle diameter from $2 \mu \mathrm{m}$ to $200 \mathrm{~nm}$ will result, assuming a similar fluorescence cross section for each dye molecule, in a decrease of the signal by a factor of 1000 . The optical detection limit, determined primarily by the readout noise of the CCD, is therefore expected to be reached and the number of unmatched current events will increase. Although the laser power can potentially be increased by several orders of magnitude to compensate for the weaker signals, this gives rise to the laser-induced noise discussed in Sec. 4.1.2. The noise is of low frequency and can be removed from the current trace using the the methods presented. A bigger problem is the formation of nano-bubbles at the pore orifice, which is connected with the noise phenomenon and can disrupt the measurement when bubbles get trapped in the pore. Therefore increasing the laser power is only feasible to a certain upper limit.

Another issue is that the detection volume cannot be decreased any further. As higher particle concentrations are necessary to achieve a sufficient throughput for smaller pore dimensions, an increase in multi events is expected. Additionally, smaller pores also lead to smaller absolute fluid velocities and the particles spend a longer time in the optical detection region. While the (on-axis) velocity at the orifice of an NP2000 pore with an applied pressure of $50 \mathrm{~mm} \mathrm{H}_{2} \mathrm{O}$ is $100 \mathrm{~mm} \mathrm{~s}^{-1}$ and a $2 \mu \mathrm{m}$ particle stays less than $1 \mathrm{~ms}$ in the FWHM of the axial optical detection region, the corresponding values for a NP200 pore and a $200 \mathrm{~nm}$ particle are $15 \mathrm{~mm} \mathrm{~s}^{-1}$ and 
$100 \mathrm{~ms}$ at the same applied pressure. This will increase the pulse heights of the optical signals, but also increase the likelihood of long multi events, analogous to those detected in Sec. 7.2.2. Reducing the concentration and increasing the pressure might be the obvious solution to this problem, but has a reduction of the signal as a consequence. This discussion shows that the experimental parameters have to be chosen very carefully for smaller pore and particle dimensions to ensure high matching rates and a sufficient throughput.

\subsubsection{Experimental Details}

To make the transition from the $\mu \mathrm{m}$-scale to the smallest beads $(\sim 200 \mathrm{~nm})$ used in this thesis, particles with a nominal diameter of $500 \mathrm{~nm}$ were measured first. The particles are labelled with a dye named "Red" by the supplier. The $514 \mathrm{~nm}$ line of the Argon-ion laser with a neutral density filter D3 is used for the excitation. The low power is still sufficient to detect the optical events and has the advantage that the laser induced noise, which is significant if the filter D2 is used, does not disturb the measurement. Although a slight noise is visible in the current trace even when the filter D3 is used, a filtering step is not necessary and the event finding algorithms are able to detect the events correctly. Both the $\times 100$ BFPL500 system and the $\times 100$ objective alone were used for the experiments presented in this section. The pinhole was set to $150 \mu \mathrm{m}$ unless otherwise noted and corresponds to a square with a side length of $4.8 \mu \mathrm{m}$ centred at the pore orifice. The alignment becomes more difficult with decreasing pore size and an exact congruence of the beam centre with the middle of the pore orifice is not routinely achieved. A high stretch $(40.80 \mu \mathrm{m})$ was applied to the pore specimen (NP400) in order to minimise the regular blocking encountered at lower stretches. Although electrokinetic effects increase in relative strength with decreasing pore size, the hydrodynamic transport is still dominant. The particle concentration is $122 \times 10^{6}$ particles $/ \mathrm{mL}$, significantly higher than at the $\mu \mathrm{m}$-scale to allow for a sufficient throughput. The molarity of the electrolyte was reduced to $50 \mathrm{mM}$ to ensure a stable colloid system. A voltage of $0.5 \mathrm{~V}$ was applied.

\subsubsection{Results}

The parameters and results of six experimental runs are summarized in Tab. 7.7. The matching rates and Wilson scores at high pressures are significantly smaller compared to the experiments presented in the previous two sections, mainly due to the detection of multi events. The number of unmatched current events in experiment III2, which was conducted with a smaller beam size, is exceptionally high. This is most likely a consequence of a lateral misalignment of the beam with the 


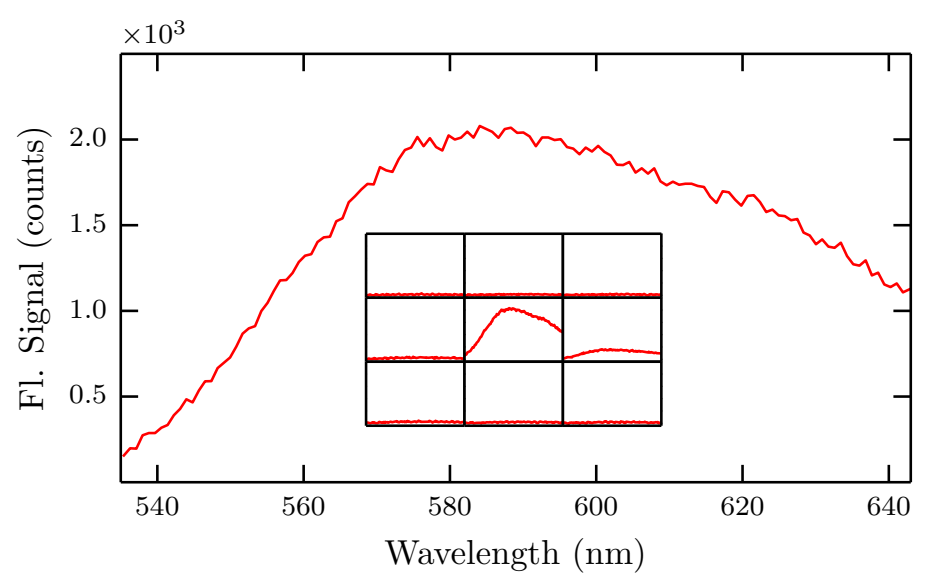

Figure 7.26: The spectrum of a "Red"-labelled particle passing through the pore and its evolution with time (experiment III4).

pore orifice. Many particles are therefore subjected to a small laser intensity and are below the detection limit. A larger beam size is therefore preferable as the results are less sensitive to slight misalignments. On the other hand, a smaller beam size has the advantage that the occurrence of multi events is less likely due to a reduced Rayleigh range. This example shows that the parameters have to be chosen very carefully at this length scale. Apart from experiment III2, the number of unmatched current and optical events is relatively low and most of the unmatched optical events are due to small "pre events" as shown in Fig. 7.1 (f). The matching rates increase for lower pressures where rates close to $90 \%$ are achieved. As usual, the optical signal increased for lower pressures. The hydrodynamic transport is still dominant at this length scale and the event frequency is appropriately proportional to the pressure. 
Table 7.7: Experimental parameters and results of a series of experiments with $500 \mathrm{~nm}$ polystyrene beads. Parameters for experiments that differ from the values described in the text are noted in the last column (here, beam width).

\begin{tabular}{|c|c|c|c|c|c|c|c|c|c|c|}
\hline Exp. & $\begin{array}{c}P \\
(\mathrm{~mm})\end{array} \mid$ & TRPS & $\begin{array}{l}\text { Number } \\
\text { Optical }\end{array}$ & $\begin{array}{l}\text { Events } \\
\text { Matching }\end{array}$ & Multi & $\begin{array}{c}\text { Wilson } \\
\text { Score }\end{array}$ & $F I$ & $d_{\text {calc }}(\mathrm{nm})$ & $c_{\text {calc }}$ & Note \\
\hline III1 & 50 & $357(4)$ & $310(7)$ & $261(73 \%)$ & 92 & $70 \%$ & $0.49(114 \%)$ & $471(10.8 \%)$ & 132 & \\
\hline III2 & 50 & $331(90)$ & $211(2)$ & $183(55 \%)$ & 12 & $51 \%$ & $0.50(75 \%)$ & $444(11.5 \%)$ & 122 & $w_{f}=0.90 \mu \mathrm{m}$ \\
\hline III3 & 30 & $179(8)$ & $170(15)$ & $141(79 \%)$ & 30 & $75 \%$ & $0.88(71 \%)$ & $446(9.3 \%)$ & 110 & \\
\hline III4 & 20 & $137(1)$ & $137(4)$ & $130(95 \%)$ & 6 & $92 \%$ & $0.80(78 \%)$ & $459(8.3 \%)$ & 127 & \\
\hline III5 & 10 & $89(3)$ & $92(9)$ & $80(90 \%)$ & 6 & $85 \%$ & $1.16(75 \%)$ & $447(8.9 \%)$ & 164 & \\
\hline III6 & -50 & $318(10)$ & $275(5)$ & $238(75 \%)$ & 70 & $72 \%$ & $0.41(79 \%)$ & $444(10.5 \%)$ & 117 & \\
\hline
\end{tabular}




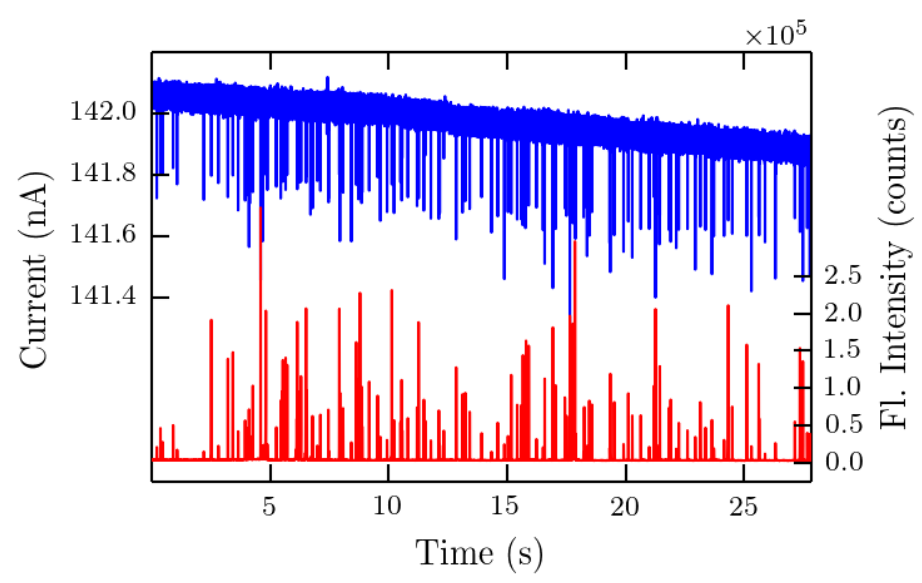

Figure 7.27: The current and optical traces for experiment III4.

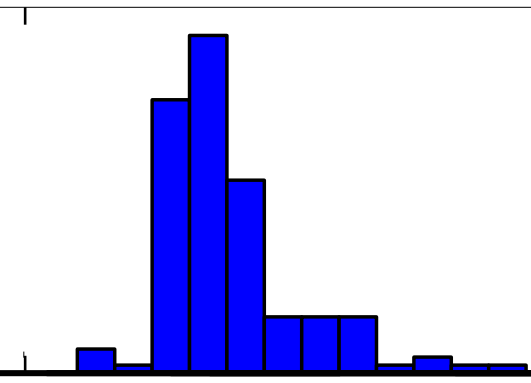

Figure 7.28: The distribution of (a) the resistive pulse heights, (b) the resistive pulse durations, (c) the fluorescence intensities and (d) the durations of the optical events of experiment III4. The inset in (c) shows the fluorescence intensities integrated over the duration of the optical events.

Figure 7.28 shows the histograms of the events detected in experiment III4 (Fig. 7.27). The resistive pulse heights and durations in Figs. 7.28 (a) and (b) show distributions typical for a mono-disperse sample. Compared to the particles at the $\mu \mathrm{m}$-scale the average duration of the optical pulses is, due to the lower parti- 


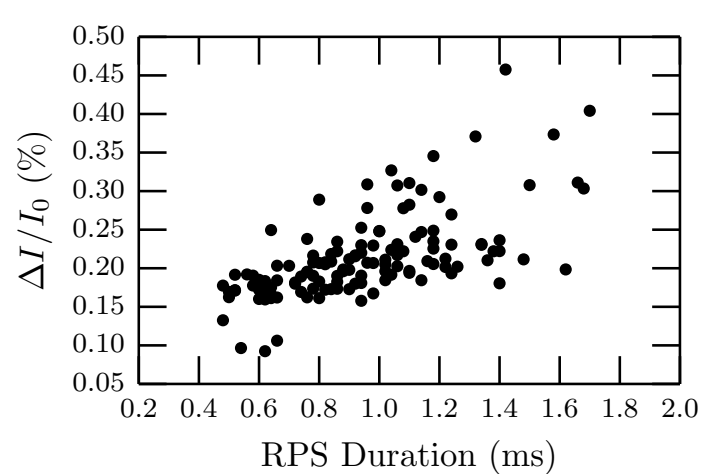

(a)

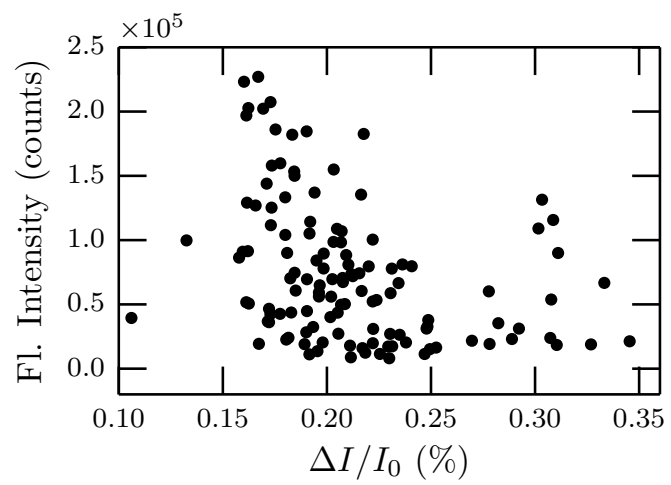

(c)

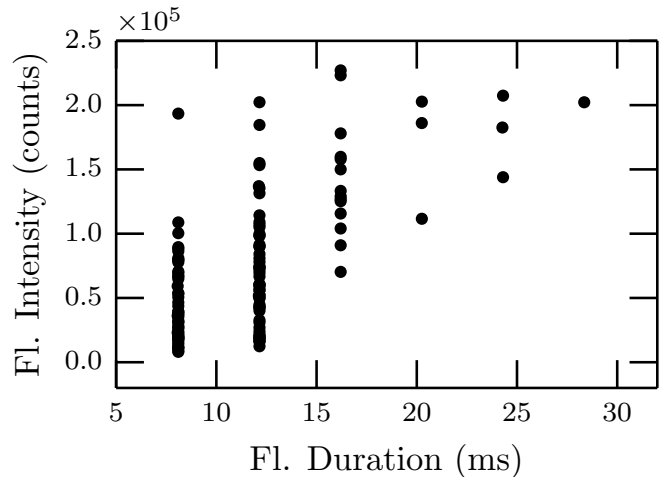

(b)

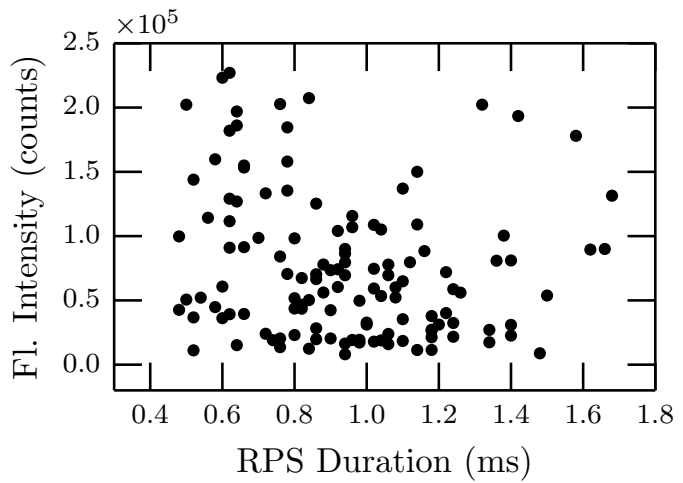

(d)

Figure 7.29: (a) The correlation between the resistive pulse durations and the resistive peak heights (Pearson coefficient: 0.58). Scatter plots of the measured fluorescence intensities with (b) the durations (0.65) of the optical events (c) the resistive peak heights $(0.02)$ and $(d)$ the resistive pulse durations $(-0.02)$ for the experiment III4.

cle velocities, longer and most events consist of two data points that are above the background signal (peak at $12 \mathrm{~ms}$ in Fig. 7.28 (d)). The distribution of the optical signals (Fig. 7.28 (c)) shows a more uniform characteristic than at the $\mu \mathrm{m}$-scale, which is both attributed to a larger beam width to pore ratio and the fact that most particles spend more than one time step in the optical detection volume. The scatter plots of both the electrical and optical pulse heights with their corresponding durations show the usual, strongly positive correlations (Pearson coefficients of 0.58 and 0.65, Figs. 7.29 (a) and (b)). The correlation between the optical pulse durations with the time integrated florescence intensities (inset in Fig. 7.28 (c)) is even stronger and has a value of 0.86 . This confirms again that on-axis particles, which spend more time in the optical detection volume, create higher optical signals. The correlations between electrical and optical data (Figs. 7.29 (c) and (d)) do not show the expected negative values. This is a consequence of the larger beam width to pore ratio, so that a slight lateral misalignment, which is likely to occur for small pores, can already reduce the cross correlation between electrical and optical data 
significantly.

\subsubsection{Particle Sizing and Concentration Measurement}

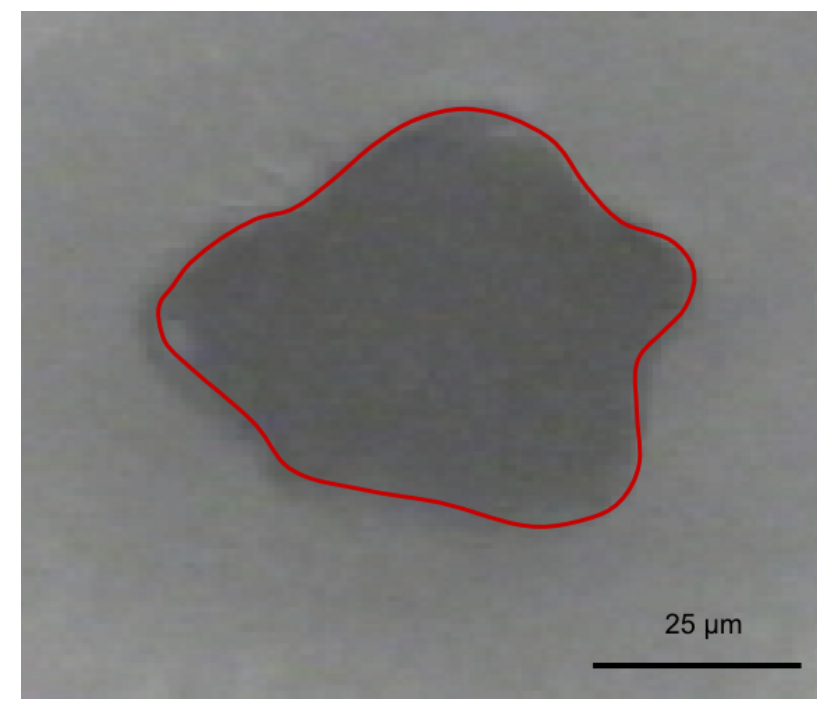

Figure 7.30: Optical image of the large pore opening of the pore specimen (NP400) used in this section. The red line shows the outline of the orifice used to determine the hydraulic radius.

An optical determination of the size of the small pore opening is not possible at this length scale and below. As an alternative, the approximate dimension of the small pore opening can be deduced with the knowledge of the large pore opening, which is obtained as usual $(23.8 \mu \mathrm{m}$, note the larger diameter caused by the increased stretch, Fig. 7.30), the thickness of the membrane $(164 \mu \mathrm{m})$, the baseline current $(140 \mathrm{nA})$, the applied voltage $(0.5 \mathrm{~V})$ and the resistivity of the electrolyte. Using Eq. 3.3, the radius of the small pore orifice was calculated to be $\sim 1.15 \mu \mathrm{m}$. The particle diameter and the concentration can then be derived with the equations Eqs. 6.1 and 6.2 and are listed in Tab. 7.7. The numbers agree surprisingly well with the nominal values, taking into account that the calculation involves high uncertainties both due to the experimental parameters and the underlying model.

One experiment (II6) was conducted at a negative pressure and therefore upwards moving particles. Neither the optical nor the electrical signals are more uniform compared to downwards particles. This indicates that a flow focussing effect, which could be observed for a NP2000 pore, is not present and is expected as the flow velocity and therefore the Reynolds number decrease with smaller pore size. 


\subsection{Co-ordinated Detection of $200 \mathrm{~nm}$ Particles}

The detection of $\sim 200 \mathrm{~nm}$ polystyrene beads is demonstrated in this section. Two sets of particles with a different fluorescent label ("Dragon-Green" (DG) and "SurfGreen" (SG) beads) and a slightly different nominal diameter (DG: $220 \mathrm{~nm}$, SG: $190 \mathrm{~nm}$ ) are used. The spectra of the two particle sets are pictured in Fig. 7.31. The oscillations at high wavelengths for the SG beads can be attributed to whispering gallery modes in the particles that modulate the emission signal.

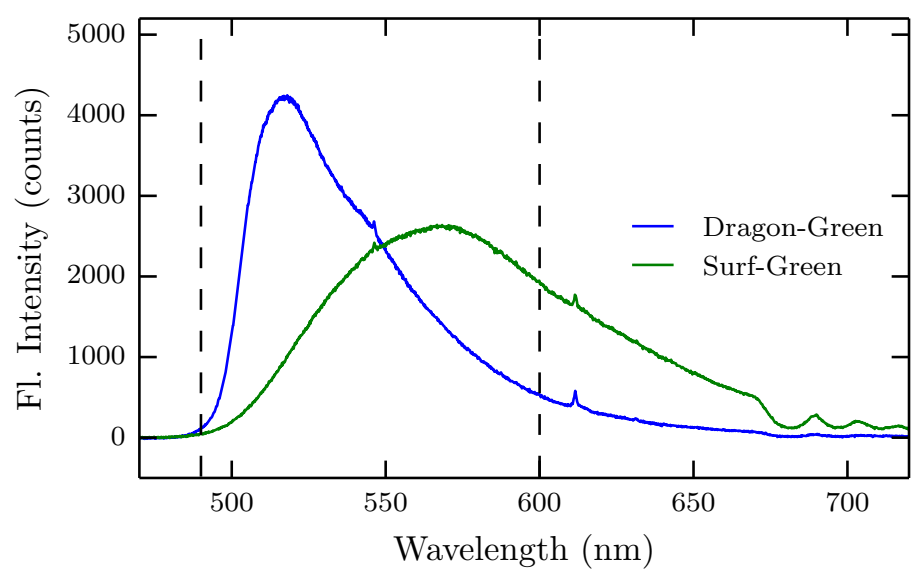

Figure 7.31: The spectra of the two particle sets ("Surf-Green" and "Dragon Green") used in this section. The concentration was $8.58 \times 10^{10}$ particles $/ \mathrm{mL}$ for the DG and $1.33 \times 10^{11}$ particles $/ \mathrm{mL}$ SG beads respectively. The $\times 100$ BFPL500 system, a pinhole of $300 \mu \mathrm{m}$ and an integration time of $1 \mathrm{~s}$ were used. The multi window feature of the spectrometer was used to achieve a measurement over the full emission region. The vertical broken lines indicate the region which is detected in the coordination experiments.

Both the $\times 100$ and a $\times 100$ BFPL500 system were used in the following experiments. The $458 \mathrm{~nm}$-line of the Ar-ion laser with a neutral density filter $D 2$ was used for the excitation. The higher laser intensity connected with a higher sensitivity of the small pores to mechanical vibrations gives rise to the low-frequency noise upon laser illumination. The noise has the usual frequency of $\sim 45 \mathrm{~Hz}$ and the current trace can be filtered with the wavelet method. Figure 7.32 (a) shows the raw and the filtered trace of experiment IV4. The noise also appears in the optical traces (Fig. 7.32 (b). Filtering is not feasible as it would introduce a significant distortion due to the relatively low sample frequency of the optical detection.

The noise can be utilised to align the beam with the pore centre and the best alignment is achieved when the noise is maximised. A purely optical alignment is difficult at this length scale as the pore orifice is barely recognisable due to its low contrast. The noise can potentially also be used to align the electrical and optical traces without the use of the correlation method by introducing "marker" events, 


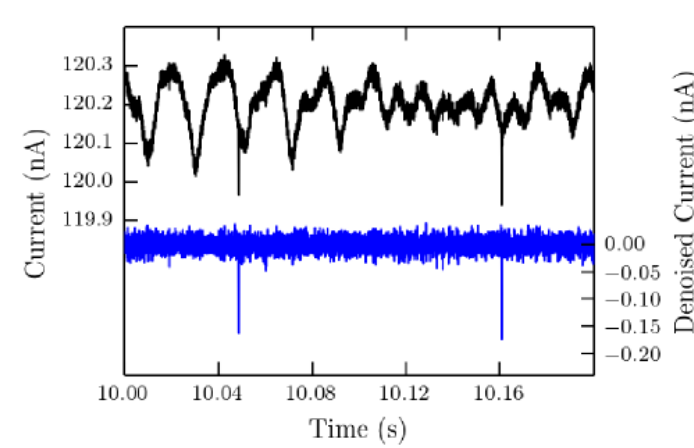

(a)

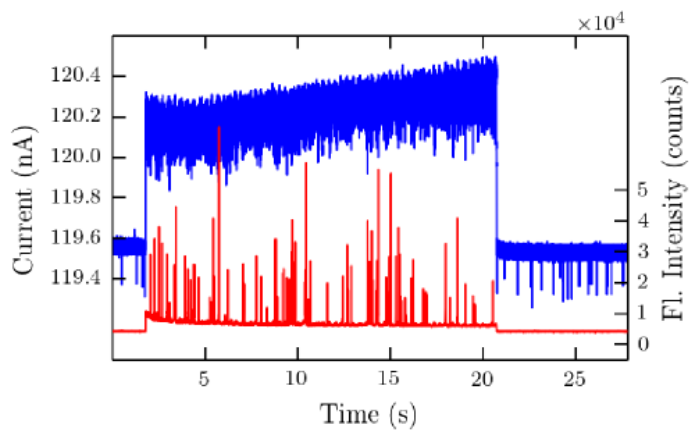

(c)

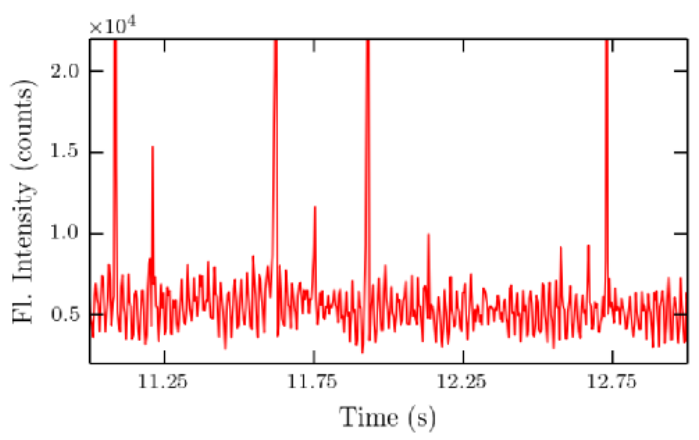

(b)

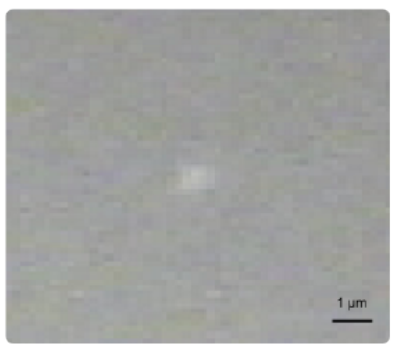

(d)

Figure 7.32: A part of the raw and filtered current trace for experiment IV $7^{*}$ using the COBRA wavelet filtering algorithm (parameters: "DB10" wavelet, 5 transform levels, 3 iterations, noise background 0.025). (b) The noise in the optical trace of experiment IV8* leads to a difficulties in the event finding algorithm for small optical signals. (c) The current and optical traces for experiment IV7*. The sharp jumps in the traces occurs when the laser is switched on/off and can be used to aligned the traces in time without the correlation method. (d) A white shimmer indicates in an optical image that an air bubble is trapped in the pore.

i.e. switching the laser on and off (Fig. 7.32 (c)).

One major problem that was encountered here was the formation of air bubbles in the pore. A freshly assembled pore shows only a small noise amplitude in the current trace upon initial illumination. After being illuminated for several tens of seconds to minutes, the noise becomes larger until the current trace eventually shows an erratic behaviour, which indicates that an air bubble is trapped in the pore. The bubble can also be observed optically as a white shimmer in the pore orifice (Fig. 7.32), which usually appears black. Many experiments had to be aborted and recorded traces fully or partly discarded due to this reason. If a bubble is trapped in the pore orifice, the pore has to be removed, carefully cleaned and put back into place. It is plausible that small bubbles created by the laser get attached to the pore walls and accumulate until a bigger bubble forms, which eventually disrupts the current measurement. The exact mechanism of the bubble formation and the mechanism that amplifies the mechanical vibrations of the system warrant further investigation. For example, the influence of carefully degassed solutions on the effect 
could be studied.

A $100 \mu \mathrm{m}$ pinhole, which corresponds to a square with a side length of $3.2 \mu \mathrm{m}$ centred at the pore orifice, was used, unless otherwise noted. A macroscopic stretch of $39 \mathrm{~mm}$ was applied to the pore specimen. A voltage of $1.00 \mathrm{~V}$, a molarity of $50 \mathrm{mM}$ and a particle concentration of $572 \times 10^{6}$ particles $/ \mathrm{mL}$ were chosen for experiments IV1 to IV4 in Tab. 7.8. Due to the formation of air bubbles in the pore, the device had to be dissembled and reassembled several times between the experiments IV4 and IV9*. In an attempt to reduce the build up of bubbles and also minimise the occurrence of pore blocking, different stretches and therefore voltages were applied. As the stretches were not accurately monitored during this procedure, the experiments cannot be directly compared and are marked with *. The collected data, however, is still beneficial to the discussion.

\subsubsection{Results for One Particle Set}

The results for nine experiments using the DG beads are summarised in Tab. 7.8. The matching rates and Wilson scores are generally lower than the ones achieved at the $\mu \mathrm{m}$-scale. However, at low pressure and therefore particle speeds matching rates close to $90 \%$ can be achieved. The number of unmatched electrical and optical events is relatively high for all pressures. The unmatched optical events are a combination of small "pre" events (Fig. 7.1 (f)), particles that diffuse in and out of the detection volume without translocating the pore, and "noise" events, that are artefacts of the event finding algorithm and do not correspond to a real particle. However the number of the latter kind of unmatched optical events is remarkably small as the concentration compared to the $\mu \mathrm{m}$-scale is $\sim 500$ times higher, while the optical detection volume is still the same in the axial direction. The unmatched current events are due to optical events which are either below the detection limit or below the threshold of the optical event finding algorithm. Due to the occurrence of the low-frequency noise, the threshold has to be chosen very carefully in order to prevent the detection of "noise" events and at the same time not to exclude small optical signals from the detection (see Fig. 7.32 (b)). 
Table 7.8: Experimental parameters and results of a series of experiments with $220 \mathrm{~nm}$ polystyrene beads with the fluorescent label "Dragon-Green". The average fluorescent intensity FI is given in counts $\times 10^{4}$ with its relative standard deviation. Experiments that are marked with a $*$ were conducted at various different pore stretches and voltages and the average resistive pulse heights are not listed for these cases. Parameters for experiments that differ from the values described in the text are noted in the last column (here, experimental duration).

\begin{tabular}{|c|c|c|c|c|c|c|c|c|c|c|}
\hline Exp. & $\begin{array}{c}P \\
(\mathrm{~mm})\end{array}$ & $\begin{array}{c}w_{f} \\
(\mu \mathrm{m})\end{array}$ & TRPS & $\begin{array}{l}\text { Number } \\
\text { Optical }\end{array}$ & $\begin{array}{l}\text { Events } \\
\text { Matching }\end{array}$ & Multi & $\begin{array}{l}\text { Wilson } \\
\text { Score }\end{array}$ & $F I$ & $\Delta I / I_{0}(\%)$ & Note \\
\hline IV1 & 80 & 1.54 & $304(88)$ & $211(21)$ & $168(55 \%)$ & 48 & $51 \%$ & $1.40(87 \%)$ & $0.18(15.8 \%)$ & \\
\hline IV2 & 50 & 1.54 & $106(37)$ & $177(11)$ & $63(59 \%)$ & 6 & $52 \%$ & $1.90(80 \%)$ & $0.20(9.3 \%)$ & $15.1 \mathrm{~s}$ \\
\hline IV3 & 20 & 1.54 & $110(40)$ & $77(7)$ & $70(64 \%)$ & 0 & $58 \%$ & $2.47(65 \%)$ & $0.26(13.1 \%)$ & \\
\hline IV4 & 20 & 1.54 & $103(3)$ & $109(11)$ & $96(93 \%)$ & 4 & $89 \%$ & $1.94(83 \%)$ & $0.25(18.0 \%)$ & \\
\hline IV5* & 50 & 0.9 & $180(88)$ & $96(12)$ & $76(42 \%)$ & 16 & $37 \%$ & $3.01(61 \%)$ & - & \\
\hline IV6* & 40 & 0.9 & $153(5)$ & $147(8)$ & $128(84 \%)$ & 20 & $80 \%$ & $1.03(51 \%)$ & - & $23.1 \mathrm{~s}$ \\
\hline $\mathrm{IV} 7^{*}$ & 20 & 0.9 & $111(30)$ & $85(8)$ & $73(66 \%)$ & 8 & $60 \%$ & $1.86(54 \%)$ & - & $19.0 \mathrm{~s}$ \\
\hline IV8* & 20 & 0.9 & $112(19)$ & $110(18)$ & $91(81 \%)$ & 2 & $76 \%$ & $2.16(61 \%)$ & - & \\
\hline IV9* & -50 & 0.9 & $40(4)$ & $42(7)$ & $34(85 \%)$ & 2 & $75 \%$ & $1.77(74 \%)$ & - & \\
\hline
\end{tabular}


Accurate lateral alignment is critical and the results are sensitive to displacements in the order of sub- $u$ m, which explains why very different matching rates can be achieved for otherwise identical experimental parameters (compare experiments IV3 and IV4). The alignment also greatly influences the optical signals so that an increase with decreasing pressure, as seen in the previous sections, is not clearly observed here. Electrokinetic effects become more significant at this length scale, which is reflected in the TRPS event frequency. The number of resistive events does not decrease with decreasing pressure in the same way as seen in the previous sections, where hydrodynamic transport was the only relevant mechanism. By extrapolating the data of experiments IV1 to IV4, approximately 40 events are expected at zero applied pressure. Although experiment IV9* was conducted at a negative pressure and cannot be directly compared with the first four runs due to a different applied stretch, it has a much reduced number of events compared to experiments with similar absolute, but positive pressure. Both observations show that electrokinetic effects are relevant, with electrophoresis dominating electroosmosis so that a bias for downwards moving particles is created. This indicates that the zeta potential of the particles is larger than that of the pore membrane, although only a qualitative statement can be made.

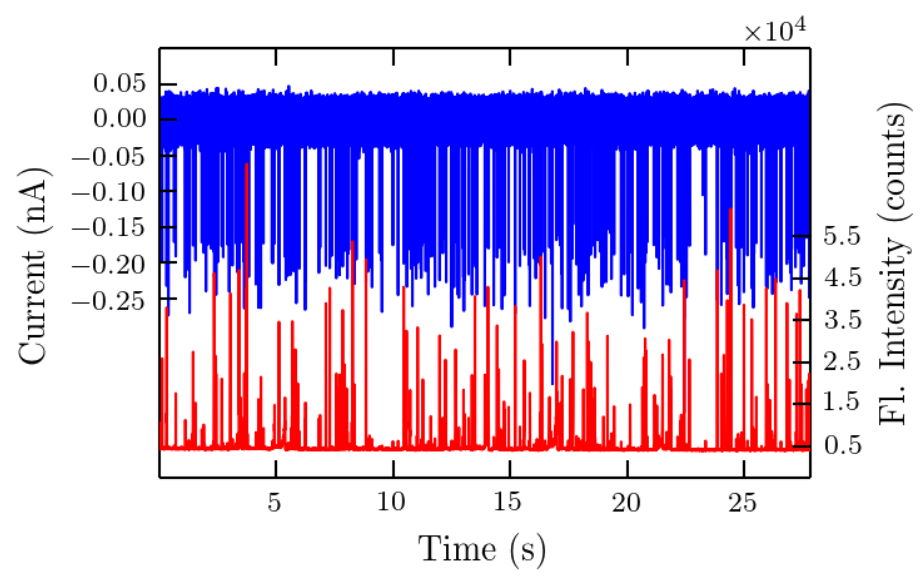

Figure 7.33: The current (filtered) and the optical traces for experiment IV1.

Figure 7.34 shows the histograms of the events detected in experiment IV1, whose electrical and optical traces are plotted in Fig. 7.33. The resistive pulse heights and durations show the typical distribution of a monodisperse sample. The pulse heights are calculated relative to the "noisy" background current, which is slightly higher than the current when the laser is switched off (see Fig. 7.32 (c)). In experiments IV1 to IV4 this difference is below $2 \mathrm{nA}$ with a background current ranging from $109 \mathrm{nA}$ to $116 \mathrm{nA}$, and therefore this does not significantly affect the determination of the relative pulse height. However, larger laser intensities and noise levels can introduce a systematic error, which has to be kept in mind if the particle size is of 
interest.

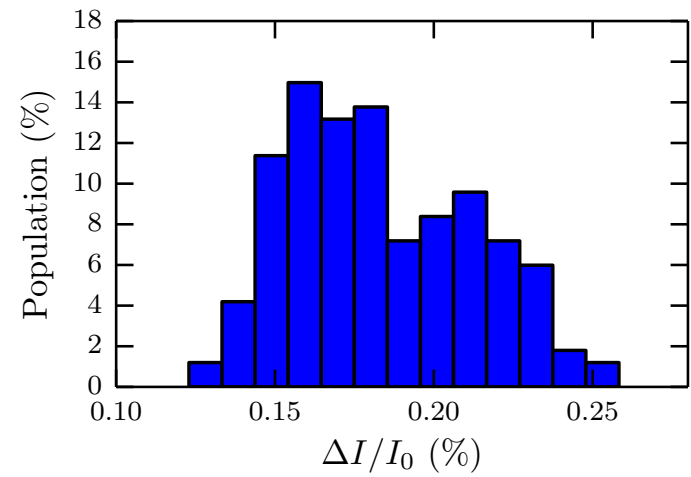

(a)

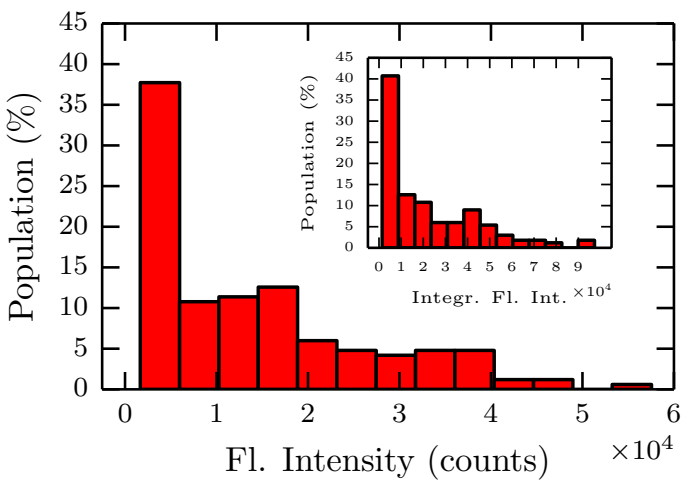

(c)

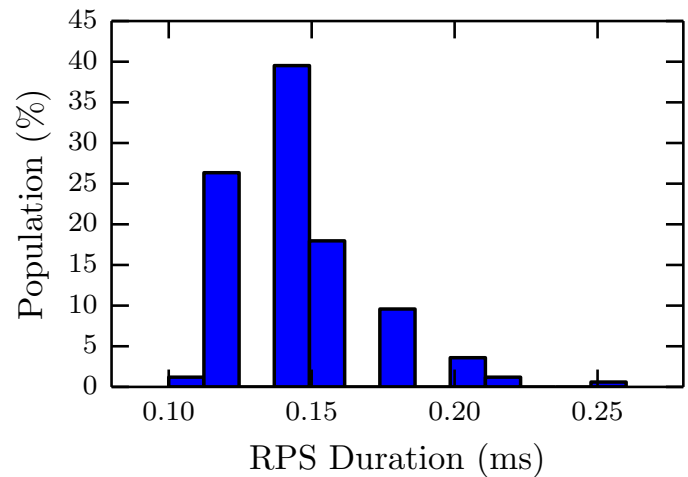

(b)

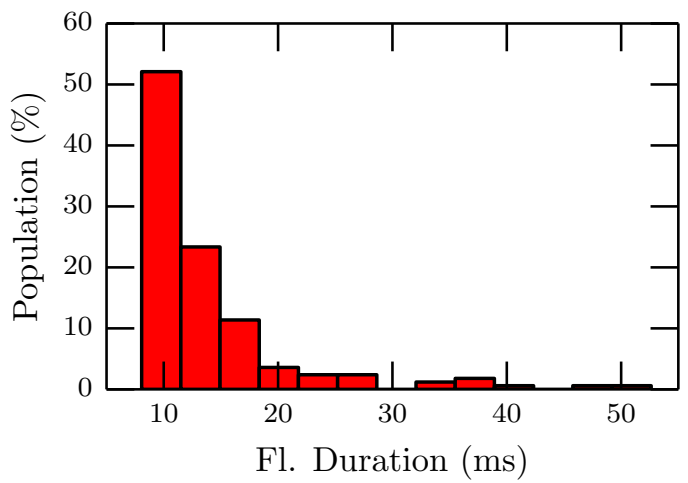

(d)

Figure 7.34: The distribution of (a) the resistive peak heights, (b) the resistive pulse durations, (c) the fluorescence intensities and (d) the durations of the optical events of experiment IV1. The inset in (c) shows the fluorescence intensities integrated over the duration of the optical events.

The resistive pulse durations in Fig. 7.34 (b) show lower values compared to the results obtained for larger pore and particle dimensions. Additionally, the distribution has gaps between the columns, which indicates that the pulse duration becomes comparable with the time resolution of the electrical measurement (0.02 ms). Therefore most pulses in experiment IV1 consist of only six data points (peak at $0.14 \mathrm{~ms}$ in the distribution. This is due to the smaller sensing region of the pore and can be understood by modelling the time-current relationship of the pulse. Here, the semi-analytic model with the geometry of the NP200 model pore was used with an applied pressure of $80 \mathrm{~mm} \mathrm{H}_{2} \mathrm{O}$, an applied voltage of $1 \mathrm{~V}$ and zeta potentials of the membrane and the particle of $\zeta_{\text {wall }}=-20 \mathrm{mV}$ and $\zeta_{\text {part }}=-30 \mathrm{mV}$ respectively. The electrical "sensing zone", the region where the particle causes a decrease of the background current below the RMS of the high-frequency noise $\left(\sim 0.01 I_{0}\right)$, is approximately $3.5 \mu \mathrm{m}$ long. It takes the particle $0.19 \mathrm{~ms}$ to cross this region, which corresponds to $\sim 10$ data points. Due to the unknown pore geometry this is not exactly what is observed in the experiment, but the calculation illustrates the ef- 
fect when compared with a $\mu \mathrm{m}$-scale pore. For a NP2000 (voltage of $0.1 \mathrm{~V}$, other parameters as above) the values increase to $50 \mu \mathrm{m}$ and $1.9 \mathrm{~ms}$ for the length of the "sensing zone" and the pulse duration. The pulse therefore consists of approximately 100 data points and the distribution of the pulse durations at the $\mu \mathrm{m}$-scale has a "continuous" appearance (Fig. 7.9 (b)).

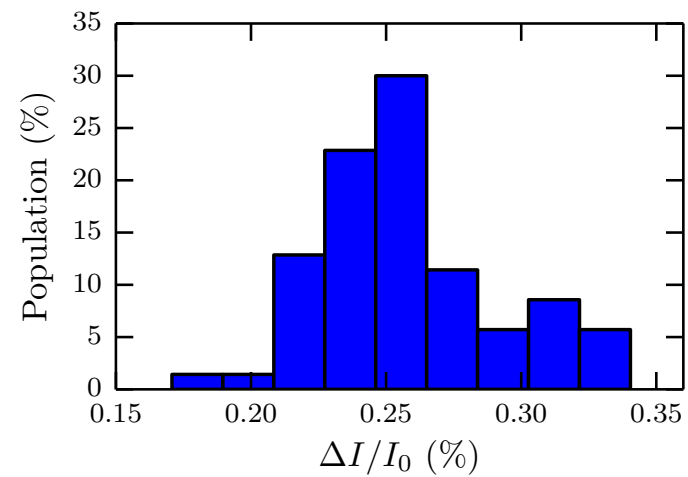

(a)

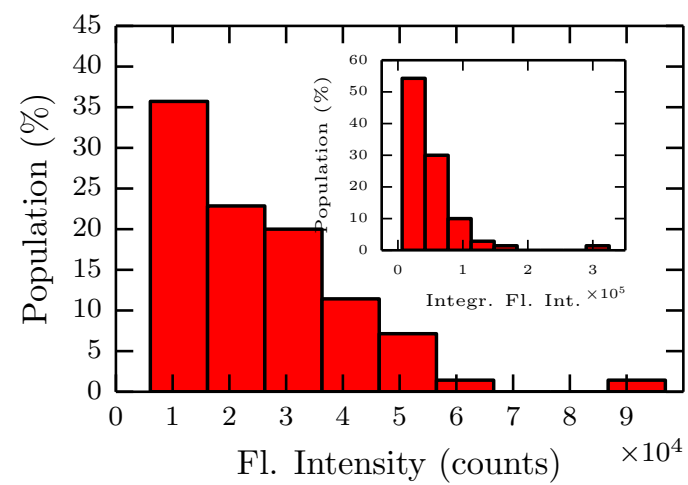

(c)

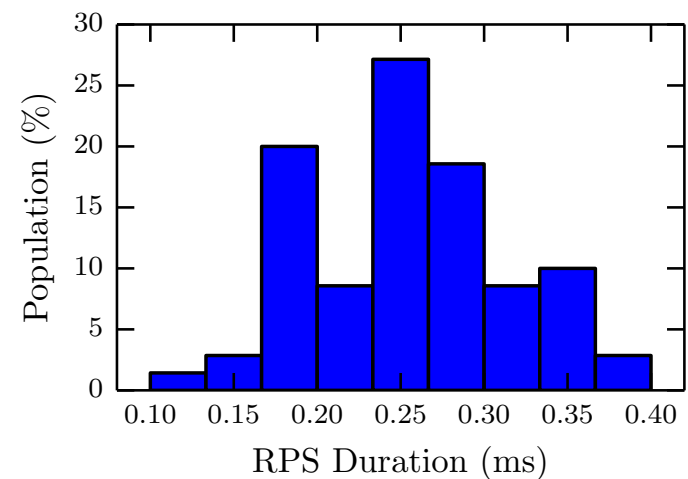

(b)

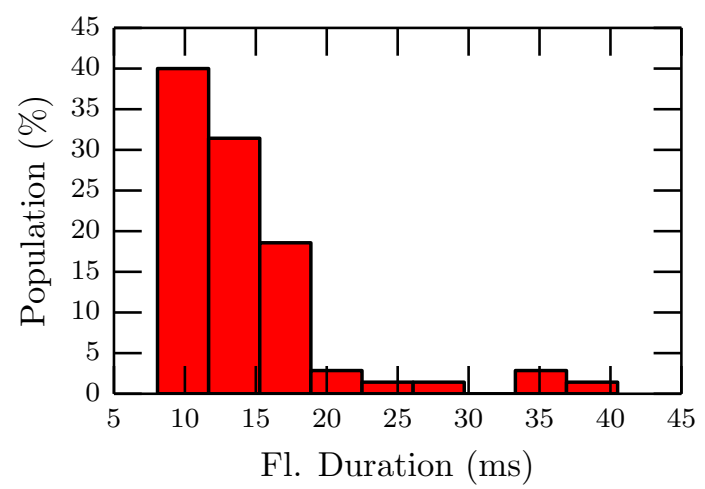

(d)

Figure 7.35: The distribution of (a) the resistive peak heights, (b) the resistive pulse duration, (c) the fluorescence intensities and (d) the durations of the optical events of experiment IV3. The inset in (c) shows the fluorescence intensities integrated over the duration of the optical events.

The low number of data points for small pores has the consequence that the resistive pulses cannot be properly resolved and their sizes are underestimated. This is a known artefact in TRPS and has to be kept in mind in the analysis of the data. Using a lower pressure and therefore particle velocity avoids this problem. The pulses then consists of a sufficient number of data points and the peak heights can be resolved. The distributions of the pulse heights and pulse durations for experiment IV3, which was conducted at the lower pressure $\left(20 \mathrm{~mm} \mathrm{H}_{2} \mathrm{O}\right)$, are shown in Figs. 7.35 (a) and (b). The pulses of most events consist now of more than 10 data points and the pulse height consequently shifts to higher values. Assuming a volumetric relationship between pulse and particle volume, the difference between the average pulse height between experiment IV1 and IV3 translates into a size 
difference of $\sim 12 \%$.

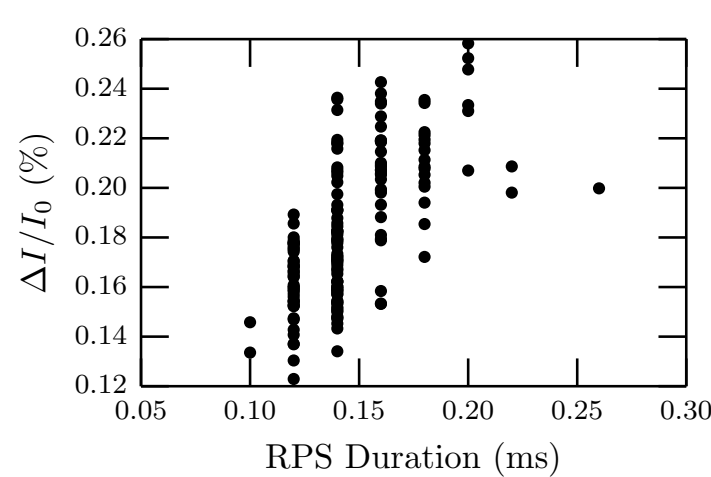

(a)

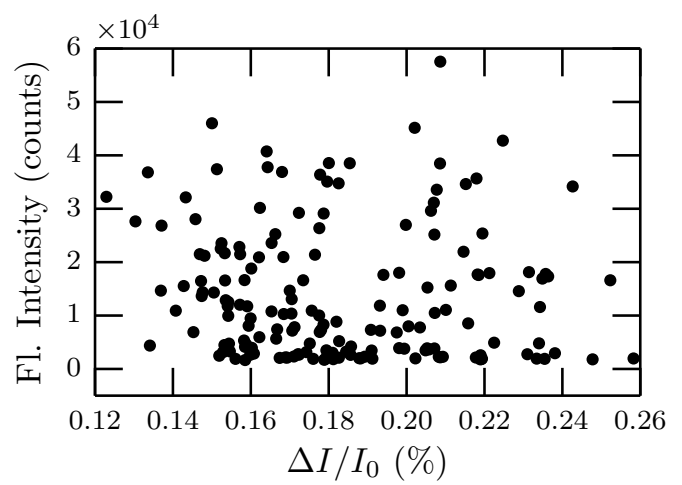

(c)

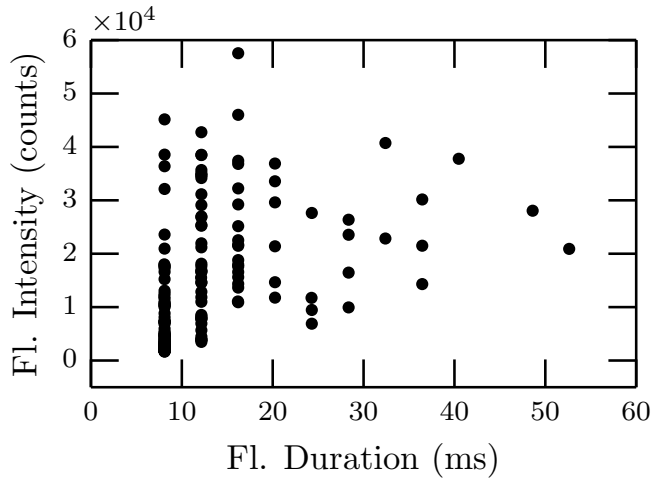

(b)

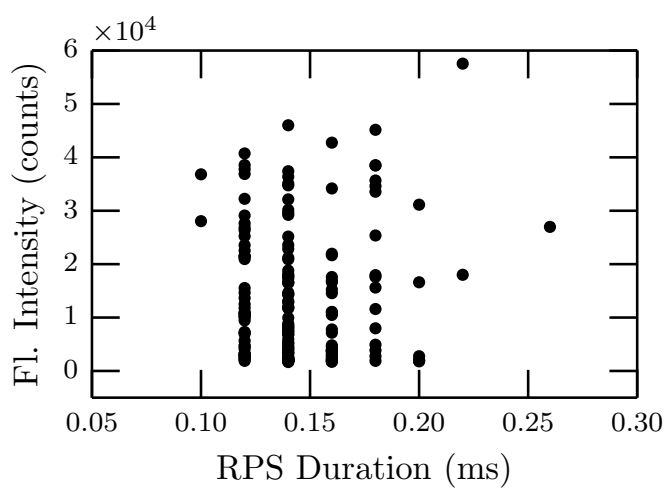

(d)

Figure 7.36: (a) The correlation between the resistive pulse durations and the resistive peak heights (Pearson coefficient: 0.64). Scatter plots of the measured fluorescence intensities with (b) the durations (0.43) of the optical events (c) the resistive peak heights $(-0.09)$ and $(d)$ the resistive pulse durations (0.07) for the experiment IV1.

The absolute velocity of the particles is smaller compared to the $\mu \mathrm{m}$-scale, which can be seen in the duration of the optical events (Figs. 7.34 and 7.35 (d)). A particle spends several time steps of $4.25 \mathrm{~ms}$ in the optical detection region. The distributions of the fluorescence intensities (Figs. 7.34 and 7.35 (c)) are more uniform due to the larger beam to pore ratio and longer optical pulse durations. The uniformity of the signals increases with decreasing pressure so that the lowest uniformity factor observed in this thesis was measured for experiment IV3, which is $\sim 5$.

The scatter plot of the electrical pulse heights with the pulse durations shows the usual strongly positive correlation (Pearson coefficients of 0.64, Fig. 7.36 (a)). The correlation between the optical pulse heights with the optical pulse durations is 0.43 (0.41 for IV3) and increases to 0.74 (0.86 for IV3) if the time integrated florescence intensities are used. This observation and the fact that the distribution in Fig. 7.34 (c) better reflects the expected particle flow distribution through the pore, shows that the integrated signals should be used in the analysis, if most of the 
optical signals consist of more than one data point. The pulse height of the optical signals does not reflect the position of the particle as clearly as seen at $\mu \mathrm{m}$ scale as they strongly depend on the start time of the signal acquisition. The correlations between electrical and optical data (Figs. 7.36 (a) and (b)) are, as already seen with the $500 \mathrm{~nm}$ particles, close to 0 . This is due to the larger beam to pore ratio, increasing difficulties in the focussing and the mechanical stability of the system and the longer optical pulse durations. The values are closer to the expected vale of -1 when a smaller beam size is used, reaching values of $-0.39(-0.57$ for the integrated signals) for the correlation between the pulse heights and $-0.50(-0.50)$ between the optical pulse heights with the resistive pulse durations in experiment IV7*.

\subsubsection{Results for Mixed Particle Sets}

A mix of two particle species (DG and SG) was used for experiments IV10 to IV13. The species of the detected matching events was identified with the linear least squares method described in Sec. 7.3.1. A pinhole of $200 \mu \mathrm{m}$, a neutral density filter D2, a $\times 100$ BFPL500 system, a pore stretch of $39.50 \mathrm{~mm}$, a molarity of $50 \mathrm{mM}$, and a voltage of $1.5 \mathrm{~V}$ were used for the experiments unless otherwise noted in Tab. 7.9. Both particle sets were diluted by the same factor (1 to 5000) from the original solution (2.5\% solid content for both species), so that, due to the different particle sizes, the concentration for the SG beads in the mixed solution is slightly higher $\left(533 \times 10^{6}\right.$ particles $\left./ \mathrm{mL}\right)$ than for the DG beads $\left(343 \times 10^{6}\right.$ particles $\left./ \mathrm{mL}\right)$. 
Table 7.9: Experimental parameters and results of a series of experiments with a mixture of $190 \mathrm{~nm}$ (SG) and $220 \mathrm{~nm}$ DG polystyrene beads. Parameters for experiments that differ from the values described in the text are noted in the last column (here, beam width, Ph $=$ pinhole, laser intensity, experimental duration).

\begin{tabular}{|c|c|c|c|c|c|c|c|c|c|c|c|c|c|c|}
\hline \multirow{2}{*}{ Exp. } & \multirow{2}{*}{$\begin{array}{c}P \\
(\mathrm{~mm})\end{array}$} & \multirow{2}{*}{$\begin{array}{c}w_{f} \\
(\mu \mathrm{m})\end{array}$} & \multicolumn{6}{|c|}{ Number of Events } & \multirow{2}{*}{$\begin{array}{c}\text { Wilson } \\
\text { Score }\end{array}$} & \multirow{2}{*}{$F I_{D G}$} & \multirow{2}{*}{$F I_{S G}$} & \multirow{2}{*}{$\begin{array}{c}F I_{D G} / \\
F I_{S G} \\
\end{array}$} & \multirow{2}{*}{$\begin{array}{c}\Delta I_{D G} / \\
\Delta I_{S G} \\
\end{array}$} & \multirow{2}{*}{ Note } \\
\hline & & & TRPS & Optical & Matching & Multi & DG & $\mathrm{SG}$ & & & & & & \\
\hline IV10 & 80 & 1.54 & $166(62)$ & $125(34)$ & $80(48 \%)$ & 24 & 40 & 40 & $43 \%$ & 1.18 & 0.4 & 2.68 & 1.64 & h 30 \\
\hline IV11 & 20 & 1.54 & $71(15)$ & $76(23)$ & $50(75 \%)$ & 6 & 24 & 26 & $67 \%$ & 9.24 & 3.11 & 2.97 & 1.42 & $D 1$ \\
\hline IV12 & 20 & 1.54 & $70(16)$ & $67(15)$ & $50(71 \%)$ & 4 & 26 & 24 & $63 \%$ & 1.93 & 0.62 & 3.11 & 1.70 & $22.8 \mathrm{~s}$ \\
\hline IV13 & 20 & 0.9 & $61(6)$ & $67(15)$ & $47(75 \%)$ & 8 & 22 & 25 & $67 \%$ & 1.41 & 0.41 & 3.44 & 1.51 & \\
\hline
\end{tabular}




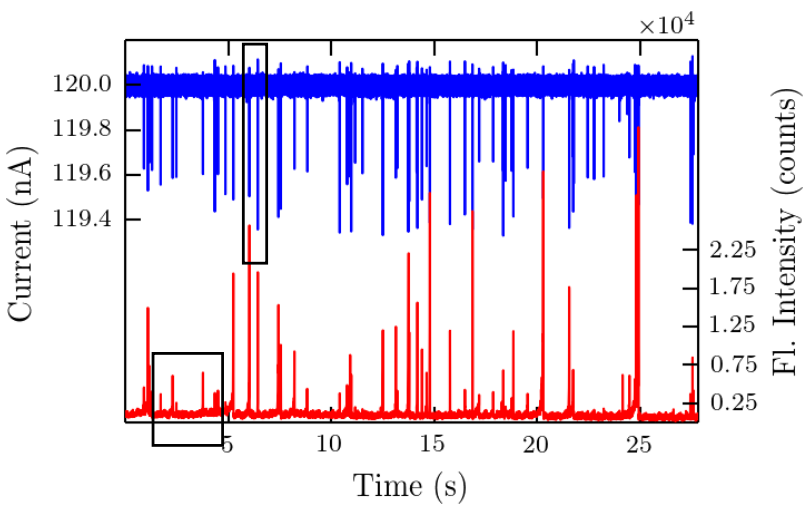

(a)

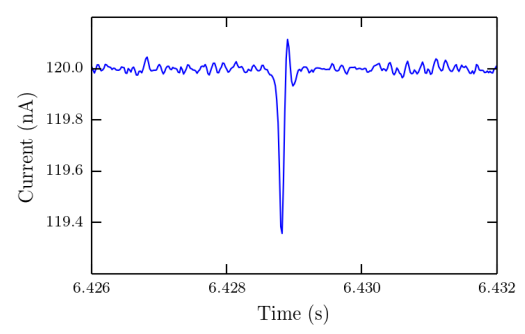

(b)

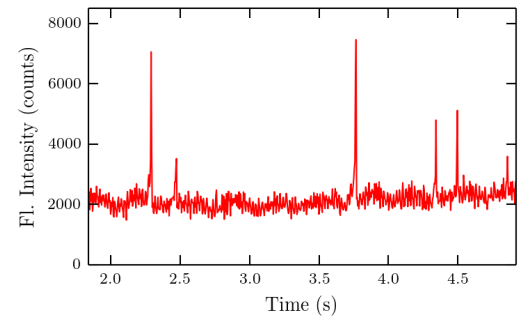

(c)

Figure 7.37: (a) The filtered current and the optical traces for experiment IV13. Magnified plots show details of the events in the black boxes and indicate a biphasic pulse (b) and the detection of a SG beads (second event) that is close to the optical detection limit (c).

The results are summarized in Tab. 7.9. Matching rates are repeatedly above $70 \%$ at a low pressure. The number of multi events is increasing for high pressure. The number of unmatched events for both traces is relatively high due to the same reasons as explained above. A higher number of SG beads is expected due to their larger concentration in the solution. However, this is not observed in the experiments and the number of detected events of each species is very similar. Apart from uncertainties in the dilution step (10\%), this is probably because more SG are undetected in the optical trace due to their lower fluorescence cross section (see Tab. 4.7).

The particle sets can clearly be distinguished due to their resistive pulse heights, where there is no overlap between the species (Fig. 7.38 (a)). The ratio $\Delta I_{D G}$ / $\Delta I_{S G}$ (Tab. 7.9) between average pulse heights is close to the expected value of 1.55 , assuming a volumetric pulse. The average optical signals are consistently larger for the DG beads, with the ratio between the species slightly larger (Tab. 7.9) than expected from fluorescence cross sections (2.08). However, as both measurements are subjected to uncertainties, a surprisingly good agreement could be found even at a low sample size of only $\sim 25$ events each. While the cross section for a single event cannot be attributed to a species due to a large overlap in the optical pulse height (Fig. 7.38 (b)) intrinsic to the method, a determination of the average fluorescence cross section of a species using calibration particles of a known cross section could be a way to quantify the emission.

Due to the higher voltage $(1.5 \mathrm{~V})$ used in IV10 to IV13, so called bi-phasic pulses 


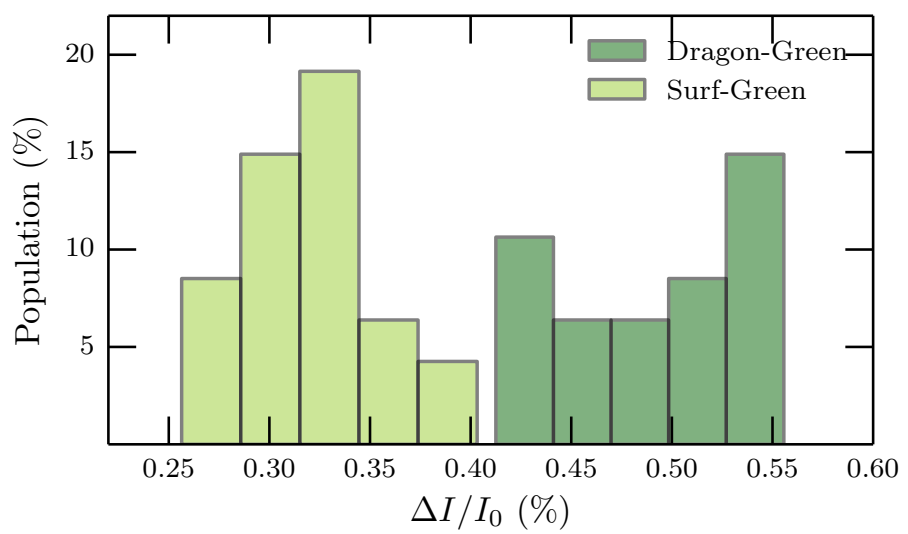

(a)

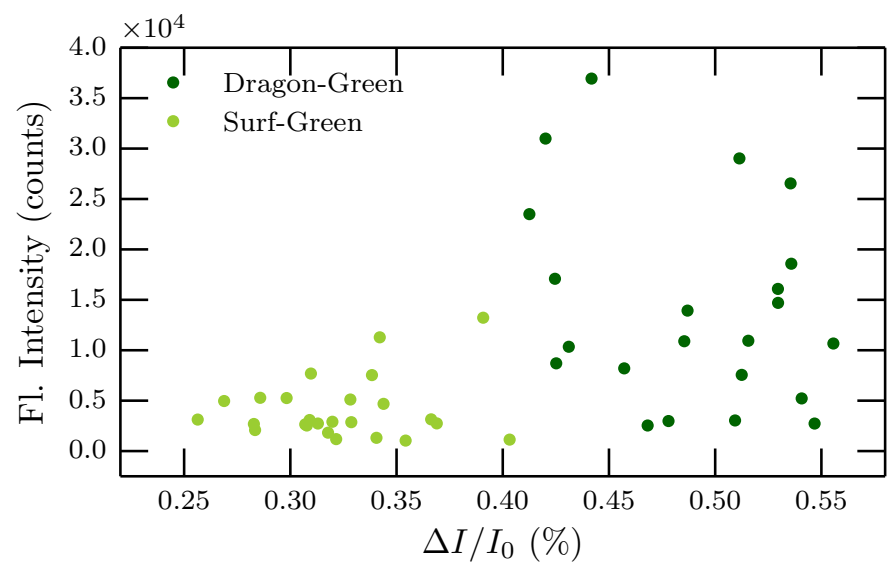

(b)

Figure 7.38: (a) The distribution of the resistive peak heights for experiment IV13, resolved by the particle species. (b) The scatter plot of resistive pulse heights and florescence intensities.

[134] in the resistive events are observed. This is not an artefact of the filtering process as the specific pulse shapes also appear in the raw trace. This observation indicates that charge polarisation effects start to play a role at this length scale at reduced molarity and high voltage. The detection of the SG beads shows the limit of the detection for this specific setup (Fig. 7.37 (c)). At an average detection height of 5000 counts, divided between 1024/8 pixels and assuming an triangular shape of the fluorescence profile, the peak height in the spectrum is $\sim 80$ counts, which can only just be distinguished from the noise level of the CCD (50 counts, see Fig. 4.25). An increase in laser power would increase the noise and formation of bubbles so that the usable time length would be reduced.

\subsection{Conclusion}

In this chapter the coordinated detection of fluorescently labelled polystyrene beads from $2 \mu \mathrm{m}$ down to to $190 \mathrm{~nm}$ has been successfully demonstrated using the second 
setup. At the $\mu \mathrm{m}$-scale the two setups have a comparable performance. However, while the first setup is limited to the $\mu \mathrm{m}$-scale the second setup is capable of detecting nm-scale particles. Additionally more accurate focusing gave rise to a better correlation between the electrical and optical data.

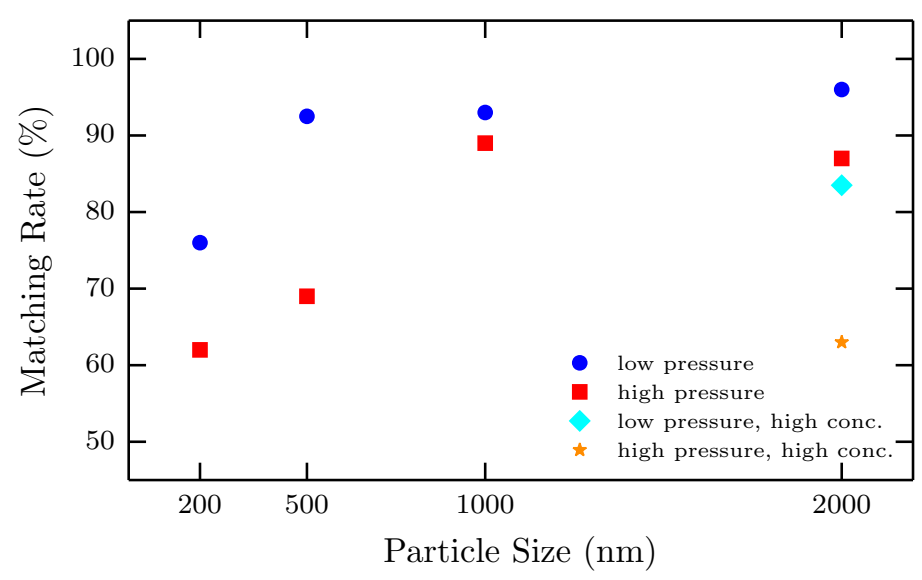

Figure 7.39: The matching rates at low and high pressure for the particle sizes tested. The values were obtained by averaging over experimental runs conducted at the highest and lowest pressures and concentrations used.

The matching rates between electrical and optically detected events are repeatedly over $90 \%$. Generally, low pressure and low concentrations (see Fig. 7.39) result in higher matching rates with a negative effect on the throughput. At the micron scale the detection of very long multi events could be reduced by using an objective with a higher magnification and therefore small optical detection region.

It has been shown that both the distributions of resistive and optical pulse heights have an intrinsic spread due to the different trajectories of the particles. While the resistive pulse distributions are relatively narrow, the distribution of the optical events is much broader and can have a uniformity factor of more than 20 due to the rapid radial decrease of the laser intensity. Several beam sizes were used to create a more uniform illumination. However, the tested systems did not result in a very uniform distribution and a certain spread is intrinsic to the technique due to the different trajectories. Therefore a quantification of the fluorescence, or more generally the emission cross section, on a particle-to-particle basis is not possible in the current setup. However as the mechanism has been well understood and as the developed optical model has proven successful, the actual optical intensity could potentially be extracted from the measured values and corrected for the trajectory effect in a post processing step. Modelling the optical signals suggests that larger beam sizes than experimentally tested would lead to a uniformity factor close to one, enabling the determination of the fluorescence cross section of single beads.

The peak distribution reflects the distribution of the particle trajectories, show- 
ing that most particles approach on an angle and on-axis trajectories are rare. However a flow distribution cannot be directly extracted from the optical data as it is convoluted with the laser intensity, the pinhole lateral detection efficiency, the time the particle spends in the optical detection volume, and also the start time for the smaller particles, where an optical pulse consists of more than one data point. In theory and with perfect central alignment finding a relationship between the signals and the trajectories is possible and could be the subject of following studies. Cross correlating the two data sets can add additional information about the trajectory of particles and can give insight about the TRPS device.

Using different labelled beads at both the micro and nanoscale a clear separation could be shown between species. The intensities were such that a potential method using calibration particles with known fluorescence cross section could be employed. Due to the spectral overlap of the emission spectra a detector that only detects the fluorescent intensity would find it difficult to distinguish between the dyes used, showing the advantage of a spectrally resolved signal.

In addition to the corresponding pulses and a gain in information about the analyte, other effects were studied using the data obtained by the coordination technique. For example, it appears that flow focussing of upward moving particles is present. Charge effects also appear to be not negligible at low molarity even for large particles. A calibration particle free method to measure the particle charge was proposed. Simulation developed in Sec. 3.4 could assist with the interpretation of results. The coordination method has the advantage that different particle sets of the same diameter but other interesting properties, e.g. charge, can be distinguished in a parallel fashion. For the qNano alone particles could only be measured after each other, introducing systemic errors.

The detection of events at the nano-scale remains challenging, especially as the laser power cannot be increased arbitrarily due to the formation of air bubbles in the pore. In order to successfully conduct experiments the experimental parameters have to be chosen carefully so that a high matching rate can be achieved. The model presented in Sec. 4.4 was successfully used to predict the optical signals and can assist in setting up an experiment. The most limiting factor of the setup is the noise induced by the laser. Hereby the noise itself is not the main problem but the inevitable formation of air bubbles in the pore orifice often leads to a premature abortion of the measurement. However, especially at the $\mu \mathrm{m}$-scale the system is reliable, easy to use and can be implemented to gain information about the analyte.

Possible improvements include the use of a confocal pinhole to reduced the chance of multi events. However, this would also cause a reduction in the signal as the particles spend less time in the optical detection volume and could be problematic for particles with a weak emission. This could be compensated by reducing the 
particle speed which, however, has a lower throughput as a consequence. Increasing the integration time will also result in a higher signal but also a higher likelihood for detecting multi events. This shows that finding the optimal parameters for specific experiments in order to achieve a high signal but also an acceptable matching rate is a complex problem. However, the developed model is able to assist in this task.

A gain in optical signal could also be achieved by increasing the collection efficiency. However, as an objective with a very high numerical aperture is already used, a significant improvement is not likely. The total detection efficiency of the spectrometer (Sec. 4.2.4) is only $\sim 3.7 \%$ and could potentially be improved with optimised optical components. The used event detection algorithm could also be refined by using not a threshold, but a differential method allowing for fluctuation in the background signal.

A manuscript presenting the results of this chapter is currently under preparation. 
7.6. Conclusion 


\section{Chapter 8}

\section{Conclusion and Outlook}

Using TRPS and optical spectroscopy the coordinated detection of fluorescently labelled polystyrene beads in the size range from $200 \mathrm{~nm}$ to $2 \mu \mathrm{m}$ has been successfully demonstrated. Matching rates between the electrical and optical signals of over $90 \%$ could repeatedly be achieved. TRPS data was used to measure particle size and concentration, while the optical measurement added high-resolution spectral information on a particle-by-particle basis.

The technique also proved successful to distinguish particles with a similar size but different fluorescence spectrum, both at the micro- and nanoscale. This showed how the two techniques can complement each other. While particles with the same diameter cannot be distinguished with TRPS alone the additional optical information makes that possible. Due to the significant spectral overlap of the fluorescent spectra of the used particles a method that detects only the overall fluorescent intensity would not be sufficient to distinguish the beads, showing the advantage of a spectrally resolved signal. TRPS provides an accurately controllable and ordered flow for guiding the analyte through the optical detection region, which is a big advantage over diffusion-based techniques. The coordination techniques also showed their potential for gaining more insight into the TRPS technique. Effects liked flow focusing and also a charge effect at low electrolyte molarities could also be observed.

Both the distributions of resistive and optical pulse heights have an intrinsic spread due to the different trajectories of the particles. While the resistive pulse distributions are relatively narrow, the distribution of the optical events is much broader. The peak distribution reflects the distribution of the particle trajectories, showing that most particles approach on an angle and on-axis trajectories are rare. A quantitative measurement of the fluorescence cross section on a particle-by-particle basis is therefore not straight-forward. However, by joining the information about the trajectory from both electrical and optical data a method to correct for the intrinsic spread could potentially be developed.

Careful experimental optimization was carried out and resulted in the develop- 
ment of two setups, both of which are portable and not restricted to a certain type of spectrometer. A careful choice of the experimental parameters is necessary in order to successfully conduct experiments and achieve high matching rates. A model was developed that successfully predicts the optical signals and can assist in setting up an experiment. In both the model and the experiments the largest influence on the intensity of the optical signals in our system is the particle velocity defined mainly by the applied hydrostatic pressure.

In an attempt to achieve a more uniform illumination of the pore a simple method to predict and engineer the beam size was adopted. A combination of two lenses can be used to continuously tune the size of a laser beam width at the focal plane of the objective over a wide range of beam sizes. This method should be useful for many other applications in the field of microscope-based laser spectroscopy.

Intensive FEM modelling was conducted to better understand the particle transport in TRPS pores. The semi-analytic models, which are conventionally used in TRPS, show good agreement with FEM for total current and flow through a pore. However, they are not capable to predicting the accurate field distribution at the pore orifice, off-axis trajectories, particle-field interactions and the ion distribution in the pore, which are important for understanding certain effects observed in TRPS. The semi-analytic model also does not capture the transport mechanisms correctly, e.g. it highly overestimates electrophoretic transport.

\subsection{Future Work}

The optical detection of events at the nano-scale remains challenging. The most limiting factor of the setup is the noise induced by the laser, so that the laser power cannot be increased arbitrarily. Hereby the noise itself is not the main problem but the inevitable formation of air bubbles in the pore orifice often leads to a premature abortion of the measurement. However, especially at the $\mu \mathrm{m}$-scale the the system is reliable, easy to use and can be implemented to gain information about the analyte.

Ongoing work should focus on experimental development in order to further improve the sensitivity of the method. The system has potential for improvement, including the use of "real" confocal detection to limit the optical detection region as well as increasing the detection efficiency by optimising the optical components. Continuing advances in detection technology, e.g. faster and more sensitive CCDchips, may open new possibilities for the technique. Also a multi-channel detection using photo detectors with optical bandpass filters instead the detection with a grating based spectrometry could be sufficient for some applications and world greatly increase the sensitivity and the time resolution of the technique. Solid state pores with a more defined shape could also potentially be beneficial for the method. 
Incorporation of wave-guides and a redesign of the pore could produce a more userfriendly device. The use of pores with plasmonic structures would intrinsically limit the detection to particles close very close to the pore due to the highly localised enhancement effect.

A future candidate for optical co-ordination with TRPS could be Surface-Enhanced Raman Spectroscopy (SERS). Studies of the ability to measure silver colloid clusters using TRPS measured would also be of beneficial for the SERS technique and give insight into the signal variation between colloids. An estimation of the required parameters to measure Raman signals was conducted in Sec. 4.4 and shows that it is challenging but possible with slight adaptations to the existing setup.

Although one aim is to push the technique towards the detection of smaller particles, the size range from several hundred nanometers to micrometers has many applications itself, e.g. industrial colloids, drug delivery particles, or on-bead analytic assays. By marking specific analytes with different fluorophores the influence of shape, charge or other particle characteristics on the resistive pulse could be identified. The influence of light-particle interactivity is also yet to be fully explored either due to pushing or light-induced charge effects. Potentially the involved forces could be derived and particles could be trapped in the pore.

The greatest limiting factor was the formation of air bubbles in the pore. Although undesired for the technique, the effect in itself is interesting and could be investigated further. Formation of bubbles in these circumstances has not been widely reported before. The observation may be important for other techniques and for other fields where lasers are used in an aqueous solution.

Apart from the future experimental development, FEM modelling, which has not been used to this extent in TRPS before, would be beneficial for a thorough understanding of the technique and particle analysis beyond this work. The ion distribution in the pore for low molarity electrolyte is not uniform and can lead to current rectification or bi-phasic pulses, which can be predicted with the FEM-ion model. Adding the particle dynamics results in a model very close to the physical reality and would help to better understand the fundamentals of the technique and potentially a new method to measure the particle charge and zeta potential could be developed from these studies. However, the computational costs of these models are very high and have to be carried out using enhanced computational resources. 
8.1. Future Work 


\section{Appendix A}

\section{Publications and Presentations}

- P. Hauer, E. C. Le Ru, and G. W. Willmott, "Co-ordinated detection of microparticles using tunable resistive pulse sensing and fluorescence spectroscopy", Biomicrofluidics, Vol 9, Number 1, Pages 104-110, Jan 2015

- P. Hauer, J. Grand, A. Djorovic, G. W. Willmott, and E. C. Le Ru, "Spot Size Engineering in Microscope-based Laser Spectroscopy", The Journal of Physical Chemistry C, July 2016, doi:10.1021/acs.jpcc.6b04574

- E. Weatherall, P. Hauer, R. Vogel, and G. W. Willmott, "Pulse Size Distributions in Tunable Resistive Pulse Sensing", The Journal of Analytical Chemistry, July 2016, doi: 10.1021/acs.analchem.6b01818.

- P. Hauer, E. C. Le Ru, and G. W. Willmott, "Simultaneous Electrical and Optical Detection of Micro- and Nanoparticles and Particle Mixtures using Tuneable Resistive Pulse Sensing and Fluorescence Spectroscopy", in preparation

- P. Hauer, E. C. Le Ru, and G. W. Willmott, "Co-ordinated Fluorescence and Resistive Pulse Sensing of Micro- and Nanoparticles", Talk given at the Seventh International Conference on Advanced Materials, and Nanotechnology (AMN-7), Nelson, New Zealand, Feb 2015

- P. Hauer, E. C. Le Ru, and G. W. Willmott, "Co-ordinated Detection of Micro- and Nano-particles using Tuneable Resistive Pulse Sensing and Fluorescence Spectroscopy", Talk given at the Fifth Australia and New Zealand Micro/Nanofluidics Symposium (ANZMNF-5), Hobart, Australia, April 2014

- P. Hauer, P. Etchegoin, E. C. Le Ru, and G. W. Willmott, "Simultaneous Electrical and Optical Detection of Micro- and Nanospheres Passing Through Tunable Pores", Talk given at the Sixth International Conference on Advanced Materials, and Nanotechnology (AMN-6), Auckland, New Zealand, Feb 2013 
- P. Hauer, P. Etchegoin, and G. W. Willmott, "Towards Co-ordinated Nanoparticle Detection using Raman Spectroscopy and Resistive Pulse Sensing", Poster presented at the Third Australia and New Zealand Micro/Nanofluidics and Bionano Symposium (ANZMNF-3), Wellington, New Zealand, April 2012 


\section{Bibliography}

[1] W. H. Coulter, "Means for counting particles suspended in a fluid," 1953. US Patent No. 2656508.

[2] S. Howorka and Z. Siwy, "Nanopore analytics: Sensing of single molecules," Chemical Society Reviews, vol. 38, no. 8, pp. 2360-2384, 2009.

[3] A. Ivanov, K. Wilson, F. Doğan, D. Japrung, J. Edel, et al., "Single molecule sensing with solid-state nanopores: Novel materials, methods, and applications," Chemical Society Reviews, 2012.

[4] M. Wanunu, "Nanopores: A journey towards DNA sequencing," Physics of Life Reviews, vol. 9, no. 2, pp. 125-158, 2012.

[5] Y. Feng, Y. Zhang, C. Ying, D. Wang, and C. Du, "Nanopore-based fourthgeneration DNA sequencing technology," Genomics, Proteomics 85 Bioinformatics, vol. 13, no. 1, pp. 4-16, 2015.

[6] B. J. Berne and R. Pecora, Dynamic light scattering: With applications to chemistry, biology, and physics. Courier Dover Publications, 2000.

[7] E. C. Gregg and K. D. Steidley, "Electrical counting and sizing of mammalian cells in suspension," Biophysical Journal, vol. 5, no. 4, p. 393, 1965.

[8] R. DeBlois and C. Bean, "Counting and sizing of submicron particles by the resistive pulse technique," Review of Scientific Instruments, vol. 41, no. 7, pp. 909-916, 1970.

[9] R. W. DeBlois, E. E. Uzgiris, D. H. Cluxton, and H. M. Mazzone, "Comparative measurements of size and polydispersity of several insect viruses," Analytical Biochemistry, vol. 90, no. 1, pp. 273-288, 1978.

[10] B. N. Miles, A. P. Ivanov, K. A. Wilson, F. Doğan, D. Japrung, and J. B. Edel, "Single molecule sensing with solid-state nanopores: Novel materials, methods, and applications," Chemical Society Reviews, vol. 42, no. 1, pp. 15$28,2013$. 
[11] H. Bayley and C. R. Martin, "Resistive-pulse sensing - from microbes to molecules," Chemical Reviews, vol. 100, no. 7, pp. 2575-2594, 2000.

[12] A. Oukhaled, L. Bacri, M. Pastoriza-Gallego, J.-M. Betton, and J. Pelta, "Sensing proteins through nanopores: Fundamental to applications," ACS Chemical Biology, vol. 7, no. 12, pp. 1935-1949, 2012.

[13] K. Zhou, L. Li, Z. Tan, A. Zlotnick, and S. C. Jacobson, "Characterization of hepatitis B virus capsids by resistive-pulse sensing," Journal of the American Chemical Society, vol. 133, no. 6, pp. 1618-1621, 2011.

[14] D. A. Holden, J. J. Watkins, and H. S. White, "Resistive-pulse detection of multilamellar liposomes," Langmuir, vol. 28, no. 19, pp. 7572-7577, 2012.

[15] R. Henriquez, T. Ito, L. Sun, and R. Crooks, "The resurgence of Coulter counting for analyzing nanoscale objects," Analyst, vol. 129, no. 6, pp. 478482, 2004.

[16] Z. Siwy and A. Fuliński, "Fabrication of a synthetic nanopore ion pump," Physical Review Letters, vol. 89, no. 19, p. 198103, 2002.

[17] A. Storm, J. Chen, X. Ling, H. Zandbergen, and C. Dekker, "Fabrication of solid-state nanopores with single-nanometre precision," Nature materials, vol. 2 , no. 8, pp. 537-540, 2003.

[18] M. Aziz, J. Golovchenko, D. Branton, C. McMullan, D. Stein, and J. Li, "Ion-beam sculpting at nanometre length scales," Nature, vol. 412, no. 6843, pp. 166-169, 2001.

[19] C. Dekker, "Solid-state nanopores," Nature Nanotechnology, vol. 2, no. 4, pp. 209-215, 2007.

[20] R. E. Gyurcsnyi, "Chemically-modified nanopores for sensing," $\operatorname{Tr} A C$ - Trends in Analytical Chemistry, vol. 27, no. 7, pp. 627-639, 2008.

[21] Z. Siwy, L. Trofin, P. Kohli, L. A. Baker, C. Trautmann, and C. R. Martin, "Protein biosensors based on biofunctionalized conical gold nanotubes," Journal of the American Chemical Society, vol. 127, no. 14, pp. 5000-5001, 2005.

[22] J. Kasianowicz, E. Brandin, D. Branton, and D. Deamer, "Characterization of individual polynucleotide molecules using a membrane channel," Proceedings of the National Academy of Sciences, vol. 93, no. 24, pp. 13770-13773, 1996. 
[23] D. Branton, D. W. Deamer, A. Marziali, H. Bayley, S. A. Benner, T. Butler, M. Di Ventra, S. Garaj, A. Hibbs, X. Huang, et al., "The potential and challenges of nanopore sequencing," Nature Biotechnology, vol. 26, no. 10, pp. 1146-1153, 2008.

[24] F. Haque, J. Li, H.-C. Wu, X.-J. Liang, and P. Guo, "Solid-state and biological nanopore for real-time sensing of single chemical and sequencing of DNA," Nano Today, vol. 8, no. 1, pp. 56-74, 2013.

[25] P. M. Ashton, S. Nair, T. Dallman, S. Rubino, W. Rabsch, S. Mwaigwisya, J. Wain, and J. O'Grady, "Minion nanopore sequencing identifies the position and structure of a bacterial antibiotic resistance island," Nature Biotechnology, vol. 33, no. 3, pp. 296-300, 2015.

[26] S. Wei and Z. Williams, "Rapid short-read sequencing and aneuploidy detection using minion nanopore technology," Genetics, vol. 202, no. 1, pp. 37-44, 2016.

[27] J. Schmidt, "Membrane platforms for biological nanopore sensing and sequencing," Current Opinion in Biotechnology, vol. 39, pp. 17-27, 2016.

[28] A. C. Ward and W. Kim, "MinION"TM: New, long read, portable nucleic acid sequencing device," Journal of Bacteriology and Virology, vol. 45, no. 4, pp. 285-303, 2015.

[29] L. Luo, S. R. German, W.-J. Lan, D. A. Holden, T. L. Mega, and H. S. White, "Resistive-pulse analysis of nanoparticles," Annual Review of Analytical Chemistry, vol. 7, pp. 513-535, 2014.

[30] S. J. Sowerby, M. F. Broom, and G. B. Petersen, "Dynamically resizable nanometre-scale apertures for molecular sensing," Sensors and Actuators B: Chemical, vol. 123, no. 1, pp. 325-330, 2007.

[31] G. R. Willmott and P. W. Moore, "Reversible mechanical actuation of elastomeric nanopores," Nanotechnology, vol. 19, no. 47, 2008.

[32] D. Kozak, W. Anderson, and M. Trau, "Tuning particle velocity and measurement sensitivity by changing pore sensor dimensions," Chemistry Letters, vol. 41, no. 10, pp. 1134-1136, 2012.

[33] http://www.izon.com, 2012.

[34] G. R. Willmott, R. Vogel, S. S. C. Yu, L. G. Groenewegen, G. S. Roberts, D. Kozak, W. Anderson, and M. Trau, "Use of tunable nanopore blockade rates 
to investigate colloidal dispersions," Journal of Physics Condensed Matter, vol. 22 , no. $45,2010$.

[35] G. S. Roberts, S. Yu, Q. Zeng, L. C. L. Chan, W. Anderson, A. H. Colby, M. W. Grinstaff, S. Reid, and R. Vogel, "Tunable pores for measuring concentrations of synthetic and biological nanoparticle dispersions," Biosensors and Bioelectronics, vol. 31, no. 1, pp. 17-25, 2012.

[36] R. Vogel, G. Willmott, D. Kozak, G. S. Roberts, W. Anderson, L. Groenewegen, B. Glossop, A. Barnett, A. Turner, and M. Trau, "Quantitative sizing of nano/microparticles with a tunable elastomeric pore sensor," Analytical Chemistry, vol. 83, no. 9, pp. 3499-3506, 2011.

[37] D. Kozak, W. Anderson, R. Vogel, S. Chen, F. Antaw, and M. Trau, "Simultaneous size and zeta-potential measurements of individual nanoparticles in dispersion using size-tunable pore sensors," ACS Nano, vol. 6, no. 8, pp. 69906997, 2012.

[38] R. Vogel, W. Anderson, J. Eldridge, B. Glossop, and G. Willmott, "A variable pressure method for characterizing nanoparticle surface charge using pore sensors," Analytical Chemistry, vol. 84, no. 7, pp. 3125-3131, 2012.

[39] J. A. Eldridge, G. R. Willmott, W. Anderson, and R. Vogel, "Nanoparticle $\zeta$-potential measurements using tunable resistive pulse sensing with variable pressure," Journal of Colloid and Interface Science, vol. 429, pp. 45-52, 2014.

[40] G. R. Willmott, M. G. Fisk, and J. Eldridge, "Magnetic microbead transport during resistive pulse sensing," Biomicrofluidics, vol. 7, no. 6, p. 064106, 2013.

[41] M. Platt, G. R. Willmott, and G. U. Lee, "Resistive pulse sensing of analyteinduced multicomponent rod aggregation using tunable pores," Small, vol. 8, no. 15 , pp. 2436-2444, 2012.

[42] O. A. Alsager, S. Kumar, G. R. Willmott, K. P. McNatty, and J. M. Hodgkiss, "Small molecule detection in solution via the size contraction response of aptamer functionalized nanoparticles," Biosensors and Bioelectronics, vol. 57, pp. 262-268, 2014.

[43] G. S. Roberts, D. Kozak, W. Anderson, M. F. Broom, R. Vogel, and M. Trau, "Tunable nano/micropores for particle detection and discrimination: Scanning ion occlusion spectroscopy," Small, vol. 6, no. 23, pp. 2653-2658, 2010. 
[44] D. Kozak, W. Anderson, R. Vogel, and M. Trau, "Advances in resistive pulse sensors: Devices bridging the void between molecular and microscopic detection," Nano Today, vol. 6, no. 5, pp. 531-545, 2011.

[45] E. Garza-Licudine, D. Deo, S. Yu, A. Uz-Zaman, and W. B. Dunbar, "Portable nanoparticle quantization using a resizable nanopore instrument - the IZON qNano ${ }^{\mathrm{TM}}, "$ in Engineering in Medicine and Biology Society (EMBC), 2010 Annual International Conference of the IEEE, pp. 5736-5739, IEEE, 2010.

[46] W. Anderson, R. E. Lane, D. Korbie, and M. Trau, "Observations of tunable resistive pulse sensing for exosome analysis: Improving system sensitivity and stability," Langmuir, 2015.

[47] E. Van Der Pol, A. Hoekstra, A. Sturk, C. Otto, T. Van Leeuwen, and R. Nieuwland, "Optical and non-optical methods for detection and characterization of microparticles and exosomes," Journal of Thrombosis and Haemostasis, vol. 8, no. 12, pp. 2596-2607, 2010.

[48] E. Pol, F. Coumans, Z. Varga, M. Krumrey, and R. Nieuwland, "Innovation in detection of microparticles and exosomes," Journal of Thrombosis and Haemostasis, vol. 11, no. s1, pp. 36-45, 2013.

[49] E. Weatherall and G. R. Willmott, "Applications of tunable resistive pulse sensing," Analyst, vol. 140, no. 10, pp. 3318-3334, 2015.

[50] E. L. Blundell, L. J. Mayne, E. R. Billinge, and M. Platt, "Emergence of tunable resistive pulse sensing as a biosensor," Analytical Methods, 2015.

[51] G. Willmott, M. Broom, M. Jansen, R. Young, and W. Arnold, "Tunable elastomeric nanopores," in Molecular-and Nano-Tubes, pp. 209-261, Springer, 2011.

[52] W. Anderson, D. Kozak, V. A. Coleman, A. K. Jämting, and M. Trau, "A comparative study of submicron particle sizing platforms: Accuracy, precision and resolution analysis of polydisperse particle size distributions," Journal of Colloid and Interface Science, 2013.

[53] S. Weiss, "Fluorescence spectroscopy of single biomolecules," Science, vol. 283, no. 5408, pp. 1676-1683, 1999.

[54] R. Alfano, D. Tata, J. Cordero, P. Tomashefsky, F. Longo, and M. Alfano, "Laser induced fluorescence spectroscopy from native cancerous and normal tissue," Quantum Electronics, IEEE Journal of, vol. 20, no. 12, pp. 1507-1511, 1984. 
[55] G. A. T. Chansin, R. Mulero, J. Hong, M. J. Kim, A. J. DeMello, and J. B. Edel, "Single-molecule spectroscopy using nanoporous membranes," Nano Letters, vol. 7, no. 9, pp. 2901-2906, 2007.

[56] B. McNally, A. Singer, Z. Yu, Y. Sun, Z. Weng, and A. Meller, "Optical recognition of converted DNA nucleotides for single-molecule DNA sequencing using nanopore arrays," Nano Letters, vol. 10, no. 6, pp. 2237-2244, 2010.

[57] A. J. Heron, J. R. Thompson, B. Cronin, H. Bayley, and M. I. Wallace, "Simultaneous measurement of ionic current and fluorescence from single protein pores," Journal of the American Chemical Society, vol. 131, no. 5, pp. 16521653, 2009.

[58] G. V. Soni, A. Singer, Z. Yu, Y. Sun, B. McNally, and A. Meller, "Synchronous optical and electrical detection of biomolecules traversing through solid-state nanopores," Review of Scientific Instruments, vol. 81, no. 1, 2010.

[59] W. Song, P. Pang, J. He, and S. Lindsay, "Optical and electrical detection of single-molecule translocation through carbon nanotubes," ACS Nano, vol. 7, no. 1, pp. 689-694, 2012.

[60] V. Kurz, E. M. Nelson, J. Shim, and G. Timp, "Direct visualization of single molecule translocations through a synthetic nanopores comparable in size to a molecule," ACS Nano, 2013.

[61] N. Di Fiori, A. Squires, D. Bar, T. Gilboa, T. D. Moustakas, and A. Meller, "Optoelectronic control of surface charge and translocation dynamics in solidstate nanopores," Nature Nanotechnology, vol. 8, no. 12, pp. 946-951, 2013.

[62] E. Angeli, A. Volpe, P. Fanzio, L. Repetto, G. Firpo, P. Guida, R. Lo Savio, M. Wanunu, and U. Valbusa, "Simultaneous electro-optical tracking for nanoparticle recognition and counting," Nano Letters, 2015.

[63] X. Wu, C. H. Chon, Y.-N. Wang, Y. Kang, and D. Li, "Simultaneous particle counting and detecting on a chip," Lab on a Chip, vol. 8, no. 11, pp. 1943-1949, 2008.

[64] J. Guo, L. Chen, X. Huang, C. M. Li, Y. Ai, and Y. Kang, "Dual characterization of biological cells by optofluidic microscope and resistive pulse sensor," Electrophoresis, vol. 36, no. 3, pp. 420-423, 2015.

[65] B. N. Anderson, O. N. Assad, T. Gilboa, A. H. Squires, D. Bar, and A. Meller, "Probing solid-state nanopores with light for the detection of unlabeled analytes," ACS Nano, vol. 8, no. 11, pp. 11836-11845, 2014. 
[66] A. Ivankin, R. Y. Henley, J. Larkin, S. Carson, M. L. Toscano, and M. Wanunu, "Label-free optical detection of biomolecular translocation through nanopore arrays," ACS Nano, vol. 8, no. 10, pp. 10774-10781, 2014.

[67] W. H. Pitchford, H.-J. Kim, A. P. Ivanov, H.-M. Kim, J.-S. Yu, R. J. Leatherbarrow, T. Albrecht, K.-B. Kim, and J. B. Edel, "Synchronized optical and electronic detection of biomolecules using a low noise nanopore platform," ACS Nano, vol. 9, no. 2, pp. 1740-1748, 2015.

[68] S. Liu, Y. Zhao, J. W. Parks, D. W. Deamer, A. R. Hawkins, and H. Schmidt, "Correlated electrical and optical analysis of single nanoparticles and biomolecules on a nanopore-gated optofluidic chip," Nano Letters, vol. 14, no. 8, pp. 4816-4820, 2014.

[69] I. Notingher, "Raman spectroscopy cell-based biosensors," Sensors, vol. 7, no. 8, pp. 1343-1358, 2007.

[70] K. C. Bantz, A. F. Meyer, N. J. Wittenberg, H. Im, Ö. Kurtuluş, S. H. Lee, N. C. Lindquist, S.-H. Oh, and C. L. Haynes, "Recent progress in SERS biosensing," Physical Chemistry Chemical Physics, vol. 13, no. 24, pp. 1155111567, 2011.

[71] C. Chen, J. Ye, Y. Li, L. Lagae, T. Stakenborg, and P. Van Dorpe, "Detection of DNA bases and oligonucleotides in plasmonic nanoslits using fluidic SERS," IEEE Journal of Selected Topics in Quantum Electronics, vol. 19, no. 3, pp. 4600707-4600707, 2013.

[72] M. Belkin, S.-H. Chao, M. P. Jonsson, C. Dekker, and A. Aksimentiev, "Plasmonic nanopores for trapping, controlling displacement, and sequencing of DNA," ACS Nano, vol. 9, no. 11, pp. 10598-10611, 2015.

[73] N. L. Thompson, "Fluorescence correlation spectroscopy," in Topics in fluorescence spectroscopy, pp. 337-378, Springer, 2002.

[74] P. Schwille, "Fluorescence correlation spectroscopy," in Encyclopedic Reference of Genomics and Proteomics in Molecular Medicine, pp. 576-578, Springer, 2005.

[75] J. P. Nolan and D. Condello, "Spectral flow cytometry," Current Protocols in Cytometry, pp. 1-27, 2013.

[76] J. C. Maxwell and J. J. Thompson, A treatise on electricity and magnetism, vol. 1. Clarendon, 1892. 
[77] G. R. Willmott, M. Platt, and G. U. Lee, "Resistive pulse sensing of magnetic beads and supraparticle structures using tunable pores," Biomicrofluidics, vol. 6, no. 1, p. 014103, 2012.

[78] S. W. Kowalczyk, A. Y. Grosberg, Y. Rabin, and C. Dekker, "Modeling the conductance and DNA blockade of solid-state nanopores," Nanotechnology, vol. 22, no. 31, p. 315101, 2011.

[79] G. Stober, L. Steinbock, and U. Keyser, "Modeling of colloidal transport in capillaries," Journal of Applied Physics, vol. 105, no. 8, p. 084702, 2009.

[80] D. Kozak, W. Anderson, M. Grevett, and M. Trau, "Modeling elastic pore sensors for quantitative single particle sizing," The Journal of Physical Chemistry $C$, vol. 116, no. 15, pp. 8554-8561, 2012.

[81] J. E. Hall, "Access resistance of a small circular pore," The Journal of General Physiology, vol. 66, no. 4, pp. 531-532, 1975.

[82] J. Wang, J. Ma, Z. Ni, L. Zhang, and G. Hu, "Effects of access resistance on the resistive-pulse caused by translocating of a nanoparticle through a nanopore," RSC Advances, vol. 4, no. 15, pp. 7601-7610, 2014.

[83] R. B. Schoch, J. Han, and P. Renaud, "Transport phenomena in nanofluidics," Reviews of modern physics, vol. 80, no. 3, p. 839, 2008.

[84] B. Hille, "Ionic channels in nerve membranes," Progress in Biophysics and Molecular Biology, vol. 21, pp. 1-32, 1970.

[85] E. A. Heins, Z. S. Siwy, L. A. Baker, and C. R. Martin, "Detecting single porphyrin molecules in a conically shaped synthetic nanopore," Nano Letters, vol. 5, no. 9, pp. 1824-1829, 2005.

[86] L. Bacri, A. Oukhaled, B. Schiedt, G. Patriarche, E. Bourhis, J. Gierak, J. Pelta, and L. Auvray, "Dynamics of colloids in single solid-state nanopores," The Journal of Physical Chemistry B, vol. 115, no. 12, pp. 2890-2898, 2011.

[87] G. Willmott and B. Smith, "Modelling of resistive pulse sensing: Flexible methods for submicron particles," The ANZIAM Journal, vol. 55, no. 03, pp. 197-213, 2014.

[88] G. R. Willmott and B. E. T. Parry, "Resistive pulse asymmetry for nanospheres passing through tunable submicron pores," Journal of Applied Physics, vol. 109, no. 9, 2011. 
[89] A. E. Velasco, S. G. Friedman, M. Pevarnik, Z. S. Siwy, and P. Taborek, "Pressure-driven flow through a single nanopore," Physical Review E - Statistical, Nonlinear, and Soft Matter Physics, vol. 86, no. 2, 2012.

[90] K. P. Travis, B. Todd, and D. J. Evans, "Departure from navier-stokes hydrodynamics in confined liquids," Physical Review E, vol. 55, no. 4, pp. 4288-4295, 1997.

[91] G. K. Batchelor, An introduction to fluid dynamics. Cambridge University Press, 2000.

[92] D. R. Lide, CRC Handbook of chemistry and physics. CRC press, 2004.

[93] F. Durst, S. Ray, B. Ünsal, and O. Bayoumi, "The development lengths of laminar pipe and channel flows," Journal of Fluids Engineering, vol. 127, no. 6, pp. 1154-1160, 2005.

[94] Z. Dagan, S. Weinbaum, and R. Pfeffer, "An infinite-series solution for the creeping motion through an orifice of finite length," Journal of Fluid Mechanics, vol. 115, pp. 505-523, 1982.

[95] R. A. Sampson, "On stokes's current function," Philosophical Transactions of the Royal Society of London. A, pp. 449-518, 1891.

[96] W. Wang-yi and R. Skalak, "The stokes flow from half-space into semi-infinite circular cylinder," Applied Mathematics and Mechanics, vol. 6, no. 1, pp. 9-23, 1985.

[97] T. M. Squires and S. R. Quake, "Microfluidics: Fluid physics at the nanoliter scale," Reviews of Modern Physics, vol. 77, no. 3, p. 977, 2005.

[98] T. Z. Jubery, A. S. Prabhu, M. J. Kim, and P. Dutta, "Modeling and simulation of nanoparticle separation through a solid-state nanopore," Electrophoresis, vol. 33, no. 2, pp. 325-333, 2012.

[99] C. E. Brennen, Fundamentals of multiphase flow. Cambridge University Press, 2005.

[100] J. N. Israelachvili, Intermolecular and surface forces: Revised third edition. Academic Press, 2011.

[101] M. Gouy, "Sur la constitution de la charge electrique a la surface d'un electrolyte," J. Phys. Theor. Appl., vol. 9, no. 1, pp. 457-468, 1910. 
[102] D. L. Chapman, "LI. A contribution to the theory of electrocapillarity," The London, Edinburgh, and Dublin Philosophical Magazine and Journal of Science, vol. 25, no. 148, pp. 475-481, 1913.

[103] C. Malmberg and A. Maryott, "Dielectric constant of water from $0^{\circ}$ to $1000^{\circ}$ C," Journal of Research of the National Bureau of Standards, vol. 56, pp. 1-8, 1956.

[104] P. Attard, D. Antelmi, and I. Larson, "Comparison of the zeta potential with the diffuse layer potential from charge titration," Langmuir, vol. 16, no. 4, pp. 1542-1552, 2000.

[105] G. Torrie and J. Valleau, "Electrical double layers. 4. limitations of the Gouy-Chapman theory," The Journal of Physical Chemistry, vol. 86, no. 16, pp. 3251-3257, 1982.

[106] S. L. Carnie, D. Y. Chan, and J. Stankovich, "Computation of forces between spherical colloidal particles: Nonlinear Poisson-Boltzmann theory," Journal of Colloid and Interface Science, vol. 165, no. 1, pp. 116-128, 1994.

[107] Á. V. Delgado, F. González-Caballero, R. Hunter, L. Koopal, and J. Lyklema, "Measurement and interpretation of electrokinetic phenomena," Journal of Colloid and Interface Science, vol. 309, no. 2, pp. 194-224, 2007.

[108] W. K. Chandler, A. Hodgkin, and H. Meves, "The effect of changing the internal solution on sodium inactivation and related phenomena in giant axons.," The Journal of Physiology, vol. 180, no. 4, p. 821, 1965.

[109] S. H. Behrens and D. G. Grier, "The charge of glass and silica surfaces," The Journal of Chemical Physics, vol. 115, no. 14, pp. 6716-6721, 2001.

[110] B. J. Kirby and E. F. Hasselbrink, "Zeta potential of microfluidic substrates: 1. theory, experimental techniques, and effects on separations," Electrophoresis, vol. 25, no. 2, pp. 187-202, 2004.

[111] R. J. Hunter, Zeta potential in colloid science: Principles and applications, vol. 2. Academic Press, 2013.

[112] C. Rice and R. Whitehead, "Electrokinetic flow in a narrow cylindrical capillary," The Journal of Physical Chemistry, vol. 69, no. 11, pp. 4017-4024, 1965.

[113] M. Smoluchowski, "Contribution to the theory of electro-osmosis and related phenomena," Bull Int Acad Sci Cracovie, vol. 3, pp. 184-199, 1903. 
[114] S. Schulz, T. Gisler, M. Borkovec, and H. Sticher, "Surface charge on functionalized latex spheres in aqueous colloidal suspensions," Journal of Colloid and Interface Science, vol. 164, no. 1, pp. 88-98, 1994.

[115] R. Vogel, "Personal information." Izon, 2013.

[116] L. Höfler and R. E. Gyurcsányi, "Nanosensors lost in space. a random walk study of single molecule detection with single-nanopore sensors," Analytica Chimica Acta, vol. 722, pp. 119-126, 2012.

[117] G. Goyal, K. J. Freedman, and M. J. Kim, "Gold nanoparticle translocation dynamics and electrical detection of single particle diffusion using solid-state nanopores," Analytical Chemistry, vol. 85, no. 17, pp. 8180-8187, 2013.

[118] J. Zhu, T.-R. J. Tzeng, G. Hu, and X. Xuan, "DC dielectrophoretic focusing of particles in a serpentine microchannel," Microfluidics and Nanofluidics, vol. 7, no. 6, pp. 751-756, 2009.

[119] P. K. Thwar, J. J. Linderman, and M. A. Burns, "Electrodeless direct current dielectrophoresis using reconfigurable field-shaping oil barriers," Electrophoresis, vol. 28, no. 24, pp. 4572-4581, 2007.

[120] A. R. Minerick, "DC dielectrophoresis in lab-on-a-chip devices," in Encyclopedia of Microfluidics and Nanofluidics, pp. 331-334, Springer, 2008.

[121] D. L. House and H. Luo, "Effect of direct current dielectrophoresis on the trajectory of a non-conducting colloidal sphere in a bent pore," Electrophoresis, vol. 32, no. 22, pp. 3277-3285, 2011.

[122] A. Ashkin, "Optical trapping and manipulation of neutral particles using lasers," Proceedings of the National Academy of Sciences, vol. 94, no. 10, pp. 4853-4860, 1997.

[123] M. I. Mishchenko, L. D. Travis, and A. A. Lacis, Scattering, absorption, and emission of light by small particles. Cambridge University Press, 2002.

[124] P. G. Etchegoin and E. C. Le Ru, Principles of surface enhanced Raman spectroscopy and related plasmonic effects. Elsevier, 2009.

[125] W. Lan, D. A. Holden, J. Liu, and H. S. White, "Pressure-driven nanoparticle transport across glass membranes containing a conical-shaped nanopore," Journal of Physical Chemistry C, vol. 115, no. 38, pp. 18445-18452, 2011. 
[126] S. R. German, T. S. Hurd, H. S. White, and T. L. Mega, "Sizing individual Au nanoparticles in solution with sub-nanometer resolution," ACS Nano, vol. 9, no. 7, pp. 7186-7194, 2015.

[127] S. R. German, L. Luo, H. S. White, and T. L. Mega, "Controlling nanoparticle dynamics in conical nanopores," The Journal of Physical Chemistry C, vol. 117, no. 1, pp. 703-711, 2012.

[128] W.-J. Lan, D. A. Holden, J. Liu, and H. S. White, "Pressure-driven nanoparticle transport across glass membranes containing a conical-shaped nanopore," The Journal of Physical Chemistry C, vol. 115, no. 38, pp. 18445-18452, 2011.

[129] W. Lan, D. A. Holden, B. Zhang, and H. S. White, "Nanoparticle transport in conical-shaped nanopores," Analytical Chemistry, vol. 83, no. 10, pp. 3840$3847,2011$.

[130] W. Lan, Particle transport and ion current rectification in conical-shaped nanopores. PhD thesis, The University of Utah, US, 2011.

[131] Y. Ai, S. W. Joo, Y. Jiang, X. Xuan, and S. Qian, "Pressure-driven transport of particles through a converging-diverging microchannel," Biomicrofluidics, vol. 3, no. 2, p. 022404, 2009.

[132] Z. Qin, J. Zhe, and G.-X. Wang, "Effects of particle's off-axis position, shape, orientation and entry position on resistance changes of micro Coulter counting devices," Measurement Science and Technology, vol. 22, no. 4, p. 045804, 2011.

[133] J. N. Reddy, An introduction to the finite element method, vol. 2.2. McGrawHill New York, 1993.

[134] E. Weatherall and G. R. Willmott, "Conductive and biphasic pulses in tunable resistive pulse sensing," The Journal of Physical Chemistry B, vol. 119, no. 16, pp. 5328-5335, 2015.

[135] R. Courant et al., "Variational methods for the solution of problems of equilibrium and vibrations," Bull. Amer. Math. Soc, vol. 49, no. 1, pp. 1-23, 1943.

[136] R. W. Clough, The finite element method in plane stress analysis. American Society of Civil Engineers, 1960.

[137] J. H. Argyris and S. Kelsey, Energy theorems and structural analysis, vol. 1. Springer, 1960.

[138] M. J. Turner, "Stiffness and deflection analysis of complex structures," Journal of the Aeronautical Sciences, 2012. 
[139] A. Hrennikoff, "Solution of problems of elasticity by the framework method," Journal of applied mechanics, vol. 8, no. 4, pp. 169-175, 1941.

[140] W.-J. Lan, D. A. Holden, and H. S. White, "Pressure-dependent ion current rectification in conical-shaped glass nanopores," Journal of the American Chemical Society, vol. 133, no. 34, pp. 13300-13303, 2011.

[141] W.-J. Lan, C. Kubeil, J.-W. Xiong, A. Bund, and H. S. White, "Effect of surface charge on the resistive pulse waveshape during particle translocation through glass nanopores," The Journal of Physical Chemistry C, vol. 118, no. 5, pp. 2726-2734, 2014.

[142] R. W. Deblois, C. P. Bean, and R. K. Wesley, "Electrokinetic measurements with submicron particles and pores by the resistive pulse technique," Journal of Colloid and Interface Science, vol. 61, no. 2, pp. 323-335, 1977.

[143] J. Donea, S. Giuliani, and J. Halleux, "An arbitrary Lagrangian-Eulerian finite element method for transient dynamic fluid-structure interactions," Computer Methods in Applied Mechanics and Engineering, vol. 33, no. 1, pp. 689-723, 1982.

[144] G. D'Avino, T. Tuccillo, P. Maffettone, F. Greco, and M. Hulsen, "Numerical simulations of particle migration in a viscoelastic fluid subjected to shear flow," Computers \& Fluids, vol. 39, no. 4, pp. 709-721, 2010.

[145] COMSOL Manual, 2014.

[146] G. Segre, "Radial particle displacements in Poiseuille flow of suspensions," Nature, vol. 189, pp. 209-210, 1961.

[147] G. Segre and A. Silberberg, "Behaviour of macroscopic rigid spheres in Poiseuille flow part 2. Experimental results and interpretation," Journal of Fluid Mechanics, vol. 14, no. 01, pp. 136-157, 1962.

[148] R. Cox and H. Brenner, "The lateral migration of solid particles in Poiseuille flow I Theory," Chemical Engineering Science, vol. 23, no. 2, pp. 147-173, 1968.

[149] R. Repetti and E. Leonard, "Segré-Silberberg annulus formation: A possible explanation," Nature, vol. 203, pp. 1346-1348, 1964.

[150] K. Gotoh, "Migration of a neutrally buoyant particle in Poiseuille flow: A possible explanation," 1970. 
[151] Y.-S. Choi and S.-J. Lee, "Holographic analysis of three-dimensional inertial migration of spherical particles in micro-scale pipe flow," Microfluidics and Nanofluidics, vol. 9, no. 4-5, pp. 819-829, 2010.

[152] E. Loth and A. Dorgan, "An equation of motion for particles of finite Reynolds number and size," Environmental Fluid Mechanics, vol. 9, no. 2, pp. 187-206, 2009.

[153] M. Villone, G. D'Avino, M. Hulsen, F. Greco, and P. L. Maffettone, "Numerical simulations of particle migration in a viscoelastic fluid subjected to Poiseuille flow," Computers \& Fluids, vol. 42, no. 1, pp. 82-91, 2011.

[154] X. Shao, Z. Yu, and B. Sun, "Inertial migration of spherical particles in circular Poiseuille flow at moderately high Reynolds numbers," Physics of Fluids (1994-present), vol. 20, no. 10, p. 103307, 2008.

[155] Y. Kim and J. Yoo, "Transport of solid particles in microfluidic channels," Optics and Lasers in Engineering, vol. 50, no. 1, pp. 87-98, 2012.

[156] L. I. Berge, T. Jossang, and J. Feder, "Off-axis response for particles passing through long apertures in Coulter-type counters," Measurement Science and Technology, vol. 1, no. 6, p. 471, 1990.

[157] W. Smythe, "Off-axis particles in Coulter type counters," Review of Scientific Instruments, vol. 43, no. 5, pp. 817-818, 1972.

[158] O. Saleh and L. Sohn, "Correcting off-axis effects in an on-chip resistive-pulse analyzer," Review of Scientific Instruments, vol. 73, no. 12, pp. 4396-4398, 2002.

[159] E. Weatherall, P. Hauer, R. Vogel, and G. R. Willmott, "Pulse size distributions in tunable resistive pulse sensing," Analytical Chemistry, 2016.

[160] E. W. Young and D. Li, "Dielectrophoretic force on a sphere near a planar boundary," Langmuir, vol. 21, no. 25, pp. 12037-12046, 2005.

[161] S. Balme, F. Picaud, M. Manghi, J. Palmeri, M. Bechelany, S. Cabello-Aguilar, A. Abou-Chaaya, P. Miele, E. Balanzat, and J. M. Janot, "Ionic transport through sub-10 nm diameter hydrophobic high-aspect ratio nanopores: Experiment, theory and simulation," Scientific Reports, vol. 5, 2015.

[162] D. P. Hoogerheide, S. Garaj, and J. A. Golovchenko, "Probing surface charge fluctuations with solid-state nanopores," Physical Review Letters, vol. 102, no. 25, p. 256804, 2009. 
[163] I. Vlassiouk and Z. S. Siwy, "Nanofluidic diode," Nano Letters, vol. 7, no. 3, pp. 552-556, 2007.

[164] J. Menestrina, C. Yang, M. Schiel, I. Vlassiouk, and Z. S. Siwy, "Charged particles modulate local ionic concentrations and cause formation of positive peaks in resistive-pulse-based detection," The Journal of Physical Chemistry $C$, vol. 118, no. 5, pp. 2391-2398, 2014.

[165] R. Chein and P. Dutta, "Effect of charged membrane on the particle motion through a nanopore," Colloids and Surfaces A: Physicochemical and Engineering Aspects, vol. 341, no. 1-3, pp. 1-12, 2009.

[166] E. Samson, J. Marchand, and K. Snyder, "Calculation of ionic diffusion coefficients on the basis of migration test results," Materials and Structures, vol. 36, no. 3, pp. 156-165, 2003.

[167] Y. Ai and S. Qian, "Electrokinetic particle translocation through a nanopore," Physical Chemistry Chemical Physics, vol. 13, no. 9, pp. 4060-4071, 2011.

[168] G. R. Willmott and L. H. Bauerfeind, "Detection of polystyrene sphere translocations using resizable elastomeric nanopores," arXiv preprint arXiv:1002.0611, 2010.

[169] G. Willmott and R. Young, "Analysis and finite element modelling of resizable nanopores," in Advanced Materials and Nanotechnology: Proceedings of the International Conference (AMN-4), vol. 1151, pp. 153-156, AIP Publishing, 2009.

[170] G. Willmott, R. Chaturvedi, S. Cummins, and L. Groenewegen, "Actuation of tunable elastomeric pores: Resistance measurements and finite element modelling," Experimental Mechanics, vol. 54, no. 2, pp. 153-163, 2014.

[171] D. Colquhoun and F. Sigworth, "Fitting and statistical analysis of singlechannel records," in Single-Channel Recording, pp. 483-587, Springer, 1995.

[172] J. D. Uram, K. Ke, and M. Mayer, "Noise and bandwidth of current recordings from submicrometer pores and nanopores," ACS Nano, vol. 2, no. 5, pp. 857$872,2008$.

[173] R. M. Smeets, U. F. Keyser, N. H. Dekker, and C. Dekker, "Noise in solidstate nanopores," Proceedings of the National Academy of Sciences, vol. 105, no. 2, pp. 417-421, 2008. 
[174] V. Tabard-Cossa, D. Trivedi, M. Wiggin, N. N. Jetha, and A. Marziali, "Noise analysis and reduction in solid-state nanopores," Nanotechnology, vol. 18, no. 30, p. 305505, 2007.

[175] Z. Siwy and A. Fuliński, "1/f noise in ion transport through nanopores: Origins and mechanism," in Third International Conference on Unsolved Problems of Noise and Fluctuations in Physics, Biology, and High Technology, vol. 665, pp. 273-282, AIP Publishing, 2003.

[176] D. T. Sawyer, A. Sobkowiak, and J. L. Roberts, Electrochemistry for Chemists. Wiley, 1995.

[177] U. F. Keyser, D. Krapf, B. N. Koeleman, R. M. Smeets, N. H. Dekker, and C. Dekker, "Nanopore tomography of a laser focus," Nano letters, vol. 5, no. 11, pp. 2253-2256, 2005.

[178] Y. Li, C. Chen, S. Kerman, P. Neutens, L. Lagae, G. Groeseneken, T. Stakenborg, and P. Van Dorpe, "Harnessing plasmon-induced ionic noise in metallic nanopores," Nano Letters, vol. 13, no. 4, pp. 1724-1729, 2013.

[179] R. Smeets, N. Dekker, and C. Dekker, "Low-frequency noise in solid-state nanopores," Nanotechnology, vol. 20, no. 9, p. 095501, 2009.

[180] R. Smeets, U. Keyser, M. Wu, N. Dekker, and C. Dekker, "Nanobubbles in solid-state nanopores," Physical Review Letters, vol. 97, no. 8, p. 088101, 2006.

[181] S. Weber, "Laserinduced nanobubbles," Master's thesis, University of Konstanz, Germany, 2007.

[182] T. Saramäki, "Finite impulse response filter design," Handbook for Digital Signal Processing, pp. 155-277, 1993.

[183] N. Arjmandi, W. Van Roy, L. Lagae, and G. Borghs, "Improved algorithms for nanopore signal processing," arXiv preprint arXiv:1207.2319, 2012.

[184] A. V. Jagtiani, R. Sawant, J. Carletta, and J. Zhe, "Wavelet transform-based methods for denoising of Coulter counter signals," Measurement Science and Technology, vol. 19, no. 6, p. 065102, 2008.

[185] Q. Gao, Y. Lu, D. Sun, and D. Zhang, "A multiscale products technique for denoising of dna capillary electrophoresis signals," Measurement Science and Technology, vol. 24, no. 6, p. 065004, 2013. 
[186] V. Barclay, R. Bonner, and I. Hamilton, "Application of wavelet transforms to experimental spectra: Smoothing, denoising, and data set compression," Analytical Chemistry, vol. 69, no. 1, pp. 78-90, 1997.

[187] C. Torrence and G. P. Compo, "A practical guide to wavelet analysis," Bulletin of the American Meteorological Society, vol. 79, no. 1, pp. 61-78, 1998.

[188] C. Galloway, E. Le Ru, and P. Etchegoin, "An iterative algorithm for background removal in spectroscopy by wavelet transforms," Applied Spectroscopy, vol. 63, no. 12, pp. 1370-1376, 2009.

[189] http://www.victoria.ac.nz/scps/research/research-groups/ramanlab/numerical tools, 2015.

[190] E. C. Le Ru, E. Blackie, M. Meyer, and P. G. Etchegoin, "Surface enhanced Raman scattering enhancement factors: A comprehensive study," Journal of Physical Chemistry C, vol. 111, no. 37, pp. 13794-13803, 2007.

[191] http://www.horiba.com/us/en/scientific/products/optics-tutorial/entrance optics/, 2015.

[192] W. Cai, B. Ren, X. Li, C. She, F. Liu, X. Cai, and Z.-Q. Tian, "Investigation of surface-enhanced Raman scattering from platinum electrodes using a confocal Raman microscope: Dependence of surface roughening pretreatment," Surface Science, vol. 406, no. 1, pp. 9-22, 1998.

[193] H. Schrötter and H. Klöckner, "Raman scattering cross sections in gases and liquids," in Raman Spectroscopy of Gases and Liquids, pp. 123-166, Springer, 1979.

[194] L. Mullins, "Softening of rubber by deformation," Rubber Chemistry and Technology, vol. 42, no. 1, pp. 339-362, 1969.

[195] B. L. Darby, B. Auguié, M. Meyer, A. E. Pantoja, and E. C. Le Ru, "Modified optical absorption of molecules on metallic nanoparticles at sub-monolayer coverage," Nature Photonics, vol. 10, no. 1, pp. 40-45, 2016.

[196] J. Eldridge, Nanoparticle charge and shape measurement using Tuneable Resistive Pulse Sensing. PhD thesis, Victoria University of Wellington, New Zealand, 2016.

[197] R. Pashley and M. Karaman, Applied colloid and surface chemistry. John Wiley \& Sons, 2005. 
[198] http://www.bangslabs.com/support/technical-support/spectra information, 2015.

[199] N. B. Nelson and B. B. Prézelin, "Calibration of an integrating sphere for determining the absorption coefficient of scattering suspensions," Applied Optics, vol. 32, no. 33, pp. 6710-6717, 1993.

[200] J. R. Lakowicz, Principles of fluorescence spectroscopy. Springer Science \& Business Media, 2013.

[201] S. A. Meyer, E. C. L. Ru, and P. G. Etchegoin, "Quantifying resonant Raman cross sections with SERS," The Journal of Physical Chemistry A, vol. 114, no. 17 , pp. 5515-5519, 2010.

[202] Z. Gu, Y.-L. Ying, C. Cao, P. He, and Y.-T. Long, "Accurate data process for nanopore analysis," Analytical Chemistry, vol. 87, no. 2, pp. 907-913, 2015.

[203] D. Pedone, M. Firnkes, and U. Rant, "Data analysis of translocation events in nanopore experiments," Analytical Chemistry, vol. 81, no. 23, pp. 9689-9694, 2009.

[204] K. Pearson, "Note on regression and inheritance in the case of two parents," Proceedings of the Royal Society of London, pp. 240-242, 1895.

[205] J. Shlens, "A tutorial on principal component analysis," arXiv preprint arXiv:1404.1100, 2014.

[206] T. Dieing, O. Hollricher, and J. Toporski, Confocal Raman microscopy, vol. 158. Springer Science \& Business Media, 2011.

[207] R. H. Webb, "Confocal optical microscopy," Reports on Progress in Physics, vol. 59, no. 3, p. 427, 1996.

[208] C. Eggeling, J. Widengren, R. Rigler, and C. Seidel, "Photobleaching of fluorescent dyes under conditions used for single-molecule detection: Evidence of two-step photolysis," Analytical Chemistry, vol. 70, no. 13, pp. 2651-2659, 1998.

[209] C. M. Galloway, C. Artur, J. Grand, and E. C. Le Ru, "Photobleaching of fluorophores on the surface of nanoantennas," The Journal of Physical Chemistry C, vol. 118, no. 49, pp. 28820-28830, 2014.

[210] S. A. Meyer, E. C. Le Ru, and P. G. Etchegoin, "Combining surface plasmon resonance (SPR) spectroscopy with surface-enhanced Raman scattering (SERS)," Analytical Chemistry, vol. 83, no. 6, pp. 2337-2344, 2011. 
[211] S. A. Meyer, B. Auguié, E. C. Le Ru, and P. G. Etchegoin, "Combined SPR and SERS microscopy in the Kretschmann configuration," The Journal of Physical Chemistry A, vol. 116, no. 3, pp. 1000-1007, 2012.

[212] E. Le Ru, S. Meyer, C. Artur, P. Etchegoin, J. Grand, P. Lang, and F. Maurel, "Experimental demonstration of surface selection rules for SERS on flat metallic surfaces," Chemical Communications, vol. 47, no. 13, pp. 3903-3905, 2011.

[213] A. E. Siegman, Lasers. University Science Books, 1986.

[214] S. A. Self, "Focusing of spherical Gaussian beams," Applied Optics, vol. 22, no. 5, pp. 658-661, 1983.

[215] W. H. Carter, "Focal shift and concept of effective fresnel number for a Gaussian laser beam," Applied Optics, vol. 21, no. 11, pp. 1989-1994, 1982.

[216] H. T. Yura and S. G. Hanson, "Optical beam wave propagation through complex optical systems," Journal of the Optical Society of America A, vol. 4, no. 10, pp. 1931-1948, 1987.

[217] G. D. Gillen, K. Gillen, and S. Guha, Light propagation in linear optical media. CRC Press, 2013.

[218] Y. Suzaki and A. Tachibana, "Measurement of the $\mu \mathrm{m}$ sized radius of Gaussian laser beam using the scanning knife-edge," Applied Optics, vol. 14, no. 12, pp. 2809-2810, 1975.

[219] P. Hauer, J. Grand, A. Djorovic, G. R. Willmott, and E. C. Le Ru, "Spot size engineering in microscope-based laser spectroscopy," The Journal of Physical Chemistry C, 2016.

[220] P. Hauer, E. C. Le Ru, and G. R. Willmott, "Co-ordinated detection of microparticles using tunable resistive pulse sensing and fluorescence spectroscopy," Biomicrofluidics, vol. 9, no. 1, p. 014110, 2015.

[221] E. B. Wilson, "Probable inference, the law of succession, and statistical inference," Journal of the American Statistical Association, vol. 22, no. 158, pp. 209-212, 1927.

[222] J. Lee Rodgers and W. A. Nicewander, "Thirteen ways to look at the correlation coefficient," The American Statistician, vol. 42, no. 1, pp. 59-66, 1988. 
[223] J. Kestin, M. Sokolov, and W. A. Wakeham, "Viscosity of liquid water in the range $-8^{\circ} \mathrm{C}$ to $150^{\circ} \mathrm{C}, "$ Journal of Physical and Chemical Reference Data, vol. 7 , no. 3, pp. 941-948, 1978.

[224] K. Baldwin and D. Batchelder, "Confocal Raman microspectroscopy through a planar interface," Applied Spectroscopy, vol. 55, no. 5, pp. 517-524, 2001.

[225] N. J. Everall, "Confocal Raman microscopy: Why the depth resolution and spatial accuracy can be much worse than you think," Applied Spectroscopy, vol. 54, no. 10, pp. 1515-1520, 2000.

[226] L. I. Smith, "A tutorial on principal components analysis," Cornell University, USA, vol. 51, p. 52, 2002. 